\title{
Supramolecular Helical Assemblies of Dirhodium(II) Paddlewheels with 1,4-Diazabicyclo[2.2.2] octane: A Remarkable Substituent Effect on Helical Sense Preference and Amplification of Helical Handedness Excess of Metallo-Supramolecular Helical Polymers
}

\author{
Shogo Okuda, ${ }^{\dagger}$ Naoki Ousaka, *,+, ${ }^{*}$, Takuya Iwata, ${ }^{+}$Riku Ishida, ${ }^{+}$Akio Urushima, ${ }^{\dagger}$ \\ Nozomu Suzuki, 'Shusaku Nagano, ${ }^{\dagger}$ Tomoyuki Ikai, ${ }^{\dagger}$ and Eiji Yashima*,+,t’
}

\footnotetext{
$\dagger$ Department of Molecular and Macromolecular Chemistry, Graduate School of Engineering, Nagoya University, Chikusa-ku, Nagoya 464-8603, Japan.

\$ Department of Molecular Design and Engineering, Graduate School of Engineering, Nagoya University, Chikusa-ku, Nagoya 464-8603, Japan.

$\S$ Present Address: Molecular Engineering Institute, Kyushu Institute of Technology, Tobata-ku, Kitakyushu, 804-8550, Japan.
}

E-mail: ousaka.naoki@gmail.com; yashima@chembio.nagoya-u.ac.jp 


\section{Table of Contents}

1. Instruments and Materials $\quad$ S3

2. Synthesis of Dirhodium Complexes and Supramolecular Polymers with DABCO S4

3. General Procedures for AFM Measurements of Supramolecular Homopolymers of (S)-Dirhodium(II) Paddlewheels Complexed with DABCO S30

4. Preparation of the Oriented Fibers of $\left[(S)-\mathbf{1 a}^{\left.\mathbf{P h}_{-} \text {TEG } \cdot \mathrm{DABCO}\right]_{\mathrm{n}}} \quad\right.$ S30

5. General Procedures for "Sergeants and Soldiers" and "Majority Rule" Experiments S30

6. Theoretical Analysis of Temperature-Dependent CD Intensity Changes: Supramolecular Polymerization Mechanism

7-1. CD and/or Absorption Titrations of $(S)-\mathbf{1 a}^{\mathrm{Ph}}-\mathrm{TEG}-(S)-\mathbf{2} \mathbf{b}^{\mathrm{Me}}-\mathrm{C} 12$ with QND and DABCO in $\mathrm{CHCl}_{3} / n$-Hexane $(1 / 1, \mathrm{v} / \mathrm{v})$ or $\mathrm{CHCl}_{3}$ at 25,50 , or $55^{\circ} \mathrm{C}$

7-2. Estimation of $K_{1}$ and $K_{2}$ by Absorption Titrations of $(S)-\mathbf{1 a}^{\mathbf{P h}}-\mathrm{TEG}$ and $(S)-\mathbf{1 b}^{\mathbf{M e}}-\mathrm{C} 12$ with QND in $\mathrm{CHCl}_{3}$ at $50{ }^{\circ} \mathrm{C}$

7-3. Estimation of Degree of Polymerization (n) of Supramolecular Helical Coordination Polymers by Variable-Temperature Absorption Spectral Changes of

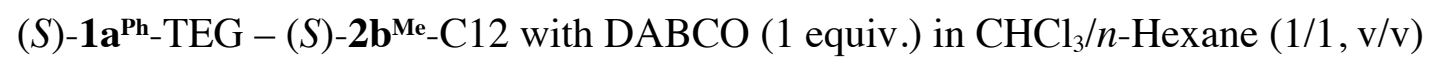

8. Supporting Data

9. Supporting References S75

10. ${ }^{1} \mathrm{H}$ and ${ }^{13} \mathrm{C}$ NMR Spectral Data 


\section{Instruments and Materials}

\section{Instruments}

The melting points were measured on a Yanaco MP-500D micromelting point apparatus (Yanako, Kyoto, Japan) and were uncorrected. The IR spectra were recorded on a JASCO FT/IR-680 spectrophotometer (JASCO, Tokyo, Japan). The NMR spectra were measured using a Bruker Ascend 500 (Bruker Biospin, Billerica, MA, USA), a Bruker Avance III HD 600, or a Varian 500AS (Agilent Technologies, Santa Clara, CA, USA) spectrometer operating at $500 \mathrm{MHz}$ for ${ }^{1} \mathrm{H}$ and 126 or 151 $\mathrm{MHz}$ for ${ }^{13} \mathrm{C}$ using tetramethylsilane (TMS) or a solvent residual peak as the internal standard. The absorption and CD spectra were measured in a 0.01-, 0.1-, or 1-cm quartz cell using a JASCO V-570 spectrophotometer and a JASCO J-1500 spectropolarimeter, respectively. The temperature was controlled with a JASCO PTC-423L or a PTC-510 apparatus. The electrospray ionization (ESI) mass spectra were recorded using a JEOL JMS-T100CS mass spectrometer (JEOL, Akishima, Japan). The size exclusion chromatography (SEC) fractionations were performed using an LC-908W-C60 liquid chromatograph (Japan Analytical Industry, Tokyo, Japan) equipped with two SEC columns (JALGEL-1H-40 (4 (i.d.) $\times 60 \mathrm{~cm})$ and JALGEL-2H-40 (4 (i.d.) $\times 60 \mathrm{~cm}))$ in series and UV-visible (254 nm, JAI UV-3702) and RI (JAI RI-5) detectors; $\mathrm{CHCl}_{3}$ was used as the eluent at a flow rate of $3.5 \mathrm{~mL} / \mathrm{min}$. The atomic force microscopy (AFM) measurements were performed using a Nanoscope V microscope (Bruker AXS, Santa Barbara, CA) or a Cypher ES microscope (Oxford InstrumentsAsylum Research, Santa Barbara, CA) in air at room temperature or $30^{\circ} \mathrm{C}$ with standard cantilevers (TESP-V2, Bruker AXS) or cantilevers for high-speed imaging (FS1500AuD, Oxford InstrumentsAsylum Research) in the tapping mode, respectively. The differential scanning calorimetry (DSC) measurements were performed on a TA Instruments Q2000 (TA Instruments, New Castle, DE) under a nitrogen atmosphere. The X-ray diffraction (XRD) measurements were carried out using a Rigaku FR-E/R-AXIS IV X-ray diffractometer (Rigaku, Tokyo, Japan) with a rotating-anode generator with graphite monochromated $\mathrm{Cu} K \alpha$ radiation $(\lambda=0.15418 \mathrm{~nm})$ focused through a $0.3 \mathrm{~mm}$ pinhole collimator, which was supplied at $45 \mathrm{kV}$ and $45 \mathrm{~mA}$ current, equipped with a flat imaging plate camera having a specimen-to-plate distance of $300 \mathrm{~mm}$. The polarizing optical microscopic (POM) observations were carried out with an E600POL polarizing optical microscope (Nikon, Tokyo, Japan) equipped with a DS-Fi2 CCD camera (Nikon) connected to a DS-L3 control unit (Nikon) and a Mettler Toledo FP82HT hot stage (Mettler Toledo, Greifensee, Switzerland) connected to a Mettler Toledo FP90 central processor unit. The dynamic light scattering (DLS) measurements were performed on a Photal ELS-Z2 spectrometer (Otsuka Electronics Co. Ltd., Osaka, Japan) equipped with a semiconductor laser $(660 \mathrm{~nm})$. The elemental analyses were performed by the laboratory of elemental analyses in the Department of Agriculture, Nagoya University. 


\section{Materials}

All starting materials and dehydrated solvents were purchased from commercial suppliers and were used without further purification unless otherwise noted. $(S)-4,7,10,13$-Tetraoxatetradecan-2amine $\quad\left((S)-\mathbf{4}^{\mathrm{Me}}\right.$-TEG $),{ }^{\mathrm{S} 1} \quad(R)-4,7,10,13$-tetraoxatetradecan-2-amine $\quad\left((R)-4^{\mathrm{Me}}\right.$-TEG $),{ }^{\mathrm{S} 1} \quad 3,6,9,12-$ tetraoxatridecan-1-amine $\left(4^{\mathrm{H}}-\mathrm{TEG}\right),{ }^{\mathrm{S} 2}$ and 2 -(dodecyloxy)ethanamine $\left(\mathbf{4}^{\mathrm{H}}-\mathrm{C} 12\right)^{\mathrm{S} 2}$ were prepared according to the literatures.

\section{Synthesis of Dirhodium Complexes and Supramolecular Polymers with DABCO}

Eight chiral dirhodium(II) paddlewheels with TEG $((R)$ - and $(S)$-1a and -2a) or $n$-dodecyl chains $((R)$ - and $(S)$-1b and -2b) and two achiral ones with TEG (3a) or $n$-dodecyl chains (3b) were synthesized according to Scheme 1 through the ligand exchange reactions of dirhodium(II) tetrakis(trifluoroacetate) with the corresponding enantiopure or achiral $m$-terphenyl carboxylic acids bearing the chiral or achiral amide functional groups at both edges in chlorobenzene at $130{ }^{\circ} \mathrm{C}$ during which the solvents were gradually evaporated to remove the resulting free trifluoroacetic acid.

Synthesis of $(\boldsymbol{S})-\mathbf{4}^{\mathrm{Ph}}$-TEG. Into a three-neck round bottom flask containing $\mathrm{NaH}(60 \%$ in mineral<smiles>NC(COCCOCCOCCOCCOCCO)c1ccccc1</smiles>

$(S)-4^{\text {Ph }}$-TEG oil) (1.46 g, $36.5 \mathrm{mmol})$ was added dry THF $(100 \mathrm{~mL})$ at room temperature under argon. After $20 \mathrm{~min}$, to this was added $(S)-(+)$-phenyl glycinol $(5.00$ $\mathrm{g}, 36.5 \mathrm{mmol})$ at $0{ }^{\circ} \mathrm{C}$ under argon. The reaction mixture was stirred at $0{ }^{\circ} \mathrm{C}$ for $1 \mathrm{~h}$ and further at room temperature for $1 \mathrm{~h}$. After cooling to $0{ }^{\circ} \mathrm{C}$, to this was added diethylene glycol 2-bromoethyl methyl ether $(7.00 \mathrm{~mL}, 40.4 \mathrm{mmol})$ under argon. The reaction mixture was stirred at $0{ }^{\circ} \mathrm{C}$ for $1 \mathrm{~h}$ and then at room temperature for $26 \mathrm{~h}$, and then to this was added $\mathrm{H}_{2} \mathrm{O}$ (ca. $100 \mu \mathrm{L}$ ). After removal of insoluble salts by filtration, the filtrate was evaporated to dryness under reduced pressure. The crude product was purified by flash column chromatography $\left(\mathrm{SiO}_{2}\right.$, EtOAc to $\left.\mathrm{CHCl}_{3} / \mathrm{MeOH}=9 / 1, \mathrm{v} / \mathrm{v}\right)$ to give $(S)-4^{\mathrm{Ph}}-\mathrm{TEG}(5.14 \mathrm{~g}, 50 \%)$ as a yellow oil. IR $(\mathrm{KBr}$, $\left.\mathrm{cm}^{-1}\right): 1110$ ( $\left.v_{\mathrm{C}-\mathrm{O}-\mathrm{C}}\right) .{ }^{1} \mathrm{H}$ NMR $\left(500 \mathrm{MHz}, \mathrm{CD}_{3} \mathrm{CN}, 25^{\circ} \mathrm{C}\right): \delta 7.40-7.39(\mathrm{~m}, 2 \mathrm{H}, \mathrm{ArH}), 7.34-7.31(\mathrm{~m}$, 2H, ArH), 7.25-7.22 (m, 1H, ArH), $4.11(\mathrm{dd}, J=8.0,4.5 \mathrm{~Hz}, 1 \mathrm{H}, \mathrm{NCH}), 3.61-3.35\left(\mathrm{~m}, 14 \mathrm{H}, \mathrm{OCH}_{2}\right)$, $3.29\left(\mathrm{~s}, 3 \mathrm{H}, \mathrm{OCH}_{3}\right) .{ }^{13} \mathrm{C} \mathrm{NMR}\left(126 \mathrm{MHz}, \mathrm{CD}_{3} \mathrm{CN}, 25^{\circ} \mathrm{C}\right): \delta 144.66,129.15,127.95,127.90,78.23$, 72.62, 71.22, 71.16, 71.15, 71.07, 71.00, 58.91, 56.09. HRMS (ESI+): $m / z$ calcd for $\mathrm{C}_{15} \mathrm{H}_{26} \mathrm{NO}_{4}$ $\left(\mathrm{M}+\mathrm{H}^{+}\right), 284.1856$; found 284.1855.

$(R)-4^{\mathrm{Ph}}-\mathrm{TEG}$ was also prepared from $(R)-(-)$-phenyl glycinol in the same way for the synthesis of $(S)-4^{\text {Ph }}$-TEG. 
Spectroscopic data of $(R)-4^{\text {Ph }}$-TEG: Yellow oil. Yield: 42\%. IR (neat, $\left.\mathrm{cm}^{-1}\right): 1107\left(v_{\mathrm{C}-\mathrm{O}-\mathrm{C}) .}{ }^{1} \mathrm{H}\right.$

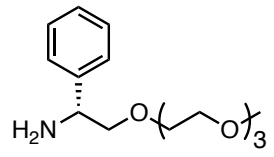

$(R)-4^{\mathrm{Ph}}$-TEG NMR (500 MHz, $\left.\mathrm{CD}_{3} \mathrm{CN}, 25^{\circ} \mathrm{C}\right): \delta 7.40-7.38(\mathrm{~m}, 2 \mathrm{H}, \mathrm{ArH}), 7.33-7.30(\mathrm{~m}$, $2 \mathrm{H}, \mathrm{ArH}), 7.25-7.22(\mathrm{~m}, 1 \mathrm{H}, \mathrm{ArH}), 4.10(\mathrm{dd}, J=8.0,4.5 \mathrm{~Hz}, 1 \mathrm{H}, \mathrm{NCH})$, 3.60-3.35 (m, 14H, $\left.\mathrm{OCH}_{2}\right), 3.28\left(\mathrm{~s}, 3 \mathrm{H}, \mathrm{OCH}_{3}\right) \cdot{ }^{13} \mathrm{C} \mathrm{NMR}(126 \mathrm{MHz}$, $\left.\mathrm{CD}_{3} \mathrm{CN}, 25^{\circ} \mathrm{C}\right): \delta 144.76,129.16,127.95,127.92,78.25,72.63,71.23$, 71.18, 71.16, 71.08, 71.01, 58.89, 56.10. HRMS (ESI+): $m / z$ calcd for $\mathrm{C}_{15} \mathrm{H}_{25} \mathrm{NNaO}_{4}\left(\mathrm{M}+\mathrm{Na}^{+}\right)$, 306.1676 ; found 306.1668 .

Synthesis of $(\boldsymbol{S})-\mathbf{4}^{\mathrm{Ph}}-\mathbf{C 1 2}$. Into a three-neck round bottom flask containing $\mathrm{NaH}(60 \%$ in mineral<smiles>NC(O[AlH2])c1ccccc1</smiles>

$(S)-4^{\mathrm{Ph}}-\mathrm{C} 12$ oil) (572 mg, $14.3 \mathrm{mmol})$ was added dry THF $(40 \mathrm{~mL})$ at room temperature under argon. After $20 \mathrm{~min}$, to this was added $(S)-(+)$-phenyl glysinol (1.89 $\mathrm{g}, 13.8 \mathrm{mmol})$ at $0{ }^{\circ} \mathrm{C}$ under argon. The reaction mixture was stirred at $0{ }^{\circ} \mathrm{C}$ for $1 \mathrm{~h}$ and further at room temperature for $1 \mathrm{~h}$. After cooling to $0^{\circ} \mathrm{C}$, to this was added 1-bromododecane $(3.80 \mathrm{~mL}, 15.8 \mathrm{mmol})$ under argon. The reaction mixture was stirred at $0{ }^{\circ} \mathrm{C}$ for $1 \mathrm{~h}$ and then at room temperature for $10 \mathrm{~h}$, and then to this was added $\mathrm{H}_{2} \mathrm{O}$ (ca. $1 \mathrm{~mL}$ ). After evaporating the solvent under reduced pressure, the residue was suspended in $n$-hexane, and the suspension was washed with $\mathrm{H}_{2} \mathrm{O}$ and brine. The organic layer was dried over anhydrous $\mathrm{MgSO}_{4}$, filtered, and concentrated under reduced pressure. The crude product was purified by flash column chromatography $\left(\mathrm{SiO}_{2}, \mathrm{CHCl}_{3}\right)$ to give $(S)-4^{\mathrm{Ph}}-\mathrm{C} 12(1.06 \mathrm{~g}, 25 \%)$ as a pale yellow oil. IR (neat, $\mathrm{cm}^{-}$ $\left.{ }^{1}\right): 1110\left(v_{\mathrm{C}-\mathrm{O}-\mathrm{C}}\right) .{ }^{1} \mathrm{H}$ NMR $\left(500 \mathrm{MHz}, \mathrm{CDCl}_{3}, 25^{\circ} \mathrm{C}\right): \delta 7.38-7.39(\mathrm{~m}, 2 \mathrm{H}, \mathrm{ArH}), 7.31-7.34(\mathrm{~m}, 2 \mathrm{H}$, ArH), 7.23-7.27 (m, 1H, ArH), 4.19 (dd, $J=9.1,3.5 \mathrm{~Hz}, 1 \mathrm{H}, \mathrm{NCH}), 3.54(\mathrm{dd}, J=9.3,3.7 \mathrm{~Hz}, 1 \mathrm{H}$, $\left.\mathrm{OCH}_{2} \mathrm{CH}\right), 3.41-3.50\left(\mathrm{~m}, 2 \mathrm{H}, \mathrm{OCH}_{2} \mathrm{CH}_{2}\right), 3.36\left(\mathrm{t}, J=9.1 \mathrm{~Hz}, 1 \mathrm{H}, \mathrm{OCH}_{2} \mathrm{CH}\right), 1.55-1.61(\mathrm{~m}, 2 \mathrm{H}$, $\left.\mathrm{OCH}_{2} \mathrm{CH}_{2}\right), 1.26-1.35\left(\mathrm{~m}, 18 \mathrm{H}, \mathrm{CH}_{2}\right), 0.88\left(\mathrm{t}, J=7.1 \mathrm{~Hz}, 3 \mathrm{H}, \mathrm{CH}_{3}\right) .{ }^{13} \mathrm{C} \mathrm{NMR}\left(126 \mathrm{MHz}, \mathrm{CDCl}_{3}\right.$, $\left.25^{\circ} \mathrm{C}\right): \delta 142.69,128.38,127.32,126.85,77.15,71.42,55.58,31.93,29.71,29.69,29.65,29.63$, 29.51, 29.37, 26.19, 22.70, 14.13. HRMS (ESI+): $m / z$ calcd for $\mathrm{C}_{20} \mathrm{H}_{36} \mathrm{NO}\left(\mathrm{M}+\mathrm{H}^{+}\right), 306.2791$; found 306.2795 .

$(R)-4^{\mathrm{Ph}}-\mathrm{C} 12$ and $(S)$ - and $(R)-\mathbf{4}^{\mathrm{Me}}-\mathrm{C} 12$ were also prepared from $(R)-(-)$-phenyl glycinol, $(S)-(+)-$ alaninol, and $(R)-(-)$-alaninol, respectively, in the same way for the synthesis of $(S)-4^{\mathbf{P h}_{-}} \mathrm{C} 12$. 
Spectroscopic data of $(R)-4^{\mathrm{Ph}}-\mathrm{C} 12$ : Pale yellow oil. Yield: $25 \%$. IR (neat, $\left.\mathrm{cm}^{-1}\right)$ : $1111\left(v_{\mathrm{C}-\mathrm{O}-\mathrm{C}) .}{ }^{1} \mathrm{H}\right.$

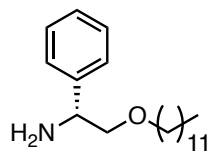

$(R)-4^{\mathrm{Ph}}-\mathrm{C} 12$

NMR (500 MHz, $\left.\mathrm{CDCl}_{3}, 25^{\circ} \mathrm{C}\right): \delta 7.36-7.38(\mathrm{~m}, 2 \mathrm{H}, \mathrm{ArH}), 7.29-7.32(\mathrm{~m}$, 2H, ArH), 7.21-7.25 (m, 1H, ArH), 4.18 (dd, $J=9.1,3.5 \mathrm{~Hz}, 1 \mathrm{H}, \mathrm{NCH})$, $3.52\left(\mathrm{dd}, J=9.4,3.8 \mathrm{~Hz}, 1 \mathrm{H}, \mathrm{OCH}_{2} \mathrm{CH}\right), 3.40-3.49\left(\mathrm{~m}, 2 \mathrm{H}, \mathrm{OCH}_{2} \mathrm{CH}_{2}\right)$, $3.34\left(\mathrm{t}, J=9.1 \mathrm{~Hz}, 1 \mathrm{H}, \mathrm{OCH}_{2} \mathrm{CH}\right), 1.55-1.60\left(\mathrm{~m}, 2 \mathrm{H}, \mathrm{OCH}_{2} \mathrm{CH}_{2}\right), 1.26-1.35$ $\left(\mathrm{m}, 18 \mathrm{H}, \mathrm{CH}_{2}\right), 0.88\left(\mathrm{t}, J=7.1 \mathrm{~Hz}, 3 \mathrm{H}, \mathrm{CH}_{3}\right) .{ }^{13} \mathrm{C} \mathrm{NMR}\left(126 \mathrm{MHz}, \mathrm{CDCl}_{3}, 25^{\circ} \mathrm{C}\right): \delta 142.80,128.50$, 127.44, 126.96, 77.26, 71.54, 55.70, 32.05, 29.83, 29.80, 29.77, 29.74, 29.63, 29.48, 26.30, 22.82, 14.24. HRMS (ESI+): $m / z$ calcd for $\mathrm{C}_{20} \mathrm{H}_{36} \mathrm{NO}\left(\mathrm{M}+\mathrm{H}^{+}\right)$, 306.2791; found 306.2797.

Spectroscopic data of $(S)-4^{\text {Me }}{ }_{-C} 12$ : Pale yellow oil. Yield: $27 \%$. IR (neat, $\left.\mathrm{cm}^{-1}\right): 1115\left(v_{\text {C-O-C }}\right) .{ }^{1} \mathrm{H}$<smiles>CC(N)CO[Al]</smiles>

$(S)-4^{\mathrm{Me}}-\mathrm{C} 12$ $\operatorname{NMR}\left(500 \mathrm{MHz}, \mathrm{CDCl}_{3}, 25^{\circ} \mathrm{C}\right): \delta 3.47-3.38\left(\mathrm{~m}, 2 \mathrm{H}, \mathrm{OCH}_{2}\right), 3.35-3.31(\mathrm{~m}$, $1 \mathrm{H}, \mathrm{NCH}), 3.14-3.07$ (m, 2H, $\left.\mathrm{OCH}_{2}\right), 1.60-1.54\left(\mathrm{~m}, 2 \mathrm{H}, \mathrm{CH}_{2}\right), 1.35-1.23(\mathrm{~m}$, $\left.18 \mathrm{H}, \mathrm{CH}_{2}\right), 1.03\left(\mathrm{~d}, J=6.1 \mathrm{~Hz}, 3 \mathrm{H}, \mathrm{CH}_{3}\right), 0.88\left(\mathrm{t}, J=7.0 \mathrm{~Hz}, 3 \mathrm{H}, \mathrm{CH}_{3}\right) .{ }^{13} \mathrm{C}$ NMR (126 MHz, $\left.\mathrm{CDCl}_{3}, 25^{\circ} \mathrm{C}\right): \delta 77.81,71.41,46.56,31.99,29.78,29.74,29.70,29.69,29.57$, 29.42, 26.25, 22.75, 19.85, 14.17. HRMS (ESI+): $m / z$ calcd for $\mathrm{C}_{15} \mathrm{H}_{34} \mathrm{NO}\left(\mathrm{M}+\mathrm{H}^{+}\right), 244.2635$; found 244.2634 .

Spectroscopic data of $(R)-4^{\text {Me }}-\mathrm{C} 12$ : Pale yellow oil. Yield: $50 \%$. IR (neat, $\left.\mathrm{cm}^{-1}\right): 1115\left(v_{\mathrm{C}-\mathrm{O}-\mathrm{C}}\right) .{ }^{1} \mathrm{H}$<smiles>CC(N)COCCO</smiles>
$\operatorname{NMR}\left(500 \mathrm{MHz}, \mathrm{CDCl}_{3}, 25^{\circ} \mathrm{C}\right): \delta 3.47-3.38\left(\mathrm{~m}, 2 \mathrm{H}, \mathrm{OCH}_{2}\right), 3.35-3.31(\mathrm{~m}$, $1 \mathrm{H}, \mathrm{NCH}), 3.14-3.08\left(\mathrm{~m}, 2 \mathrm{H}, \mathrm{OCH}_{2}\right), 1.60-1.54\left(\mathrm{~m}, 2 \mathrm{H}, \mathrm{CH}_{2}\right), 1.35-1.23(\mathrm{~m}$, $\left.18 \mathrm{H}, \mathrm{CH}_{2}\right), 1.03\left(\mathrm{~d}, J=5.9 \mathrm{~Hz}, 3 \mathrm{H}, \mathrm{CH}_{3}\right), 0.88\left(\mathrm{t}, J=7.0 \mathrm{~Hz}, 3 \mathrm{H}, \mathrm{CH}_{3}\right) .{ }^{13} \mathrm{C}$ NMR (126 MHz, $\left.\mathrm{CDCl}_{3}, 25^{\circ} \mathrm{C}\right): \delta 77.84,71.37,46.52,31.93,29.73,29.68,29.65,29.64,29.52$, 29.37, 26.20, 22.70, 19.88, 14.12. HRMS (ESI+): $m / z$ calcd for $\mathrm{C}_{15} \mathrm{H}_{34} \mathrm{NO}\left(\mathrm{M}+\mathrm{H}^{+}\right), 244.2635$; found 244.2636 .

Synthesis of $(\boldsymbol{S}) \mathbf{5}^{\mathrm{Ph}}$-TEG. To a solution of 4-bromobenzoic acid (3.00 g, $\left.14.8 \mathrm{mmol}\right)$ and 1-<smiles>O=C(COCC(NC(=O)c1ccc(Br)cc1)c1ccccc1)OCCO</smiles>

$(S)-5^{\text {Ph }}-$ TEG hydroxybenzotriazole monohydrate $\left(\mathrm{HOBt} \cdot \mathrm{H}_{2} \mathrm{O}\right)(2.96 \mathrm{~g}, 19.2 \mathrm{mmol})$ in dry DMF $(70 \mathrm{~mL})$ was added 1-ethyl-3-(3-dimethylaminopropyl)carbodiimide hydrochloride $(\mathrm{EDC} \cdot \mathrm{HCl})(2.84 \mathrm{~g}, 19.2 \mathrm{mmol})$ at $0{ }^{\circ} \mathrm{C}$ under nitrogen, and the reaction mixture was stirred at $0{ }^{\circ} \mathrm{C}$ for $0.5 \mathrm{~h}$. After $(S)-4^{\text {Ph }}$-TEG (4.21 g, $\left.14.8 \mathrm{mmol}\right)$ was added dropwise to this at $0{ }^{\circ} \mathrm{C}$, the mixture was warmed to room temperature, and stirred for $14 \mathrm{~h}$. After evaporating the solvent under reduced pressure, the residue was dissolved in EtOAc, and the solution was washed with $1 \mathrm{~N}$ aqueous $\mathrm{HCl}, 5 \%$ aqueous $\mathrm{NaHCO}_{3}$, and brine. The organic layer was dried over anhydrous $\mathrm{Na}_{2} \mathrm{SO}_{4}$, 
filtered, and concentrated under reduced pressure. The crude product was purified by flash column chromatography $\left(\mathrm{SiO}_{2}, \mathrm{EtOAc} / n\right.$-hexane $\left.=4 / 1-1 / 0, \mathrm{v} / \mathrm{v}\right)$ to give $(S)-5^{\mathrm{Ph}}-\mathrm{TEG}(5.59 \mathrm{~g}, 81 \%)$ as a colorless oil. IR (neat, $\left.\mathrm{cm}^{-1}\right): 1643\left(v_{\mathrm{C}=\mathrm{O}}\right), 1538\left(v_{\mathrm{N}-\mathrm{H}}\right) .{ }^{1} \mathrm{H} \mathrm{NMR}\left(500 \mathrm{MHz}, \mathrm{CD}_{3} \mathrm{CN}, 25{ }^{\circ} \mathrm{C}\right): \delta 7.75$ (d, $J=8.7 \mathrm{~Hz}, 2 \mathrm{H}, \mathrm{ArH}), 7.63$ (d, $J=8.7 \mathrm{~Hz}, 2 \mathrm{H}, \mathrm{ArH}), 7.57$ (d, $J=7.3 \mathrm{~Hz}, 1 \mathrm{H}, \mathrm{NH}), 7.41-7.40$ (m, 2H, ArH), 7.36-7.33 (m, 2H, ArH), 7.29-7.26 (m, 1H, ArH), 5.26-5.22 (m, 1H, NCH), 3.80-3.73 (m, $\left.2 \mathrm{H}, \mathrm{OCH}_{2}\right), 3.63-3.60\left(\mathrm{~m}, 2 \mathrm{H}, \mathrm{OCH}_{2}\right), 3.57-3.55\left(\mathrm{~m}, 2 \mathrm{H}, \mathrm{OCH}_{2}\right), 3.54-3.52\left(\mathrm{~m}, 2 \mathrm{H}, \mathrm{OCH}_{2}\right), 3.50-$ $3.48\left(\mathrm{~m}, 4 \mathrm{H}, \mathrm{OCH}_{2}\right), 3.42-3.40\left(\mathrm{~m}, 2 \mathrm{H}, \mathrm{OCH}_{2}\right), 3.25\left(\mathrm{~s}, 3 \mathrm{H}, \mathrm{OCH}_{3}\right) .{ }^{13} \mathrm{C} \mathrm{NMR}\left(126 \mathrm{MHz}, \mathrm{CD}_{3} \mathrm{CN}\right.$, $\left.25^{\circ} \mathrm{C}\right): \delta 166.84,141.52,134.81,132.51,130.18,129.39,128.29,127.90,126.31,74.26,72.57,71.16$, 71.15, 71.12, 71.05, 70.98, 58.87, 54.85. HRMS (ESI+): $m / z$ calcd for $\mathrm{C}_{22} \mathrm{H}_{28} \mathrm{BrNNaO}_{5}\left(\mathrm{M}+\mathrm{Na}^{+}\right)$, 488.1043; found 488.1072.

$(R)-5^{\text {Ph }}$-TEG, $(S)$ - and $(R)-5^{\mathbf{P h}}-\mathrm{C} 12,(S)$ - and $(R)-5^{\text {Me }}-\mathrm{TEG},(S)$ - and $(R)-5^{\mathbf{M e}}-\mathrm{C} 12, \mathbf{5}^{\mathbf{H}}-\mathrm{TEG}$, and $\mathbf{5}^{\mathrm{H}}-\mathrm{C} 12$ were also prepared from $(R)-\mathbf{4}^{\mathrm{Ph}}-\mathrm{TEG},(S)-\mathbf{4}^{\mathrm{Ph}}-\mathrm{C} 12,(R)-\mathbf{4}^{\mathrm{Ph}}-\mathrm{C} 12,(S)-\mathbf{4}^{\mathrm{Me}}-\mathrm{TEG},(R)-\mathbf{4}^{\mathrm{Me}}-\mathrm{TEG}$, $(S)-\mathbf{4}^{\mathrm{Me}}-\mathrm{C} 12,(R)-\mathbf{4}^{\mathrm{Me}}-\mathrm{C} 12, \mathbf{4}^{\mathrm{H}}-\mathrm{TEG}$, and $\mathbf{4}^{\mathrm{H}}-\mathrm{C} 12$, respectively, in the same way for the synthesis of $(S)-5^{\text {Ph }}-$ TEG.

Spectroscopic data of $(R)-5^{\text {Ph }}$-TEG: Colorless oil. Yield: 99\%. IR (neat, $\left.\mathrm{cm}^{-1}\right): 1643\left(\nu_{\mathrm{C}=0}\right), 1538$

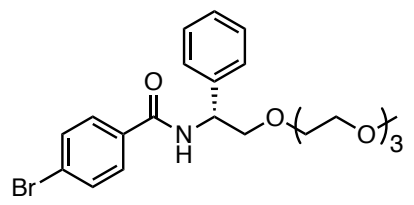

$(R)-5^{\text {Ph }}$-TEG

$\left(v_{\mathrm{N}-\mathrm{H}}\right) .{ }^{1} \mathrm{H} \mathrm{NMR}\left(500 \mathrm{MHz}, \mathrm{CD}_{3} \mathrm{CN}, 25^{\circ} \mathrm{C}\right): \delta 7.75(\mathrm{~d}, J=8.7 \mathrm{~Hz}, 2 \mathrm{H}$, $\operatorname{ArH}), 7.63(\mathrm{~d}, J=8.7 \mathrm{~Hz}, 2 \mathrm{H}, \mathrm{ArH}), 7.55$ (d, $J=7.3 \mathrm{~Hz}, 1 \mathrm{H}, \mathrm{NH}), 7.41-$ 7.40 (m, 2H, ArH), 7.36-7.33 (m, 2H, ArH), 7.29-7.26 (m, 1H, ArH), 5.26-5.22 (m, 1H, NCH), 3.80-3.73 (m, 2H, $\left.\mathrm{OCH}_{2}\right), 3.63-3.60(\mathrm{~m}, 2 \mathrm{H}$, $\left.\mathrm{OCH}_{2}\right), 3.57-3.55\left(\mathrm{~m}, 2 \mathrm{H}, \mathrm{OCH}_{2}\right), 3.54-3.52\left(\mathrm{~m}, 2 \mathrm{H}, \mathrm{OCH}_{2}\right), 3.49-3.47$ (m, 4H, $\left.\mathrm{OCH}_{2}\right), 3.41-3.39$ $\left(\mathrm{m}, 2 \mathrm{H}, \mathrm{OCH}_{2}\right), 3.25\left(\mathrm{~s}, 3 \mathrm{H}, \mathrm{OCH}_{3}\right) .{ }^{13} \mathrm{C} \mathrm{NMR}\left(126 \mathrm{MHz}, \mathrm{CD}_{3} \mathrm{CN}, 25{ }^{\circ} \mathrm{C}\right): \delta 166.84,141.53,134.82$, $132.52,130.19,129.39,128.30,127.90,126.31,74.27,72.57,71.16,71.15,71.12,71.06,70.99$, 58.87, 54.86. HRMS (ESI+): $m / z$ calcd for $\mathrm{C}_{22} \mathrm{H}_{28} \mathrm{BrNNaO}_{5}\left(\mathrm{M}+\mathrm{Na}^{+}\right), 488.1043$; found 488.1039 .

Spectroscopic data of $(S)-5^{\mathrm{Ph}}-\mathrm{C} 12$ : White solid. Yield: $80 \%$. Mp: $92.2-93.0{ }^{\circ} \mathrm{C}, \mathrm{IR}\left(\mathrm{KBr}, \mathrm{cm}^{-1}\right)$ :

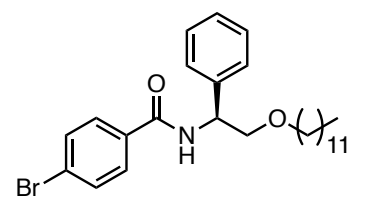

$(S)-5^{\mathrm{Ph}}-\mathrm{C} 12$

$1638\left(v_{\mathrm{C}=\mathrm{O}}\right), 1541\left(v_{\mathrm{N}-\mathrm{H}}\right) .{ }^{1} \mathrm{H} \mathrm{NMR}\left(500 \mathrm{MHz}, \mathrm{CDCl}_{3}, 25{ }^{\circ} \mathrm{C}\right): \delta 7.66-$ 7.69 (m, 2H, ArH), 7.55-7.58 (m, 2H, ArH), 7.39 (d, $J=7.4 \mathrm{~Hz}, 2 \mathrm{H}$, $\operatorname{ArH}), 7.32-7.35$ (m, 2H, ArH), 7.25-7.28 (m, 1H, ArH), 6.92 (d, $J=7.1$ $\mathrm{Hz}, 1 \mathrm{H}, \mathrm{NH}), 5.28-5.32(\mathrm{~m}, 1 \mathrm{H}, \mathrm{NCH}), 3.74-3.81\left(\mathrm{~m}, 2 \mathrm{H}, \mathrm{OCH}_{2} \mathrm{CH}\right)$, 3.41-3.51 (m, 2H, $\left.\mathrm{OCH}_{2} \mathrm{CH}_{2}\right), 1.53-1.58\left(\mathrm{~m}, 2 \mathrm{H}, \mathrm{OCH}_{2} \mathrm{CH}_{2}\right), 1.24-1.31$ $\left(\mathrm{m}, 18 \mathrm{H}, \mathrm{CH}_{2}\right), 0.88\left(\mathrm{t}, J=6.9 \mathrm{~Hz}, 3 \mathrm{H}, \mathrm{CH}_{3}\right) .{ }^{13} \mathrm{C} \mathrm{NMR}\left(126 \mathrm{MHz}, \mathrm{CDCl}_{3}, 25^{\circ} \mathrm{C}\right): \delta 166.03,140.04$, $133.38,131.77,128.77,128.55,127.55,126.95,126.18,72.93,71.42,53.34,31.99,29.75,29.71$, 
29.67, 29.59, 29.49, 29.42, 26.24, 22.76, 14.20. HRMS (ESI+): $m / z$ calcd for $\mathrm{C}_{27} \mathrm{H}_{38} \mathrm{BrNNaO}_{2}$ $\left(\mathrm{M}+\mathrm{Na}^{+}\right), 510.1978$; found 510.2007.

Spectroscopic data of $(R)-5^{\mathrm{Ph}}-\mathrm{C} 12$ : White solid. Yield: $83 \%$. $(R)-5^{\mathrm{Ph}}-\mathrm{C} 12$ was synthesized in the

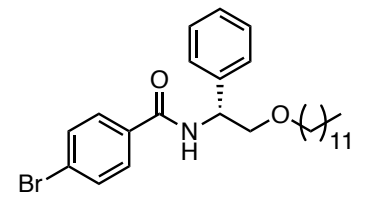

$(R)-5^{\mathrm{Ph}}-\mathrm{C} 12$ same manner to that for the corresponding $(S)$-enantiomer $(1.04 \mathrm{~g}, 83 \%)$. IR $\left(\mathrm{KBr}, \mathrm{cm}^{-1}\right): 1638\left(v_{\mathrm{C}=\mathrm{O}}\right), 1542\left(v_{\mathrm{C}-\mathrm{O}-\mathrm{C}}\right) .{ }^{1} \mathrm{H} \mathrm{NMR}\left(500 \mathrm{MHz}, \mathrm{CDCl}_{3}\right.$, $\left.25^{\circ} \mathrm{C}\right): \delta 7.61-7.64(\mathrm{~m}, 2 \mathrm{H}, \mathrm{ArH}), 7.46-7.49(\mathrm{~m}, 2 \mathrm{H}, \mathrm{ArH}), 7.37(\mathrm{~d}, J=$ 7.4 Hz, 2H, ArH), 7.28-7.31 (m, 2H, ArH), 7.22-7.25 (m, 1H, ArH), $7.18(\mathrm{~d}, J=7.4 \mathrm{~Hz}, 1 \mathrm{H}, \mathrm{NH}), 5.27-5.31(\mathrm{~m}, 1 \mathrm{H}, \mathrm{NCH}), 3.73$ (d, $J=4.9$ $\left.\mathrm{Hz}, 2 \mathrm{H}, \mathrm{OCH}_{2} \mathrm{CH}\right), 3.38-3.49\left(\mathrm{~m}, 2 \mathrm{H}, \mathrm{OCH}_{2} \mathrm{CH}_{2}\right), 1.51-1.56\left(\mathrm{~m}, 2 \mathrm{H}, \mathrm{OCH}_{2} \mathrm{CH}_{2}\right), 1.24-1.31(\mathrm{~m}, 18 \mathrm{H}$, $\left.\mathrm{CH}_{2}\right), 0.88\left(\mathrm{t}, J=6.9 \mathrm{~Hz}, 3 \mathrm{H}, \mathrm{CH}_{3}\right) .{ }^{13} \mathrm{C} \mathrm{NMR}\left(126 \mathrm{MHz}, \mathrm{CDCl}_{3}, 25{ }^{\circ} \mathrm{C}\right): \delta 166.03,140.04,133.36$, 131.68, 128.76, 128.51, 127.50, 126.94, 126.11, 72.90, 71.36, 53.34, 31.96, 29.73, 29.68, 29.64, 29.57, 29.47, 29.40, 26.22, 22.73, 14.18. HRMS (ESI+): $m / z$ calcd for $\mathrm{C}_{27} \mathrm{H}_{38} \mathrm{BrNNaO}_{2}\left(\mathrm{M}+\mathrm{Na}^{+}\right)$, 510.1978; found 510.1999.

Spectroscopic data of $(S)-5^{\text {Me }}$-TEG: Colorless oil. Yield: 88\%. IR (neat, $\left.\mathrm{cm}^{-1}\right): 1639\left(v_{\mathrm{C}=0}\right), 1541$

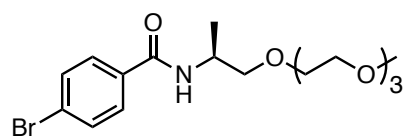

$(S)-5^{\text {Me }}$-TEG $\left({ }_{\mathrm{N}-\mathrm{H}}\right) .{ }^{1} \mathrm{H} \mathrm{NMR}\left(500 \mathrm{MHz}, \mathrm{CDCl}_{3}, 25^{\circ} \mathrm{C}\right): \delta 7.69(\mathrm{~d}, J=8.5 \mathrm{~Hz}, 2 \mathrm{H}$, ArH), $7.54(\mathrm{~d}, J=8.5 \mathrm{~Hz}, 2 \mathrm{H}, \mathrm{ArH}), 6.83(\mathrm{~d}, J=7.6 \mathrm{~Hz}, 1 \mathrm{H}, \mathrm{NH}), 4.36-$ 4.29 (m, 1H, NCH), 3.69-3.56 (m, 12H, $\left.\mathrm{OCH}_{2}\right), 3.51$ (t, $J=4.8 \mathrm{~Hz}, 2 \mathrm{H}$, $\left.\mathrm{OCH}_{2}\right), 3.34\left(\mathrm{~s}, 3 \mathrm{H}, \mathrm{OCH}_{3}\right), 1.28\left(\mathrm{~d}, J=6.8 \mathrm{~Hz}, 3 \mathrm{H}, \mathrm{CH}_{3}\right) \cdot{ }^{13} \mathrm{C} \mathrm{NMR}\left(126 \mathrm{MHz}, \mathrm{CDCl}_{3}, 25{ }^{\circ} \mathrm{C}\right): \delta$ 165.92, 133.67, 131.54, 128.78, 125.80, 77.37, 73.86, 71.86, 70.63, 70.54, 70.51, 70.48, 58.93, 45.70, 17.57. HRMS (ESI+): $m / z$ calcd for $\mathrm{C}_{17} \mathrm{H}_{26} \mathrm{BrNNaO}_{5}\left(\mathrm{M}+\mathrm{Na}^{+}\right), 426.0887$; found 426.0897 .

Spectroscopic data of $(R)-5^{\text {Me }}$-TEG: Colorless oil. Yield: 95\%. IR (neat, $\left.\mathrm{cm}^{-1}\right): 1638\left(v_{\mathrm{C}=0}\right), 1541$

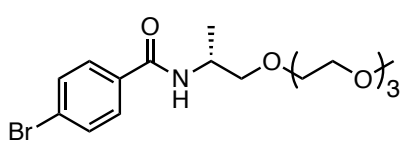

$(R)-5^{\mathrm{Me}}-\mathrm{TEG}$
$\left(w_{\mathrm{N}-\mathrm{H}}\right) .{ }^{1} \mathrm{H} \mathrm{NMR}\left(500 \mathrm{MHz}, \mathrm{CDCl}_{3}, 25^{\circ} \mathrm{C}\right): \delta 7.69(\mathrm{~d}, J=6.6 \mathrm{~Hz}, 2 \mathrm{H}$, ArH), $7.56(\mathrm{~d}, J=8.5 \mathrm{~Hz}, 2 \mathrm{H}, \mathrm{ArH}), 6.68(\mathrm{~d}, J=7.8 \mathrm{~Hz}, 1 \mathrm{H}, \mathrm{NH}), 4.37-$ $4.30(\mathrm{~m}, 1 \mathrm{H}, \mathrm{NCH}), 3.70-3.55\left(\mathrm{~m}, 12 \mathrm{H}, \mathrm{OCH}_{2}\right), 3.51$ (t, $J=4.6 \mathrm{~Hz}, 2 \mathrm{H}$, $\left.\mathrm{OCH}_{2}\right), 3.35\left(\mathrm{~s}, 3 \mathrm{H}, \mathrm{OCH}_{3}\right), 1.29\left(\mathrm{~d}, J=6.8 \mathrm{~Hz}, 3 \mathrm{H}, \mathrm{CH}_{3}\right) .{ }^{13} \mathrm{C} \mathrm{NMR}$ $\left(126 \mathrm{MHz}, \mathrm{CDCl}_{3}, 25^{\circ} \mathrm{C}\right): \delta 165.92,133.67,131.54,128.78,125.80,77.37,73.86,71.86,70.63$, 70.54, 70.51, 70.48, 58.93, 45.70, 17.57. HRMS (ESI+): $m / z$ calcd for $\mathrm{C}_{17} \mathrm{H}_{26} \mathrm{BrNNaO}_{5}\left(\mathrm{M}^{+} \mathrm{Na}^{+}\right)$, 426.0887; found 426.0903 . 
Spectroscopic data of $(S)-5^{\mathrm{Me}}-\mathrm{C} 12$ : White solid. Yield: $94 \%$. Mp: 89.9-90.4 ${ }^{\circ} \mathrm{C}$. IR $\left(\mathrm{KBr}, \mathrm{cm}^{-1}\right)$ :<smiles>CC(CO[Al])NC(=O)c1ccc(Br)cc1</smiles>

$(S)-5^{\mathrm{Me}}-\mathrm{C} 12$

$1635\left(v_{\mathrm{C}=\mathrm{O}}\right), 1544\left(v_{\mathrm{N}-\mathrm{H}}\right) .{ }^{1} \mathrm{H} \mathrm{NMR}\left(500 \mathrm{MHz}, \mathrm{CDCl}_{3}, 25^{\circ} \mathrm{C}\right): \delta 7.63$ $(\mathrm{d}, J=8.8 \mathrm{~Hz}, 2 \mathrm{H}, \mathrm{ArH}), 7.56(\mathrm{~d}, J=6.6 \mathrm{~Hz}, 2 \mathrm{H}, \mathrm{ArH}), 6.38$ (d, $J=7.6$ $\mathrm{Hz}, 1 \mathrm{H}, \mathrm{NH}), 4.36-4.29(\mathrm{~m}, 1 \mathrm{H}, \mathrm{NCH}), 3.53-3.42\left(\mathrm{~m}, 4 \mathrm{H}, \mathrm{OCH}_{2}\right), 1.60-$ $1.54\left(\mathrm{~m}, 2 \mathrm{H}, \mathrm{CH}_{2}\right), 1.34-1.25\left(\mathrm{~m}, 21 \mathrm{H}, \mathrm{CH}_{2}, \mathrm{CH}_{3}\right), 0.88(\mathrm{t}, J=7.0 \mathrm{~Hz}$, $\left.3 \mathrm{H}, \mathrm{CH}_{3}\right) .{ }^{13} \mathrm{C} \mathrm{NMR}\left(126 \mathrm{MHz}, \mathrm{CDCl}_{3}, 25{ }^{\circ} \mathrm{C}\right): \delta 165.79,133.71,131.73,128.54,125.96,73.28$, $71.48,45.56,31.92,29.69,29.65,29.62,29.58,29.47,29.36,26.21,22.69,17.86,14.12$. HRMS (ESI+): $m / z$ calcd for $\mathrm{C}_{22} \mathrm{H}_{36} \mathrm{BrNNaO}_{2}\left(\mathrm{M}+\mathrm{Na}^{+}\right), 448.1822$; found 448.1837 .

Spectroscopic data of $(R)-5^{\mathrm{Me}}-\mathrm{C} 12$ : White solid. Yield: $99 \%$. Mp: 89.3-90.0 ${ }^{\circ} \mathrm{C}$. IR $\left(\mathrm{KBr}, \mathrm{cm}^{-1}\right)$ :

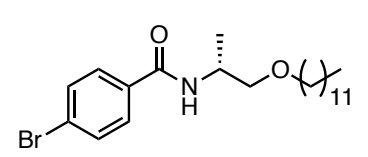

$(R)-5^{\mathrm{Me}}-\mathrm{C} 12$

$1635\left(v_{\mathrm{C}=\mathrm{O}}\right), 1544\left(v_{\mathrm{N}-\mathrm{H}}\right) .{ }^{1} \mathrm{H}$ NMR $\left(500 \mathrm{MHz}, \mathrm{CDCl}_{3}, 25^{\circ} \mathrm{C}\right): \delta 7.63(\mathrm{~d}$, $J=8.5 \mathrm{~Hz}, 2 \mathrm{H}, \operatorname{ArH}), 7.56(\mathrm{~d}, J=8.8 \mathrm{~Hz}, 2 \mathrm{H}, \operatorname{ArH}), 6.37$ (d, $J=7.6 \mathrm{~Hz}$, $1 \mathrm{H}, \mathrm{NH}), 4.37-4.29(\mathrm{~m}, 1 \mathrm{H}, \mathrm{NCH}), 3.54-3.43\left(\mathrm{~m}, 4 \mathrm{H}, \mathrm{OCH}_{2}\right), 1.60-1.54$ (m, $\left.2 \mathrm{H}, \mathrm{CH}_{2}\right), 1.34-1.25\left(\mathrm{~m}, 21 \mathrm{H}, \mathrm{CH}_{2}, \mathrm{CH}_{3}\right), 0.88(\mathrm{t}, J=7.0 \mathrm{~Hz}, 3 \mathrm{H}$, $\left.\mathrm{CH}_{3}\right) \cdot{ }^{13} \mathrm{C} \mathrm{NMR}\left(126 \mathrm{MHz}, \mathrm{CDCl}_{3}, 25^{\circ} \mathrm{C}\right): \delta 165.78,133.70,131.73,128.54,125.96,73.28,71.48$, 45.56, 31.92, 29.69, 29.65, 29.62, 29.58, 29.47, 29.36, 26.21, 22.69, 17.86, 14.12. HRMS (ESI+): $m / z$ calcd for $\mathrm{C}_{22} \mathrm{H}_{36} \mathrm{BrNNaO}_{2}\left(\mathrm{M}+\mathrm{Na}^{+}\right), 448.1822$; found 448.1807 .

Spectroscopic data of $\mathbf{5}^{\mathbf{H}}$-TEG: Colorless oil. Yield: 73\%. IR (neat, $\left.\mathrm{cm}^{-1}\right): 1644\left(v_{\mathrm{C}=0}\right), 1541$

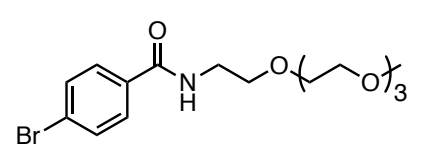

$\mathbf{5}^{\mathbf{H}}$-TEG $\left(u_{\mathrm{N}-\mathrm{H}}\right) .{ }^{1} \mathrm{H} \mathrm{NMR}\left(500 \mathrm{MHz}, \mathrm{CDCl}_{3}, 25^{\circ} \mathrm{C}\right): \delta 7.70(\mathrm{~d}, J=8.8 \mathrm{~Hz}, 2 \mathrm{H}$, $\operatorname{ArH}), 7.56(\mathrm{~d}, J=8.8 \mathrm{~Hz}, 2 \mathrm{H}, \mathrm{ArH}), 6.98(\mathrm{br} \mathrm{s}, 1 \mathrm{H}, \mathrm{NH}), 3.68-3.59$ (m, $\left.14 \mathrm{H}, \mathrm{OCH}_{2}\right), 3.51-3.50\left(\mathrm{~m}, 2 \mathrm{H}, \mathrm{NCH}_{2}\right), 3.34\left(\mathrm{~s}, 3 \mathrm{H}, \mathrm{OCH}_{3}\right) .{ }^{13} \mathrm{C} \mathrm{NMR}$ $\left(126 \mathrm{MHz}, \mathrm{CDCl}_{3}, 2{ }^{\circ} \mathrm{C}\right): \delta 166.51,133.48,131.64,128.80,125.95,71.88,70.53,70.52,70.48$, 70.23, 69.72, 58.98, 39.89. HRMS (ESI+): $m / z$ calcd for $\mathrm{C}_{16} \mathrm{H}_{24} \mathrm{BrNNaO}_{5}\left(\mathrm{M}+\mathrm{Na}^{+}\right), 414.0715$; found 414.0710 .

Spectroscopic data of $\mathbf{5}^{\mathbf{H}}-\mathrm{C} 12$ : White solid. Yield: $96 \%$. Mp: 71.6-72.0 ${ }^{\circ} \mathrm{C}$. IR $\left(\mathrm{KBr}, \mathrm{cm}^{-1}\right): 1631$

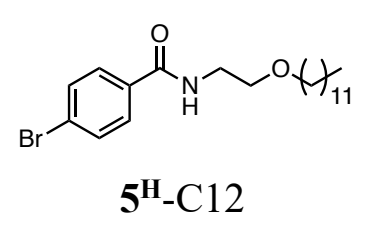
$\left(v_{\mathrm{C}=0}\right), 1544\left(\nu_{\mathrm{N}-\mathrm{H}}\right) .{ }^{1} \mathrm{H} \mathrm{NMR}\left(500 \mathrm{MHz}, \mathrm{CDCl}_{3}, 25^{\circ} \mathrm{C}\right): \delta 7.63-7.66$ (m, 2H, ArH), 7.56-7.58 (m, 2H, ArH), 6.48 (brs, 1H, NH), 3.64 (q, $J=$ $\left.5.0 \mathrm{~Hz}, 2 \mathrm{H}, \mathrm{NCH}_{2}\right), 3.59$ (t, $\left.J=4.7 \mathrm{~Hz}, 2 \mathrm{H}, \mathrm{NCH}_{2} \mathrm{CH}_{2}\right), 3.46$ (t, $J=6.6$ $\mathrm{Hz}, 2 \mathrm{H}, \mathrm{OCH}_{2}$ ), 1.58 (quint, $J=6.8 \mathrm{~Hz}, 2 \mathrm{H}, \mathrm{OCH}_{2} \mathrm{CH}_{2}$ ), 1.25-1.35 (m, $\left.18 \mathrm{H}, \mathrm{CH}_{2}\right), 0.88\left(\mathrm{t}, J=6.9 \mathrm{~Hz}, 3 \mathrm{H}, \mathrm{CH}_{3}\right) .{ }^{13} \mathrm{C} \mathrm{NMR}\left(126 \mathrm{MHz}, \mathrm{CDCl}_{3}, 25{ }^{\circ} \mathrm{C}\right): \delta 166.55,133.59$, 131.93, 128.70, 126.23, 71.46, 69.17, 40.02, 32.06, 29.83, 29.79, 29.76, 29.61, 29.51, 26.35, 22.84, 14.27. HRMS (ESI+): $m / z$ calcd for $\mathrm{C}_{21} \mathrm{H}_{34} \mathrm{BrNNaO}_{2}\left(\mathrm{M}+\mathrm{Na}^{+}\right)$, 434.1665; found 434.1665. 
Synthesis of (S)-6 ${ }^{\text {Ph }}$-TEG. A mixture of $(S)-5^{\text {Ph }}$-TEG (5.30 g, $\left.11.3 \mathrm{mmol}\right)$, bis(pinacolato)diboron

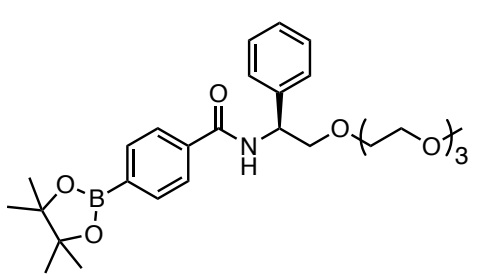

$(S)-6^{\text {Ph }}-$ TEG
$(3.14$

g,

12.3

mmol),

$\left[1,1^{\prime}-\right.$

bis(diphenylphosphino)ferrocene]palladium(II)

dichloride

dichloromethane adduct $\left(\mathrm{Pd}(\mathrm{dppf}) \mathrm{Cl}_{2} \cdot \mathrm{CH}_{2} \mathrm{Cl}_{2}\right)(818 \mathrm{mg}, 1.00$ $\mathrm{mmol})$, and KOAc $(3.34 \mathrm{~g}, 34.0 \mathrm{mmol})$ in dry 1,4-dioxane $(70 \mathrm{~mL})$ was stirred at $80^{\circ} \mathrm{C}$ for $32 \mathrm{~h}$ under nitrogen. After evaporating the solvent under reduced pressure, the residue was suspended in $\mathrm{Et}_{2} \mathrm{O}$ reduced pressure and the residue was dissolved in $\mathrm{CHCl}_{3}$. The solution was washed with $\mathrm{H}_{2} \mathrm{O}$ and brine, dried over anhydrous $\mathrm{Na}_{2} \mathrm{SO}_{4}$, filtered, and concentrated under reduced pressure. The crude product was purified by SEC $\left(\mathrm{CHCl}_{3}\right.$ as eluent) to give $(S)-6^{\mathrm{Ph}}$-TEG $(5.45 \mathrm{~g}, 94 \%)$ as a yellow oil. IR (neat, $\left.\mathrm{cm}^{-1}\right): 1642\left(v_{\mathrm{C}=\mathrm{O}}\right), 1537\left(v_{\mathrm{N}-\mathrm{H}}\right) .{ }^{1} \mathrm{H}$ NMR $\left(500 \mathrm{MHz}, \mathrm{CD}_{3} \mathrm{CN}, 25{ }^{\circ} \mathrm{C}\right): \delta 7.83(\mathrm{~d}, J=8.3$ $\mathrm{Hz}, 2 \mathrm{H}, \mathrm{ArH}), 7.79$ (d, $J=8.3 \mathrm{~Hz}, 2 \mathrm{H}, \mathrm{ArH}), 7.56$ (d, $J=7.5 \mathrm{~Hz}, 1 \mathrm{H}, \mathrm{NH}), 7.42-7.41$ (m, 2H, ArH), 7.36-7.33 (m, 2H, ArH), 7.29-7.26 (m, 1H, ArH), 5.28-5.24 (m, 1H, NCH), 3.81-3.75 (m, 2H, $\left.\mathrm{OCH}_{2}\right)$, 3.63-3.60 (m, 2H, $\left.\mathrm{OCH}_{2}\right), 3.58-3.55\left(\mathrm{~m}, 2 \mathrm{H}, \mathrm{OCH}_{2}\right), 3.53-3.51\left(\mathrm{~m}, 2 \mathrm{H}, \mathrm{OCH}_{2}\right), 3.49-3.47(\mathrm{~m}, 4 \mathrm{H}$, $\left.\mathrm{OCH}_{2}\right), 3.41-3.39\left(\mathrm{~m}, 2 \mathrm{H}, \mathrm{OCH}_{2}\right), 3.25\left(\mathrm{~s}, 3 \mathrm{H}, \mathrm{OCH}_{3}\right), 1.34\left(\mathrm{~s}, 12 \mathrm{H}, \mathrm{C}\left(\mathrm{CH}_{3}\right)_{2}\right) .{ }^{13} \mathrm{C} \mathrm{NMR}(126 \mathrm{MHz}$, $\left.\mathrm{CD}_{3} \mathrm{CN}, 25{ }^{\circ} \mathrm{C}\right): \delta 167.65,141.64,138.13,135.42,129.38,128.27,127.90,127.51,85.14,74.30$, 72.57, 71.16, 71.11, 71.06, 70.97, 58.87, 54.75, 25.20. HRMS (ESI+): $m / z$ calcd for $\mathrm{C}_{28} \mathrm{H}_{40} \mathrm{BNNaO}_{7}$ $\left(\mathrm{M}+\mathrm{Na}^{+}\right), 536.2790$; found 536.2800.

$(R)-6^{\mathrm{Ph}}$-TEG, $(S)$ - and $(R)-\mathbf{6}^{\mathrm{Me}}-\mathrm{TEG},(S)$ - and $(R)-\mathbf{6}^{\mathrm{Me}}-\mathrm{C} 12, \mathbf{6}^{\mathrm{H}}-\mathrm{TEG}$, and $\mathbf{6}^{\mathrm{H}}-\mathrm{C} 12$ were also prepared from $(R)-5^{\text {Ph }}-$ TEG, $(S)-5^{\text {Me }}-$ TEG, $(R)-5^{\text {Me }}-$ TEG, $(S)-5^{\text {Me }}-\mathrm{C} 12,(R)-5^{\text {Me }}-\mathrm{C} 12,5^{\mathbf{H}}-\mathrm{TEG}$, and $\mathbf{5}^{\mathbf{H}}-\mathrm{C} 12$, respectively, in the same way for the synthesis of $(S)-6^{\mathbf{P h}_{-}}$TEG.

Spectroscopic data of $(R)-6^{\text {Ph }}$-TEG: Yellow oil. Yield: 93\%. IR (neat, $\left.\mathrm{cm}^{-1}\right): 1643\left(v_{\mathrm{C}=0}\right), 1537$

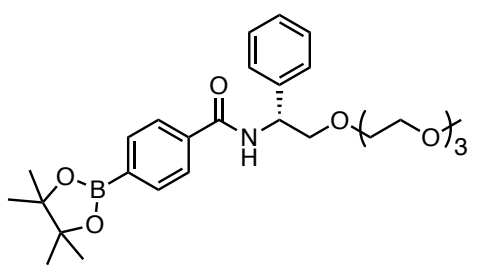

$(R)-6^{\text {Ph }}$-TEG $\left(\nu_{\mathrm{N}-\mathrm{H}}\right) .{ }^{1} \mathrm{H} \mathrm{NMR}\left(500 \mathrm{MHz}, \mathrm{CD}_{3} \mathrm{CN}, 25^{\circ} \mathrm{C}\right): \delta 7.83(\mathrm{~d}, J=8.3 \mathrm{~Hz}$, 2H, ArH), 7.79 (d, $J=8.3 \mathrm{~Hz}, 2 \mathrm{H}, \mathrm{ArH}), 7.55$ (d, $J=7.5 \mathrm{~Hz}, 1 \mathrm{H}$, $\mathrm{NH})$, 7.42-7.41 (m, 2H, ArH), 7.37-7.34 (m, 2H, ArH), 7.29-7.26 (m, 1H, ArH), 5.28-5.24 (m, 1H, NCH), 3.81-3.75 (m, 2H, $\left.\mathrm{OCH}_{2}\right)$, 3.63-3.60 (m, 2H, $\left.\mathrm{OCH}_{2}\right), 3.57-3.55\left(\mathrm{~m}, 2 \mathrm{H}, \mathrm{OCH}_{2}\right), 3.53-3.51(\mathrm{~m}$, 2H, $\left.\mathrm{OCH}_{2}\right), 3.49-3.47\left(\mathrm{~m}, 4 \mathrm{H}, \mathrm{OCH}_{2}\right), 3.41-3.39\left(\mathrm{~m}, 2 \mathrm{H}, \mathrm{OCH}_{2}\right), 3.24\left(\mathrm{~s}, 3 \mathrm{H}, \mathrm{OCH}_{3}\right), 1.33(\mathrm{~s}, 12 \mathrm{H}$, $\left.\mathrm{C}\left(\mathrm{CH}_{3}\right)_{2}\right) .{ }^{13} \mathrm{C} \mathrm{NMR}\left(126 \mathrm{MHz}, \mathrm{CD}_{3} \mathrm{CN}, 25{ }^{\circ} \mathrm{C}\right): \delta 167.75,141.65,138.14,135.43,129.39,128.28$, 127.90, 127.51, 85.15, 74.31, 72.58, 71.17, 71.12, 71.06, 70.98, 58.88, 54.76, 25.21. HRMS (ESI+): $m / z$ calcd for $\mathrm{C}_{28} \mathrm{H}_{40} \mathrm{BNNaO}_{7}\left(\mathrm{M}+\mathrm{Na}^{+}\right), 536.2790$; found 536.2821. 
Spectroscopic data of $(S)-6^{\text {Me }}$-TEG: Pale yellow oil. Yield: $77 \%$. IR (neat, $\left.\mathrm{cm}^{-1}\right): 1638\left(v_{\mathrm{C}=0}\right)$,

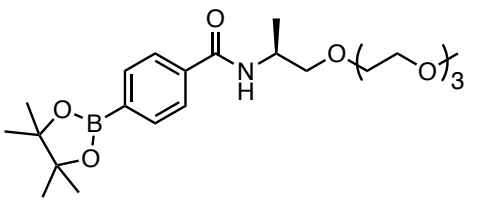

$(S)-6^{\text {Me }}$-TEG $1541\left(u_{\mathrm{N}-\mathrm{H}}\right) .{ }^{1} \mathrm{H}$ NMR $\left(500 \mathrm{MHz}, \mathrm{CDCl}_{3}, 25^{\circ} \mathrm{C}\right): \delta 7.85(\mathrm{~d}, J=8.1$ $\mathrm{Hz}, 2 \mathrm{H}, \mathrm{ArH}), 7.79$ (d, $J=8.3 \mathrm{~Hz}, 2 \mathrm{H}, \mathrm{ArH}), 6.79$ (d, $J=8.1 \mathrm{~Hz}$, $1 \mathrm{H}, \mathrm{NH}), 4.40-4.33(\mathrm{~m}, 1 \mathrm{H}, \mathrm{NCH}), 3.68-3.55\left(\mathrm{~m}, 12 \mathrm{H}, \mathrm{OCH}_{2}\right)$, $3.51\left(\mathrm{t}, J=4.6 \mathrm{~Hz}, 2 \mathrm{H}, \mathrm{OCH}_{2}\right), 3.34\left(\mathrm{~s}, 3 \mathrm{H}, \mathrm{OCH}_{3}\right), 1.35(\mathrm{~s}, 12 \mathrm{H}$, $\left.\mathrm{C}\left(\mathrm{CH}_{3}\right)_{2}\right), 1.30\left(\mathrm{~d}, J=6.8 \mathrm{~Hz}, 3 \mathrm{H}, \mathrm{CH}_{3}\right) \cdot{ }^{13} \mathrm{C} \mathrm{NMR}(126 \mathrm{MHz}$, $\left.\mathrm{CDCl}_{3}, 25^{\circ} \mathrm{C}\right): \delta 166.91,137.09,134.78,132.07,126.22,84.04,74.01,71.84,70.60,70.53,70.49$, 70.41, 58.92, 45.57, 24.87, 17.59. HRMS (ESI+): $m / z$ calcd for $\mathrm{C}_{23} \mathrm{H}_{38} \mathrm{BNNaO}_{7}\left(\mathrm{M}+\mathrm{Na}^{+}\right), 474.2634$; found 474.2653 .

Spectroscopic data of $(R)-6^{\text {Me }}$-TEG: Pale yellow oil. Yield: 84\%. IR (neat, $\left.\mathrm{cm}^{-1}\right): 1639\left(v_{\mathrm{C}=0}\right)$,

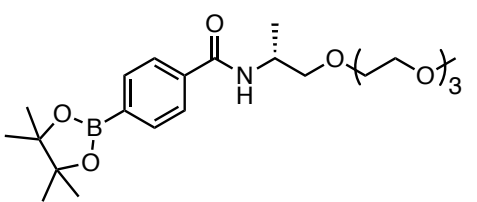

$(R)-6^{\mathrm{Me}}-\mathrm{TEG}$ $1540\left(v_{\mathrm{N}-\mathrm{H}}\right) .{ }^{1} \mathrm{H} \mathrm{NMR}\left(500 \mathrm{MHz}, \mathrm{CDCl}_{3}, 25^{\circ} \mathrm{C}\right): \delta 7.85(\mathrm{~d}, J=8.1$ $\mathrm{Hz}, 2 \mathrm{H}, \mathrm{ArH}), 7.79$ (d, $J=8.3 \mathrm{~Hz}, 2 \mathrm{H}, \mathrm{ArH}), 6.79$ (d, $J=8.1 \mathrm{~Hz}$, $1 \mathrm{H}, \mathrm{NH}), 4.40-4.33$ (m, 1H, NCH), 3.68-3.55 (m, 12H, $\left.\mathrm{OCH}_{2}\right), 3.51$ (t, $\left.J=4.6 \mathrm{~Hz}, 2 \mathrm{H}, \mathrm{OCH}_{2}\right), 3.34\left(\mathrm{~s}, 3 \mathrm{H}, \mathrm{CH}_{3}\right), 1.35\left(\mathrm{~s}, 12 \mathrm{H}, \mathrm{C}\left(\mathrm{CH}_{3}\right)_{2}\right)$, $1.30\left(\mathrm{~d}, J=6.8 \mathrm{~Hz}, 3 \mathrm{H}, \mathrm{CH}_{3}\right) \cdot{ }^{13} \mathrm{C} \mathrm{NMR}\left(126 \mathrm{MHz}, \mathrm{CDCl}_{3}, 25^{\circ} \mathrm{C}\right): \delta 166.92,137.11,134.79,132.03$, 126.25, 84.06, 74.03, 71.86, 70.61, 70.55, 70.51, 70.43, 58.95, 45.59, 24.88, 17.60. HRMS (ESI+): $m / z$ calcd for $\mathrm{C}_{23} \mathrm{H}_{38} \mathrm{BNNaO}_{7}\left(\mathrm{M}+\mathrm{Na}^{+}\right)$, 474.2634; found 474.2648.

Spectroscopic data of $(S)-6^{\mathrm{Me}}-\mathrm{C} 12$ : Pale yellow oil. Yield: $91 \%$. IR $\left(\mathrm{KBr}, \mathrm{cm}^{-1}\right): 1639\left(v_{\mathrm{C}=0}\right), 1535$

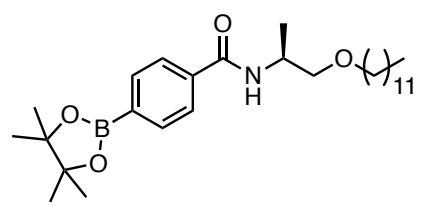

$(S)-6^{\mathrm{Me}}-\mathrm{C} 12$ $\left({ }_{\mathrm{N}-\mathrm{H}}\right) .{ }^{1} \mathrm{H} \mathrm{NMR}\left(500 \mathrm{MHz}, \mathrm{CDCl}_{3}, 25{ }^{\circ} \mathrm{C}\right): \delta 7.86(\mathrm{~d}, J=8.3 \mathrm{~Hz}$, 2H, ArH), 7.74 (d, $J=8.1 \mathrm{~Hz}, 2 \mathrm{H}, \mathrm{ArH}), 6.44$ (d, $J=7.8 \mathrm{~Hz}, 1 \mathrm{H}$, $\mathrm{NH}), 4.39-4.32(\mathrm{~m}, 1 \mathrm{H}, \mathrm{NCH}), 3.53-3.43\left(\mathrm{~m}, 4 \mathrm{H}, \mathrm{OCH}_{2}\right), 1.57$ (quint, $\left.J=6.8 \mathrm{~Hz}, 2 \mathrm{H}, \mathrm{CH}_{2}\right), 1.39-1.23\left(\mathrm{~m}, 33 \mathrm{H}, \mathrm{CH}_{2}, \mathrm{C}\left(\mathrm{CH}_{3}\right)_{2}\right.$, $\left.\mathrm{CH}_{3}\right), 0.88\left(\mathrm{t}, J=7.0 \mathrm{~Hz}, 3 \mathrm{H}, \mathrm{CH}_{3}\right) .{ }^{13} \mathrm{C} \mathrm{NMR}\left(126 \mathrm{MHz}, \mathrm{CDCl}_{3}\right.$, $\left.25^{\circ} \mathrm{C}\right): \delta 166.72,137.10,134.90,126.02,84.08,73.38,71.46,45.44,31.93,29.67,29.63,29.60$, 29.48, 29.36, 26.19, 24.88, 22.69, 17.89, 14.12. HRMS (ESI+): $m / z$ calcd for $\mathrm{C}_{28} \mathrm{H}_{48} \mathrm{BNNaO}_{4}$ $\left(\mathrm{M}+\mathrm{Na}^{+}\right), 496.3569$; found 496.3569 .

Spectroscopic data of $(R)-6^{\text {Me }}-\mathrm{C} 12$ : Pale yellow oil. Yield: 78\%. IR $\left(\mathrm{KBr}, \mathrm{cm}^{-1}\right): 1634\left(v_{\mathrm{C}=0}\right), 1552$

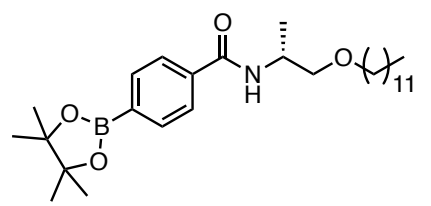

$(R)-6^{\mathrm{Me}}-\mathrm{C} 12$ $\left({ }_{\mathrm{N}-\mathrm{H}}\right) .{ }^{1} \mathrm{H} \mathrm{NMR}\left(500 \mathrm{MHz}, \mathrm{CDCl}_{3}, 25{ }^{\circ} \mathrm{C}\right): \delta 7.86(\mathrm{~d}, J=8.3 \mathrm{~Hz}$, 2H, ArH), 7.74 (d, J=8.3 Hz, 2H, ArH), 6.42 (d, J = 7.8 Hz, 1H, $\mathrm{NH}), 4.38-4.33(\mathrm{~m}, 1 \mathrm{H}, \mathrm{NCH}), 3.54-3.43\left(\mathrm{~m}, 4 \mathrm{H}, \mathrm{OCH}_{2}\right), 1.57$ (quint, $\left.J=6.8 \mathrm{~Hz}, 2 \mathrm{H}, \mathrm{CH}_{2}\right), 1.35-1.25\left(\mathrm{~m}, 33 \mathrm{H}, \mathrm{CH}_{2}, \mathrm{C}\left(\mathrm{CH}_{3}\right)_{2}\right.$, $\left.\mathrm{CH}_{3}\right), 0.88\left(\mathrm{t}, J=7.0 \mathrm{~Hz}, 3 \mathrm{H}, \mathrm{CH}_{3}\right) .{ }^{13} \mathrm{C} \mathrm{NMR}\left(126 \mathrm{MHz}, \mathrm{CDCl}_{3}\right.$, 
$\left.25^{\circ} \mathrm{C}\right): \delta 166.72,137.10,134.90,126.02,84.08,73.38,71.46,45.44,31.93,29.67,29.63,29.60$, 29.48, 29.36, 26.19, 24.88, 22.69, 17.89, 14.12. HRMS (ESI+): $m / z$ calcd for $\mathrm{C}_{28} \mathrm{H}_{48} \mathrm{BNNaO}_{4}$ $\left(\mathrm{M}+\mathrm{Na}^{+}\right), 496.3569$; found 496.3557 .

Spectroscopic data of $6^{\mathrm{H}}$-TEG: Yellow oil. Yield: 91\%. IR (neat, $\left.\mathrm{cm}^{-1}\right): 1646\left(v_{\mathrm{C}=\mathrm{O}}\right), 1540\left(v_{\mathrm{N}-\mathrm{H}}\right)$.

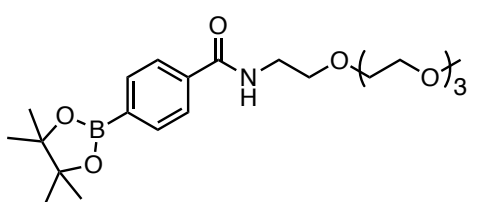

$\mathbf{6}^{\mathrm{H}}$-TEG

${ }^{1} \mathrm{H} \mathrm{NMR}\left(500 \mathrm{MHz}, \mathrm{CDCl}_{3}, 25^{\circ} \mathrm{C}\right): \delta 7.85(\mathrm{~d}, J=8.3 \mathrm{~Hz}, 2 \mathrm{H}, \mathrm{ArH})$, 7.79 (d, $J=8.3 \mathrm{~Hz}, 2 \mathrm{H}, \mathrm{ArH}), 6.87$ (br s, 1H, NH), 3.68-3.59 (m, $\left.14 \mathrm{H}, \mathrm{OCH}_{2}\right), 3.51-3.49\left(\mathrm{~m}, 2 \mathrm{H}, \mathrm{NCH}_{2}\right), 3.34\left(\mathrm{~s}, 3 \mathrm{H}, \mathrm{OCH}_{3}\right), 1.35$ $\left(\mathrm{s}, 12 \mathrm{H}, \mathrm{C}\left(\mathrm{CH}_{3}\right)_{2}\right) .{ }^{13} \mathrm{C} \mathrm{NMR}\left(126 \mathrm{MHz}, \mathrm{CDCl}_{3}, 25^{\circ} \mathrm{C}\right): \delta 167.40$, 136.86, 134.86, 126.19, 84.08, 71.90, 70.59, 70.57, 70.56, 70.51, 70.27, 69.80, 58.99, 39.81, 24.89. HRMS (ESI+): $m / z$ calcd for $\mathrm{C}_{22} \mathrm{H}_{36} \mathrm{BNNaO}_{7}\left(\mathrm{M}+\mathrm{Na}^{+}\right)$, 460.2477; found 460.2500.

Spectroscopic data of $\mathbf{6}^{\mathrm{H}}-\mathrm{C} 12$ : Yellow oil. Yield: 67\%. IR $\left(\mathrm{KBr}, \mathrm{cm}^{-1}\right): 1635\left(v_{\mathrm{C}=\mathrm{O}}\right), 1551\left(\nu_{\mathrm{N}-\mathrm{H}}\right)$.

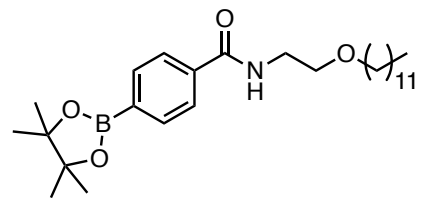

$6^{\mathrm{H}-\mathrm{C} 12}$

${ }^{1} \mathrm{H} \mathrm{NMR}\left(500 \mathrm{MHz}, \mathrm{CDCl}_{3}, 25^{\circ} \mathrm{C}\right): \delta 7.87(\mathrm{~d}, J=8.2 \mathrm{~Hz}, 2 \mathrm{H}, \mathrm{ArH})$, $7.76(\mathrm{~d}, J=8.2 \mathrm{~Hz}, 2 \mathrm{H}, \mathrm{ArH}), 6.55$ (t, $J=4.7 \mathrm{~Hz}, 1 \mathrm{H}, \mathrm{NH}), 3.65$ (q, $\left.J=5.0 \mathrm{~Hz}, 2 \mathrm{H}, \mathrm{NCH}_{2}\right), 3.60\left(\mathrm{t}, J=4.7 \mathrm{~Hz}, 2 \mathrm{H}, \mathrm{NCH}_{2} \mathrm{CH}_{2}\right), 3.47$ (t, $J=6.6 \mathrm{~Hz}, 2 \mathrm{H}, \mathrm{OCH}_{2}$ ), 1.58 (quint, $J=7.9 \mathrm{~Hz}, 2 \mathrm{H}, \mathrm{OCH}_{2} \mathrm{CH}_{2}$ ), $1.35\left(\mathrm{~s}, 12 \mathrm{H}, \mathrm{CCH}_{3}\right), 1.25-1.30\left(\mathrm{~m}, 18 \mathrm{H}, \mathrm{CH}_{2}\right), 0.88$ (t, $\left.J=6.9 \mathrm{~Hz}, 3 \mathrm{H}, \mathrm{CH}_{3}\right) .{ }^{13} \mathrm{C} \mathrm{NMR}(126 \mathrm{MHz}$, $\left.\mathrm{CDCl}_{3}, 25{ }^{\circ} \mathrm{C}\right): \delta 167.48,136.97,135.08,126.19,84.25,71.44,69.30,39.96,32.06,29.80,29.76$, 29.61, 29.49, 26.32, 25.03, 22.83, 14.26. HRMS (ESI+): $m / z$ calcd for $\mathrm{C}_{27} \mathrm{H}_{46} \mathrm{BNNaO}_{4}\left(\mathrm{M}+\mathrm{Na}^{+}\right)$, 482.3412; found 482.3439 .

Synthesis of $\mathbf{P h} \mathbf{h}_{\mathrm{Br} 2}$. To a solution of 3,5-dibromobenzoic acid (9.26 g, $\left.33.1 \mathrm{mmol}\right)$ in dry THF (80<smiles>COCOC(=O)c1cc(Br)cc(Br)c1</smiles>

$\mathrm{Ph}_{\mathrm{Br} 2}$ $\mathrm{mL})$ were added $\mathrm{Et}_{3} \mathrm{~N}(5.5 \mathrm{~mL})$ and chloromethyl methyl ether $(3.0 \mathrm{~mL}, 40$ $\mathrm{mmol}$ ) at $0{ }^{\circ} \mathrm{C}$ under nitrogen, and the reaction mixture was stirred at $0{ }^{\circ} \mathrm{C}$ for 1 $\mathrm{h}$ and further at room temperature for $12 \mathrm{~h}$. After evaporating the solvent under reduced pressure, the residue was dissolved in EtOAc, and the solution was washed with $5 \%$ aqueous $\mathrm{NaHCO}_{3}$ and brine, dried over $\mathrm{MgSO}_{4}$, filtered, and concentrated under reduced pressure, affording $\mathrm{Ph}_{\mathrm{Br} 2}(10.1 \mathrm{~g}, 94 \%)$ as a white solid. Mp: $64-67^{\circ} \mathrm{C}$. IR $\left(\mathrm{KBr}, \mathrm{cm}^{-1}\right): 1729$ $\left(v_{\mathrm{C}=0}\right) .{ }^{1} \mathrm{H} \mathrm{NMR}\left(500 \mathrm{MHz}, \mathrm{CD}_{3} \mathrm{CN}, 25^{\circ} \mathrm{C}\right): \delta 8.14(\mathrm{~d}, J=1.8 \mathrm{~Hz}, 2 \mathrm{H}, \mathrm{ArH}), 8.01(\mathrm{t}, J=1.8 \mathrm{~Hz}, 1 \mathrm{H}$, $\mathrm{ArH}), 5.43\left(\mathrm{~s}, 2 \mathrm{H}, \mathrm{OCH}_{2}\right), 3.50\left(\mathrm{~s}, 3 \mathrm{H}, \mathrm{OCH}_{3}\right) .{ }^{13} \mathrm{C} \mathrm{NMR}\left(126 \mathrm{MHz}, \mathrm{CD}_{3} \mathrm{CN}, 25{ }^{\circ} \mathrm{C}\right): \delta 164.28$, 139.34, 134.63, 132.25, 123.82, 92.71, 58.25. Anal. Calcd for $\mathrm{C}_{9} \mathrm{H}_{8} \mathrm{Br}_{2} \mathrm{O}_{3}$ : C, 33.37; H, 2.49; Found: C, 33.43; H, 2.32 . 
Synthesis of $(\boldsymbol{S})-7^{\text {Ph }}$-TEG. A mixture of $(S)-6^{\text {Ph }}$-TEG (2.26 g, $\left.4.40 \mathrm{mmol}\right), \mathrm{Ph}_{\mathrm{Br} 2}(579 \mathrm{mg}, 1.78$

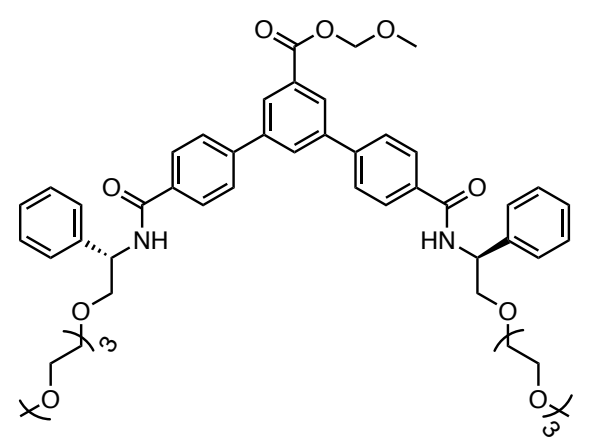

$(S)-7^{\text {Ph }}-\mathrm{TEG}$ $\mathrm{mmol}), \mathrm{Pd}\left(\mathrm{PPh}_{3}\right)_{4}(270 \mathrm{mg}, 233 \mu \mathrm{mol})$, and $\mathrm{K}_{3} \mathrm{PO}_{4}(945$ $\mathrm{mg}, 4.45 \mathrm{mmol})$ in dry 1,4-dioxane $(30 \mathrm{~mL})$ was stirred at $80{ }^{\circ} \mathrm{C}$ for $48 \mathrm{~h}$ under nitrogen. After the solvent was evaporated to dryness under reduced pressure, the crude product was purified by flash column chromatography $\left(\mathrm{SiO}_{2}\right.$, EtOAc and then $\left.\mathrm{CHCl}_{3} / \mathrm{MeOH}=19 / 1, \mathrm{v} / \mathrm{v}\right)$ and further by $\mathrm{SEC}\left(\mathrm{CHCl}_{3}\right.$ as eluent) to give $(S)-7^{\mathrm{Ph}}-\mathrm{TEG}$ $(770 \mathrm{mg}, 46 \%)$ as a yellow oil. IR (neat, $\left.\mathrm{cm}^{-1}\right): 1725$ $\left(v_{\mathrm{C}=\mathrm{O}}\right), 1643\left(v_{\mathrm{C}=\mathrm{O}}\right), 1538\left(v_{\mathrm{N}-\mathrm{H}}\right) .{ }^{1} \mathrm{H} \mathrm{NMR}\left(500 \mathrm{MHz}, \mathrm{CD}_{3} \mathrm{CN}, 25^{\circ} \mathrm{C}\right): \delta 8.33(\mathrm{~d}, J=1.8 \mathrm{~Hz}, 2 \mathrm{H}$, ArH), 8.20 (t, $J=1.8 \mathrm{~Hz}, 1 \mathrm{H}, \mathrm{ArH}), 7.97$ (d, $J=8.5 \mathrm{~Hz}, 4 \mathrm{H}, \operatorname{ArH}), 7.85$ (d, $J=8.5 \mathrm{~Hz}, 4 \mathrm{H}, \operatorname{ArH})$, $7.66(\mathrm{~d}, J=7.5 \mathrm{~Hz}, 2 \mathrm{H}, \mathrm{NH}), 7.45-7.44$ (m, 4H, ArH), 7.38-7.35 (m, $J=7.5 \mathrm{~Hz}, 4 \mathrm{H}, \mathrm{ArH}), 7.30-7.27$ (m, 2H, ArH), $5.50\left(\mathrm{~s}, 2 \mathrm{H}, \mathrm{OCH}_{2}\right), 5.32-5.27(\mathrm{~m}, 2 \mathrm{H}, \mathrm{NCH}), 3.82-3.79\left(\mathrm{~m}, 4 \mathrm{H}, \mathrm{OCH}_{2}\right), 3.65-3.62(\mathrm{~m}$, $\left.4 \mathrm{H}, \mathrm{OCH}_{2}\right), 3.59-3.57\left(\mathrm{~m}, 4 \mathrm{H}, \mathrm{OCH}_{2}\right), 3.55-3.53\left(\mathrm{~m}, 7 \mathrm{H}, \mathrm{OCH}_{2}, \mathrm{OCH}_{3}\right), 3.50-3.47\left(\mathrm{~m}, 8 \mathrm{H}, \mathrm{OCH}_{2}\right)$, 3.40-3.38 (m, 4H, $\left.\mathrm{OCH}_{2}\right), 3.22\left(\mathrm{~s}, 6 \mathrm{H}, \mathrm{OCH}_{3}\right) .{ }^{13} \mathrm{C} \mathrm{NMR}\left(126 \mathrm{MHz}, \mathrm{CD}_{3} \mathrm{CN}, 25{ }^{\circ} \mathrm{C}\right): \delta 167.37$, $166.41,143.33,142.19,141.71,135.26,132.63,131.58,129.41,128.99,128.44,128.31,128.28$, 127.94, 92.24, 74.36, 72.58, 71.19, 71.18, 71.14, 71.08, 70.99, 58.86, 58.16, 54.84. HRMS (ESI+): $m / z$ calcd for $\mathrm{C}_{53} \mathrm{H}_{64} \mathrm{~N}_{2} \mathrm{NaO}_{13}\left(\mathrm{M}+\mathrm{Na}^{+}\right)$, 959.4301; found, 959.4336.

$(R)-7^{\mathrm{Ph}}$-TEG, $(S)$ - and $(R)-7^{\mathrm{Me}}-\mathrm{TEG},(S)$ - and $(R)-7^{\mathrm{Me}}-\mathrm{C} 12,7^{\mathbf{H}}-\mathrm{TEG}$, and $7^{\mathrm{H}_{-} \mathrm{C} 12}$ were also prepared from $(R)-6^{\mathbf{P h}}-\mathrm{TEG},(S)-6^{\mathrm{Me}}-\mathrm{TEG},(R)-\mathbf{6}^{\mathrm{Me}}-\mathrm{TEG},(S)-\mathbf{6}^{\mathrm{Me}}-\mathrm{C} 12,(R)-\mathbf{6}^{\mathrm{Me}}-\mathrm{C} 12, \mathbf{6}^{\mathrm{H}}-\mathrm{TEG}$, and

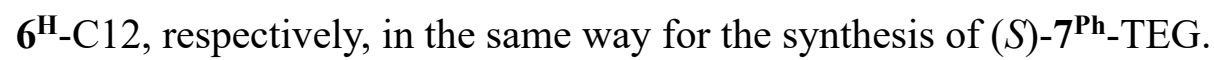

Spectroscopic data of $(R)-7^{\text {Ph }}$-TEG: Yellow oil. Yield: 35\%. IR (neat, $\left.\mathrm{cm}^{-1}\right): 1725\left(v_{\mathrm{C}=0}\right), 1643$

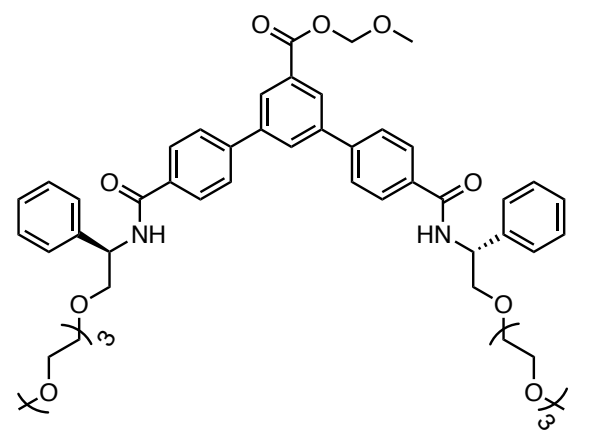

$(R)-7^{\text {Ph }}$-TEG $\left(v_{\mathrm{C}=\mathrm{O}}\right), 1538\left(v_{\mathrm{N}-\mathrm{H}}\right) .{ }^{1} \mathrm{H} \mathrm{NMR}\left(500 \mathrm{MHz}, \mathrm{CD}_{3} \mathrm{CN}, 25^{\circ} \mathrm{C}\right)$ : $\delta 8.32(\mathrm{~d}, J=1.8 \mathrm{~Hz}, 2 \mathrm{H}, \operatorname{ArH}), 8.18(\mathrm{t}, J=1.8 \mathrm{~Hz}, 1 \mathrm{H}$, ArH), 7.96 (d, $J=8.5 \mathrm{~Hz}, 4 \mathrm{H}, \mathrm{ArH}), 7.85$ (d, $J=8.5 \mathrm{~Hz}$, $4 \mathrm{H}, \mathrm{ArH}), 7.63$ (d, J=7.5 Hz, 2H, NH), 7.45-7.44 (m, 4H, ArH), 7.38-7.35 (m, 4H, ArH), 7.30-7.27 (m, 2H, ArH), $5.50\left(\mathrm{~s}, 2 \mathrm{H}, \mathrm{OCH}_{2}\right), 5.32-5.28(\mathrm{~m}, 2 \mathrm{H}, \mathrm{NCH}), 3.84-3.77$ (m, 4H, $\left.\mathrm{OCH}_{2}\right), 3.65-3.63$ (m, 4H, $\left.\mathrm{OCH}_{2}\right), 3.59-3.57$ (m, $\left.4 \mathrm{H}, \mathrm{OCH}_{2}\right), 3.55-3.53\left(\mathrm{~m}, 7 \mathrm{H}, \mathrm{OCH}_{2}, \mathrm{OCH}_{3}\right), 3.50-3.47$ (m, 8H, $\left.\mathrm{OCH}_{2}\right), 3.40-3.38\left(\mathrm{~m}, 4 \mathrm{H}, \mathrm{OCH}_{2}\right), 3.22\left(\mathrm{~s}, 6 \mathrm{H}, \mathrm{OCH}_{3}\right) .{ }^{13} \mathrm{C} \mathrm{NMR}\left(126 \mathrm{MHz}, \mathrm{CD}_{3} \mathrm{CN}, 25{ }^{\circ} \mathrm{C}\right)$ : $\delta 167.34,166.40,143.32,142.18,141.71,135.26,132.62,131.57,129.42,128.97,128.43,128.31$, 
$128.29,127.94,92.24,74.36,72.58,71.19,71.18,71.15,71.08,71.00,58.86,58.16,54.83$. HRMS (ESI+): $m / z$ calcd for $\mathrm{C}_{53} \mathrm{H}_{64} \mathrm{~N}_{2} \mathrm{NaO}_{13}\left(\mathrm{M}+\mathrm{Na}^{+}\right), 959.4301$; found 959.4306 .

Spectroscopic data of $(S)-7^{\text {Me }}$-TEG: Pale yellow oil. Yield: 38\%. IR (neat, $\left.\mathrm{cm}^{-1}\right): 1725\left(\nu_{\mathrm{C}=0}\right)$,

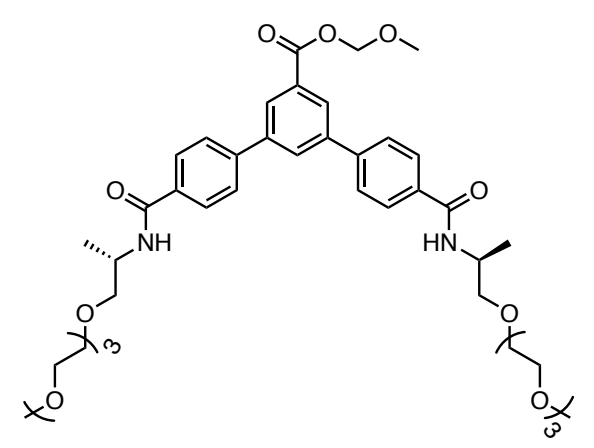

$(S)-7^{\mathrm{Me}}-\mathrm{TEG}$ $1638\left(v_{\mathrm{C}=\mathrm{O}}\right), 1541\left(v_{\mathrm{N}-\mathrm{H}}\right) .{ }^{1} \mathrm{H}$ NMR $\left(500 \mathrm{MHz}, \mathrm{CDCl}_{3}\right.$, $\left.25{ }^{\circ} \mathrm{C}\right): \delta 8.33(\mathrm{~d}, J=1.5 \mathrm{~Hz}, 2 \mathrm{H}, \operatorname{ArH}), 8.03(\mathrm{t}, J=1.6$ $\mathrm{Hz}, 1 \mathrm{H}, \mathrm{ArH}), 7.95$ (d, $J=8.3 \mathrm{~Hz}, 4 \mathrm{H}, \mathrm{ArH}), 7.74$ (d, $J=$ $8.3 \mathrm{~Hz}, 4 \mathrm{H}, \mathrm{ArH}), 6.79$ (d, $J=8.1 \mathrm{~Hz}, 2 \mathrm{H}, \mathrm{NH}), 5.56$ (s, $\left.2 \mathrm{H}, \mathrm{OCH}_{2}\right), 4.44-4.37(\mathrm{~m}, 2 \mathrm{H}, \mathrm{NCH}), 3.72-3.56(\mathrm{~m}, 27 \mathrm{H}$, $\left.\mathrm{OCH}_{2}, \mathrm{OCH}_{3}\right), 3.51\left(\mathrm{t}, J=4.6 \mathrm{~Hz}, 4 \mathrm{H}, \mathrm{OCH}_{2}\right), 3.33(\mathrm{~s}$, $\left.6 \mathrm{H}, \mathrm{OCH}_{3}\right), 1.34\left(\mathrm{~d}, J=6.8 \mathrm{~Hz}, 6 \mathrm{H}, \mathrm{CH}_{3}\right) .{ }^{13} \mathrm{C} \mathrm{NMR}(126$ $\left.\mathrm{MHz}, \mathrm{CDCl}_{3}, 25^{\circ} \mathrm{C}\right): \delta 166.46,165.68,142.44,141.34$, $134.32,131.20,130.56,127.95,127.72,127.15,91.27,77.36,74.05,71.83,70.59,70.53,70.51$, 70.48, 70.39, 58.89, 57.92, 45.66, 17.60. HRMS (ESI+): $m / z$ calcd for $\mathrm{C}_{43} \mathrm{H}_{60} \mathrm{~N}_{2} \mathrm{NaO}_{13}\left(\mathrm{M}+\mathrm{Na}^{+}\right)$, 835.3988; found 835.3978.

Spectroscopic data of $(R)-7^{\mathrm{Me}}$-TEG: White solid. Yield: 66\%. IR $\left(\mathrm{KBr}, \mathrm{cm}^{-1}\right): 1725\left(v_{\mathrm{C}=0}\right), 1635$

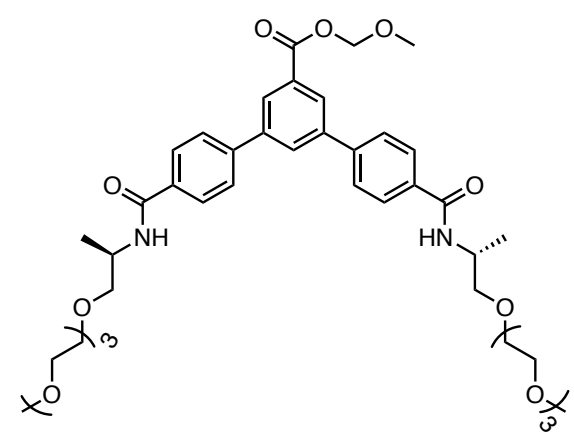

$(R)-7^{\text {Me }}-$ TEG $\left(v_{\mathrm{C}=\mathrm{O}}\right), 1544\left(v_{\mathrm{N}-\mathrm{H}}\right) .{ }^{1} \mathrm{H} \mathrm{NMR}\left(500 \mathrm{MHz}, \mathrm{CDCl}_{3}, 25^{\circ} \mathrm{C}\right)$ : $\delta 8.30(\mathrm{~d}, J=1.7 \mathrm{~Hz}, 2 \mathrm{H}, \mathrm{ArH}), 8.01(\mathrm{t}, J=1.6 \mathrm{~Hz}, 1 \mathrm{H}$, $\operatorname{ArH}), 7.95(\mathrm{~d}, J=8.3 \mathrm{~Hz}, 4 \mathrm{H}, \mathrm{ArH}), 7.72(\mathrm{~d}, J=8.3 \mathrm{~Hz}$, 4H, ArH), 6.93 (d, $J=7.8 \mathrm{~Hz}, 2 \mathrm{H}, \mathrm{NH}), 5.56$ (s, 2H, $\left.\mathrm{OCH}_{2}\right), 4.44-4.37(\mathrm{~m}, 2 \mathrm{H}, \mathrm{NCH}), 3.71-3.59(\mathrm{~m}, 27 \mathrm{H}$, $\left.\mathrm{OCH}_{2}, \mathrm{OCH}_{3}\right), 3.51\left(\mathrm{t}, J=4.8 \mathrm{~Hz}, 4 \mathrm{H}, \mathrm{OCH}_{2}\right), 3.33(\mathrm{~s}$, $\left.6 \mathrm{H}, \mathrm{OCH}_{3}\right), 1.34\left(\mathrm{~d}, J=6.8 \mathrm{~Hz}, 6 \mathrm{H}, \mathrm{CH}_{3}\right) .{ }^{13} \mathrm{C} \mathrm{NMR}(126$ $\left.\mathrm{MHz}, \mathrm{CDCl}_{3}, 25{ }^{\circ} \mathrm{C}\right): \delta 166.46,165.67,142.45,141.34$, 134.32, 131.20, 130.55, 127.94, 127.72, 127.15, 91.27, 77.36, 74.06, 71.83, 70.59, 70.53, 70.51,

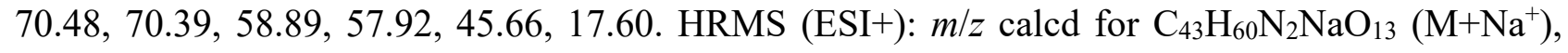
835.3988; found 835.3971. 
Spectroscopic data of $(S)-7^{\mathrm{Me}}-\mathrm{C} 12$ : White solid. Yield: $59 \%$. Mp: $79.0-81.3^{\circ} \mathrm{C}$. IR $\left(\mathrm{KBr}, \mathrm{cm}^{-1}\right)$ :

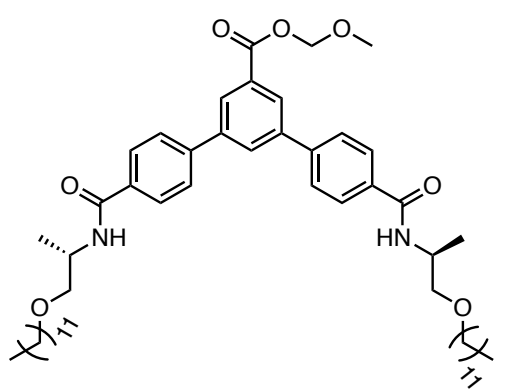

$(S)-7^{\mathrm{Me}}-\mathrm{C} 12$ $1733\left(v_{\mathrm{C}=\mathrm{O}}\right), 1631\left(v_{\mathrm{C}=\mathrm{O}}\right), 1546\left(v_{\mathrm{N}-\mathrm{H}}\right) .{ }^{1} \mathrm{H}$ NMR $(500$ $\left.\mathrm{MHz}, \mathrm{CDCl}_{3}, 25^{\circ} \mathrm{C}\right): \delta 8.33(\mathrm{~d}, J=1.7 \mathrm{~Hz}, 2 \mathrm{H}, \mathrm{ArH})$, $8.02(\mathrm{t}, J=1.8 \mathrm{~Hz}, 1 \mathrm{H}, \mathrm{ArH}), 7.90(\mathrm{~d}, J=8.5 \mathrm{~Hz}, 4 \mathrm{H}$, ArH), 7.74 (d, $J=8.3 \mathrm{~Hz}, 4 \mathrm{H}, \mathrm{ArH}), 6.47$ (d, $J=8.1 \mathrm{~Hz}$, $2 \mathrm{H}, \mathrm{NH}), 5.56\left(\mathrm{~s}, 2 \mathrm{H}, \mathrm{OCH}_{2}\right), 4.43-4.35(\mathrm{~m}, 2 \mathrm{H}, \mathrm{NCH})$, 3.59-3.45 (m, $\left.11 \mathrm{H}, \mathrm{OCH}_{2}, \mathrm{OCH}_{3}\right), 1.60$ (quint, $J=6.8 \mathrm{~Hz}$, $\left.4 \mathrm{H}, \mathrm{CH}_{2}\right), 1.37-1.23\left(\mathrm{~m}, 42 \mathrm{H}, \mathrm{CH}_{2}, \mathrm{CH}_{3}\right), 0.86(\mathrm{t}, J=7.1$

$\left.\mathrm{Hz}, 6 \mathrm{H}, \mathrm{CH}_{3}\right) .{ }^{13} \mathrm{C} \mathrm{NMR}\left(126 \mathrm{MHz}, \mathrm{CDCl}_{3}, 25^{\circ} \mathrm{C}\right): \delta 166.25,165.71,142.71,141.37,134.36,131.33$, $130.63,127.89,127.68,127.39,91.36,73.40,71.52,57.98,45.55,31.90,29.68,29.65,29.64,29.62$, 29.49, 29.34, 26.20, 22.68, 17.94, 14.11. HRMS (ESI+): $m / z$ calcd for $\mathrm{C}_{53} \mathrm{H}_{80} \mathrm{~N}_{2} \mathrm{NaO}_{7}\left(\mathrm{M}^{+} \mathrm{Na}^{+}\right)$, 879.5857; found 879.5827 .

Spectroscopic data of $(R)-7^{\mathrm{Me}}-\mathrm{C} 12$ : White solid. Yield: $28 \%$. Mp: 83.5-84.6 ${ }^{\circ} \mathrm{C}$. IR $\left(\mathrm{KBr}, \mathrm{cm}^{-1}\right)$ :

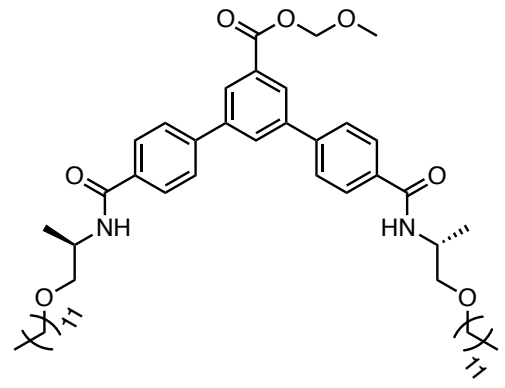

$(R)-7^{\mathrm{Me}}-\mathrm{C} 12$ $1726\left(v_{\mathrm{C}=\mathrm{O}}\right), 1636\left(v_{\mathrm{C}=\mathrm{O}}\right), 1543\left(v_{\mathrm{N}-\mathrm{H}}\right) .{ }^{1} \mathrm{H}$ NMR $(500$ $\left.\mathrm{MHz}, \mathrm{CDCl}_{3}, 25^{\circ} \mathrm{C}\right): \delta 8.33(\mathrm{~d}, J=1.7 \mathrm{~Hz}, 2 \mathrm{H}, \mathrm{ArH})$, 8.02 (t, $J=1.7 \mathrm{~Hz}, 1 \mathrm{H}, \mathrm{ArH}), 7.90$ (d, $J=8.5 \mathrm{~Hz}, 4 \mathrm{H}$, $\operatorname{ArH}), 7.74$ (d, $J=8.5 \mathrm{~Hz}, 4 \mathrm{H}, \operatorname{ArH}), 6.47$ (d, $J=7.8 \mathrm{~Hz}$, $2 \mathrm{H}, \mathrm{NH}), 5.56\left(\mathrm{~s}, 2 \mathrm{H}, \mathrm{OCH}_{2}\right), 4.43-4.35$ (m, 2H, $\left.\mathrm{NCH}\right)$, 3.59-3.45 (m, 11H, $\left.\mathrm{OCH}_{2}, \mathrm{OCH}_{3}\right), 1.60$ (quint, $J=6.8 \mathrm{~Hz}$, $\left.4 \mathrm{H}, \mathrm{CH}_{2}\right), 1.37-1.23\left(\mathrm{~m}, 42 \mathrm{H}, \mathrm{CH}_{2}, \mathrm{CH}_{3}\right), 0.86(\mathrm{t}, J=7.0$ $\left.\mathrm{Hz}, 6 \mathrm{H}, \mathrm{CH}_{3}\right){ }^{13} \mathrm{C} \mathrm{NMR}\left(126 \mathrm{MHz}, \mathrm{CDCl}_{3}, 25^{\circ} \mathrm{C}\right): \delta 166.24,165.70,142.70,141.37,134.36,131.32$, 130.63, 127.89, 127.68, 127.39, 91.36, 73.40, 71.52, 57.98, 45.54, 31.90, 29.68, 29.65, 29.64, 29.62, 29.49, 29.34, 26.20, 22.68, 17.94, 14.11. HRMS (ESI+): $m / z$ calcd for $\mathrm{C}_{53} \mathrm{H}_{80} \mathrm{~N}_{2} \mathrm{NaO}_{7}\left(\mathrm{M}^{+} \mathrm{Na}^{+}\right)$, 879.5857 ; found 879.5832 .

Spectroscopic data of $7^{\mathrm{H}}$-TEG: Yellow oil. Yield: 40\%. IR (neat, $\left.\mathrm{cm}^{-1}\right): 1725\left(v_{\mathrm{C}=0}\right), 1651\left(v_{\mathrm{C}=0}\right)$,

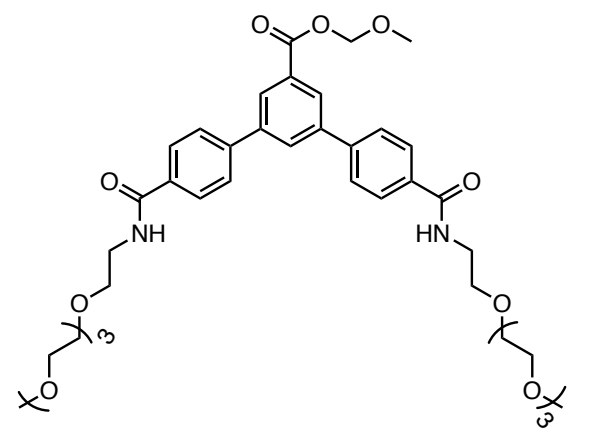

$7^{\mathbf{H}_{-}}$TEG $1540\left(v_{\mathrm{N}-\mathrm{H}}\right) .{ }^{1} \mathrm{H}$ NMR $\left(500 \mathrm{MHz}, \mathrm{CDCl}_{3}, 25^{\circ} \mathrm{C}\right): \delta 8.33$ (d, $J=1.9 \mathrm{~Hz}, 2 \mathrm{H}, \mathrm{ArH}), 8.03$ (t, $J=1.8 \mathrm{~Hz}, 1 \mathrm{H}, \mathrm{ArH}$ ), 7.95 (d, $J=8.7 \mathrm{~Hz}, 4 \mathrm{H}, \mathrm{ArH}), 7.74$ (d, $J=8.7 \mathrm{~Hz}, 4 \mathrm{H}$, ArH), 6.97 (br, 2H, NH), 5.56 (s, 2H, $\left.\mathrm{OCH}_{2}\right)$. 3.72-3.59 $\left(\mathrm{m}, 31 \mathrm{H}, \mathrm{OCH}_{2}, \mathrm{OCH}_{3}\right), 3.51-3.50\left(\mathrm{~m}, 4 \mathrm{H}, \mathrm{NCH}_{2}\right), 3.32$ $\left(\mathrm{s}, 6 \mathrm{H}, \mathrm{OCH}_{3}\right) \cdot{ }^{13} \mathrm{C} \mathrm{NMR}\left(126 \mathrm{MHz}, \mathrm{CDCl}_{3}, 25{ }^{\circ} \mathrm{C}\right): \delta$ $167.94,165.70,142.66,141.35,134.11,131.28,130.63$, 
$127.87,127.84,127.31,91.32,71.90,70.60,70.58,70.56,70.52,70.29,69.82,58.98,57.98,39.90$. HRMS (ESI+): $m / z$ calcd for $\mathrm{C}_{41} \mathrm{H}_{56} \mathrm{~N}_{2} \mathrm{NaO}_{13}\left(\mathrm{M}+\mathrm{Na}^{+}\right)$, 807.3675; found 807.3646.

Spectroscopic data of $7^{\mathrm{H}}-\mathrm{C} 12$ : White solid. Yield: $40 \%$. Mp: $56.5-58.9^{\circ} \mathrm{C}$. IR $\left(\mathrm{KBr}, \mathrm{cm}^{-1}\right): 1725$

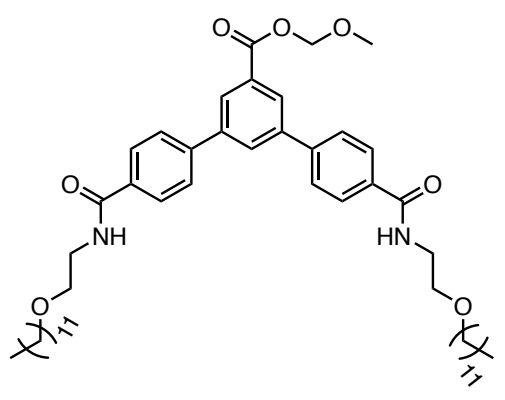

$7^{\mathrm{H}_{-} \mathrm{C} 12}$ $\left(v_{\mathrm{C}=\mathrm{O}}\right), 1638\left(v_{\mathrm{C}=\mathrm{O}}\right), 1543\left(\mathrm{w}_{\mathrm{N}-\mathrm{H}}\right) .{ }^{1} \mathrm{H}$ NMR $(500 \mathrm{MHz}$, $\left.\mathrm{CDCl}_{3}, 25^{\circ} \mathrm{C}\right): \delta 8.33(\mathrm{~d}, J=1.8 \mathrm{~Hz}, 2 \mathrm{H}, \mathrm{ArH}), 8.03(\mathrm{t}, J$ $=1.8 \mathrm{~Hz}, 1 \mathrm{H}, \mathrm{ArH}), 7.90-7.92(\mathrm{~m}, 4 \mathrm{H}, \mathrm{ArH}), 7.75(\mathrm{~m}, 4 \mathrm{H}$, $\mathrm{ArH}), 6.59(\mathrm{t}, J=5.1 \mathrm{~Hz}, 2 \mathrm{H}, \mathrm{NH}), 5.56\left(\mathrm{~s}, 2 \mathrm{H}, \mathrm{OCH}_{2} \mathrm{O}\right)$, 3.70 (q, $\left.J=5.0 \mathrm{~Hz}, 4 \mathrm{H}, \mathrm{NCH}_{2}\right), 3.63$ (t, $J=4.9 \mathrm{~Hz}, 4 \mathrm{H}$, $\left.\mathrm{NCH}_{2} \mathrm{CH}_{2}\right), 3.59\left(\mathrm{~s}, 3 \mathrm{H}, \mathrm{OCH}_{3}\right), 3.49$ (t, $J=6.8 \mathrm{~Hz}, 4 \mathrm{H}$, $\mathrm{OCH}_{2} \mathrm{CH}_{2}$ ), 1.59-1.63 (quint, $J=6.8 \mathrm{~Hz}, 4 \mathrm{H}, \mathrm{OCH}_{2} \mathrm{CH}_{2}$ ), 1.23-1.37 (m, 36H, $\left.\mathrm{CH}_{2}\right), 0.86\left(\mathrm{t}, J=7.1 \mathrm{~Hz}, 6 \mathrm{H}, \mathrm{CH}_{3}\right) .{ }^{13} \mathrm{C} \mathrm{NMR}\left(126 \mathrm{MHz}, \mathrm{CDCl}_{3}, 25^{\circ} \mathrm{C}\right): \delta 167.00$, $165.82,142.92$, 141.47, 134.25, 131.48, 130.77, 128.05, 127.85, 127.56, 91.50, 71.49, 69.31, 58.12, 40.04, 32.04, 29.81, 29.78, 29.62, 29.48, 26.33, 22.81, 14.24. HRMS (ESI+): $\mathrm{m} / z$ calcd for $\mathrm{C}_{51} \mathrm{H}_{76} \mathrm{~N}_{2} \mathrm{NaO}_{7}\left(\mathrm{M}+\mathrm{Na}^{+}\right)$, 851.5545; found 851.5515.

Synthesis of $(\boldsymbol{S})-\mathbf{8}^{\mathrm{Ph}}$-TEG. To a solution of $(S)-7^{\mathrm{Ph}}-\mathrm{TEG}(728 \mathrm{mg}, 777 \mu \mathrm{mol})$ in $\mathrm{CH}_{2} \mathrm{Cl}_{2}(8.0 \mathrm{~mL})$

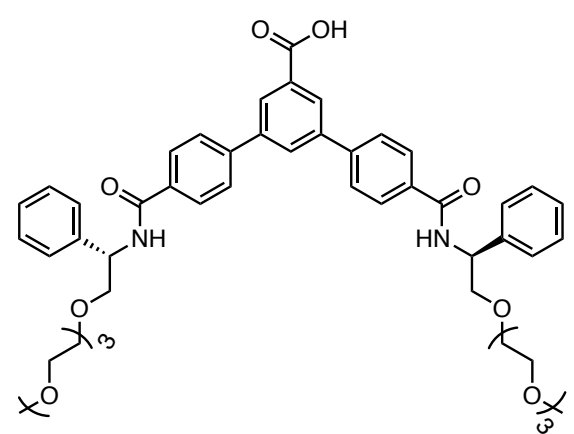

$(S)-8^{\mathrm{Ph}}-\mathrm{TEG}$ was added trifluoroacetic acid (TFA) $(1.2 \mathrm{~mL}, 15 \mathrm{mmol})$ at $0{ }^{\circ} \mathrm{C}$ under nitrogen. The mixture was warmed to room temperature, and stirred for $12 \mathrm{~h}$. The solution was diluted with $\mathrm{CH}_{2} \mathrm{Cl}_{2}$, and the diluted solution was washed with $\mathrm{H}_{2} \mathrm{O}$ and brine, dried over $\mathrm{Na}_{2} \mathrm{SO}_{4}$, filtered, and evaporated to dryness under reduced pressure, affording $(S)-\mathbf{8}^{\text {Ph }}$-TEG (639 mg, 92\%) as a yellow oil. IR (neat, $\mathrm{cm}^{-}$ $\left.{ }^{1}\right): 1716\left(v_{\mathrm{C}=\mathrm{O}}\right), 1636\left(v_{\mathrm{C}=\mathrm{O}}\right), 1540\left(v_{\mathrm{N}-\mathrm{H}}\right) .{ }^{1} \mathrm{H} \mathrm{NMR}(500$ $\left.\mathrm{MHz}, \mathrm{CD}_{3} \mathrm{CN}, 25^{\circ} \mathrm{C}\right): \delta 8.22(\mathrm{~d}, J=1.8 \mathrm{~Hz}, 2 \mathrm{H}, \mathrm{ArH}), 8.07(\mathrm{t}, J=1.8 \mathrm{~Hz}, 1 \mathrm{H}, \mathrm{ArH}), 7.92(\mathrm{~d}, J=8.5$ $\mathrm{Hz}, 4 \mathrm{H}, \mathrm{ArH}), 7.77$ (d, $J=8.5 \mathrm{~Hz}, 4 \mathrm{H}, \mathrm{ArH}), 7.74$ (d, $J=7.5 \mathrm{~Hz}, 2 \mathrm{H}, \mathrm{NH}), 7.45-7.43$ (m, 4H, ArH), 7.37-7.34 (m, 4H, ArH), 7.29-7.26 (m, 2H, ArH), 5.32-5.28 (m, 2H, NCH), 3.84-3.76 (m, 4H, $\left.\mathrm{OCH}_{2}\right)$, 3.65-3.62 (m, 4H, $\left.\mathrm{OCH}_{2}\right), 3.59-3.56\left(\mathrm{~m}, 4 \mathrm{H}, \mathrm{OCH}_{2}\right), 3.54-3.52\left(\mathrm{~m}, 4 \mathrm{H}, \mathrm{OCH}_{2}\right), 3.49-3.46(\mathrm{~m}, 8 \mathrm{H}$, $\left.\mathrm{OCH}_{2}\right), 3.39-3.37\left(\mathrm{~m}, 4 \mathrm{H}, \mathrm{OCH}_{2}\right), 3.21\left(\mathrm{~s}, 6 \mathrm{H}, \mathrm{OCH}_{3}\right) \cdot{ }^{13} \mathrm{C} \mathrm{NMR}\left(126 \mathrm{MHz}, \mathrm{CD}_{3} \mathrm{CN}, 25{ }^{\circ} \mathrm{C}\right): \delta$ $167.41,167.36,143.19,141.78,141.48,134.94,132.52$, 131.02, 129.25, 128.81, 128.35, 128.13, $128.04,127.77,74.19,72.39,71.01,71.00,70.95,70.90,70.80,58.69,54.69$. HRMS (ESI+): $\mathrm{m} / z$ calcd for $\mathrm{C}_{51} \mathrm{H}_{60} \mathrm{~N}_{2} \mathrm{NaO}_{12}\left(\mathrm{M}+\mathrm{Na}^{+}\right)$, 915.4039; found, 915.4068. 
$(R)-\mathbf{8}^{\mathbf{P h}}$-TEG, $(S)$ - and $(R)-\mathbf{8}^{\mathrm{Me}}-\mathrm{TEG},(S)$ - and $(R)-\mathbf{8}^{\mathrm{Me}}-\mathrm{C} 12, \mathbf{8}^{\mathbf{H}}-\mathrm{TEG}$, and $\mathbf{8}^{\mathbf{H}}-\mathrm{C} 12$ were also

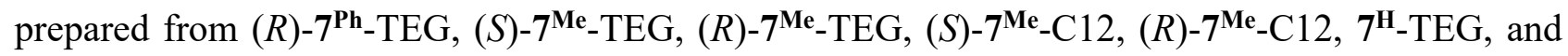
$7^{\mathbf{H}}-\mathrm{C} 12$, respectively, in the same way for the synthesis of $(S)-\mathbf{8}^{\mathbf{P h}}-\mathrm{TEG}$.

Spectroscopic data of $(R)-8^{\text {Ph }}$-TEG: Yellow oil. Yield: 94\%. IR (neat, $\left.\mathrm{cm}^{-1}\right): 1715\left(v_{\mathrm{C}=0}\right), 1638$

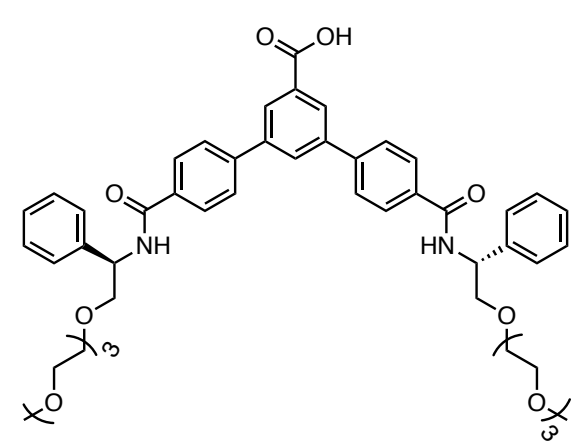

$(R)-\mathbf{8}^{\text {Ph }}$-TEG $\left(v_{\mathrm{C}=\mathrm{O}}\right), 1539\left(v_{\mathrm{N}-\mathrm{H}}\right) .{ }^{1} \mathrm{H} \mathrm{NMR}\left(500 \mathrm{MHz}, \mathrm{CD}_{3} \mathrm{CN}, 25^{\circ} \mathrm{C}\right)$ : $\delta 8.27(\mathrm{~d}, J=1.8 \mathrm{~Hz}, 2 \mathrm{H}, \operatorname{ArH}), 8.15(\mathrm{t}, J=1.8 \mathrm{~Hz}, 1 \mathrm{H}$, $\operatorname{ArH}), 7.95(\mathrm{~d}, J=8.5 \mathrm{~Hz}, 4 \mathrm{H}, \operatorname{ArH}), 7.83(\mathrm{~d}, J=8.5 \mathrm{~Hz}$, $4 \mathrm{H}, \mathrm{ArH}), 7.63$ (d, $J=7.5 \mathrm{~Hz}, 2 \mathrm{H}, \mathrm{NH}), 7.45-7.44(\mathrm{~m}, 4 \mathrm{H}$, ArH), 7.38-7.35 (m, 4H, ArH), 7.30-7.27 (m, 2H, ArH), 5.32-5.28 (m, 2H, ArH), 3.84-3.77 (m, 4H, $\left.\mathrm{OCH}_{2}\right), 3.65-$ $3.63\left(\mathrm{~m}, 4 \mathrm{H}, \mathrm{OCH}_{2}\right), 3.59-3.57\left(\mathrm{~m}, 4 \mathrm{H}, \mathrm{OCH}_{2}\right), 3.55-3.53$ $\left(\mathrm{m}, 4 \mathrm{H}, \mathrm{OCH}_{2}\right), 3.50-3.47\left(\mathrm{~m}, 8 \mathrm{H}, \mathrm{OCH}_{2}\right), 3.40-3.38(\mathrm{~m}$, $\left.4 \mathrm{H}, \mathrm{OCH}_{2}\right), 3.22\left(\mathrm{~s}, 6 \mathrm{H}, \mathrm{OCH}_{3}\right) .{ }^{13} \mathrm{C} \mathrm{NMR}\left(126 \mathrm{MHz}, \mathrm{CD}_{3} \mathrm{CN}, 25{ }^{\circ} \mathrm{C}\right): \delta 167.40,167.39,143.40$, 142.06, 141.70, 135.18, 132.64, 131.31, 129.42, 128.97, 128.56, 128.29, 128.27, 127.93, 74.36, 72.57, 71.19, 71.18, 71.14, 71.08, 70.99, 58.85, 54.83. HRMS (ESI+): $m / z$ calcd for $\mathrm{C}_{51} \mathrm{H}_{60} \mathrm{~N}_{2} \mathrm{NaO}_{12}$ $\left(\mathrm{M}+\mathrm{Na}^{+}\right), 915.4039$; found 915.4070.

Spectroscopic data of $(S)-8^{\text {Me }}$-TEG: Pale yellow oil. Yield: $62 \%$. IR (neat, $\left.\mathrm{cm}^{-1}\right): 1715\left(v_{\mathrm{C}=0}\right)$,

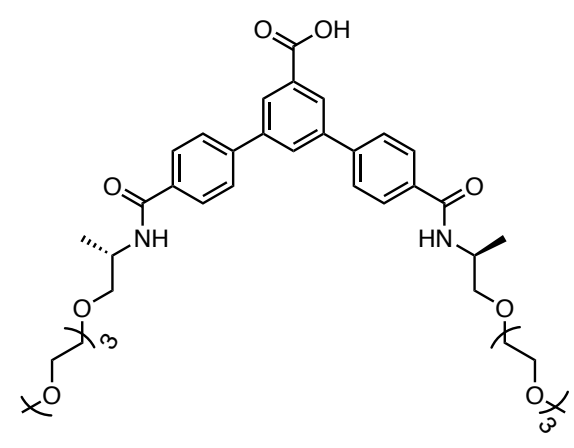

$(S)-\mathbf{8}^{\mathrm{Me}}-\mathrm{TEG}$ $1635\left(v_{\mathrm{C}=\mathrm{O}}\right), 1540\left(\nu_{\mathrm{N}-\mathrm{H}}\right) .{ }^{1} \mathrm{H} \mathrm{NMR}\left(500 \mathrm{MHz}, \mathrm{CD}_{3} \mathrm{CN}\right.$, $\left.25{ }^{\circ} \mathrm{C}\right): \delta 8.25(\mathrm{~d}, J=1.7 \mathrm{~Hz}, 2 \mathrm{H}, \mathrm{ArH}), 8.12(\mathrm{t}, J=1.7$ $\mathrm{Hz}, 1 \mathrm{H}, \mathrm{ArH}), 7.89$ (d, $J=8.3 \mathrm{~Hz}, 4 \mathrm{H}, \operatorname{ArH}), 7.81$ (d, $J=$ $8.3 \mathrm{~Hz}, 4 \mathrm{H}, \mathrm{ArH}), 7.26$ (d, J=7.6 Hz, 2H, NH), 4.35-4.27 $(\mathrm{m}, 2 \mathrm{H}, \mathrm{NCH}), 3.63-3.50\left(\mathrm{~m}, 24 \mathrm{H}, \mathrm{OCH}_{2}\right), 3.43-3.41(\mathrm{~m}$, $\left.4 \mathrm{H}, \mathrm{OCH}_{2}\right), 3.25\left(\mathrm{~s}, 6 \mathrm{H}, \mathrm{OCH}_{3}\right), 1.25(\mathrm{~d}, J=6.8 \mathrm{~Hz}, 6 \mathrm{H}$, $\left.\mathrm{CH}_{3}\right) \cdot{ }^{13} \mathrm{C} \mathrm{NMR}\left(126 \mathrm{MHz}, \mathrm{CDCl}_{3}, 25{ }^{\circ} \mathrm{C}\right): \delta 167.64$, $167.47,143.13,141.81,135.23,132.67,131.00,128.83$, 128.42, 128.07, 74.65, 72.56, 71.27, 71.17, 71.12, 71.09, 70.96, 58.87, 46.59, 17.80. HRMS (ESI+): $m / z$ calcd for $\mathrm{C}_{41} \mathrm{H}_{56} \mathrm{~N}_{2} \mathrm{NaO}_{12}\left(\mathrm{M}+\mathrm{Na}^{+}\right)$, 791.3726; found 791.3713. 
Spectroscopic data of $(R)-8^{\text {Me }}$-TEG: Pale yellow oil. Yield: 97\%. IR (neat, $\left.\mathrm{cm}^{-1}\right): 1715\left(v_{\mathrm{C}=0}\right)$,

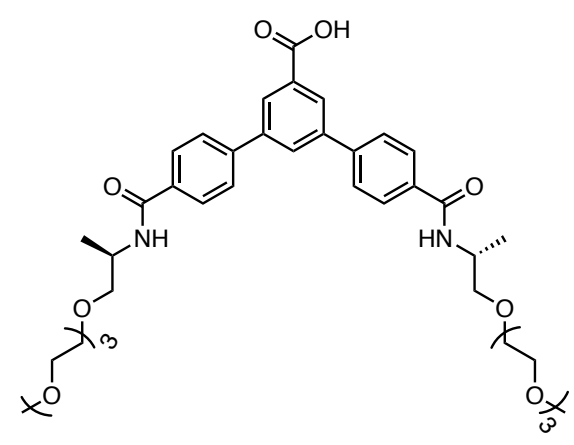

$(R)-\mathbf{8}^{\mathrm{Me}}-\mathrm{TEG}$ $1644\left(v_{\mathrm{C}=\mathrm{O}}\right), 1540\left(v_{\mathrm{N}-\mathrm{H}}\right) .{ }^{1} \mathrm{H} \mathrm{NMR}\left(500 \mathrm{MHz}, \mathrm{CD}_{3} \mathrm{CN}\right.$, $\left.25{ }^{\circ} \mathrm{C}\right): \delta 8.19(\mathrm{~d}, J=1.7 \mathrm{~Hz}, 2 \mathrm{H}, \mathrm{ArH}), 8.04(\mathrm{t}, J=1.7$ $\mathrm{Hz}, 1 \mathrm{H}, \mathrm{ArH}), 7.87$ (d, $J=8.3 \mathrm{~Hz}, 4 \mathrm{H}, \mathrm{ArH}), 7.73$ (d, $J=$ $8.3 \mathrm{~Hz}, 4 \mathrm{H}, \mathrm{ArH}), 7.10$ (d, $J=8.1 \mathrm{~Hz}, 2 \mathrm{H}, \mathrm{NH}), 4.33-4.25$ (m, 2H, NCH), 3.65-3.45 (m, 24H, $\left.\mathrm{OCH}_{2}\right), 3.42-3.40(\mathrm{~m}$, $\left.4 \mathrm{H}, \mathrm{OCH}_{2}\right), 3.23\left(\mathrm{~s}, 6 \mathrm{H}, \mathrm{OCH}_{3}\right), 1.23(\mathrm{~d}, J=6.8 \mathrm{~Hz}, 6 \mathrm{H}$, $\left.\mathrm{CH}_{3}\right) \cdot{ }^{13} \mathrm{C}$ NMR $\left(126 \mathrm{MHz}, \mathrm{CDCl}_{3}, 25{ }^{\circ} \mathrm{C}\right): \delta 167.64$, $167.47,143.13,141.81,135.23,132.67,131.00,128.83$, 128.42, 128.07, 74.65, 72.56, 71.27, 71.17, 71.12, 71.09, 70.96, 58.87, 46.59, 17.80. HRMS (ESI+): $m / z$ calcd for $\mathrm{C}_{41} \mathrm{H}_{56} \mathrm{~N}_{2} \mathrm{NaO}_{12}\left(\mathrm{M}+\mathrm{Na}^{+}\right), 791.3726$; found 791.3719.

Spectroscopic data of $(S)-8^{\mathrm{Me}}-\mathrm{C} 12$ : White solid. Yield: $85 \%$. Mp: $173.9-174.3{ }^{\circ} \mathrm{C}$. IR $\left(\mathrm{KBr}, \mathrm{cm}^{-}\right.$

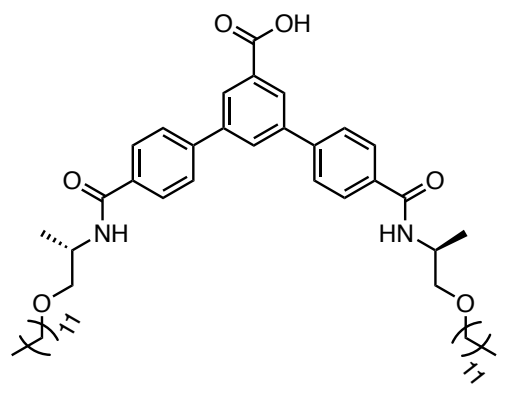

$(S)-8^{\mathrm{Me}}-\mathrm{C} 12$

$\left.{ }^{1}\right): 1709\left(v_{\mathrm{C}=\mathrm{O}}\right), 1635\left(v_{\mathrm{C}=\mathrm{O}}\right), 1543\left(\mathrm{v}_{\mathrm{N}-\mathrm{H}}\right) .{ }^{1} \mathrm{H}$ NMR $(500$ $\left.\mathrm{MHz}, \mathrm{CDCl}_{3}, 25^{\circ} \mathrm{C}\right): \delta 8.25(\mathrm{~d}, J=1.7 \mathrm{~Hz}, 2 \mathrm{H}, \mathrm{ArH})$, $7.90(\mathrm{~d}, J=8.3 \mathrm{~Hz}, 4 \mathrm{H}, \operatorname{ArH}), 7.80(\mathrm{t}, \mathrm{J}=1.7 \mathrm{~Hz}, 1 \mathrm{H}$, $\operatorname{ArH}), 7.62(\mathrm{~d}, J=8.3 \mathrm{~Hz}, 4 \mathrm{H}, \operatorname{ArH}), 6.89$ (d, $J=8.1 \mathrm{~Hz}$, $2 \mathrm{H}, \mathrm{NH}), 4.50-4.43(\mathrm{~m}, 2 \mathrm{H}, \mathrm{NCH}), 3.65-3.50(\mathrm{~m}, 8 \mathrm{H}$, $\mathrm{OCH}_{2}$ ), 1.62 (quint, $\left.J=6.8 \mathrm{~Hz}, 4 \mathrm{H}, \mathrm{CH}_{2}\right), 1.38-1.22(\mathrm{~m}$, $\left.42 \mathrm{H}, \mathrm{CH}_{2}, \mathrm{CH}_{3}\right), 0.84\left(\mathrm{t}, J=7.0 \mathrm{~Hz}, 6 \mathrm{H}, \mathrm{CH}_{3}\right) .{ }^{13} \mathrm{C} \mathrm{NMR}$ (126 MHz, $\left.\mathrm{CDCl}_{3}, 25^{\circ} \mathrm{C}\right): \delta 169.00,166.71,142.33,140.76,134.22,131.07,130.39,127.91,127.87$, 126.98, 73.41, 71.57, 45.40, 31.90, 29.67, 29.63, 29.61, 29.45, 29.33, 26.09, 22.67, 17.78, 14.11. HRMS (ESI-): $m / z$ calcd for $\mathrm{C}_{51} \mathrm{H}_{75} \mathrm{~N}_{2} \mathrm{O}_{6}\left(\mathrm{M}-\mathrm{H}^{+}\right), 811.5631$; found 811.5595.

Spectroscopic data of $(R)-8^{\mathrm{Me}}-\mathrm{C} 12$ : White solid. Yield: 92\%. Mp: 172.3-174.0 ${ }^{\circ} \mathrm{C}$. IR $\left(\mathrm{KBr}, \mathrm{cm}^{-}\right.$

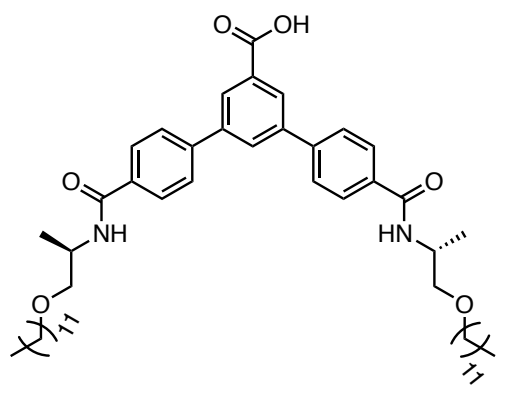

$(R)-\mathbf{8}^{\mathrm{Me}}-\mathrm{C} 12$ $\left.{ }^{1}\right): 1701\left(v_{\mathrm{C}=\mathrm{O}}\right), 1636\left(v_{\mathrm{C}=\mathrm{O}}\right), 1542\left(v_{\mathrm{N}-\mathrm{H}}\right) .{ }^{1} \mathrm{H}$ NMR $(500$ $\left.\mathrm{MHz}, \mathrm{CDCl}_{3}, 25^{\circ} \mathrm{C}\right): \delta 8.25(\mathrm{~d}, J=1.2 \mathrm{~Hz}, 2 \mathrm{H}, \mathrm{ArH})$, $7.90(\mathrm{~d}, J=8.1 \mathrm{~Hz}, 4 \mathrm{H}, \operatorname{ArH}), 7.82(\mathrm{t}, J=1.6 \mathrm{~Hz}, 1 \mathrm{H}$, $\operatorname{ArH}), 7.64(\mathrm{~d}, J=8.3 \mathrm{~Hz}, 4 \mathrm{H}, \mathrm{ArH}), 6.84(\mathrm{~d}, J=8.1 \mathrm{~Hz}$, $2 \mathrm{H}, \mathrm{NH}), 4.49-4.42(\mathrm{~m}, 2 \mathrm{H}, \mathrm{NCH}), 3.65-3.49(\mathrm{~m}, 8 \mathrm{H}$, $\mathrm{OCH}_{2}$ ), 1.62 (quint, $\left.J=6.8 \mathrm{~Hz}, 4 \mathrm{H}, \mathrm{CH}_{2}\right), 1.38-1.22$ (m, $\left.42 \mathrm{H}, \mathrm{CH}_{2}, \mathrm{CH}_{3}\right), 0.85\left(\mathrm{t}, J=7.0 \mathrm{~Hz}, 6 \mathrm{H}, \mathrm{CH}_{3}\right) .{ }^{13} \mathrm{C} \mathrm{NMR}$

$\left(126 \mathrm{MHz}, \mathrm{CDCl}_{3}, 25^{\circ} \mathrm{C}\right): \delta 169.00,166.69,142.30,140.76,134.22,131.05,130.39,127.91,127.86$, 
$126.97,73.41,71.56,45.40,31.90,29.67,29.63,29.61,29.45,29.33,26.10,22.67,17.78,14.11$. HRMS (ESI-): $m / z$ calcd for $\mathrm{C}_{51} \mathrm{H}_{75} \mathrm{~N}_{2} \mathrm{O}_{6}\left(\mathrm{M}-\mathrm{H}^{+}\right), 811.5631$; found 811.5590 .

Spectroscopic data of $\mathbf{8}^{\mathrm{H}}$-TEG: Yellow oil. Yield: 98\%. IR (neat, $\left.\mathrm{cm}^{-1}\right): 1716\left(\nu_{\mathrm{C}=0}\right), 1644\left(\nu_{\mathrm{C}=0}\right)$,

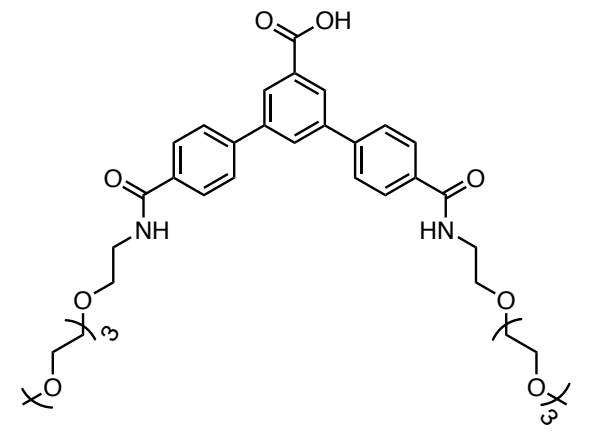

$\mathbf{8}^{\mathbf{H}}$-TEG $1541\left(u_{\mathrm{N}-\mathrm{H}}\right) .{ }^{1} \mathrm{H} \mathrm{NMR}\left(500 \mathrm{MHz}, \mathrm{CD}_{3} \mathrm{CN}, 25^{\circ} \mathrm{C}\right): \delta 8.22$ (d, $J=1.8 \mathrm{~Hz}, 2 \mathrm{H}, \mathrm{ArH}), 8.09$ (t, $J=1.8 \mathrm{~Hz}, 1 \mathrm{H}, \operatorname{ArH})$, $7.88(\mathrm{~d}, J=8.5 \mathrm{~Hz}, 4 \mathrm{H}, \mathrm{ArH}), 7.78(\mathrm{~d}, J=8.5 \mathrm{~Hz}, 4 \mathrm{H}$, ArH), 7.27 (t, $J=5.5 \mathrm{~Hz}, 2 \mathrm{H}, \mathrm{NH}), 3.64-3.49$ (m, 28H, $\left.\mathrm{OCH}_{2}\right), 3.41-3.40\left(\mathrm{~m}, 4 \mathrm{H}, \mathrm{NCH}_{2}\right), 3.24\left(\mathrm{~s}, 6 \mathrm{H}, \mathrm{OCH}_{3}\right)$. ${ }^{13} \mathrm{C}$ NMR $\left(126 \mathrm{MHz}, \mathrm{CD}_{3} \mathrm{CN}, 25^{\circ} \mathrm{C}\right): \delta 167.59,167.47$, $143.20,141.97,135.21,132.65,131.17,128.76,128.47$, 128.20, 72.58, 71.19, 71.14, 71.12, 70.99, 70.98, 70.13, 58.86, 40.52. HRMS (ESI+): $m / z$ calcd for $\mathrm{C}_{39} \mathrm{H}_{52} \mathrm{~N}_{2} \mathrm{NaO}_{12}\left(\mathrm{M}+\mathrm{Na}^{+}\right)$, 763.3413; found 763.3417.

Spectroscopic data of $\mathbf{8}^{\mathrm{H}}$-C12: White solid. Yield: $85 \%$. Mp: 139.5-140.3 ${ }^{\circ} \mathrm{C}$. IR $\left(\mathrm{KBr}, \mathrm{cm}^{-1}\right): 1701$

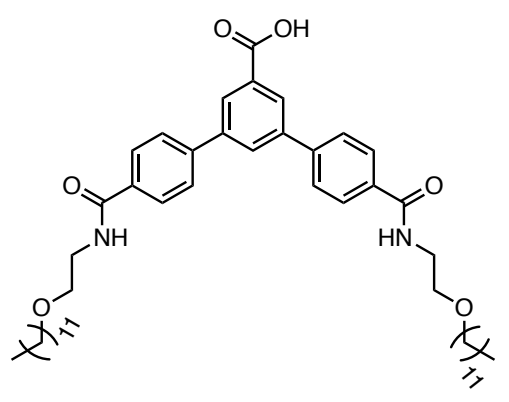

$\mathbf{8}^{\mathrm{H}_{-} \mathrm{C} 12}$ $\left(v_{\mathrm{C}=\mathrm{O}}\right), 1637\left(v_{\mathrm{C}=\mathrm{O}}\right), 1543\left(v_{\mathrm{N}-\mathrm{H}}\right) .{ }^{1} \mathrm{H}$ NMR $(500 \mathrm{MHz}$, $\left.\mathrm{CDCl}_{3}, 25^{\circ} \mathrm{C}\right): \delta 8.33(\mathrm{~d}, J=1.8 \mathrm{~Hz}, 2 \mathrm{H}, \mathrm{ArH}), 7.97(\mathrm{t}, J$ $=1.8 \mathrm{~Hz}, 1 \mathrm{H}, \mathrm{ArH}), 7.95(\mathrm{~d}, J=8.5 \mathrm{~Hz}, 4 \mathrm{H}, \mathrm{ArH}), 7.72$ (d, $J=8.5 \mathrm{~Hz}, 4 \mathrm{H}, \mathrm{ArH}), 6.98(\mathrm{t}, J=5.1 \mathrm{~Hz}, 2 \mathrm{H}, \mathrm{NH})$, 3.69-3.75 (m, 8H, $\left.\mathrm{NCH}_{2} \mathrm{CH}_{2}\right), 3.54$ (t, $J=6.6 \mathrm{~Hz}, 4 \mathrm{H}$, $\mathrm{OCH}_{2}$ ), 1.60-1.65 (quint, $J=7.9 \mathrm{~Hz}, 4 \mathrm{H}, \mathrm{OCH}_{2} \mathrm{CH}_{2}$ ), 1.22-1.37 (m, 36H, $\left.\mathrm{CH}_{2}\right), 0.85\left(\mathrm{t}, J=6.9 \mathrm{~Hz}, 6 \mathrm{H}, \mathrm{CH}_{3}\right)$. ${ }^{13} \mathrm{C}$ NMR $\left(126 \mathrm{MHz}, \mathrm{CDCl}_{3}, 25{ }^{\circ} \mathrm{C}\right): \delta 168.74,167.25,142.69,141.25,134.19,131.00,130.77$, $128.25,128.03$, 127.34, 71.52, 69.51, 39.96, 32.04, 29.81, 29.77, 29.75, 29.61, 29.60, 29.48, 26.26, 22.81, 14.25. HRMS (ESI-): $m / z$ calcd for $\mathrm{C}_{49} \mathrm{H}_{71} \mathrm{~N}_{2} \mathrm{O}_{6}\left(\mathrm{M}-\mathrm{H}^{+}\right), 783.5318$; found 783.5300 .

$(S)$ - and $(R)-\mathbf{8}^{\mathbf{P h}}-\mathrm{C} 12$ were prepared according to Scheme 1 with a slight modification. A typical experimental procedure for the synthesis of $(S)-\mathbf{8}^{\mathbf{P h}}-\mathrm{C} 12$ is described below. 
Synthesis of $(\boldsymbol{S})-\mathbf{8}^{\mathrm{Ph}}-\mathbf{C 1 2}$. A mixture of $(S)-\mathbf{5}^{\mathrm{Ph}}-\mathrm{C} 12(530 \mathrm{mg}, 1.08 \mathrm{mmol})$, bis(pinacolato)diboron

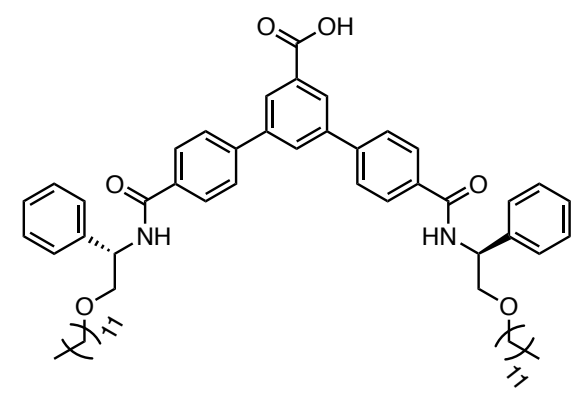

$(S)-\mathbf{8}^{\text {Ph }}-\mathrm{C} 12$ (314 mg, $1.24 \mathrm{mmol}), \mathrm{Pd}(\mathrm{dppf}) \mathrm{Cl}_{2} \cdot \mathrm{CH}_{2} \mathrm{Cl}_{2}(81 \mathrm{mg}, 0.10$ $\mathrm{mmol}$ ), and KOAc (331 $\mathrm{mg}, 3.37 \mathrm{mmol}$ ) in dry 1,4dioxane $(15 \mathrm{~mL})$ was stirred at $80{ }^{\circ} \mathrm{C}$ for $10 \mathrm{~h}$ under nitrogen. After evaporating the solvent under reduced pressure, the residue was suspended in $n$-hexane $(100$ $\mathrm{mL}$ ) and insoluble salts were removed by filtration. The filtrate was evaporated to dryness under reduced pressure and the residue was passed through a short pad of silica gel using $n$-hexane-EtOAc $(7 / 3, \mathrm{v} / \mathrm{v})$ as the eluent. After concentrating in vacuo, the crude product $(S)-6^{\mathbf{P h}}$-C12 (554 mg) was obtained as a pale yellow oil and was used without further purification. A mixture of the obtained $(S)-6^{\mathbf{P h}_{-} \mathrm{C} 12}(500 \mathrm{mg}), \mathrm{Ph}_{\mathrm{Br} 2}(137 \mathrm{mg}, 423 \mu \mathrm{mol}), \mathrm{Pd}\left(\mathrm{PPh}_{3}\right)_{4}(62 \mathrm{mg}, 54 \mu \mathrm{mol})$, and $\mathrm{K}_{3} \mathrm{PO}_{4}(462 \mathrm{mg}, 2.16 \mathrm{mmol})$ in dry 1,4-dioxane $(4.0 \mathrm{~mL})$ was stirred at $80{ }^{\circ} \mathrm{C}$ for $24 \mathrm{~h}$ under nitrogen. After the solvent was evaporated to dryness under reduced pressure, the residue was passed through a short pad of silica gel using $n$-hexane-EtOAc $(7 / 3, \mathrm{v} / \mathrm{v})$ as the eluent. After concentrating in vacuo, the crude product $(S)-7^{\mathbf{P h}}-\mathrm{C} 12(380 \mathrm{mg})$ was obtained as a pale yellow solid and was used without further purification. To a solution of the obtained $(S)-7^{\mathbf{P h}}-\mathrm{C} 12(300 \mathrm{mg})$ in $\mathrm{CH}_{2} \mathrm{Cl}_{2}(5 \mathrm{~mL})$ was added TFA $(0.24 \mathrm{~mL}, 3.1 \mathrm{mmol})$ at $0{ }^{\circ} \mathrm{C}$ under nitrogen. The mixture was warmed to room temperature, and stirred for $12 \mathrm{~h}$. The solution was diluted with $\mathrm{CH}_{2} \mathrm{Cl}_{2}$, and the diluted solution was washed with $\mathrm{H}_{2} \mathrm{O}$ and brine, dried over $\mathrm{Na}_{2} \mathrm{SO}_{4}$, filtered, and evaporated to dryness under reduced pressure, affording $(S)-8^{\text {Ph }}-\mathrm{C} 12$ (286 mg, 79\% over 3 steps) as a yellow oil. IR $\left(\mathrm{KBr}, \mathrm{cm}^{-1}\right): 1702$ $\left(v_{\mathrm{C}=\mathrm{O}}\right), 1636\left(v_{\mathrm{C}=\mathrm{O}}\right), 1541\left(v_{\mathrm{N}-\mathrm{H}}\right) .{ }^{1} \mathrm{H} \mathrm{NMR}\left(500 \mathrm{MHz}, \mathrm{CDCl}_{3}, 25^{\circ} \mathrm{C}\right): \delta 8.27(\mathrm{~s}, 2 \mathrm{H}, \mathrm{ArH}), 7.97(\mathrm{~d}, J$ $=8.2 \mathrm{~Hz}, 4 \mathrm{H}, \mathrm{ArH}), 7.79$ (s, 1H, ArH), 7.64 (d, J=8.2 Hz, 4H, ArH), 7.55 (d, J=7.1 Hz, 2H, NH), $7.46(\mathrm{~d}, J=7.4 \mathrm{~Hz}, 4 \mathrm{H}, \mathrm{ArH}), 7.36$ (t, $J=7.5 \mathrm{~Hz}, 4 \mathrm{H}, \mathrm{ArH}), 7.28$ (t, $J=7.4 \mathrm{~Hz}, 2 \mathrm{H}, \mathrm{ArH}), 5.46$ (q, $J$ $=4.7 \mathrm{~Hz}, 2 \mathrm{H}, \mathrm{NCH}), 3.95\left(\mathrm{dd}, J=10.7,6.6 \mathrm{~Hz}, 2 \mathrm{H}, \mathrm{OCH}_{2} \mathrm{CH}\right), 3.85(\mathrm{dd}, J=10.7,4.3 \mathrm{~Hz}, 2 \mathrm{H}$, $\mathrm{OCH}_{2} \mathrm{CH}$ ), 3.49-3.65 (m, 4H, $\left.\mathrm{OCH}_{2} \mathrm{CH}_{2}\right), 1.58-1.63\left(\mathrm{~m}, 4 \mathrm{H}, \mathrm{OCH}_{2} \mathrm{CH}_{2}\right), 1.21-1.32\left(\mathrm{~m}, 36 \mathrm{H}, \mathrm{CH}_{2}\right)$, $0.84\left(\mathrm{t}, J=7.1 \mathrm{~Hz}, 6 \mathrm{H}, \mathrm{CH}_{3}\right) .{ }^{13} \mathrm{C} \mathrm{NMR}\left(126 \mathrm{MHz} \mathrm{CDCl}_{3}, 25^{\circ} \mathrm{C}\right): \delta 169.23,166.91,142.58,140.86$, 139.94, 134.00, 131.21, 130.57, 128.74, 128.17, 128.06, 127.70, 127.11, 127.05, 73.07, 71.57, 53.26, $32.00,29.78,29.73,29.69,29.51,29.44,26.16,22.78,14.22$. HRMS (ESI +$): m / z$ calcd for $\mathrm{C}_{61} \mathrm{H}_{81} \mathrm{~N}_{2} \mathrm{O}_{6}\left(\mathrm{M}+\mathrm{H}^{+}\right)$, 937. 6089; found 937.6071.

$(R)-\mathbf{8}^{\mathbf{P h}}-\mathrm{C} 12$ was also prepared from $(R)-5^{\mathbf{P h}_{-}} \mathrm{C} 12$ in the same way for the synthesis of $(S)-\mathbf{8}^{\mathbf{P h}}$ $\mathrm{C} 12$. 
Spectroscopic data of $(R)-\mathbf{8}^{\mathrm{Ph}}-\mathrm{C} 12$ : Yellow oil. Yield: $52 \%$ over 3 steps. IR $\left(\mathrm{KBr}, \mathrm{cm}^{-1}\right): 1702$

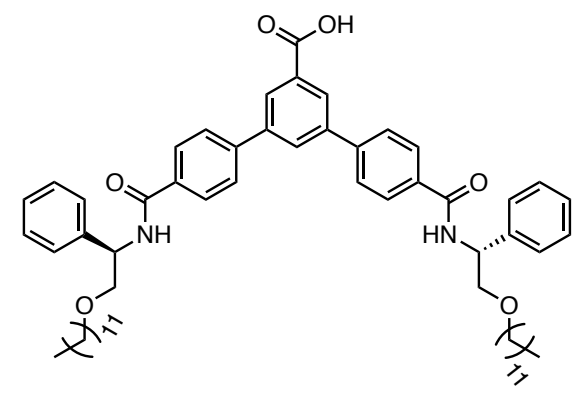

(R)-8 $\mathbf{8}^{\mathbf{P h}}-\mathrm{C} 12$ $\left(v_{\mathrm{C}=\mathrm{O}}\right), 1637\left(v_{\mathrm{C}=\mathrm{O}}\right), 1541\left(v_{\mathrm{N}-\mathrm{H}}\right) .{ }^{1} \mathrm{H}$ NMR $(500 \mathrm{MHz}$, $\left.\mathrm{CDCl}_{3}, 25^{\circ} \mathrm{C}\right): \delta 8.27(\mathrm{~s}, 2 \mathrm{H}, \mathrm{ArH}), 7.97(\mathrm{~d}, J=8.2 \mathrm{~Hz}$, 4H, ArH), 7.79 (s, 1H, ArH), $7.64(\mathrm{~d}, J=8.2 \mathrm{~Hz}, 4 \mathrm{H}, \mathrm{ArH})$, 7.55 (d, $J=7.4 \mathrm{~Hz}, 2 \mathrm{H}, \mathrm{NH}), 7.46$ (d, $J=7.6 \mathrm{~Hz}, 4 \mathrm{H}$, $\operatorname{ArH}), 7.36$ (t, $J=7.5 \mathrm{~Hz}, 4 \mathrm{H}, \operatorname{ArH}), 7.28$ (t, $J=7.4 \mathrm{~Hz}$, $2 \mathrm{H}, \mathrm{ArH}), 5.46(\mathrm{q}, J=4.7 \mathrm{~Hz}, 2 \mathrm{H}, \mathrm{NCH}), 3.95(\mathrm{dd}, J=$ $\left.10.4,6.6 \mathrm{~Hz}, 2 \mathrm{H}, \mathrm{OCH}_{2} \mathrm{CH}\right), 3.85(\mathrm{dd}, J=10.6,4.4 \mathrm{~Hz}$,

$\left.2 \mathrm{H}, \mathrm{OCH}_{2} \mathrm{CH}\right), 3.49-3.65\left(\mathrm{~m}, 4 \mathrm{H}, \mathrm{OCH}_{2} \mathrm{CH}_{2}\right), 1.58-1.63\left(\mathrm{~m}, 4 \mathrm{H}, \mathrm{OCH}_{2} \mathrm{CH}_{2}\right), 1.21-1.33(\mathrm{~m}, 36 \mathrm{H}$, $\left.\mathrm{CH}_{2}\right), 0.84\left(\mathrm{t}, J=7.1 \mathrm{~Hz}, 6 \mathrm{H}, \mathrm{CH}_{3}\right) .{ }^{13} \mathrm{C} \mathrm{NMR}\left(126 \mathrm{MHz}, \mathrm{CDCl}_{3}, 25{ }^{\circ} \mathrm{C}\right): \delta 169.23,166.91,142.58$, 140.86, 139.94, 134.00, 131.21, 130.57, 128.74, 128.17, 128.05, 127.70, 127.10, 127.05, 73.07, 71.57, 53.26, 32.00, 29.77, 29.73, 29.69, 29.51, 29.44, 26.16, 22.78, 14.22. HRMS (ESI+): $\mathrm{m} / z$ calcd for $\mathrm{C}_{61} \mathrm{H}_{80} \mathrm{~N}_{2} \mathrm{NaO}_{6}\left(\mathrm{M}+\mathrm{Na}^{+}\right), 959.5909$; found 959.5872.

Synthesis of $(S)-1 a^{\text {Ph }}$-TEG. $(S)-1 \mathbf{a}^{\text {Ph }}$-TEG was prepared according to a procedure reported by

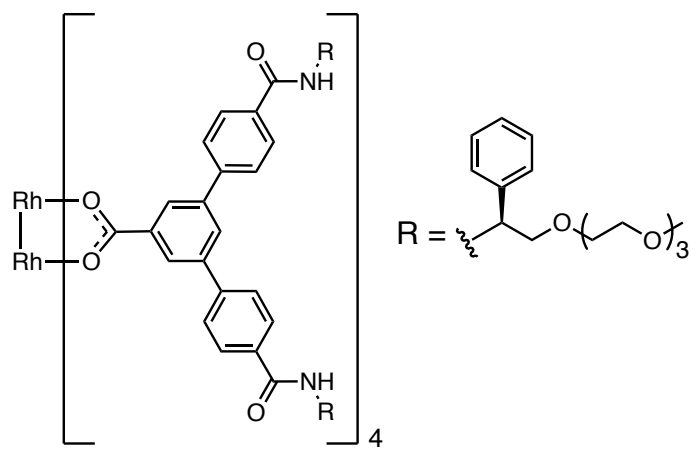

$(S)-\mathbf{1} \mathbf{a}^{\mathbf{P h}}-\mathrm{TEG}$ Callot et al. ${ }^{\mathrm{S} 3}$ A mixture of $(S)-\mathbf{8}^{\mathrm{Ph}}$-TEG $(580 \mathrm{mg}$, $582 \mu \mathrm{mol})$ and $\mathrm{Rh}_{2}(\mathrm{TFA})_{4}(64.6 \mathrm{mg}, 98.1 \mu \mathrm{mol})$ in chlorobenzene $(5 \mathrm{~mL})$ was stirred at $130{ }^{\circ} \mathrm{C}$ under a gentle nitrogen stream, during which the solvent was distilled off at a rate of ca. $1 \mathrm{~mL}$ per $10 \mathrm{~min}$. After $30 \mathrm{~min}$, an additional $3 \mathrm{~mL}$ of chlorobenzene was added and this procedure was repeated four times to remove TFA dissociated from $\mathrm{Rh}_{2}(\mathrm{TFA})_{4}$.

After the solvent was evaporated to dryness under reduced pressure, the crude product was then purified by flash column chromatography $\left(\mathrm{SiO}_{2}, \mathrm{CHCl}_{3} / \mathrm{MeOH}=19 / 1\right.$, v/v) and further by SEC $\left(\mathrm{CHCl}_{3}\right.$ as eluent $)$ to give $(S)-1 \mathbf{a}^{\mathrm{Ph}}-\mathrm{TEG}(282 \mathrm{mg}, 76 \%)$ as a green solid. Mp: $126.8-127.6^{\circ} \mathrm{C} . \mathrm{IR}(\mathrm{KBr}$, $\left.\mathrm{cm}^{-1}\right): 1638\left(v_{\mathrm{C}=\mathrm{O}}\right), 1577\left(v_{\mathrm{COO}}{ }^{-}\right), 1542\left(v_{\mathrm{N}-\mathrm{H}}\right), 1385\left(v_{\mathrm{COO}^{-}}\right) .{ }^{1} \mathrm{H}$ NMR $\left(500 \mathrm{MHz}, \mathrm{DMSO}-d_{6}, 25{ }^{\circ} \mathrm{C}\right)$ : $\delta 8.90(\mathrm{~d}, J=8.0 \mathrm{~Hz}, 8 \mathrm{H}, \mathrm{NH}), 8.11(\mathrm{br}, 12 \mathrm{H}, \mathrm{ArH}), 8.00$ (d, $J=7.8 \mathrm{~Hz}, 16 \mathrm{H}, \mathrm{ArH}), 7.83$ (br, 16H, ArH), 7.44 (d, $J=7.4 \mathrm{~Hz}, 16 \mathrm{H}, \operatorname{ArH}), 7.34$ (t, $J=7.6 \mathrm{~Hz}, 16 \mathrm{H}, \operatorname{ArH}), 7.25$ (t, J=7.3 Hz, 8H, ArH), 5.31-5.26 (m, 8H, NCH), 3.81-3.40 (m, 96H, $\left.\mathrm{OCH}_{2}\right), 3.32-3.30\left(\mathrm{~m}\right.$, partially overlapping with $\mathrm{H}_{2} \mathrm{O}$ signal, $\left.\mathrm{OCH}_{2}\right), 3.12\left(\mathrm{~s}, 24 \mathrm{H}, \mathrm{OCH}_{3}\right) .{ }^{13} \mathrm{C} \mathrm{NMR}\left(126 \mathrm{MHz}, \mathrm{DMSO}-d_{6}, 25{ }^{\circ} \mathrm{C}\right): \delta 184.35,165.53$, $141.57,140.76,140.29$, 133.85, 131.85, 129.60, 128.22, 128.13, 127.07, 126.98, 126.89, 126.11, 
73.03, 71.19, 69.75, 69.74, 69.72, 69.64, 69.51, 57.94, 52.98. HRMS (ESI+): $\mathrm{m} / z$ calcd for $\mathrm{C}_{204} \mathrm{H}_{236} \mathrm{~N}_{8} \mathrm{Na}_{3} \mathrm{O}_{48} \mathrm{Rh}_{2}\left(\mathrm{M}+3 \mathrm{Na}^{+}\right), 1280.1353$; found 1280.1371 .

$(R)-\mathbf{1} \mathbf{a}^{\mathbf{P h}}$-TEG, $(S)$ - and $(R)-\mathbf{1 b}^{\mathbf{P h}}-\mathrm{C} 12,(S)$ - and $(R)-\mathbf{2}^{\mathrm{Me}}-\mathrm{TEG},(S)$ - and $(R)-\mathbf{2} \mathbf{b}^{\mathrm{Me}}-\mathrm{C} 12,(S)-\mathbf{2} \mathbf{b}^{\mathrm{Me}}-$ $\mathrm{C} 12_{\mathrm{NM}}, \mathbf{3 a}^{\mathbf{H}}-\mathrm{TEG}$, and $\mathbf{3} \mathbf{b}^{\mathbf{H}}-\mathrm{C} 12$ were also prepared from $(R)-\mathbf{8}^{\mathbf{P h}}-\mathrm{TEG},(S)-\mathbf{8}^{\mathbf{P h}}-\mathrm{C} 12,(R)-\mathbf{8}^{\mathbf{P h}}-\mathrm{C} 12$, $(S)-\mathbf{8}^{\mathrm{Me}}-\mathrm{TEG},(R)-\mathbf{8}^{\mathrm{Me}}-\mathrm{TEG},(S)-\mathbf{8}^{\mathrm{Me}}-\mathrm{C} 12,(R)-\mathbf{8}^{\mathrm{Me}}-\mathrm{C} 12,(S)-\mathbf{8}^{\mathrm{Me}}-\mathrm{C} 12_{\mathrm{NMe}}, \mathbf{8}^{\mathbf{H}}-\mathrm{TEG}$, and $\mathbf{8}^{\mathbf{H}}-\mathrm{C} 12$, respectively, in the same way for the synthesis of $(S)-\mathbf{1} \mathbf{a}^{\mathbf{P h}}-\mathrm{TEG}$.

Spectroscopic data of (R)-1a ${ }^{\text {Ph }}$-TEG: Green solid. Yield: 94\%. Mp: 126.5-127.4 ${ }^{\circ} \mathrm{C}$. IR $\left(\mathrm{KBr}, \mathrm{cm}^{-}\right.$

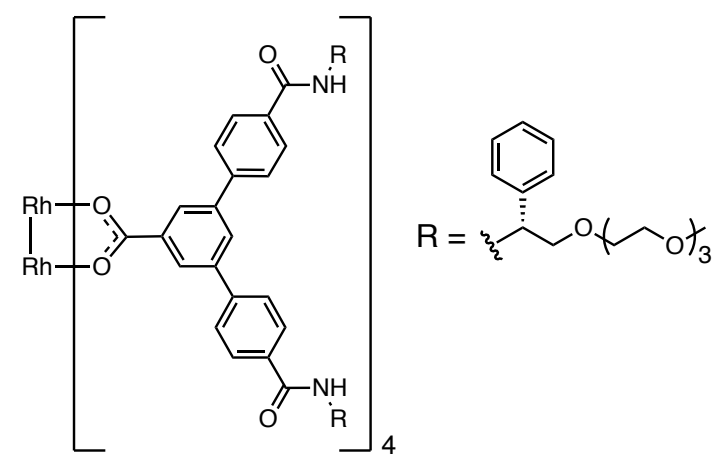

$(R)-\mathbf{1 a}^{\text {Ph }}$-TEG

$\left.{ }^{1}\right): 1639\left(v_{\mathrm{C}=\mathrm{O}}\right), 1576\left(v_{\mathrm{COO}^{-}}\right), 1540\left(v_{\mathrm{N}-\mathrm{H}}\right), 1385$ $\left(v_{\mathrm{COO}}{ }^{-}\right) .{ }^{1} \mathrm{H}$ NMR $\left(500 \mathrm{MHz}, \mathrm{DMSO}-d_{6}, 25^{\circ} \mathrm{C}\right): \delta$ $8.90(\mathrm{~d}, J=8.0 \mathrm{~Hz}, 8 \mathrm{H}, \mathrm{NH}), 8.11$ (br, $12 \mathrm{H}, \mathrm{ArH})$, $8.00(\mathrm{~d}, J=7.8 \mathrm{~Hz}, 16 \mathrm{H}, \mathrm{ArH}), 7.83$ (br, 16H, ArH), 7.44 (d, $J=7.4 \mathrm{~Hz}, 16 \mathrm{H}, \mathrm{ArH}), 7.34$ (t, $J=7.6 \mathrm{~Hz}$, $16 \mathrm{H}, \mathrm{ArH}), 7.25$ (t, $J=7.3 \mathrm{~Hz}, 8 \mathrm{H}, \mathrm{ArH}), 5.31-5.26$ $(\mathrm{m}, 8 \mathrm{H}, \mathrm{NCH}), 3.81-3.40\left(\mathrm{~m}, 96 \mathrm{H}, \mathrm{OCH}_{2}\right), 3.32-$ 3.30 (m, partially overlapping with $\mathrm{H}_{2} \mathrm{O}$ signal, $\left.\mathrm{OCH}_{2}\right), 3.12\left(\mathrm{~s}, 24 \mathrm{H}, \mathrm{OCH}_{3}\right) .{ }^{13} \mathrm{C}$ NMR $\left(126 \mathrm{MHz}, \mathrm{DMSO}-d_{6}, 25{ }^{\circ} \mathrm{C}\right): \delta 184.36,165.55,141.58$, $140.76,140.31,133.85,131.85,129.61,128.23,128.14,127.08,126.99,126.90,126.14,73.04,71.20$, 69.76, 69.74, 69.73, 69.65, 69.52, 57.95, 52.99. HRMS (ESI+): $m / z$ calcd for $\mathrm{C}_{204} \mathrm{H}_{236} \mathrm{~N}_{8} \mathrm{Na}_{3} \mathrm{O}_{48} \mathrm{Rh}_{2}$ $\left(\mathrm{M}+3 \mathrm{Na}^{+}\right), 1280.1353$; found 1280.1356 .

Spectroscopic data of $(S)-\mathbf{1 b}^{\mathrm{Ph}}-\mathrm{C} 12$ : Green solid. Yield: $73 \%$. Mp: $189.7-189.9^{\circ} \mathrm{C}$. IR $\left(\mathrm{KBr}, \mathrm{cm}^{-}\right.$

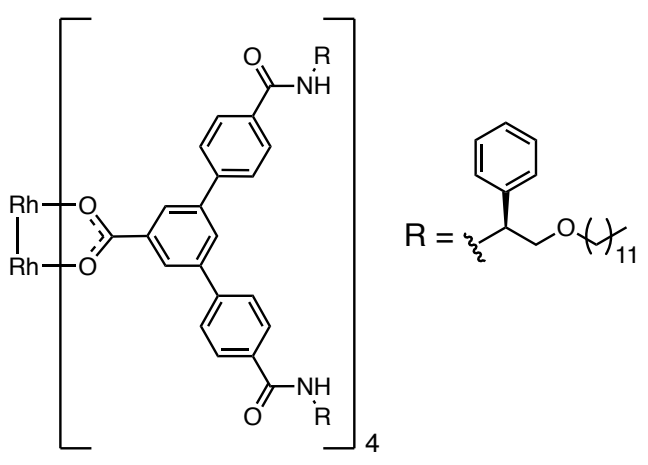

$(S)-\mathbf{1} \mathbf{b}^{\mathrm{Ph}}-\mathrm{C} 12$ $\left.{ }^{1}\right): 1639\left(v_{\mathrm{C}=\mathrm{O}}\right), 1576\left(v_{\mathrm{COO}^{-}}\right), 1534\left(v_{\mathrm{N}-\mathrm{H}}\right), 1385$ $\left(v_{\mathrm{COO}^{-}}\right) \cdot{ }^{1} \mathrm{H}$ NMR $\left(500 \mathrm{MHz}, \mathrm{CDCl}_{3} / \mathrm{DMSO}-d_{6}=\right.$ $\left.3 / 7(\mathrm{v} / \mathrm{v}), 50^{\circ} \mathrm{C}\right): \delta 8.65(\mathrm{~d}, J=8.3 \mathrm{~Hz}, 8 \mathrm{H}, \mathrm{NH})$, 8.12 (s, 8H, ArH), 7.99-7.98 (m, 20H, ArH), 7.70 (s, 16H, ArH), 7.43 (d, $J=7.6 \mathrm{~Hz}, 16 \mathrm{H}, \mathrm{ArH}), 7.31$ (t, $J=7.7 \mathrm{~Hz}, 16 \mathrm{H}, \mathrm{ArH}), 7.23$ (t, $J=7.3 \mathrm{~Hz}, 8 \mathrm{H}, \mathrm{ArH})$, $5.31(\mathrm{q}, J=7.2 \mathrm{~Hz}, 8 \mathrm{H}, \mathrm{NCH}), 3.78$ (t, $J=9.3 \mathrm{~Hz}$, $\left.8 \mathrm{H}, \mathrm{OCH}_{2} \mathrm{CH}\right), 3.65(\mathrm{dd}, J=10.2,5.6 \mathrm{~Hz}, 8 \mathrm{H}$, $\mathrm{OCH}_{2} \mathrm{CH}$ ), 3.50-3.42 (m, $16 \mathrm{H}, \mathrm{OCH}_{2} \mathrm{CH}_{2}$ ), 1.49 (quint, $J=6.9 \mathrm{~Hz}, 16 \mathrm{H}, \mathrm{OCH}_{2} \mathrm{CH}_{2}$ ), 1.27-1.11 (m, $\left.144 \mathrm{H}, \mathrm{CH}_{2}\right), 0.75\left(\mathrm{t}, J=7.1 \mathrm{~Hz}, 24 \mathrm{H}, \mathrm{CH}_{3}\right) .{ }^{13} \mathrm{C} \mathrm{NMR}\left(151 \mathrm{MHz}, \mathrm{CDCl}_{3} / \mathrm{DMSO}^{-} d_{6}=3 / 7(\mathrm{v} / \mathrm{v})\right.$, $\left.50{ }^{\circ} \mathrm{C}\right): \delta 184.53,165.64,141.74,140.82,140.23,133.90,132.07,129.15,128.02,126.98,126.87$, 
126.59, 126.29, 72.80, 70.16, 52.94, 31.25, 29.25, 29.11, 29.01, 28.89, 28.69, 25.73, 22.03, 13.77 . HRMS (ESI+): $m / z$ calcd for $\mathrm{C}_{244} \mathrm{H}_{316} \mathrm{~N}_{8} \mathrm{Na}_{3} \mathrm{O}_{24} \mathrm{Rh}_{2}\left(\mathrm{M}+3 \mathrm{Na}^{+}\right), 1339.0513$; found 1339.0511.

Spectroscopic data of $(R)-\mathbf{1 b}^{\mathrm{Ph}}-\mathrm{C} 12$ : Green solid. Yield: $61 \%$. Mp: $189.9-190.1^{\circ} \mathrm{C} . \mathrm{IR}\left(\mathrm{KBr}, \mathrm{cm}^{-}\right.$

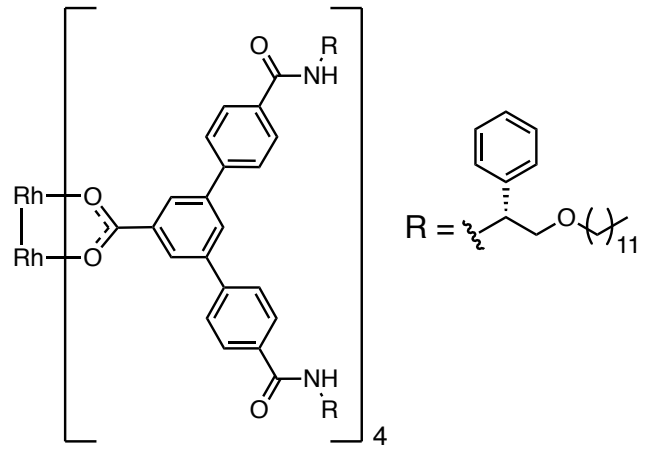

$(R)-\mathbf{1 b}^{\mathrm{Ph}}-\mathrm{C} 12$

$\left.{ }^{1}\right): 1639\left(v_{\mathrm{C}=\mathrm{O}}\right), 1577\left(v_{\mathrm{COO}^{-}}\right), 1534\left(v_{\mathrm{N}-\mathrm{H}}\right), 1385$ $\left(v_{\mathrm{COO}^{-}}\right) \cdot{ }^{1} \mathrm{H} \mathrm{NMR}\left(500 \mathrm{MHz}, \mathrm{CDCl}_{3} / \mathrm{DMSO}^{-} d_{6}=\right.$ $\left.3 / 7(\mathrm{v} / \mathrm{v}), 50{ }^{\circ} \mathrm{C}\right): \delta 8.65(\mathrm{~d}, J=8.1 \mathrm{~Hz}, 8 \mathrm{H}, \mathrm{NH})$, 8.11 (s, 8H, ArH), 7.99-7.98 (m, 20H, ArH), 7.70 (s, $16 \mathrm{H}, \mathrm{ArH}), 7.43(\mathrm{~d}, J=7.6 \mathrm{~Hz}, 16 \mathrm{H}, \mathrm{ArH}), 7.31(\mathrm{t}$, $J=7.6 \mathrm{~Hz}, 16 \mathrm{H}, \mathrm{ArH}), 7.23$ (t, $J=7.3 \mathrm{~Hz}, 8 \mathrm{H}, \mathrm{ArH})$, $5.31(\mathrm{q}, J=7.4 \mathrm{~Hz}, 8 \mathrm{H}, \mathrm{NCH}), 3.78(\mathrm{t}, J=9.3 \mathrm{~Hz}$, $\left.8 \mathrm{H}, \mathrm{OCH}_{2} \mathrm{CH}\right), 3.65(\mathrm{dd}, J=10.1,5.5 \mathrm{~Hz}, 8 \mathrm{H}$, $\mathrm{OCH}_{2} \mathrm{CH}$ ), 3.50-3.42 (m, $\left.16 \mathrm{H}, \mathrm{OCH}_{2} \mathrm{CH}_{2}\right), 1.48$ (quint, $J=6.9 \mathrm{~Hz}, 16 \mathrm{H}, \mathrm{OCH}_{2} \mathrm{CH}_{2}$ ), 1.28-1.11 (m, 144H, $\left.\mathrm{CH}_{2}\right), 0.75\left(\mathrm{t}, J=7.2 \mathrm{~Hz}, 24 \mathrm{H}, \mathrm{CH}_{3}\right) .{ }^{13} \mathrm{C} \mathrm{NMR}\left(151 \mathrm{MHz}, \mathrm{CDCl}_{3} / \mathrm{DMSO}^{-} d_{6}=3 / 7\right.$ (v/v), $\left.50{ }^{\circ} \mathrm{C}\right): \delta 184.53,165.64,141.74,140.82,140.23,133.90,132.07,129.15,128.02,126.98,126.87$, 126.59, 126.29, 72.80, 70.16, 52.94, 31.25, 29.25, 29.11, 29.01, 28.89, 28.69, 25.73, 22.03, 13.77. HRMS (ESI+): $m / z$ calcd for $\mathrm{C}_{244} \mathrm{H}_{316} \mathrm{~N}_{8} \mathrm{Na}_{3} \mathrm{O}_{24} \mathrm{Rh}_{2}\left(\mathrm{M}+3 \mathrm{Na}^{+}\right), 1339.0513$; found 1339.0498 .

Spectroscopic data of (S)-2a $\mathbf{a}^{\mathrm{Me}}$-TEG: Green solid. Yield: 53\%. Mp: 123.1-123.9 ${ }^{\circ} \mathrm{C}$. IR $\left(\mathrm{KBr}, \mathrm{cm}^{-}\right.$

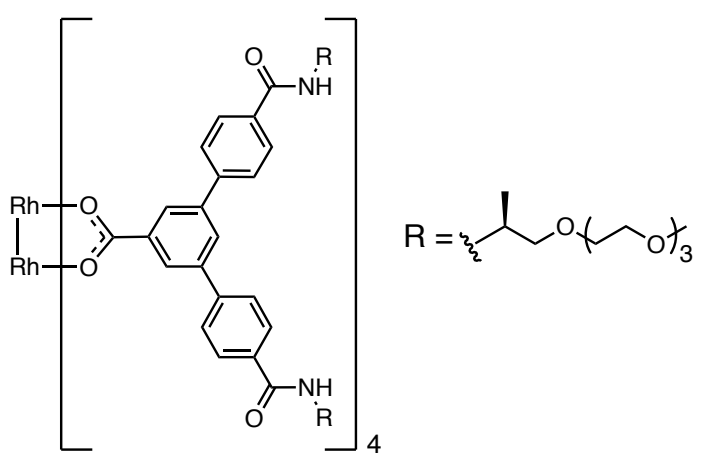

$(S)-\mathbf{2} \mathbf{a}^{\mathbf{M e}}-\mathrm{TEG}$ $\left.{ }^{1}\right): 1638\left(v_{\mathrm{C}=\mathrm{O}}\right), 1577\left(v_{\mathrm{COO}^{-}}\right), 1542\left(\nu_{\mathrm{N}-\mathrm{H}}\right), 1400$ $\left(v_{\mathrm{COO}}{ }^{-}\right) \cdot{ }^{1} \mathrm{H} \mathrm{NMR}\left(500 \mathrm{MHz}, \mathrm{DMSO}-d_{6}, 25^{\circ} \mathrm{C}\right): \delta$ $8.28(\mathrm{~d}, J=7.3 \mathrm{~Hz}, 8 \mathrm{H}, \mathrm{NH}), 8.05-7.71(\mathrm{~m}, 44 \mathrm{H}$, ArH), 4.23-4.18 (m, 8H, NCH), 3.54-3.34 (m, $\left.112 \mathrm{H}, \mathrm{OCH}_{2}\right), 3.16\left(\mathrm{~s}, 24 \mathrm{H}, \mathrm{OCH}_{3}\right), 1.17$ (d, $J=6.6$ $\left.\mathrm{Hz}, 24 \mathrm{H}, \mathrm{CH}_{3}\right) .{ }^{13} \mathrm{C}$ NMR $\left(126 \mathrm{MHz}, \mathrm{DMSO}-d_{6}\right.$, $\left.25{ }^{\circ} \mathrm{C}\right): \delta 184.35,165.25,141.33,140.19,133.98$, $131.75,129.43,127.99,126.77,126.07,73.38$, 71.23, 69.81, 69.76, 69.72, 69.55, 57.98, 44.73, 17.35. HRMS (ESI+): $m / z$ calcd for $\mathrm{C}_{164} \mathrm{H}_{220} \mathrm{~N}_{8} \mathrm{Na}_{3} \mathrm{O}_{48} \mathrm{Rh}_{2}\left(\mathrm{M}+3 \mathrm{Na}^{+}\right), 1114.7602$; found 1114.7622 . 
Spectroscopic data of $(R)-\mathbf{2 a}^{\mathrm{Me}}$-TEG: Green solid. Yield: 40\%. Mp: 122.8-123.8 ${ }^{\circ} \mathrm{C}$. IR $\left(\mathrm{KBr}, \mathrm{cm}^{-}\right.$

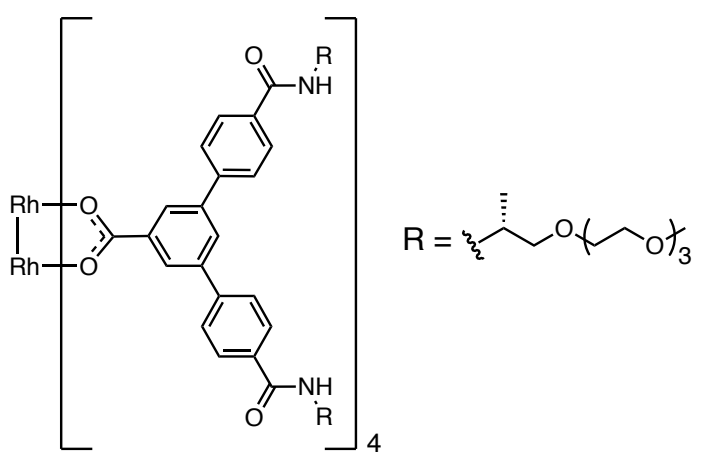

$(R)-\mathbf{2} \mathbf{a}^{\mathrm{Me}}-\mathrm{TEG}$ $\left.{ }^{1}\right): 1637\left(v_{\mathrm{C}=\mathrm{O}}\right), 1578\left(v_{\mathrm{COO}^{-}}\right), 1543\left(v_{\mathrm{N}-\mathrm{H}}\right), 1400$ $\left(v_{\mathrm{COO}}{ }^{-}\right) .{ }^{1} \mathrm{H}$ NMR $\left(500 \mathrm{MHz}, \mathrm{DMSO}-d_{6}, 25^{\circ} \mathrm{C}\right): \delta$ $8.27(\mathrm{~d}, J=7.3 \mathrm{~Hz}, 8 \mathrm{H}, \mathrm{NH}), 8.04-7.70(\mathrm{~m}, 44 \mathrm{H}$, $\mathrm{ArH}), 4.23-4.17(\mathrm{~m}, 8 \mathrm{H}, \mathrm{NCH}), 3.54-3.33(\mathrm{~m}$, $\left.112 \mathrm{H}, \mathrm{OCH}_{2}\right), 3.15\left(\mathrm{~s}, 24 \mathrm{H}, \mathrm{OCH}_{3}\right), 1.16(\mathrm{~d}, J=6.6$ $\left.\mathrm{Hz}, 24 \mathrm{H}, \mathrm{CH}_{3}\right) .{ }^{13} \mathrm{C}$ NMR $\left(126 \mathrm{MHz}, \mathrm{DMSO}-d_{6}\right.$, $\left.25^{\circ} \mathrm{C}\right): \delta 184.33,165.24,141.31,140.17,133.97$, $131.72,129.36,127.97,126.75,126.05,73.38$, 71.23, 69.80, 69.76, 69.72, 69.54, 57.97, 44.72, 17.35. HRMS (ESI+): $\mathrm{m} / z$ calcd for $\mathrm{C}_{164} \mathrm{H}_{220} \mathrm{~N}_{8} \mathrm{Na}_{3} \mathrm{O}_{48} \mathrm{Rh}_{2}\left(\mathrm{M}+3 \mathrm{Na}^{+}\right), 1114.7602$; found 1114.7591 .

Spectroscopic data of $(S)-2 \mathbf{b}^{\mathrm{Me}}-\mathrm{C} 12$ : Green solid. Yield: $55 \%$. Mp: decomposed at $>240{ }^{\circ} \mathrm{C}$. IR

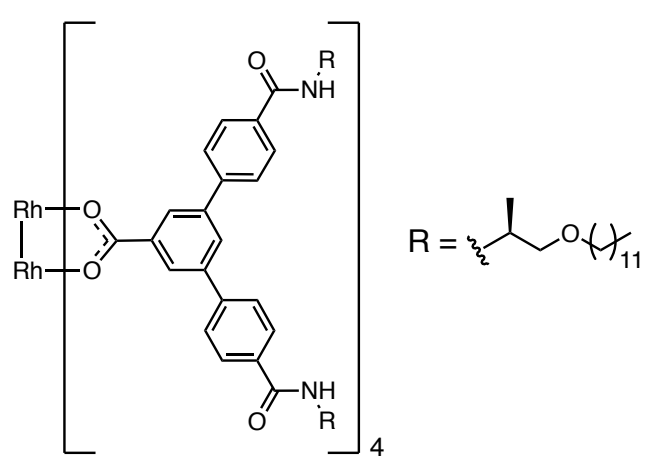

$(S)-\mathbf{2} \mathbf{b}^{\mathrm{Me}}-\mathrm{C} 12$ $\left(\mathrm{KBr}, \mathrm{cm}^{-1}\right): 1638\left(v_{\mathrm{C}=0}\right), 1577\left(v_{\mathrm{COO}^{-}}\right), 1542\left(\nu_{\mathrm{N}-}\right.$ H), $1404\left(v_{\mathrm{COO}^{-}}\right) .{ }^{1} \mathrm{H} \quad \mathrm{NMR} \quad(500 \quad \mathrm{MHz}$, $\left.\mathrm{CDCl}_{3} / \mathrm{DMSO}-d_{6}=1 / 1(\mathrm{v} / \mathrm{v}), 50{ }^{\circ} \mathrm{C}\right): \delta 8.09(\mathrm{~s}, 8 \mathrm{H}$, ArH), 7.92-7.88 (m, 28H, NH, ArH), 7.59 (s, 16H, ArH), 4.29-4.24 (m, 8H, NCH), 3.53-3.37 (m, 32H, $\mathrm{OCH}_{2}$ ), 1.53 (quint, $J=7.0 \mathrm{~Hz}, 16 \mathrm{H}, \mathrm{OCH}_{2} \mathrm{CH}_{2}$ ), 1.34-1.18 (m, 168H, $\left.\mathrm{CH}_{2}, \mathrm{NCHCH}_{3}\right), 0.81$ (t, $J=$ $\left.7.1 \mathrm{~Hz}, \quad 24 \mathrm{H}, \mathrm{CH}_{3}\right) .{ }^{13} \mathrm{C}$ NMR $(151 \mathrm{MHz}$, $\left.\mathrm{CDCl}_{3} / \mathrm{DMSO}-d_{6}=1 / 1(\mathrm{v} / \mathrm{v}), 50{ }^{\circ} \mathrm{C}\right): \delta 184.67,165.64,141.83,140.31,134.17,131.89,128.77$, 127.95, 126.61, 126.44, 73.28, 70.54, 44.94, 31.40, 29.36, 29.21, 29.14, 29.03, 28.82, 25.80, 22.17, 17.39, 13.86. HRMS (ESI+): $m / z$ calcd for $\mathrm{C}_{204} \mathrm{H}_{300} \mathrm{~N}_{8} \mathrm{Na}_{3} \mathrm{O}_{24} \mathrm{Rh}_{2}\left(\mathrm{M}+3 \mathrm{Na}^{+}\right), 1173.6762$; found 1173.6761.

Spectroscopic data of $(R)-2 \mathbf{b}^{\mathrm{Me}}-\mathrm{C} 12$ : Green solid. Yield: $25 \%$. Mp: decomposed at $>240{ }^{\circ} \mathrm{C}$. IR

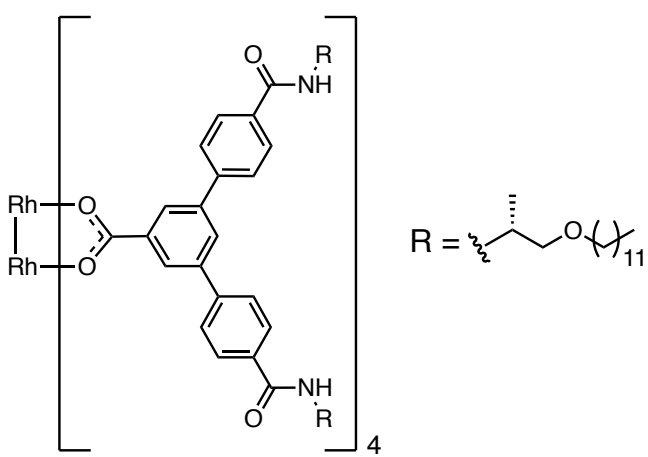

$(R)-\mathbf{2} \mathbf{b}^{\mathrm{Me}}-\mathrm{C} 12$ $\left(\mathrm{KBr}, \mathrm{cm}^{-1}\right): 1637\left(v_{\mathrm{C}=0}\right), 1577\left(v_{\mathrm{COO}^{-}}\right), 1542\left(v_{\mathrm{N}-}\right.$ H), $\quad 1400\left(v_{\mathrm{COO}^{-}}\right) . \quad{ }^{1} \mathrm{H} \quad \mathrm{NMR} \quad(500 \quad \mathrm{MHz}$, $\left.\mathrm{CDCl}_{3} / \mathrm{DMSO}-d_{6}=1 / 1(\mathrm{v} / \mathrm{v}), 50{ }^{\circ} \mathrm{C}\right): \delta 8.12(\mathrm{~s}, 8 \mathrm{H}$, ArH), 7.94-7.88 (m, 28H, NH, ArH), 7.64 (s, 16H, ArH), 4.29-4.23 (m, 8H, NCH), 3.52-3.36 (m, 32H, $\mathrm{OCH}_{2}$ ), 1.53 (quint, $J=7.0 \mathrm{~Hz}, 16 \mathrm{H}, \mathrm{OCH}_{2} \mathrm{CH}_{2}$ ), 1.32-1.18 (m, 168H, $\left.\mathrm{CH}_{2}, \mathrm{NCHCH}_{3}\right), 0.81$ (t, $J=$ 
$\left.7.3 \mathrm{~Hz}, 24 \mathrm{H}, \mathrm{CH}_{3}\right) .{ }^{13} \mathrm{C} \mathrm{NMR}\left(151 \mathrm{MHz}, \mathrm{CDCl}_{3} / \mathrm{DMSO}-d_{6}=1 / 1(\mathrm{v} / \mathrm{v}), 50{ }^{\circ} \mathrm{C}\right): \delta 184.67,165.67$, $141.84,140.31,134.17,131.89,128.77,127.93,126.61,126.46,73.29,70.56,44.96,31.40,29.37$, 29.22, 29.14, 29.04, 28.83, 25.81, 22.18, 17.40, 13.86. HRMS (ESI+): $\mathrm{m} / \mathrm{z}$ calcd for $\mathrm{C}_{204} \mathrm{H}_{300} \mathrm{~N}_{8} \mathrm{Na}_{3} \mathrm{O}_{24} \mathrm{Rh}_{2}\left(\mathrm{M}+3 \mathrm{Na}^{+}\right)$, 1173.6762; found 1173.6752 .

Spectroscopic data of $\mathbf{3 a}^{\mathrm{H}}$-TEG: Green solid. Yield: $68 \%$. Mp: 123.9-124.8 ${ }^{\circ} \mathrm{C}$. IR $\left(\mathrm{KBr}, \mathrm{cm}^{-1}\right)$ :

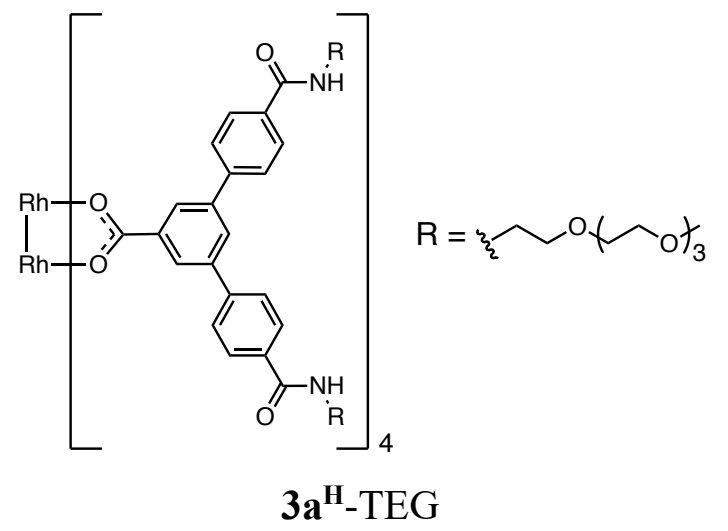
$1646\left(v_{\mathrm{C}=\mathrm{O}}\right), 1577\left(v_{\mathrm{COO}^{-}}\right), 1542\left(v_{\mathrm{N}-\mathrm{H}}\right), 1385$ $\left(v_{\mathrm{COO}^{-}}\right) \cdot{ }^{1} \mathrm{H}$ NMR $\left(500 \mathrm{MHz}, \mathrm{DMSO}-d_{6}, 25^{\circ} \mathrm{C}\right): \delta$ $8.59(\mathrm{t}, J=5.5 \mathrm{~Hz}, 8 \mathrm{H}, \mathrm{NH}), 8.08-8.05(\mathrm{~m}, 12 \mathrm{H}$, ArH), 7.95 (d, $J=8.5 \mathrm{~Hz}, 16 \mathrm{H}, \mathrm{ArH}), 7.78$ (d, $J=$ $6.5 \mathrm{~Hz}, 16 \mathrm{H}, \mathrm{ArH}), 3.57-3.45\left(\mathrm{~m}, 112 \mathrm{H}, \mathrm{OCH}_{2}\right)$, 3.38-3.35 (m, 16H, $\left.\mathrm{OCH}_{2}\right), 3.17\left(\mathrm{~s}, 24 \mathrm{H}, \mathrm{OCH}_{3}\right)$. ${ }^{13} \mathrm{C}$ NMR (126 MHz, DMSO- $\left.d_{6}, 25^{\circ} \mathrm{C}\right): \delta 184.29$, $165.74,141.43,140.21,133.74,131.85,129.47$, 127.91, 126.89, 126.05, 71.23, 69.79, 69.76, 69.73, 69.64, 69.54, 68.86, 57.98. One of ${ }^{13} \mathrm{C}$ signals could not be identified because of overlap with the solvent. HRMS (ESI+): $\mathrm{m} / \mathrm{z}$ calcd for $\mathrm{C}_{156} \mathrm{H}_{204} \mathrm{~N}_{8} \mathrm{Na}_{3} \mathrm{O}_{48} \mathrm{Rh}_{2}\left(\mathrm{M}+3 \mathrm{Na}^{+}\right)$, 1077.3852; found 1077.3843 .

Spectroscopic data of $3 \mathbf{b}^{\mathrm{H}}-\mathrm{C} 12$ : Green solid. Yield: $51 \%$. Mp: decomposed at $>240{ }^{\circ} \mathrm{C}$. IR $(\mathrm{KBr}$,

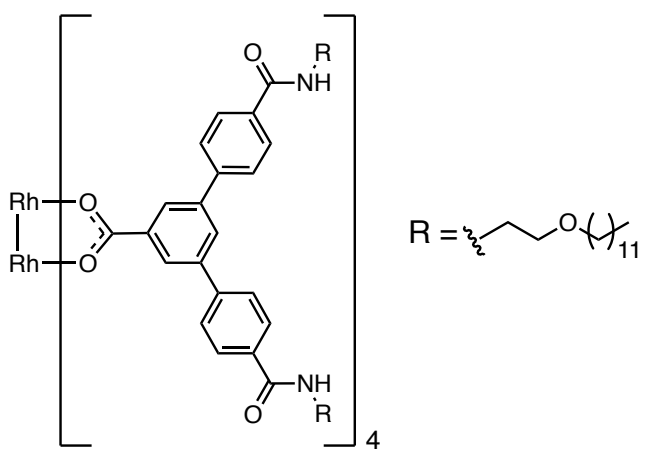

$3 \mathbf{b}^{\mathrm{H}}-\mathrm{C} 12$ $\left.\mathrm{cm}^{-1}\right): 1638\left(v_{\mathrm{C}=\mathrm{O}}\right), 1542\left(v_{\mathrm{COO}^{-}}\right), 1507\left(v_{\mathrm{N}-\mathrm{H}}\right), 1403$ $\left(v_{\mathrm{COO}^{-}}\right) .{ }^{1} \mathrm{H} \mathrm{NMR}\left(500 \mathrm{MHz}, \mathrm{CDCl}_{3} / \mathrm{DMSO}^{-} d_{6}(1 / 1\right.$, $\left.\mathrm{v} / \mathrm{v}), 50^{\circ} \mathrm{C}\right): \delta 8.21(\mathrm{t}, J=5.4 \mathrm{~Hz}, 8 \mathrm{H}, \mathrm{NH}), 8.13(\mathrm{~d}$, $J=1.5 \mathrm{~Hz}, 8 \mathrm{H}, \mathrm{ArH}), 7.95-7.88(\mathrm{~m}, 20 \mathrm{H}, \mathrm{ArH})$, $7.65(\mathrm{~d}, J=8.1 \mathrm{~Hz}, 16 \mathrm{H}, \operatorname{ArH}), 3.56(\mathrm{t}, J=6.0 \mathrm{~Hz}$, $\left.16 \mathrm{H}, \mathrm{NCH}_{2} \mathrm{CH}_{2}\right), 3.50\left(\mathrm{q}, J=5.5 \mathrm{~Hz}, 16 \mathrm{H}, \mathrm{NCH}_{2}\right)$, $3.44\left(\mathrm{t}, J=6.6 \mathrm{~Hz}, 16 \mathrm{H}, \mathrm{OCH}_{2} \mathrm{CH}_{2}\right), 1.56-1.51$ (m, $\left.16 \mathrm{H}, \mathrm{OCH}_{2} \mathrm{CH}_{2}\right), 1.32-1.11\left(\mathrm{~m}, 144 \mathrm{H}, \mathrm{CH}_{2}\right), 0.81$ $\left(\mathrm{t}, J=7.0 \mathrm{~Hz}, 24 \mathrm{H}, \mathrm{CH}_{3}\right) .{ }^{13} \mathrm{C} \mathrm{NMR}\left(126 \mathrm{MHz}, \mathrm{CDCl}_{3} / \mathrm{DMSO}^{-} d_{6}(1 / 1, \mathrm{v} / \mathrm{v}), 50{ }^{\circ} \mathrm{C}\right): \delta 185.03,166.54$, $142.20,140.60,134.27,132.55,129.37,128.17,126.97,126.73,79.07,70.84,69.06,39.84,31.70$, 29.67, 29.50, 29.44, 29.36, 29.11, 26.09, 22.47, 14.13. HRMS (ESI+): $m / z$ calcd for $\mathrm{C}_{196} \mathrm{H}_{284} \mathrm{~N}_{8} \mathrm{Na}_{3} \mathrm{O}_{24} \mathrm{Rh}_{2}\left(\mathrm{M}+3 \mathrm{Na}^{+}\right), 1136.3012$; found 1136.3031 . 
An $N$-methylated analogue of $(S)-\mathbf{2} \mathbf{b}^{\mathrm{Me}}-\mathrm{C} 12\left((S)-\mathbf{2} \mathbf{b}^{\mathrm{Me}}-\mathrm{C} 12_{\mathrm{NMe}}\right)$ and its complex with DABCO were synthesized according to Scheme S1.

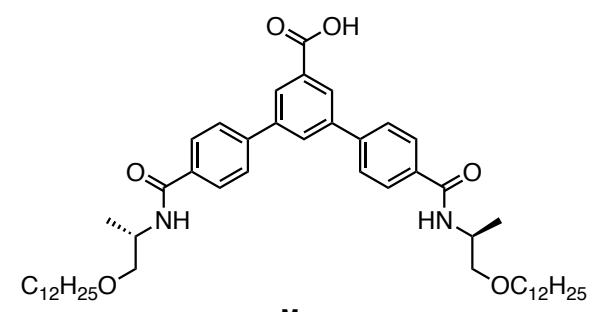

(S)-8 $\mathbf{8}_{-\mathrm{C}} 12$

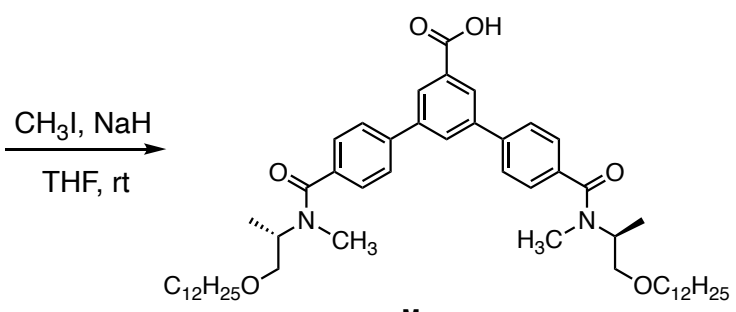

$(S)-8^{\mathrm{Me}}-\mathrm{C} 12_{\mathrm{NMe}}$
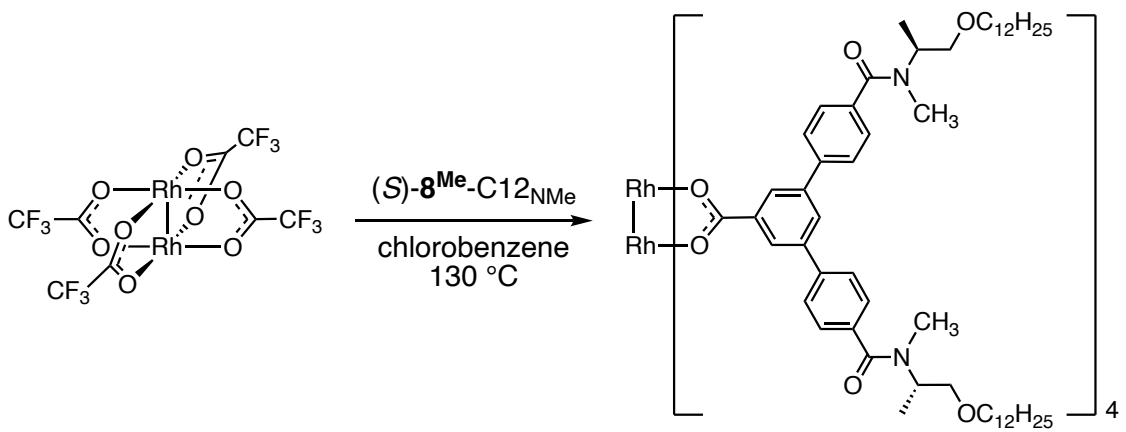

(S) $-\mathbf{2} \mathbf{b}^{\mathrm{Me}}-\mathrm{C} 12_{\mathrm{NMe}}$

Scheme S1. Syntheses of an optically active dirhodium(II) paddlewheel complex $\left((S)-\mathbf{2} \mathbf{b}^{\mathrm{Me}}-\mathrm{C} 12_{\mathrm{NMe}}\right)$.

Synthesis of $(\boldsymbol{S})-8^{\mathrm{Me}}-\mathbf{C 1 2} \mathbf{N M e}_{\mathrm{N}}$. Into a three-neck round bottom flask containing $\mathrm{NaH}(60 \%$ in<smiles>CC(COC(C)(C)C)N(C)C(=O)c1ccc(-c2ccc(-c3ccc(C(=O)O)cc3)cc2)cc1</smiles>

$(S)-\mathbf{8}^{\mathrm{Me}}-\mathrm{C} 12_{\mathrm{NMe}}$ mineral oil) (25 mg, $0.61 \mathrm{mmol})$ was added dry THF (5 mL) at room temperature under argon. After $20 \mathrm{~min}$, to this was added $(S)-8^{\mathrm{Me}}-\mathrm{C} 12(120 \mathrm{mg}, 145 \mu \mathrm{mol})$ at $0{ }^{\circ} \mathrm{C}$ under nitrogen. The reaction mixture was stirred at room temperature for $30 \mathrm{~min}$. To this was added iodomethane $(45 \mathrm{mg}, 0.32 \mathrm{mmol})$ under nitrogen and the reaction mixture was stirred at room temperature for $5 \mathrm{~h}$. After quenching the reaction with water and evaporating the solvent, the residue was diluted with $\mathrm{CHCl}_{3}$ and the solution was washed with water and brine. The organic layer was dried over anhydrous $\mathrm{MgSO}_{4}$, filtered, and concentrated under reduced pressure. The crude product was purified by flash column chromatography $\left(\mathrm{SiO}_{2}, \mathrm{CHCl}_{3} / \mathrm{MeOH}=19 / 1\right.$, v/v) to give $(S)-\mathbf{8}^{\mathrm{Me}}-\mathrm{C} 12_{\mathrm{NMe}}(110 \mathrm{mg}, 89 \%)$ as a yellow oil. IR (neat, $\left.\mathrm{cm}^{-1}\right): 1709\left(v_{\mathrm{C}=0}\right), 1635\left(v_{\mathrm{C}=0}\right)$, $1543\left(v_{\mathrm{N}-\mathrm{H}}\right) .{ }^{1} \mathrm{H}$ NMR $\left(500 \mathrm{MHz}, \mathrm{DMSO}-d_{6}, 80{ }^{\circ} \mathrm{C}\right): \delta 8.22(\mathrm{~d}, J=1.8 \mathrm{~Hz}, 2 \mathrm{H}, \mathrm{ArH}), 8.20(\mathrm{~s}, 1 \mathrm{H}$, $\mathrm{COOH}), 8.17(\mathrm{t}, J=1.7 \mathrm{~Hz}, 1 \mathrm{H}, \mathrm{ArH}), 7.83(\mathrm{~d}, J=8.2 \mathrm{~Hz}, 4 \mathrm{H}, \mathrm{ArH}), 7.49$ (d, $J=8.2 \mathrm{~Hz}, 4 \mathrm{H}, \operatorname{ArH})$, $4.29(\mathrm{~s}, 2 \mathrm{H}, \mathrm{NCH}), 3.54-3.34\left(\mathrm{~m}, 8 \mathrm{H}, \mathrm{OCH}_{2} \mathrm{CH}_{2}\right), 2.86\left(\mathrm{~s}, 6 \mathrm{H}, \mathrm{NCH}_{3}\right), 1.53$ (quint, $J=7.0 \mathrm{~Hz}, 4 \mathrm{H}$, 
$\left.\mathrm{OCH}_{2} \mathrm{CH}_{2}\right), 1.33-1.23\left(\mathrm{~m}, 36 \mathrm{H}, \mathrm{CH}_{2}\right), 1.14\left(\mathrm{~d}, J=7.0 \mathrm{~Hz}, 6 \mathrm{H}, \mathrm{NCHCH}_{3}\right), 0.84(\mathrm{t}, J=7.0 \mathrm{~Hz}, 6 \mathrm{H}$, $\left.\mathrm{CH}_{3}\right) .{ }^{13} \mathrm{C}$ NMR $\left(126 \mathrm{MHz}, \mathrm{DMSO}-d_{6}, 80{ }^{\circ} \mathrm{C}\right): \delta 170.00,166.36,140.25,139.31,136.58,132.30$, $128.64,126.83,126.31,126.16,78.61,70.54,69.95,30.66,28.69,28.41,28.35,28.23,28.02,25.12$, 21.38, 13.85, 13.11. HRMS (ESI-): $m / z$ calcd for $\mathrm{C}_{53} \mathrm{H}_{79} \mathrm{~N}_{2} \mathrm{O}_{6}\left(\mathrm{M}-\mathrm{H}^{+}\right), 839.5944$; found 839.5935.

Synthesis of $(\boldsymbol{S})-2 \mathbf{b}^{\mathrm{Me}}-\mathbf{C} 12_{\mathrm{NMe}}(S)-2 \mathbf{b}^{\mathrm{Me}}-\mathrm{C} 12_{\mathrm{NMe}}$ was prepared from $(S)-\mathbf{8}^{\mathrm{Me}}-\mathrm{C} 12_{\mathrm{NMe}}$ in the same way for the synthesis of $(S)-\mathbf{1} \mathbf{a}^{\text {Ph }}-$ TEG.

Spectroscopic data of $(S)-\mathbf{2} \mathbf{b}^{\mathrm{Me}}-\mathrm{C} 12_{\mathrm{NMe}}$ : Green solid. Yield: $62 \%$. Mp: decomposed at $>240{ }^{\circ} \mathrm{C}$.

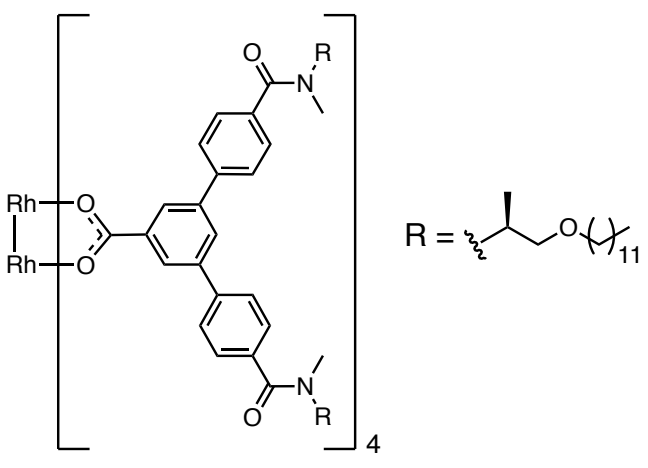

$(S)-\mathbf{2} \mathbf{b}^{\mathrm{Me}}-\mathrm{C} 12_{\mathrm{NMe}}$ IR $\left(\mathrm{KBr}, \mathrm{cm}^{-1}\right): 1637\left(v_{\mathrm{C}=\mathrm{O}}\right), 1577\left(v_{\mathrm{COO}^{-}}\right), 1400\left(v_{\mathrm{COO}^{-}}\right)$. ${ }^{1} \mathrm{H}$ NMR $\left(500 \mathrm{MHz}, \mathrm{CDCl}_{3} / \mathrm{DMSO}-d_{6}=1 / 1(\mathrm{v} / \mathrm{v})\right.$, $\left.50{ }^{\circ} \mathrm{C}\right): \delta 8.24(\mathrm{~d}, J=1.7 \mathrm{~Hz}, 8 \mathrm{H}, \mathrm{ArH}), 8.08(\mathrm{t}, J=1.7$ $\mathrm{Hz}, 4 \mathrm{H}, \mathrm{ArH}), 7.77$ (d, $J=8.3 \mathrm{~Hz}, 16 \mathrm{H}, \mathrm{ArH}), 7.49$ (d, $J$ $=8.3 \mathrm{~Hz}, 16 \mathrm{H}, \mathrm{ArH}), 5.10-4.59$ (br, $2 \mathrm{H}, \mathrm{NCH}), 4.23-3.80$ (br, 6H, NCH), 3.65-3.20 (br, 32H, $\mathrm{OCH}_{2}$ ), 2.89 (s, 24H, $\mathrm{NCH}_{3}$ ), 1.56-1.51 (quint, $J=7.0 \mathrm{~Hz}, 16 \mathrm{H}, \mathrm{OCH}_{2} \mathrm{CH}_{2}$ ), 1.32-1.15 (m, 168H, $\left.\mathrm{NCHCH}_{3}, \mathrm{CH}_{2}\right), 0.84$ (t, $J=7.1 \mathrm{~Hz}$, $\left.24 \mathrm{H}, \mathrm{CH}_{3}\right) .{ }^{13} \mathrm{C} \mathrm{NMR}\left(126 \mathrm{MHz}, \mathrm{CDCl}_{3} / \mathrm{DMSO}_{-} d_{6}=1 / 1(\mathrm{v} / \mathrm{v}), 50{ }^{\circ} \mathrm{C}\right): \delta 184.70,167.21,140.86$, $140.24,136.83$, 132.68, 129.32, 127.39, 127.02, 126.87, 78.80, 71.08, 70.73, 53.29, 31.45, 29.37, 29.20, 29.16, 29.01, 28.84, 25.81, 22.21, 14.74, 13.87. HRMS (ESI+): $\mathrm{m} / z$ calcd for $\mathrm{C}_{212} \mathrm{H}_{316} \mathrm{~N}_{8} \mathrm{Na}_{3} \mathrm{O}_{24} \mathrm{Rh}_{2}\left(\mathrm{M}+3 \mathrm{Na}^{+}\right), 1211.0513$; found 1211.0541 . 
Polymerization. Coordination-driven polymerization of dirhodium complexes (1-3) with 1,4diazabicyclo[2.2.2] octane (DABCO) were carried out according to Scheme S2 in a dry Schlenk flask under a dry nitrogen atmosphere. A typical procedure for the polymerization of $(S)$-1 $\mathbf{a}^{\mathbf{P h}}$-TEG with DABCO is described as follows.
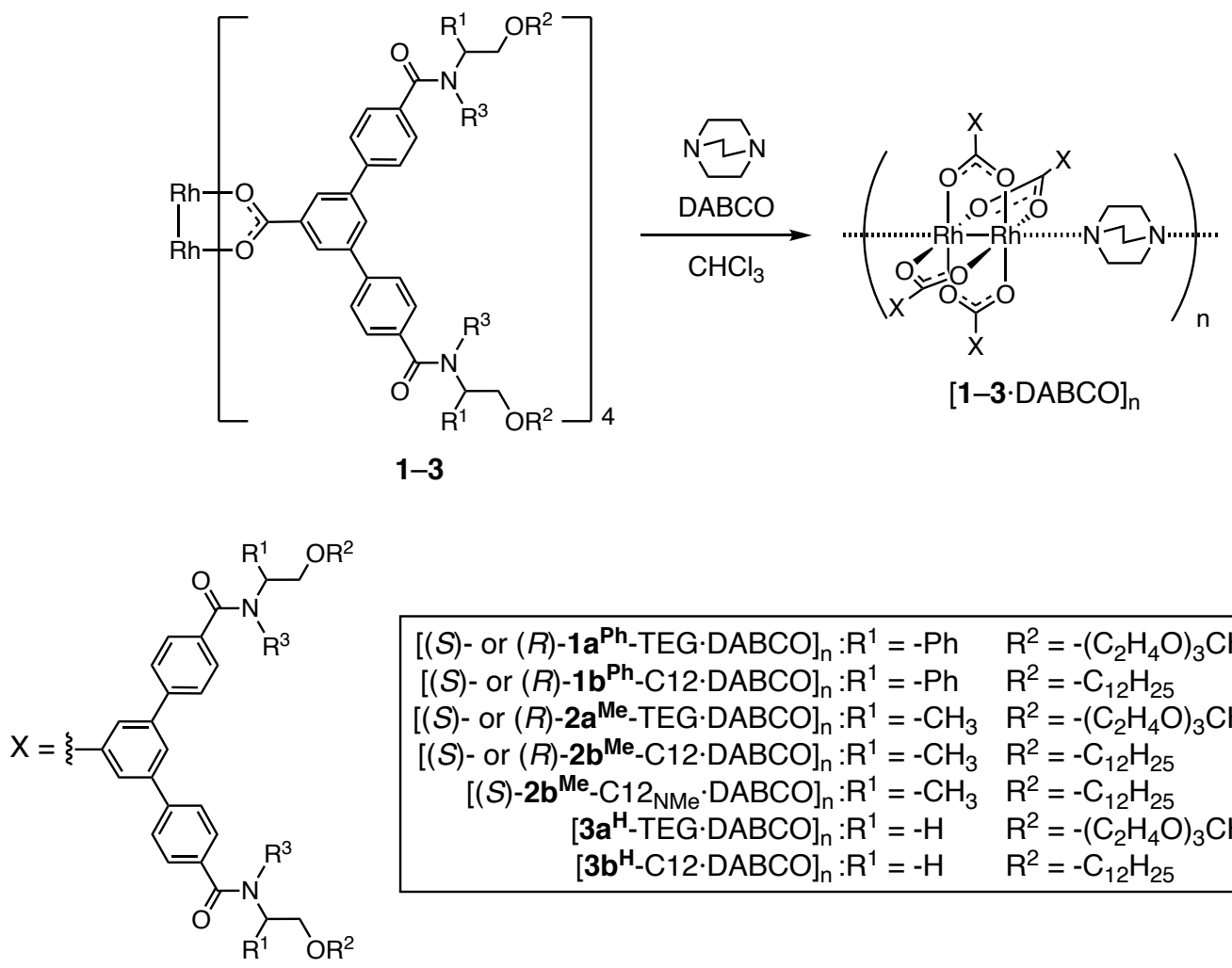

\begin{tabular}{|c|c|c|}
\hline $\begin{aligned} {\left[(S)-\text { or }(R)-1 \mathbf{a}^{\mathrm{Ph}}-\mathrm{TEG} \cdot \mathrm{DABCO}\right]_{\mathrm{n}}: \mathrm{R}^{1} } & =-\mathrm{Ph} \\
{\left[(S)-\text { or }(R)-\mathbf{1} \mathbf{b}^{\mathrm{Ph}}-\mathrm{C} 12 \cdot \mathrm{DABCO}\right]_{\mathrm{n}}: \mathrm{R}^{1} } & =-\mathrm{Ph} \\
{\left[(S)-\text { or }(R)-\mathbf{2} \mathbf{a}^{\mathrm{Me}}-\mathrm{TEG} \cdot \mathrm{DABCO}\right]_{\mathrm{n}}: \mathrm{R}^{1} } & =-\mathrm{CH}_{3} \\
{\left[(S)-\text { or }(R)-\mathbf{2} \mathbf{b}^{\mathrm{Me}}-\mathrm{C} 12 \cdot \mathrm{DABCO}\right]_{\mathrm{n}}: \mathrm{R}^{1} } & =-\mathrm{CH}_{3} \\
{\left[(S)-\mathbf{2} \mathbf{b}^{\mathrm{Me}}-\mathrm{C} 1 \mathrm{NMe}_{\mathrm{NMe}} \cdot \mathrm{DABCO}\right]_{\mathrm{n}}: \mathrm{R}^{1} } & =-\mathrm{CH}_{3} \\
{\left[3 \mathbf{a}^{\mathrm{H}}-\mathrm{TEG} \cdot \mathrm{DABCO}\right]_{\mathrm{n}}: \mathrm{R}^{1} } & =-\mathrm{H} \\
{\left[3 \mathbf{b}^{\mathrm{H}}-\mathrm{C} 12 \cdot \mathrm{DABCO}\right]_{\mathrm{n}}: \mathrm{R}^{1} } & =-\mathrm{H}\end{aligned}$ & $\begin{array}{l}R^{2}=-\left(\mathrm{C}_{2} \mathrm{H}_{4} \mathrm{O}\right)_{3} \mathrm{CH}_{3} \\
R^{2}=-\mathrm{C}_{12} \mathrm{H}_{25} \\
\mathrm{R}^{2}=-\left(\mathrm{C}_{2} \mathrm{H}_{4} \mathrm{O}\right)_{3} \mathrm{CH}_{3} \\
\mathrm{R}^{2}=-\mathrm{C}_{12} \mathrm{H}_{25} \\
\mathrm{R}^{2}=-\mathrm{C}_{12} \mathrm{H}_{25} \\
\mathrm{R}^{2}=-\left(\mathrm{C}_{2} \mathrm{H}_{4} \mathrm{O}\right)_{3} \mathrm{CH}_{3} \\
\mathrm{R}^{2}=-\mathrm{C}_{12} \mathrm{H}_{25}\end{array}$ & $\begin{array}{l}\mathrm{R}^{3}=-\mathrm{H} \\
\mathrm{R}^{3}=-\mathrm{H} \\
\mathrm{R}^{3}=-\mathrm{H} \\
\mathrm{R}^{3}=-\mathrm{H} \\
\mathrm{R}^{3}=-\mathrm{CH}_{3} \\
\mathrm{R}^{3}=-\mathrm{H} \\
\mathrm{R}^{3}=-\mathrm{H}\end{array}$ \\
\hline
\end{tabular}

Scheme S2. Syntheses of optically active $\left(\left[(S) \text { - or }(R)-\mathbf{1} \mathbf{a}^{\mathbf{P h}}-\mathrm{TEG} \cdot \mathrm{DABCO}\right]_{\mathrm{n}},\left[(S)-\right.\right.$ or $(R)-\mathbf{1 b}^{\mathbf{P h}}$ $\mathrm{C} 12 \cdot \mathrm{DABCO}]_{\mathrm{n}},\left[(S)-\text { or }(R)-\mathbf{2} \mathbf{a}^{\mathbf{M e}}-\mathrm{TEG} \cdot \mathrm{DABCO}\right]_{\mathrm{n}},\left[(S)-\text { or }(R)-\mathbf{2} \mathbf{b}^{\mathbf{M e}}-\mathrm{C} 12 \cdot \mathrm{DABCO}\right]_{\mathrm{n}}$, and $\left[(S)-\mathbf{2} \mathbf{b}^{\mathbf{M e}}\right.$ $\left.\left.\mathrm{C} 12_{\mathrm{NMe}} \cdot \mathrm{DABCO}\right]_{\mathrm{n}}\right)$ and achiral $\left(\left[\mathbf{3 a}^{\mathbf{H}}-\mathrm{TEG} \cdot \mathrm{DABCO}\right]_{\mathrm{n}}\right.$ and $\left.\left[\mathbf{3 b}^{\mathbf{H}}-\mathrm{C} 12 \cdot \mathrm{DABCO}\right]_{\mathrm{n}}\right)$ dirhodium(II) paddlewheel-based coordination polymers.

$(S)-1 a^{\text {Ph }}$-TEG $(54.0 \mathrm{mg}, 14.3 \mu \mathrm{mol})$ and an excess amount of DABCO (4.5 mg, $\left.40 \mu \mathrm{mol}\right)$ were placed in a dry Schlenk flask, which was then evacuated on a vacuum line and flushed with dry nitrogen. After this evacuation-flush procedure was repeated three times, anhydrous $\mathrm{CHCl}_{3}(2 \mathrm{~mL})$ was added using a syringe. After stirring at room temperature for $5 \mathrm{~min}$, the solvent was evaporated to dryness under reduced pressure. To this was added a mixture of ethanol/n-hexane (ca. 1/3, v/v) (ca. $10 \mathrm{~mL}$ ) and the resulting suspension was sonicated. The residue was collected by centrifugation to remove excess amounts of DABCO by decantation. This procedure was repeated twice and the residual solid was then dried under vacuum to give $\left[(S)-\mathbf{1} \mathbf{a}^{\mathbf{P h}}-\mathrm{TEG} \cdot \mathrm{DABCO}\right]_{\mathrm{n}}(53.6 \mathrm{mg}, \mathbf{9 6} \%)$ as a 
pink solid. Anal. Calcd for $\mathrm{C}_{204} \mathrm{H}_{236} \mathrm{~N}_{8} \mathrm{O}_{48} \mathrm{Rh}_{2} \cdot \mathrm{C}_{6} \mathrm{H}_{12} \mathrm{~N}_{2}$ : C, 64.91; H, 6.43; N, 3.60; Found: C, 64.93; $\mathrm{H}, 6.46 ; \mathrm{N}, 3.61$.

$\left[(R)-\mathbf{1} \mathbf{a}^{\mathbf{P h}}-\mathrm{TEG} \cdot \mathrm{DABCO}\right]_{\mathrm{n}}, \quad\left[(S)-\mathbf{1 b}^{\mathbf{P h}}-\mathrm{C} 12 \cdot \mathrm{DABCO}\right]_{\mathrm{n}}, \quad\left[(R)-\mathbf{1 b}^{\mathbf{P h}}-\mathrm{C} 12 \cdot \mathrm{DABCO}\right]_{\mathrm{n}}, \quad\left[(S)-\mathbf{2 a}^{\mathbf{M e}}-\right.$ $\mathrm{TEG} \cdot \mathrm{DABCO}]_{\mathrm{n}},\left[(R)-\mathbf{2} \mathbf{a}^{\mathbf{M e}}-\mathrm{TEG} \cdot \mathrm{DABCO}\right]_{\mathrm{n}},\left[(S)-\mathbf{2 b}^{\mathbf{M e}}-\mathrm{C} 12 \cdot \mathrm{DABCO}\right]_{\mathrm{n}},\left[(R)-\mathbf{2} \mathbf{b}^{\mathbf{M e}}-\mathrm{C} 12 \cdot \mathrm{DABCO}\right]_{\mathrm{n}}$, $\left[(S)-\mathbf{2} \mathbf{b}^{\mathrm{Me}}-\mathrm{C} 12_{\mathrm{NMe}} \cdot \mathrm{DABCO}\right]_{\mathrm{n}},\left[\mathbf{3 a}^{\mathbf{H}}-\mathrm{TEG} \cdot \mathrm{DABCO}\right]_{\mathrm{n}}$, and $\left[\mathbf{3 b}^{\mathbf{H}}-\mathrm{C} 12 \cdot \mathrm{DABCO}\right]_{\mathrm{n}}$ were also prepared from $(R)-\mathbf{1} \mathbf{a}^{\mathbf{P h}}-\mathrm{TEG},(S)-\mathbf{1 b}^{\mathbf{P h}}-\mathrm{C} 12,(R)-\mathbf{1 b}^{\mathbf{P h}}-\mathrm{C} 12,(S)-\mathbf{2 a}^{\mathrm{Me}}-\mathrm{TEG},(R)-\mathbf{a}^{\mathbf{M e}}-\mathrm{TEG},(S)-\mathbf{2} \mathbf{b}^{\mathrm{Me}}-\mathrm{C} 12,(R)$ $\mathbf{2} \mathbf{b}^{\mathrm{Me}}-\mathrm{C} 12,(S)-\mathbf{2} \mathbf{b}^{\mathrm{Me}}-\mathrm{C} 12_{\mathrm{NMe}}, \mathbf{3} \mathbf{a}^{\mathbf{H}}-\mathrm{TEG}$, and $\mathbf{3} \mathbf{b}^{\mathbf{H}}-\mathrm{C} 12$, respectively, in the same way for the synthesis of $\left[(S)-\mathbf{1} \mathbf{a}^{\mathbf{P h}}-\mathrm{TEG} \cdot \mathrm{DABCO}\right]_{\mathrm{n}}$.

$\left[(R)-\mathbf{1 a}^{\mathrm{Ph}}-\mathrm{TEG} \cdot \mathrm{DABCO}\right]_{\mathrm{n}}$ : Pink solid. Yield: 94\%. Anal. Calcd for $\mathrm{C}_{204} \mathrm{H}_{236} \mathrm{~N}_{8} \mathrm{O}_{48} \mathrm{Rh}_{2} \cdot \mathrm{C}_{6} \mathrm{H}_{12} \mathrm{~N}_{2}$ : C, 64.91; H, 6.43; N, 3.60; Found: C, 64.92; H, 6.32; N, 3.65.

[(S)-1b $\left.{ }^{\mathrm{Ph}}-\mathrm{C} 12 \cdot \mathrm{DABCO}\right]_{\mathrm{n}}$ : Pink solid. Yield: $98 \%$. Anal. Calcd for $\mathrm{C}_{244} \mathrm{H}_{316} \mathrm{~N}_{8} \mathrm{O}_{24} \mathrm{Rh}_{2} \cdot \mathrm{C}_{6} \mathrm{H}_{12} \mathrm{~N}_{2}: \mathrm{C}$, 73.94; H, 8.16; N, 3.44; Found: C, 73.94; H, 8.34; N, 3.51.

[(R)-1 $\left.\mathbf{b}^{\mathrm{Ph}}-\mathrm{C} 12 \cdot \mathrm{DABCO}\right]_{\mathrm{n}}$ : Pink solid. Yield: $97 \%$. Anal. Calcd for $\mathrm{C}_{244} \mathrm{H}_{316} \mathrm{~N}_{8} \mathrm{O}_{24} \mathrm{Rh}_{2} \cdot \mathrm{C}_{6} \mathrm{H}_{12} \mathrm{~N}_{2}: \mathrm{C}$, 73.94; H, 8.16; N, 3.44; Found: C, 73.93; H, 8.27; N, 3.50.

[(S)-2a $\mathbf{a}^{\text {Me }}$ TEG $\left.\cdot \mathrm{DABCO}\right]_{\mathrm{n}}$ : Pink solid. Yield: $98 \%$. Anal. Calcd for $\mathrm{C}_{164} \mathrm{H}_{220} \mathrm{~N}_{8} \mathrm{O}_{48} \mathrm{Rh}_{2} \cdot \mathrm{C}_{6} \mathrm{H}_{12} \mathrm{~N}_{2}$ : C, 60.24; H, 6.90; N, 4.13; Found: C, 60.20; H, 7.19; N, 4.37.

$\left[(R)-\mathbf{2 a} \mathbf{a}^{\mathrm{Me}}-\mathrm{TEG} \cdot \mathrm{DABCO}\right]_{\mathrm{n}}$ : Pink solid. Yield: $98 \%$. Anal. Calcd for $\mathrm{C}_{164} \mathrm{H}_{220} \mathrm{~N}_{8} \mathrm{O}_{48} \mathrm{Rh}_{2} \cdot \mathrm{C}_{6} \mathrm{H}_{12} \mathrm{~N}_{2}$ : C, 60.24; H, 6.90; N, 4.13; Found: C, 60.21; H, 6.91; N, 4.24.

[(S)-2b $\left.\mathbf{b}^{\mathrm{Me}}-\mathrm{C} 12 \cdot \mathrm{DABCO}\right]_{\mathrm{n}}$ : Pink solid. Yield: $98 \%$. Anal. Calcd for $\mathrm{C}_{204} \mathrm{H}_{300} \mathrm{~N}_{8} \mathrm{O}_{24} \mathrm{Rh}_{2} \cdot \mathrm{C}_{6} \mathrm{H}_{12} \mathrm{~N}_{2}: \mathrm{C}_{\text {, }}$ 70.72; H, 8.82; N, 3.93; Found: C,70.73; H, 8.76; N, 3.88 .

[(R)-2b $\left.\mathbf{b}^{\mathrm{Me}}-\mathrm{C} 12 \cdot \mathrm{DABCO}\right]_{\mathrm{n}}$ : Pink solid. Yield: $98 \%$. Anal. Calcd for $\mathrm{C}_{204} \mathrm{H}_{300} \mathrm{~N}_{8} \mathrm{O}_{24} \mathrm{Rh}_{2} \cdot \mathrm{C}_{6} \mathrm{H}_{12} \mathrm{~N}_{2}$ : C, 70.72; H, 8.82; N, 3.93; Found: C, 70.71; H, 8.94; N, 3.90.

$\left[(S)-\mathbf{2 b}^{\mathrm{Me}}-\mathrm{C} 12_{\mathrm{NMe}} \cdot \mathrm{DABCO}\right]_{\mathrm{n}}$ : Pink solid. Yield: $98 \%$. Anal. Calcd for $\mathrm{C}_{212} \mathrm{H}_{316} \mathrm{~N}_{8} \mathrm{O}_{24} \mathrm{Rh}_{2} \cdot \mathrm{C}_{6} \mathrm{H}_{12} \mathrm{~N}_{2}$ : C, 71.17; H, 8.99; N, 3.81; Found: C, 71.19; H, 9.00; N, 3.90.

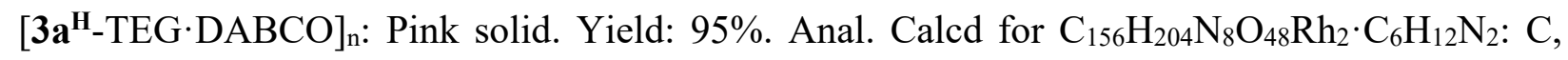
59.37; H, 6.64; N, 4.27; Found: C, 59.38; H, 6.52; N, 4.11.

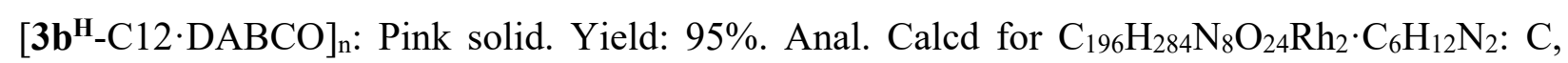
70.23; H, 8.64; N, 4.05; Found: C, 70.19; H, 8.67; N, 4.10. 
Dehydrated solvents were used for all measurements.

3. General Procedures for AFM Measurements of Supramolecular Homopolymers of (S)Dirhodium(II) Paddlewheels Complexed with DABCO

A typical experimental procedure for the AFM measurements of $\left[(S)-\mathbf{1 a}^{\mathbf{P h}}-\mathrm{TEG} \cdot \mathrm{DABCO}\right]_{\mathrm{n}}$ is described below. A stock solution of $\left[(S)-\mathbf{1} \mathbf{a}^{\mathbf{P h}}-\mathrm{TEG} \cdot \mathrm{DABCO}\right]_{\mathrm{n}}$ in $\mathrm{CHCl}_{3} / n$-hexane $(1 / 1, \mathrm{v} / \mathrm{v})(0.70$ $\mathrm{mM})$ was prepared. Samples for the AFM measurements of $\left[(S)-\mathbf{1} \mathbf{a}^{\mathbf{P h}}-\mathrm{TEG} \cdot \mathrm{DABCO}\right]_{\mathrm{n}}$ were prepared by spin casting $(1000 \mathrm{rpm}) 10 \mu \mathrm{L}$ aliquots of the stock solution on freshly cleaved highly oriented pyrolytic graphite (HOPG) at room temperature (ca. $25^{\circ} \mathrm{C}$ ). After the substrates were dried under vacuum for $1 \mathrm{~h}$, the HOPG substrates were then exposed to $\mathrm{CHCl}_{3}$ vapors at room temperature for 36 $\mathrm{h}$, and the substrates were dried under vacuum for $2 \mathrm{~h}$. The organic solvent vapor was prepared by putting $1 \mathrm{~mL}$ of $\mathrm{CHCl}_{3}$ into a $2 \mathrm{~mL}$ flask that was inside a $50 \mathrm{~mL}$ flask, and the HOPG substrates were then placed in the $50 \mathrm{~mL}$ flask. The typical settings of the AFM measurements for the highmagnification observations using a Nanoscope V microscope and a Cypher ES microscope were as follows: a free amplitude of the oscillation frequency of 280 and $30-600 \mathrm{mV}$, a set-point amplitude of 180-250 and 30-600 mV, and a scan rate of 2.5 and $1.0 \mathrm{~Hz}$, respectively. The Nanoscope image processing software (Nanoscope V) or Igor Pro version 16 (Cypher ES) software was used for the image analysis. The AFM images of $\left[(S)-\mathbf{1 a}^{\mathbf{P h}}-\mathrm{TEG} \cdot \mathrm{DABCO}\right]_{\mathrm{n}},\left[(S)-\mathbf{1} \mathbf{b}^{\mathbf{P h}}-\mathrm{C} 12 \cdot \mathrm{DABCO}\right]_{\mathrm{n}},\left[(S)-\mathbf{2 a}^{\mathbf{M e}}\right.$ TEG·DABCO $]_{\mathrm{n}}$, and $\left[(S)-\mathbf{2} \mathbf{b}^{\mathbf{M e}}-\mathrm{C} 12 \cdot \mathrm{DABCO}\right]_{\mathrm{n}}$ shown in Figures $3 \mathrm{a}$ and S16 were measured using Nanoscope V and/or Cypher ES microscopes, respectively. AFM images showing similar 2D bundle structures were observed independent of the microscopes.

\section{Preparation of the Oriented Fibers of $\left[(S)-1 a^{\mathrm{Ph}}-\mathrm{TEG} \cdot \mathrm{DABCO}\right]_{\mathrm{n}}$}

The oriented fibers of $\left[(S)-\mathbf{1} \mathbf{a}^{\mathbf{P h}}-\mathrm{TEG} \cdot \mathrm{DABCO}\right]_{\mathrm{n}}$ were prepared by pulling a highly viscous liquid crystalline polymer at ca. $200{ }^{\circ} \mathrm{C}$ with tweezers followed by rapid cooling to room temperature, and then the WAXD of the fibers was measured.

\section{General Procedures for "Sergeants and Soldiers" and "Majority Rule" Experiments}

A typical experimental procedure is described below. Stock solutions of $(S)-\mathbf{1 a}^{\mathbf{P h}}$-TEG DABCO

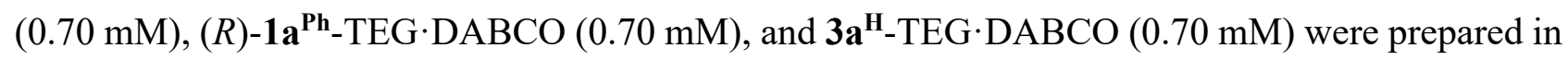
$\mathrm{CHCl}_{3} / n$-hexane $(1 / 1, \mathrm{v} / \mathrm{v})$. For the sergeants and soldiers experiments, aliquots of the stock solutions of $(S)-\mathbf{1} \mathbf{a}^{\mathbf{P h}}$-TEG.DABCO and $\mathbf{3} \mathbf{a}^{\mathbf{H}}-$ TEG $\cdot$ DABCO were placed in eight vessels at room temperature so that the molar fraction of the $(S)-\mathbf{1 a}^{\mathrm{Ph}}$-TEG in the mixtures became $1,2.5,5,10,30,50,70$, and $90 \mathrm{~mol} \%$, respectively. After the solutions were mixed using a vibrator, the absorption and CD spectra 
were then measured at 50,25 , and $-10^{\circ} \mathrm{C}$ in this order for each vessel (Figures $8 b(\mathrm{i})$ and S27a). For the majority rule experiments, aliquots of the stock solutions of $(S)-\mathbf{1 a}^{\mathbf{P h}}-\mathrm{TEG} \cdot \mathrm{DABCO}$ and $(R)-\mathbf{1 a}^{\mathbf{P h}}$ TEG.DABCO were placed in four vessels at room temperature so that the percent ee of the mixtures ( $S$-rich) was 25, 50, 75, and 90, respectively. After the solutions were mixed using a vibrator, the absorption and $\mathrm{CD}$ spectra were measured at 50,25 , and $-10^{\circ} \mathrm{C}$ in this order for each vessel (Figures 8c(i) and S32a). In a similar way, the sergeants and soldiers and majority rule experiments were performed for other $(S)$ - and achiral-dirhodium-DABCO mixtures and $(S)$ - and $(R)$ dirhodium $\cdot \mathrm{DABCO}$ mixtures $\left(S\right.$-rich) in $\mathrm{CHCl}_{3} / n$-hexane $(1 / 1, \mathrm{v} / \mathrm{v})$, respectively (Figures $8, \mathrm{~S} 27, \mathrm{~S} 29$, and S32). For the $(S)-\mathbf{1 b}^{\mathbf{P h}}-\mathrm{C} 12 \cdot \mathrm{DABCO}$ and $\mathbf{3} \mathbf{b}^{\mathbf{H}}-\mathrm{C} 12 \cdot \mathrm{DABCO}$ and the $(S)-\mathbf{1 b}^{\mathbf{P h}}-\mathrm{C} 12 \cdot \mathrm{DABCO}$ and $(R)-\mathbf{1 b}^{\mathbf{P h}}-\mathrm{C} 12 \cdot \mathrm{DABCO}$ mixed systems, $\mathrm{CHCl}_{3} / n$-hexane $(1 / 2, \mathrm{v} / \mathrm{v})$ was also used as a mixed solvent for the sergeants and soldiers and majority rule experiments (Figures $8 \mathrm{~b}(\mathrm{v})$ and $\mathrm{S} 30$ ).

\section{Theoretical Analysis of Temperature-Dependent CD Intensity Changes: Supramolecular}

\section{Polymerization Mechanism}

Temperature (T)-dependent CD intensity changes at the 1st Cotton effect (295 or $288 \mathrm{~nm}$ for $(S)$ 1 and $(S)$-2, respectively) were monitored at a cooling rate of $0.1 \mathrm{~K} / \mathrm{min}$ except for $(S)-\mathbf{1 a} \cdot \mathrm{DABCO}$ $(0.2 \mathrm{~K} / \mathrm{min})$ to identify the supramolecular polymerization mechanisms of four $(S)$-dirhodium(II) paddlewheels with $\mathrm{DABCO}$ in $\mathrm{CHCl}_{3} / n$-hexane $(1 / 1, \mathrm{v} / \mathrm{v})$ according to the methods reported by Schenning, Meijer, and co-workers. ${ }^{\text {S4-5 }}$ The $T$-dependent CD intensity changes were first converted to the normalized degree of polymerization $\left(\phi_{\mathrm{n}}\right)\left(\Delta \varepsilon / \Delta \varepsilon_{\max }\right.$ at 295 or $\left.288 \mathrm{~nm}\right)$ based on the observed $(\Delta \varepsilon)$ and maximum molar ellipticities $\left(\Delta \varepsilon_{\max }\right)$ for each sample, which were then plotted as a function of temperature $(T)$ (Figure 5). The resulting $\phi_{\mathrm{n}}-T$ profiles were then fitted with the theoretical curves for the isodesmic and cooperative (nucleation-elongation) supramolecular polymerization models according to equation (eq) S1 and eqs S2 and S3, respectively, from which the thermodynamic parameters characterizaing the supramolecular polymerization process can be obtained. ${ }^{\text {S4-5 }}$ Graphing and analysis software (DataGraph ver. 4.6.1) was used to fit eqs S1-S3 to the experimentally observed $\phi_{\mathrm{n}}-T$ profiles. Based on the absorption titration results of $(S)-\mathbf{1 a}^{\mathbf{P h}}-\mathrm{TEG}$ and $(S)-\mathbf{1 b}^{\mathbf{M e}}-\mathrm{C} 12$ with QND in $\mathrm{CHCl}_{3}$ at $50{ }^{\circ} \mathrm{C}$ (Figures S21 and S22) along with the variable-temperature absorption spectral changes of the supramolecular helical coordination polymers in $\mathrm{CHCl}_{3}$ (Figure S24), we assumed that the supramolecular helical coordination polymers dissociate into a 1:1 complex with DABCO in $\mathrm{CHCl}_{3} / n$-hexane $(1 / 1, \mathrm{v} / \mathrm{v})$ at high temperatures, but do not dissociate into free dirhodium carboxylates and DABCO. 


\section{Isodesmic model}

$$
\phi_{n}=\frac{1}{1+\exp \left(-0.908 \Delta H \frac{T-T_{m}}{R T_{m}^{2}}\right)}
$$

in which $T$ is the absolute temperature, $T_{\mathrm{m}}$ is the melting temperature defined as the temperature for which $\phi_{\mathrm{n}}=0.5, \Delta H$ is the molar enthalpy release related to the formation of noncovalent intermolecular interactions, and $R$ is the gas constant. ${ }^{\mathrm{S} 5}$

\section{Cooperative (Nucleation-elongation) model}

In the elongation regime $\left(T<T_{\mathrm{e}}\right)$, the $\phi_{\mathrm{n}}$ is given by eq $\mathrm{S} 2$

$$
\phi_{n}=\phi_{S A T}\left\{1-\exp \left[\frac{-h_{e}}{R T_{e}{ }^{2}}\left(T-T_{e}\right)\right]\right\}
$$

in which $T_{\mathrm{e}}$ is the elongation temperature, $h_{\mathrm{e}}$ is the molecular enthalpy release due to noncovalent interactions during elongation, and $\phi_{\mathrm{SAT}}$, which is introduced as a parameter to ensure that $\phi_{\mathrm{n}} / \phi_{\mathrm{SAT}}$ does not exceed unity.

In the nucleation regime $\left(T>T_{\mathrm{e}}\right)$, that is, at temperatures above the elongation temperature $T_{\mathrm{e}}$, the $\phi_{\mathrm{n}}$ is described by eq $\mathrm{S} 3$

$$
\phi_{n}=\sqrt[3]{K_{a}} \exp \left[\left(\frac{2}{3 \cdot \sqrt[3]{K_{a}}}-1\right) \frac{h_{e}}{R T_{e}{ }^{2}}\left(T-T_{e}\right)\right]
$$

in which three parameters $\left(h_{\mathrm{e}}, T_{\mathrm{e}}\right.$, and $\left.\phi_{\mathrm{SAT}}\right)$ can be determined with eq $\mathrm{S} 2$ and $K_{\mathrm{a}}$ is the dimensionless equilibrium constant of the activation step at $T_{\mathrm{e}}$., which displays the degree of cooperativity and can be estimated by modelling the onset of the $\phi_{\mathrm{n}}$ at $T_{\mathrm{e}}{ }^{\mathrm{S} 4-5}$

The average length of the stack, $N_{\mathrm{n}}\left(T_{\mathrm{e}}\right)$, averaged over the nucleated species, at $T_{\mathrm{e}}$ was estimated according to eq S4 derived from eq S3 with four parameters $\left(h_{\mathrm{e}}, T_{\mathrm{e}}, \phi_{\mathrm{SAT}}\right.$, and $\left.K_{\mathrm{a}}\right)$.

$$
<N_{n}\left(T_{e}\right)>=\frac{1}{\sqrt[3]{K_{a}}}
$$


In the elongation regime, the number-averaged degree of polymerization, averaged over all active species, $N_{\mathrm{n}}$, was estimated according to eq S5 derived from eq S2 with three parameters $\left(h_{\mathrm{e}}, T_{\mathrm{e}}\right.$, and $\left.\phi_{\mathrm{SAT}}\right)$.

$$
<N_{n}(T)>=\frac{1}{\sqrt{K_{a}}} \sqrt{\frac{\phi_{n}}{\phi_{S A T}-\phi_{n}}}
$$


Table S1. Thermodynamic Parameters for Supramolecular Coordination Polymers of $(S)-1 \mathrm{a}-(S)-2 \mathrm{~b}$ with $\mathrm{DABCO}_{\text {in }} \mathrm{CHCl} / \mathrm{n}-\mathrm{Hexane}$ $(1 / 1, v / v)$ Determined by Cooperative (Nucleation-Elongation) Model

\begin{tabular}{|c|c|c|c|c|c|c|c|c|}
\hline complex & $\begin{array}{l}\text { cooling } \\
\text { rate } \\
\left({ }^{\circ} \mathrm{C} / \mathrm{min}\right)\end{array}$ & $\begin{array}{c}\text { monitering } \\
\text { wavelength } \\
(\mathrm{nm})\end{array}$ & $\begin{array}{c}c_{\mathrm{T}} \\
(\mathrm{mM})\end{array}$ & $\phi \phi_{\mathrm{SAT}}$ & $\begin{array}{c}T_{\mathrm{e}} \\
(\mathrm{K})\end{array}$ & $\begin{array}{c}h_{\mathrm{e}} \\
(\mathrm{kJ} / \mathrm{mol})\end{array}$ & $K_{\mathrm{a}}$ & $<N_{n}\left(T_{\mathrm{e}}\right)>$ \\
\hline$\left[(S)-\mathbf{1} \mathbf{a}^{\mathbf{P h}}-\mathrm{TEG} \cdot \mathrm{DABCO}\right]_{\mathrm{n}}$ & 0.2 & $295^{a}$ & 0.7 & 1.052 & 308 & -66.4 & $1.94 \times 10^{-3}$ & 8 \\
\hline$\left[(S)-\mathbf{1 b}^{\mathbf{P h}}-\mathrm{C} 12 \cdot \mathrm{DABCO}\right]_{\mathrm{n}}$ & 0.1 & $295^{a}$ & 0.7 & 1.004 & 318 & -57.6 & $1.27 \times 10^{-4}$ & 20 \\
\hline$\left[(S)-\mathbf{2} \mathbf{a}^{\mathbf{M e}}-\mathrm{TEG} \cdot \mathrm{DABCO}\right]_{\mathrm{n}}$ & 0.1 & $288^{a}$ & 0.7 & 1.111 & 315 & -37.0 & $2.13 \times 10^{-4}$ & 17 \\
\hline$\left[(S)-\mathbf{2} \mathbf{b}^{\mathbf{M e}}-\mathrm{C} 12 \cdot \mathrm{DABCO}\right]_{\mathrm{n}}$ & 0.1 & $288^{a}$ & 0.7 & 1.032 & 318 & -49.5 & $1.20 \times 10^{-4}$ & 13 \\
\hline$\left[(S)-\mathbf{2} \mathbf{b}^{\mathbf{M e}}-\mathrm{C} 12 \cdot \mathrm{DABCO}\right]_{\mathrm{n}}{ }^{b}$ & - & $538^{c}$ & 0.7 & 1.021 & 320 & -52.8 & $-d$ & $-d$ \\
\hline
\end{tabular}

$\overline{a_{m}}$-Terphenyl region. ${ }^{b}$ Estimated based on the $T$-dependent $\mathrm{CD}$ spectral changes in the visible absorption region measured at every $2{ }^{\circ} \mathrm{C}$ in the cooling process from 55 to $-11{ }^{\circ} \mathrm{C}$ (Figure S11). ${ }^{c}$ Visible absorption region. ${ }^{d}$ Not estimated due to a small number of data points. 


\subsection{CD and/or Absorption Titrations of $(S)-1 \mathrm{a}^{\mathrm{Ph}}-\mathrm{TEG}-(S)-2 \mathrm{~b}^{\mathrm{Me}}-\mathrm{C} 12$ with $\mathrm{QND}$ and DABCO in $\mathrm{CHCl}_{3} / n$-Hexane $(1 / 1, \mathrm{v} / \mathrm{v})$ or $\mathrm{CHCl}_{3}$ at 25,50 , or $55^{\circ} \mathrm{C}$}

A typical experimental procedure for the absorption titration of $(S)-\mathbf{1 a}^{\mathbf{P h}}-\mathrm{TEG}$ with QND in $\mathrm{CHCl}_{3}$ at $50{ }^{\circ} \mathrm{C}$ is described below. Stock solutions of $(S)-\mathbf{1 a}^{\mathrm{Ph}}$-TEG $(0.70 \mathrm{mM} / 4.5 \mathrm{~mL})$ in $\mathrm{CHCl}_{3}$ and QND $(7.0 \mathrm{mM} / 3.0 \mathrm{~mL})$ in $\mathrm{CHCl}_{3}$ containing $(S)-\mathbf{1} \mathbf{a}^{\mathbf{P h}}$-TEG $(0.70 \mathrm{mM})$ were prepared. A $1.0 \mathrm{~mL}$ aliquot of the stock solution of $(S)-\mathbf{1} \mathbf{a}^{\mathbf{P h}}$-TEG was placed into a $1.0-\mathrm{cm}$ quartz cell. To this was added increasing amounts of the stock solution of QND $(0.1-6.1$ equiv. $)$ so as to maintain the $(S)-\mathbf{1 a}^{\mathbf{P h}}$ TEG concentration at $0.70 \mathrm{mM}$. The absorption spectra were measured for each addition of QND at $50{ }^{\circ} \mathrm{C}$ (Figure S21). In a similar manner, the absorption titrations of $(S)-\mathbf{1 a}^{\mathbf{P h}}-\mathrm{TEG}-(S)-\mathbf{2} \mathbf{b}^{\mathbf{M e}}-\mathrm{C} 12$ with QND in $\mathrm{CHCl}_{3} / n$-hexane $(1 / 1, \mathrm{v} / \mathrm{v})$ or $\mathrm{CHCl}_{3}$ at $50{ }^{\circ} \mathrm{C}$ (Figures $\mathrm{S} 22$ and $\mathrm{S} 23$ ) and $\mathrm{CD}$ and absorption titrations of $(S)-\mathbf{1} \mathbf{a}^{\mathbf{P h}}-\mathrm{TEG}-(S)-\mathbf{2} \mathbf{b}^{\mathbf{M e}}-\mathrm{C} 12$ with DABCO in $\mathrm{CHCl}_{3} / n$-hexane (1/1, v/v) at 25 and $50^{\circ} \mathrm{C}$ (Figures S19 and S20) were also performed.

\subsection{Estimation of $K_{1}$ and $K_{2}$ by Absorption Titrations of $(S)-1 a^{\mathrm{Ph}}-\mathrm{TEG}$ and $(S)-1 b^{\mathrm{Me}}-\mathrm{C} 12$ with QND in $\mathrm{CHCl}_{3}$ at $50^{\circ} \mathrm{C}$}

As reported in the literature, ${ }^{\mathrm{S} 6-10}$ the dirhodium(II) carboxylates form 1:1 and 1:2 complexes with Lewis bases, such as piperidine, in nonpolar solvents, such as benzene and dichloromethane; the first binding constants $\left(K_{1}\right)$ are very high (ca. $10^{9}$ and ca. $10^{8}$, respectively) and the second binding constants $\left(K_{2}\right)$ are more than four to five orders of magnitude lower than those of the $K_{1}$ values. If this is the case, the absorption spectra of the dirhodium(II) carboxylates change in a stepwise reaction manner with an increase in the concentration of Lewis bases through two specific isosbestic points corresponding to the 1:1 complex formation from the free dirhodium followed by the 1:2 complex formation, ${ }^{\mathrm{S} 6-9}$ as observed in the absorption titrations of $(S)-\mathbf{1 a} \mathbf{a}^{\mathbf{P h}}-\mathrm{TEG}$ with QND in $\mathrm{CHCl}_{3}$ at $50{ }^{\circ} \mathrm{C}$ (Figure S21a,b). The first binding constant $K_{1}$ of $(S)-\mathbf{1 a}^{\mathbf{P h}}-\mathrm{TEG}$ with QND was then roughly estimated based on a 1:1 equilibrium system using the absorbance intensity changes at 608 and $570 \mathrm{~nm}$ in the presence of $0-1.0$ equiv. of QND that correspond to the maximum absorption wavelength $\left(\lambda_{\max }\right)$ values for the free $(S)-\mathbf{1 a}^{\mathbf{P h}}-\mathrm{TEG}$ and its 1:1 complex with QND $\left(\mathrm{Rh}_{2} \cdot \mathrm{QND}\right)$, respectively, to be greater than $10^{7} \mathrm{M}^{-1}$ by comparison with the simulated absorbance intensity changes with different $K_{1}$ values according to eq S6 (Figure S21c,d). 


$$
\begin{aligned}
& \mathrm{Rh}_{2}+\mathrm{QND} \underset{\mathrm{CHCl}_{3}}{\stackrel{K_{1}}{\rightleftarrows}} \mathrm{Rh}_{2} \cdot \mathrm{QND} \quad K_{1}=\frac{\left[\mathrm{Rh}_{2} \cdot \mathrm{QND}\right]}{\left[\mathrm{Rh}_{2}\right][\mathrm{QND}]} \\
& \Delta A b s_{1}=A b S_{o b s}-A b S_{R h} \\
& =\frac{\left(\mathrm{AbS}_{\mathrm{Rh} \cdot \mathrm{QND}}-\mathrm{AbS \textrm {Rh }}\right)}{2 K_{1}\left[\mathrm{Rh}_{2}\right]_{0}}\left[1+K_{1}\left[\mathrm{Rh}_{2}\right]_{0}+K_{1}[\mathrm{QND}]_{0}-\left\{\left(1+K_{1}\left[\mathrm{Rh}_{2}\right]_{0}+K_{\mathrm{a}}[\mathrm{QND}]_{0}\right)^{2}-4 K_{1}^{2}\left[\mathrm{Rh}_{2}\right]_{0}[\mathrm{QND}]_{0}\right\}^{1 / 2}\right]
\end{aligned}
$$

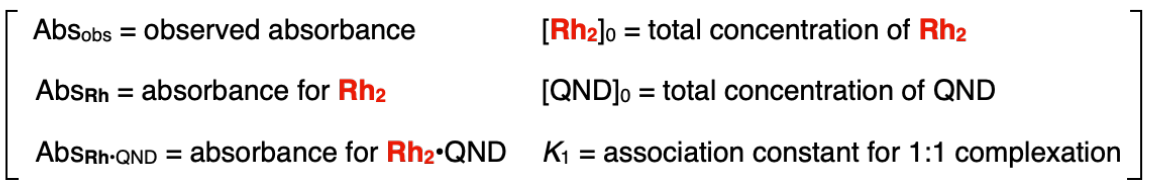

The second binding constant $K_{2}$ was then estimated based on a 1:1 equilibrium system between the 1:1 and 1:2 complex formations using the absorbance intensity changes at 570 and $527 \mathrm{~nm}$ in the presence of $1.0-6.1$ equiv. of QND that correspond to the maximum absorption wavelength $\left(\lambda_{\max }\right)$ values for the 1:1 $\left(\mathrm{Rh}_{2} \cdot \mathrm{QND}\right)$ and 1:2 $\left(\mathrm{Rh}_{2} \cdot(\mathrm{QND})_{2}\right)$ complexes of $(S)-\mathbf{a}^{\mathbf{P h}}-\mathrm{TEG}$ with QND, respectively, by the nonlinear least-squares curve-fittings to be $1.22 \times 10^{4}$ and $6.96 \times 10^{3} \mathrm{M}^{-1}$, respectively, according to eq S7 (Figure S21e,f). During the calculation of the $K_{2}$ values, we presumed that the 1:1 complex of $(S)-\mathbf{1} \mathbf{a}^{\mathrm{Ph}}-\mathrm{TEG}$ with $\mathrm{QND}\left(\mathrm{Rh}_{2} \cdot \mathrm{QND}\right)$ would be quantitatively formed in the presence of 1.0 equiv. of QND because of the sufficiently high $K_{1}\left(>10^{7}\right)$.

$$
\begin{aligned}
& \mathrm{Rh}_{2} \cdot \mathrm{QND}+\mathrm{QND} \underset{\mathrm{CHCl}_{3}}{\stackrel{K_{2}}{\rightleftarrows}} \mathrm{Rh}_{2} \cdot(\mathrm{QND})_{2} \quad K_{2}=\frac{\left[\mathrm{Rh}_{2} \cdot(\mathrm{QND})_{2}\right]}{\left[\mathrm{Rh}_{2} \cdot \mathrm{QND}\right][\mathrm{QND}]} \\
& \Delta A b s_{2 .}=A b S_{\text {obs }}-A b S_{\mathrm{Rh} \cdot \mathrm{QND}} \\
& =\frac{\left(\mathrm{AbS}_{\mathrm{Rh} \cdot(\mathrm{QND}) 2}-\mathrm{AbS} \mathrm{Rh} \cdot \mathrm{QND}\right)}{2 K_{2}\left[\mathrm{Rh} h_{2} \cdot \mathrm{QND}\right]_{0}}\left[1+K_{2}\left[\mathrm{Rh}_{2} \cdot \mathrm{QND}\right]_{0}+K_{2}[\mathrm{QND}]_{0}-\left\{\left(1+K_{2}\left[\mathrm{Rh} h_{2} \cdot \mathrm{QND}\right]_{0}+K_{2}[\mathrm{QND}]_{0}\right)^{2}-4 K_{2} 2\left[\mathrm{Rh} h_{2} \cdot \mathrm{QND}\right]_{0}[\mathrm{QND}]_{0}\right\}^{1 / 2}\right] \\
& {\left[\begin{array}{ll}
\mathrm{Abs}_{\mathrm{obs}}=\text { observed absorbance } & {\left[\mathrm{Rh}_{2} \cdot \mathrm{QND}\right]_{0}=\text { total concentration of } \mathrm{Rh}_{2} \cdot \mathrm{QND}} \\
\mathrm{AbS} \mathrm{Rh}_{\mathrm{RND}}=\text { absorbance for } \mathrm{Rh}_{2} \cdot \mathrm{QND} & {[\mathrm{QND}]_{0}=\text { total concentration of QND }} \\
\mathrm{AbSh} \cdot(\mathrm{QND}) 2=\text { absorbance for } \mathrm{Rh}_{2} \cdot(\mathrm{QND})_{2} & K_{2}=\text { association constant for 1:2 complexation }
\end{array}\right]}
\end{aligned}
$$

In the same way, the $K_{1}$ and $K_{2}$ values for $(S)-\mathbf{1 b}^{\mathbf{P h}}-\mathrm{C} 12$ with QND in $\mathrm{CHCl}_{3}$ at $50{ }^{\circ} \mathrm{C}$ were also estimated as shown in Figure S22, giving the $K_{1}$ and $K_{2}$ to be greater than $10^{7} \mathrm{M}^{-1}$ and 1.36 or $1.69 \times$ $10^{4} \mathrm{M}^{-1}$, respectively. 


\subsection{Estimation of Degree of Polymerization (n) of Supramolecular Helical Coordination Polymers by Variable-Temperature Absorption Spectral Changes of $(S)-1 a^{\mathrm{Ph}}-\mathrm{TEG}-(S)-2 b^{\mathrm{Me}}$ C12 with DABCO (1 equiv.) in $\mathrm{CHCl}_{3} / n$-Hexane $(1 / 1, \mathrm{v} / \mathrm{v})$}
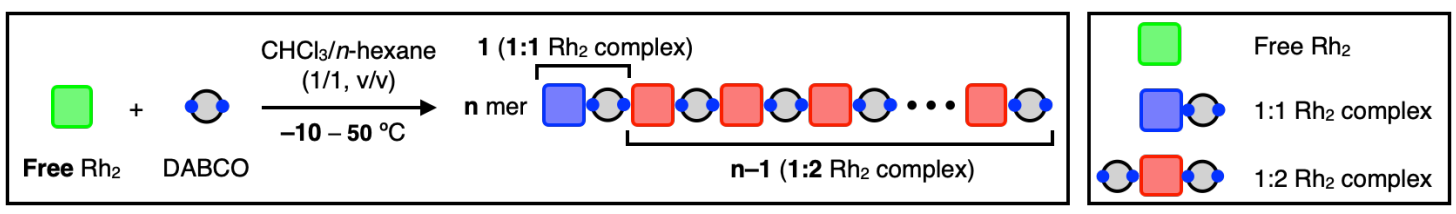

$$
A_{\text {simu. }}=\operatorname{Abs}_{1: 1} \times \frac{1}{n}+\operatorname{Abs}_{1: 2} \times \frac{n-1}{n}
$$

The supramolecular helical coordination polymer backbones formed from an equimolar mixture of the dirhodium(II) paddlewheels $\left(\mathrm{Rh}_{2}\right)$ and DABCO in $\mathrm{CHCl}_{3} / n$-hexane $(1 / 1, \mathrm{v} / \mathrm{v})$ are mostly composed of the 1:2 complexes of $\mathrm{Rh}_{2}$ with two $\mathrm{DABCO}\left(\mathrm{Rh}_{2} \cdot(\mathrm{DABCO})_{2}\right)$, in which the two axial binding sites of the dirhodium metals are occupied with $\mathrm{DABCO}$, while each coordination polymer possesses a 1:1 complex of $\mathrm{Rh}_{2}$ with one $\mathrm{DABCO}\left(\mathrm{Rh}_{2} \cdot \mathrm{DABCO}\right)$ at the terminal end. The absorption spectra for the 1:1 and 1:2 complexes of $\mathrm{Rh}_{2}$ with $\mathrm{DABCO}$ are expected to be considerably different from each other as evidenced by the absorption titration results of $\mathrm{Rh}_{2}$ with QND (Figures $\mathrm{S} 21$ and S22). Hence, we can estimate the degree of polymerization (n) of the coordination polymers at different temperatures based on the absorption spectra of the polymers when the absorption spectra for the corresponding 1:1 (Abs1:1) and 1:2 (Abs $1: 2)$ complexes of $\mathrm{Rh}_{2}$ with DABCO are available (eq $\mathrm{S} 8)$. The absorption spectra for the 1:2 complexes of $\mathrm{Rh}_{2}$ with $\mathrm{DABCO}$ in $\mathrm{CHCl}_{3} / n$-hexane $(1 / 1, \mathrm{v} / \mathrm{v})$ were readily obtained by measuring the absorption spectra of $\mathrm{Rh}_{2}$ in the presence of a large excess amount of DABCO at $25{ }^{\circ} \mathrm{C}$ during the titration experiments (Figures 6b,c and S19). However, the absorption spectra for the $1: 1$ complexes $\left(\mathrm{Rh}_{2} \cdot \mathrm{DABCO}\right)$ in $\mathrm{CHCl}_{3} / n$-hexane $(1 / 1, \mathrm{v} / \mathrm{v})$ could not be obtained because of higher $K_{1}$ and $K_{2}$ values in the solvent mixture than those in $\mathrm{CHCl}_{3}$, resulting in the formation of a mixture of the 1:1 and 1:2 complexes with no clear isosbestic points for the formation of the 1:1 complex during the titration experiments (Figure S19) as we anticipated from the absorption titrations of $\mathrm{Rh}_{2}$ with QND in $\mathrm{CHCl}_{3} / n$-hexane $(1 / 1, \mathrm{v} / \mathrm{v})$ at $50{ }^{\circ} \mathrm{C}$ (Figure $\left.\mathrm{S} 23\right)$. In $\mathrm{CHCl}_{3}$ at higher temperatures, the supramolecular helical coordination polymers $\left(\left[\mathrm{Rh}_{2} \cdot \mathrm{DABCO}\right]_{\mathrm{n}}\right)$ would mostly dissociate into the $1: 1$ complex $\left(\mathrm{Rh}_{2} \cdot \mathrm{DABCO}\right)$ as supported by the variabletemperature absorption spectral changes, which were accompanied by a gradual red shift of $\lambda_{\max }$, reaching almost constant $\lambda_{\max }$ values $(572-573 \mathrm{~nm})$ at 50 or $55^{\circ} \mathrm{C}$ (Figures $6 \mathrm{~d}$,e and S24), which 
are in good agreement with those observed in the $1: 1$ complex formation of $\mathrm{Rh}_{2}$ with QND in $\mathrm{CHCl}_{3}$ at $50{ }^{\circ} \mathrm{C}$ (Figures S21 and S22).

Using the observed absorption spectra for the 1:1 (Abs1:1) and 1:2 (Abs:2) complexes of $\mathrm{Rh}_{2}$ with DABCO (Figures $6 f$ and S25), we then estimated the degree of polymerization (n) of the coordination polymers at different temperatures by comparison with the simulated absorption spectra calculated as a function of $\mathbf{n}$ according to eq S8 (Figures 7 and S26). The results are summarized in Table S4. Because of the accuracy of the spectrophotometer $( \pm 0.0015)$, the simulated $\mathbf{n}$ values at the lower temperatures showed a relatively large distribution. 
8. Supporting Data

(a) (S)-1 $\mathrm{a}^{\text {Ph-TEG }}$
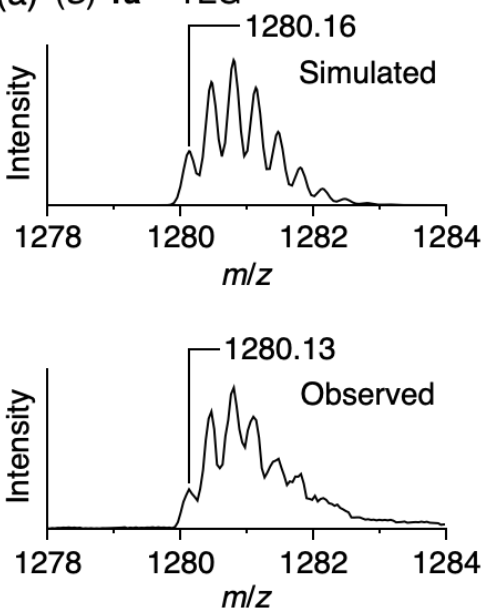

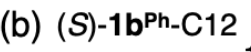
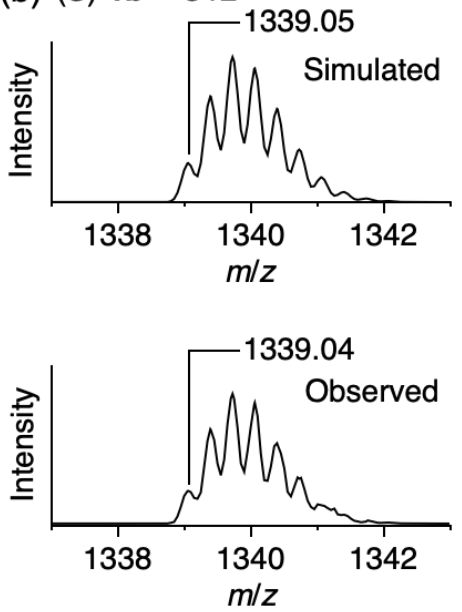

(c) (S)-2aMe-TEG
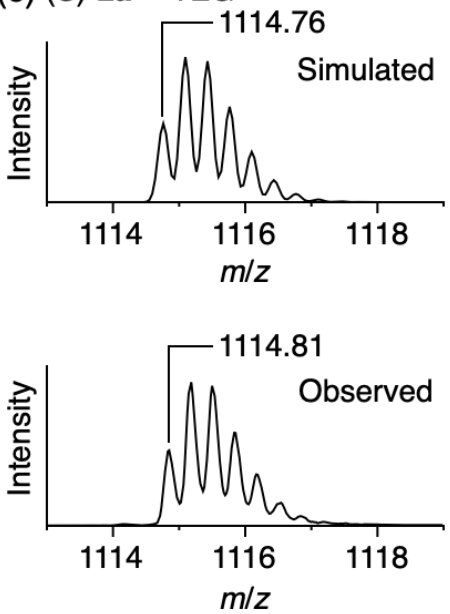

(d) (S)-2bMe-C12
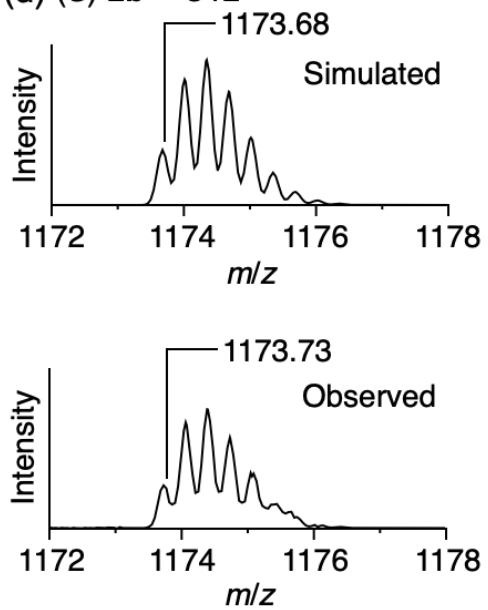

(f) $3 \mathbf{b}$ H-C12
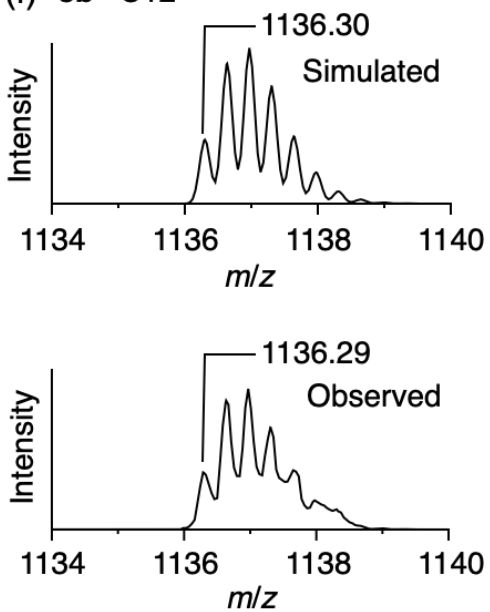

(e) $3 \mathbf{a}^{\mathrm{H}-\mathrm{TEG}}$
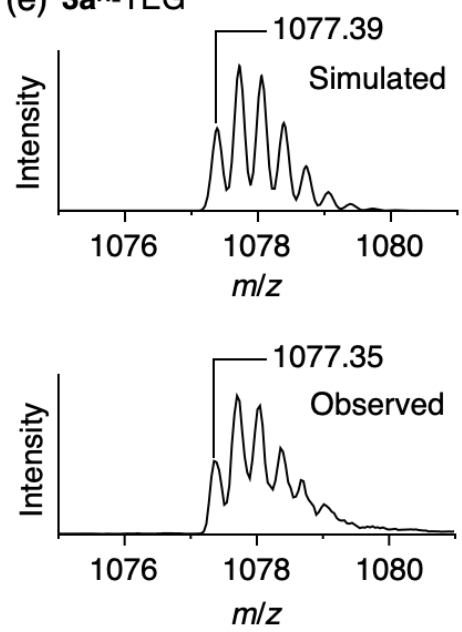

(g) (S)-2bMe-C12NMe
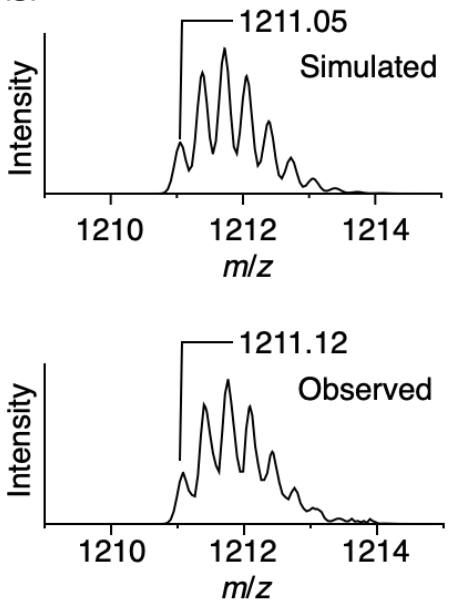

Figure S1. Positive mode ESI mass spectra of $(S)-\mathbf{1 a}^{\mathbf{P h}}-\mathrm{TEG}(\mathrm{a}),(S)-\mathbf{1 b}^{\mathbf{P h}}-\mathrm{C} 12(\mathrm{~b}),(S)-\mathbf{2} \mathbf{a}^{\mathbf{M e}}-\mathrm{TEG}(\mathrm{c})$, $(S)-2 \mathbf{b}^{\mathrm{Me}}-\mathrm{C} 12(\mathrm{~d}), \mathbf{3}^{\mathrm{H}}-\mathrm{TEG}(\mathrm{e}), \mathbf{3 b}^{\mathrm{H}}-\mathrm{C} 12(\mathrm{f})$, and $(S)-\mathbf{2 b}^{\mathrm{Me}}-\mathrm{C} 12_{\mathrm{NMe}}(\mathrm{g})$. 

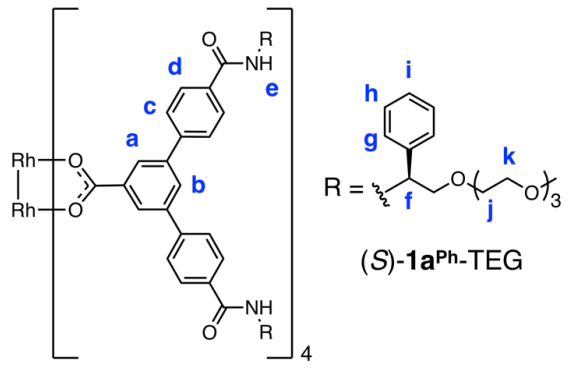

(S)-1aPh-TEG

(a) $\left[(S)-1 a^{\mathrm{Ph}}-\mathrm{TEG} \cdot \mathrm{DABCO}\right]_{\mathrm{n}}\left(0.70 \mathrm{mM}\right.$, in $\left.\mathrm{CDCl}_{3}\right)$

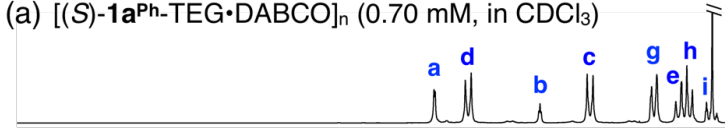
$\mathrm{HCl}_{3}$

(b) (S)-1 $\mathrm{a}^{\mathrm{Ph}}$-TEG $\left(0.70 \mathrm{mM}\right.$, in $\left.\mathrm{CDCl}_{3}\right)$ g $\mathrm{h}_{\mathrm{i}} \mathrm{i}$

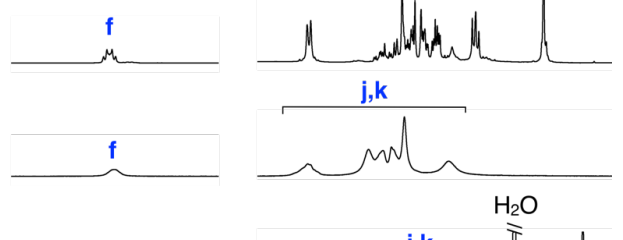

(c) (S)-1 $\mathrm{a}^{\text {Ph-TEG }}\left(0.70 \mathrm{mM}\right.$, in DMSO- $\left.d_{6}\right)$

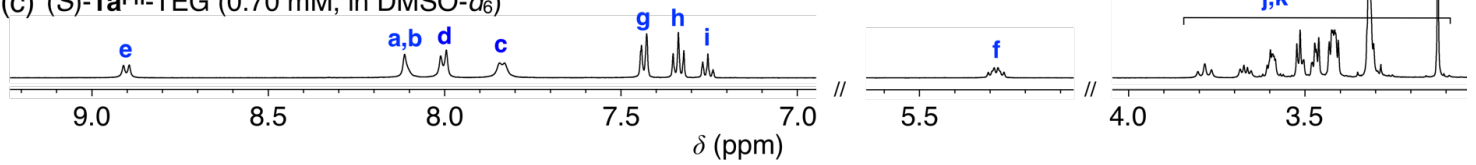

Figure S2. ${ }^{1} \mathrm{H}$ NMR $\left(500 \mathrm{MHz}, 25^{\circ} \mathrm{C}\right)$ spectra of $\left[(S)-1 \mathbf{a}^{\mathbf{P h}}-\mathrm{TEG} \cdot \mathrm{DABCO}\right]_{\mathrm{n}}$ in $\mathrm{CDCl}_{3}$ (a) and $(S)$ $\mathbf{1 a}^{\mathbf{P h}}-\mathrm{TEG}$ in $\mathrm{CDCl}_{3}$ (b) and DMSO-d $(\mathrm{c}) .[1]=0.70 \mathrm{mM}$.

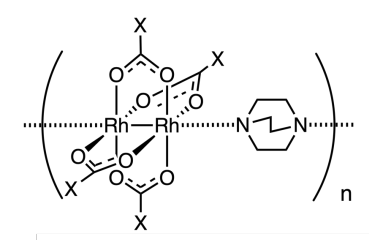

$\left[(S)-1 a^{\text {Ph-TEG}} \cdot \mathrm{DABCO}\right]_{\mathrm{n}}$

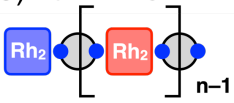

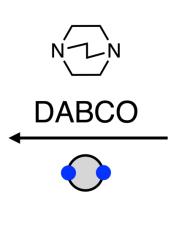

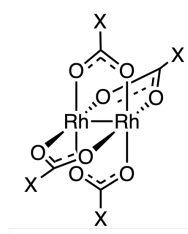

(S)-1aPh-TEG

$R h_{2}$
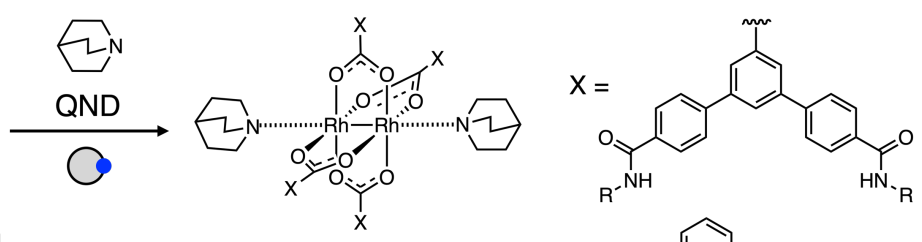

(S)-1a $\mathrm{a}^{\mathrm{Ph}}-\mathrm{TEG} \cdot(\mathrm{QND})_{2}$
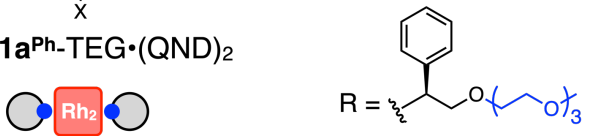

(a) $\left[(S)-1 a^{\text {Ph-TEG }} \cdot \mathrm{DABCO}\right]_{\mathrm{n}}$ in $\mathrm{CDCl}_{3} / \boldsymbol{n}$-hexane- $\boldsymbol{d}_{14}$ at $25^{\circ} \mathrm{C}$
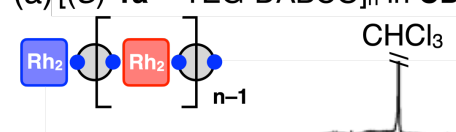

(b) (S)-1a ${ }^{\text {Ph-TEG }} \cdot(\mathrm{QND})_{2}$ in $\mathrm{CDCl}_{3} / \boldsymbol{n}$-hexane- $\boldsymbol{d}_{14}$ at $25^{\circ} \mathrm{C}$

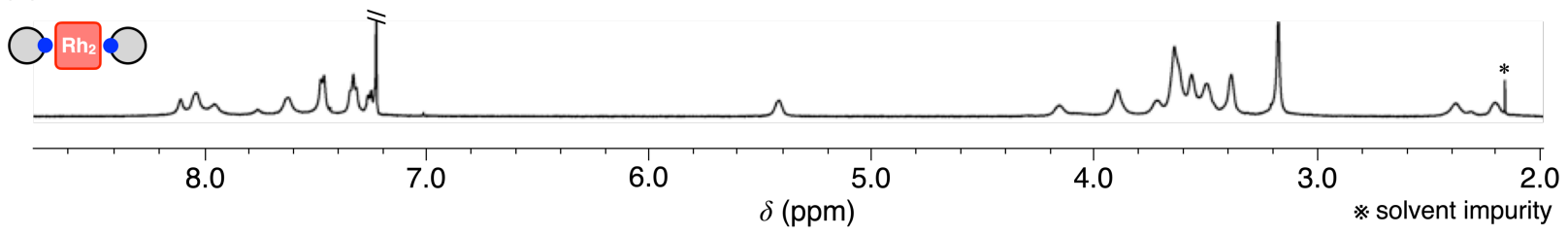

Figure S3. ${ }^{1} \mathrm{H}$ NMR $\left(500 \mathrm{MHz}, \mathrm{CDCl}_{3} / n\right.$-hexane- $\left.d_{14}(1 / 1, \mathrm{v} / \mathrm{v}), 25^{\circ} \mathrm{C}\right)$ spectra of $\left[(S)-\mathbf{1 a}^{\mathbf{P h}}{ }_{-}\right.$ TEG $\cdot \mathrm{DABCO}]_{\mathrm{n}}(\mathrm{a})$ and $(S)-\mathbf{1} \mathbf{a}^{\mathrm{Ph}}-\mathrm{TEG} \cdot(\mathrm{QND})_{2}(\mathrm{~b}) \cdot[\mathbf{1}]=0.70 \mathrm{mM}$. 


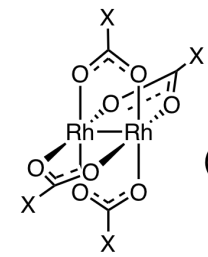

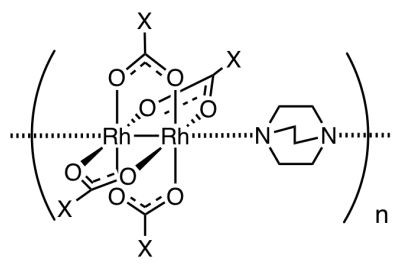

$\left[(S)-1 a^{P h}-T E G \cdot D A B C O\right]_{n}$

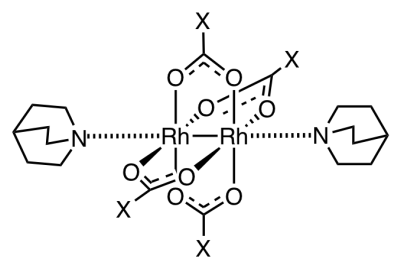

$(S)-1 a^{P h}-T E G \cdot(Q N D)_{2}$<smiles>[X]C(=O)Nc1ccc(-c2cc(-c3ccc(C(=O)NP)cc3)cc(C(C)C)c2)cc1</smiles>

(b)

$0.70 \mathrm{mM}$ in $\mathrm{CHCl}_{3} / n$-hexane $(1 / 1, \mathrm{v} / \mathrm{v})$ at $25^{\circ} \mathrm{C}$

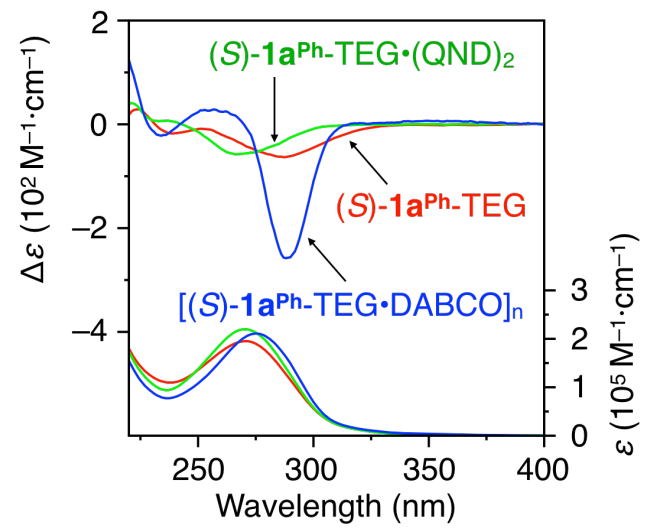

(a) $\quad 0.70 \mathrm{mM}$

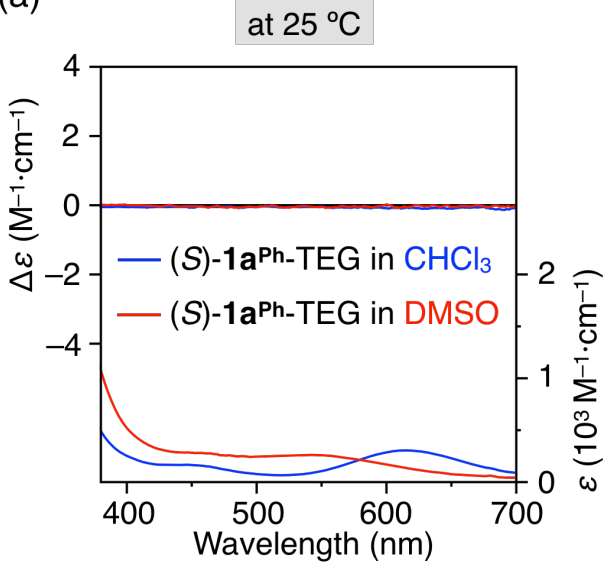

(c)
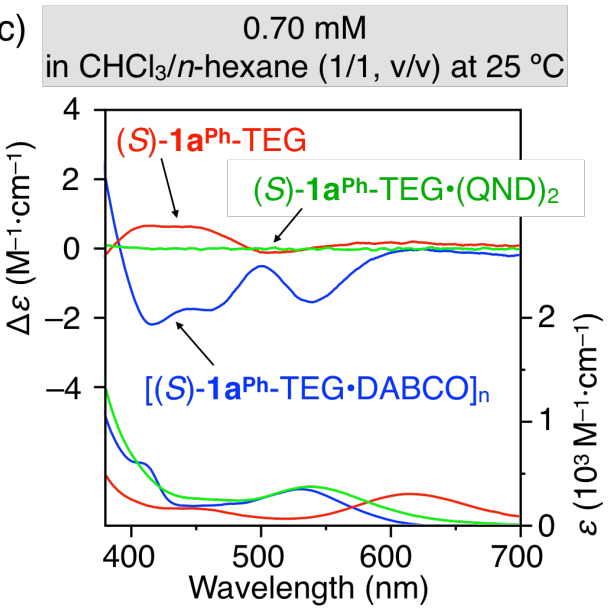

Figure S4. $\mathrm{CD}$ and absorption spectra of $(S)-\mathbf{1 a}^{\mathbf{P h}}-\mathrm{TEG}$ in $\mathrm{CHCl}_{3}$ (blue lines) and DMSO (red lines) in visible region at $25^{\circ} \mathrm{C}(\mathrm{a})$ and those of $(S)-\mathbf{1} \mathbf{a}^{\mathbf{P h}}$-TEG (red lines), $\left[(S)-\mathbf{1 a}^{\mathbf{P h}}-\mathrm{TEG} \cdot \mathrm{DABCO}\right]_{\mathrm{n}}(\mathrm{blue}$ lines), and (S)-1a $\mathbf{a}^{\mathbf{P h}}-\mathrm{TEG} \cdot(\mathrm{QND})_{2}$ (green lines) in $\mathrm{CHCl}_{3} / n$-hexane $(1 / 1, \mathrm{v} / \mathrm{v})$ in UV (b) and visible (c) regions at $25^{\circ} \mathrm{C} .[1]=0.70 \mathrm{mM}$. 


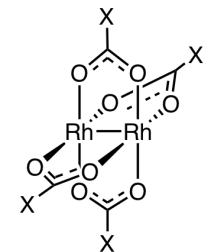

(S)-1a $\mathrm{a}^{\text {Ph-TEG }}$

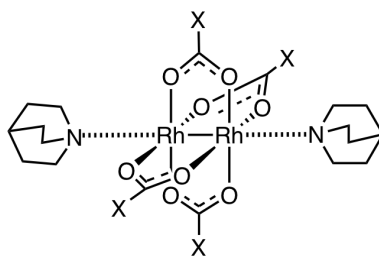

(S)-1aPh-TEG·(QND) 2

$$
0.70 \mathrm{mM}
$$

in $\mathrm{CHCl}_{3} / n$-hexane $(1 / 1, \mathrm{v} / \mathrm{v})$

(a)

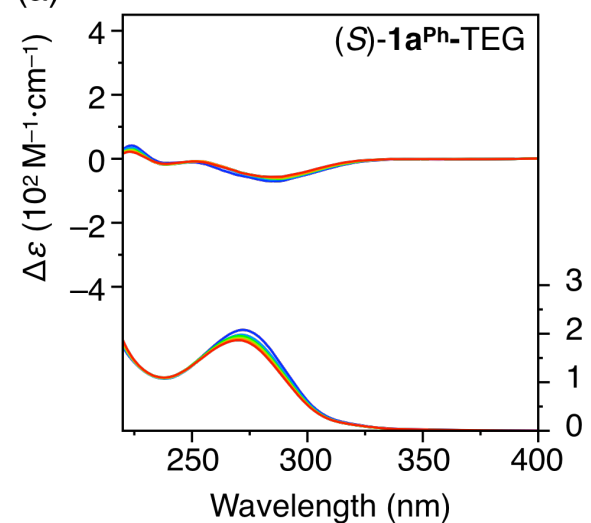

(c)

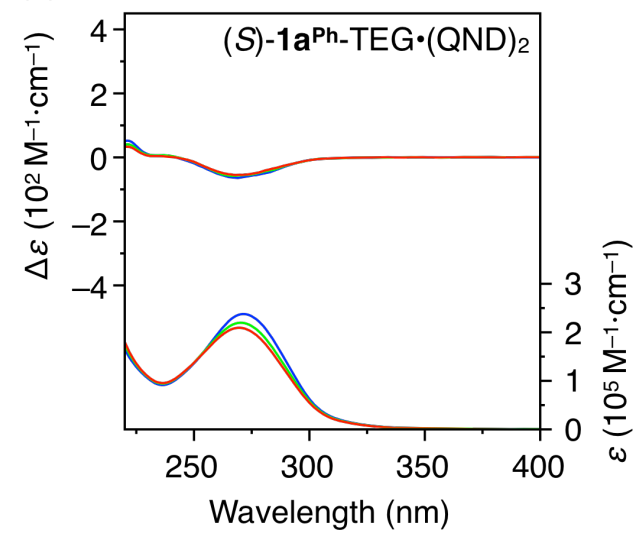<smiles>[R]NC(=O)c1ccc(-c2cc(C)cc(-c3ccc(C=[Y])cc3)c2)cc1</smiles>

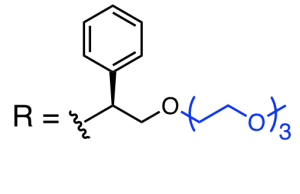

(b)

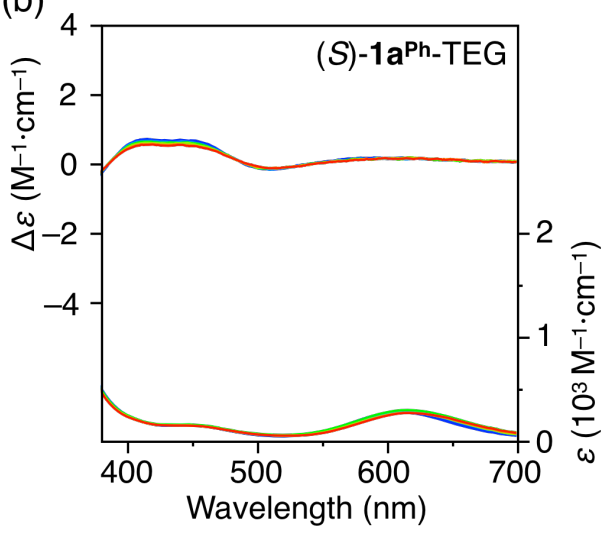

(d)

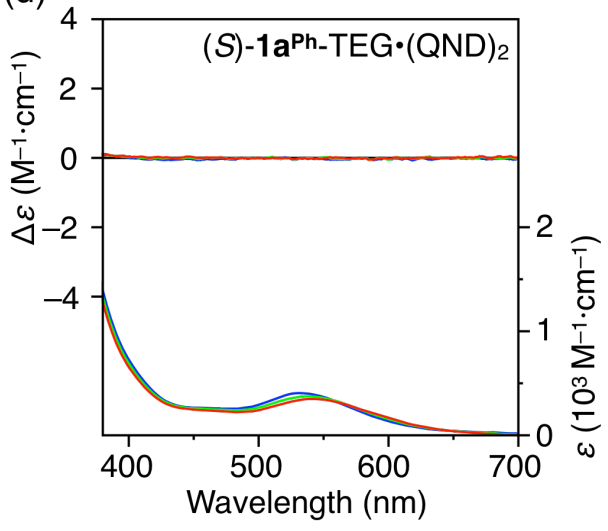

Figure S5. $T$-dependent $C D$ and absorption spectral changes of $(S)-\mathbf{1 a}^{\mathbf{P h}_{-}}$-TEG $(\mathrm{a}, \mathrm{b})$ and $(S)-\mathbf{1 a}^{\mathbf{P h}}$ TEG $(\mathrm{QND})_{2}(\mathrm{c}, \mathrm{d})$ in $\mathrm{CHCl}_{3} / n$-hexane $(1 / 1, \mathrm{v} / \mathrm{v})$ in $\mathrm{UV}(\mathrm{a}, \mathrm{c})$ and visible $(\mathrm{b}, \mathrm{d})$ regions. $[\mathbf{1}]=0.70$ mM. 

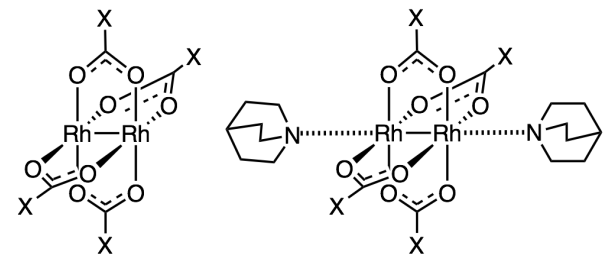

(S)-1 $\mathrm{b}^{\mathrm{Ph}}-\mathrm{C} 12$

(S)-1bPh-C12·(QND) 2 $0.70 \mathrm{mM}$

in $\mathrm{CHCl}_{3} / n$-hexane $(1 / 1, \mathrm{v} / \mathrm{v})$

(a)
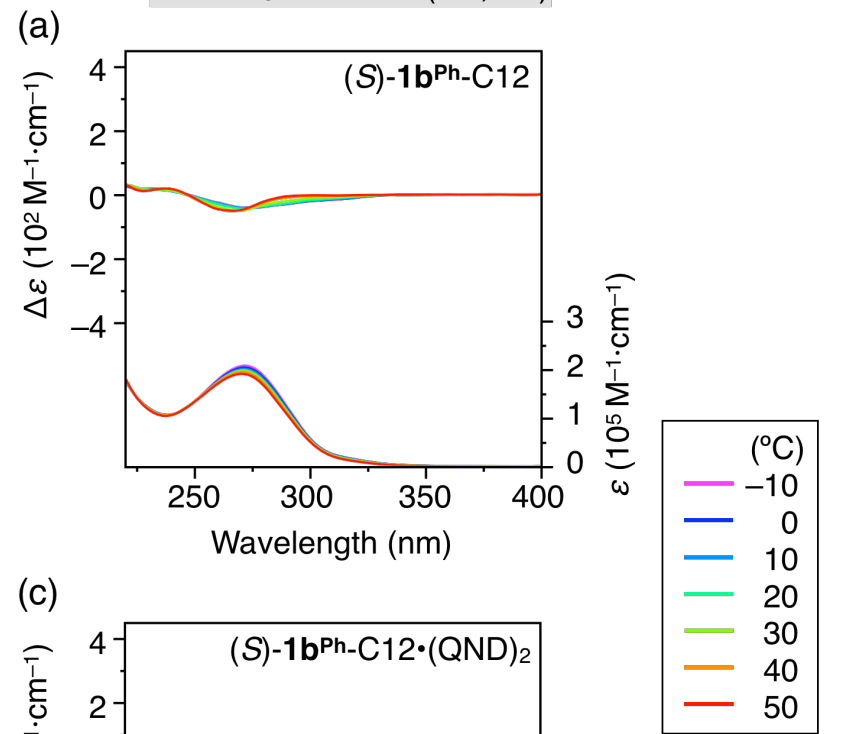

(c)

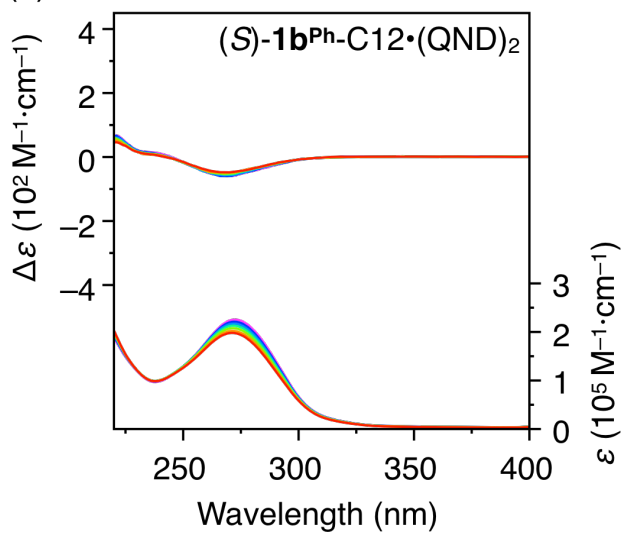

(b)

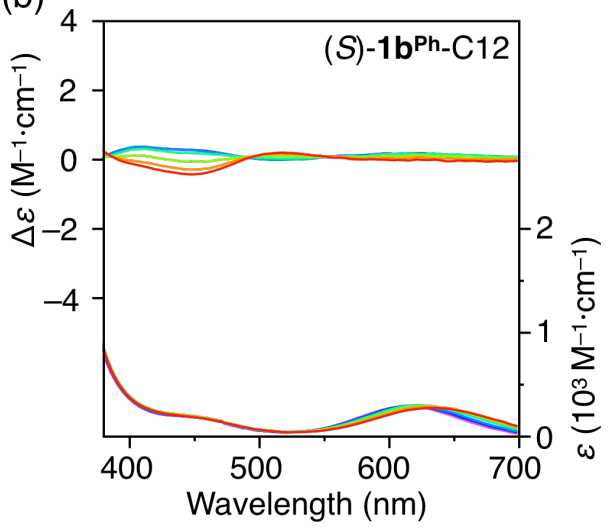

(d)

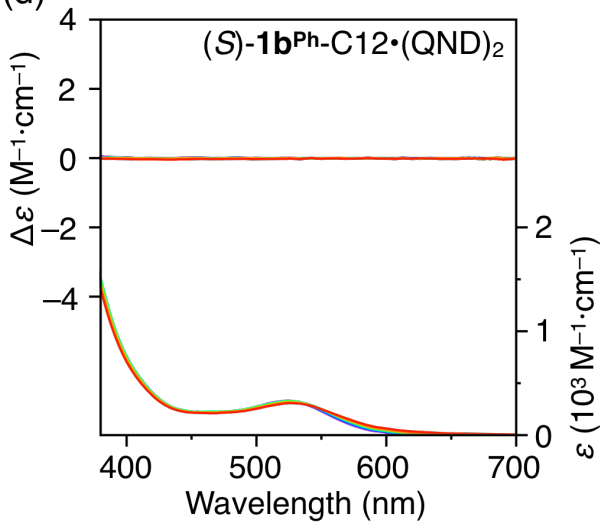

Figure S6. $T$-dependent $C D$ and absorption spectral changes of $(S)-\mathbf{1 b}^{\mathbf{P h}}-\mathrm{C} 12(\mathrm{a}, \mathrm{b})$ and $(S)-\mathbf{1} \mathbf{b}^{\mathbf{P h}}$ $\mathrm{C} 12 \cdot(\mathrm{QND})_{2}(\mathrm{c}, \mathrm{d})$ in $\mathrm{CHCl}_{3} / n$-hexane $(1 / 1, \mathrm{v} / \mathrm{v})$ in $\mathrm{UV}(\mathrm{a}, \mathrm{c})$ and visible $(\mathrm{b}, \mathrm{d})$ regions. $[\mathbf{1}]=0.70$ $\mathrm{mM}$. 


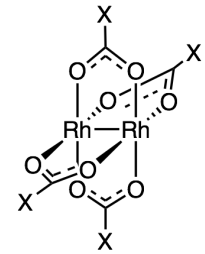

(S)-2aMe-TEG

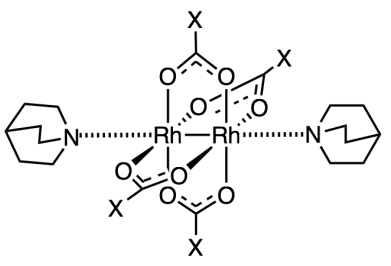

(S)-2aMe-TEG·(QND) 2

$$
0.70 \mathrm{mM}
$$

in $\mathrm{CHCl}_{3} / n$-hexane $(1 / 1, \mathrm{v} / \mathrm{v})$

(a)

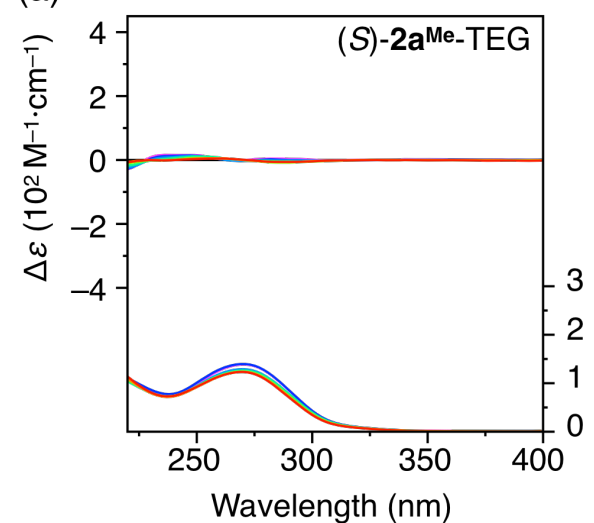

(c)

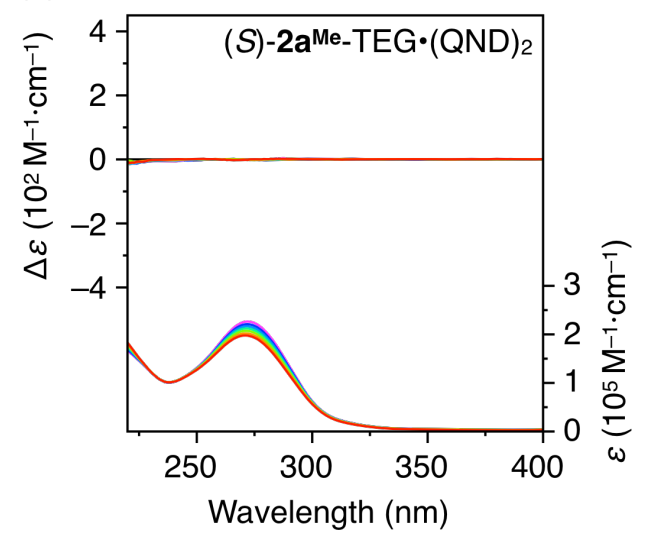

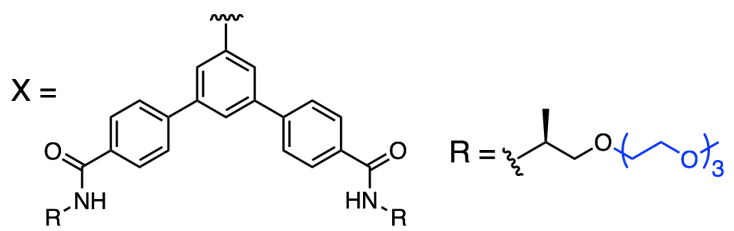

(b)

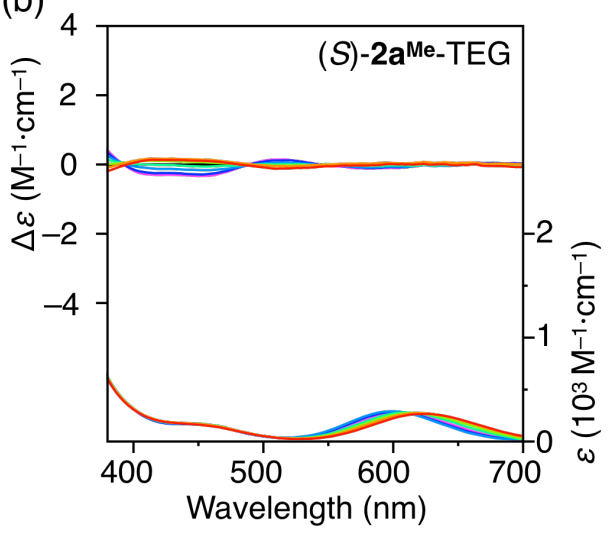

(d)

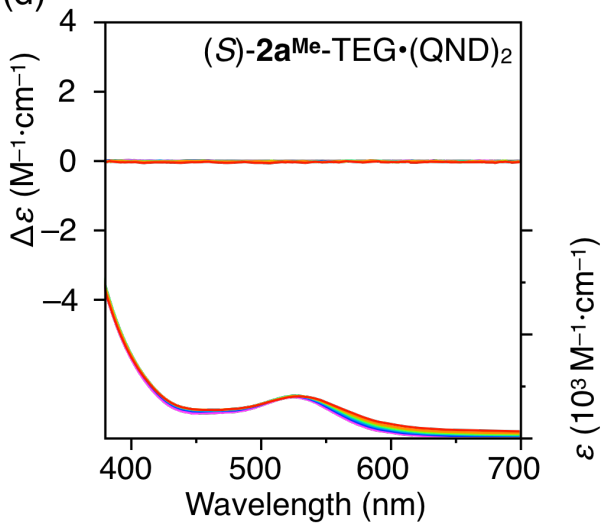

Figure S7. $T$-dependent $\mathrm{CD}$ and absorption spectral changes of $(S)-\mathbf{2} \mathbf{a}^{\mathbf{M e}}-\mathrm{TEG}(\mathrm{a}, \mathrm{b})$ and $(S)-\mathbf{2} \mathbf{a}^{\mathbf{M e}}$ TEG $(\mathrm{QND})_{2}(\mathrm{c}, \mathrm{d})$ in $\mathrm{CHCl}_{3} / n$-hexane $(1 / 1, \mathrm{v} / \mathrm{v})$ in UV $(\mathrm{a}, \mathrm{c})$ and visible $(\mathrm{b}, \mathrm{d})$ regions. [2] $=0.70$ $\mathrm{mM}$. 


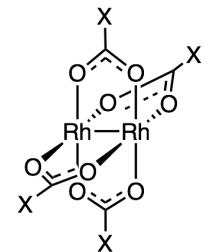

(S)-2b'Me-C12

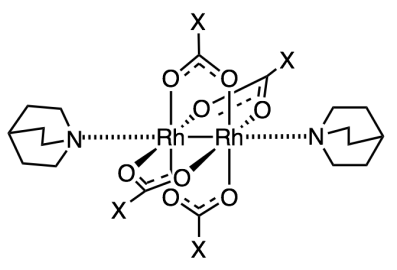

$(S)-2 b^{M e}-C 12 \cdot(Q N D)_{2}$

$0.70 \mathrm{mM}$

in $\mathrm{CHCl}_{3} / n$-hexane $(1 / 1, \mathrm{v} / \mathrm{v})$

(a)

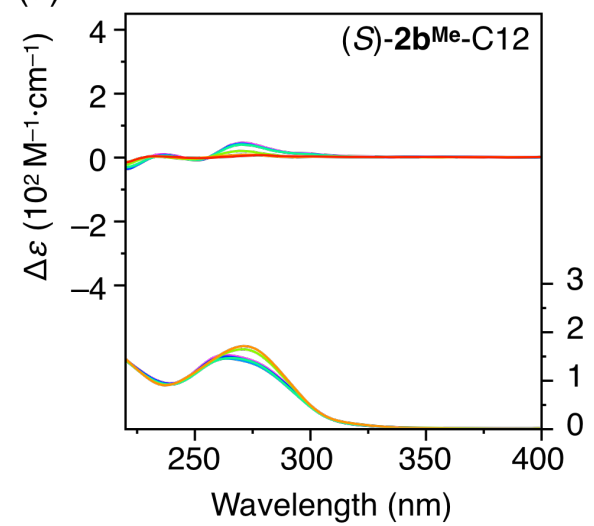

(c)

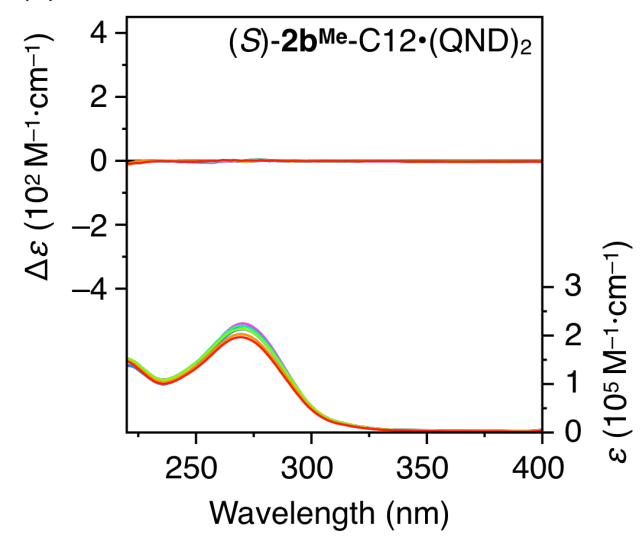<smiles></smiles>

(b)

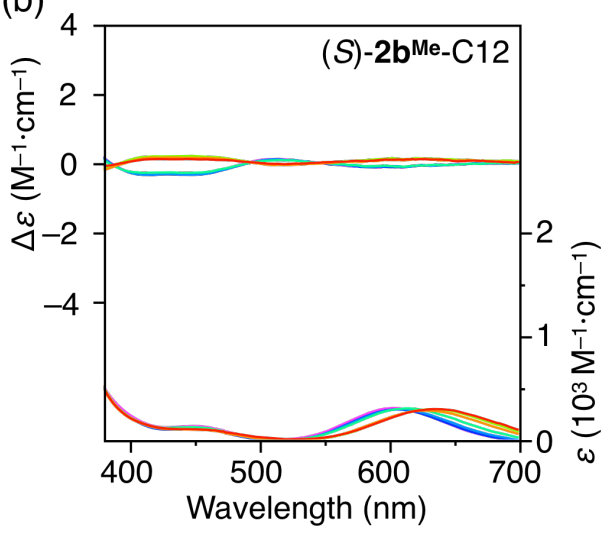

(d)

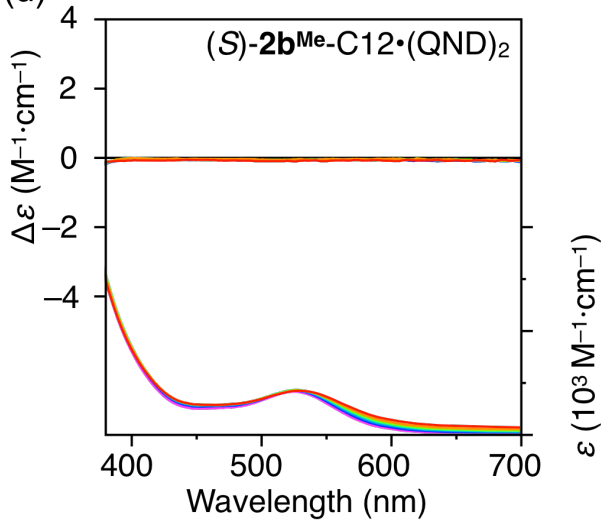

Figure S8. $T$-dependent $C D$ and absorption spectral changes of $(S)-\mathbf{2} \mathbf{b}^{\mathbf{M e}}-\mathrm{C} 12(\mathrm{a}, \mathrm{b})$ and $(S)-\mathbf{2 b}^{\mathbf{M e}}$ $\mathrm{C} 12 \cdot(\mathrm{QND})_{2}(\mathrm{c}, \mathrm{d})$ in $\mathrm{CHCl}_{3} / n$-hexane $(1 / 1, \mathrm{v} / \mathrm{v})$ in $\mathrm{UV}(\mathrm{a}, \mathrm{c})$ and visible $(\mathrm{b}, \mathrm{d})$ regions. [2] $=0.70$ $\mathrm{mM}$. 


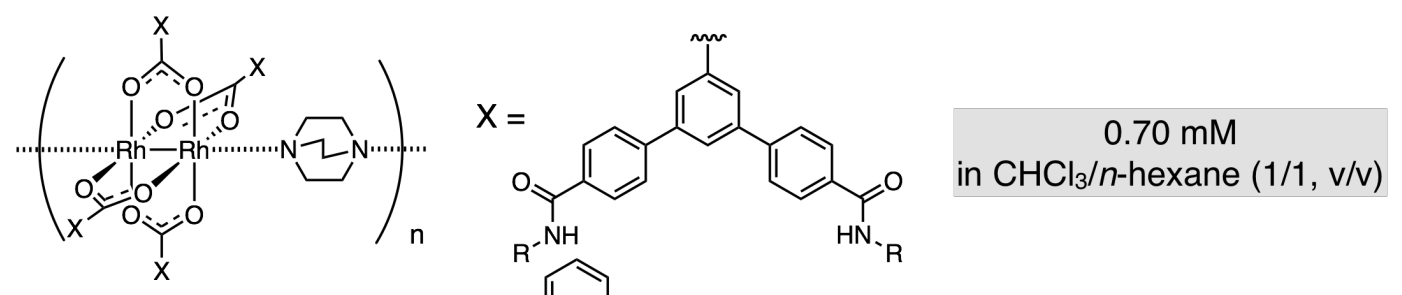

(a) $\left[(S)-1 b^{\text {Ph-C } 12 \cdot D A B C O}\right]_{n} \quad R=\xi 0+t_{11}$

(b)
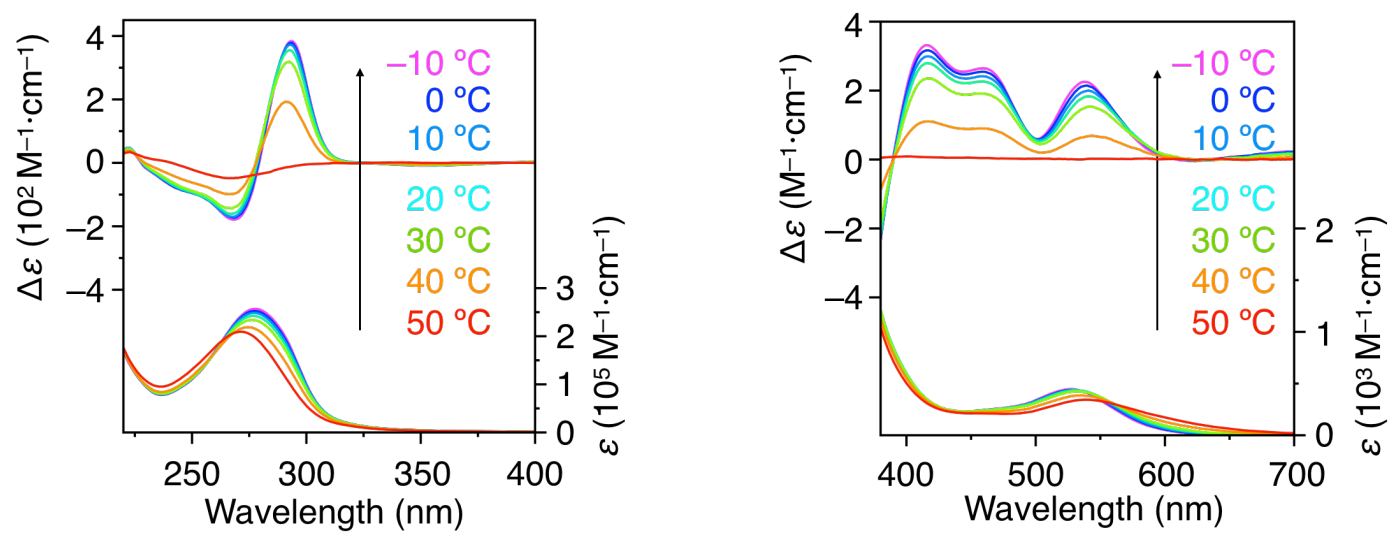

(c) $\left[(S)-2 a^{M e-T E G} \cdot \mathrm{DABCO}\right]_{\mathrm{n}} \mathrm{R}=\overbrace{\varepsilon} \mathrm{O} \mathrm{O}_{\mathrm{O}} \mathrm{\gamma}_{3}(\mathrm{~d})$
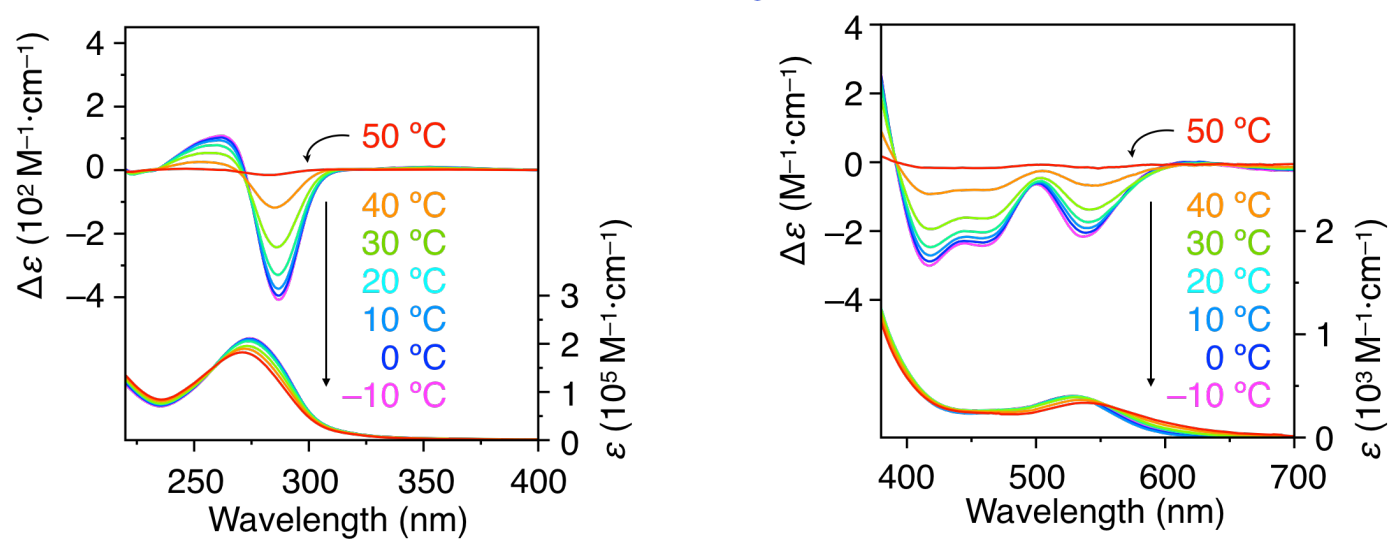

(e) $\left[(S)-2 b^{M e-C 12 \cdot D A B C O}\right]_{n} \quad R=\xi$ CO $_{11}$

(f)
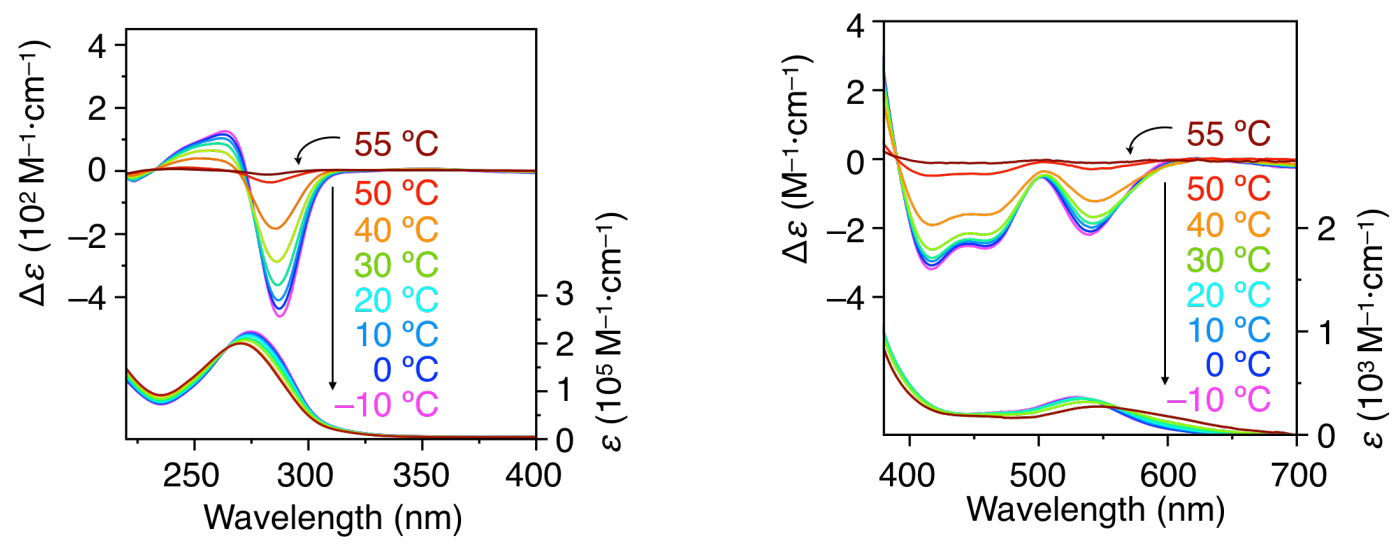

Figure S9. $T$-dependent $\mathrm{CD}$ and absorption spectral changes of $\left[(S)-\mathbf{1 b}^{\mathbf{P h}}-\mathrm{C} 12 \cdot \mathrm{DABCO}\right]_{\mathrm{n}}(\mathrm{a}, \mathrm{b}),[(S)$ $\left.\mathbf{2 a}^{\mathrm{Me}}-\mathrm{TEG} \cdot \mathrm{DABCO}\right]_{\mathrm{n}}(\mathrm{c}, \mathrm{d})$, and $\left[(S)-\mathbf{2} \mathbf{b}^{\mathrm{Me}}-\mathrm{C} 12 \cdot \mathrm{DABCO}\right]_{\mathrm{n}}(\mathrm{e}, \mathrm{f})$ in $\mathrm{CHCl}_{3} / n$-hexane $(1 / 1, \mathrm{v} / \mathrm{v})$ in UV $(\mathrm{a}, \mathrm{c}, \mathrm{e})$ and visible $(\mathrm{b}, \mathrm{d}, \mathrm{f})$ regions. [1 $\mathbf{1}^{\circ}$ or $\left.\mathbf{2} \cdot \mathrm{DABCO}\right]=0.70 \mathrm{mM}$. 


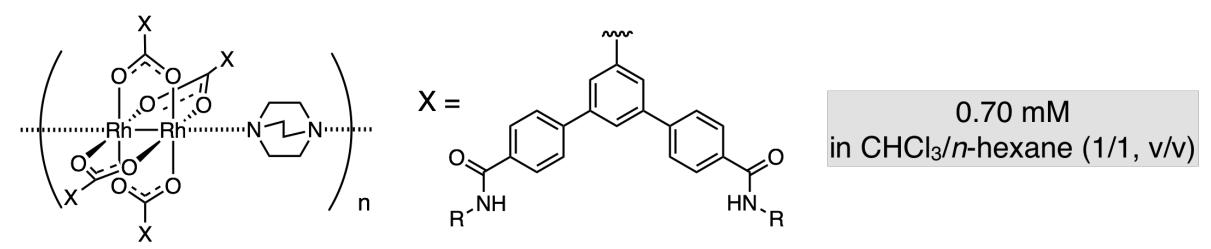

(a)

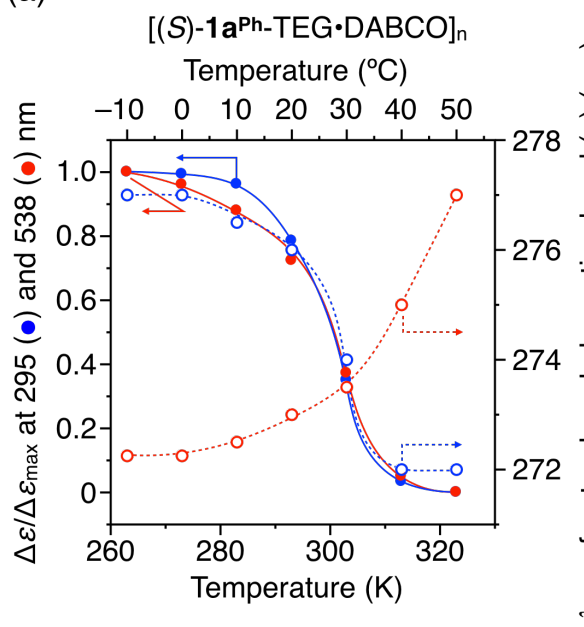

(c)

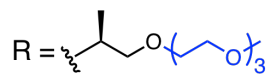

[(S)-2aMe-TEG・DABCO $]_{n}$

Temperature $\left({ }^{\circ} \mathrm{C}\right)$

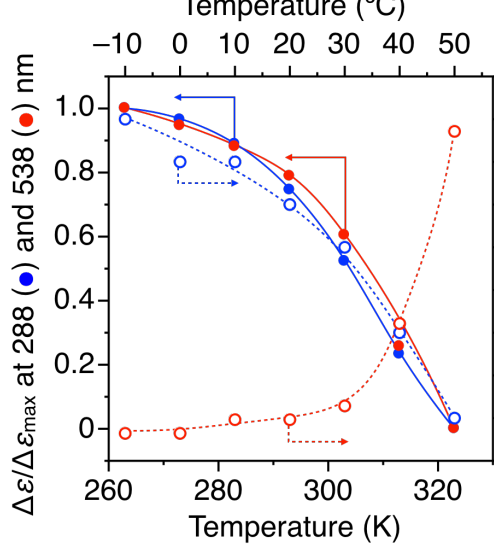

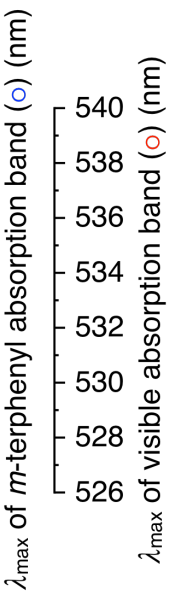

(d)

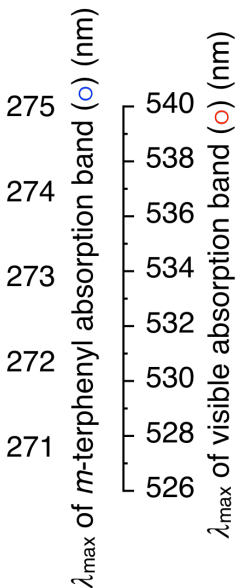

(b)<smiles>[R]=CC(CO[AlH2])c1ccccc1</smiles>

[(S)-1 $\mathbf{b}^{\text {Ph-C12·DABCO }}$ n

Temperature $\left({ }^{\circ} \mathrm{C}\right)$

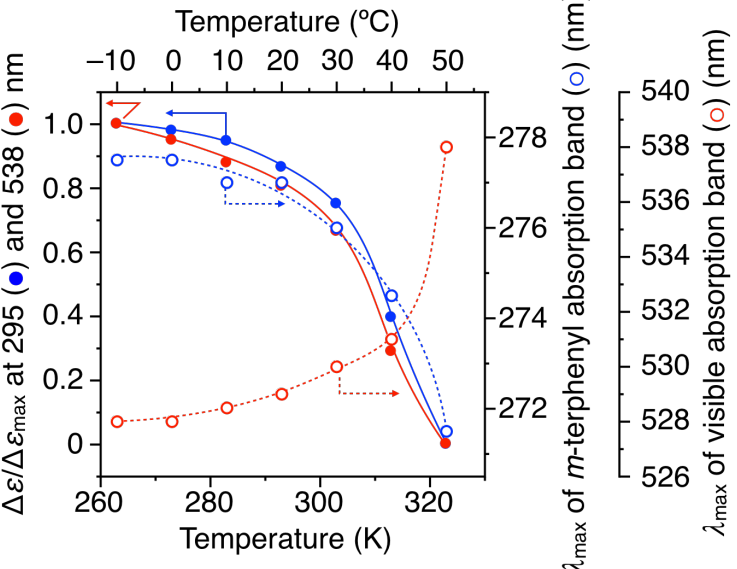

$\mathrm{R}=\overbrace{2} \mathrm{O})_{11}$

[(S)-2bMe-C12-DABCO ]

Temperature $\left({ }^{\circ} \mathrm{C}\right)$

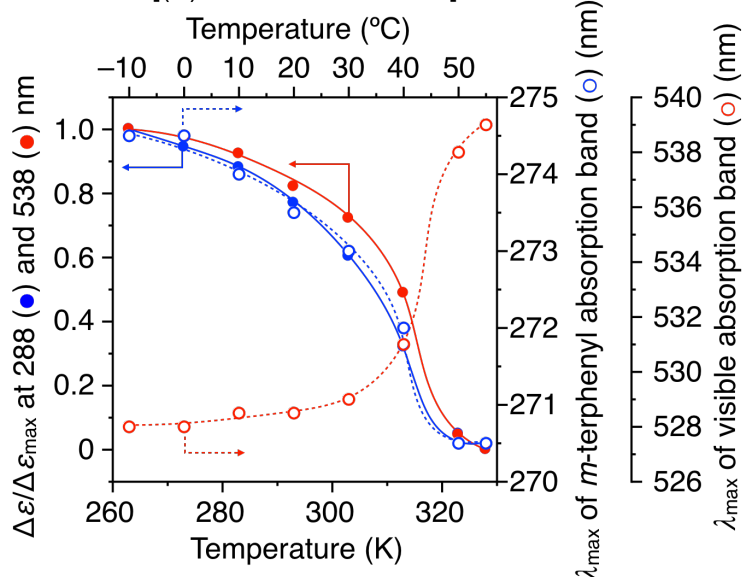

Figure S10. Plots of the normalized CD intensity $\left(\Delta \varepsilon / \Delta \varepsilon_{\max }\right)$ of the $m$-terphenyl $(\mathbf{O} ; 295(\mathrm{a}, \mathrm{b})$ and $288(\mathrm{c}, \mathrm{d}) \mathrm{nm})$ and visible $(0 ; 538 \mathrm{~nm})$ chromophore regions and the maximum absorption

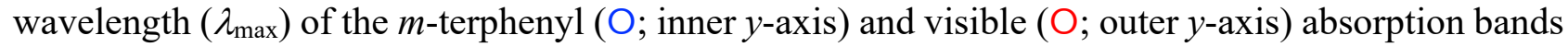
of $\left[(S)-\mathbf{1} \mathbf{a}^{\mathbf{P h}}-\mathrm{TEG} \cdot \mathrm{DABCO}\right]_{\mathrm{n}}(\mathrm{a}),\left[(S)-\mathbf{1} \mathbf{b}^{\mathrm{Ph}}-\mathrm{C} 12 \cdot \mathrm{DABCO}\right]_{\mathrm{n}}(\mathrm{b}),\left[(S)-\mathbf{2} \mathbf{a}^{\mathrm{Me}}-\mathrm{TEG} \cdot \mathrm{DABCO}\right]_{\mathrm{n}}(\mathrm{c})$, and $\left[(S)-2 \mathbf{b}^{\mathbf{M e}}-\mathrm{C} 12 \cdot \mathrm{DABCO}\right]_{\mathrm{n}}(\mathrm{d})$ in $\mathrm{CHCl}_{3} / n$-hexane $(1 / 1, \mathrm{v} / \mathrm{v})$ versus temperature. The $\Delta \varepsilon_{\text {max }}$ represents the maximum $\mathrm{CD}$ intensity of the corresponding one-handed helical coordination polymers in $\mathrm{CHCl}_{3} / n$-hexane $(1 / 1, \mathrm{v} / \mathrm{v})$ at $263 \mathrm{~K}\left(-10^{\circ} \mathrm{C}\right) .[1 \cdot$ or $2 \cdot \mathrm{DABCO}]=0.70 \mathrm{mM}$. 


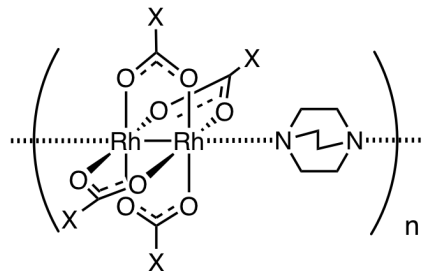

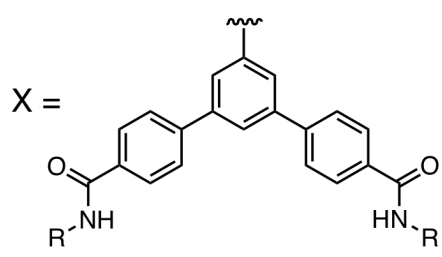<smiles>[R]=CCC(C)CO[Al]</smiles>

$0.70 \mathrm{mM}$ in $\mathrm{CHCl}_{3} / n$-hexane $(1 / 1, \mathrm{v} / \mathrm{v})$

Experimental $(\bullet)$ Isodesmic model $(-)$ Cooperative model $(-)$

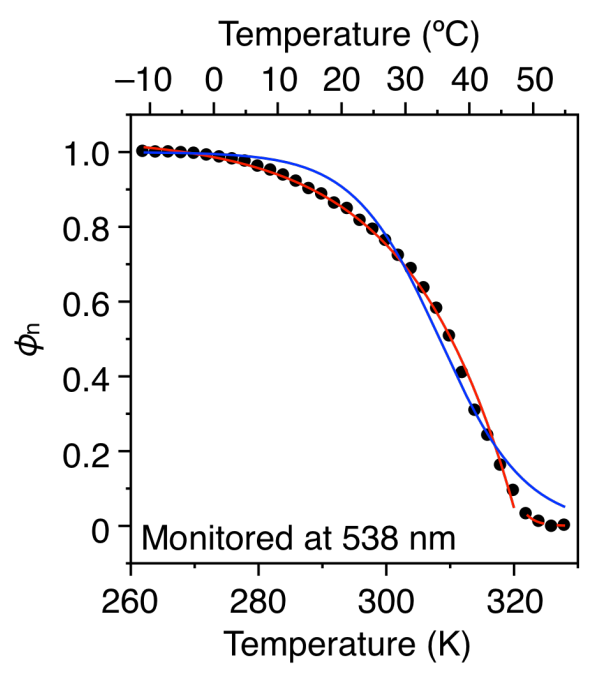

Figure S11. Normalized degree of polymerization $\left(\phi_{\mathrm{n}}\right)$ of $(S)-\mathbf{2} \mathbf{b}^{\mathrm{Me}}-\mathrm{C} 12$ with $\mathrm{DABCO}$ in $\mathrm{CHCl}_{3} / n$ hexane $(1 / 1, v / v)$ determined from $T$-dependent $C D$ intensity changes at visible chromophore region $(\mathbf{O} ; 538 \mathrm{~nm})$ fitted with the isodesmic (blue line) and cooperative models (red line). 


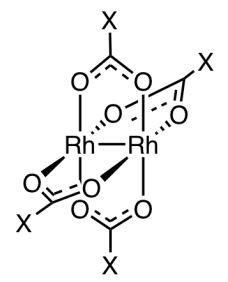

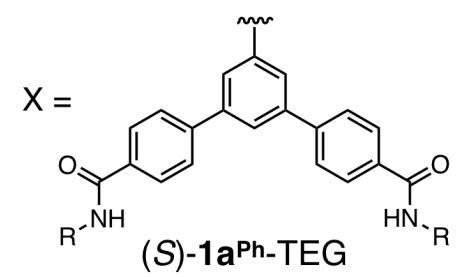

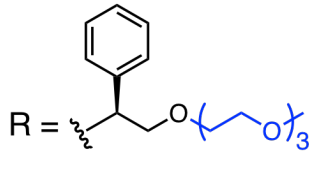

in $\mathrm{CHCl}_{3} / n$-hexane $(1 / 1, \mathrm{v} / \mathrm{v})$ at $25^{\circ} \mathrm{C}$

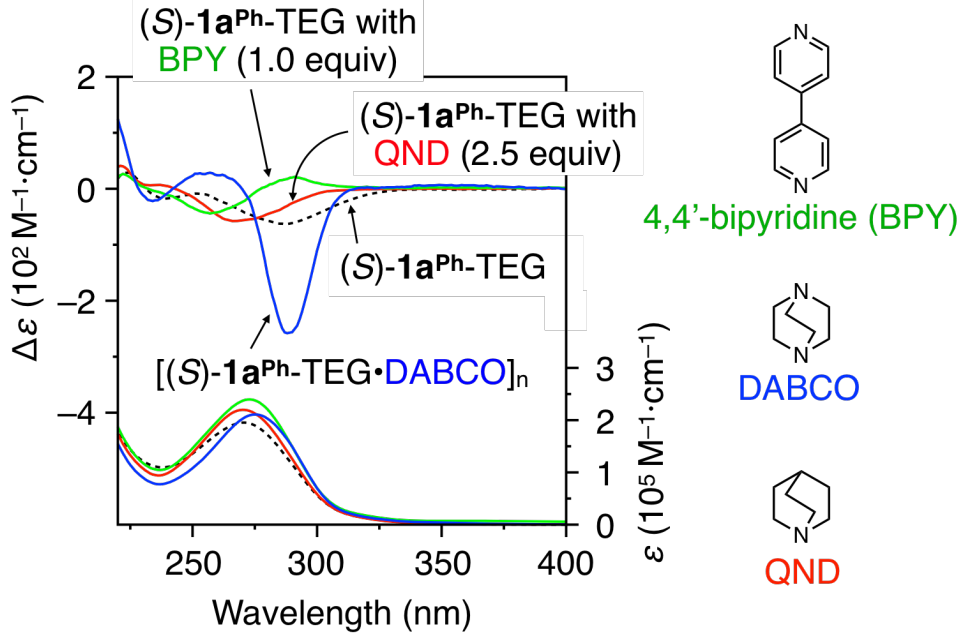

Figure S12. CD and absorption spectra of $(S)-1 \mathbf{a}^{\mathbf{P h}}-\mathrm{TEG}$ in the presence of QND (2.5 equiv) (red lines) and BPY (1.0 equiv) (green lines) in $\mathrm{CHCl}_{3} / n$-hexane $(1 / 1, \mathrm{v} / \mathrm{v})$ at $25^{\circ} \mathrm{C}$. $\mathrm{CD}$ and absorption spectra of $(S)-\mathbf{1} \mathbf{a}^{\mathbf{P h}}-$ TEG (black dashed lines) and $\left[(S)-\mathbf{1 a}^{\mathbf{P h}}-\mathrm{TEG} \cdot \mathrm{DABCO}\right]_{\mathrm{n}}$ (blue lines) are also shown. [1] $=0.70 \mathrm{mM}$. 


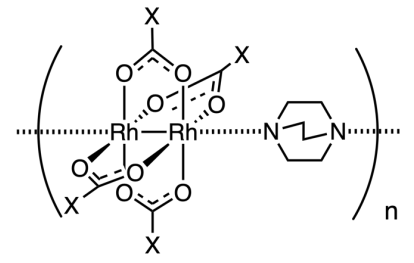

$\left[(S)-\mathbf{1} \mathbf{a}^{\text {Ph }} \text { or } \mathbf{2} \mathrm{a}^{\text {Me-TEG }} \cdot \mathrm{DABCO}\right]_{\mathrm{n}}$

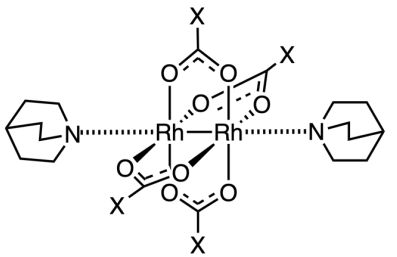

$(S)-1 \mathbf{a}^{\text {Ph}}$-TEG $(Q N D)_{2}$<smiles>[Y]=C(NC(=O)c1ccc(-c2cc(C)cc(-c3ccc(C(N)=O)cc3)c2)cc1)c1ccccc1</smiles>

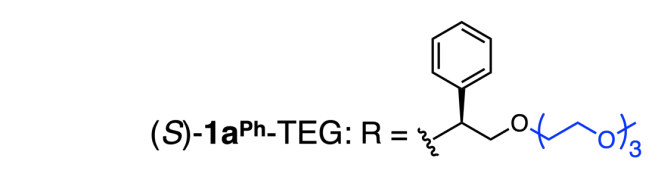

(S)-2aMe-TEG: $\mathrm{R}=\mathrm{L}_{2} \mathrm{O} \mathrm{Not}_{3}$
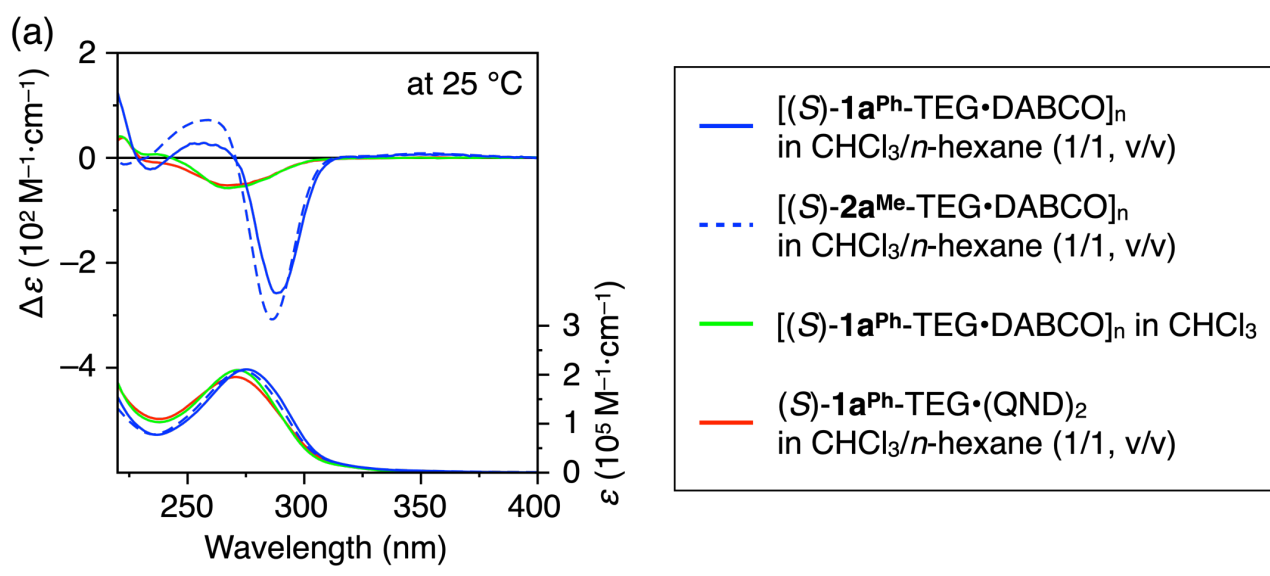

(b)

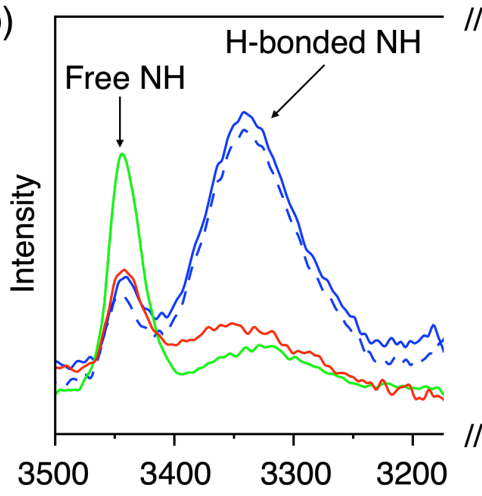

Amide I at r.t.

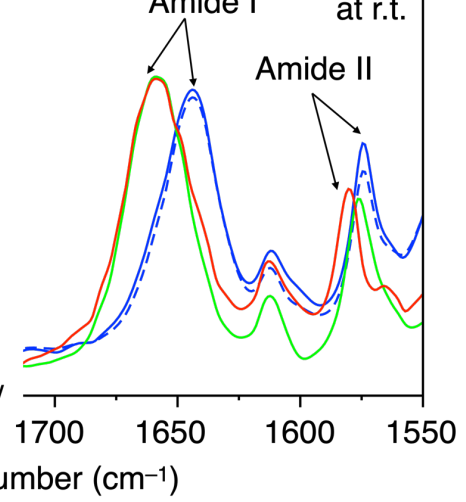

\begin{tabular}{|c|c|c|c|c|}
\hline Complex & Solvent & $\begin{array}{l}\text { Amide I } \\
\left(\mathrm{cm}^{-1}\right)\end{array}$ & $\begin{array}{c}\text { H-bonded NH } \\
\left(\mathrm{cm}^{-1}\right)\end{array}$ & $\begin{array}{c}\text { Free NH } \\
\left(\mathrm{cm}^{-1}\right)\end{array}$ \\
\hline$\left[(S)-1 \mathrm{a}^{\mathrm{Ph}}-\mathrm{TEG} \cdot \mathrm{DABCO}\right]_{\mathrm{n}}$ & $\mathrm{CHCl}_{3} / n$-hexane $(1 / 1, \mathrm{v} / \mathrm{v})$ & 1644 & 3341 & 3440 \\
\hline$\left[(S)-2 \mathbf{a}^{M e-T E G} \cdot D A B C O\right]_{n}$ & $\mathrm{CHCl}_{3} / n$-hexane $(1 / 1, \mathrm{v} / \mathrm{v})$ & 1644 & 3341 & 3442 \\
\hline$\left[(S)-1 a^{\text {Ph-TEG.DABCO }]_{n}}\right.$ & $\mathrm{CHCl}_{3}$ & 1658 & 3350 (broad) & 3435 \\
\hline$(S)-1 a^{\text {Ph-TEG }}(\mathrm{QND})_{2}$ & $\mathrm{CHCl}_{3} / n$-hexane $(1 / 1, \mathrm{v} / \mathrm{v})$ & 1658 & 3325 (broad) & 3435 \\
\hline
\end{tabular}

Figure S13. CD and absorption (a) and IR (b) spectra of $\left[(S)-\mathbf{1 a}{ }^{\mathrm{Ph}}-\mathrm{TEG} \cdot \mathrm{DABCO}\right]_{\mathrm{n}}$ in $\mathrm{CHCl}_{3} / n$ hexane $(1 / 1, \mathrm{v} / \mathrm{v})$ (blue lines) and $\mathrm{CHCl}_{3}$ (green lines), $\left[(S)-\mathbf{2} \mathbf{a}^{\mathrm{Me}}-\mathrm{TEG} \cdot \mathrm{DABCO}\right]_{\mathrm{n}}$ in $\mathrm{CHCl}_{3} / n$-hexane $(1 / 1, \mathrm{v} / \mathrm{v})$ (blue dashed lines), and $(S)-\mathbf{1} \mathbf{a}^{\mathbf{P h}}-\mathrm{TEG} \cdot(\mathrm{QND})_{2}$ in $\mathrm{CHCl}_{3} / n$-hexane $(1 / 1, \mathrm{v} / \mathrm{v})$ (red lines) at $25^{\circ} \mathrm{C}$ (a) and room temperature (b). [1 or 2] $=0.70 \mathrm{mM}$. 


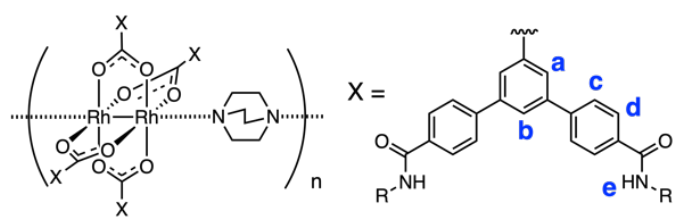

(a) $0.70 \mathrm{mM}$ in $\mathrm{CHCl}_{3} / n$-hexane $(1 / 1, \mathrm{v} / \mathrm{v})$ at r.t.

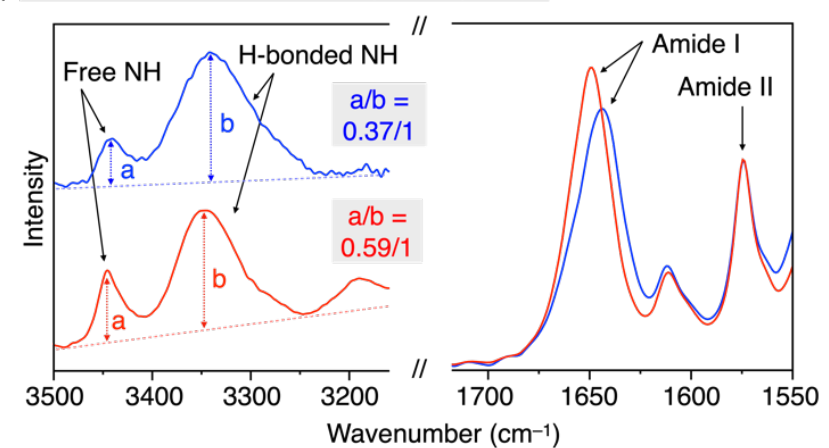

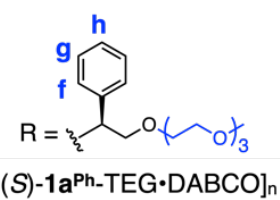

$($-)

(b) $0.70 \mathrm{mM}$ in $\mathrm{CHCl}$ at $\mathrm{rt}$.

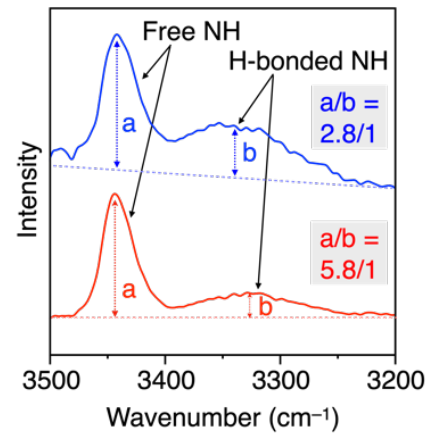

\begin{tabular}{|c|c|c|c|c|}
\hline Complex & Solvent & $\begin{array}{l}\text { Amide I } \\
\left(\mathrm{cm}^{-1}\right)\end{array}$ & $\begin{array}{c}\text { H-bonded NH } \\
\left(\mathrm{cm}^{-1}\right)\end{array}$ & $\begin{array}{c}\text { Free } \mathrm{NH} \\
\left(\mathrm{cm}^{-1}\right)\end{array}$ \\
\hline$\left[(S)-1 a^{\text {Ph-TEG }} \cdot \mathrm{DABCO}\right]_{\mathrm{n}}$ & $\mathrm{CHCl}_{3} / n$-hexane $(1 / 1, \mathrm{v} / \mathrm{v})$ & 1644 & 3341 & 3440 \\
\hline 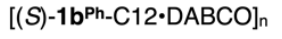 & $\mathrm{CHCl}_{3} / n$-hexane $(1 / 1, \mathrm{v} / \mathrm{v})$ & 1649 & 3345 & 3440 \\
\hline$\left[(S)-1 a^{P h-T E G} \cdot D A B C O\right]_{n}$ & $\mathrm{CHCl}_{3}$ & 1658 & 3350 (broad) & 3435 \\
\hline$\left[(S)-\mathbf{1} \mathbf{b}^{\mathrm{Ph}}-\mathrm{C} 12 \cdot \mathrm{DABCO}\right]_{\mathrm{n}}$ & $\mathrm{CHCl}_{3}$ & 1658 & 3329 (broad) & 3435 \\
\hline
\end{tabular}

(c) $\left[(S)-1 a^{\text {Ph-TEG} \cdot D A B C O}\right]_{n}$ in $\mathrm{CDCl}_{3}$ at $25^{\circ} \mathrm{C}$

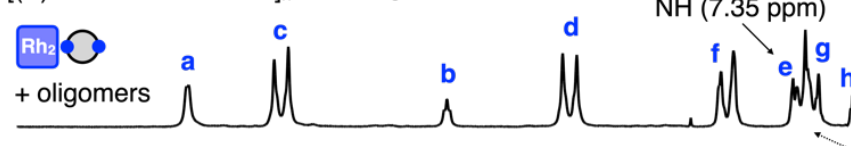

(d) $\left[(S)-1 b^{\text {Ph- }-C 12 \cdot D A B C O}\right]_{n}$ in $\mathrm{CDCl}_{3}$ at $25^{\circ} \mathrm{C}$

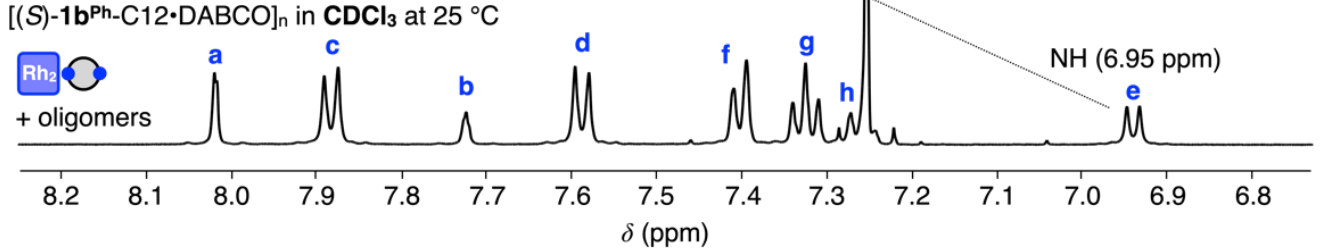

Figure S14. $(\mathrm{a}, \mathrm{b})$ IR spectra of $\left[(S)-\mathbf{1 a}^{\mathbf{P h}}-\mathrm{TEG} \cdot \mathrm{DABCO}\right]_{\mathrm{n}}$ (blue lines) and $\left[(S)-\mathbf{1 b}^{\mathbf{P h}}-\mathrm{C} 12 \cdot \mathrm{DABCO}\right]_{\mathrm{n}}$ (red lines) in $\mathrm{CHCl}_{3} / n$-hexane $\left(1 / 1, \mathrm{v} / \mathrm{v}\right.$ ) (a) and $\mathrm{CHCl}_{3}$ (b) at room temperature. (c, d) ${ }^{1} \mathrm{H} \mathrm{NMR}$ spectra $\left(500 \mathrm{MHz}, \mathrm{CDCl}_{3}, 25^{\circ} \mathrm{C}\right)$ of $\left[(S)-\mathbf{1} \mathbf{a}^{\mathbf{P h}}-\mathrm{TEG} \cdot \mathrm{DABCO}\right]_{\mathrm{n}}(\mathrm{c})$ and $\left[(S)-\mathbf{1} \mathbf{b}^{\mathrm{Ph}}-\mathrm{C} 12 \cdot \mathrm{DABCO}\right]_{\mathrm{n}}(\mathrm{d})$. $[1]=0.70 \mathrm{mM}$.

Based on the changes in the relative peak intensities between the hydrogen-bonded (3341-3345 $\mathrm{cm}^{-}$ $\left.{ }^{1}\right)$ and free amide N-H $\left(3440 \mathrm{~cm}^{-1}\right)$ stretch bands of $\left[(S)-\mathbf{1 a}^{\mathbf{P h}}-\mathrm{TEG} \cdot \mathrm{DABCO}\right]_{\mathrm{n}}$ and $\left[(S)-\mathbf{1} \mathbf{b}^{\mathbf{P h}}\right.$ $\mathrm{C} 12 \cdot \mathrm{DABCO}]_{\mathrm{n}}$ measured in $\mathrm{CHCl}_{3} / n$-hexane $(1 / 1, \mathrm{v} / \mathrm{v})$ and $\mathrm{CHCl}_{3}$, in which they exist as polymeric and monomeric/oligomeric states, respectively, the relative ratio of the hydrogen-bonded amide $\mathrm{NH}$ band appears to be higher for $\left[(S)-\mathbf{1 b}^{\mathbf{P h}}-\mathrm{C} 12 \cdot \mathrm{DABCO}\right]_{\mathrm{n}}$ (Figure $\mathrm{S} 14 \mathrm{a}, \mathrm{b}$ ), resulting from an intramolecular hydrogen bonding with the ether oxygen atoms of the peripheral TEG chains of $[(S)$ $\mathbf{1 a}^{\mathbf{P h}}$-TEG $\left.\cdot \mathrm{DABCO}\right]_{\mathrm{n}}$. This is also supported by their ${ }^{1} \mathrm{H}$ NMR spectra in $\mathrm{CDCl}_{3}$, in which the amide $\mathrm{NH}$ proton resonances of the monomeric $\left[(S)-\mathbf{1} \mathbf{a}^{\mathbf{P h}}-\mathrm{TEG} \cdot \mathrm{DABCO}\right]_{\mathrm{n}}$ showed a $0.4 \mathrm{ppm}$ downfield shift relative to those of $\left[(S)-1 \mathbf{b}^{\mathrm{Ph}}-\mathrm{C} 12 \cdot \mathrm{DABCO}\right]_{\mathrm{n}}$ (Figure $\left.\mathrm{S} 14 \mathrm{c}, \mathrm{d}\right)$. Such a downfield shift is expected for the protons involved in hydrogen bonding with the PEG chains. 


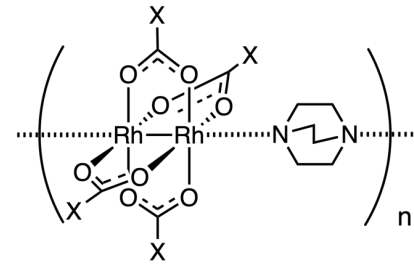

\section{$0.70 \mathrm{mM}$}

in $\mathrm{CHCl}_{3} / n$-hexane $(1 / 1, \mathrm{v} / \mathrm{v})$

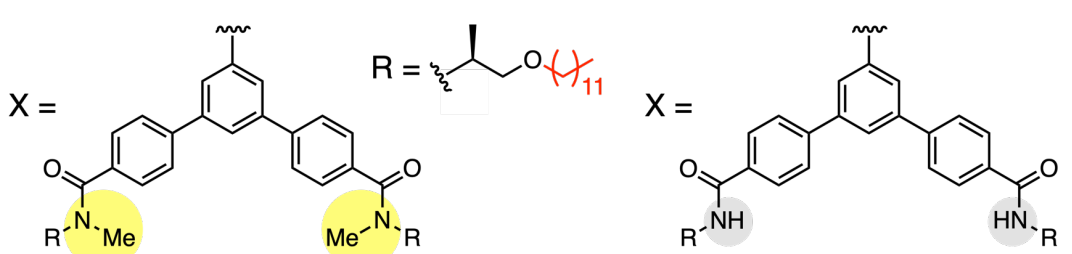

$\left[(S)-2 \mathbf{b}^{M e}-\mathrm{C} 12_{\mathrm{NMe}} \cdot \mathrm{DABCO}\right]_{\mathrm{n}}$ (a)

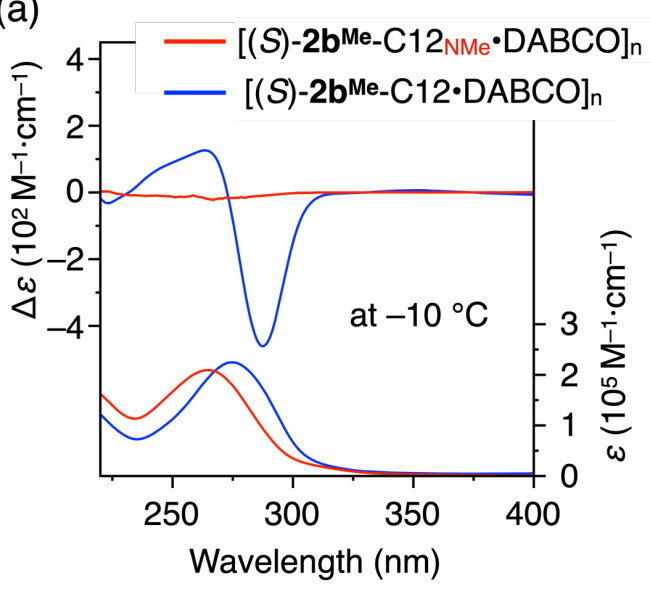

(b)

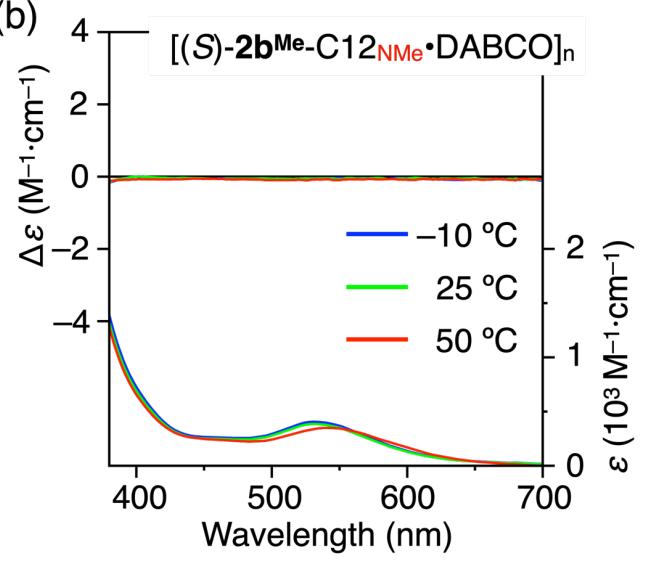

(c)

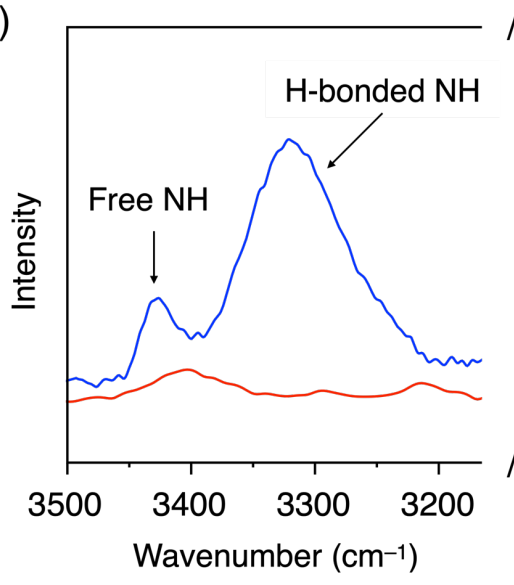

$/ / \quad-\quad\left[(S)-2 b^{M e}-C 12_{\mathrm{NMe}} \cdot \mathrm{DABCO}\right]_{\mathrm{n}}$

$\left[(S)-2 b^{M e}-C 12 \cdot D A B C O\right]_{n}$

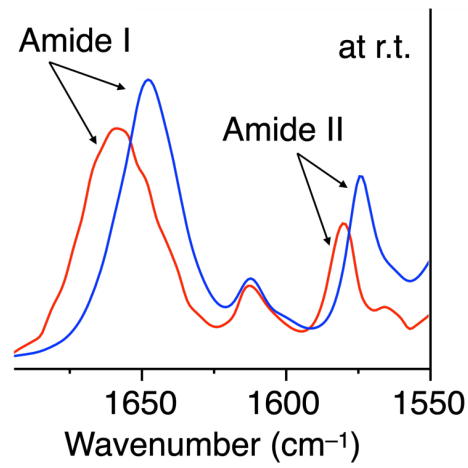

\begin{tabular}{ccccc}
\hline Complex & Solvent & $\begin{array}{c}\text { Amide I } \\
\left(\mathrm{cm}^{-1}\right)\end{array}$ & $\begin{array}{c}\mathrm{H} \text {-bonded NH } \\
\left(\mathrm{cm}^{-1}\right)\end{array}$ & $\begin{array}{c}\text { Free } \mathrm{NH} \\
\left(\mathrm{cm}^{-1}\right)\end{array}$ \\
\hline$[(S) \text {-2bMe-C12NMe-DABCO }]_{\mathrm{n}}$ & $\mathrm{CHCl}_{3} / n$-hexane $(1 / 1, \mathrm{v} / \mathrm{v})$ & 1659 & - & - \\
{$\left[(S)-2 b^{M e}-\mathrm{C} 12 \cdot \mathrm{DABCO}\right]_{\mathrm{n}}$} & $\mathrm{CHCl}_{3} / n$-hexane $(1 / 1, \mathrm{v} / \mathrm{v})$ & 1648 & $\sim 3350(\mathrm{broad})$ & 3441 \\
\hline
\end{tabular}

(d) $50^{\circ} \mathrm{C}$

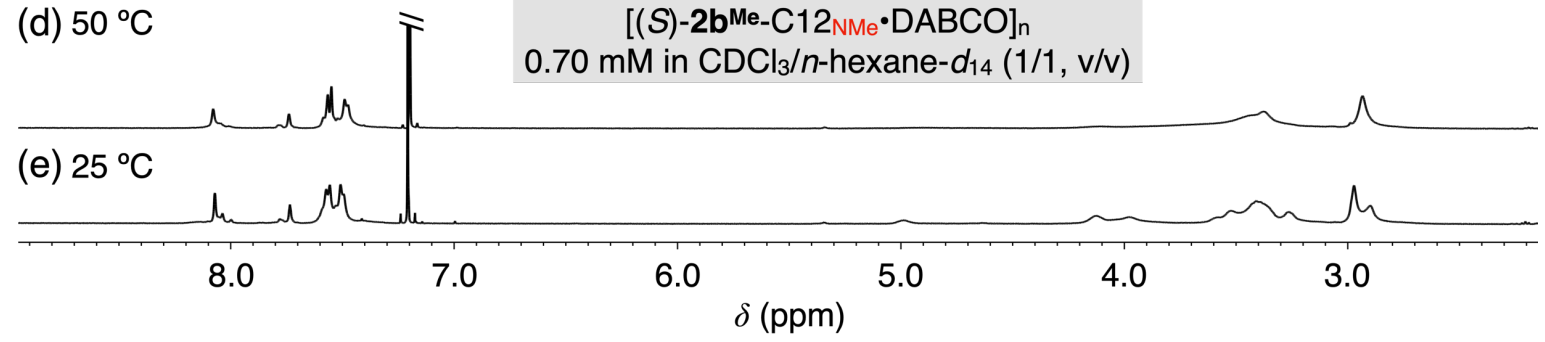


Figure S15. CD and absorption (a) and IR (c) spectra of $\left[(S)-\mathbf{2} \mathbf{b}^{\mathbf{M e}}-\mathrm{C} 12_{\mathrm{NMe}} \cdot \mathrm{DABCO}\right]_{\mathrm{n}}$ (red lines) and $\left[(S)-2 b^{\mathrm{Me}}-\mathrm{C} 12 \cdot \mathrm{DABCO}\right]_{\mathrm{n}}$ (blue lines) in $\mathrm{CHCl}_{3} / n$-hexane $(1 / 1, \mathrm{v} / \mathrm{v})$ at $-10{ }^{\circ} \mathrm{C}$ (a) and room temperature (c). (b) $T$-dependent $\mathrm{CD}$ and absorption spectral changes of $\left[(S)-\mathbf{2} \mathbf{b}^{\mathbf{M e}}-\mathrm{C} 12_{\mathrm{NMe}} \cdot \mathrm{DABCO}\right]_{\mathrm{n}}$ in $\mathrm{CHCl}_{3} / n$-hexane $(1 / 1, \mathrm{v} / \mathrm{v})$ in visible region. [2] $=0.70 \mathrm{mM} .{ }^{1} \mathrm{H} \mathrm{NMR}\left(500 \mathrm{MHz}, \mathrm{CDCl}_{3} / n\right.$-hexane$\left.d_{14}\right)$ spectra of $\left[(S)-2 \mathbf{b}^{\mathrm{Me}}-\mathrm{C} 12_{\mathrm{NMe}} \cdot \mathrm{DABCO}\right]_{\mathrm{n}}$ at $50^{\circ} \mathrm{C}(\mathrm{d})$ and $25^{\circ} \mathrm{C}(\mathrm{e})$. 


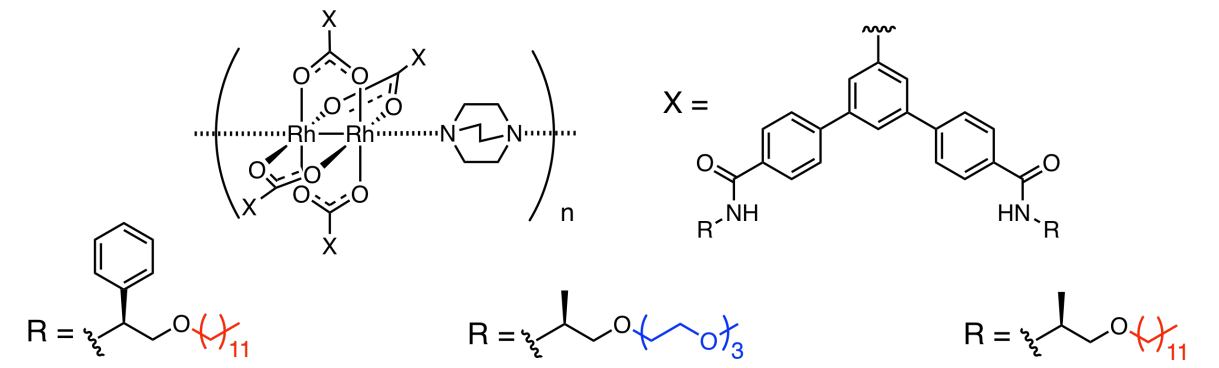

(a) $\left[(S)-\mathbf{1} \mathbf{b}^{\mathrm{Ph}}-\mathrm{C} 12 \cdot \mathrm{DABCO}\right]_{\mathrm{n}}$

Phase image $250 \mathrm{~nm} \times 250 \mathrm{~nm}$

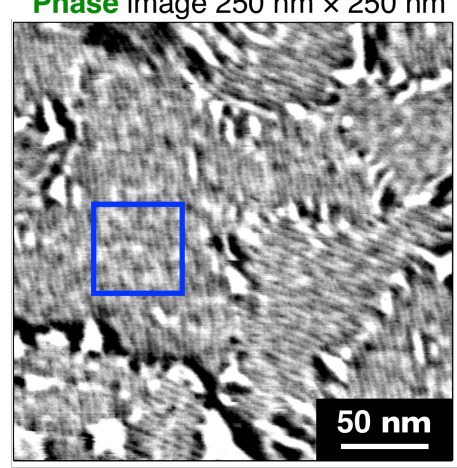

ca. $3.8 \mathrm{~nm}$

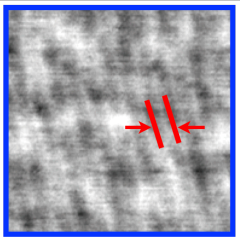

(d) $\left[(S)-1 a^{\text {Ph }}-\mathrm{TEG} \cdot \mathrm{DABCO}\right]_{\mathrm{n}}$ Height image $1 \mu \mathrm{m} \times 1 \mu \mathrm{m}$

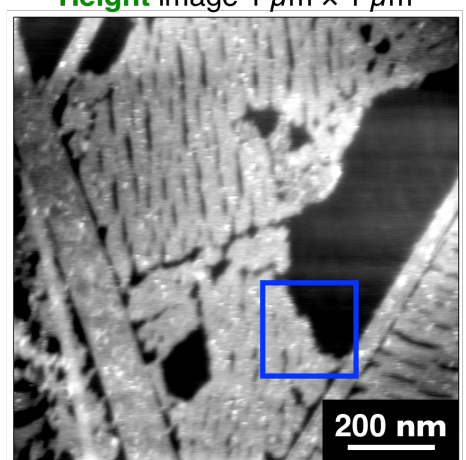

(f)

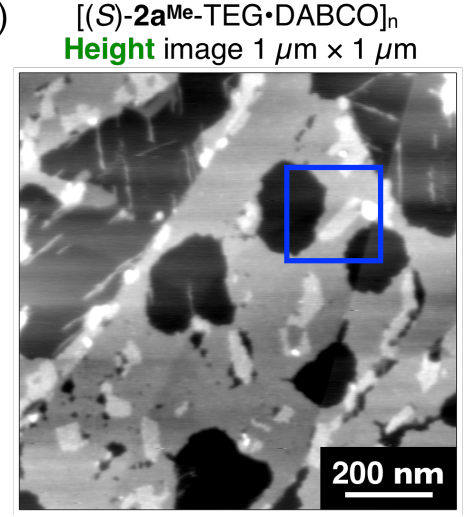

(b) $\left[(S)-2 a^{M e-T E G} \cdot D A B C O\right]_{n}$

Phase image $250 \mathrm{~nm} \times 250 \mathrm{~nm}$

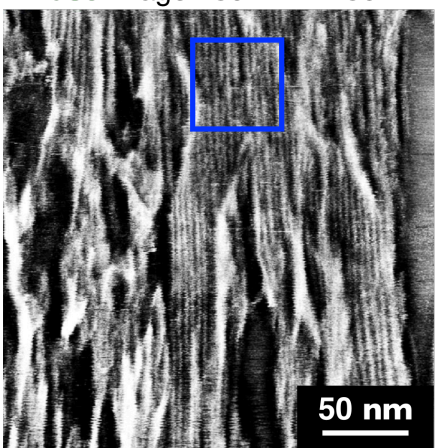

ca. $4.1 \mathrm{~nm}$

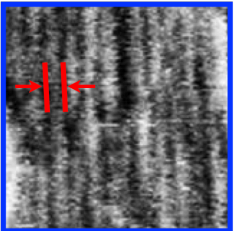

(c) $\left[(S)-2 b^{M e}-C 12 \cdot D A B C O\right]_{n}$

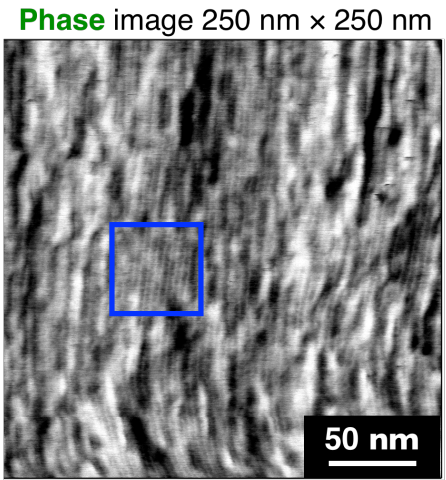

ca. $3.6 \mathrm{~nm}$

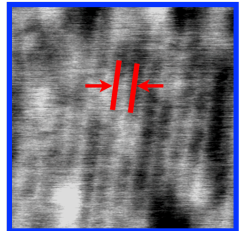

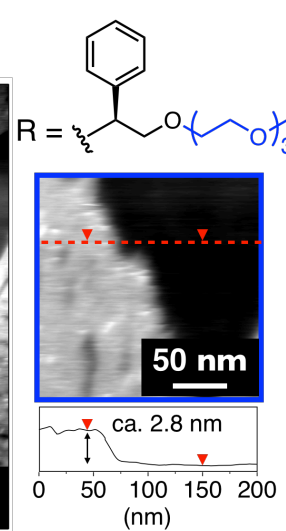

(e)
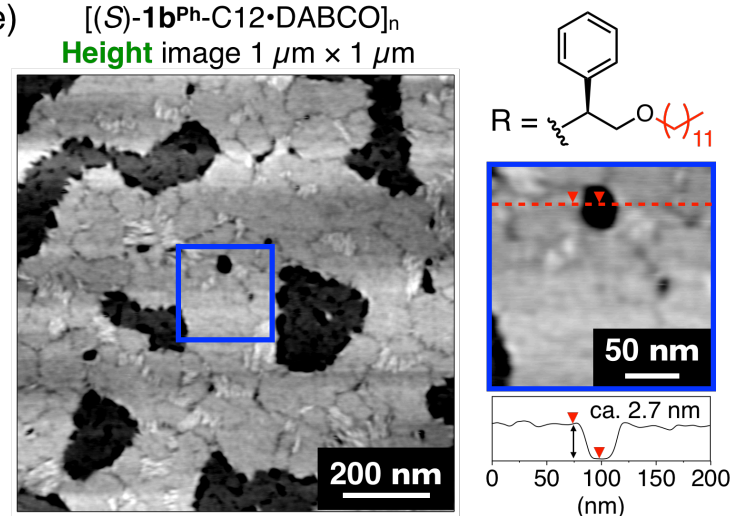

(g)
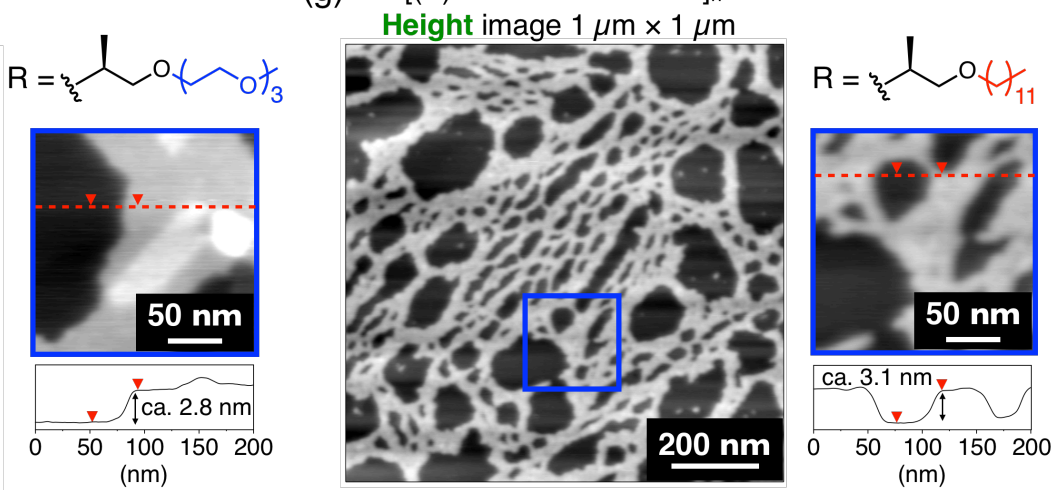

Figure S16. AFM phase $(\mathrm{a}-\mathrm{c})$ and height $(\mathrm{d}-\mathrm{g})$ images of $\left[(S)-\mathbf{1} \mathbf{a}^{\mathbf{P h}}-\mathrm{TEG} \cdot \mathrm{DABCO}\right]_{\mathrm{n}}(\mathrm{d}),\left[(S)-\mathbf{1} \mathbf{b}^{\mathbf{P h}}\right.$ $\mathrm{C} 12 \cdot \mathrm{DABCO}]_{\mathrm{n}}(\mathrm{a}, \mathrm{e}),\left[(S)-\mathbf{2} \mathbf{a}^{\mathrm{Me}}-\mathrm{TEG} \cdot \mathrm{DABCO}\right]_{\mathrm{n}}(\mathrm{b}, \mathrm{f})$, and $\left[(S)-\mathbf{2} \mathbf{b}^{\mathbf{M e}}-\mathrm{C} 12 \cdot \mathrm{DABCO}\right]_{\mathrm{n}}(\mathrm{c}, \mathrm{g})$ cast on HOPG. The magnified images indicated by the blue squares are also shown in $(\mathrm{a}-\mathrm{g})$. 
Table S2. AFM Measurement Results of Supramolecular Helical Polymers of Dirhodium(II) Paddlewheels with DABCO

\begin{tabular}{ccc}
\hline complex & $\begin{array}{c}\text { average height }^{a} \\
(\mathrm{~nm})\end{array}$ & $\begin{array}{c}\text { average chain-to-chain distance }^{a} \\
(\mathrm{~nm})\end{array}$ \\
\hline$\left[(S)-\mathbf{1} \mathbf{a}^{\mathbf{P h}} \text {-TEG} \cdot \mathrm{DABCO}\right]_{\mathrm{n}}$ & $2.77 \pm 0.18$ & $3.39 \pm 0.32$ \\
{$\left[(S)-\mathbf{1} \mathbf{b}^{\mathbf{P h}}-\mathrm{C} 12 \cdot \mathrm{DABCO}\right]_{\mathrm{n}}$} & $2.67 \pm 0.12$ & $3.79 \pm 0.25$ \\
{$\left[(S)-\mathbf{2} \mathbf{a}^{\mathbf{M e}}-\mathrm{TEG} \cdot \mathrm{DABCO}\right]_{\mathrm{n}}$} & $2.80 \pm 0.19$ & $4.10 \pm 0.18$ \\
{$\left[(S)-\mathbf{2} \mathbf{b}^{\mathbf{M e}}-\mathrm{C} 12 \cdot \mathrm{DABCO}\right]_{\mathrm{n}}$} & $3.14 \pm 0.20$ & $3.58 \pm 0.33$ \\
\hline
\end{tabular}

${ }^{a}$ Based on an evaluation of 10 (height) and 60 (chain-to-chain distance) polymers, the average height and chain-to-chain distance were estimated. 
(a)
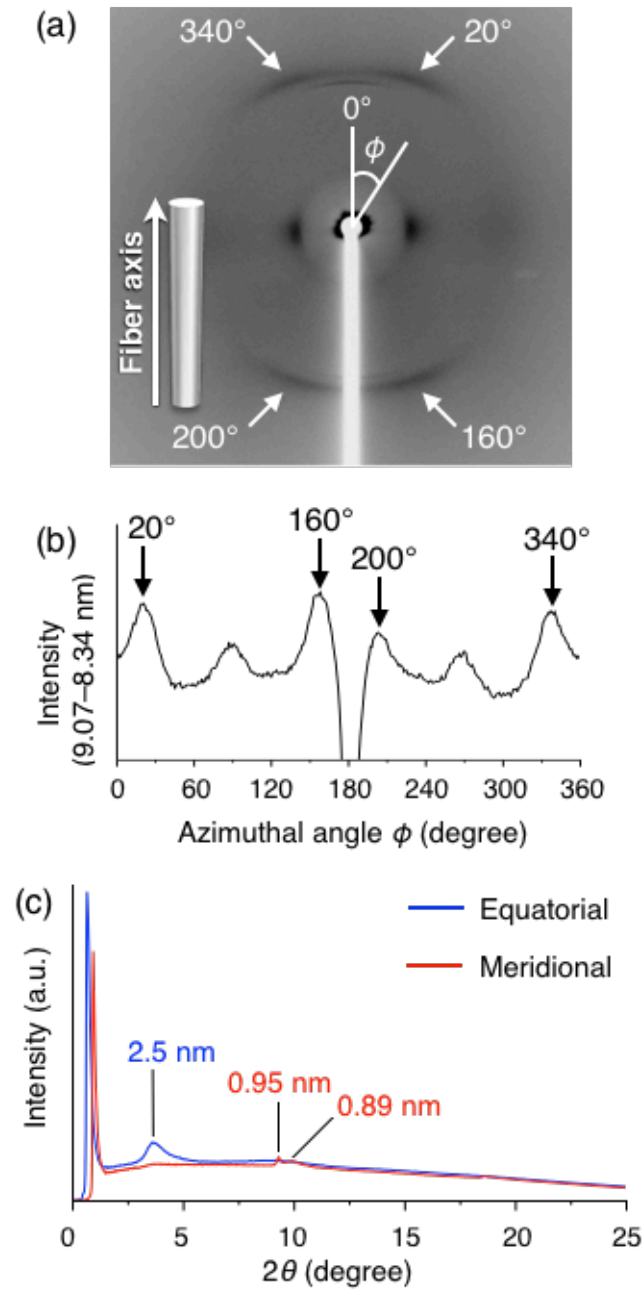

Figure S17. (a) 2D-WAXD pattern of the oriented fibers of $\left[(S)-1 \mathbf{a}^{\mathbf{P h}}-\mathrm{TEG} \cdot \mathrm{DABCO}\right]_{\mathrm{n}}$ at $25^{\circ} \mathrm{C}$. (b) Azimuthal angle $(\phi)$ versus intensity plot of the fiber pattern from (a). (c) Meridional (red line) and equatorial (blue line) profiles of the fiber pattern from (a). 

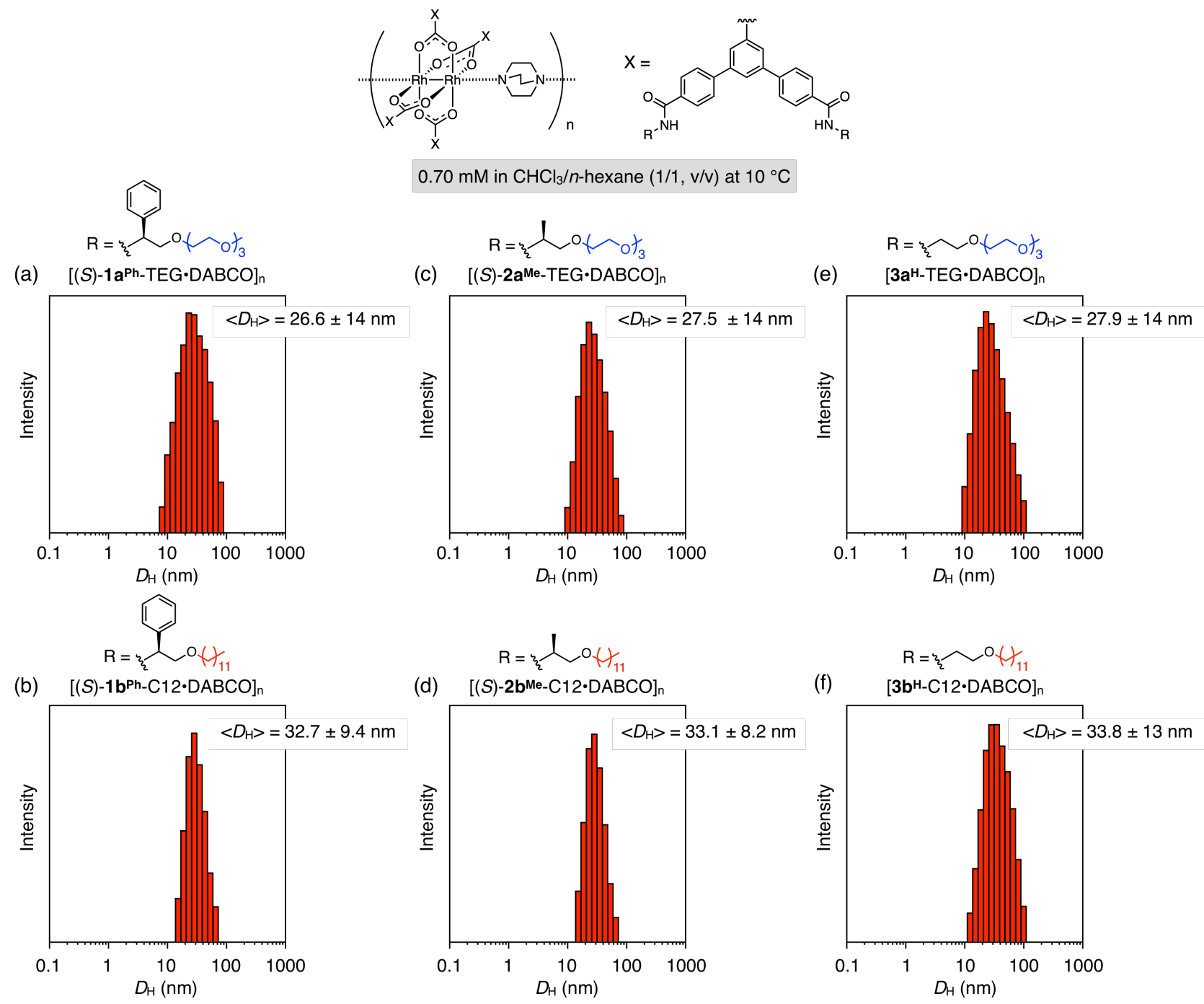

Figure S18. Histogram analyses of DLS measurements of $\left[(S)-\mathbf{1 a}^{\mathbf{P h}}-\mathrm{TEG} \cdot \mathrm{DABCO}\right]_{\mathrm{n}}(\mathbf{a}),\left[(S)-\mathbf{1} \mathbf{b}^{\mathbf{P h}}\right.$

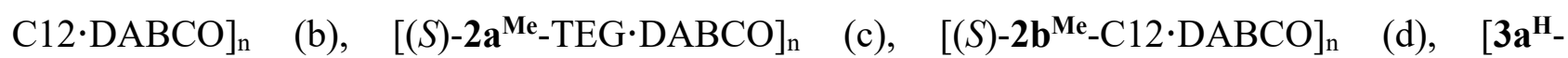
$\mathrm{TEG} \cdot \mathrm{DABCO}]_{\mathrm{n}}(\mathrm{e})$, and $\left[\mathbf{3 b}^{\mathrm{H}}-\mathrm{C} 12 \cdot \mathrm{DABCO}\right]_{\mathrm{n}}$ (f) in $\mathrm{CHCl}_{3} / n$-hexane $(1 / 1, \mathrm{v} / \mathrm{v})$ at $10{ }^{\circ} \mathrm{C}$. [dirhodium(II) $\cdot \mathrm{DABCO}]=0.70 \mathrm{mM}$. 
Table S3. Hydrodynamic Diameter $\left.\left(<D_{\mathrm{H}}\right\rangle\right)$ Values of Coordination Polymers Estimated by DLS Measurements in $\mathrm{CHCl}_{3} / n$-Hexane $(1 / 1, \mathrm{v} / \mathrm{v})$ at Different Temperatures

\begin{tabular}{|c|c|c|c|c|c|}
\hline \multirow{3}{*}{ complex } & \multicolumn{4}{|c|}{ temperature } & \multirow{3}{*}{$\mathrm{DP}(\mathbf{n})$ at $10^{\circ} \mathrm{C}^{a}$} \\
\hline & $40^{\circ} \mathrm{C}$ & $30^{\circ} \mathrm{C}$ & $20^{\circ} \mathrm{C}$ & $10^{\circ} \mathrm{C}$ & \\
\hline & $\begin{array}{c}<\boldsymbol{D}_{\mathrm{H}}> \\
(\mathrm{nm})\end{array}$ & $\begin{array}{l}<\boldsymbol{D}_{\mathbf{H}}> \\
(\mathrm{nm})\end{array}$ & $\begin{array}{c}<\boldsymbol{D}_{\mathbf{H}}> \\
(\mathrm{nm})\end{array}$ & $\begin{array}{c}<\boldsymbol{D}_{\mathbf{H}}> \\
(\mathrm{nm})\end{array}$ & \\
\hline$\left[(S)-\mathbf{1 a}^{\mathbf{P h}}-\mathrm{TEG} \cdot \mathrm{DABCO}\right]_{\mathrm{n}}$ & $-b$ & $-b$ & $18.1 \pm 8.1$ & $26.6 \pm 14$ & $28 \pm 15$ \\
\hline$\left[(S)-\mathbf{1 b}^{\mathbf{P h}}-\mathrm{C} 12 \cdot \mathrm{DABCO}\right]_{\mathrm{n}}$ & $-b$ & $14.7 \pm 6.3$ & $20.9 \pm 10$ & $32.7 \pm 9.4$ & $34 \pm 10$ \\
\hline$\left[(S)-\mathbf{2} \mathbf{a}^{\mathbf{M e}}-\mathrm{TEG} \cdot \mathrm{DABCO}\right]_{\mathrm{n}}$ & $-b$ & $13.8 \pm 5.8$ & $18.8 \pm 9.1$ & $27.5 \pm 14$ & $29 \pm 15$ \\
\hline$\left[(S)-\mathbf{2} \mathbf{b}^{\mathbf{M e}}-\mathrm{C} 12 \cdot \mathrm{DABCO}\right]_{\mathrm{n}}$ & $-b$ & $16.1 \pm 6.2$ & $21.1 \pm 11$ & $33.1 \pm 8.2$ & $35 \pm 9$ \\
\hline$\left[(S)-\mathbf{2} \mathbf{b}^{\mathbf{M e}}-\mathrm{C} 12 \cdot \mathrm{DABCO}\right]_{\mathrm{n}}{ }^{c}$ & - & - & $-b$ & $-b$ & - \\
\hline$\left[(S)-\mathbf{2} \mathbf{b}^{\mathbf{M e}}-\mathrm{C} 12_{\mathrm{NMe}} \cdot \mathrm{DABCO}\right]_{\mathrm{n}}$ & $-b$ & $-b$ & $-b$ & $-b$ & - \\
\hline$\left[\mathbf{3} \mathbf{a}^{\mathbf{H}}-\mathrm{TEG} \cdot \mathrm{DABCO}\right]_{\mathrm{n}}$ & $-b$ & $14.6 \pm 6.0$ & $20.8 \pm 9.8$ & $27.9 \pm 14$ & $29 \pm 15$ \\
\hline$\left[3 \mathbf{b}^{\mathbf{H}}-\mathrm{C} 12 \cdot \mathrm{DABCO}\right]_{\mathrm{n}}$ & $-b$ & $18.8 \pm 7.9$ & $24.4 \pm 11$ & $33.8 \pm 13$ & $36 \pm 14$ \\
\hline
\end{tabular}

${ }^{a}$ Estimated based on the optimized polymer structure (Figure $3 \mathrm{e}$ ). ${ }^{b}$ Not detected. ${ }^{c}$ In $\mathrm{CHCl}_{3}$. 


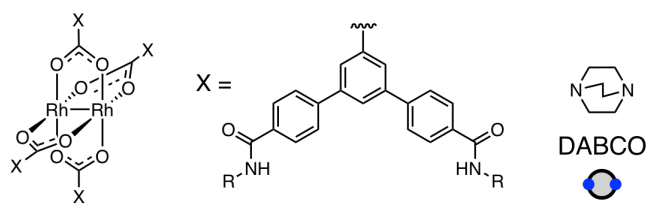

$0.70 \mathrm{mM}$ in $\mathrm{CHCl}_{3} / n$-hexane $(1 / 1, \mathrm{v} / \mathrm{v})$ at $25^{\circ} \mathrm{C}$
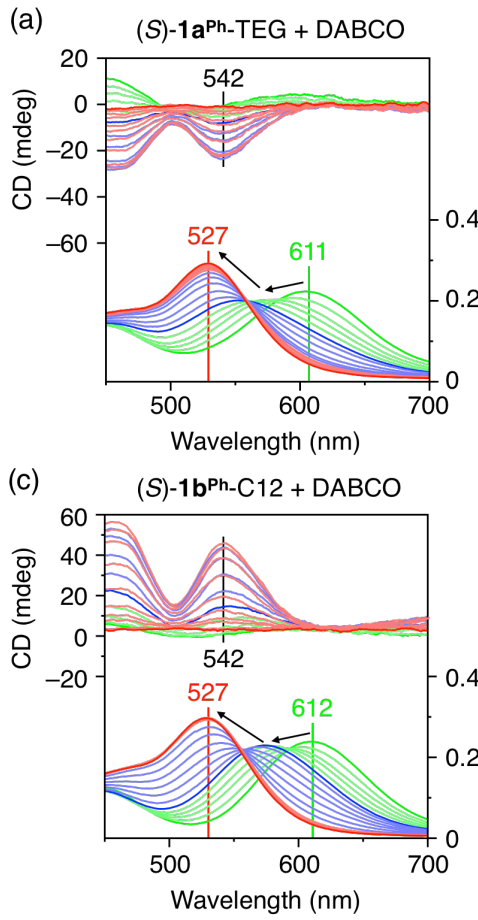

(e) (S)-2aMe-TEG + DABCO

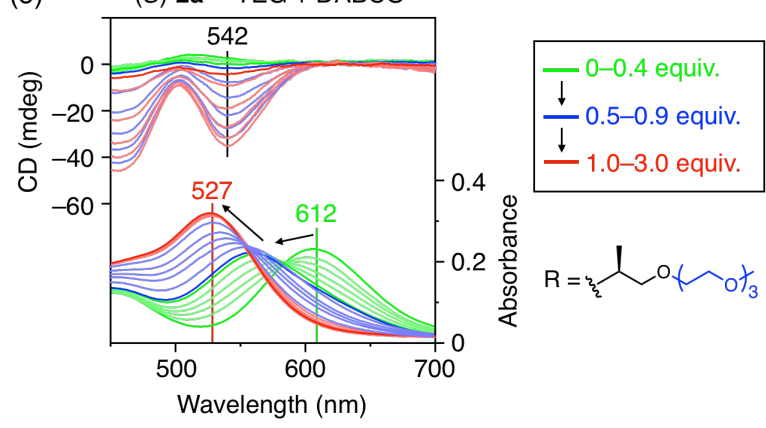

(g) $\quad(S)-2 b^{\text {Me-C}}$ 12 + DABCO
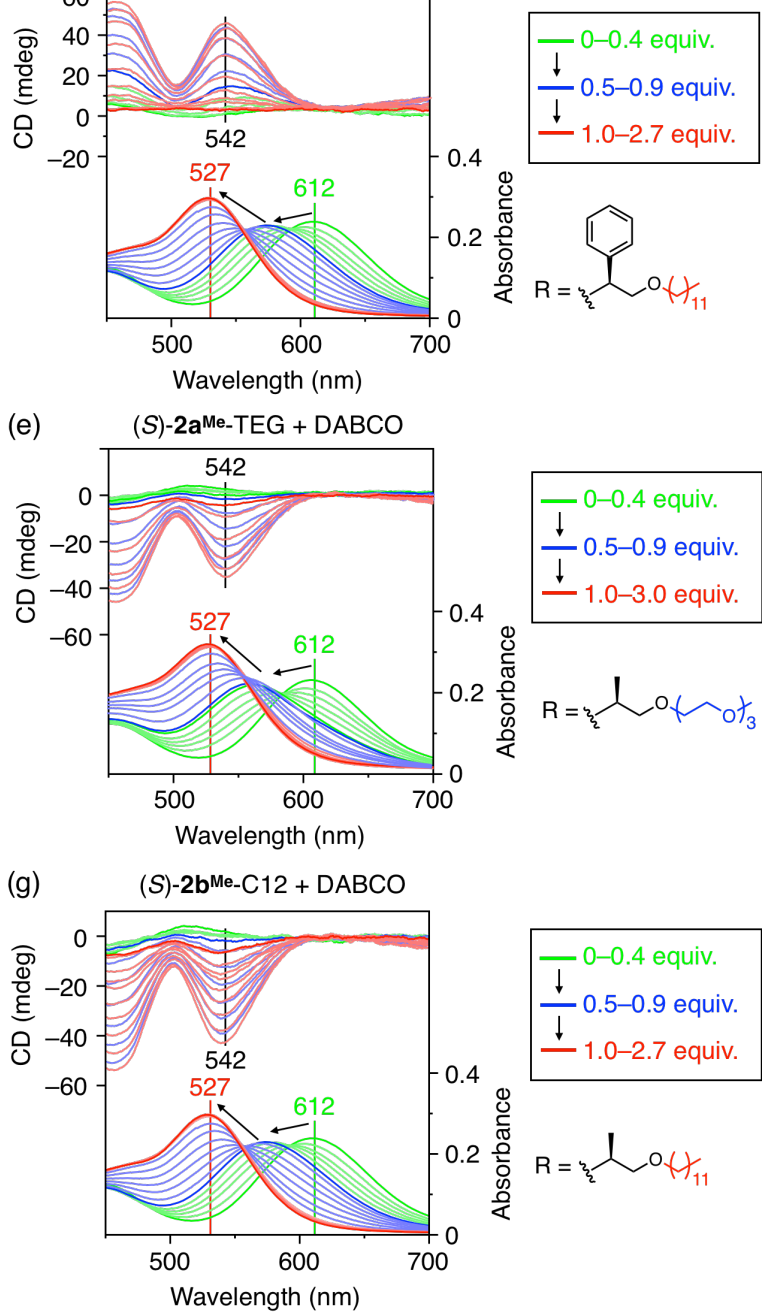

(b)

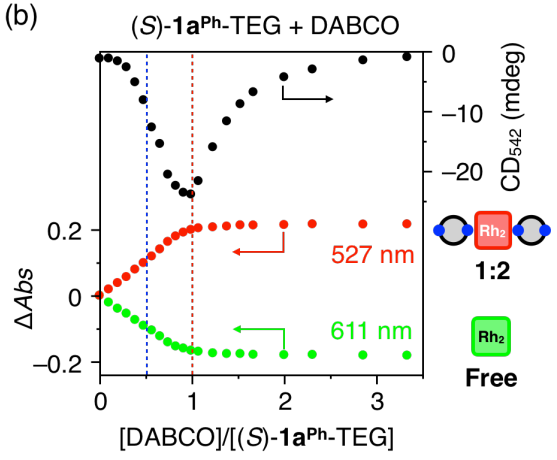

(d)

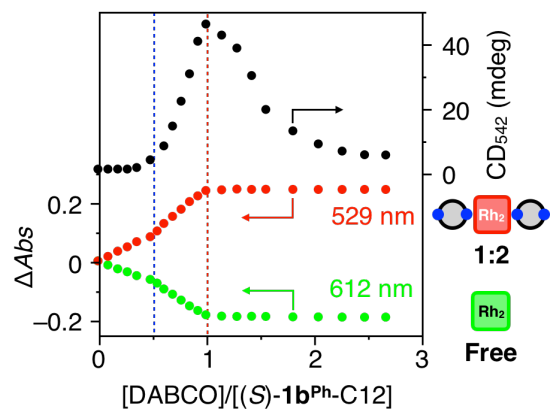

(f)

(S)-2aMe-TEG + DABCO

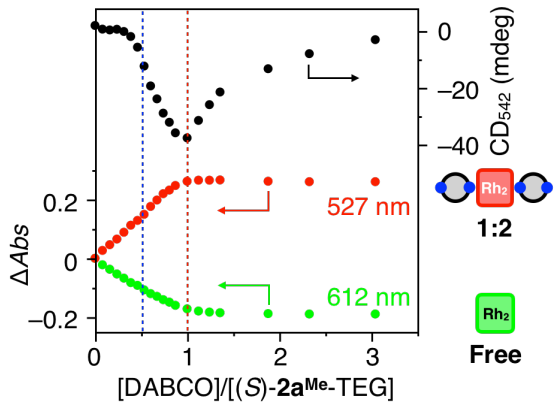

(h) $\quad(S)-2 b^{\mathrm{Me}-\mathrm{C} 12}+\mathrm{DABCO}$

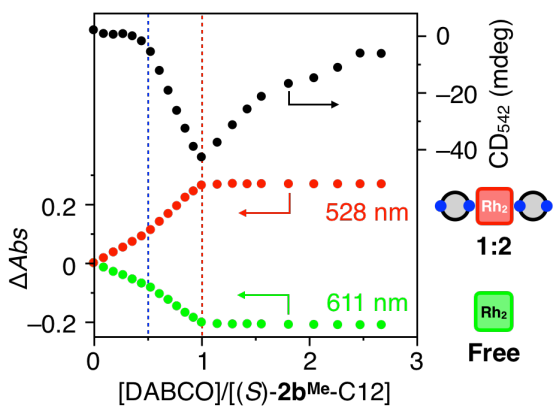

Figure S19. $\mathrm{CD}$ and visible absorption spectral changes of $(S)-\mathbf{1} \mathbf{a}^{\mathbf{P h}}-\mathrm{TEG}(\mathrm{a}),(S)-\mathbf{1 b}^{\mathbf{P h}}-\mathrm{C} 12(\mathrm{c}),(S)$ $\mathbf{2 a}^{\text {Me }}$-TEG (e), and $(S)-2 \mathbf{b}^{\text {Me }}-\mathrm{C} 12(\mathrm{~g})$ upon the addition of DABCO $(0-0.4$ equiv. (green color), 0.5 -1.0 equiv. (blue color), and $\geq 1$.0equiv. (red color)) in $\mathrm{CHCl}_{3} / n$-hexane $(1 / 1, \mathrm{v} / \mathrm{v})$ at $25^{\circ} \mathrm{C}$. (b, d, f, h) Plots of the $\mathrm{CD}$ intensities (O) at $542 \mathrm{~nm}$ vs $[\mathrm{DABCO}] /\left[\mathrm{Rh}_{2}\right]$ (top) and those of the absorbance

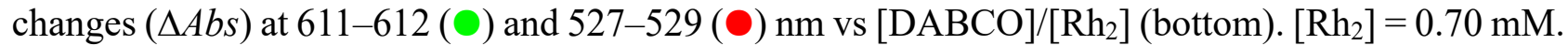




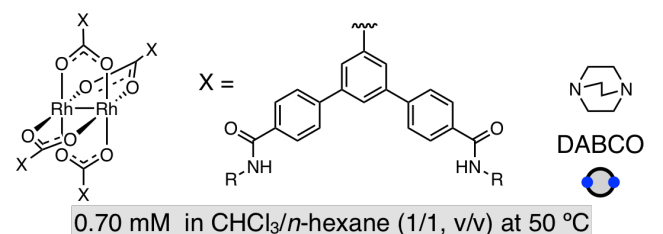

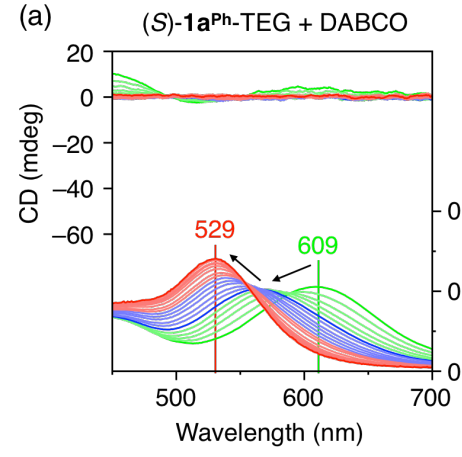
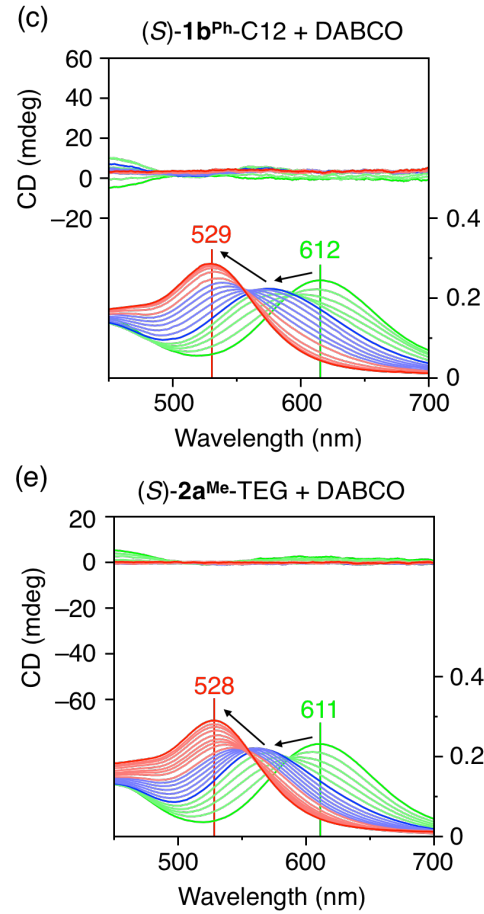

(g)
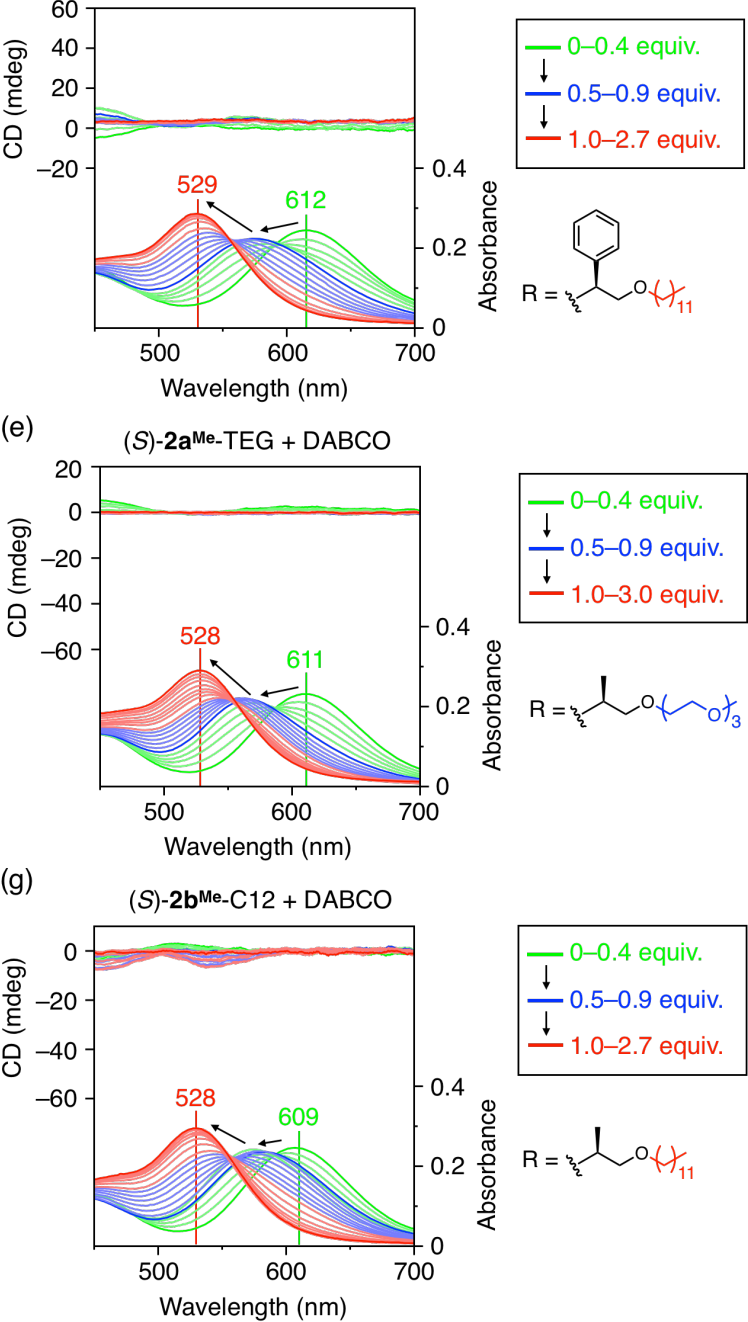

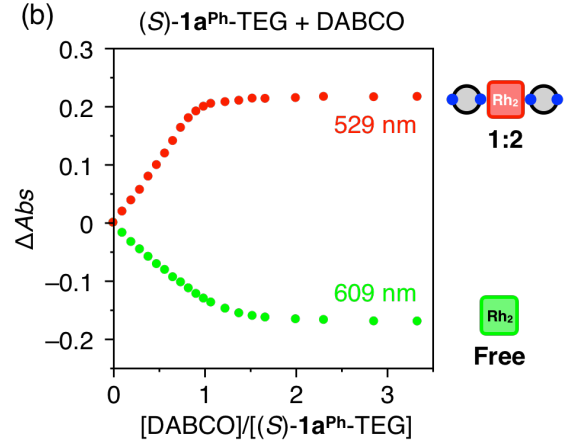

(d)

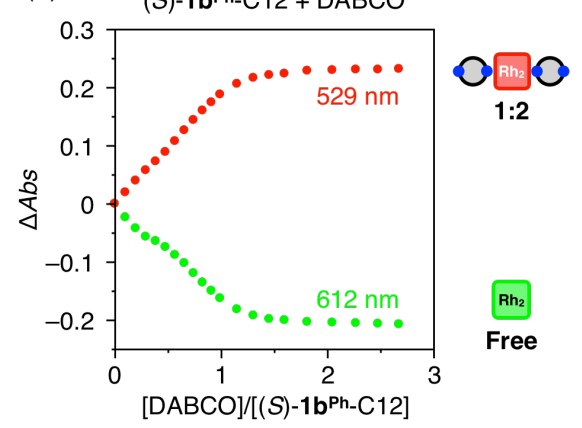

(f)

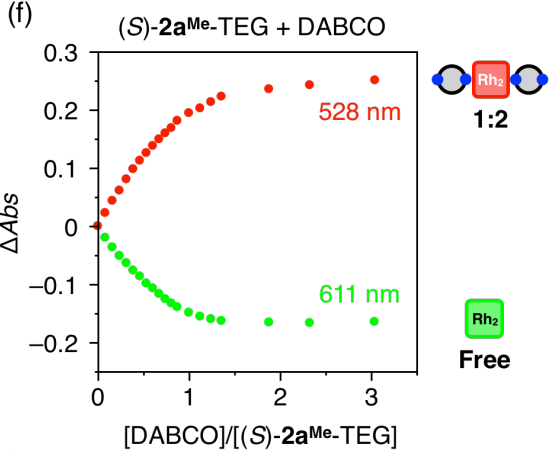

(h)

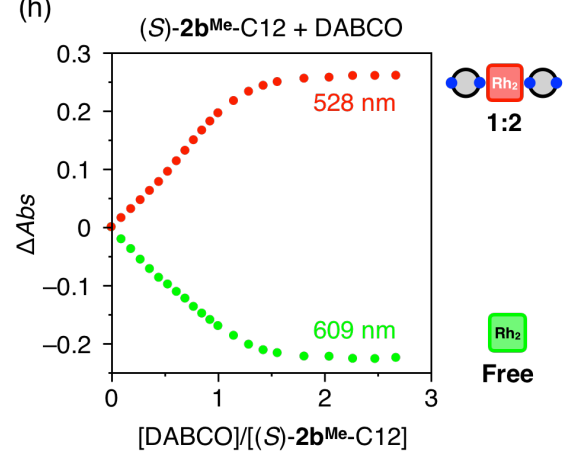

Figure S20. CD and visible absorption spectral changes of $(S)-\mathbf{1 a}^{\mathbf{P h}}-\mathrm{TEG}(\mathrm{a}),(S)-\mathbf{1} \mathbf{b}^{\mathbf{P h}}-\mathrm{C} 12(\mathrm{c}),(S)-$ $\mathbf{2 a}^{\text {Me }}$-TEG (e), and $(S)$-2 $\mathbf{b}^{\text {Me }}-\mathrm{C} 12(\mathrm{~g})$ upon the addition of DABCO $(0-0.4$ equiv. (green color), 0.5 - 1.0 equiv. (blue color), and $\geq 1.0$ equiv. (red color)) in $\mathrm{CHCl}_{3} / n$-hexane $(1 / 1, \mathrm{v} / \mathrm{v})$ at $50^{\circ} \mathrm{C}$. $(\mathrm{b}, \mathrm{d}, \mathrm{f}$, h) Plots of the absorbance changes $(\triangle A b s)$ at 609-612(○) and 528-529(○) nm vs $[\mathrm{DABCO}] /\left[\mathrm{Rh}_{2}\right]$. $\left[\mathrm{Rh}_{2}\right]=0.70 \mathrm{mM}$. 


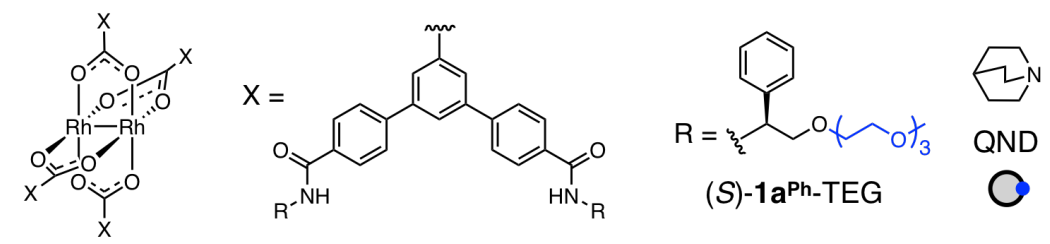

$0.70 \mathrm{mM}$ in $\mathrm{CHCl}_{3} 50^{\circ} \mathrm{C}$

(a)

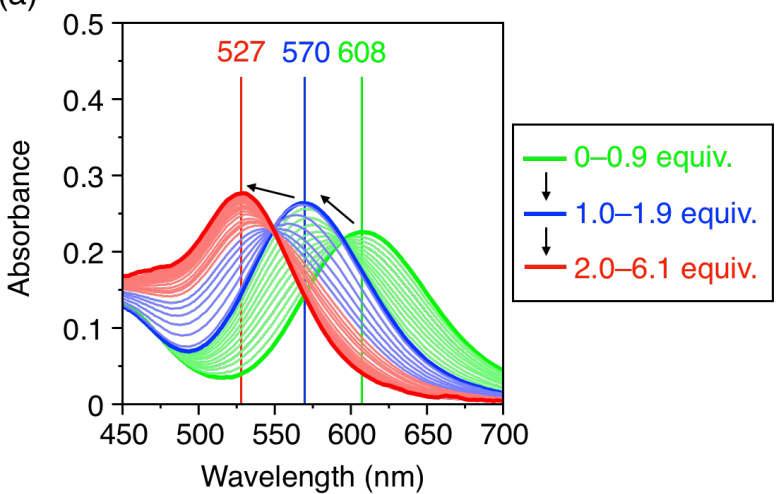

(c)

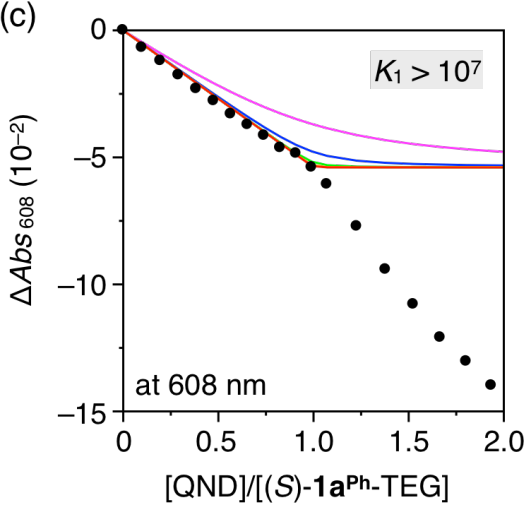

(e)

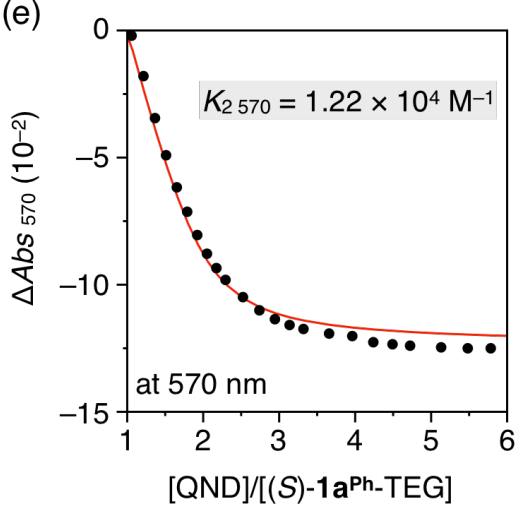

(b)

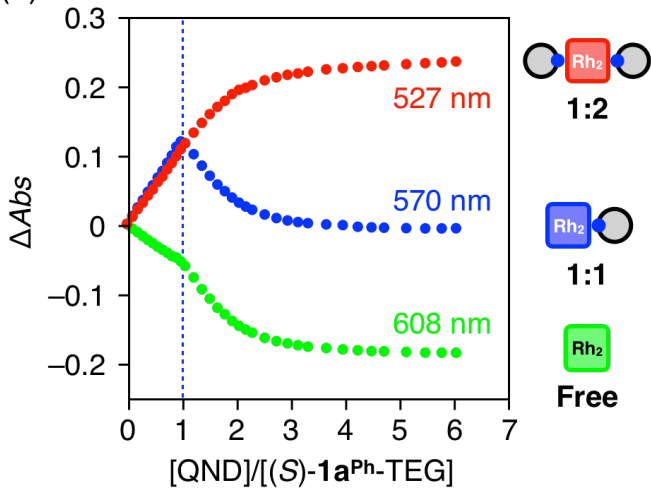

(d)
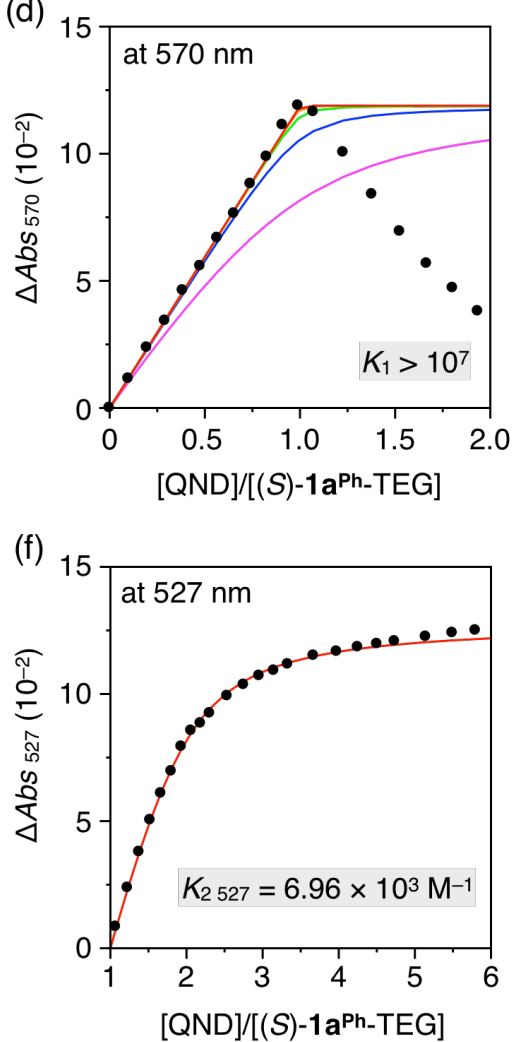

Figure S21. (a) Visible absorption spectral changes of (S)-1a $\mathbf{a}^{\mathbf{P h}}-\mathrm{TEG}(0.70 \mathrm{mM})$ upon the addition of QND (0 - 0.9 equiv. (green color), $1.0-1.9$ equiv. (blue color), and $2.0-6.1$ equiv. (red color)) in $\mathrm{CHCl}_{3}$ at $50{ }^{\circ} \mathrm{C}$. (b) Plots of the absorbance changes $(\triangle A b s)$ at $608(\bigcirc), 570(\odot)$, and $527 \mathrm{~nm}(\odot)$ vs $[Q N D] /\left[(S)-1 a^{\text {Ph}}-T E G\right]$. (c,d) Plots of the observed $\triangle A b s$ values at 608 (c) and $570 \mathrm{~nm}$ (d) vs $[\mathrm{QND}] /\left[(S)-1 \mathbf{a}^{\mathrm{Ph}}-\mathrm{TEG}\right]=0-2.0$. The curves in the plots were simulated ones as a function of $K_{1}$ values, giving the first binding constant $\left(K_{1}\right)$ to be approximately larger than $10^{7} \mathrm{M}^{-1}$. (e,f) Plots of the observed $\triangle A b s$ values at 570 and $527 \mathrm{~nm}(\mathrm{f}) \mathrm{vs}[\mathrm{QND}] /\left[(S)-\mathbf{1} \mathbf{a}^{\mathbf{P h}}-\mathrm{TEG}\right]=1.0-6.0$. The curves in the plots were obtained by the nonlinear least-squares curve-fitting, giving the second binding constant $\left(K_{2}\right)$ to be $1.22 \times 10^{4}(\mathrm{e})$ and $6.96 \times 10^{3} \mathrm{M}^{-1}(\mathrm{f})$, respectively. 


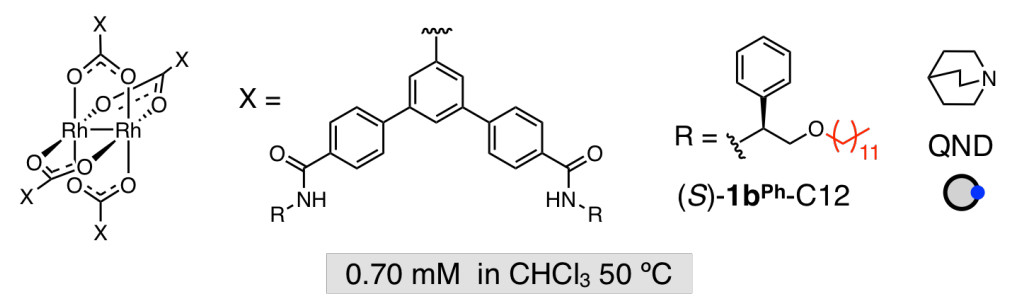

(a)

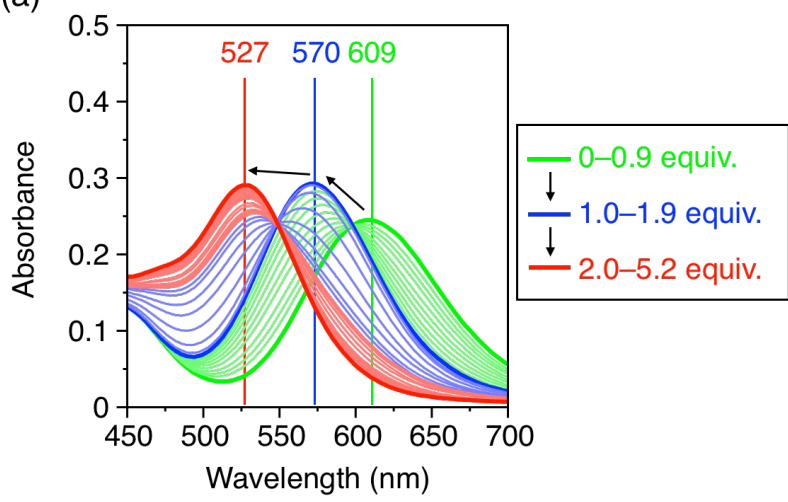

(c)

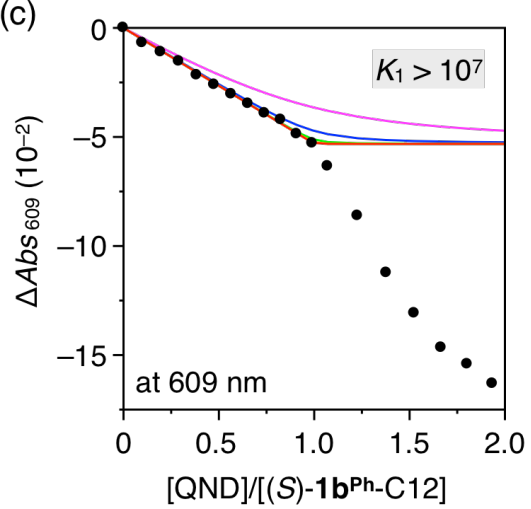

(e)

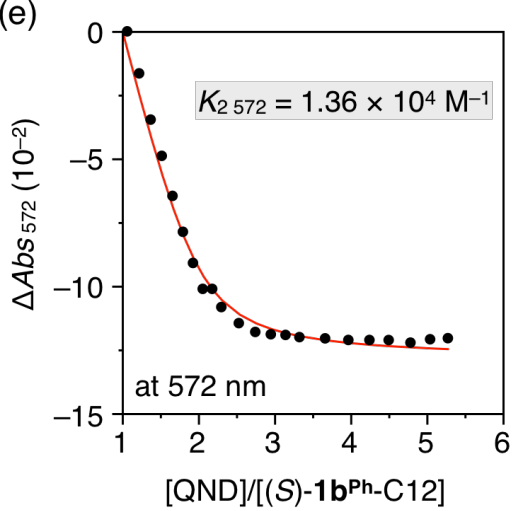

(b)

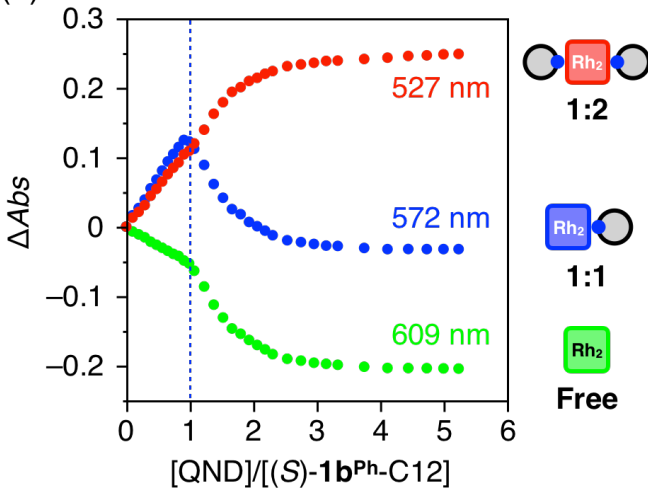

(d)
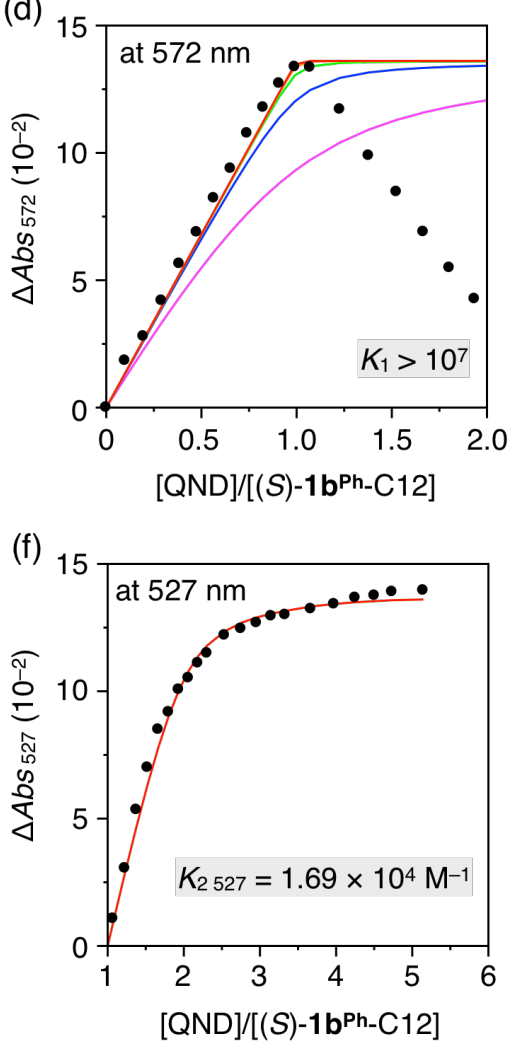

Figure S22. (a) Visible absorption spectral changes of $(S)-\mathbf{1 b}^{\mathbf{P h}}-\mathrm{C} 12(0.70 \mathrm{mM})$ upon the addition of QND ( 0 - 0.9 equiv. (green color), 1.0 - 1.9 equiv. (blue color), and $2.0-5.2$ equiv. (red color)) in $\mathrm{CHCl}_{3}$ at $50^{\circ} \mathrm{C}$. (b) Plots of the absorbance changes $(\triangle A b s)$ at $609(\bigcirc), 572(\bigcirc)$, and $527 \mathrm{~nm}\left(\mathrm{O}^{\circ}\right) \mathrm{vs}$ $[Q N D] /\left[(S)-1 \mathbf{b}^{\left.\text {Ph }_{-} \mathrm{C} 12\right]}\right.$. (c,d) Plots of the observed $\triangle A b s$ values at 609 (c) and $572 \mathrm{~nm}$ (d) vs $[\mathrm{QND}] /\left[(S)-\mathbf{1 b}^{\mathrm{Ph}}-\mathrm{C} 12\right]=0-2.0$. The curves in the plots were simulated ones as a function of $K_{1}$ values, giving the first binding constant $\left(K_{1}\right)$ to be approximately larger than $10^{7} \mathrm{M}^{-1}$. (e,f) Plots of the observed $\triangle A b s$ values at $572(\mathrm{e})$ and $527 \mathrm{~nm}(\mathrm{f}) \mathrm{vs}[\mathrm{QND}] /\left[(S)-\mathbf{1} \mathbf{b}^{\mathbf{P h}}-\mathrm{C} 12\right]=1.0-6.0$. The curves in the plots were obtained by the nonlinear least-squares curve-fitting, giving the second binding constant $\left(K_{2}\right)$ to be $1.36 \times 10^{4}(\mathrm{e})$ and $1.69 \times 10^{4} \mathrm{M}^{-1}(\mathrm{f})$, respectively. 

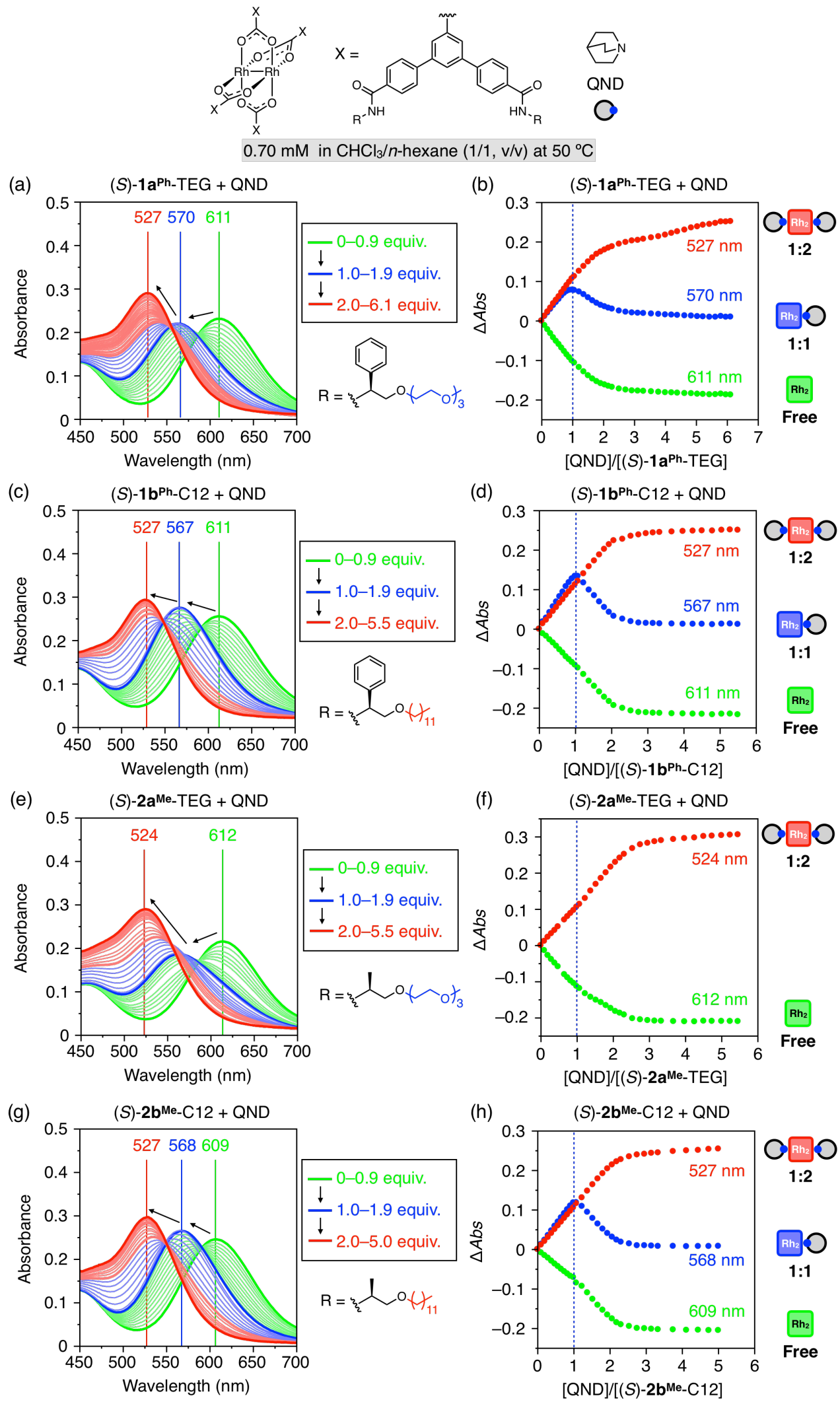

Figure S23. Visible absorption spectral changes of $(S)-\mathbf{1 a}^{\mathbf{P h}}-\mathrm{TEG}(\mathrm{a}),(S)-\mathbf{1 b}^{\mathbf{P h}}-\mathrm{C} 12(\mathrm{c}),(S)-\mathbf{2} \mathbf{a}^{\mathbf{M e}}-$ TEG (e), and $(S)-2 \mathbf{b}^{\text {Me }}-\mathrm{C} 12(\mathrm{~g})$ upon the addition of QND $(0-0.9$ equiv. (green color), $1.0-1.9$ equiv. (blue color), and $\geq 2.0$ equiv. (red color)) in $\mathrm{CHCl}_{3} / n$-hexane $(1 / 1, \mathrm{v} / \mathrm{v})$ at $50{ }^{\circ} \mathrm{C}$. $(\mathrm{b}, \mathrm{d}, \mathrm{f}, \mathrm{h})$ Plots of the absorbance changes $(\triangle A b s)$ at 609-612 (O), 567-570 (๑), and 524-527(○) nm vs $[\mathrm{QND}] /\left[\mathrm{Rh}_{2}\right] .\left[\mathrm{Rh}_{2}\right]=0.70 \mathrm{mM}$. 

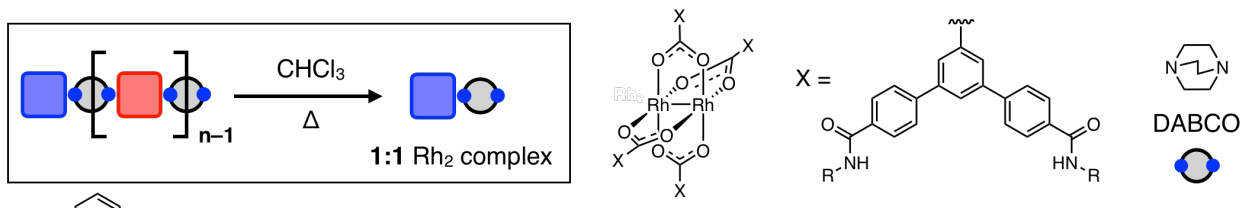

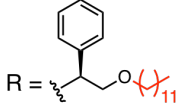

(a) (S) -1 b $^{\text {Ph- }}-\mathrm{C} 12+\mathrm{DABCO}(1.0$ equiv. $)$

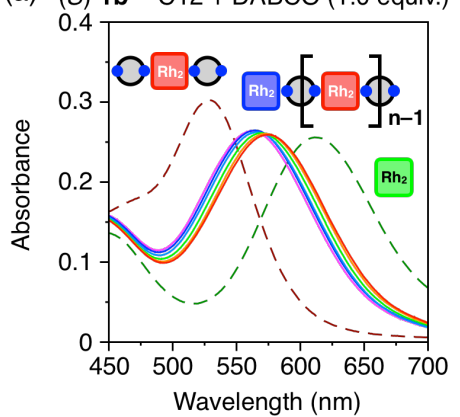

(d)

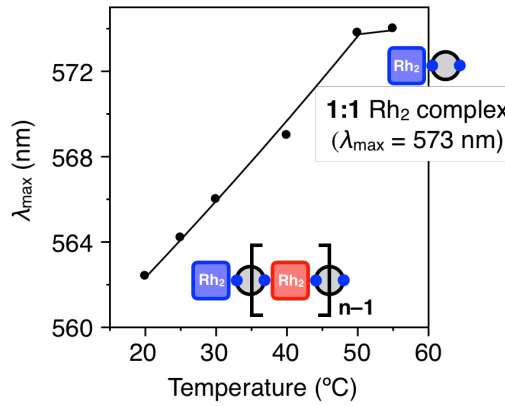

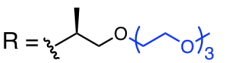

(b) (S)-2ame-TEG + DABCO (1.0 equiv.)

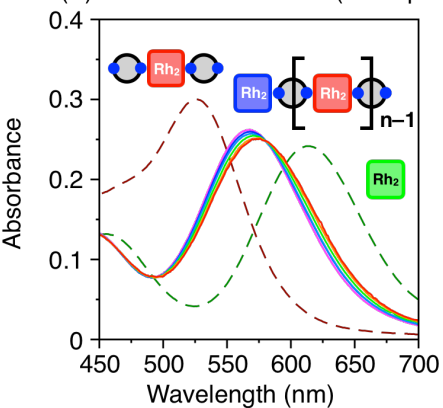

(e)

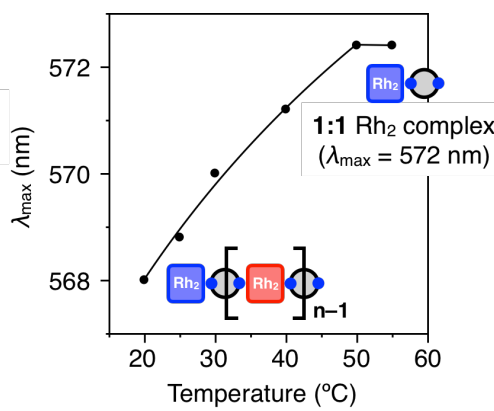

$R=$ 立

(c) (S)-2bMe-C12 + DABCO (1.0 equiv.)

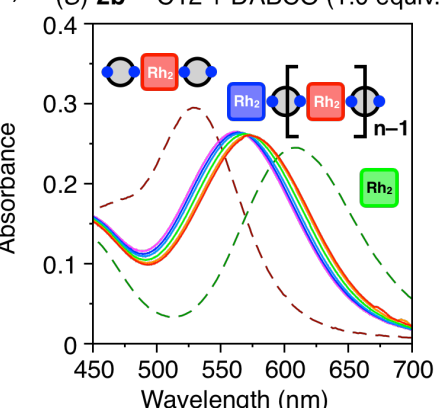

(f)

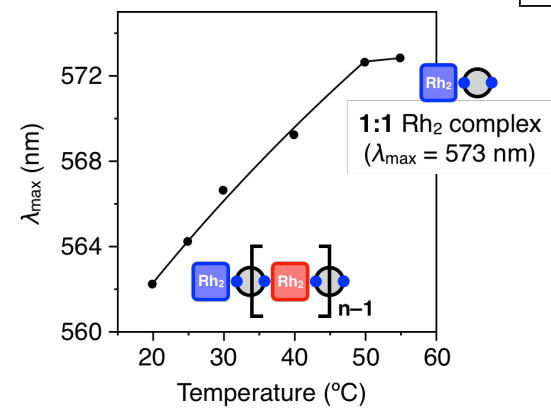

Figure S24. (a-c) $T$-dependent visible absorption spectral changes of $(S)-\mathbf{1 b}^{\mathbf{P h}}-\mathrm{C} 12$ (a), $(S)-\mathbf{2 a}^{\mathbf{M e}}$ TEG (b), and (S)-2b $\mathbf{b}^{\mathrm{Me}}-\mathrm{C} 12$ (c) with DABCO (1.0 equiv.) in $\mathrm{CHCl}_{3}$. Visible absorption spectra of free $\mathrm{Rh}_{2}\left(\mathbf{A b s}_{\text {free }}\right)$ (green dashed lines) and its 1:2 complexes with DABCO (Absi:2) (blown dashed lines) estimated by the absorption titration experiments (Figure S19) are also shown. (d-f) Plots of the maximum absorption wavelength $\left(\lambda_{\max }\right)$ of $(S)-\mathbf{1 b}^{\mathbf{P h}}-\mathrm{C} 12(\mathrm{~d}),(S)-\mathbf{2} \mathbf{a}^{\mathbf{M e}}-\mathrm{TEG}(\mathrm{e})$, and $(S)-\mathbf{2} \mathbf{b}^{\mathbf{M e}}-\mathrm{C} 12(\mathrm{f})$ with $\mathrm{DABCO}$ (1.0 equiv.) in $\mathrm{CHCl}_{3}$ vs temperature. 

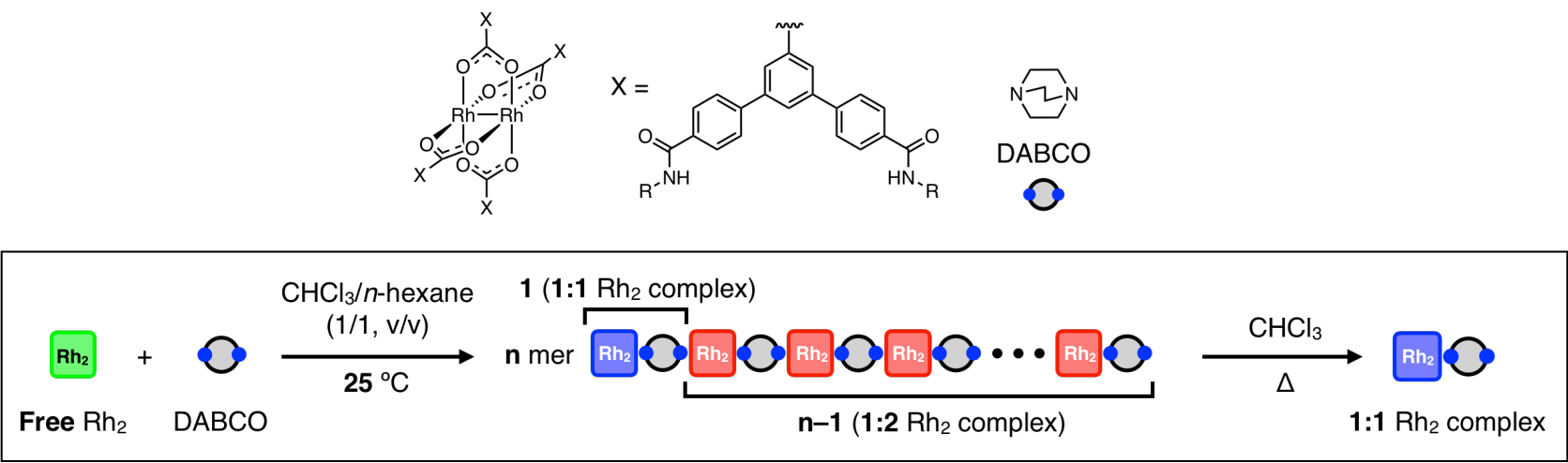

(i) -: 0 equiv. DABCO in $\mathrm{CHCl}_{3} / n$-hexane $(1 / 1, \mathrm{v} / \mathrm{v})$ at $25^{\circ} \mathrm{C}$

(ii) $-: 1.0$ equiv. $\mathrm{DABCO}$ in $\mathrm{CHCl}_{3}$ at $55^{\circ} \mathrm{C}$

(iii) - : $>2.7$ equiv. DABCO in $\mathrm{CHCl}_{3} / n$-hexane $(1 / 1, v / v)$ at $25^{\circ} \mathrm{C}$<smiles></smiles>

(a)

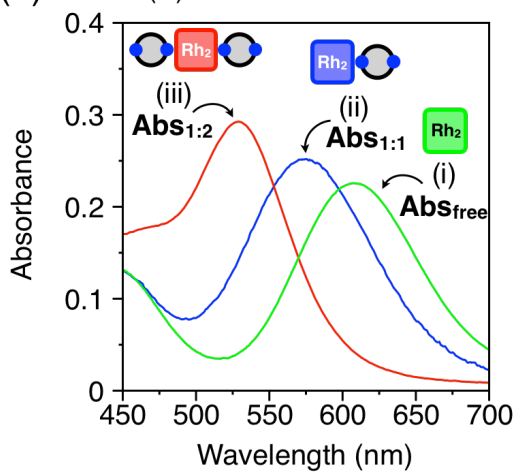

$\mathrm{R}=$ 久 $\mathrm{Nothot}_{3}$

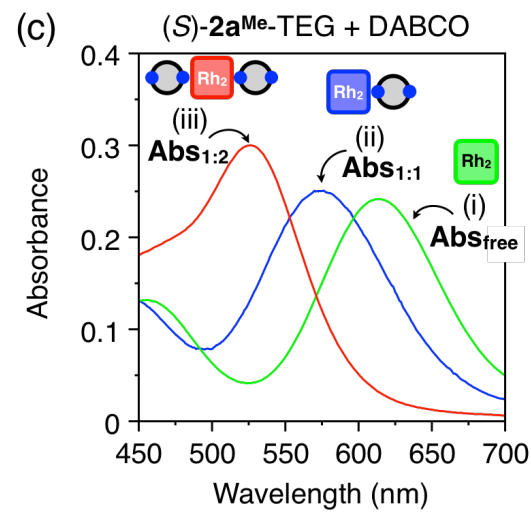

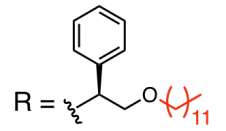

(b)

(S) $-1 \mathbf{b}^{\text {Ph}}-\mathrm{C} 12+\mathrm{DABCO}$

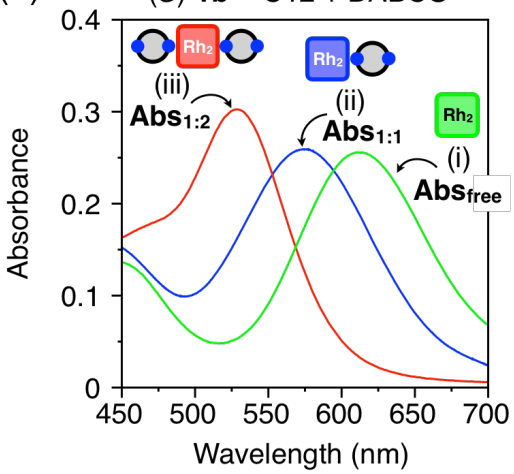

$\mathrm{R}=$ \&

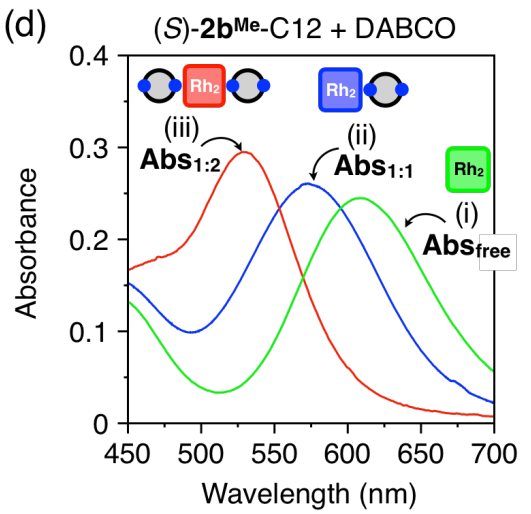

Figure S25. Visible absorption spectra of free (Absfree) (green line) (i) $(S)-\mathbf{1 a}^{\mathbf{P h}}-$ TEG $(\mathbf{a}),(S)-\mathbf{1 b}^{\mathbf{P h}}$ -

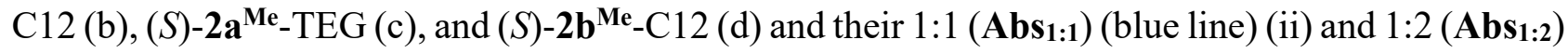
(red line) (iii) complexes with DABCO estimated by the absorption titrations (i and iii) (Figure S19) and $T$-dependent visible absorption spectral changes (ii) (Figures 6d,e and S24). 

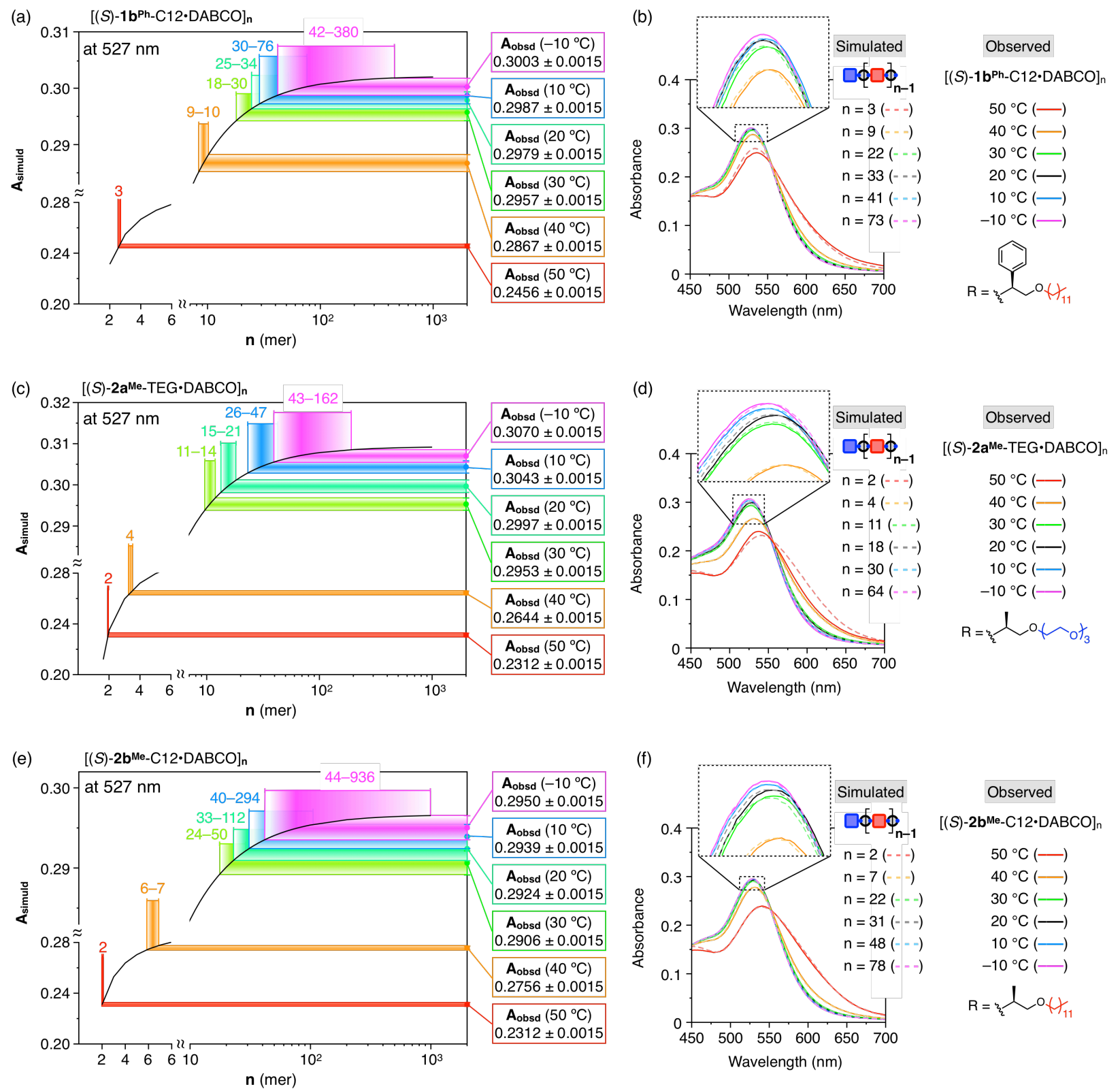

Figure S26. (a, c, e) $T$-dependent visible absorption spectral changes of $\left[(S)-\mathbf{1 b}^{\mathbf{P h}}-\mathrm{C} 12 \cdot \mathrm{DABCO}\right]_{\mathrm{n}}(\mathrm{a})$, $\left[(S)-\mathbf{2} \mathbf{a}^{\mathrm{Me}}-\mathrm{TEG} \cdot \mathrm{DABCO}\right]_{\mathrm{n}}(\mathrm{c})$, and $\left[(S)-\mathbf{2} \mathbf{b}^{\mathbf{M e}}-\mathrm{C} 12 \cdot \mathrm{DABCO}\right]_{\mathrm{n}}(\mathrm{e})$ in $\mathrm{CHCl}_{3} / n$-hexane $(1 / 1, \mathrm{v} / \mathrm{v})$ (solid lines) and their simulated visible absorption spectra calculated as a function of $\mathbf{n}$ according to eq 1 (Figure 7c) (dashed lines) (see Table S4). (b, d, f) Plots of the simulated absorption intensities (A $\mathbf{A}_{\text {simuld }}$ ) of $\left[(S)-\mathbf{1} \mathbf{b}^{\mathbf{P h}}-\mathrm{C} 12 \cdot \mathrm{DABCO}\right]_{\mathrm{n}}(\mathrm{b}),\left[(S)-\mathbf{2} \mathbf{a}^{\mathrm{Me}}-\mathrm{TEG} \cdot \mathrm{DABCO}\right]_{\mathrm{n}}(\mathrm{d})$, and $\left[(S)-\mathbf{2} \mathbf{b}^{\mathrm{Me}}-\mathrm{C} 12 \cdot \mathrm{DABCO}\right]_{\mathrm{n}}(\mathrm{f})$ at $527 \mathrm{~nm}$ vs the $\mathbf{n}$ value (open circles). The observed absorption intensities ( $\left.\mathbf{A}_{\mathbf{o b s d}}\right)$ of the corresponding coordination polymers at different temperatures $\left(-10-50{ }^{\circ} \mathrm{C}\right)$ in $\mathrm{CHCl}_{3} / n$-hexane $(1 / 1, \mathrm{v} / \mathrm{v})$ at 527 $\mathrm{nm}$ are also shown. Because of the accuracy of the spectrophotometer $( \pm 0.0015)$, the simulated $\mathbf{n}$ values at lower temperatures showed a relatively large distribution. 
Table S4. Observed Absorption Intensities $\left(\mathrm{A}_{\mathrm{obsd}}\right)$ at $527 \mathrm{~nm}$ of the Coordination Polymers at Different Temperatures and the Degree of Polymerization (n) Estimated by Simulation Using Eq $1^{a}$

\begin{tabular}{|c|c|c|c|c|c|c|c|c|}
\hline \multirow{2}{*}{$\begin{array}{c}\text { Temperature } \\
\left({ }^{\circ} \mathrm{C}\right)\end{array}$} & \multicolumn{2}{|c|}{$\left[(S)-\mathbf{1 a}^{\mathbf{P h}}-\mathrm{TEG} \cdot \mathrm{DABCO}\right]_{\mathrm{n}}$} & \multicolumn{2}{|c|}{$\left[(S)-\mathbf{1 b}^{\mathbf{P h}}-\mathrm{C} 12 \cdot \mathrm{DABCO}\right]_{\mathrm{n}}$} & \multicolumn{2}{|c|}{$\left[(S)-\mathbf{2} \mathbf{a}^{\mathbf{M e}}-\mathrm{TEG} \cdot \mathrm{DABCO}\right]_{\mathrm{n}}$} & \multicolumn{2}{|c|}{$\left[(S)-\mathbf{2} \mathbf{b}^{\mathbf{M e}}-\mathrm{C} 12 \cdot \mathrm{DABCO}\right]_{\mathrm{n}}$} \\
\hline & $\begin{array}{c}\mathbf{A}_{\text {obsd }} \\
( \pm 0.0015)\end{array}$ & $\begin{array}{c}\mathbf{n} \\
(\mathrm{mer})\end{array}$ & 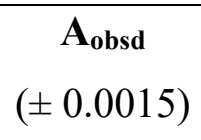 & $\begin{array}{c}\mathbf{n} \\
\text { (mer) }\end{array}$ & $\begin{array}{c}\text { A } \begin{array}{c}\text { obsd } \\
( \pm 0.0015)\end{array}\end{array}$ & $\begin{array}{c}\mathbf{n} \\
(\mathrm{mer})\end{array}$ & $\begin{array}{c}\text { A } \begin{array}{c}\text { obsd } \\
( \pm 0.0015)\end{array}\end{array}$ & $\begin{array}{c}\mathbf{n} \\
(\mathrm{mer})\end{array}$ \\
\hline 40 & 0.2524 & 4 & 0.2867 & $9-10$ & 0.2644 & 4 & 0.2756 & $6-7$ \\
\hline 30 & 0.2744 & $8-9$ & 0.2957 & $18-30$ & 0.2953 & $11-14$ & 0.2906 & $18-31$ \\
\hline 10 & 0.2854 & $19-31$ & 0.2987 & $30-76$ & 0.3043 & $26-47$ & 0.2939 & $33-112$ \\
\hline 0 & 0.2886 & $32-83$ & 0.2998 & $38-174$ & 0.3063 & $37-103$ & 0.2947 & 40-294 \\
\hline-10 & 0.2894 & $39-152$ & 0.3003 & $42-380$ & 0.3070 & $43-162$ & 0.2950 & 44-936 \\
\hline
\end{tabular}

${ }^{a}$ The degree of polymerization (n) values are estimated by simulation using eq 1 in Figure 7c (see Figure S26 and also the section 7.3 in the SI, in which eq S8 is identical to eq 1). 

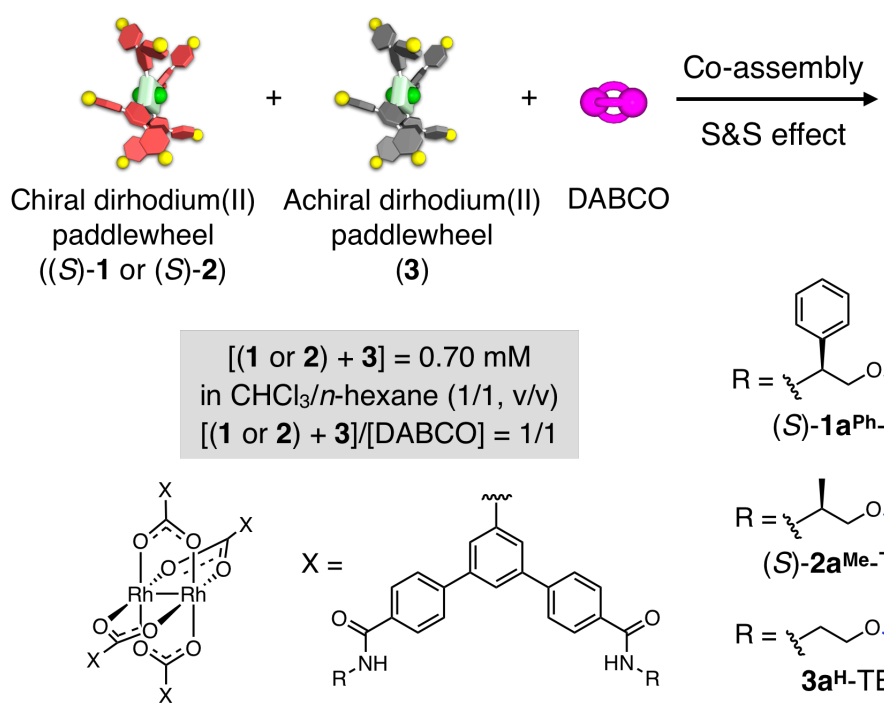

S\&S effect

Chiral dirhodium(II) Achiral dirhodium(II) DABCO

paddlewhee

$(S)-1$ or $(S)-2)$

(3)<smiles>O=CCC(C=O)c1ccccc1</smiles>

(S)-1a $\mathrm{a}^{\mathrm{Ph}}-\mathrm{TEG}$

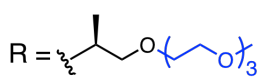

(S)-2aMe-TEG

\section{Preferred-handed helical} coordination polymers

(a) (S)-1 $\mathbf{a}^{\text {Ph}}-$ TEG / $3 \mathbf{a}^{\mathrm{H}}-\mathrm{TEG} / \mathrm{DABCO}$

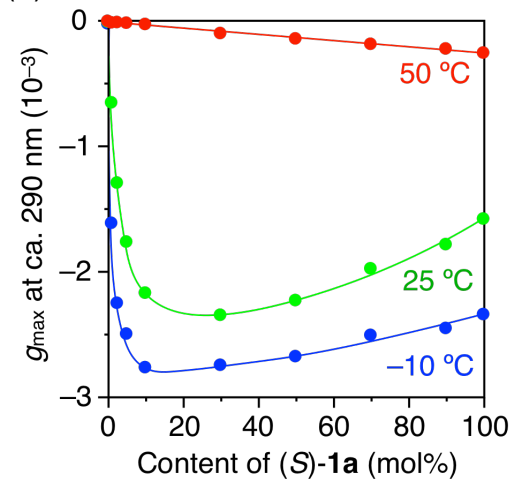

(b) (S) -1 $\mathbf{b}^{\text {Ph-C12 }}$ / 3 $\mathbf{b}^{\mathrm{H}-\mathrm{C} 12} / \mathrm{DABCO}$

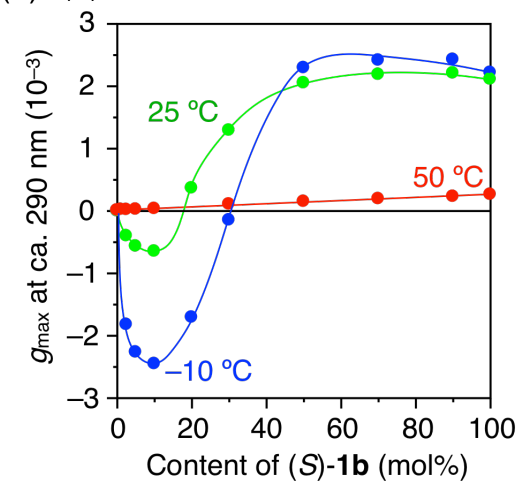

(c) (S)-2aMe-TEG / 3a $\mathrm{a}^{\mathrm{H}}$-TEG / DABCO

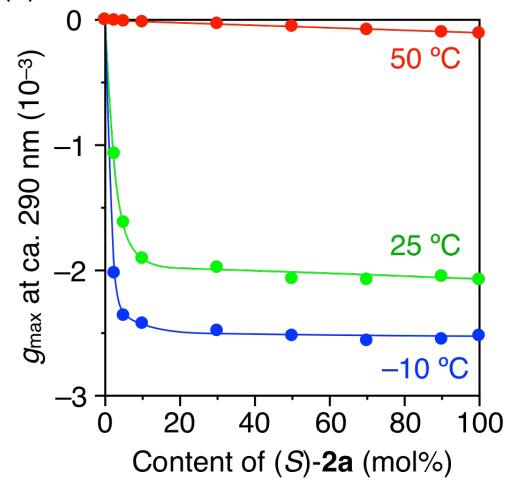

(d) (S)-2bMe-C12 / 3b b-C12 / DABCO

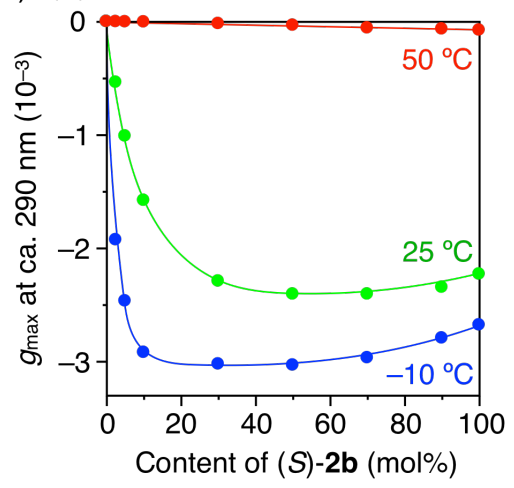

Figure S27. Plots of the Kuhn's dissymmetry factors at the maximum CD intensities $\left(g_{\max }\right)$ at ca. 290 $\mathrm{nm}$ of the helical coordination polymers prepared from the ternary mixtures of $(S)-\mathbf{1} \mathbf{a}^{\mathbf{P h}}-\mathrm{TEG} / \mathbf{3 a}^{\mathbf{H}_{-}}$

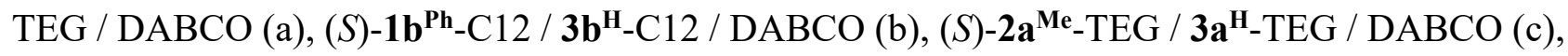
and $(S)-2 b^{\text {Me }}$-C12 / 3b ${ }^{\mathbf{H}}$-C12 / DABCO (d) ([dirhodium(II) paddlewheel] / [DABCO] = 1/1) in $\mathrm{CHCl}_{3} / n$-hexane $(1 / 1, \mathrm{v} / \mathrm{v})$ at $-10(\bigcirc), 25(\bigcirc)$, and $50(\bigcirc){ }^{\circ} \mathrm{C}$ versus the content of $(S)$-1 or $(S)-2$. [dirhodium(II) paddlewheel] $=0.70 \mathrm{mM}$. 


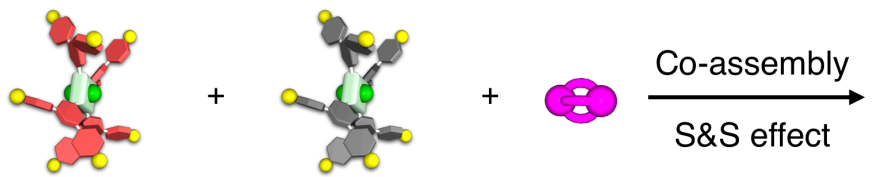

Chiral dirhodium(II) Achiral dirhodium(II) DABCO paddlewheel $((S)-1 \mathbf{b})$ paddlewheel

\section{Preferred-handed helical coordination polymers}

(3b)

$$
\begin{aligned}
{[\mathbf{1 b}+\mathbf{3 b}]=} & 0.70 \mathrm{mM} \text { in } \mathrm{CHCl}_{3} / n \text {-hexane }(1 / 1, \mathrm{v} / \mathrm{v}) \\
& {[\mathbf{1 b}+\mathbf{3 b}] /[\mathrm{DABCO}]=1 / 1 }
\end{aligned}
$$

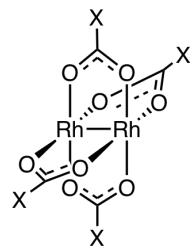

(a)

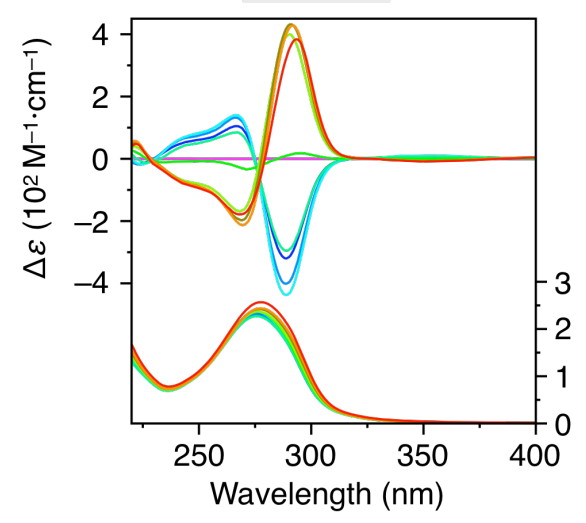

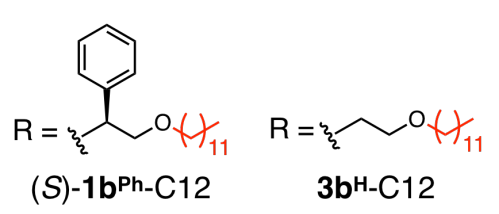

Figure S28. CD and absorption spectra of ternary mixtures of $(S)-\mathbf{1} \mathbf{b}^{\mathbf{P h}}-\mathrm{C} 12 / \mathbf{3 b}^{\mathbf{H}}-\mathrm{C} 12 / \mathrm{DABCO}$ ([dirhodium(II) paddlewheel] / [DABCO] = 1/1) in $\mathrm{CHCl}_{3} / n$-hexane $(1 / 1, \mathrm{v} / \mathrm{v})$ at -10 (a) and 25 (b) ${ }^{\circ} \mathrm{C}$. [dirhodium(II) paddlewheel $]=0.70 \mathrm{mM}$. 


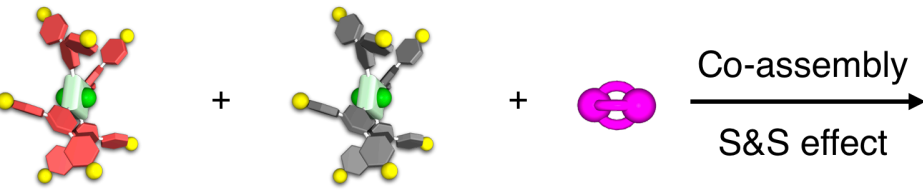

Chiral dirhodium(II) Achiral dirhodium(II) DABCO paddlewheel $((S)-1$ or $(S)-2)$
(3) paddlewheel

\section{Preferred-handed helical coordination polymers}

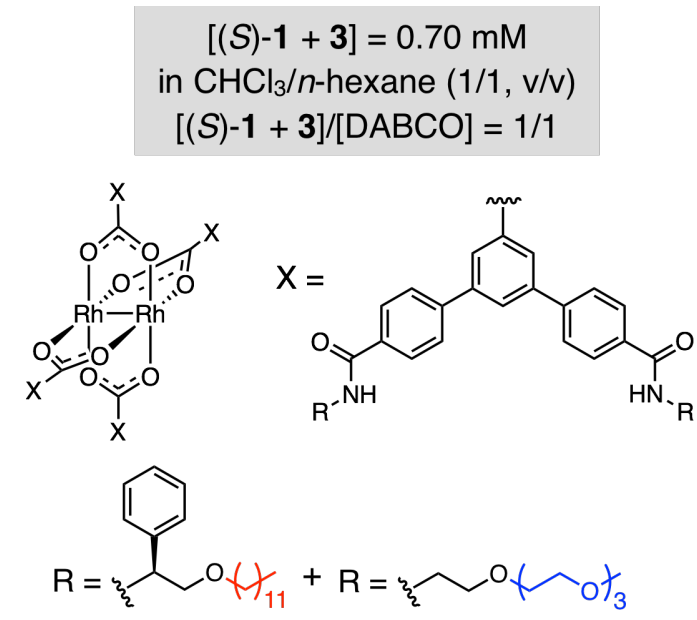<smiles>[R]CC(CO[Ga])c1ccccc1</smiles>

(S) $-1 \mathbf{b}^{\text {Ph}}-\mathrm{C} 12$<smiles></smiles>

(S)-1aPh-TEG

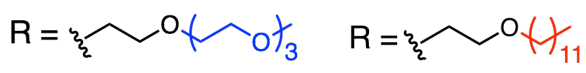

$$
\begin{aligned}
& 3 b^{H}-\mathrm{C} 12
\end{aligned}
$$

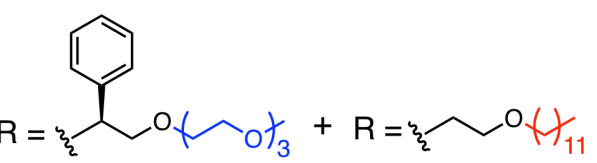
(a) (S)-1 $\mathbf{b}^{\mathrm{Ph}-\mathrm{C} 12} / \mathbf{3} \mathbf{a}^{\mathrm{H}-\mathrm{TEG}} / \mathrm{DABCO}$

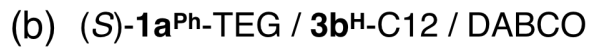
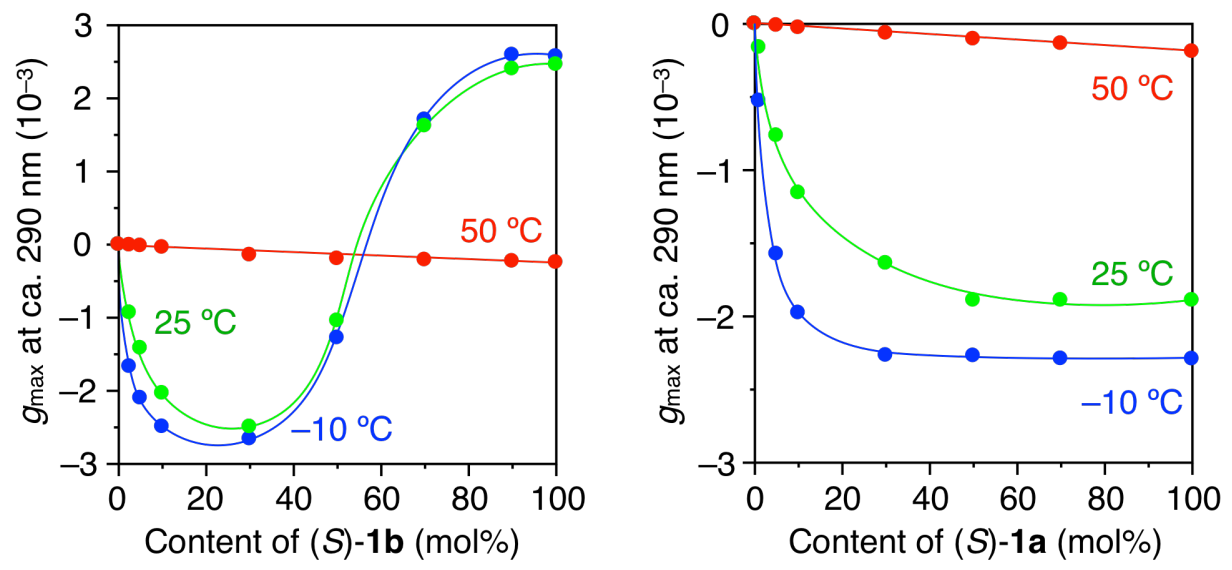

Figure S29. Plots of the $g_{\max }$ value at ca. $290 \mathrm{~nm}$ of the helical coordination polymers prepared from the ternary mixtures of $(S)-\mathbf{1 b}^{\mathbf{P h}}-\mathrm{C} 12 / \mathbf{3 a}^{\mathbf{H}}-\mathrm{TEG} / \mathrm{DABCO}(\mathrm{a})$ and $(S)-\mathbf{1} \mathbf{a}^{\mathbf{P h}}-\mathrm{TEG} / \mathbf{3 b}^{\mathbf{H}}-\mathrm{C} 12$ / DABCO (b) $\left(\left[\right.\right.$ dirhodium(II) paddlewheel] / [DABCO] = 1/1) in $\mathrm{CHCl}_{3} / n$-hexane $(1 / 1, \mathrm{v} / \mathrm{v})$ at $-10(\bigcirc), 25(\bigcirc)$, and $50(\bigcirc){ }^{\circ} \mathrm{C}$ versus the content of $(S)-\mathbf{1 a}$ or $(S)-\mathbf{1 b}$. [dirhodium(II) paddlewheel] $=0.70 \mathrm{mM}$. 


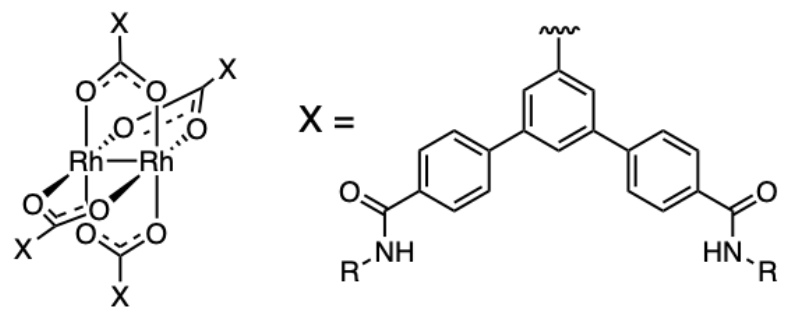

(a) S\&S effect

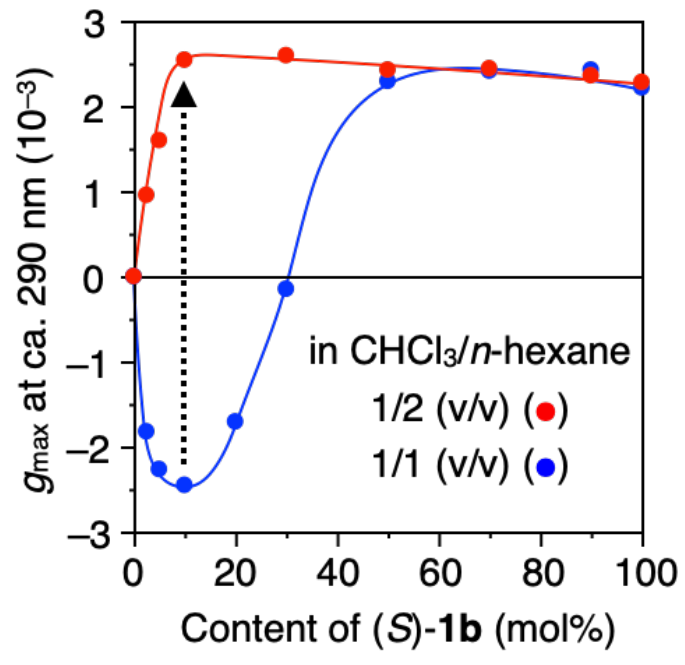

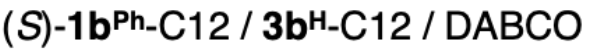

$[(S)-1 b+3 b]=0.70 \mathrm{mM}$ in $\mathrm{CHCl}_{3} / n$-hexane at $-10{ }^{\circ} \mathrm{C}$ $[(S)-1 \mathbf{b}+\mathbf{3 b}] /[\mathrm{DABCO}]=1 / 1$

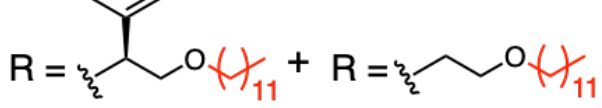

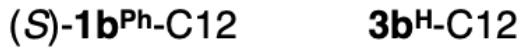

(b) MR effect

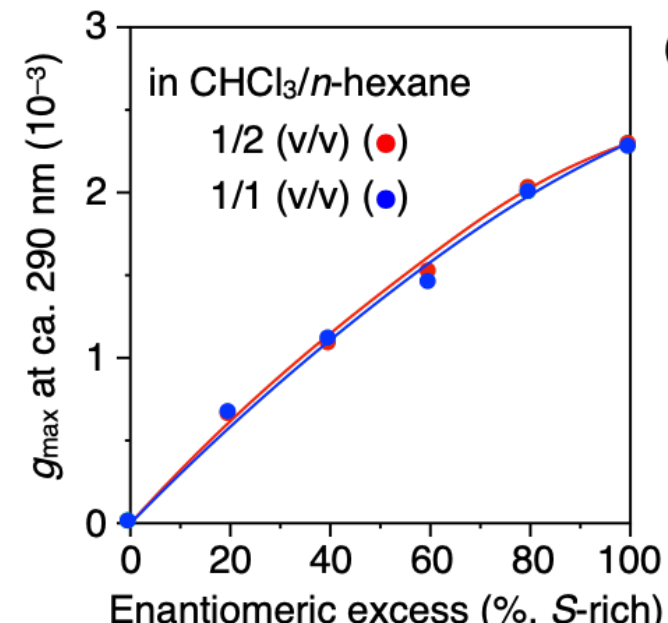

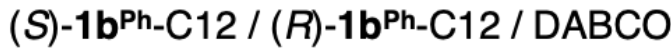

[nonracemic 1b] $=0.70 \mathrm{mM}$ in $\mathrm{CHCl}_{3} / n$-hexane at $-10^{\circ} \mathrm{C}$ [nonracemic 1b]/[DABCO] = 1/1

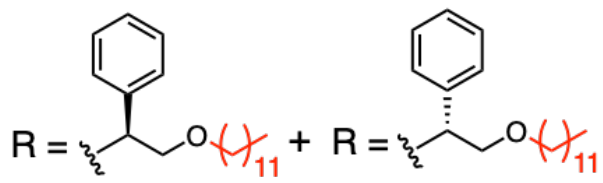

$(S)-1 b^{\text {Ph-C12 }} \quad(R)-$ 1 $^{\text {Ph-C12 }}$

Figure S30. (a) Plots of the $g_{\max }$ value at ca. $290 \mathrm{~nm}$ of the helical coordination polymers prepared from the ternary mixtures of $(S)$ - $\mathbf{1} \mathbf{b}^{\mathrm{Ph}}-\mathrm{C} 12 / \mathbf{3 b}^{\mathrm{H}_{-}} \mathrm{C} 12$ / DABCO $(\mathrm{a})$ and $(S)-\mathbf{1} \mathbf{b}^{\mathrm{Ph}}-\mathrm{C} 12 /(R)-\mathbf{1 b}^{\mathbf{P h}}-\mathrm{C} 12$ / DABCO ([dirhodium(II) paddlewheel] / [DABCO] = 1/1) (b) in $\mathrm{CHCl}_{3} / n$-hexane $(1 / 1(\bullet)$ and $1 / 2$ (๑), v/v) at $-10{ }^{\circ} \mathrm{C}$ versus the content of $(S)-\mathbf{1 b}$ (a) and \% ee of $\mathbf{1 b}$ (S-rich) (b). [dirhodium(II) paddlewheel $]=0.70 \mathrm{mM}$. 


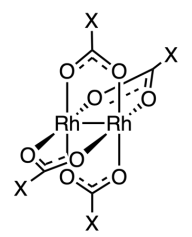<smiles>[X]C(C)(C)C(=O)Nc1ccc(-c2ccc(C(=O)NP)cc2)cc1</smiles>

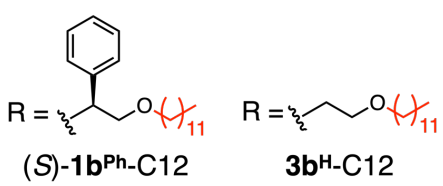
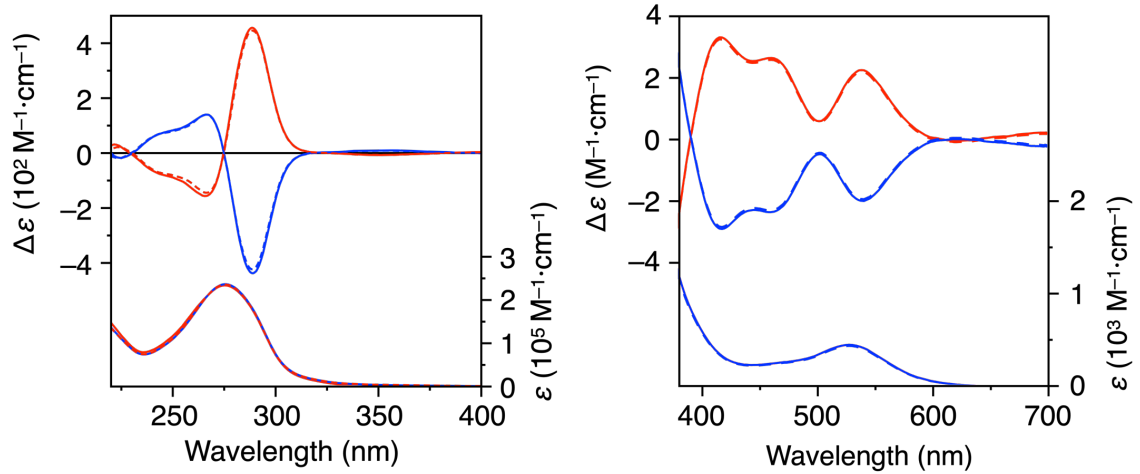

$10 \mathrm{~mol} \%(S)-1 b^{\text {Ph}}-\mathrm{C} 12$

$[(S)-1 \mathbf{b}+3 \mathbf{b}]=0.70 \mathrm{mM}$, at $-10^{\circ} \mathrm{C}$ $[(S)-1 b+3 b] /[D A B C O]=1 / 1$

in $\mathrm{CHCl}_{3} / n$-hexane $1 / 2(v / v)(-)$ $1 / 1(\mathrm{v} / \mathrm{v})(-)$

in water-saturated- $\mathrm{CHCl}_{3} / n$-hexane $1 / 2(v / v)(--)$ $1 / 1(v / v)(--)$

Figure S31. CD and absorption spectra of a $(S)-\mathbf{1 b}^{\mathbf{P h}}-\mathrm{C} 12$ / 3 $\mathbf{b}^{\mathbf{H}}-\mathrm{C} 12$ / DABCO ternary mixture (0.1 / $0.9 / 1.0, \mathrm{~mol} / \mathrm{mol} / \mathrm{mol}$ ) in $1 / 1$ (blue color) and $1 / 2$ (red color) dry $\mathrm{CHCl}_{3} / n$-hexane (solid lines) and water-saturated $\mathrm{CHCl}_{3}$ and $n$-hexane mixtures (v/v) (dashed lines) at $-10{ }^{\circ} \mathrm{C}$. 


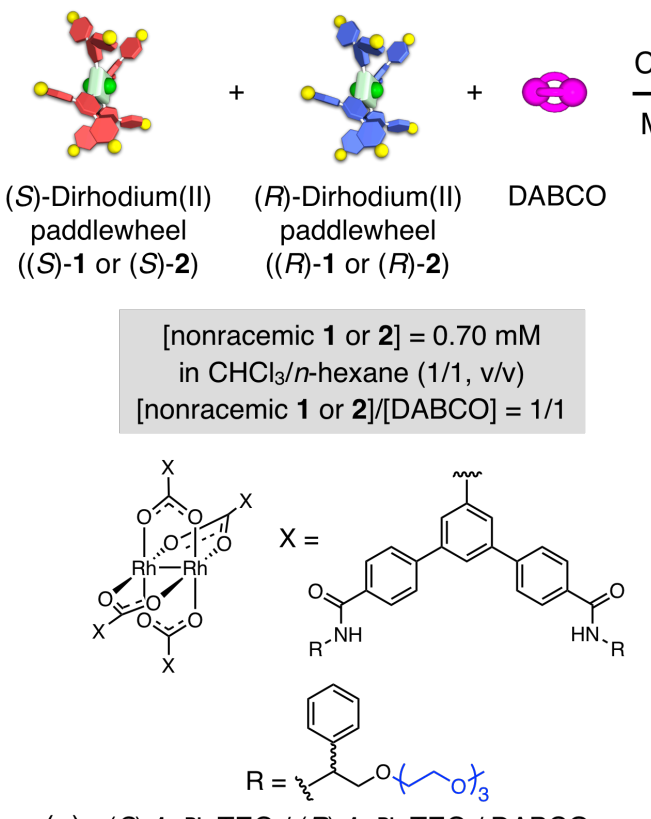

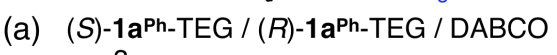

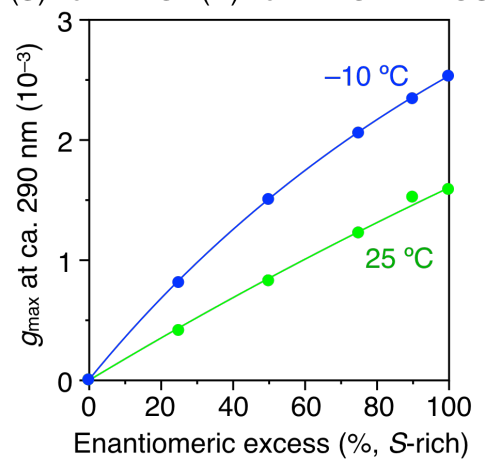

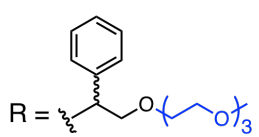

(S)- or $(R)-1 \mathrm{a}^{\text {Ph-TEG }}$
Preferred-handed helical coordination polymers

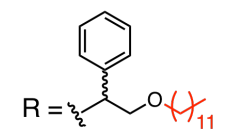

$(S)$ - or $(R)-1 b^{\text {Ph-C12 }}$

$$
\begin{aligned}
& \mathrm{R}=\overbrace{2} \mathrm{O} \mathrm{Not}_{3} \\
& \mathrm{R}=\boldsymbol{\varepsilon}_{2} \mathrm{~s}+\mathrm{H}_{11}
\end{aligned}
$$

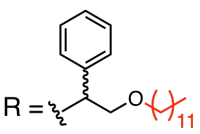

(b) (S)-1 $\mathbf{b}^{\text {Ph}}-\mathrm{C} 12 /(R)-\mathbf{1} \mathbf{b}^{\text {Ph}}-\mathrm{C} 12 /$ DABCO

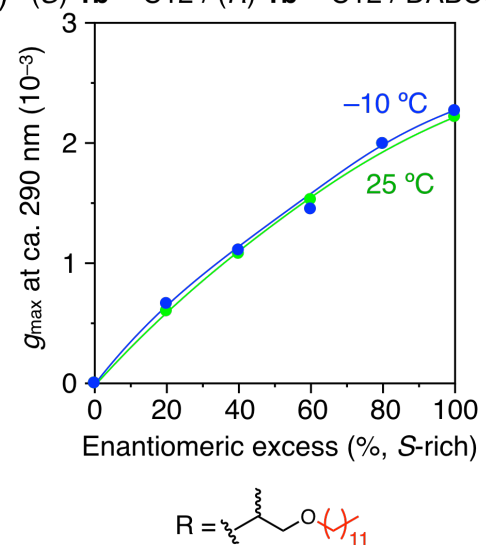

(d) (S)-2bMe-C12 / (R)-2b'Me-C12 / DABCO

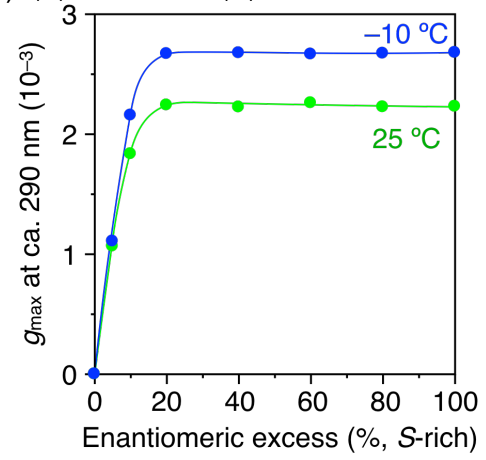

Figure S32. Plots of the $g_{\max }$ value at ca. $290 \mathrm{~nm}$ of the helical coordination polymers prepared from

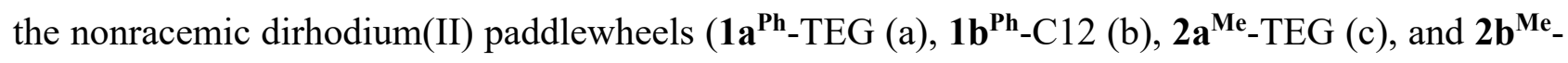
$\mathrm{C} 12$ (d)) and DABCO ([dirhodium(II) paddlewheel] / [DABCO] = 1/1) in $\mathrm{CHCl}_{3} / n$-hexane $(1 / 1, \mathrm{v} / \mathrm{v})$ at $-10(\bullet)$ and $25(\bullet){ }^{\circ} \mathrm{C}$ versus the $\%$ ee of 1 or $2(S$-rich). [dirhodium(II) paddlewheel] $=0.70 \mathrm{mM}$. 


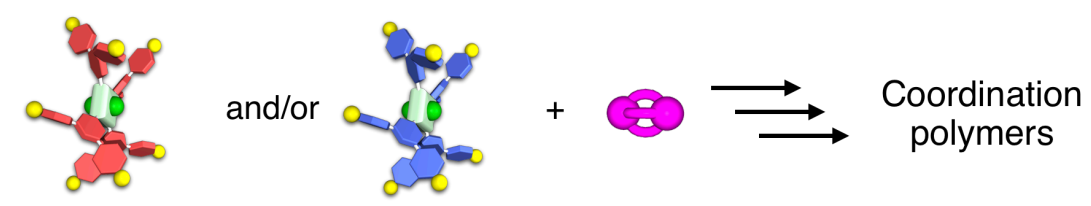

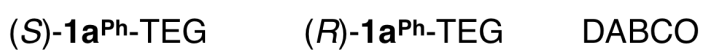
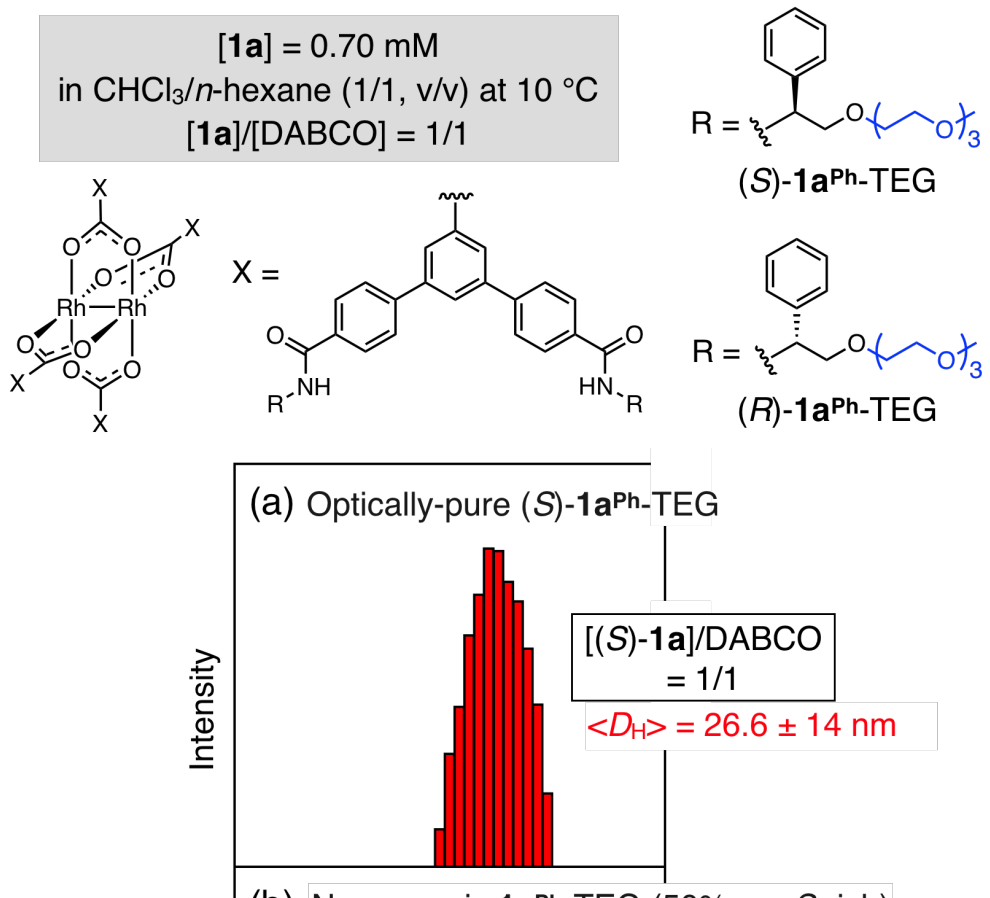

(b) Nonracemic 1aPh-TEG (50\% ee, S-rich)
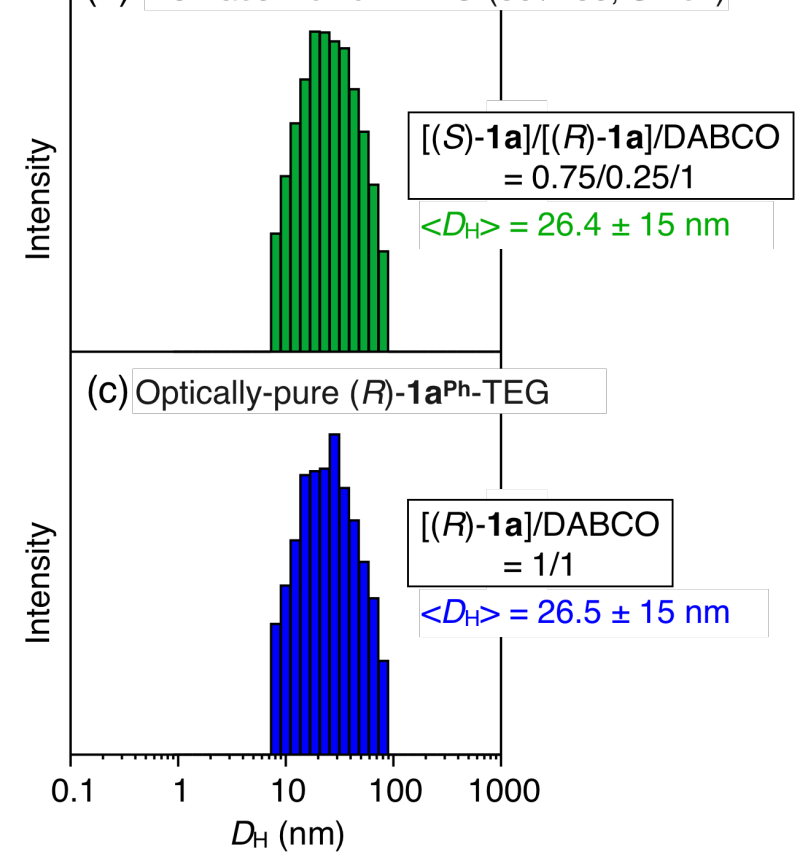

Figure S33. Histogram analyses of DLS measurements of the coordination polymers prepared from the optically-pure $\left((S)-\mathbf{1 a}^{\mathbf{P h}}\right.$-TEG (a) and $(R)-\mathbf{1 a}^{\text {Ph }}$-TEG (c)) and nonracemic $\mathbf{1 a}^{\mathbf{P h}}$-TEG (b; 50\% ee $(S$-rich $))$ and DABCO $([1] /[\mathrm{DABCO}]=1 / 1)$ in $\mathrm{CHCl}_{3} / n$-hexane $(1 / 1, \mathrm{v} / \mathrm{v})$ at $10^{\circ} \mathrm{C} .[1]=0.70 \mathrm{mM}$. 


\section{Supporting References}

(S1) Banno, M.; Yamaguchi, T.; Nagai, K.; Kaiser, C.; Hecht, S.; Yashima, E. Optically Active, Amphiphilic Poly(meta-phenylene ethynylene)s: Synthesis, Hydrogen-Bonding Enforced Helix Stability, and Direct AFM Observation of Their Helical Structures. J. Am. Chem. Soc. 2012, 134, 8718-8728.

(S2) Tuchscherer, A.; Schaarschmidt, D.; Schulze, S.; Hietschold, M.; Lang, H. Simple and Efficient: Ethylene Glycol Isonitrile Gold(I) Chlorides for the Formation and Stabilization of Gold Nanoparticles. Eur. J. Inorg. Chem. 2011, 2011, 4421-4428.

(S3) Callot, H. J.; Metz, F. Rhodium(II)2,4,6-Triarylbenzoates: Improved Catalysts for the Syn Cyclopropanation of Z-Olefins. Tetrahedron 1985, 41, 4495-4501.

(S4) Smulders, M. M. J.; Schenning, A. P. H. J.; Meijer, E. W. Insight into the Mechanisms of Cooperative Self-Assembly: The "Sergeants-and-Soldiers" Principle of Chiral and Achiral $C_{3}$-Symmetrical Discotic Triamides. J. Am. Chem. Soc. 2008, 130, 606-611, correction: J. Am. Chem. Soc. 2008, 130, 4204.

(S5) Smulders, M. M. J.; Nieuwenhuizen, M. M. L.; de Greef, T. F. A.; van der Schoot, P.; Schenning, A. P. H. J.; Meijer, E. W. How to Distinguish Isodesmic from Cooperative Supramolecular Polymerisation. Chem. - Eur. J. 2010, 16, 362-367.

(S6) Drago, R. S.; Tanner, S. P.; Richman, R. M.; Long, J. R. Quantitative Studies of Chemical Reactivity of Tetra- $\mu$-butyrato-dirhodium(II) Complexes. J. Am. Chem. Soc. 1979, 101, 28972903.

(S7) Drago, R. S.; Long, J. R.; Cosmano, R. Metal Synergism in the Coordination Chemistry of a Metal-Metal Bonded System: $\mathrm{Rh}_{2}\left(\mathrm{C}_{3} \mathrm{H}_{7} \mathrm{COO}\right)_{4}$. Inorg. Chem. 1981, 20, 2920-2927.

(S8) Drago, R. S.; Bilgrien, C. J. Inductive Transfer and Coordination of Ligands in Metal-Metal Bonded Systems. Polyhedron 1988, 7, 1453-1468.

(S9) Rusjan, M.; Donnio, B.; Guillon, D.; Cukiernik, F. D. Liquid-Crystalline Materials Based on Rhodium Carboxylate Coordination Polymers: Synthesis, Characterization and Mesomorphic Properties of Tetra(alkoxybenzoato)dirhodium(Ii) Complexes and Their Pyrazine Adducts. Chem Mater 2002, 14, 1564-1575.

(S10) Trindade, A. F.; Coelho, J. A. S.; Afonso, C. A. M.; Veiros, L. F.; Gois, P. M. P. Fine Tuning of Dirhodium(II) Complexes: Exploring the Axial Modification. ACS Catal. 2012, 2, 370383. 
10. ${ }^{1} \mathrm{H}$ and ${ }^{13} \mathrm{C}$ NMR Spectral Data

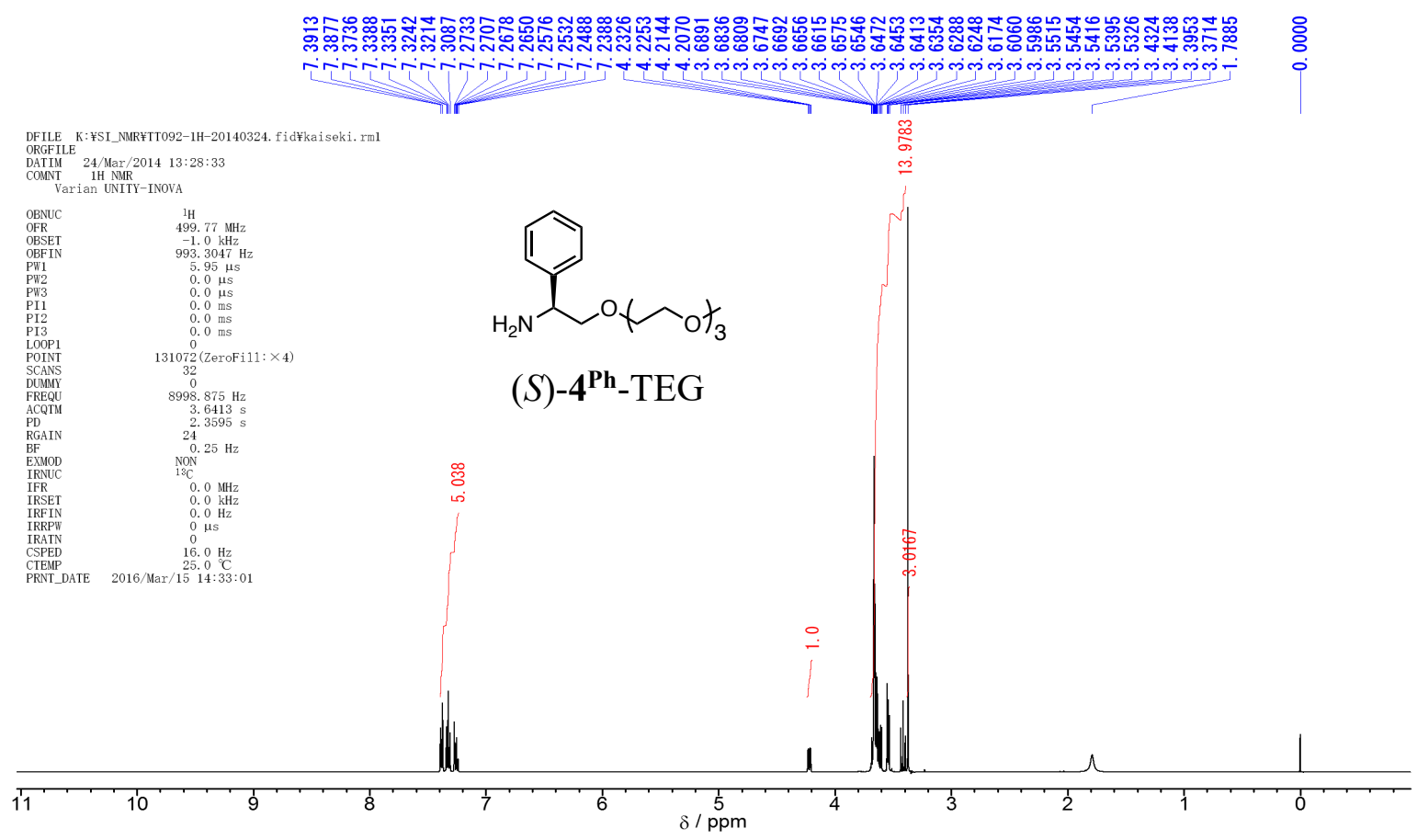

Figure S34. ${ }^{1} \mathrm{H}$ NMR spectrum of $(S)-4^{\mathrm{Ph}}-\mathrm{TEG}$ in $\mathrm{CDCl}_{3}$ at $25^{\circ} \mathrm{C}$.

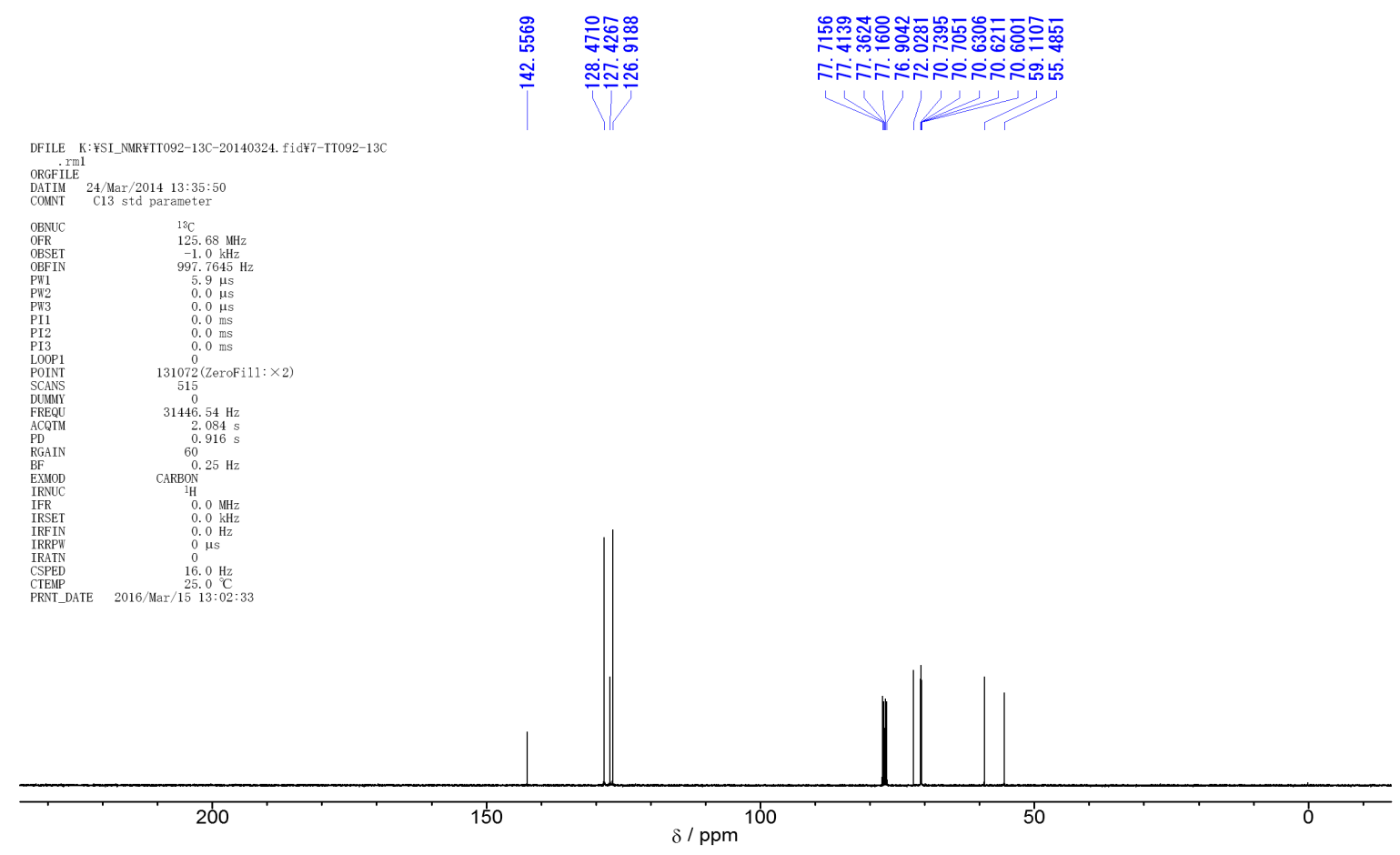

Figure S35. ${ }^{13} \mathrm{C}$ NMR spectrum of $(S)-4^{\mathrm{Ph}}-\mathrm{TEG}$ in $\mathrm{CDCl}_{3}$ at $25^{\circ} \mathrm{C}$. 


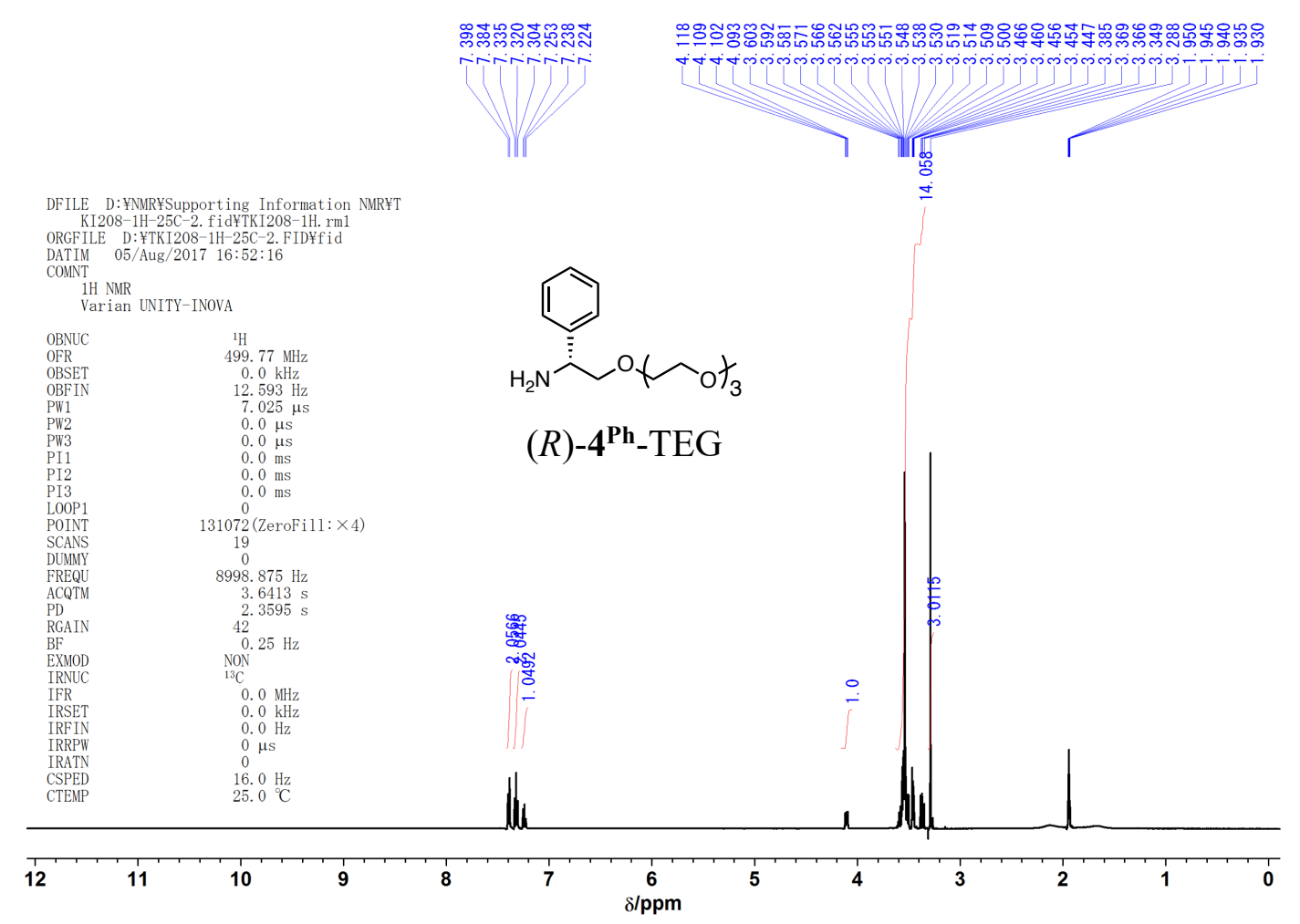

Figure S36. ${ }^{1} \mathrm{H}$ NMR spectrum of $(R)-4^{\mathrm{Ph}}-\mathrm{TEG}$ in $\mathrm{CD}_{3} \mathrm{CN}$ at $25^{\circ} \mathrm{C}$.

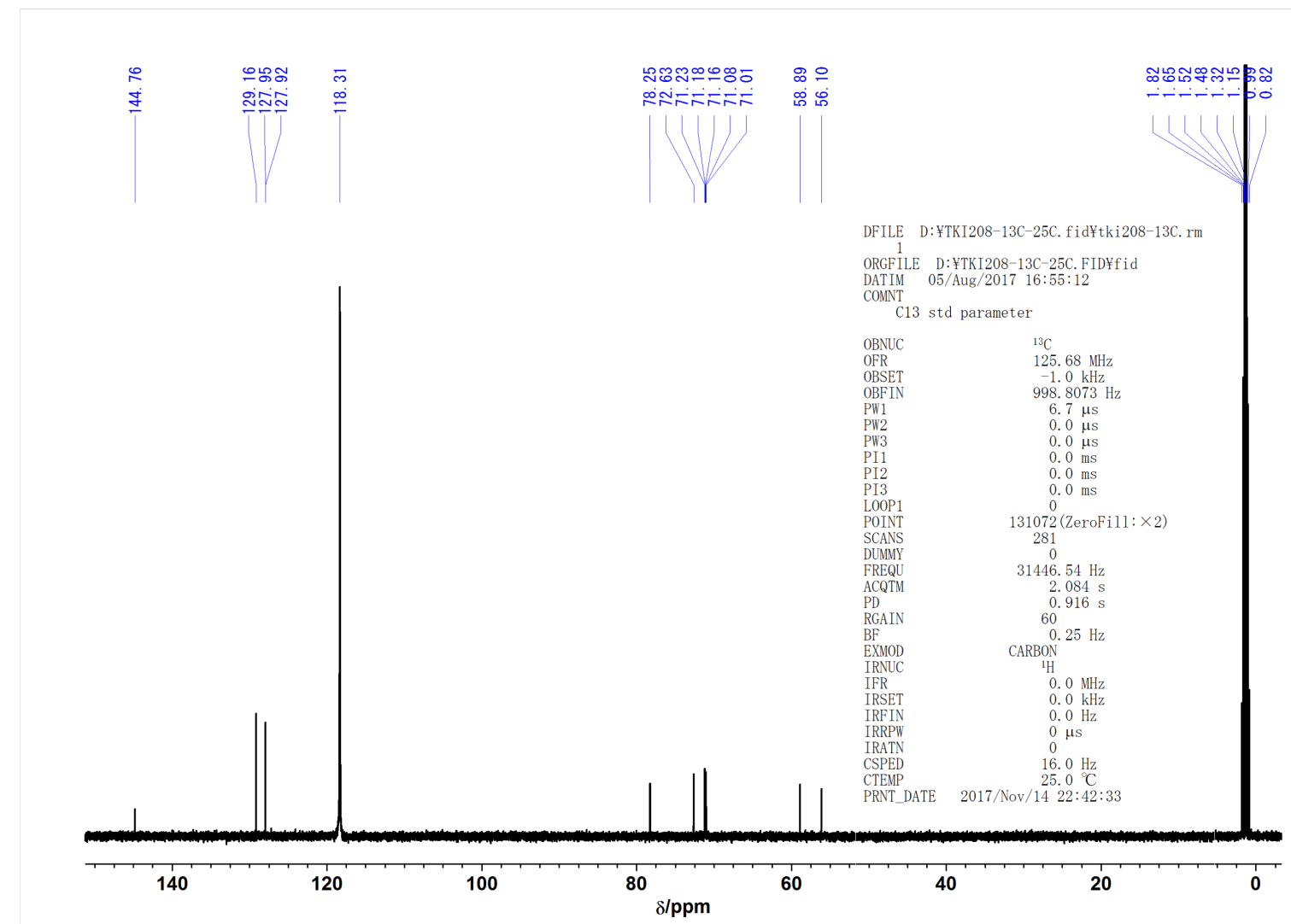

Figure S37. ${ }^{13} \mathrm{C}$ NMR spectrum of $(R)-4^{\mathrm{Ph}}-\mathrm{TEG}$ in $\mathrm{CD}_{3} \mathrm{CN}$ at $25^{\circ} \mathrm{C}$. 


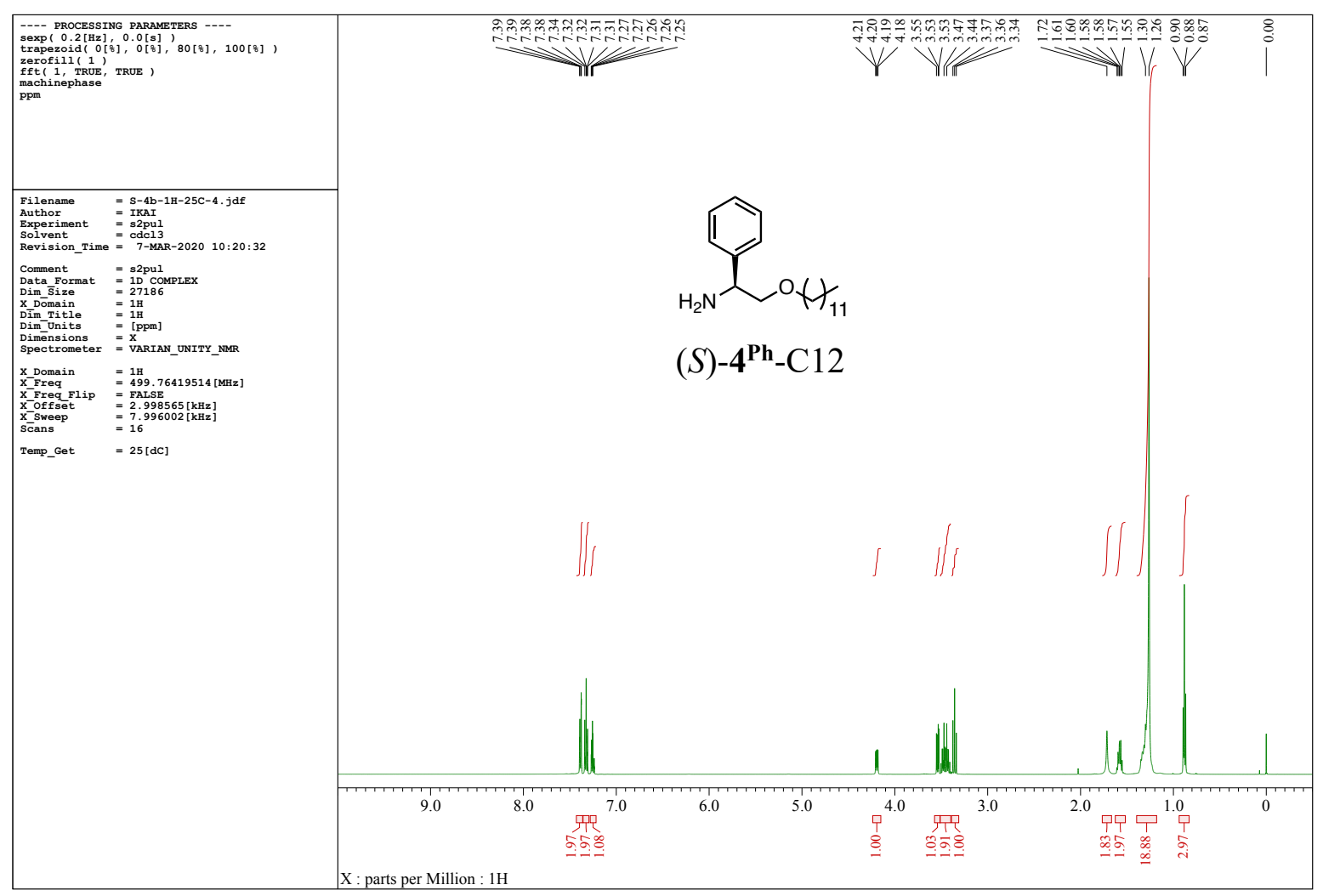

Figure S38. ${ }^{1} \mathrm{H}$ NMR spectrum of $(S)-4^{\mathrm{Ph}}-\mathrm{C} 12$ in $\mathrm{CDCl}_{3}$ at $25^{\circ} \mathrm{C}$.

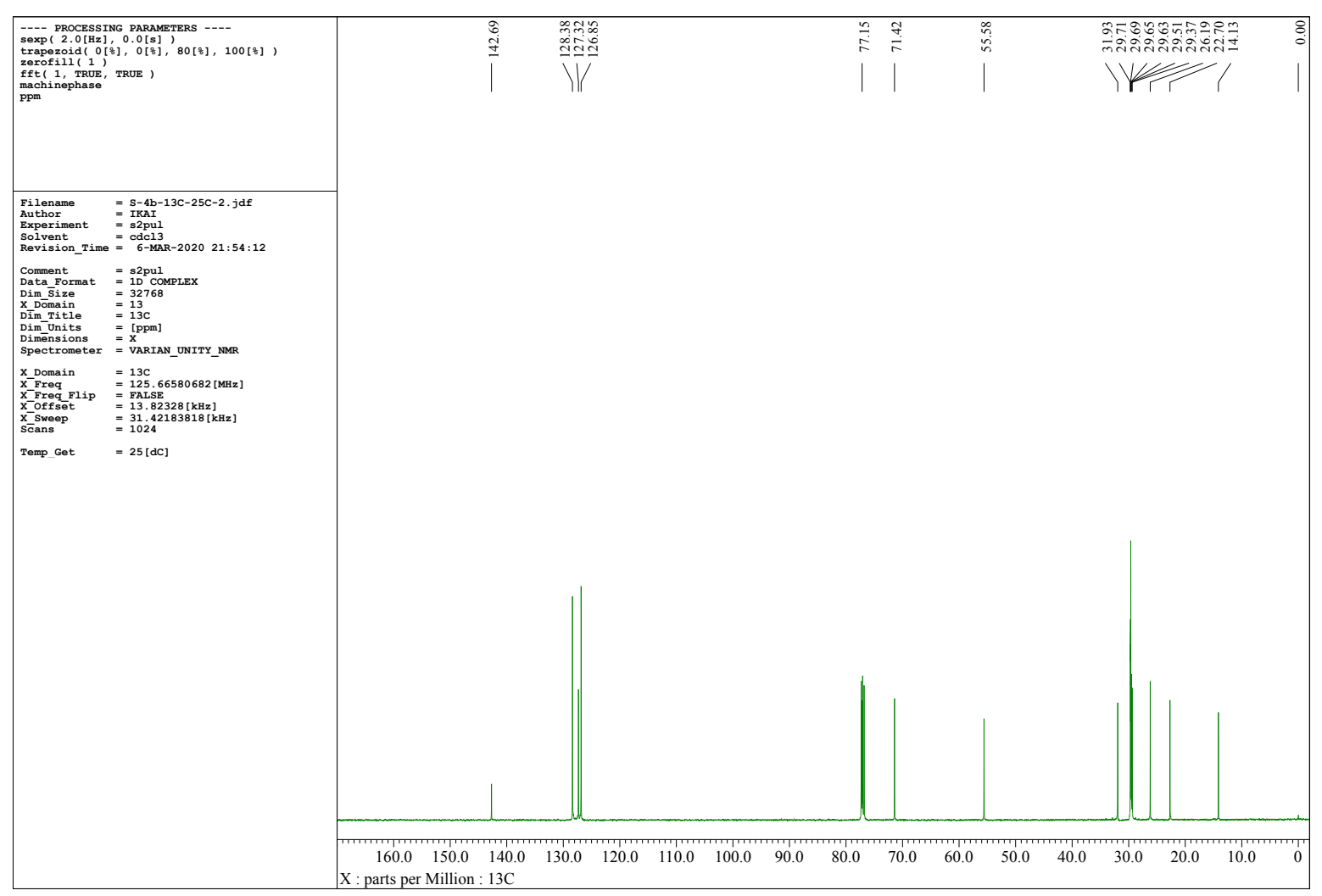

Figure S39. ${ }^{13} \mathrm{C}$ NMR spectrum of $(S)-4^{\mathrm{Ph}}-\mathrm{C} 12$ in $\mathrm{CDCl}_{3}$ at $25^{\circ} \mathrm{C}$. 


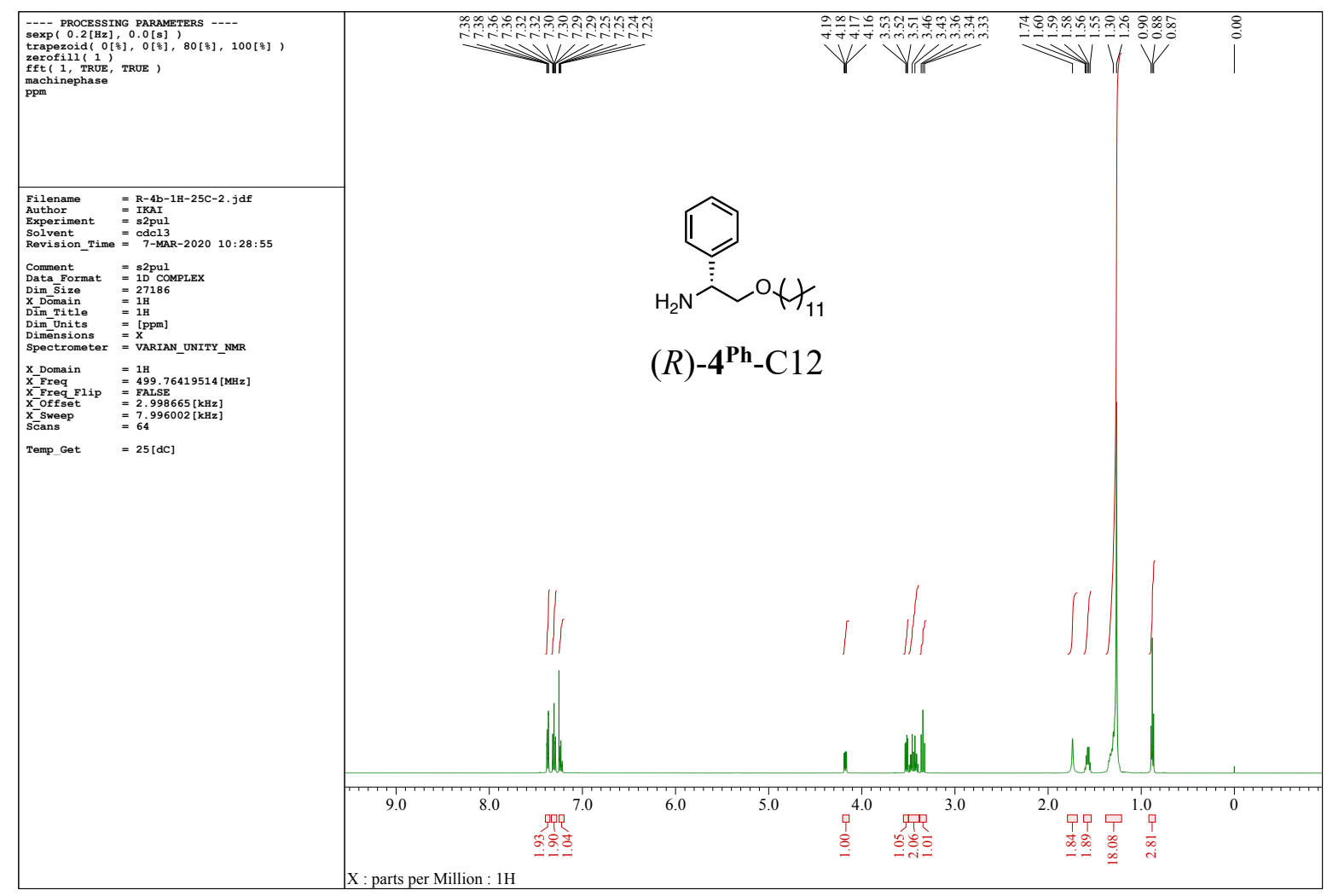

Figure S40. ${ }^{1} \mathrm{H}$ NMR spectrum of $(R)-4^{\mathrm{Ph}}-\mathrm{C} 12$ in $\mathrm{CDCl}_{3}$ at $25^{\circ} \mathrm{C}$.

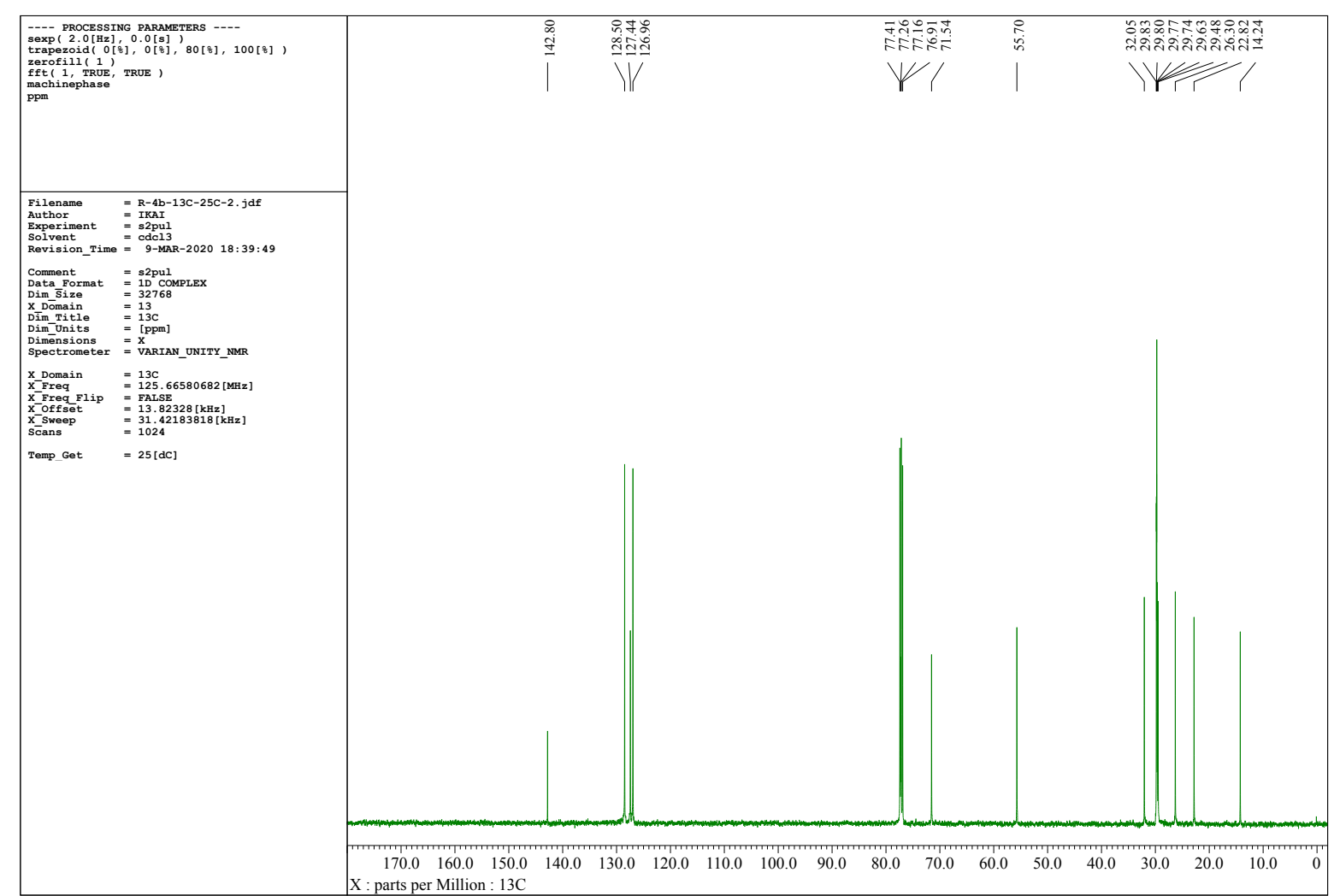

Figure S41. ${ }^{13} \mathrm{C}$ NMR spectrum of $(R)-4^{\mathrm{Ph}}-\mathrm{C} 12$ in $\mathrm{CDCl}_{3}$ at $25^{\circ} \mathrm{C}$. 


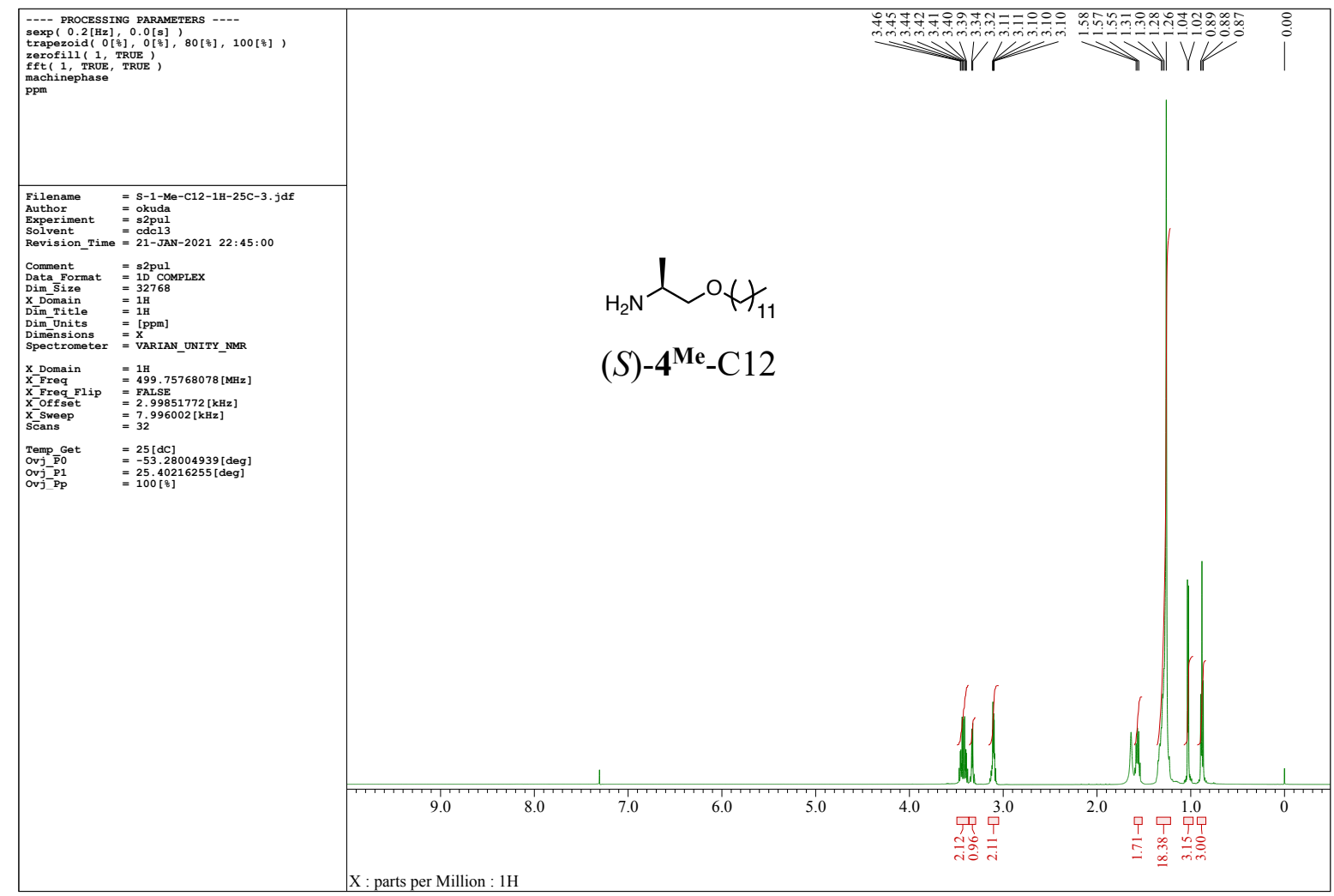

Figure S42. ${ }^{1} \mathrm{H}$ NMR spectrum of $(S)-4^{\mathrm{Me}}-\mathrm{C} 12$ in $\mathrm{CDCl}_{3}$ at $25^{\circ} \mathrm{C}$.

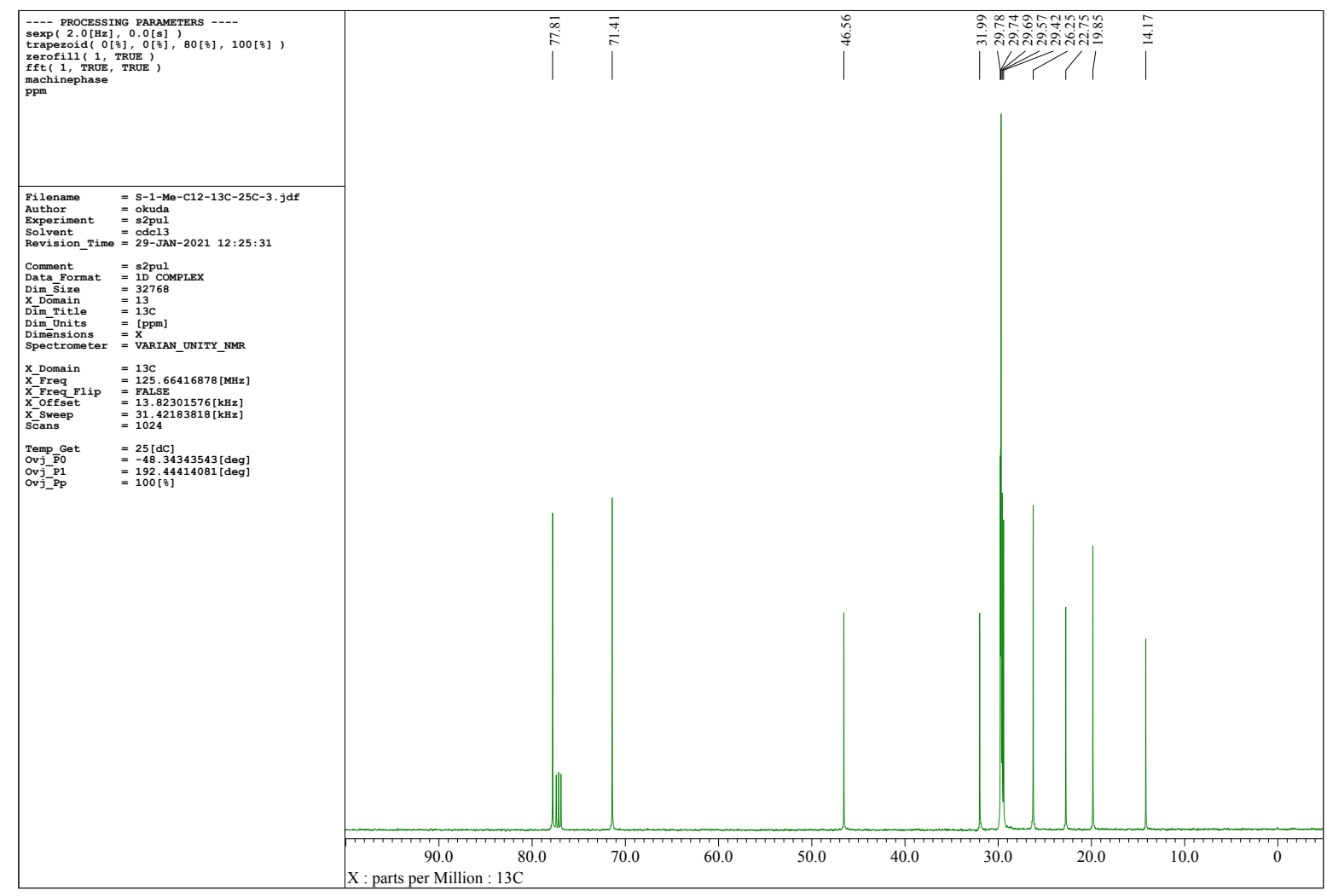

Figure S43. ${ }^{13} \mathrm{C}$ NMR spectrum of $(S)-4^{\mathrm{Me}}-\mathrm{C} 12$ in $\mathrm{CDCl}_{3}$ at $25{ }^{\circ} \mathrm{C}$. 


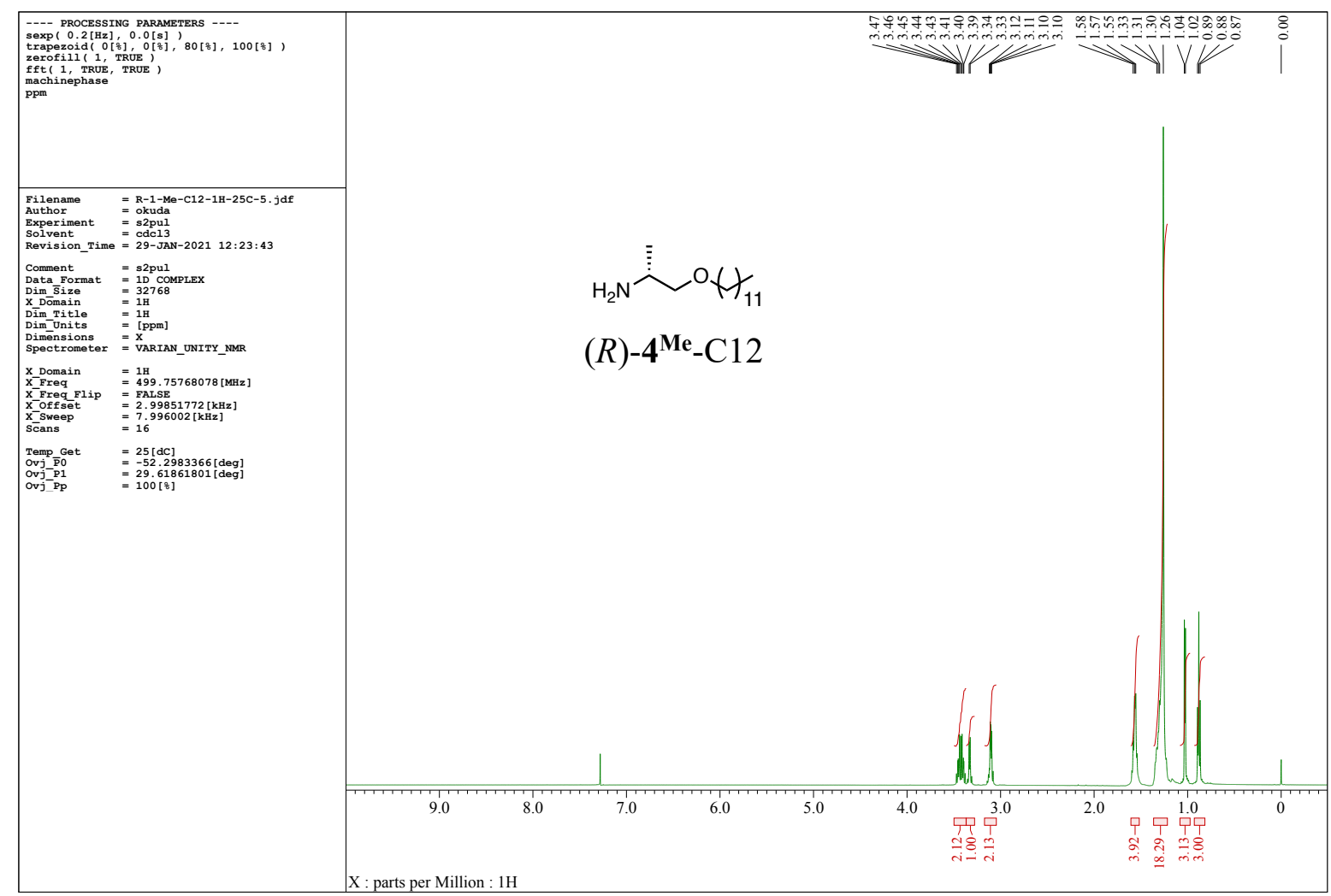

Figure S44. ${ }^{1} \mathrm{H}$ NMR spectrum of $(R)-4^{\mathrm{Me}}-\mathrm{C} 12$ in $\mathrm{CDCl}_{3}$ at $25^{\circ} \mathrm{C}$.

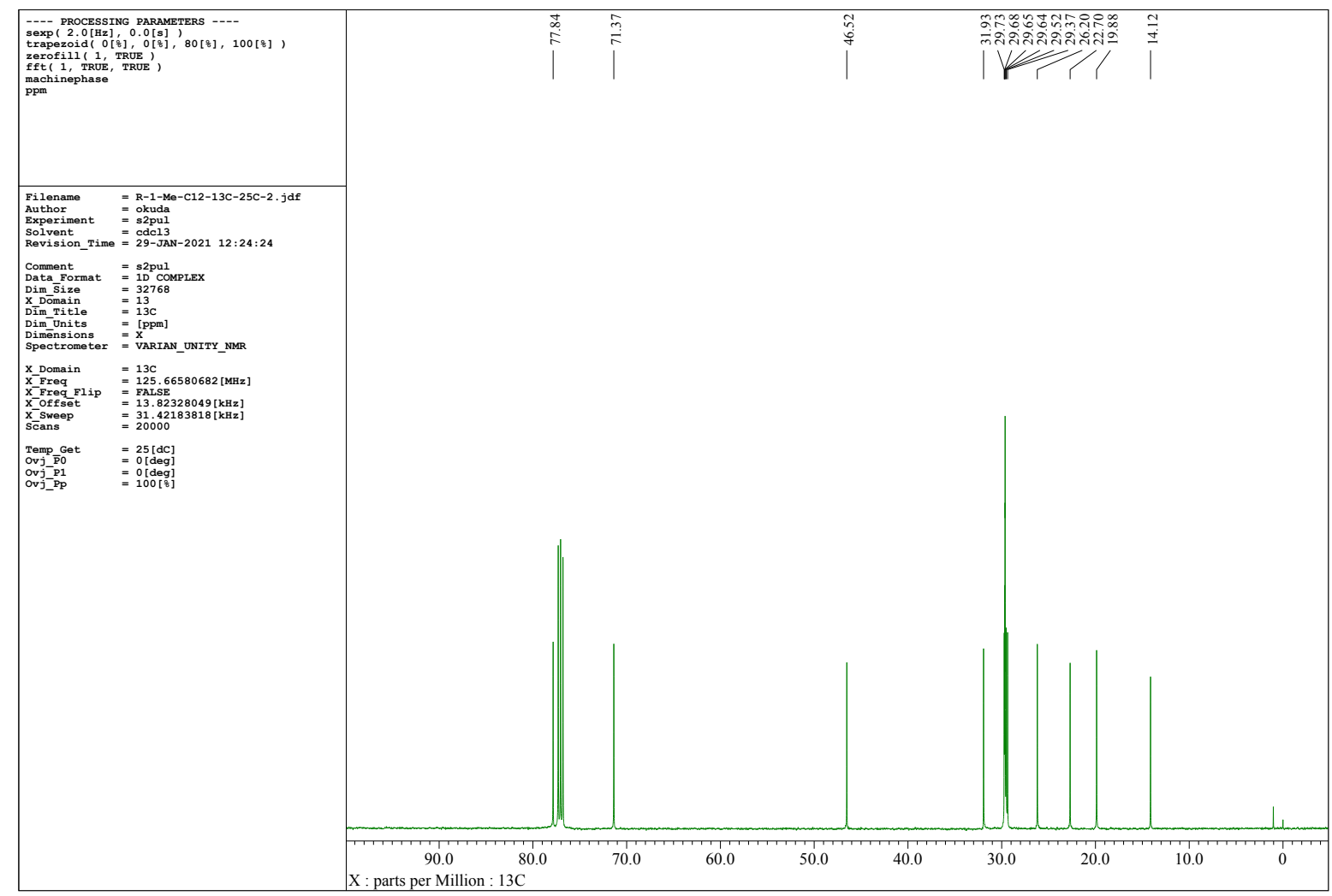

Figure $\mathbf{S 4 5} .{ }^{13} \mathrm{C}$ NMR spectrum of $(R)-4^{\mathrm{Me}}-\mathrm{C} 12$ in $\mathrm{CDCl}_{3}$ at $25{ }^{\circ} \mathrm{C}$. 
DFILE D: \#NMR:Supporting Information NMR¥T

ORGFILE J:¥TKI125-2017-1H. FID¥fid

DATIM 11/Jan/2017 12:01:07

$\begin{array}{lc}\text { COMNT } & \\ \text { OBNUC } & 1 \mathrm{H} \\ \text { OFR } & 499.77 \mathrm{MHz} \\ \text { OBSET } & 0.0 \mathrm{kHz}\end{array}$

$\begin{array}{lr}\text { OBSET } & 0.0 \mathrm{kHz} \\ \text { OBFIN } & 12.593 \mathrm{~Hz}\end{array}$

$\begin{array}{ll}\text { OBFIN } & 12.593 \mathrm{~Hz} \\ \text { PW1 } & 6.6625 \mu \mathrm{s} \\ \text { PW2 } & 0.0 \mu \mathrm{s} \\ \text { PW3 } & 0.0 \mu \mathrm{s}\end{array}$

$\begin{array}{ll}\text { PN3 } & 0.0 \mu \mathrm{\mu s} \\ \text { PI1 } & 0.0 \mathrm{~ms} \\ \text { PI2 } & 0.0 \mathrm{~ms} \\ \text { PI3 } & 0.0 \mathrm{~ms}\end{array}$

0
$131072($ ZeroFi11 $: \times 2)$
16

16

8998. $875 \mathrm{~Hz}$

$98.875 \mathrm{~Hz}$
$7.2827 \mathrm{~s}$
$2.3595 \mathrm{~s}$

$\begin{array}{lc}\text { PD } & 2.3595 \mathrm{~s} \\ \text { RGAIN } & 30 \\ \text { BF } & 0.25 \mathrm{~Hz} \\ \text { EXMOD } & \text { NON } \\ \text { IRNUC } & { }^{13} \mathrm{C} \\ \text { IFR } & 0.0 \mathrm{MHz} \\ \text { IRSET } & 0.0 \mathrm{kHz} \\ \text { IRFIN } & 0.0 \mathrm{~Hz} \\ \text { IRRPW } & 0 \mathrm{\mu s} \\ \text { IRATN } & 00 \mathrm{~Hz} \\ \text { CSPED } & 16.0 \mathrm{~Hz} \\ \text { CTEMP } & 25.0{ }^{\circ} \mathrm{C} \\ \text { PRNT_DATE } & 2017 / \mathrm{Nov} / 18.15: 27: 51\end{array}$

$\begin{array}{lc}\text { PD } & 2.3595 \mathrm{~s} \\ \text { RGAIN } & 30 \\ \text { BF } & 0.25 \mathrm{~Hz} \\ \text { EXMOD } & \text { NON } \\ \text { IRNUC } & { }^{13} \mathrm{C} \\ \text { IFR } & 0.0 \mathrm{MHz} \\ \text { IRSET } & 0.0 \mathrm{kHz} \\ \text { IRFIN } & 0.0 \mathrm{~Hz} \\ \text { IRRPW } & 0 \mathrm{\mu s} \\ \text { IRATN } & 00 \mathrm{~Hz} \\ \text { CSPED } & 16.0 \mathrm{~Hz} \\ \text { CTEMP } & 25.0{ }^{\circ} \mathrm{C} \\ \text { PRNT_DATE } & 2017 / \mathrm{Nov} / 18.15: 27: 51\end{array}$

$\begin{array}{lc}\text { PD } & 2.3595 \mathrm{~s} \\ \text { RGAIN } & 30 \\ \text { BF } & 0.25 \mathrm{~Hz} \\ \text { EXMOD } & \text { NON } \\ \text { IRNUC } & { }^{13} \mathrm{C} \\ \text { IFR } & 0.0 \mathrm{MHz} \\ \text { IRSET } & 0.0 \mathrm{kHz} \\ \text { IRFIN } & 0.0 \mathrm{~Hz} \\ \text { IRRPW } & 0 \mathrm{\mu s} \\ \text { IRATN } & 00 \mathrm{~Hz} \\ \text { CSPED } & 16.0 \mathrm{~Hz} \\ \text { CTEMP } & 25.0{ }^{\circ} \mathrm{C} \\ \text { PRNT_DATE } & 2017 / \mathrm{Nov} / 18.15: 27: 51\end{array}$

$\begin{array}{lc}\text { PD } & 2.3595 \mathrm{~s} \\ \text { RGAIN } & 30 \\ \text { BF } & 0.25 \mathrm{~Hz} \\ \text { EXMOD } & \text { NON } \\ \text { IRNUC } & { }^{13} \mathrm{C} \\ \text { IFR } & 0.0 \mathrm{MHz} \\ \text { IRSET } & 0.0 \mathrm{kHz} \\ \text { IRFIN } & 0.0 \mathrm{~Hz} \\ \text { IRRPW } & 0 \mathrm{\mu s} \\ \text { IRATN } & 00 \mathrm{~Hz} \\ \text { CSPED } & 16.0 \mathrm{~Hz} \\ \text { CTEMP } & 25.0{ }^{\circ} \mathrm{C} \\ \text { PRNT_DATE } & 2017 / \mathrm{Nov} / 18.15: 27: 51\end{array}$

$\begin{array}{lc}\text { PD } & 2.3595 \mathrm{~s} \\ \text { RGAIN } & 30 \\ \text { BF } & 0.25 \mathrm{~Hz} \\ \text { EXMOD } & \text { NON } \\ \text { IRNUC } & { }^{13} \mathrm{C} \\ \text { IFR } & 0.0 \mathrm{MHz} \\ \text { IRSET } & 0.0 \mathrm{kHz} \\ \text { IRFIN } & 0.0 \mathrm{~Hz} \\ \text { IRRPW } & 0 \mathrm{\mu s} \\ \text { IRATN } & 00 \mathrm{~Hz} \\ \text { CSPED } & 16.0 \mathrm{~Hz} \\ \text { CTEMP } & 25.0{ }^{\circ} \mathrm{C} \\ \text { PRNT_DATE } & 2017 / \mathrm{Nov} / 18.15: 27: 51\end{array}$

$\begin{array}{lc}\text { PD } & 2.3595 \mathrm{~s} \\ \text { RGAIN } & 30 \\ \text { BF } & 0.25 \mathrm{~Hz} \\ \text { EXMOD } & \text { NON } \\ \text { IRNUC } & { }^{13} \mathrm{C} \\ \text { IFR } & 0.0 \mathrm{MHz} \\ \text { IRSET } & 0.0 \mathrm{kHz} \\ \text { IRFIN } & 0.0 \mathrm{~Hz} \\ \text { IRRPW } & 0 \mathrm{\mu s} \\ \text { IRATN } & 00 \mathrm{~Hz} \\ \text { CSPED } & 16.0 \mathrm{~Hz} \\ \text { CTEMP } & 25.0{ }^{\circ} \mathrm{C} \\ \text { PRNT_DATE } & 2017 / \mathrm{Nov} / 18.15: 27: 51\end{array}$

$\begin{array}{lc}\text { PD } & 2.3595 \mathrm{~s} \\ \text { RGAIN } & 30 \\ \text { BF } & 0.25 \mathrm{~Hz} \\ \text { EXMOD } & \text { NON } \\ \text { IRNUC } & { }^{13} \mathrm{C} \\ \text { IFR } & 0.0 \mathrm{MHz} \\ \text { IRSET } & 0.0 \mathrm{kHz} \\ \text { IRFIN } & 0.0 \mathrm{~Hz} \\ \text { IRRPW } & 0 \mathrm{\mu s} \\ \text { IRATN } & 0 \\ \text { CSPED } & 16.0 \mathrm{~Hz} \\ \text { CTEMP } & 25.0{ }^{\circ} \mathrm{C} \\ \text { PRNT_DATE } & 2017 / \text { Nov } / 18.15: 27: 51\end{array}$

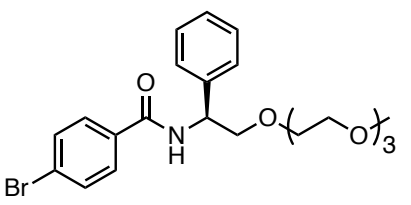

$(S)-5^{\text {Ph }}-$ TEG

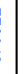

In

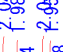

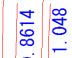

$+$
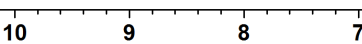

1
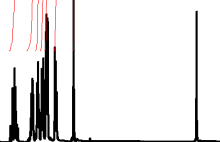

12

11

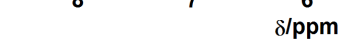

Figure S46. ${ }^{1} \mathrm{H}$ NMR spectrum of $(S)-5^{\mathrm{Ph}}-\mathrm{TEG}$ in $\mathrm{CD}_{3} \mathrm{CN}$ at $25^{\circ} \mathrm{C}$.

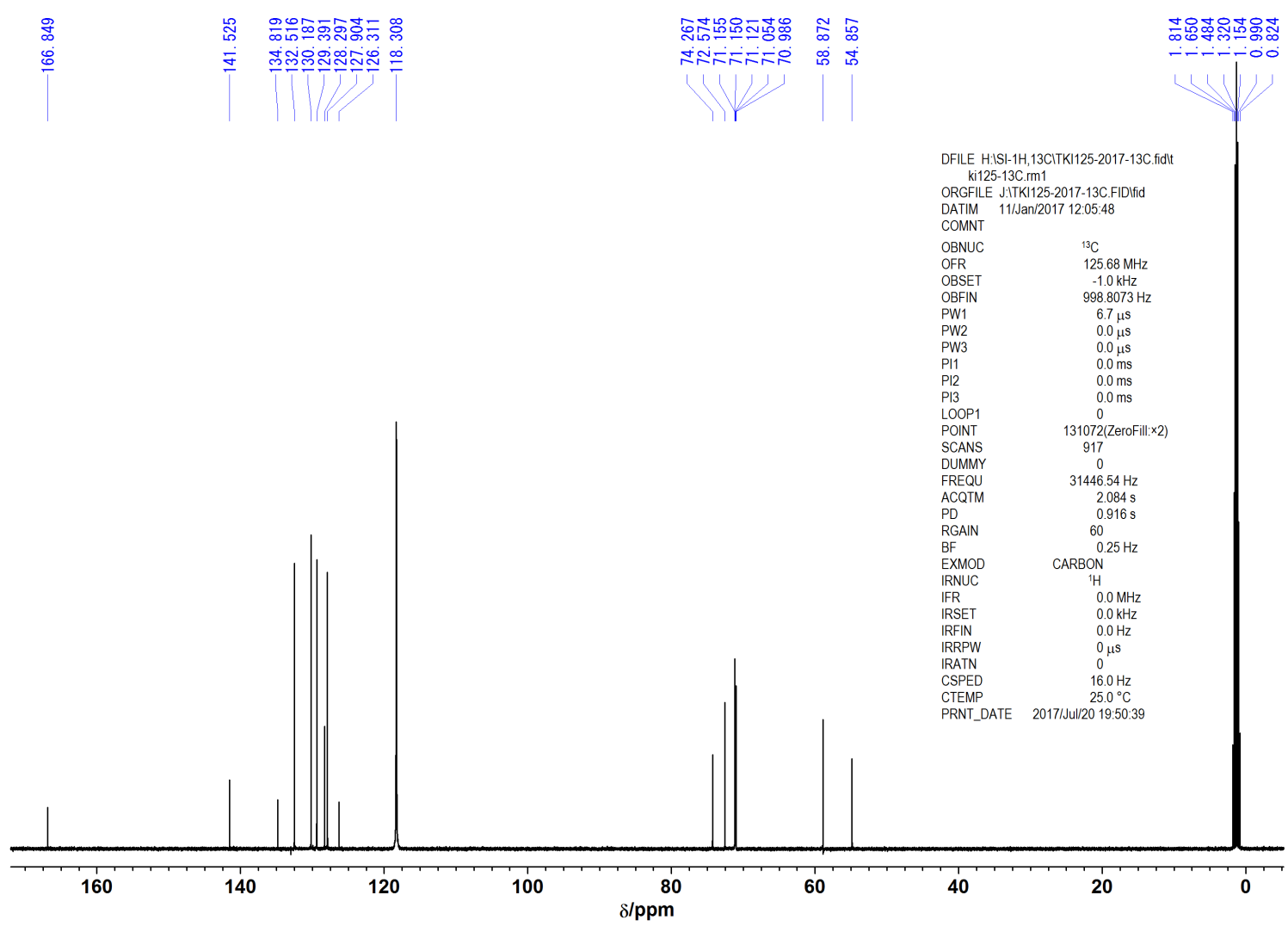

Figure $\mathbf{S 4 7 .}{ }^{13} \mathrm{C}$ NMR spectrum of $(S)-5^{\mathrm{Ph}}-\mathrm{TEG}$ in $\mathrm{CD}_{3} \mathrm{CN}$ at $25{ }^{\circ} \mathrm{C}$. 


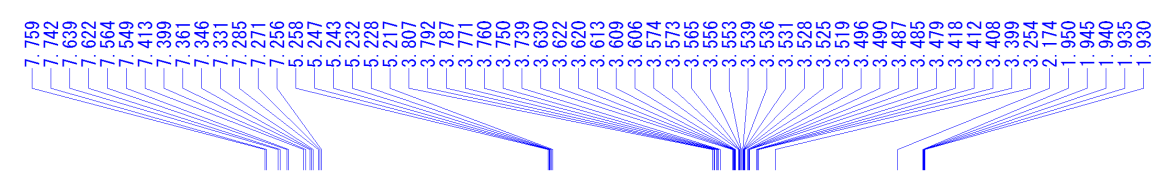

DFILE D: ¥NMR¥Supporting Information NMR¥T

ORGFILE D: :TKI210-1H-25C. FID手id

DATIM 01/Aug/2017 14:33:18

COMNT
1H NMR
Varian UNITY-INOVA

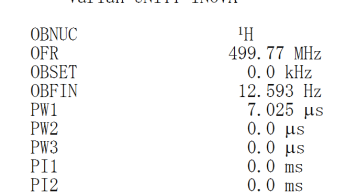

$0.0 \mathrm{~ms}$

$0.0 \mathrm{~ms}$
$0.0 \mathrm{~ms}$

131072(ZeroFi11: $\times 4)$

16
0

$\stackrel{0}{8998.875 \mathrm{~Hz}}$

$998.875 \mathrm{~Hz}$
$3.6413 \mathrm{~s}$
$2.3595 \mathrm{~s}$

$2.3595 \mathrm{~s}$
36
$0.25 \mathrm{~Hz}$

NON

${ }^{13} \mathrm{C} 0.0 \mathrm{MHz}$

$0.0 \mathrm{MHz}$
$0.0 \mathrm{kHz}$
$0.0 \mathrm{~Hz}$

$0.0 \mathrm{~Hz}$
$0 \mu \mathrm{s}$

16. $0 \mathrm{~Hz}$

8 15:33:33<smiles>COCCOCC(NC(=O)c1ccc(Br)cc1)c1ccccc1</smiles>

$(R)-5^{\text {Ph }}$-TEG

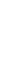




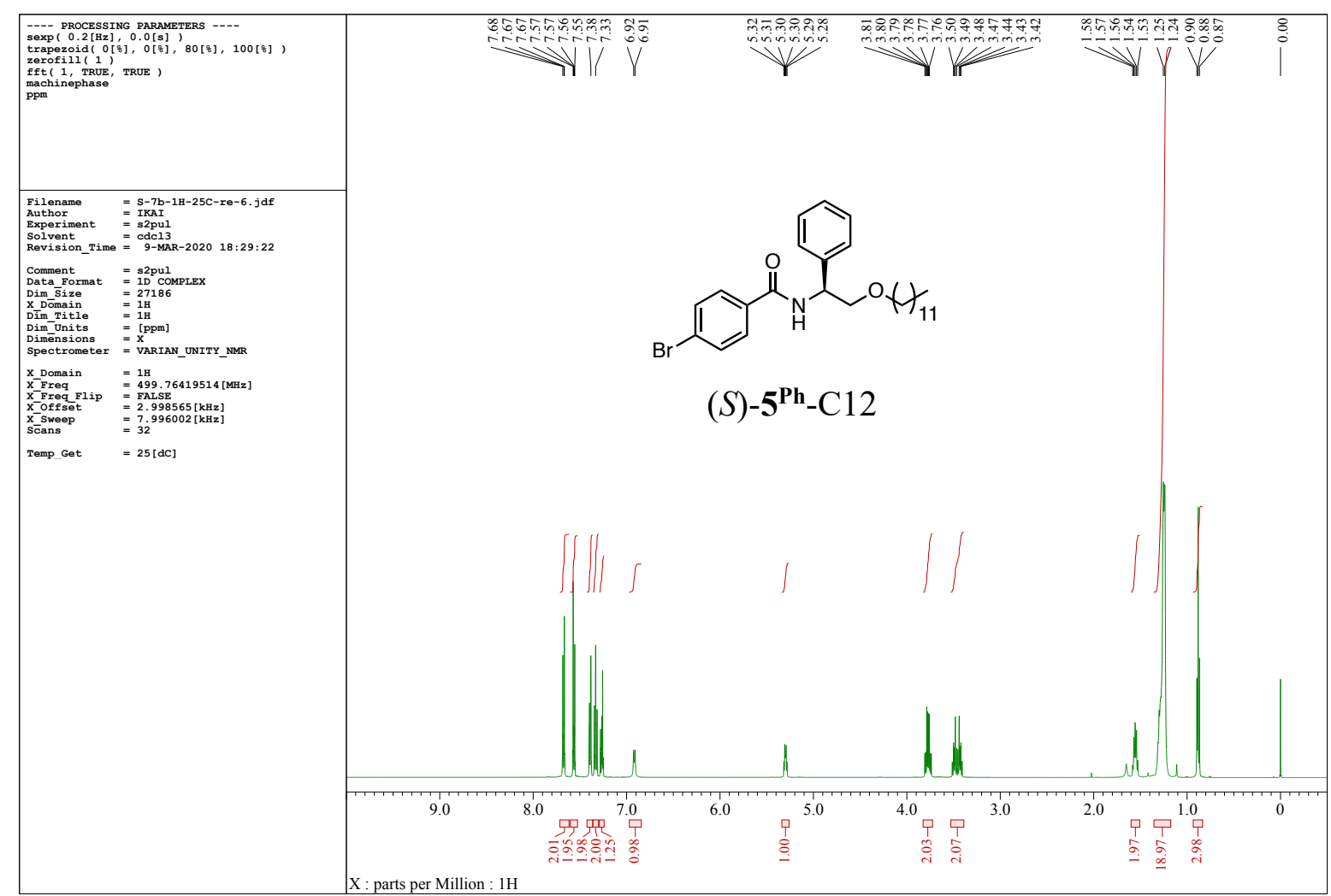

Figure S50. ${ }^{1} \mathrm{H}$ NMR spectrum of $(S)-5^{\mathrm{Ph}}-\mathrm{C} 12$ in $\mathrm{CDCl}_{3}$ at $25^{\circ} \mathrm{C}$.

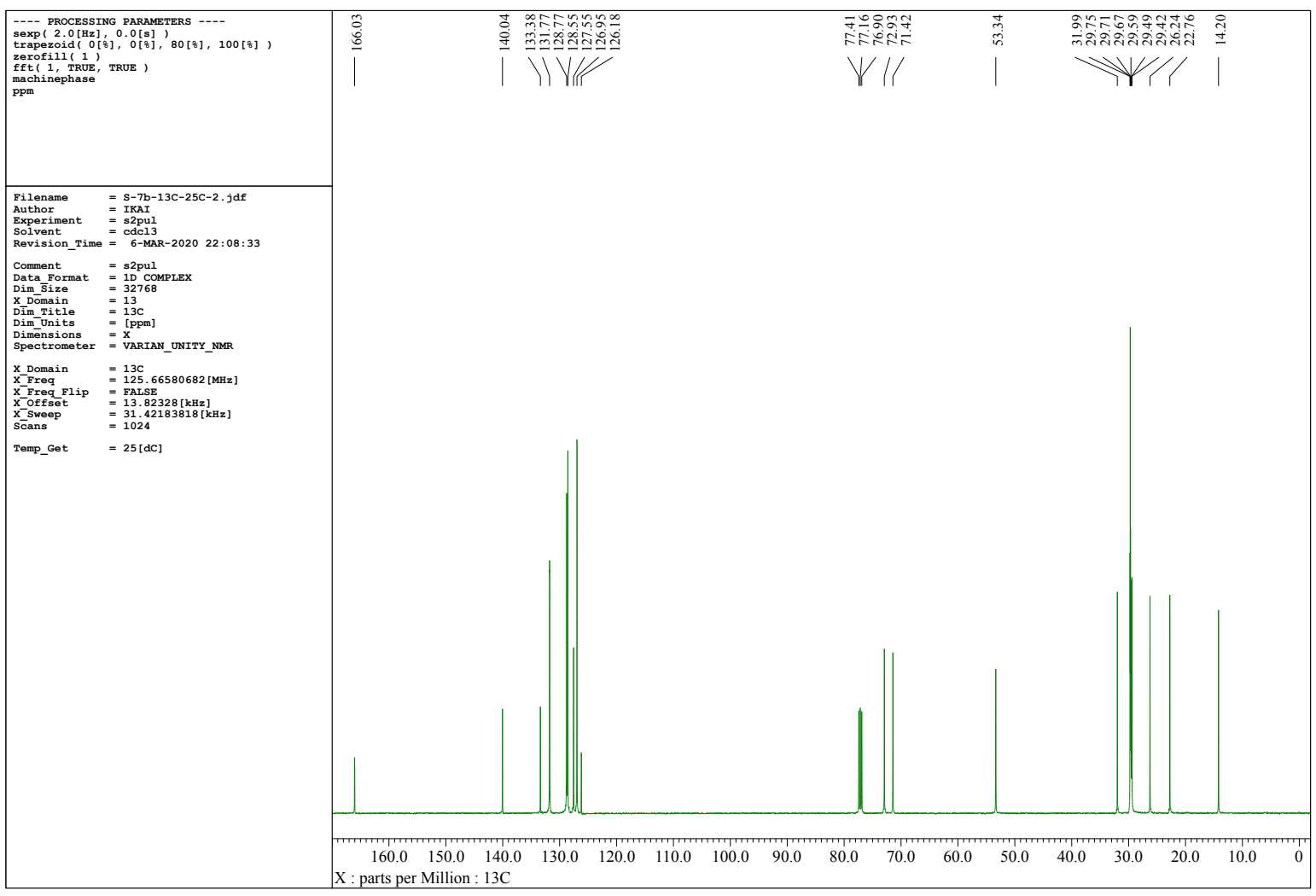

Figure S51. ${ }^{13} \mathrm{C}$ NMR spectrum of $(S)-5^{\mathbf{P h}}-\mathrm{C} 12$ in $\mathrm{CDCl}_{3}$ at $25^{\circ} \mathrm{C}$. 


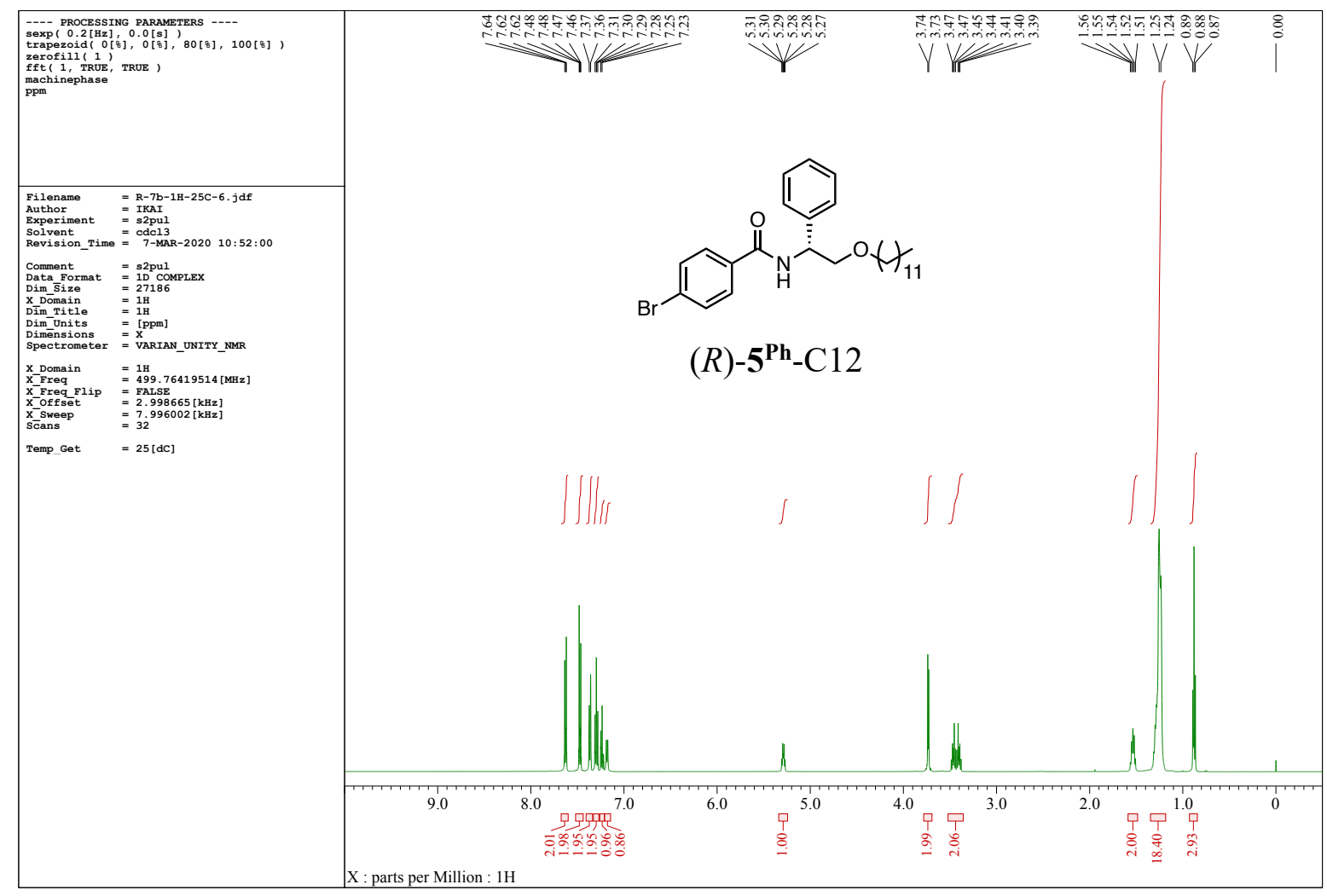

Figure S52. ${ }^{1} \mathrm{H}$ NMR spectrum of $(R)-5^{\mathrm{Ph}}-\mathrm{C} 12$ in $\mathrm{CDCl}_{3}$ at $25^{\circ} \mathrm{C}$.

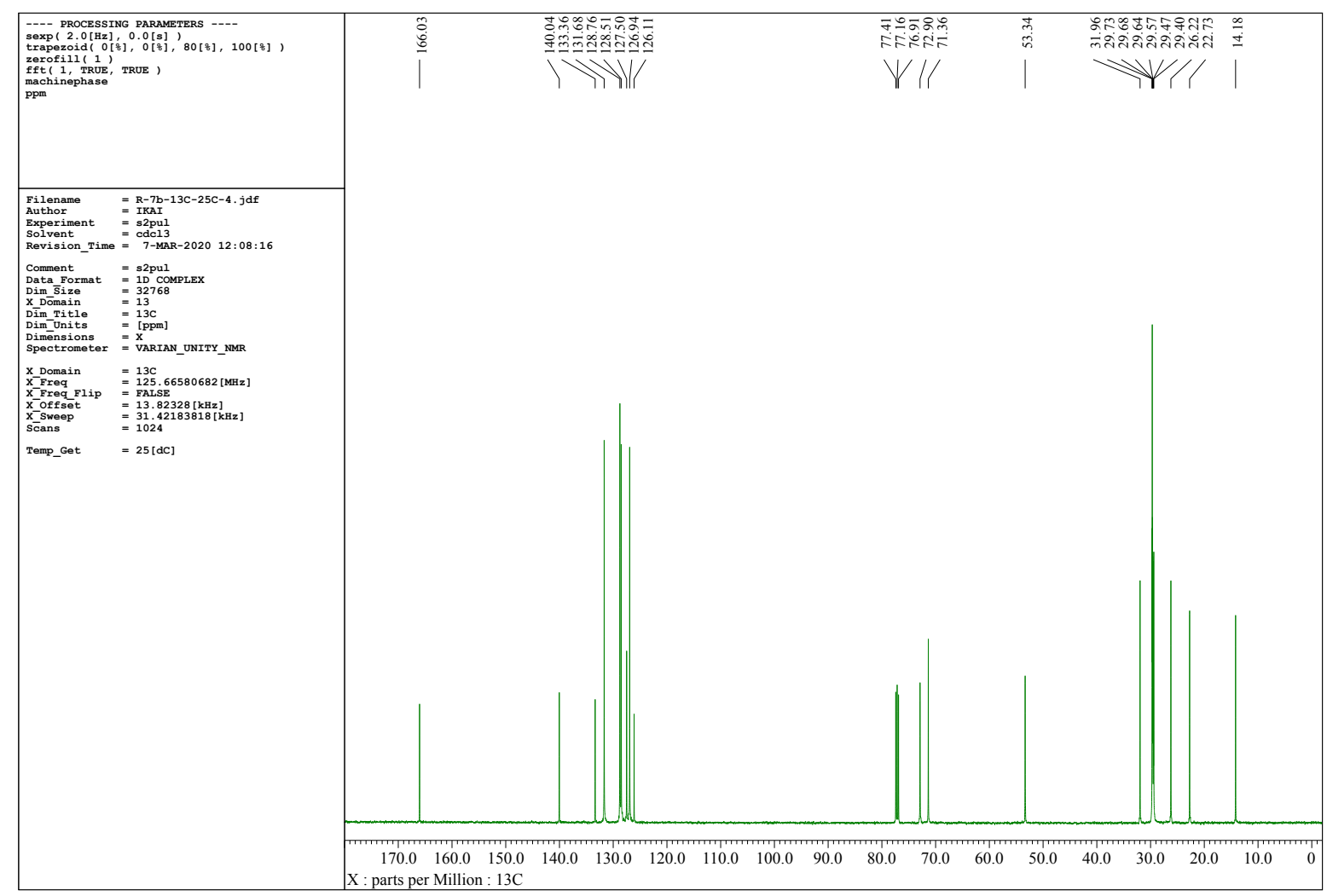

Figure S53. ${ }^{13} \mathrm{C}$ NMR spectrum of $(R)-5^{\mathrm{Ph}}-\mathrm{C} 12$ in $\mathrm{CDCl}_{3}$ at $25^{\circ} \mathrm{C}$. 


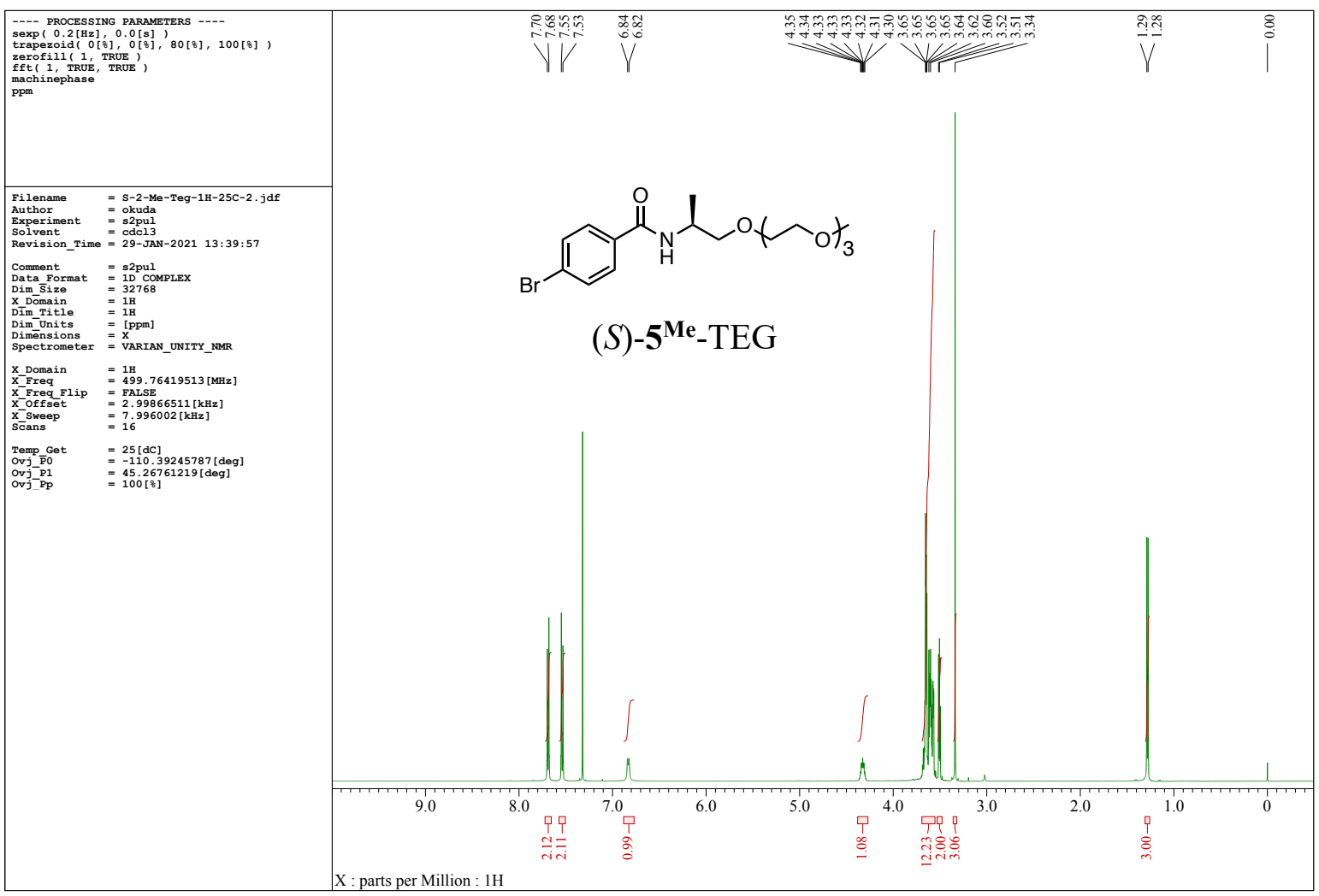

Figure S54. ${ }^{1} \mathrm{H}$ NMR spectrum of $(S)-5^{\mathrm{Me}}-\mathrm{TEG}$ in $\mathrm{CDCl}_{3}$ at $25^{\circ} \mathrm{C}$.

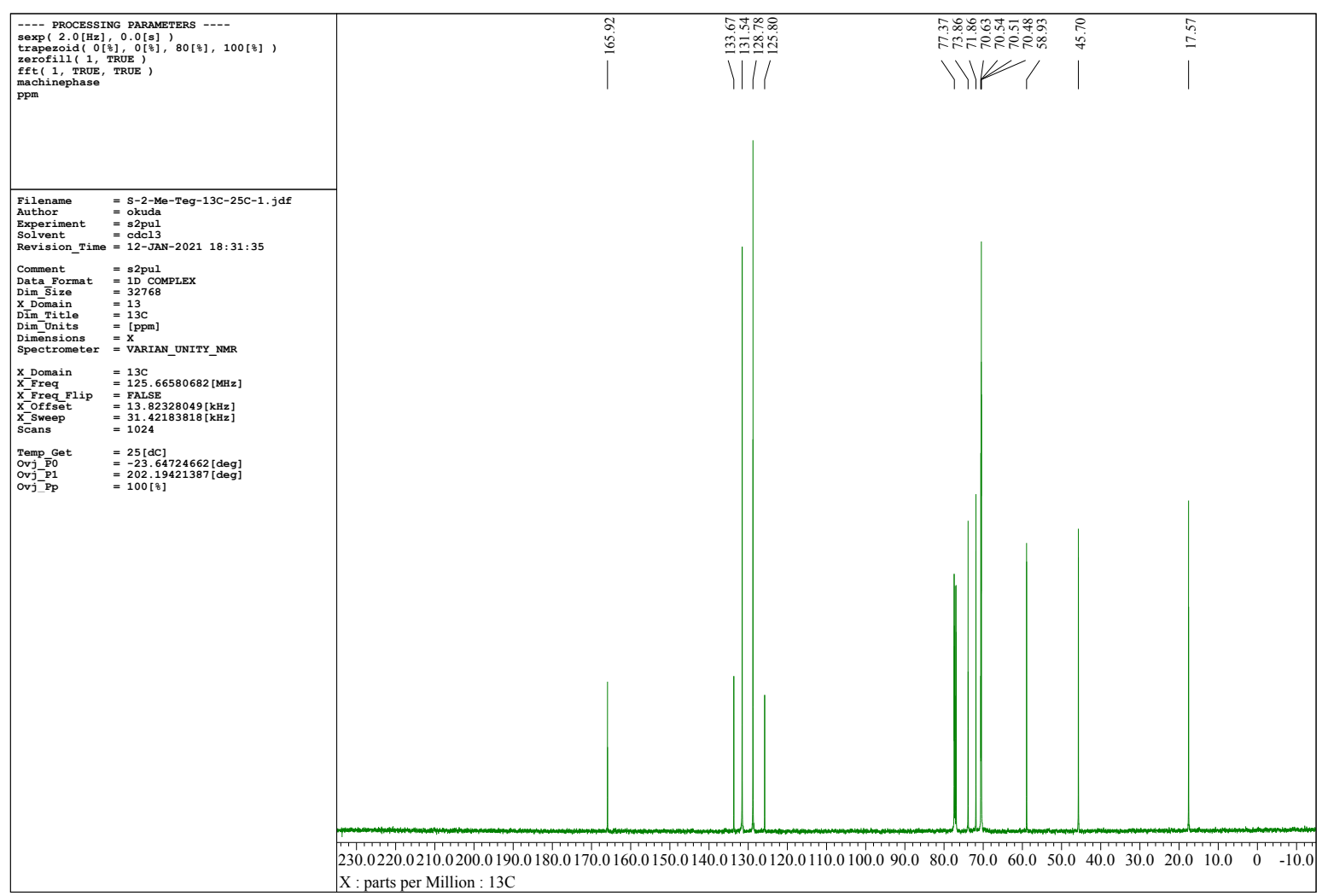

Figure S55. ${ }^{13} \mathrm{C}$ NMR spectrum of $(S)-5^{\text {Me }}-\mathrm{TEG}$ in $\mathrm{CDCl}_{3}$ at $25^{\circ} \mathrm{C}$. 


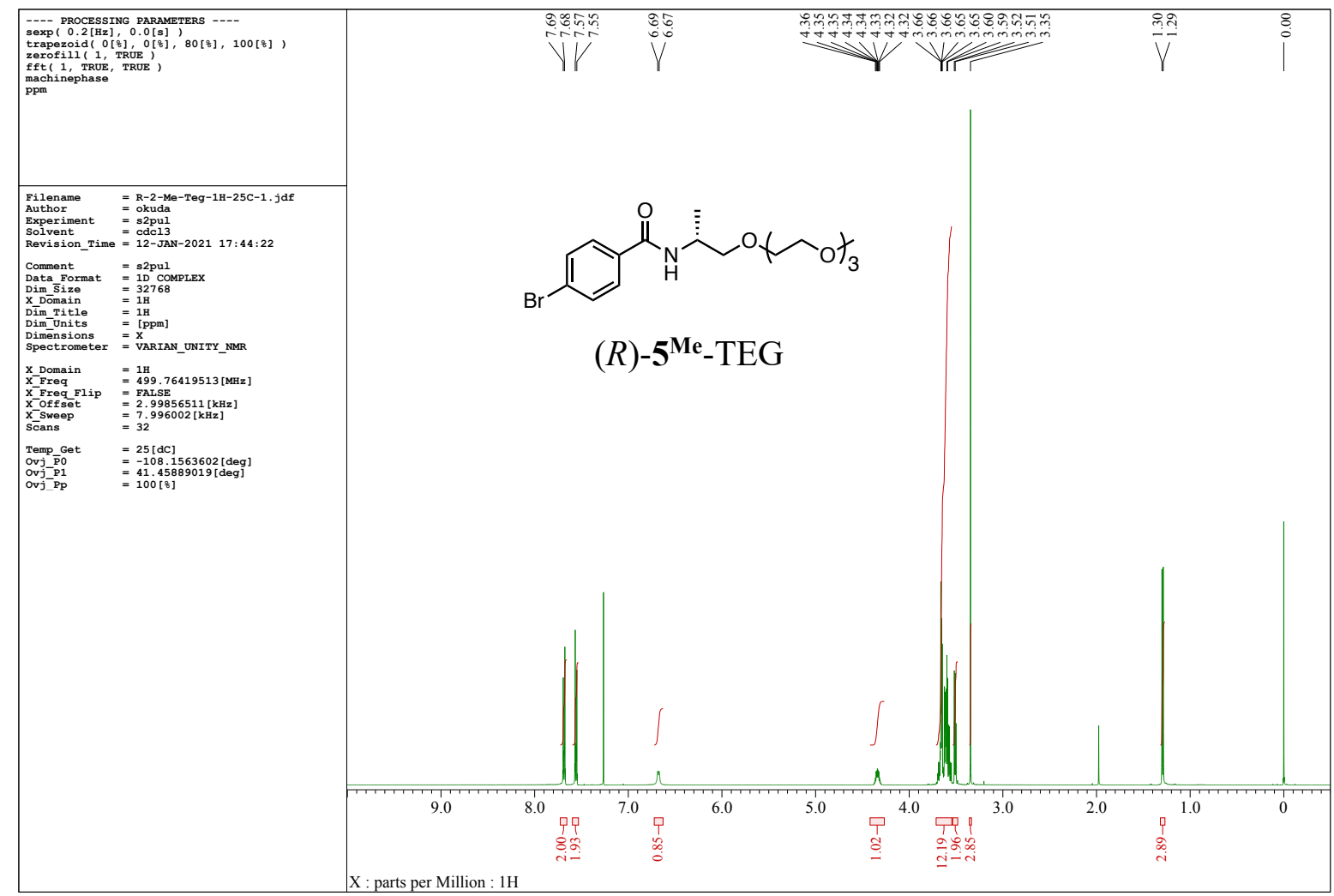

Figure S56. ${ }^{1} \mathrm{H}$ NMR spectrum of $(R)-5^{\mathrm{Me}}-\mathrm{TEG}$ in $\mathrm{CDCl}_{3}$ at $25^{\circ} \mathrm{C}$.

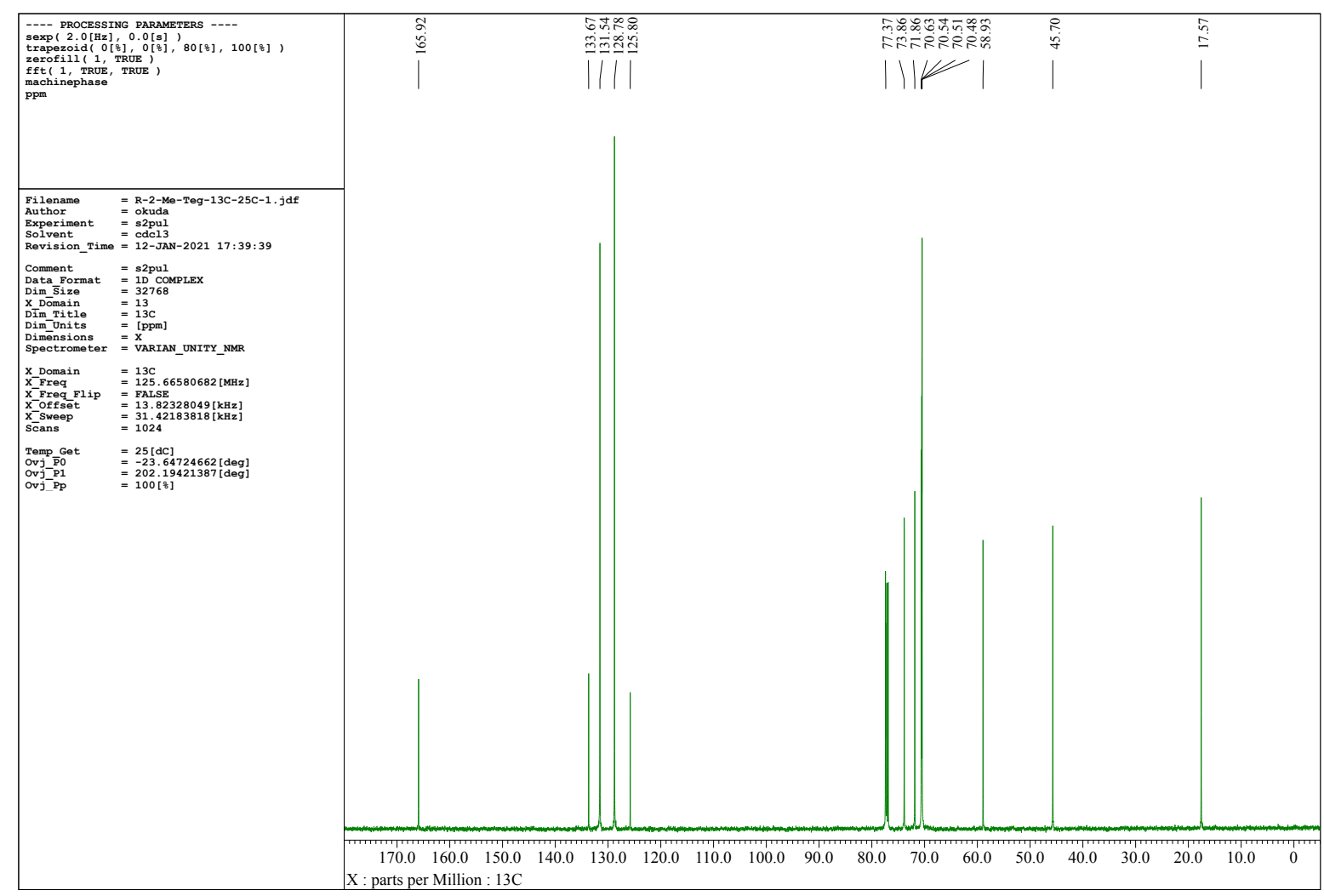

Figure S57. ${ }^{13} \mathrm{C}$ NMR spectrum of $(R)-5^{\mathrm{Me}}-\mathrm{TEG}$ in $\mathrm{CDCl}_{3}$ at $25^{\circ} \mathrm{C}$. 


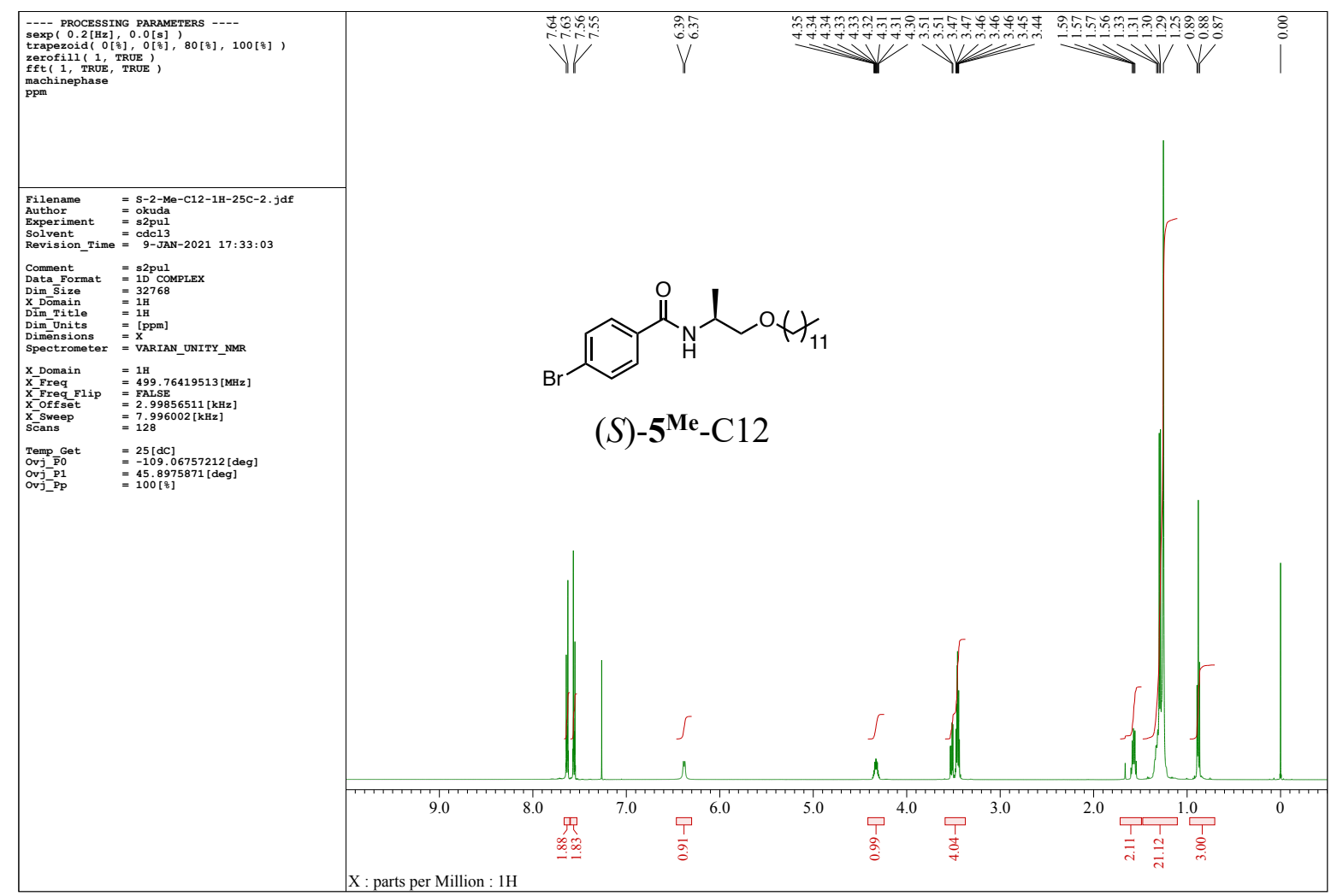

Figure S58. ${ }^{1} \mathrm{H}$ NMR spectrum of $(S)-5^{\mathrm{Me}}-\mathrm{C} 12$ in $\mathrm{CDCl}_{3}$ at $25^{\circ} \mathrm{C}$.

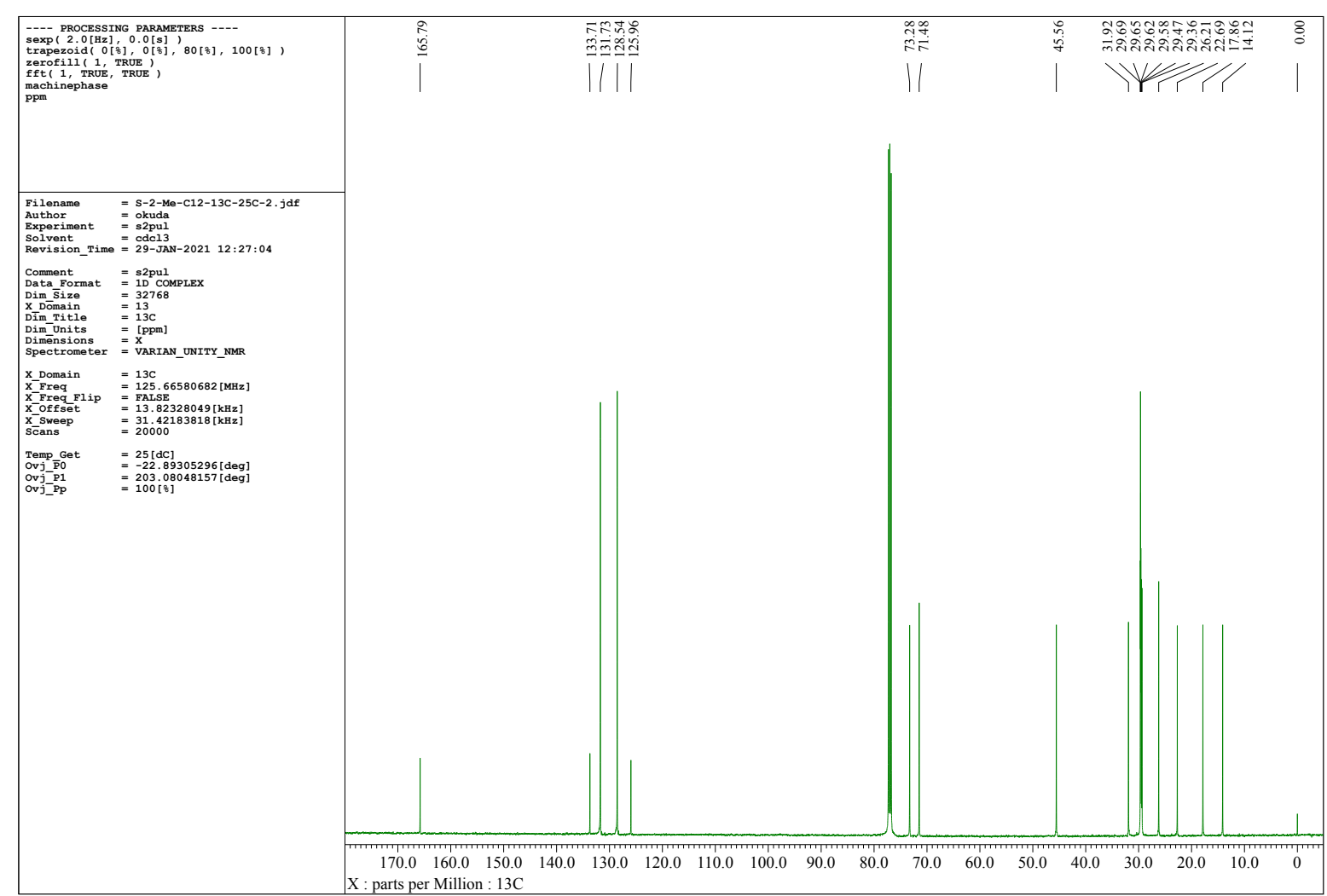

Figure S59. ${ }^{13} \mathrm{C}$ NMR spectrum of $(S)-5^{\mathrm{Me}}-\mathrm{C} 12$ in $\mathrm{CDCl}_{3}$ at $25{ }^{\circ} \mathrm{C}$. 


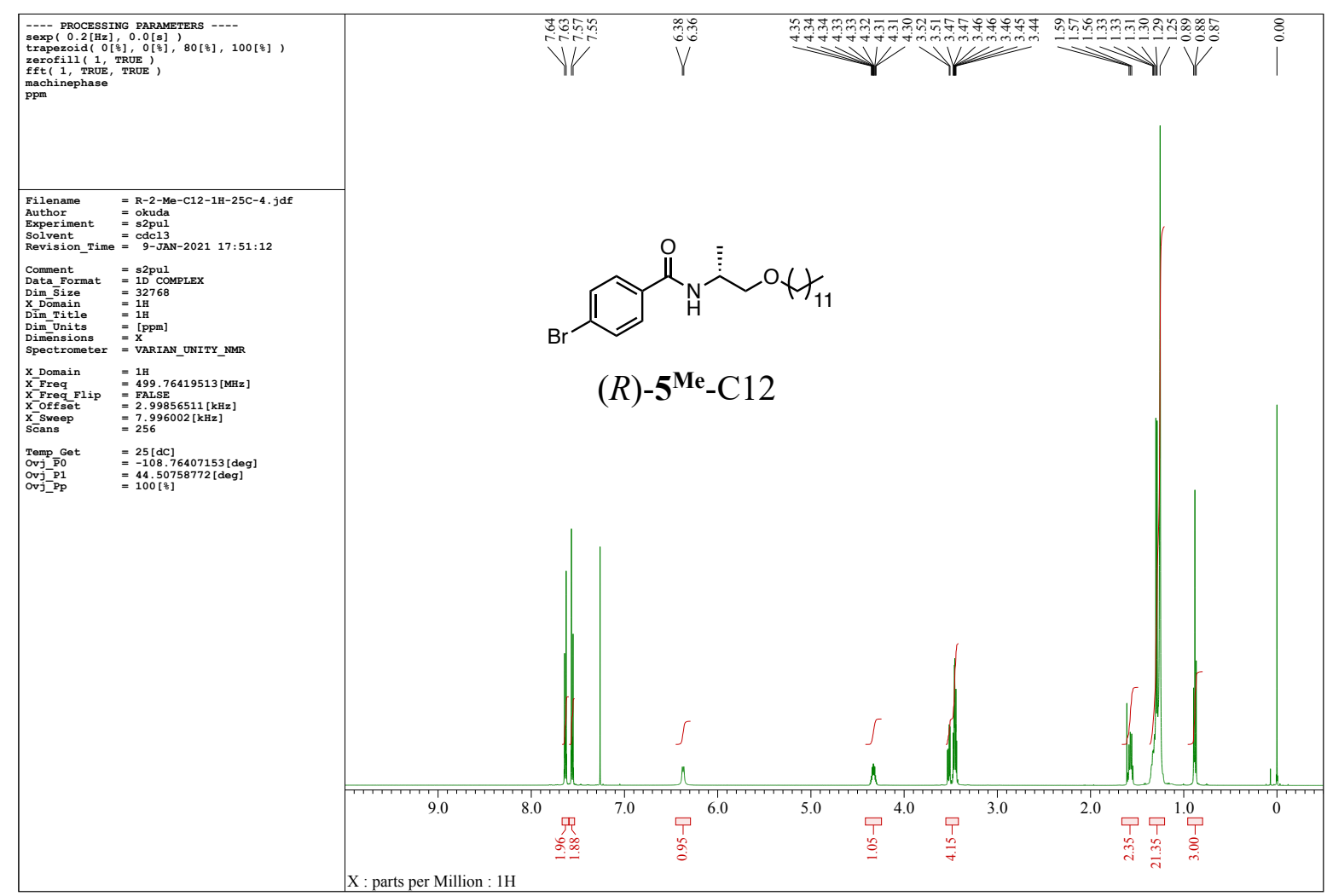

Figure S60. ${ }^{1} \mathrm{H}$ NMR spectrum of $(R)-5^{\mathrm{Me}}-\mathrm{C} 12$ in $\mathrm{CDCl}_{3}$ at $25^{\circ} \mathrm{C}$.

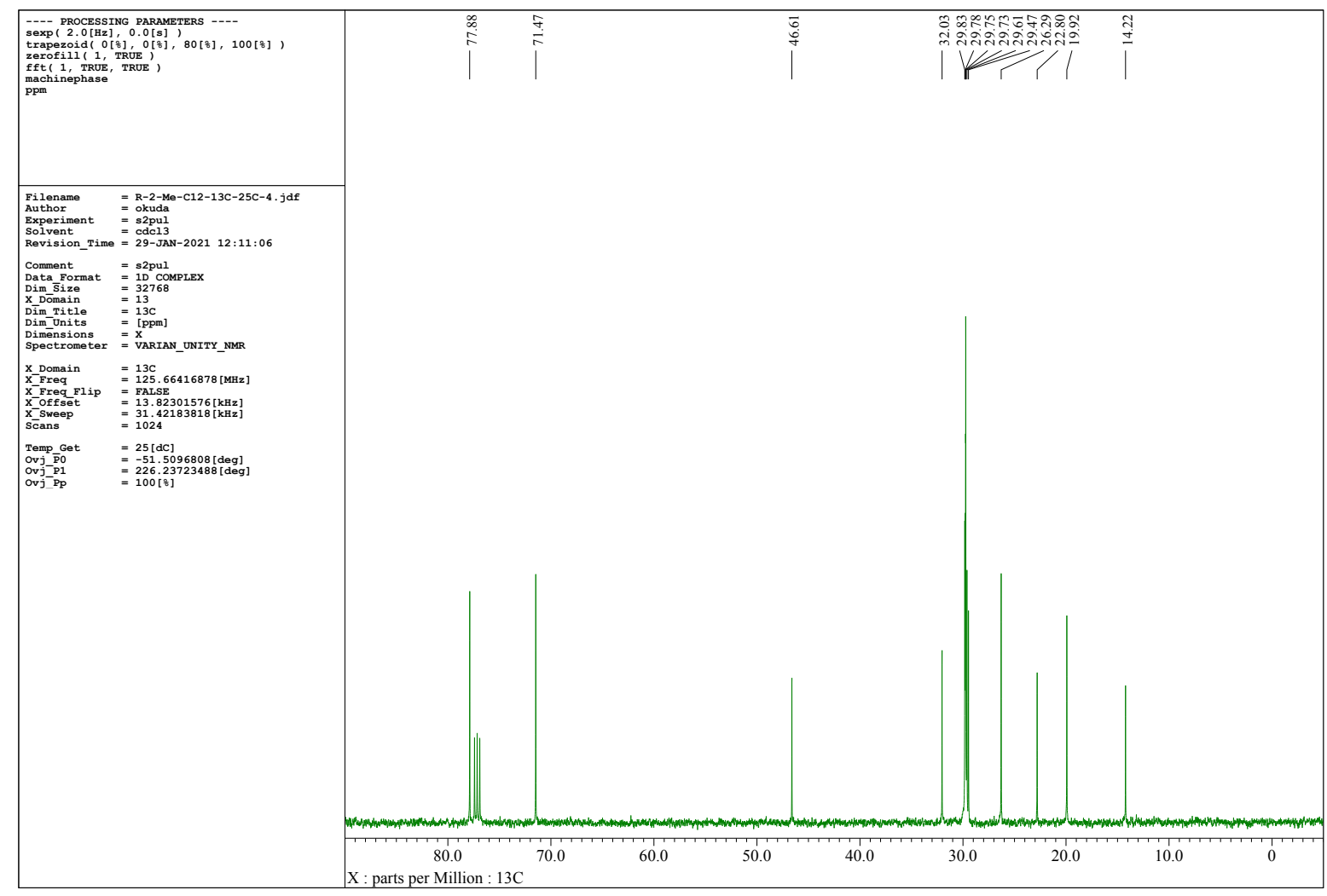

Figure S61. ${ }^{13} \mathrm{C}$ NMR spectrum of $(R)-5^{\mathrm{Me}}-\mathrm{C} 12$ in $\mathrm{CDCl}_{3}$ at $25^{\circ} \mathrm{C}$. 


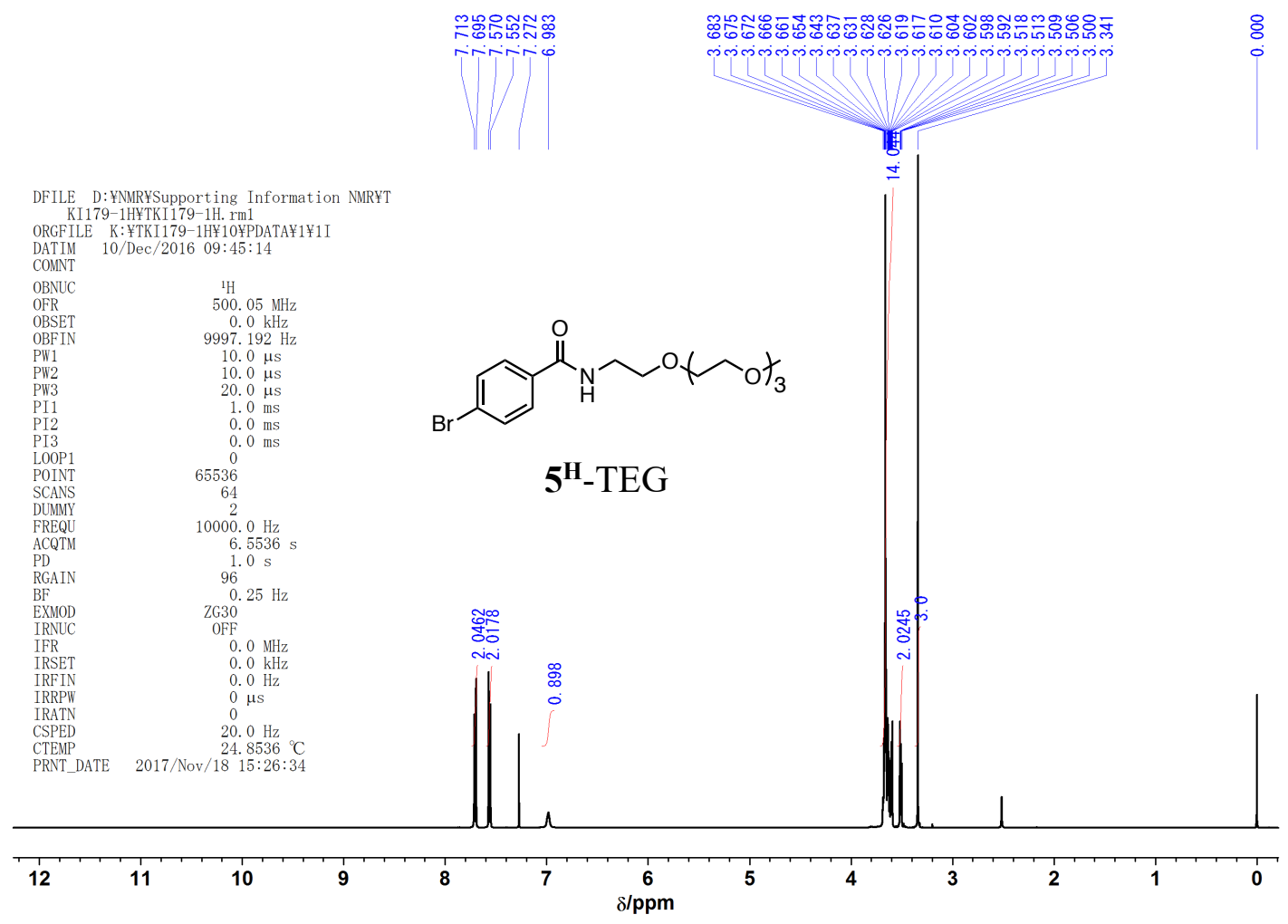

Figure S62. ${ }^{1} \mathrm{H}$ NMR spectrum of $5^{\mathrm{H}}-\mathrm{TEG}$ in $\mathrm{CDCl}_{3}$ at $25^{\circ} \mathrm{C}$.

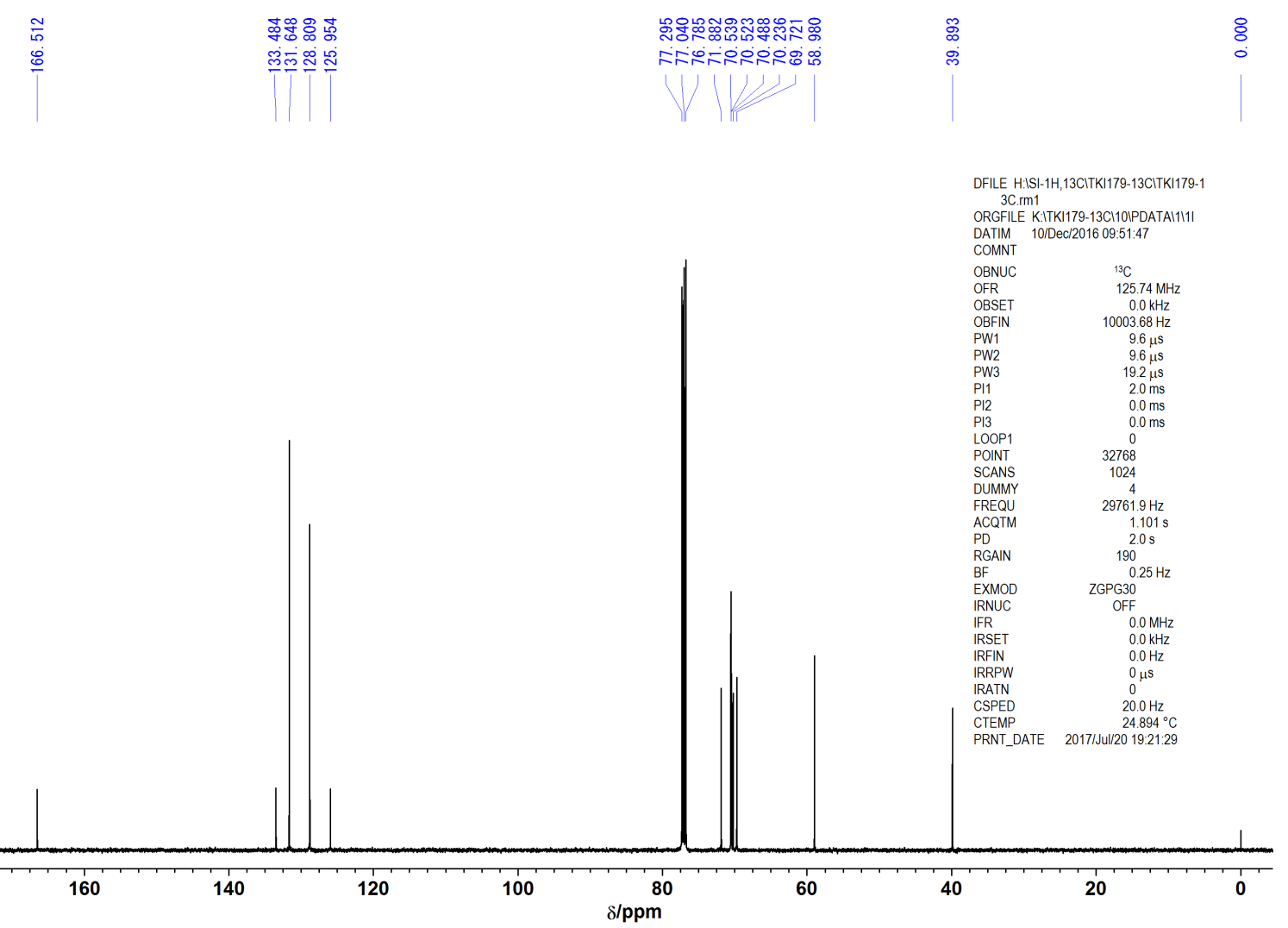

Figure S63. ${ }^{13} \mathrm{C}$ NMR spectrum of $\mathbf{5}^{\mathbf{H}}-\mathrm{TEG}$ in $\mathrm{CDCl}_{3}$ at $25^{\circ} \mathrm{C}$. 


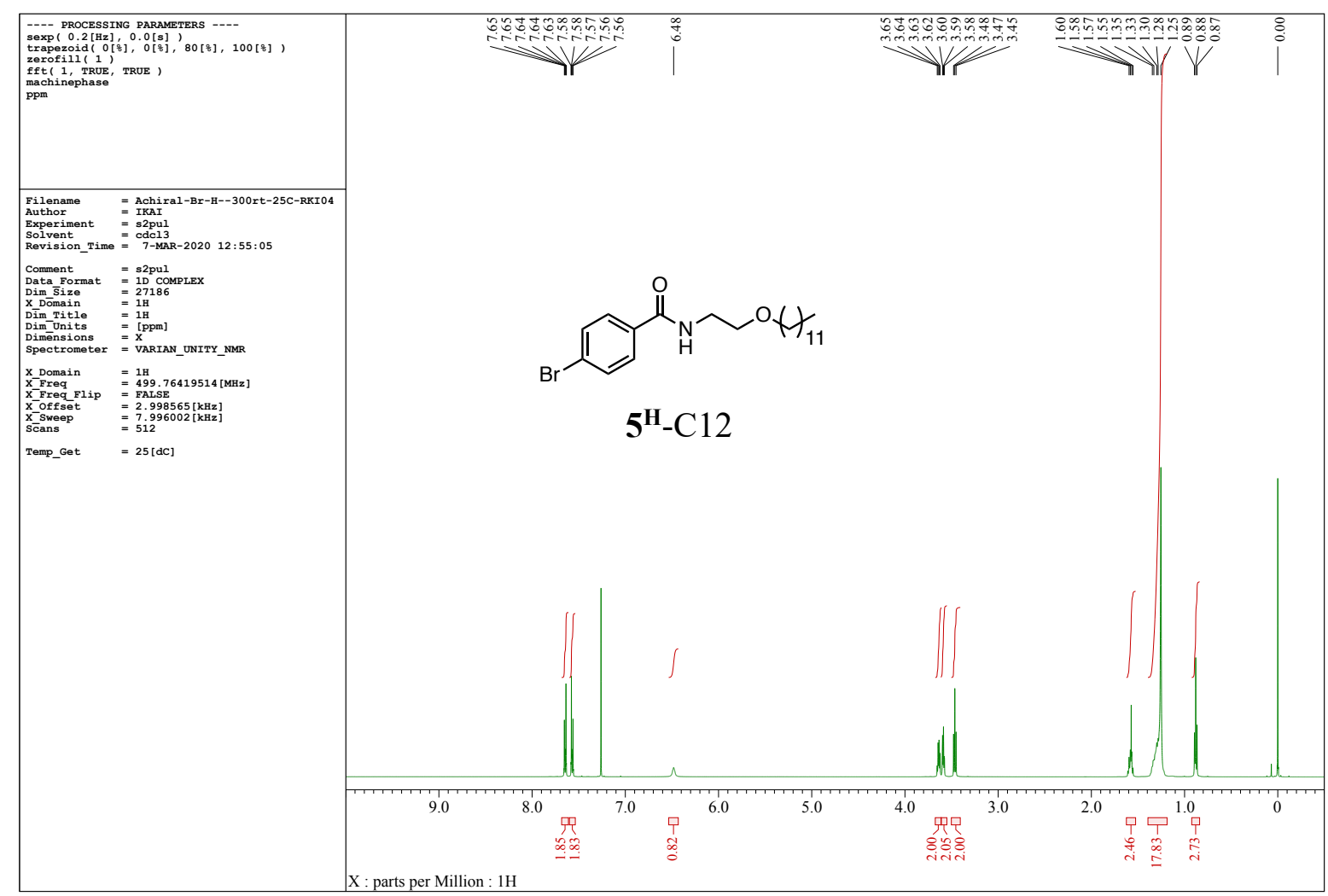

Figure S64. ${ }^{1} \mathrm{H}$ NMR spectrum of $\mathbf{5}^{\mathbf{H}}-\mathrm{C} 12$ in $\mathrm{CDCl}_{3}$ at $25^{\circ} \mathrm{C}$.

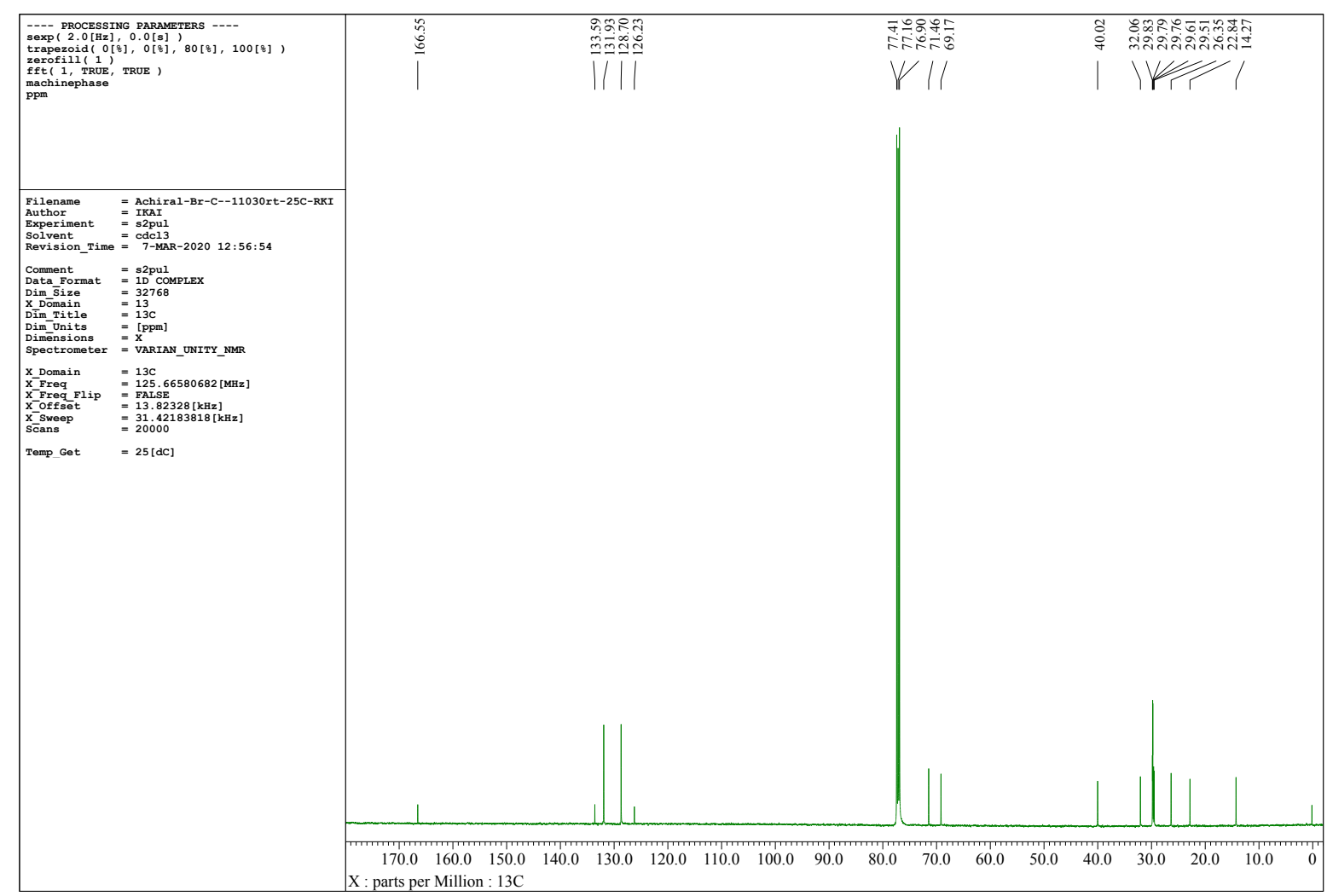

Figure S65. ${ }^{13} \mathrm{C}$ NMR spectrum of $\mathbf{5}^{\mathbf{H}}-\mathrm{C} 12$ in $\mathrm{CDCl}_{3}$ at $25{ }^{\circ} \mathrm{C}$. 


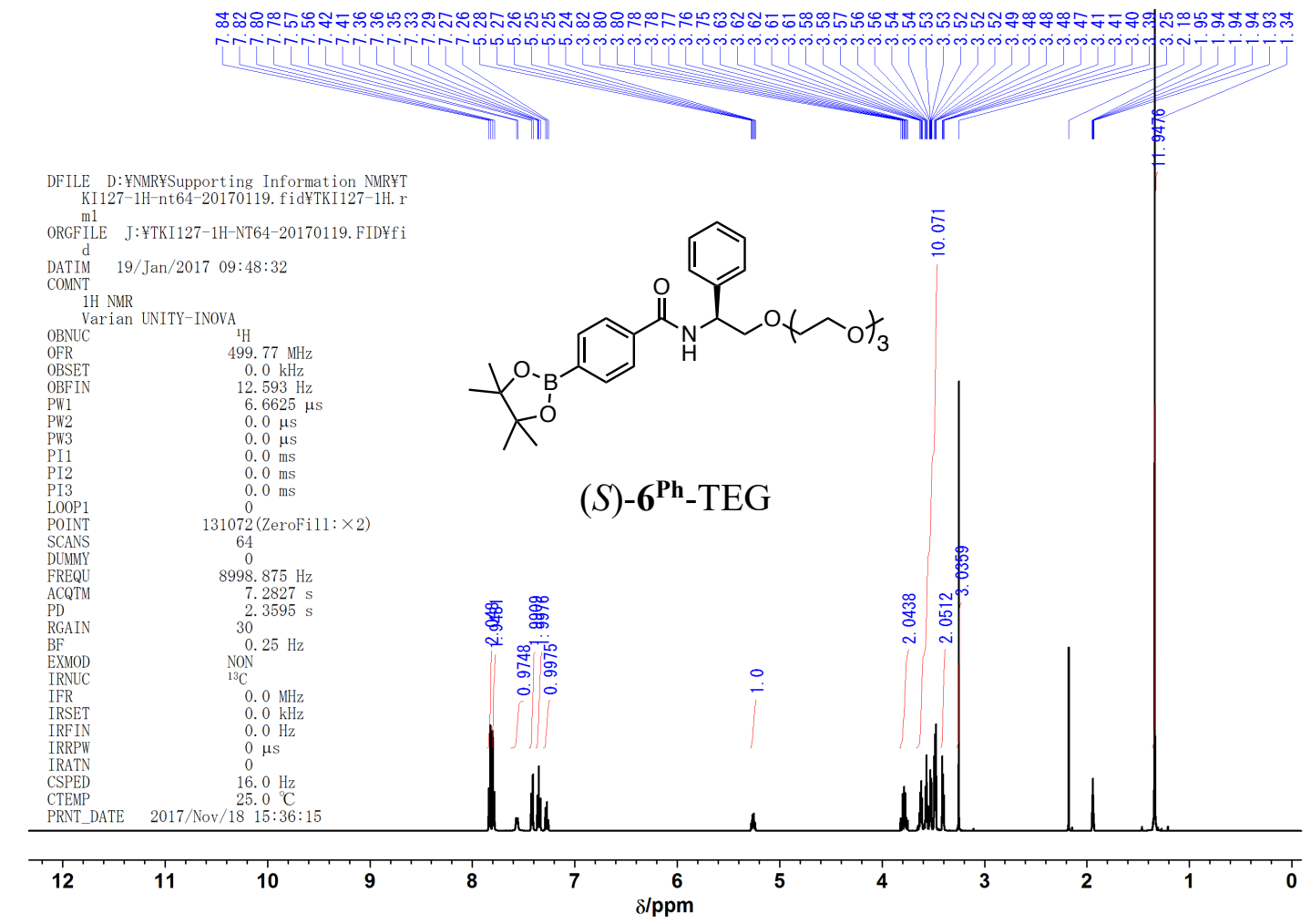

Figure S66. ${ }^{1} \mathrm{H}$ NMR spectrum of $(S)-6^{\mathbf{P h}}-\mathrm{TEG}$ in $\mathrm{CD}_{3} \mathrm{CN}$ at $25^{\circ} \mathrm{C}$.

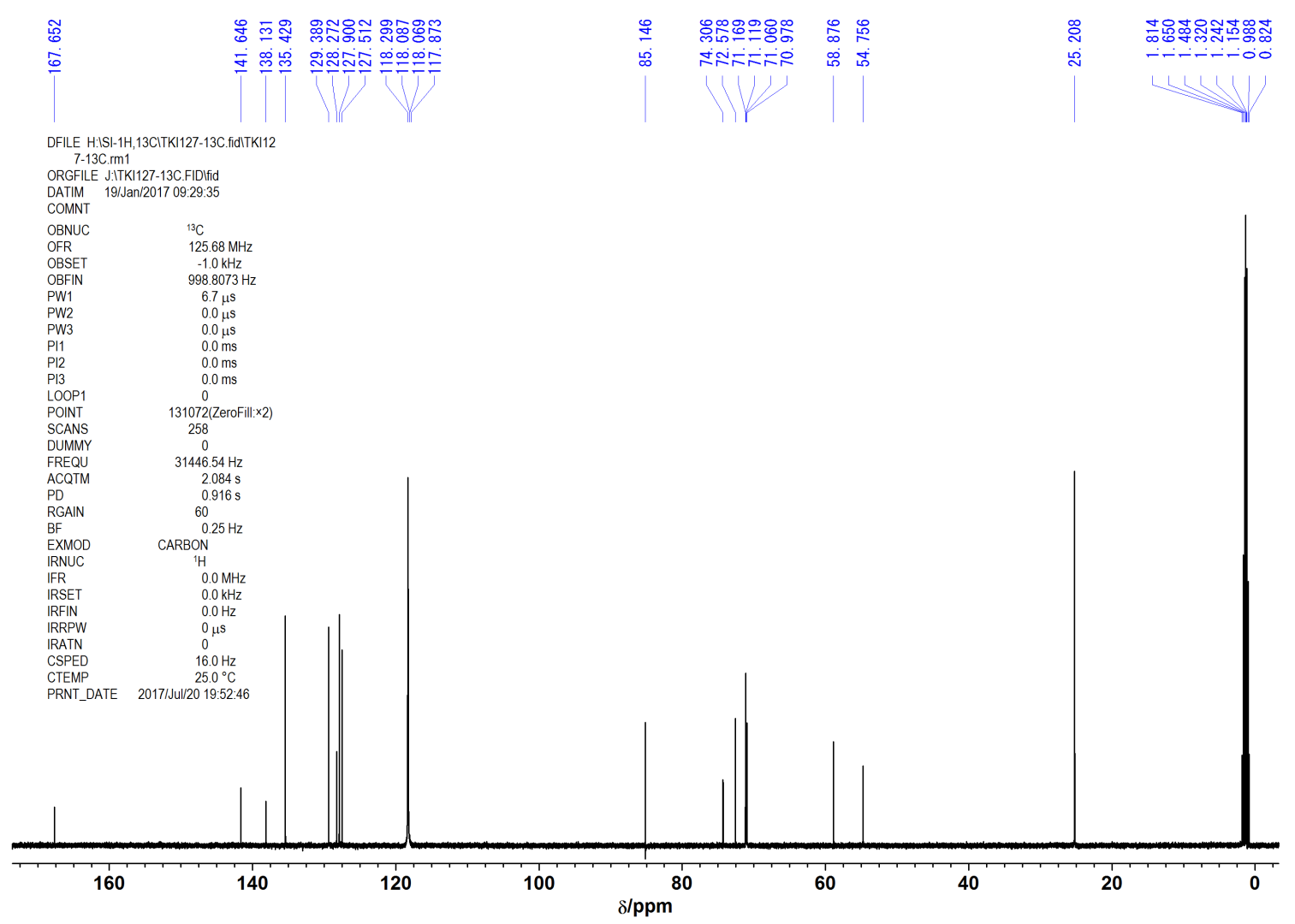

Figure S67. ${ }^{13} \mathrm{C}$ NMR spectrum of $(S)-6^{\text {Ph }}-\mathrm{TEG}$ in $\mathrm{CD}_{3} \mathrm{CN}$ at $25^{\circ} \mathrm{C}$. 

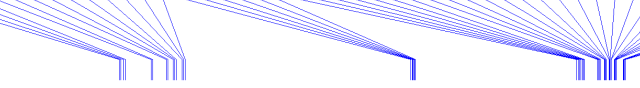

参

DFILE D: ¥NMR¥Supporting Information NMR¥T

KI212-1H-25C. fid¥TKI212-1H. rmi
ORGFILE D: ¥TKI212-1H-25C. FID¥fid

DATIM 05/Aug/2017 17:21:00

COMNT 1 H NMR

Varian UNITY-INOVA

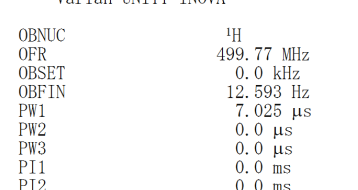

$.0 \mathrm{~ms}$

0
0
$131072($ meroFi $11: \times 4)$

4
0

4
0
$98.875 \mathrm{~Hz}$

8. $875 \mathrm{~Hz}$
$3.6413 \mathrm{~s}$

$2.3595 \mathrm{~s}$
36
$0.25 \mathrm{~Hz}$

NON

${ }^{3} \mathrm{C}$

$0.0 \mathrm{MHz}$
$0.0 \mathrm{kHz}$
$0.0 \mathrm{~Hz}$
$0.5 \mathrm{~s}$

$0 \mu \mathrm{s}$

16. $0 \mathrm{~Hz}$

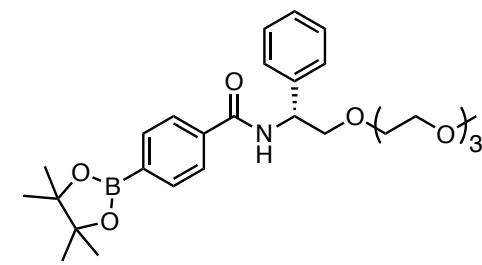

(R)-6 - $^{\text {Ph }}$ TEG

E
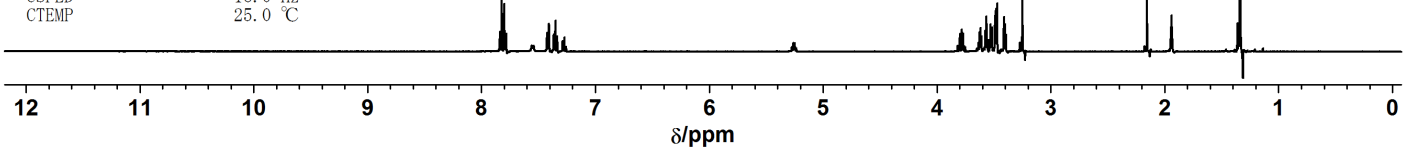

Figure S68. ${ }^{1} \mathrm{H}$ NMR spectrum of $(R)-6^{\mathrm{Ph}}-\mathrm{TEG}$ in $\mathrm{CD}_{3} \mathrm{CN}$ at $25^{\circ} \mathrm{C}$.
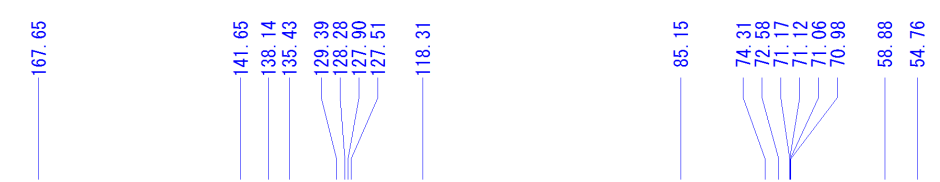

DFILE D: ¥TKI212-13C-25C. fid¥TKI212-13C. rm

ORGFILE D: ¥TKI212-13C-25C. FID¥fid

$\begin{array}{ll}\text { DATIM } & \text { D: } ¥ \text { Aug } / 2017 \text { 17:22:28 } \\ \text { CONNT } & \end{array}$

C13 std parameter

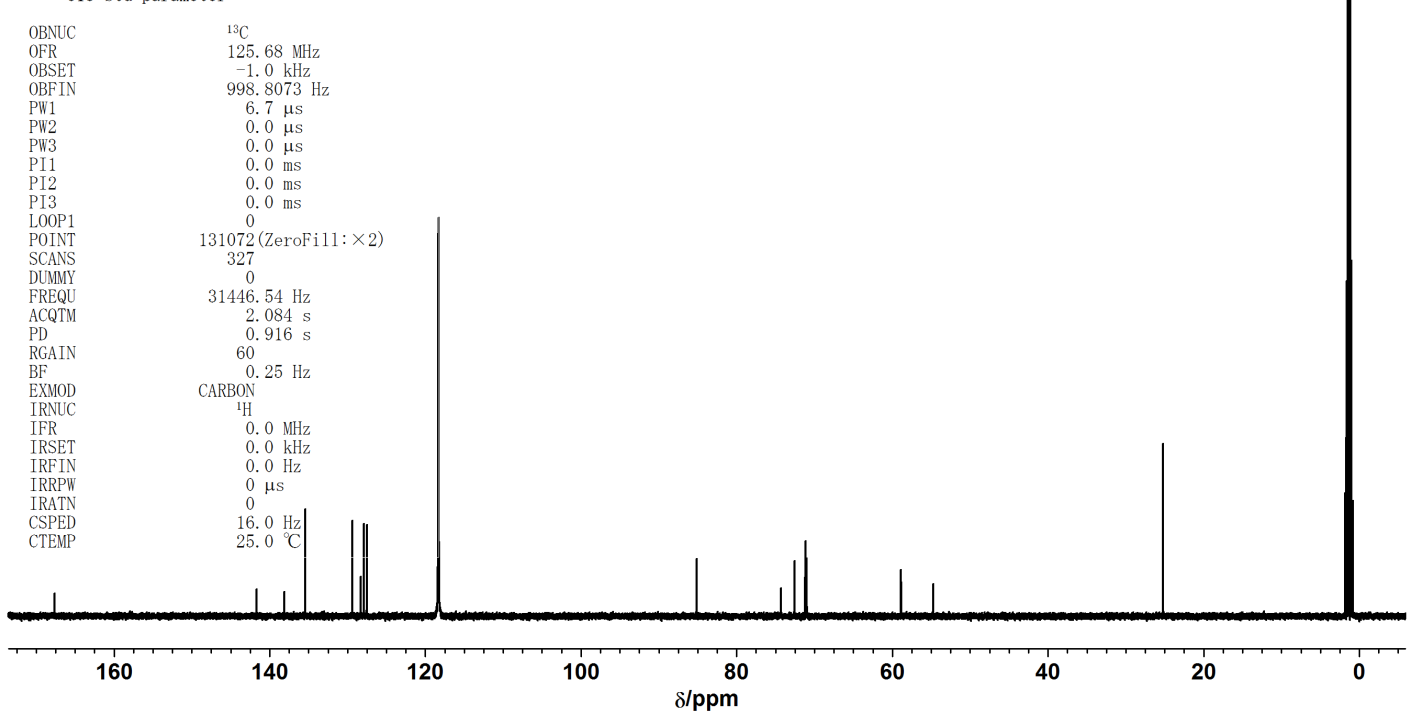

Figure S69. ${ }^{13} \mathrm{C}$ NMR spectrum of $(R)-6^{\mathrm{Ph}}-\mathrm{TEG}$ in $\mathrm{CD}_{3} \mathrm{CN}$ at $25^{\circ} \mathrm{C}$. 


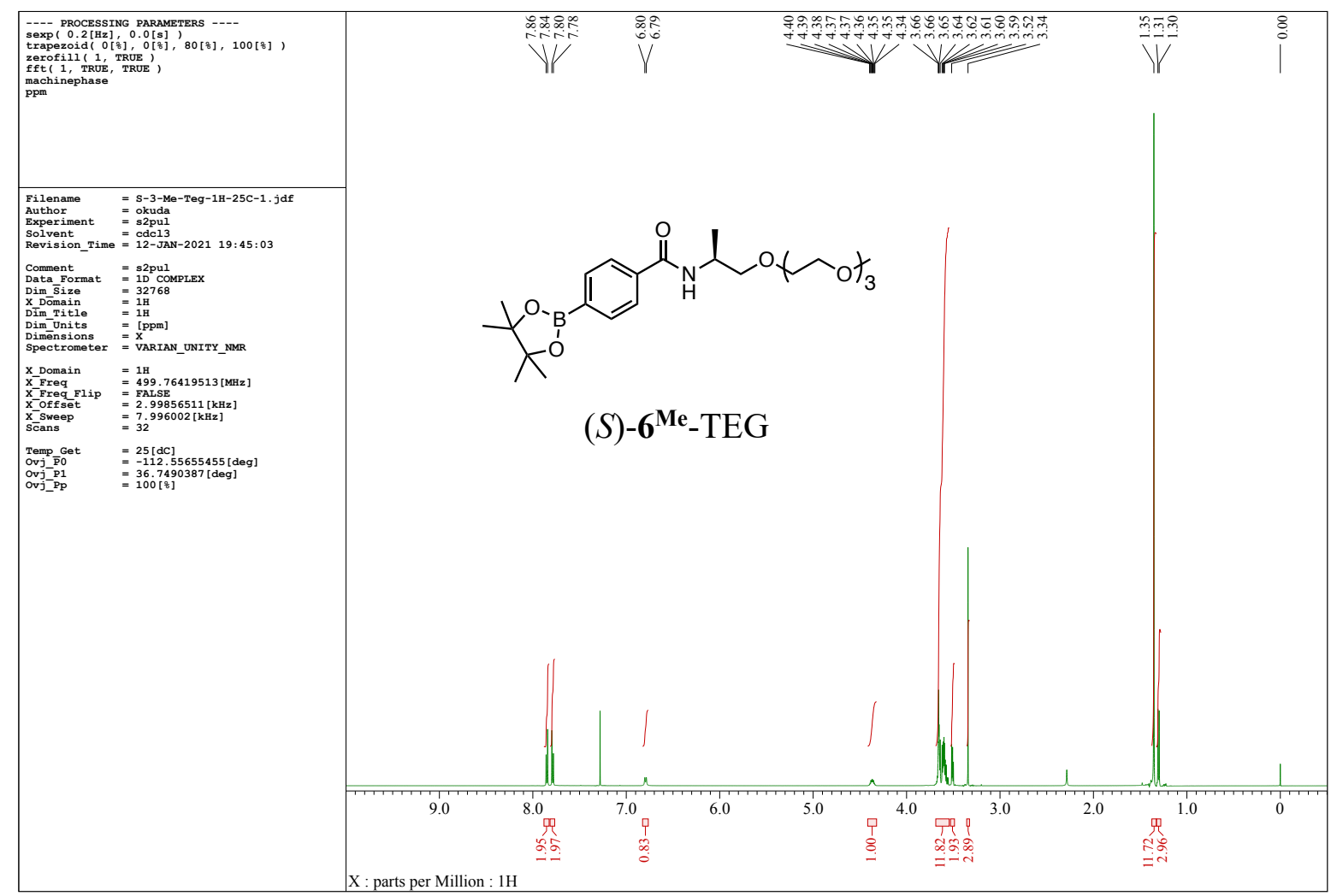

Figure S70. ${ }^{1} \mathrm{H}$ NMR spectrum of $(S)-6^{\mathrm{Me}}-\mathrm{TEG}$ in $\mathrm{CDCl}_{3}$ at $25^{\circ} \mathrm{C}$.

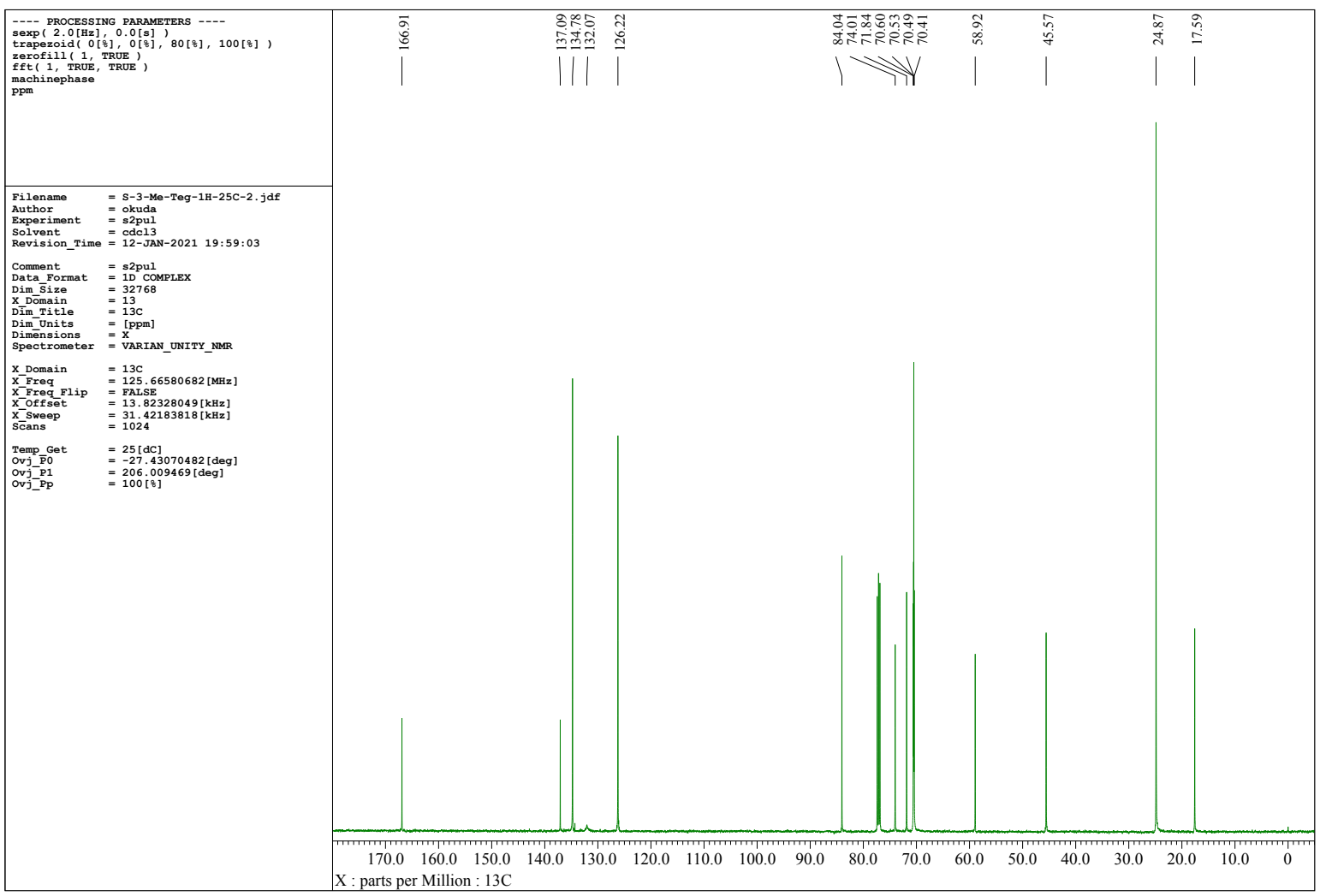

Figure S71. ${ }^{13} \mathrm{C}$ NMR spectrum of $(S)-6^{\mathrm{Me}}-\mathrm{TEG}$ in $\mathrm{CDCl}_{3}$ at $25^{\circ} \mathrm{C}$. 


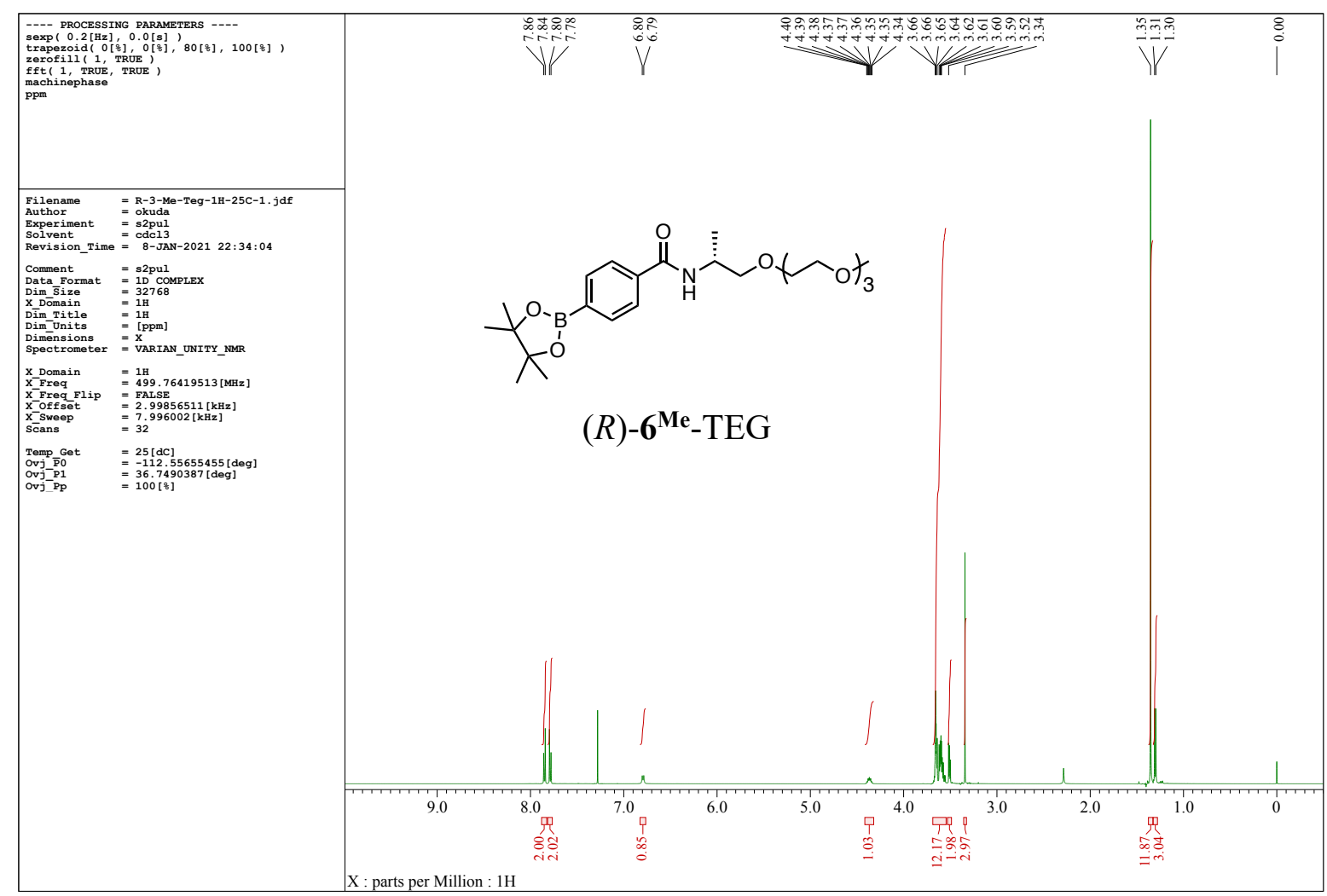

Figure S72. ${ }^{1} \mathrm{H}$ NMR spectrum of $(R)-6^{\mathrm{Me}}-\mathrm{TEG}$ in $\mathrm{CDCl}_{3}$ at $25^{\circ} \mathrm{C}$.

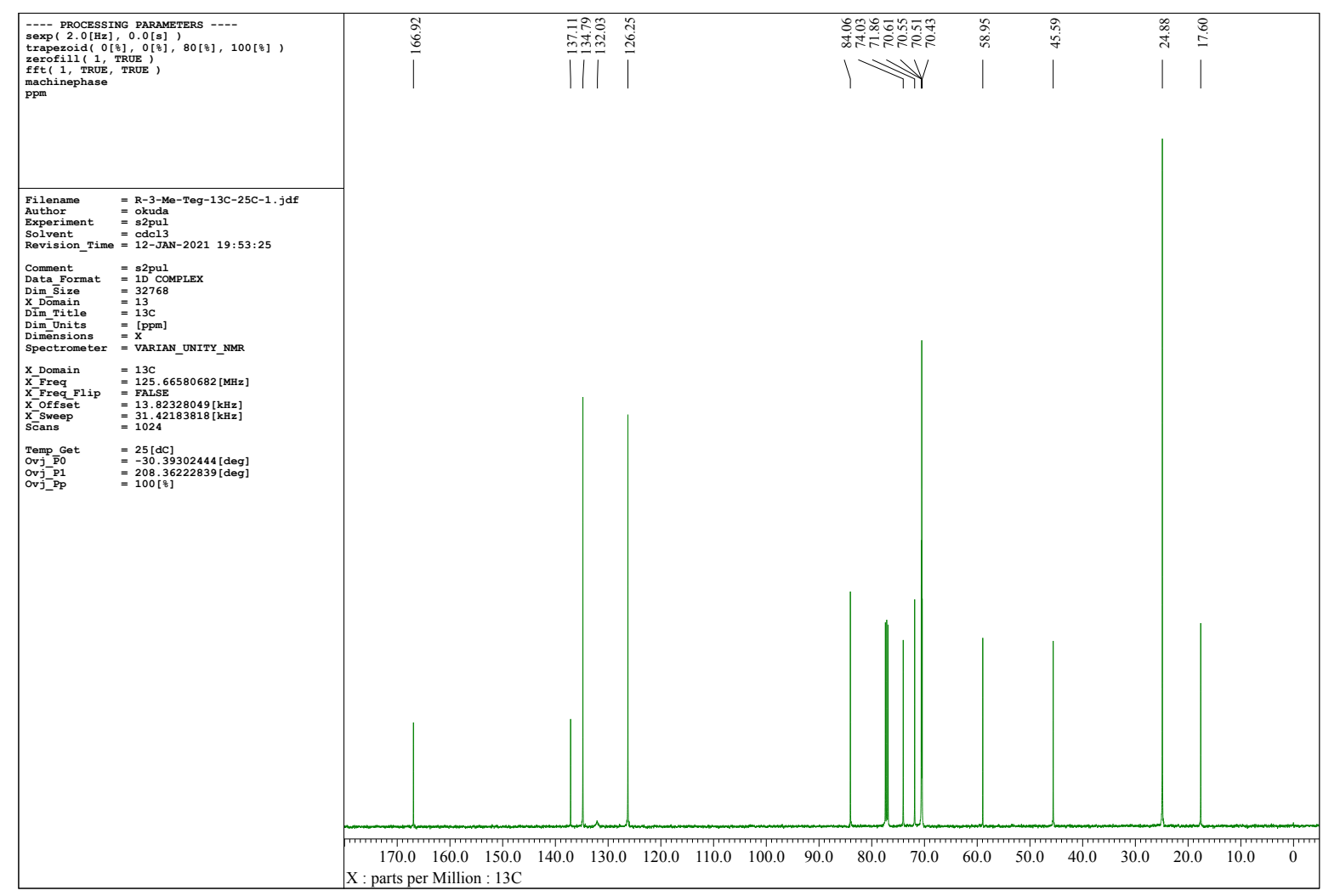

Figure S73. ${ }^{13} \mathrm{C}$ NMR spectrum of $(R)-6^{\mathrm{Me}}-\mathrm{TEG}$ in $\mathrm{CDCl}_{3}$ at $25^{\circ} \mathrm{C}$. 


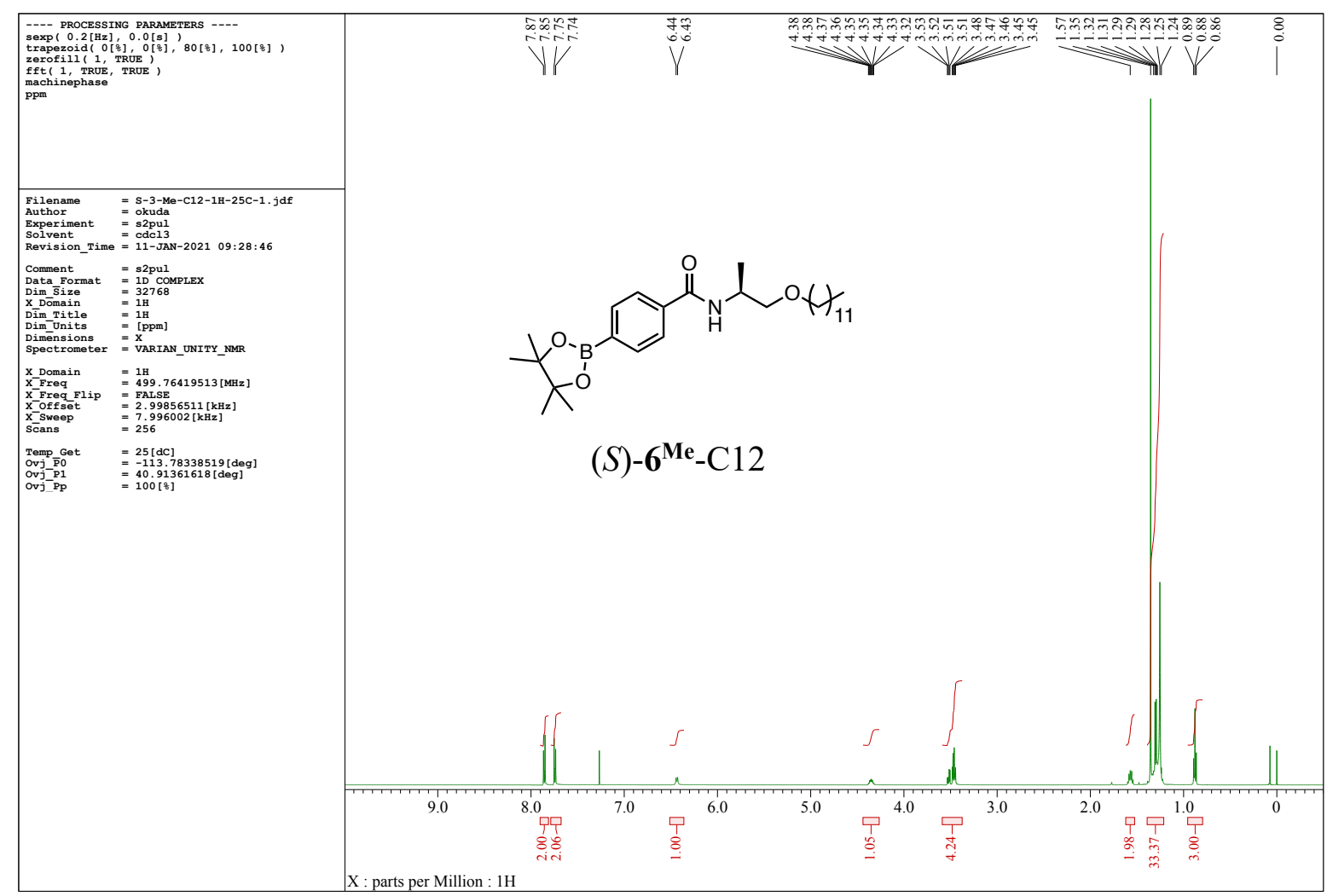

Figure S74. ${ }^{1} \mathrm{H}$ NMR spectrum of $(S)-6^{\mathrm{Me}}-\mathrm{C} 12$ in $\mathrm{CDCl}_{3}$ at $25^{\circ} \mathrm{C}$.

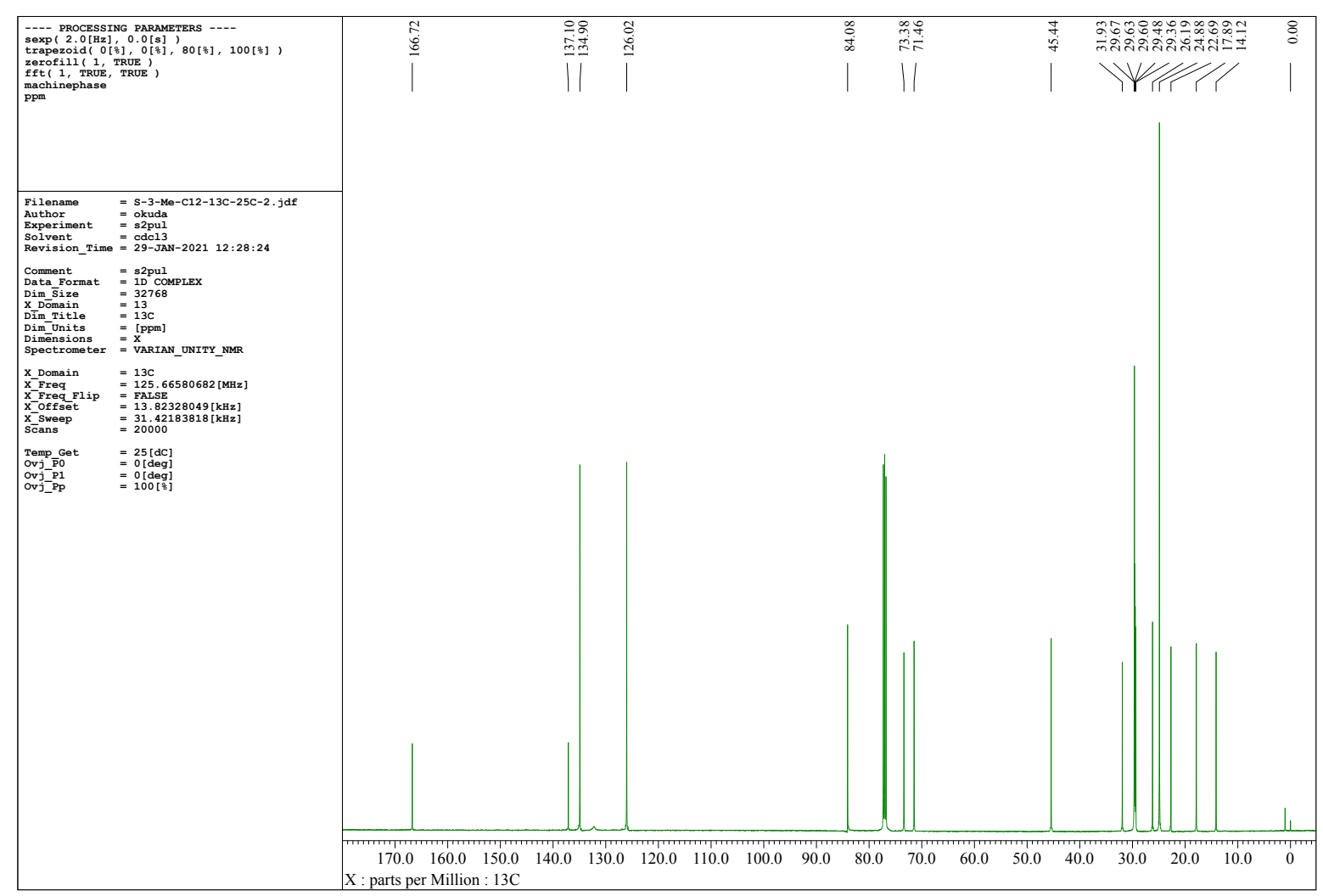

Figure S75. ${ }^{13} \mathrm{C}$ NMR spectrum of $(S)-6^{\mathrm{Me}}-\mathrm{C} 12$ in $\mathrm{CDCl}_{3}$ at $25{ }^{\circ} \mathrm{C}$. 


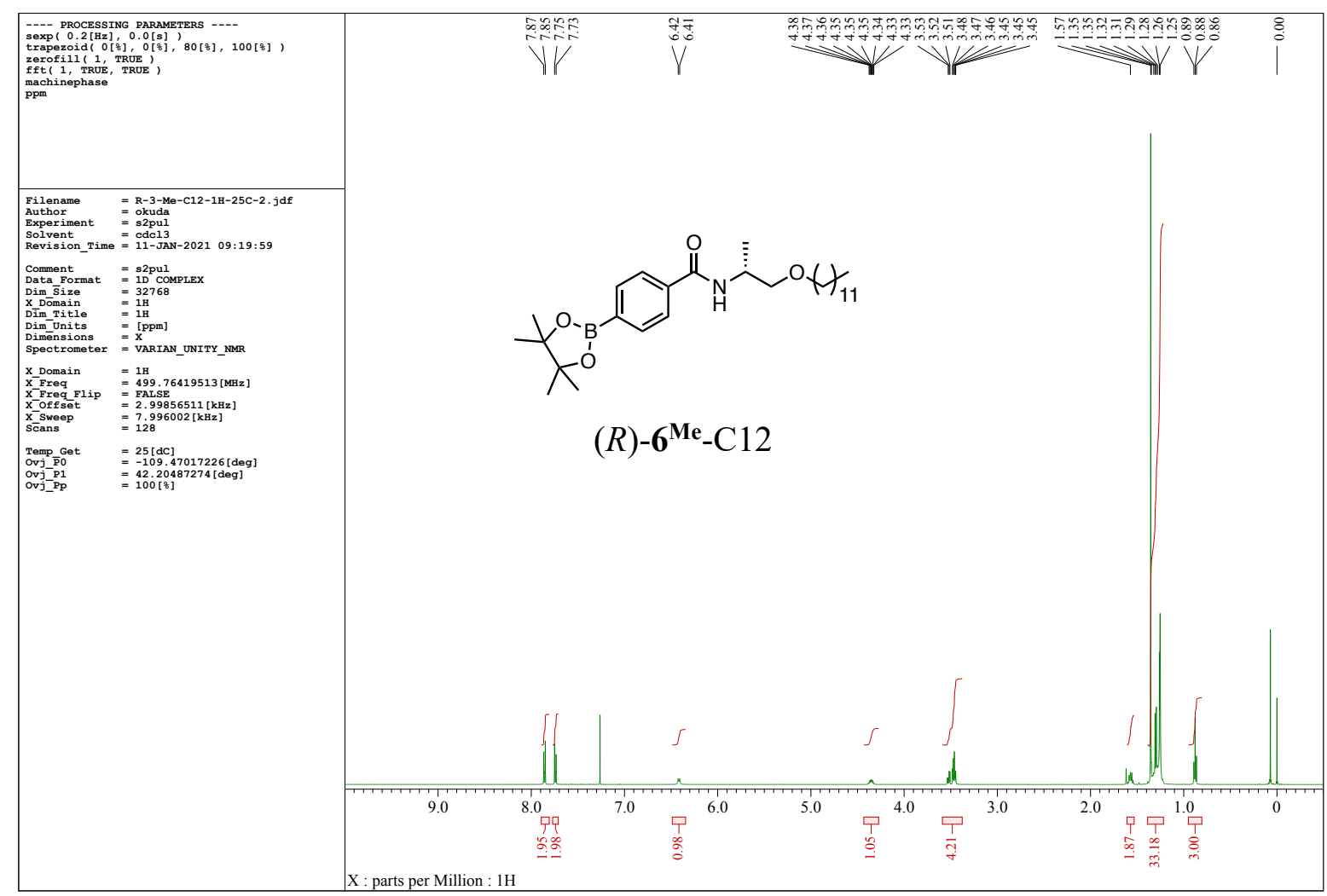

Figure S76. ${ }^{1} \mathrm{H}$ NMR spectrum of $(R)-6^{\mathrm{Me}}-\mathrm{C} 12$ in $\mathrm{CDCl}_{3}$ at $25^{\circ} \mathrm{C}$.

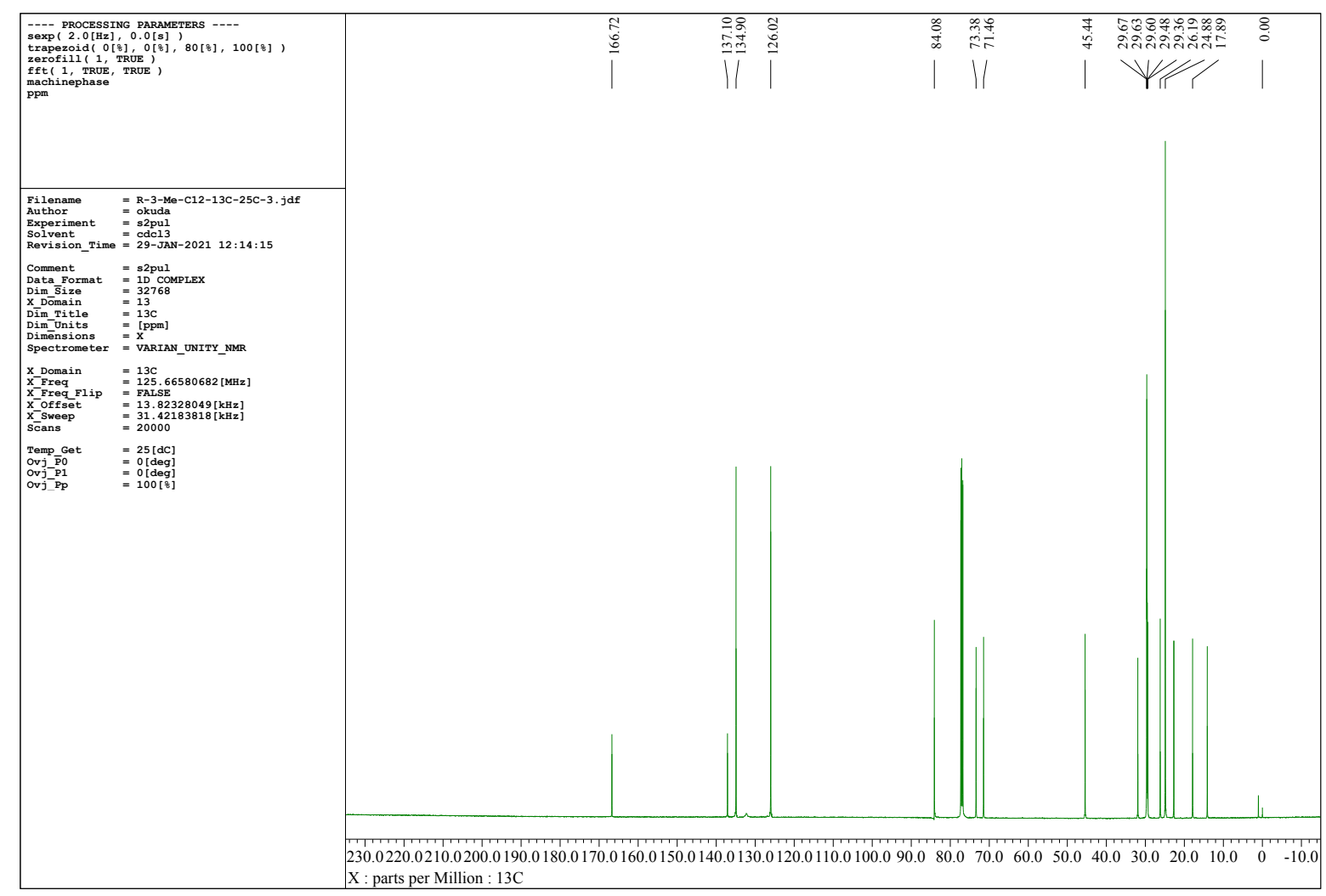

Figure S77. ${ }^{13} \mathrm{C}$ NMR spectrum of $(R)-6^{\mathrm{Me}}-\mathrm{C} 12$ in $\mathrm{CDCl}_{3}$ at $25^{\circ} \mathrm{C}$. 


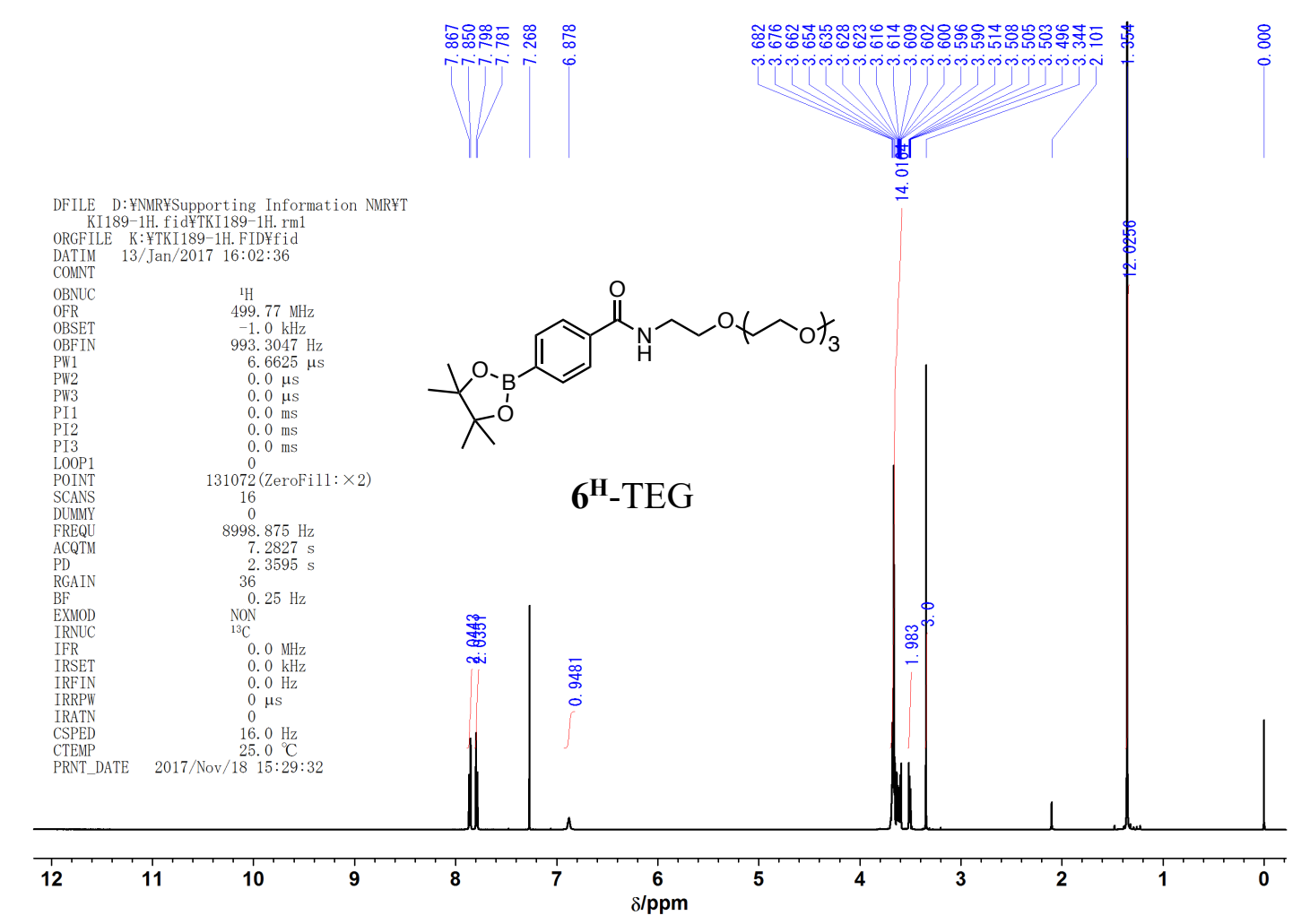

Figure S78. ${ }^{1} \mathrm{H}$ NMR spectrum of $6^{\mathrm{H}}-\mathrm{TEG}$ in $\mathrm{CDCl}_{3}$ at $25^{\circ} \mathrm{C}$.

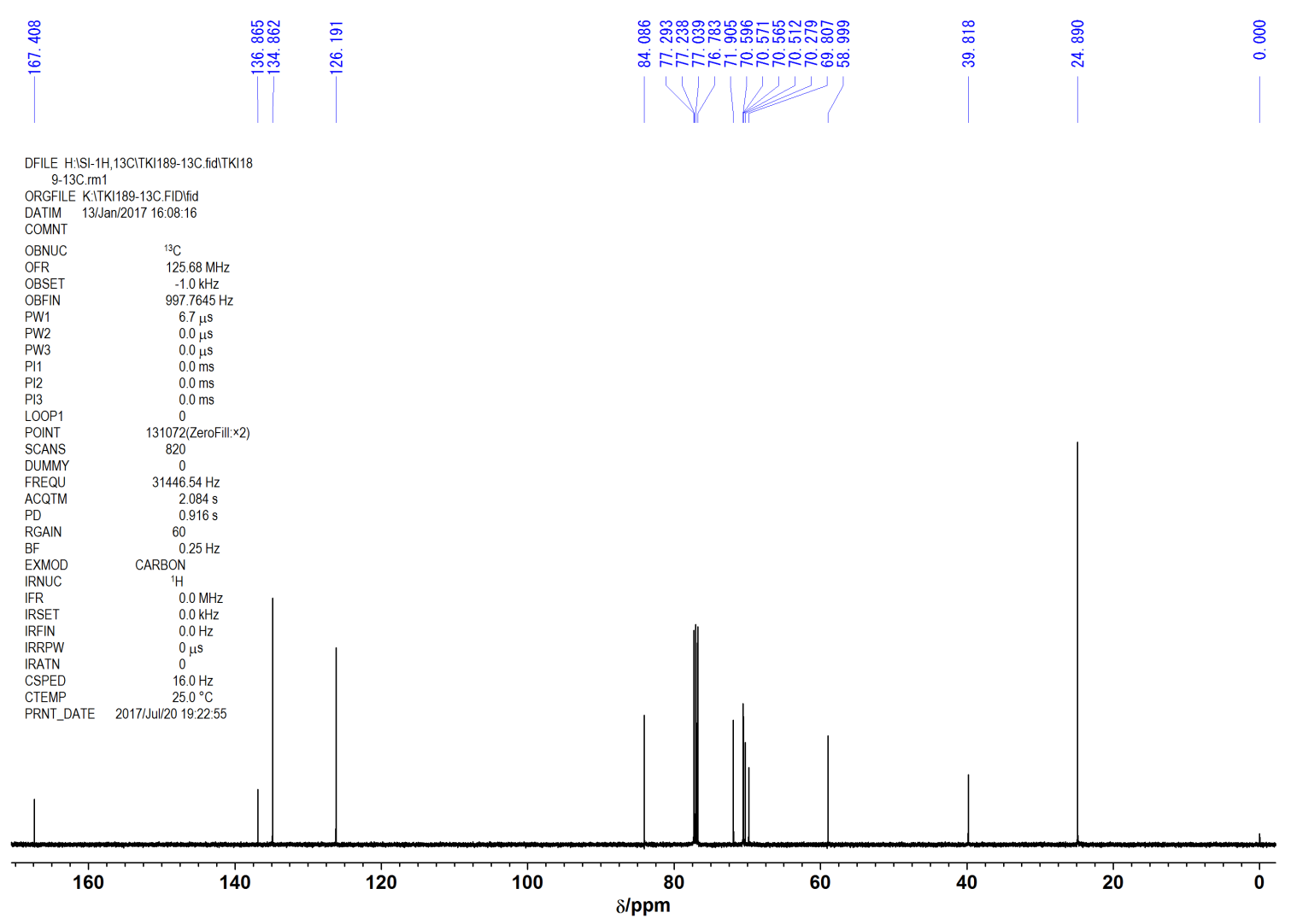

Figure S79. ${ }^{13} \mathrm{C}$ NMR spectrum of $6^{\mathrm{H}}-\mathrm{TEG}$ in $\mathrm{CDCl}_{3}$ at $25^{\circ} \mathrm{C}$. 


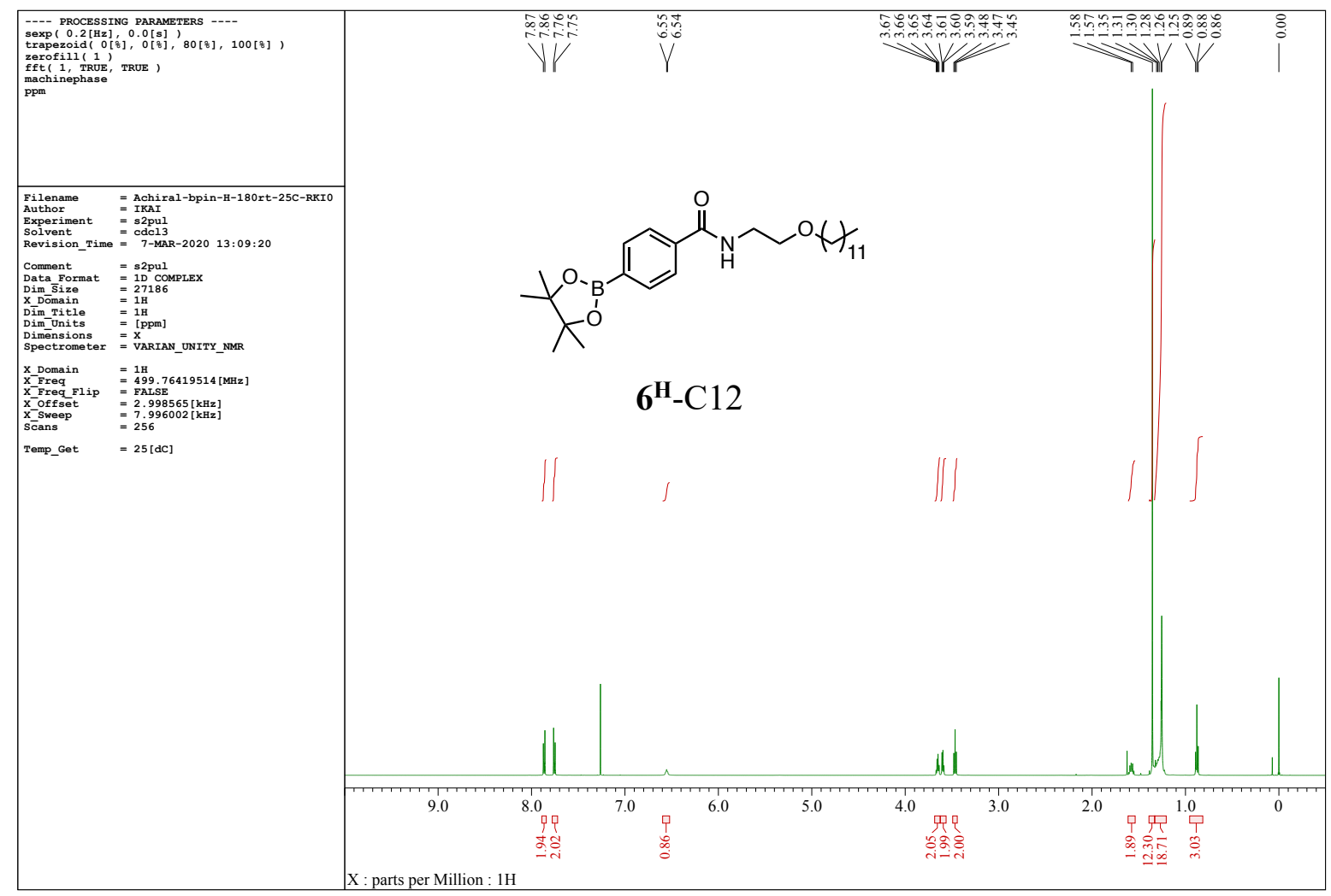

Figure S80. ${ }^{1} \mathrm{H}$ NMR spectrum of $6^{\mathbf{H}}-\mathrm{C} 12$ in $\mathrm{CDCl}_{3}$ at $25^{\circ} \mathrm{C}$.

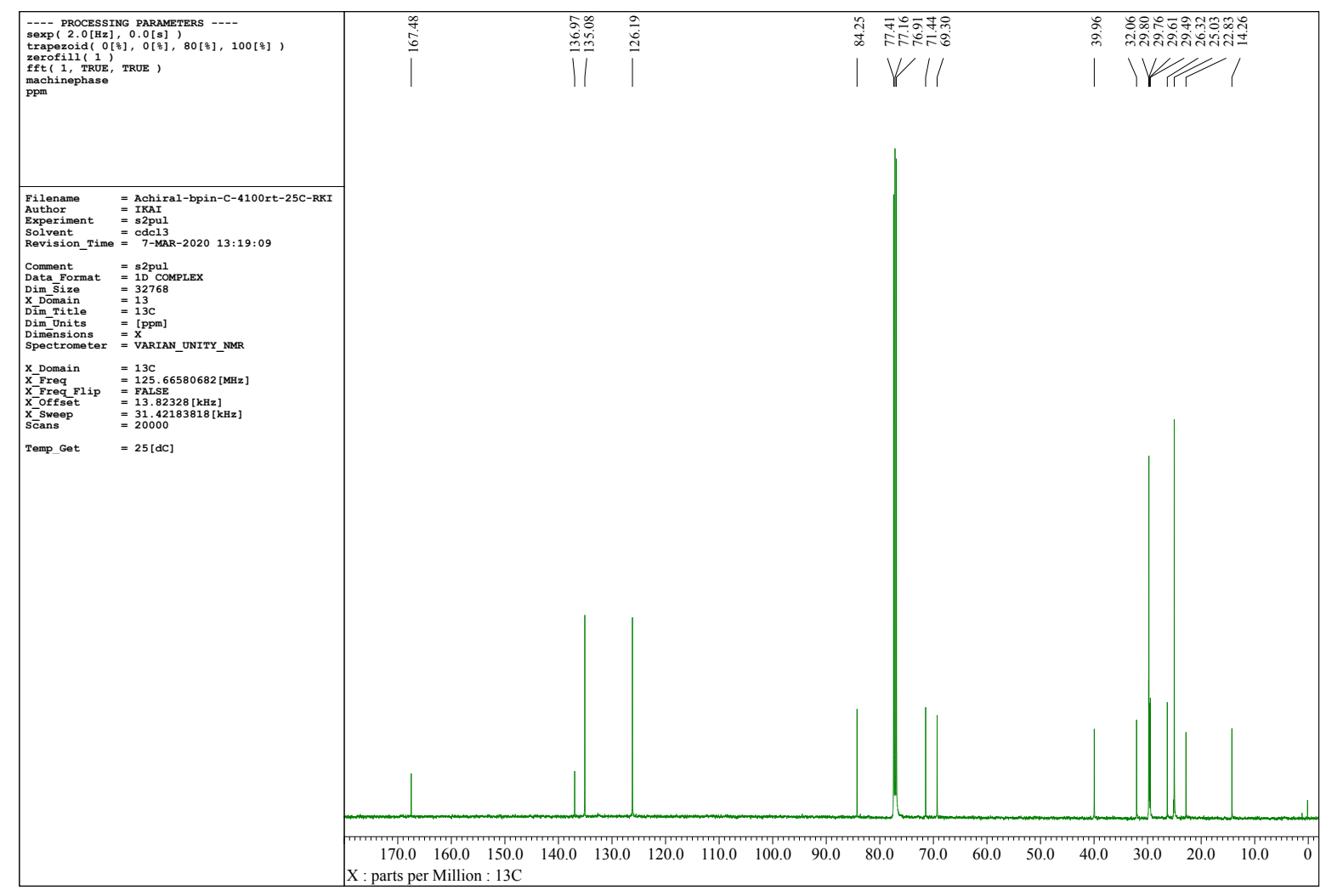

Figure S81. ${ }^{13} \mathrm{C}$ NMR spectrum of $6^{\mathbf{H}}-\mathrm{C} 12$ in $\mathrm{CDCl}_{3}$ at $25{ }^{\circ} \mathrm{C}$. 


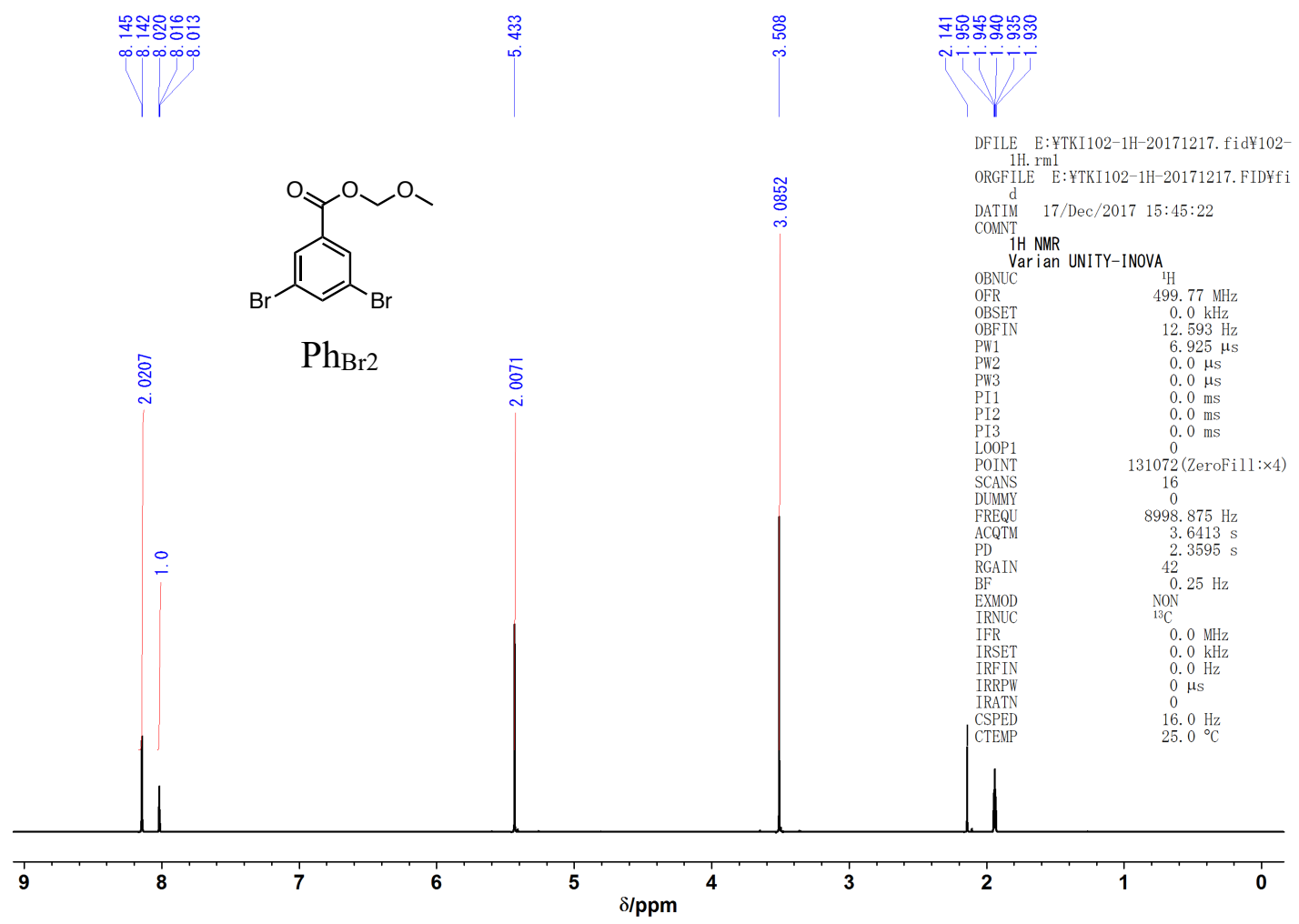

Figure S82. ${ }^{1} \mathrm{H}$ NMR spectrum of $\mathrm{Ph}_{\mathrm{Br} 2}$ in $\mathrm{CD}_{3} \mathrm{CN}$ at $25^{\circ} \mathrm{C}$.

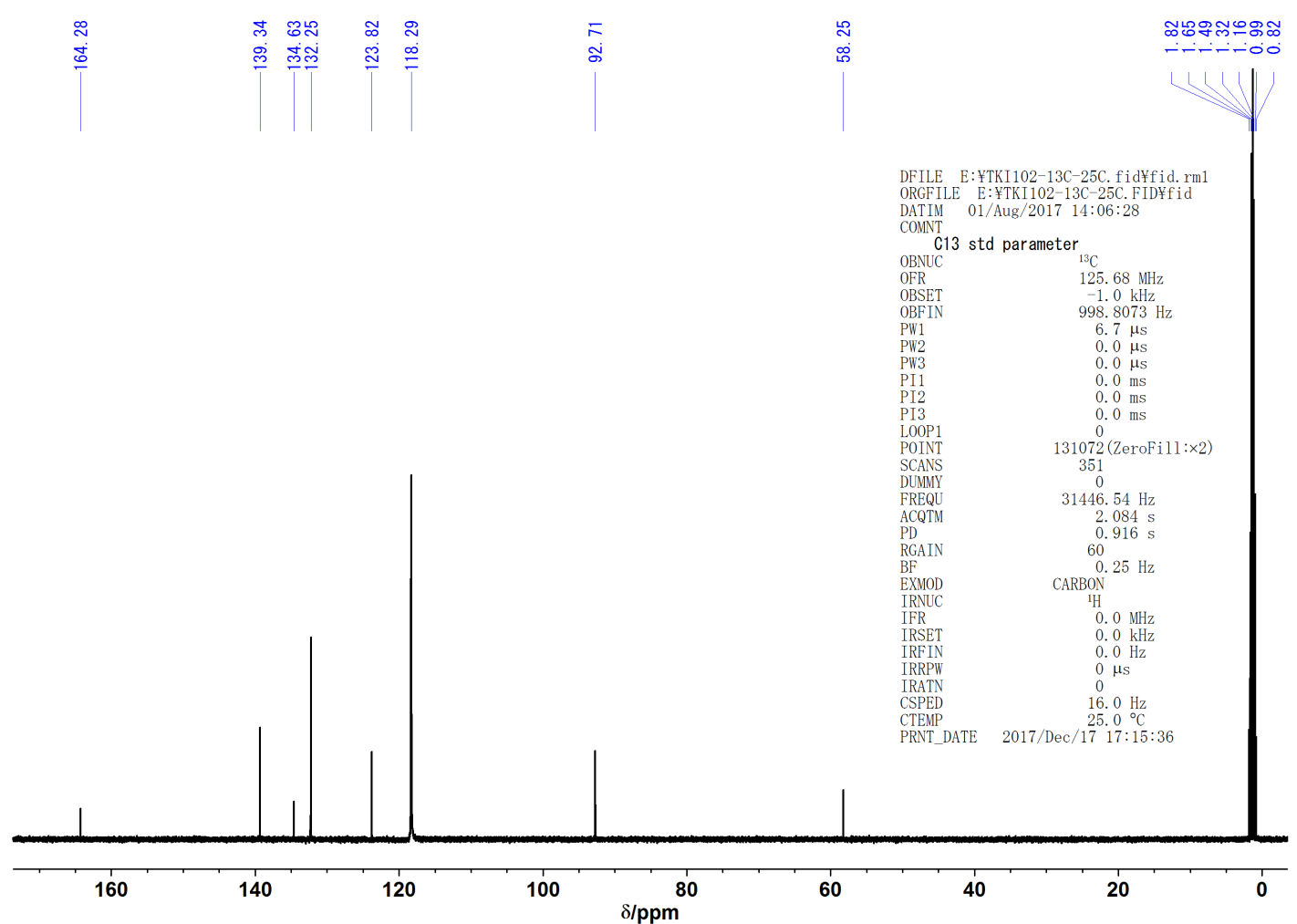

Figure S83. ${ }^{13} \mathrm{C}$ NMR spectrum of $\mathrm{Ph}_{\mathrm{Br} 2}$ in $\mathrm{CD}_{3} \mathrm{CN}$ at $25{ }^{\circ} \mathrm{C}$. 


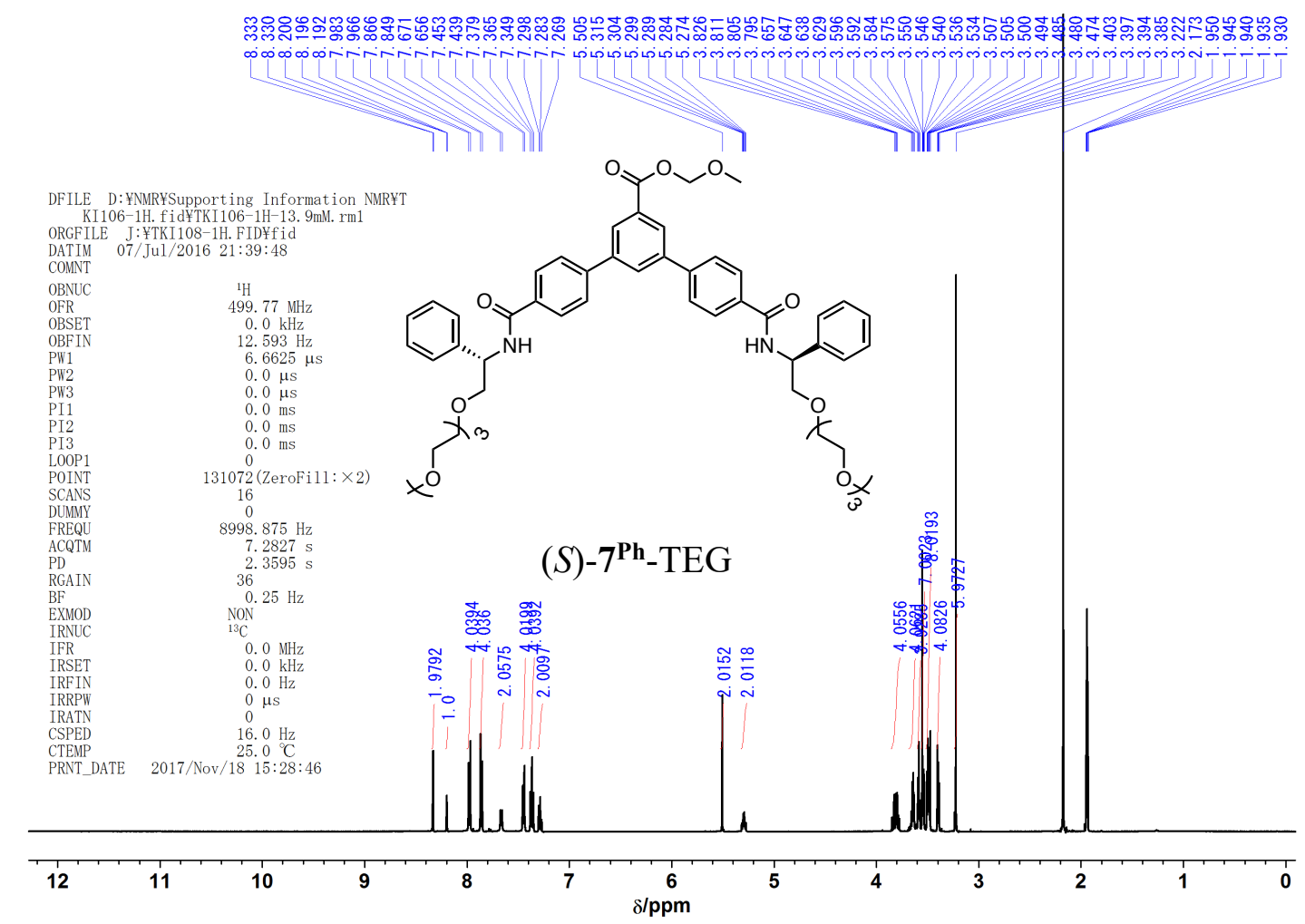

Figure S84. ${ }^{1} \mathrm{H}$ NMR spectrum of $(S)-7^{\mathbf{P h}}-\mathrm{TEG}$ in $\mathrm{CD}_{3} \mathrm{CN}$ at $25^{\circ} \mathrm{C}$.

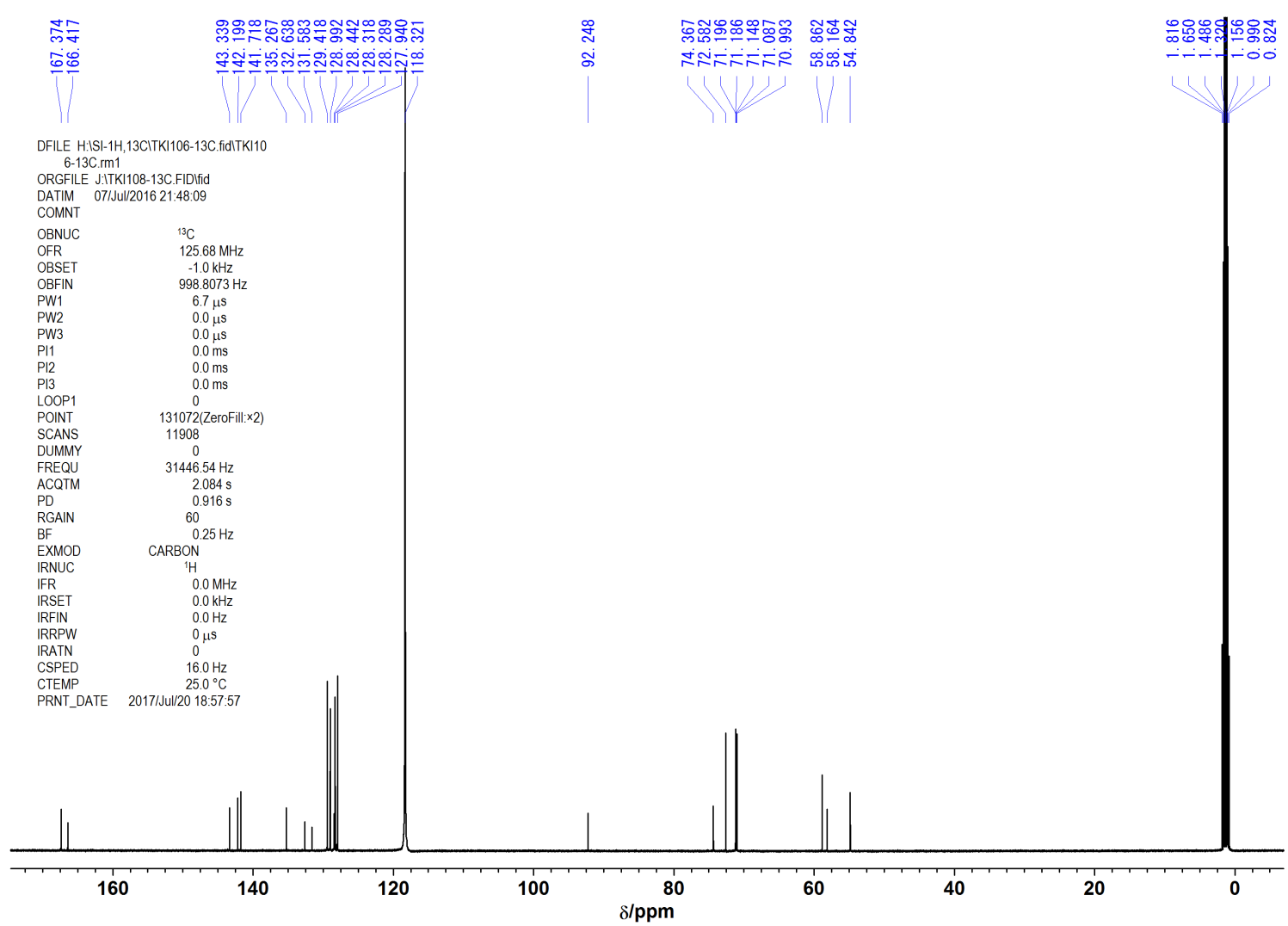

Figure S85. ${ }^{13} \mathrm{C}$ NMR spectrum of $(S)-7^{\text {Ph }}$ TEG in $\mathrm{CD}_{3} \mathrm{CN}$ at $25^{\circ} \mathrm{C}$. 


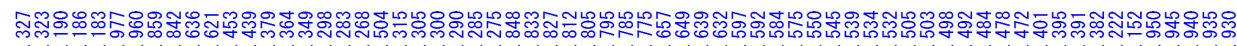

[L L

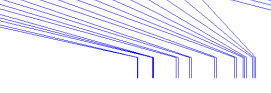

DFILE D: ¥NMR¥Supporting Information NMR¥T

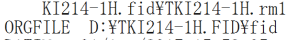

DATIM 01/Aug/2017 15:59:05

COMNT
$1 \mathrm{H}$ NMR
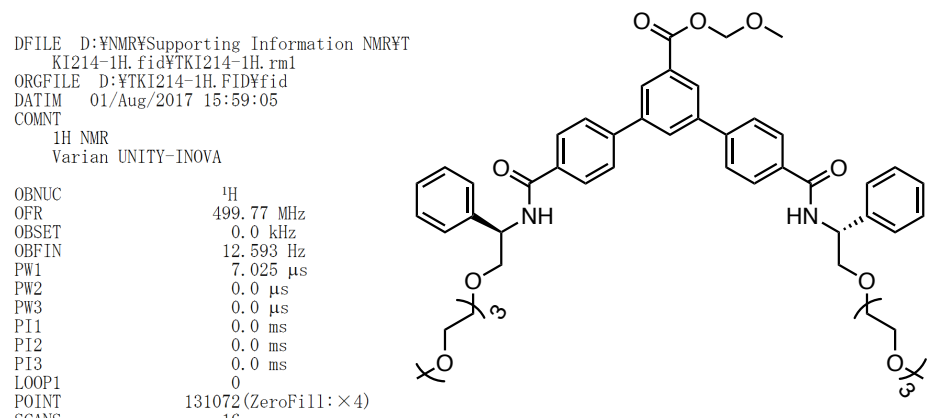

31072 (ZeroFi11: $\times 4)$

16
0

998. $875 \mathrm{~Hz}$

3. $6413 \mathrm{~s}$
2. $3595 \mathrm{~s}$

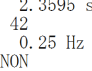

${ }_{13}^{\mathrm{NON}}$

${ }^{13} \mathrm{C} 0.0 \mathrm{MHz}$

$0.0 \mathrm{kHz}$
$0.0 \mathrm{~Hz}$

$0 \mu \mathrm{s}$

0
$16.0 \mathrm{~Hz}$
$25.0{ }^{\circ} \mathrm{C}$

$(R)-7^{\text {Ph }}$-TEG
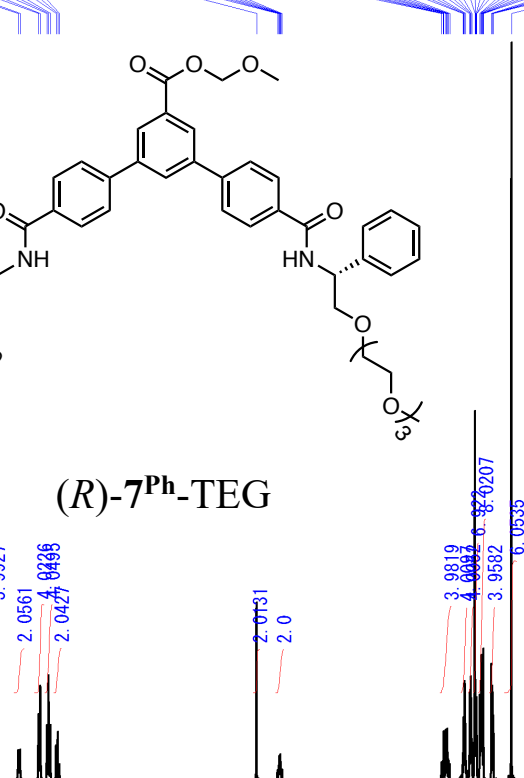

\section{.}
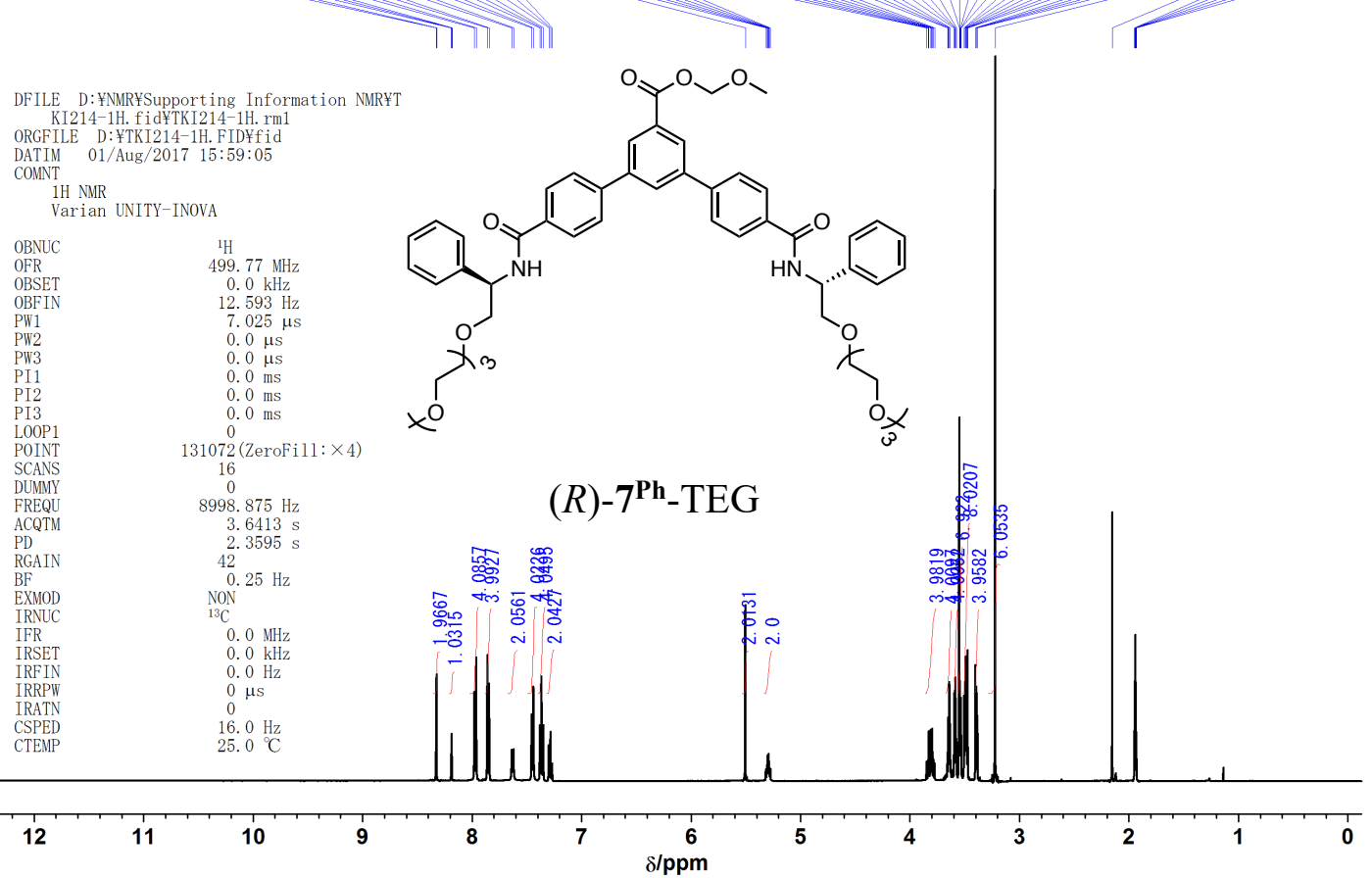

Figure S86. ${ }^{1} \mathrm{H}$ NMR spectrum of $(R)-7^{\text {Ph }}-\mathrm{TEG}$ in $\mathrm{CD}_{3} \mathrm{CN}$ at $25^{\circ} \mathrm{C}$.

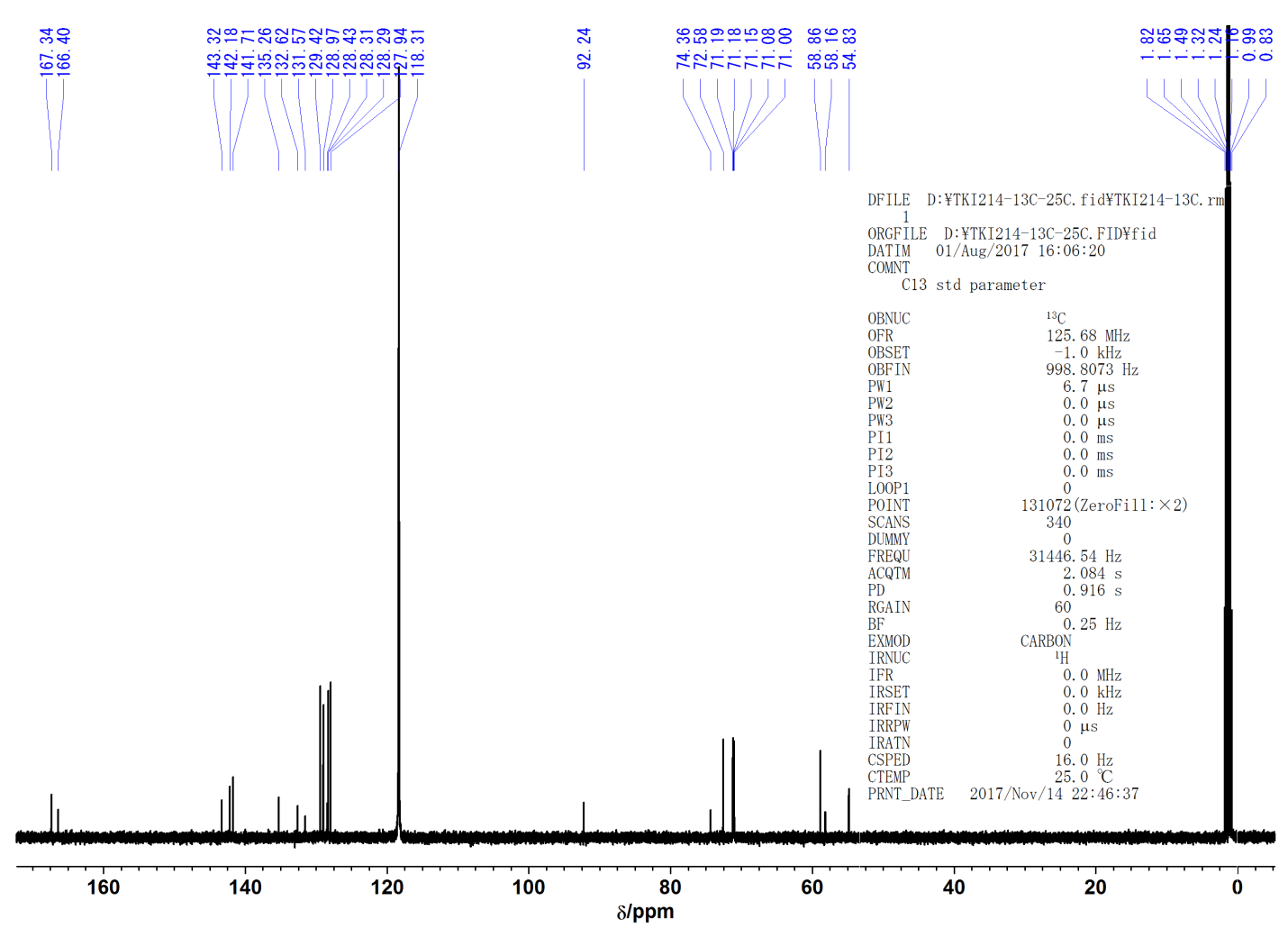

Figure S87. ${ }^{13} \mathrm{C}$ NMR spectrum of $(R)-7^{\mathrm{Ph}}-\mathrm{TEG}$ in $\mathrm{CD}_{3} \mathrm{CN}$ at $25{ }^{\circ} \mathrm{C}$. 


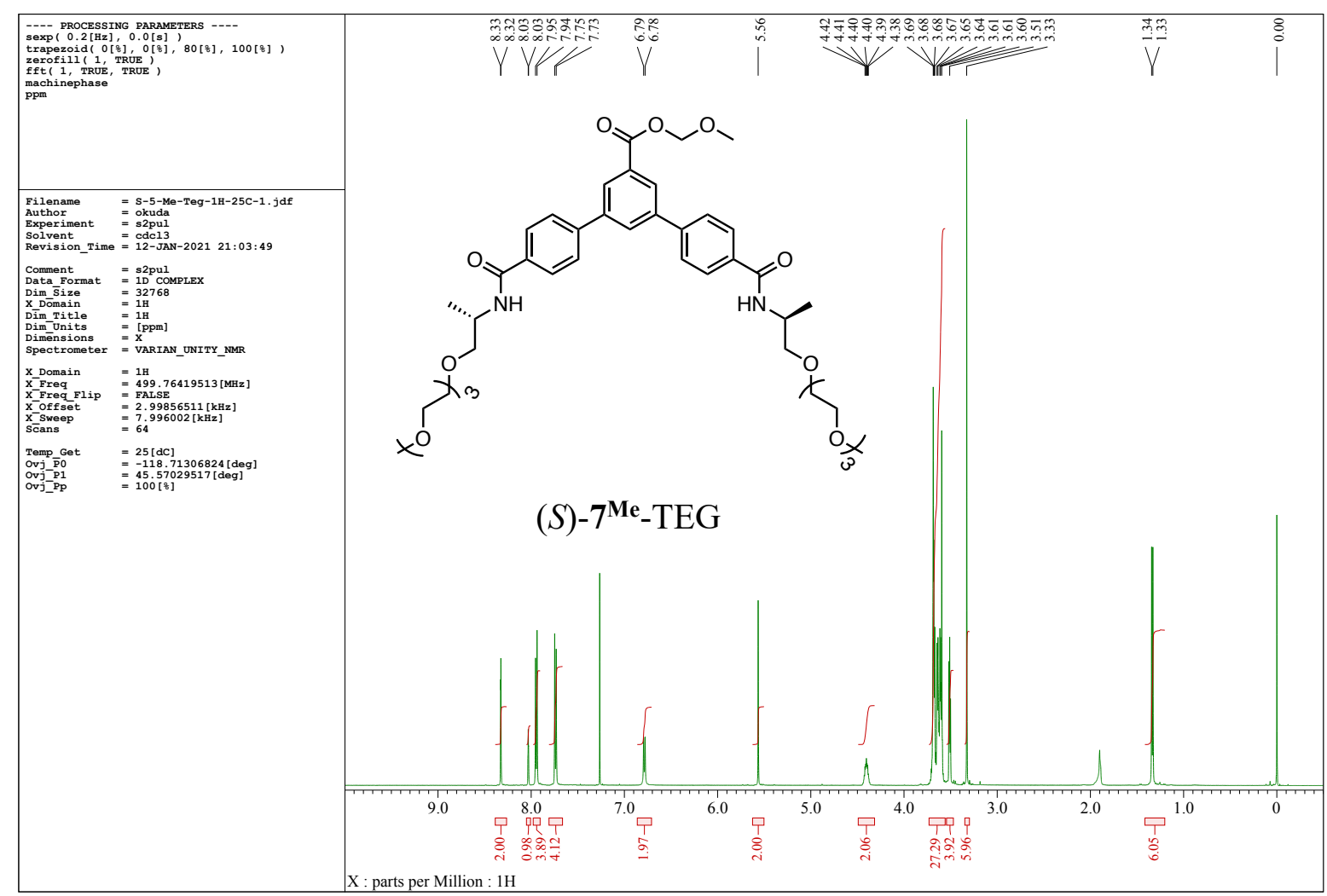

Figure S88. ${ }^{1} \mathrm{H}$ NMR spectrum of $(S)-7^{\mathrm{Me}}-\mathrm{TEG}$ in $\mathrm{CDCl}_{3}$ at $25^{\circ} \mathrm{C}$.

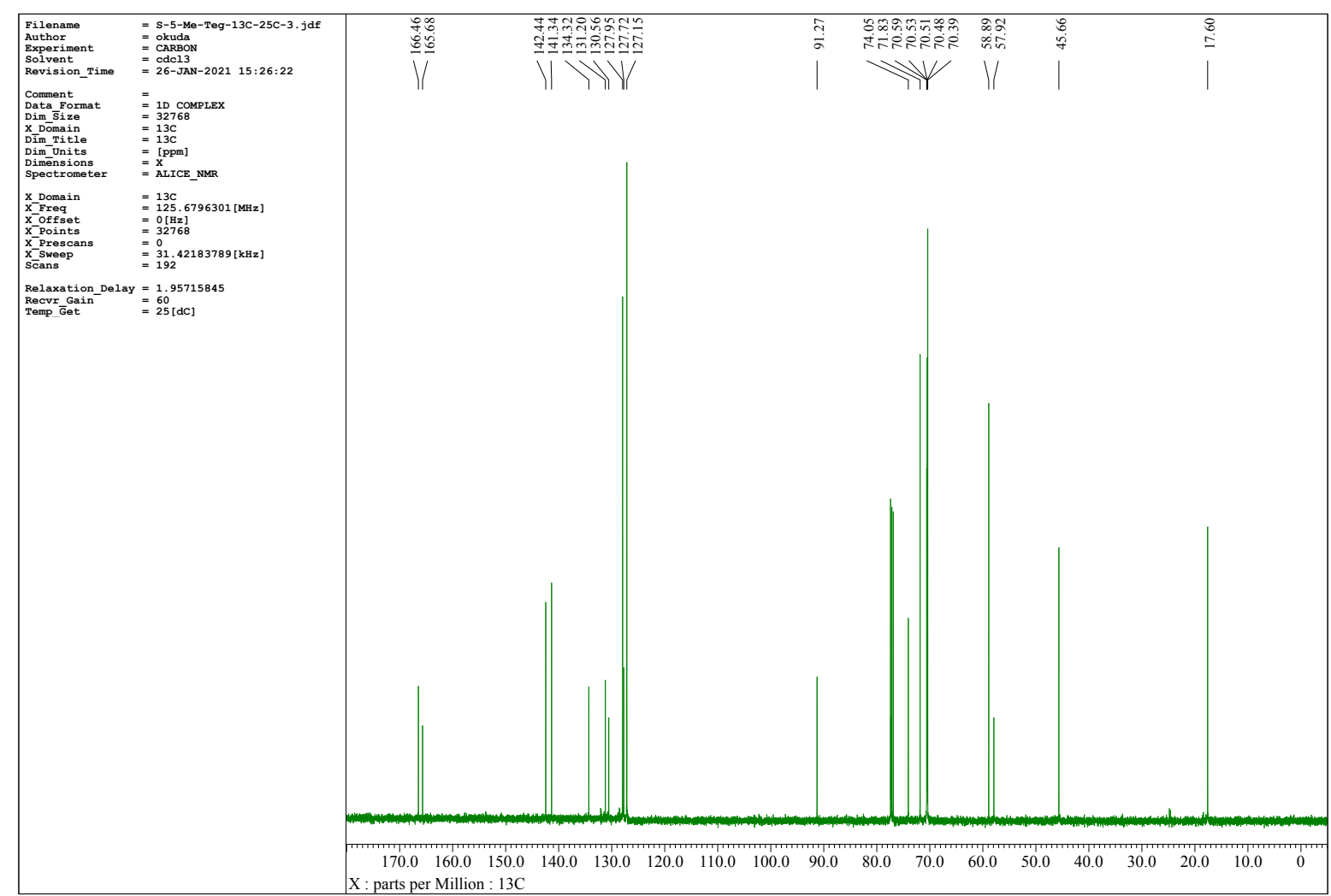

Figure S89. ${ }^{13} \mathrm{C}$ NMR spectrum of $(S)-7^{\mathrm{Me}}-\mathrm{TEG}$ in $\mathrm{CDCl}_{3}$ at $25^{\circ} \mathrm{C}$. 


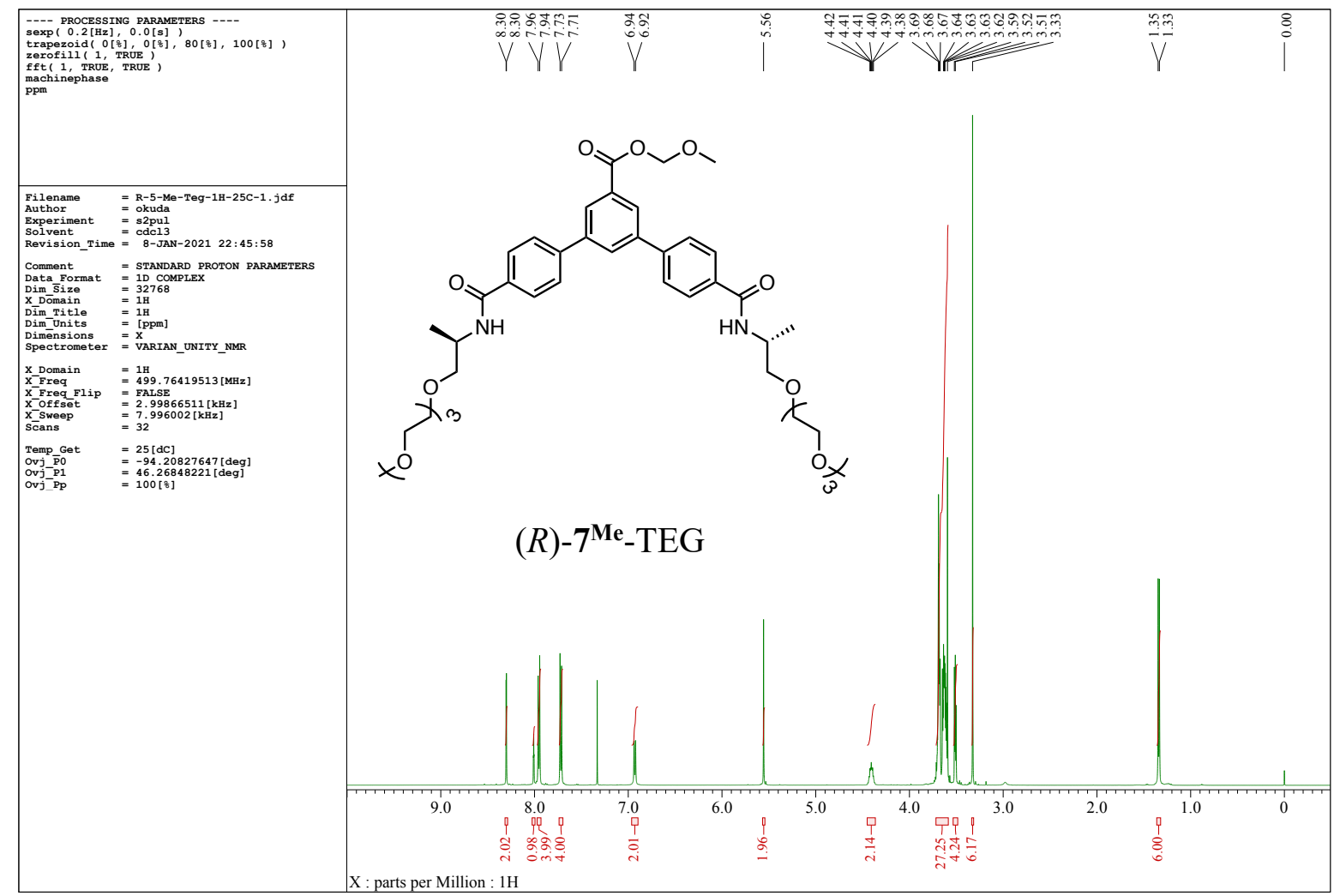

Figure S90. ${ }^{1} \mathrm{H}$ NMR spectrum of $(R)-7^{\mathrm{Me}}-\mathrm{TEG}$ in $\mathrm{CDCl}_{3}$ at $25^{\circ} \mathrm{C}$.

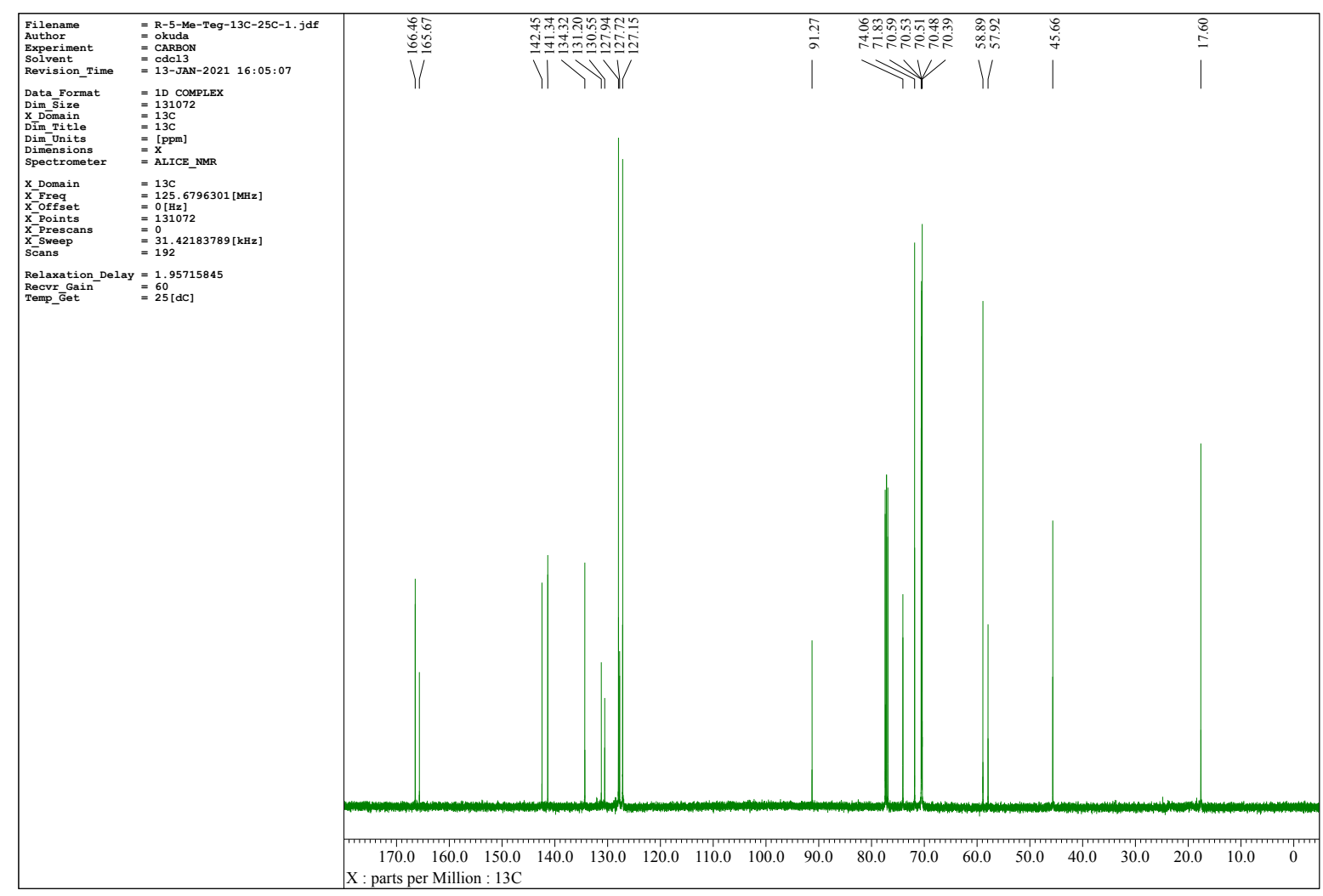

Figure S91. ${ }^{13} \mathrm{C}$ NMR spectrum of $(R)-7^{\text {Me }}-\mathrm{TEG}$ in $\mathrm{CDCl}_{3}$ at $25^{\circ} \mathrm{C}$. 


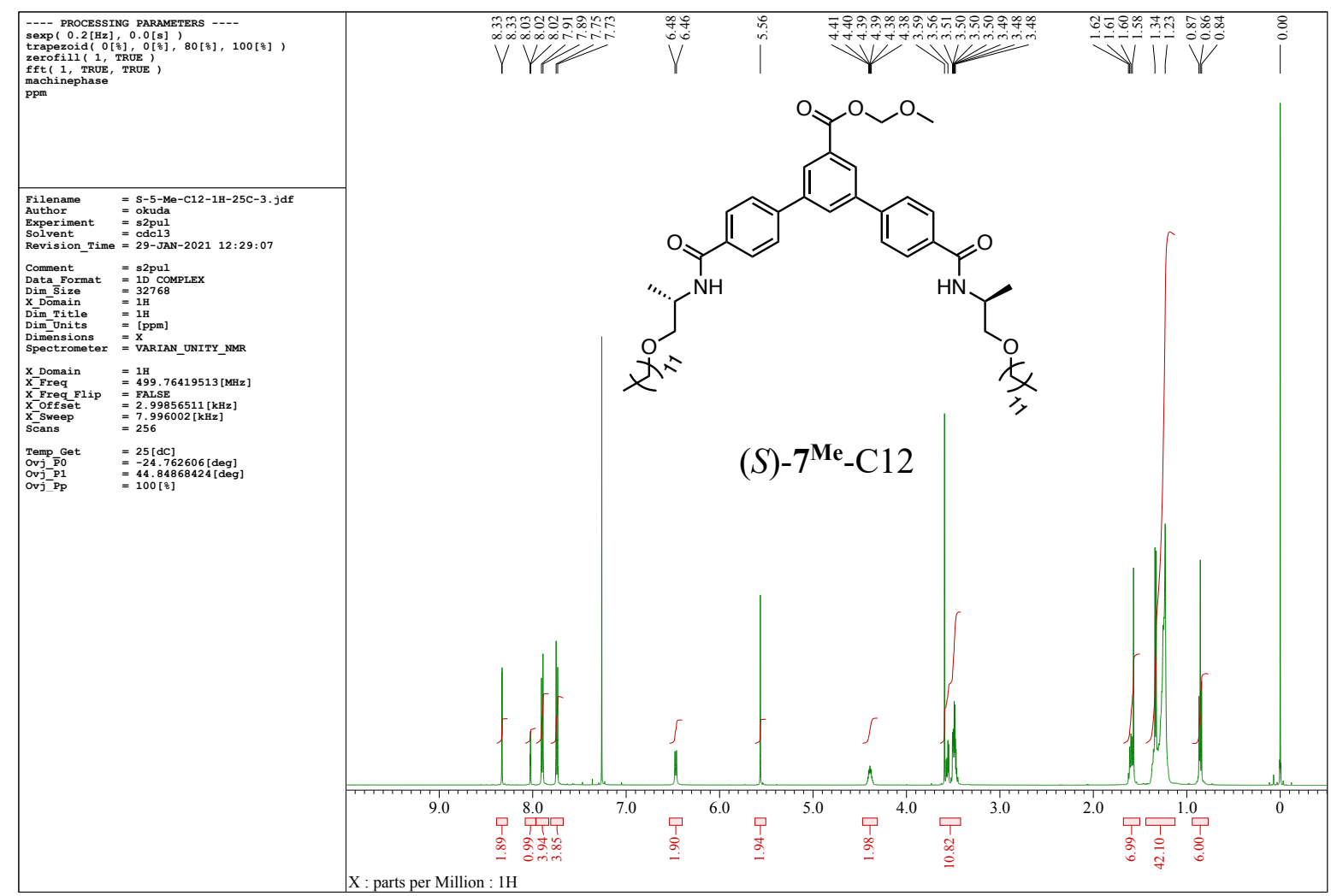

Figure S92. ${ }^{1} \mathrm{H}$ NMR spectrum of $(S)-7^{\mathrm{Me}}-\mathrm{C} 12$ in $\mathrm{CDCl}_{3}$ at $25^{\circ} \mathrm{C}$.

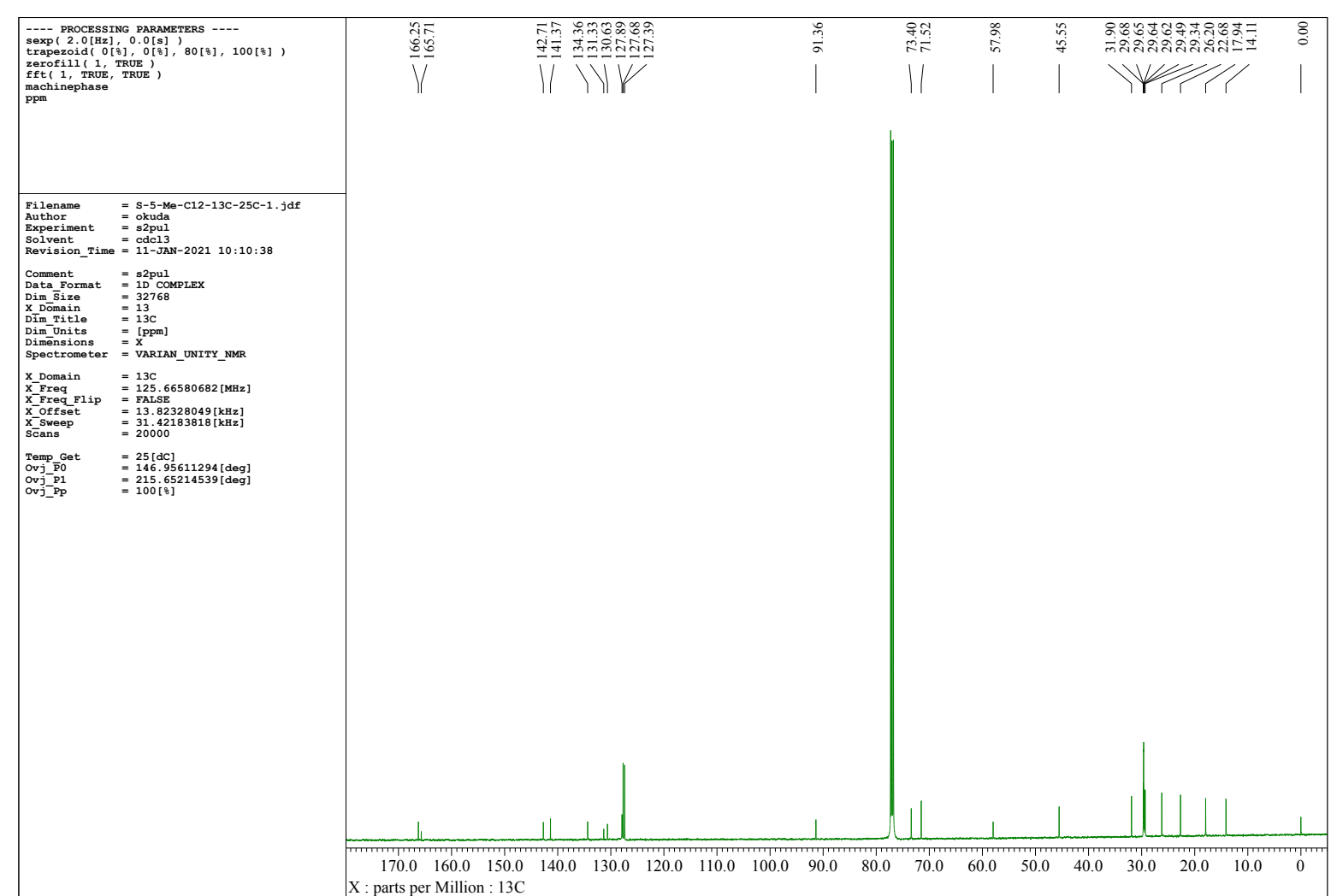

Figure S93. ${ }^{13} \mathrm{C}$ NMR spectrum of $(S)-7^{\mathrm{Me}}-\mathrm{C} 12$ in $\mathrm{CDCl}_{3}$ at $25^{\circ} \mathrm{C}$. 


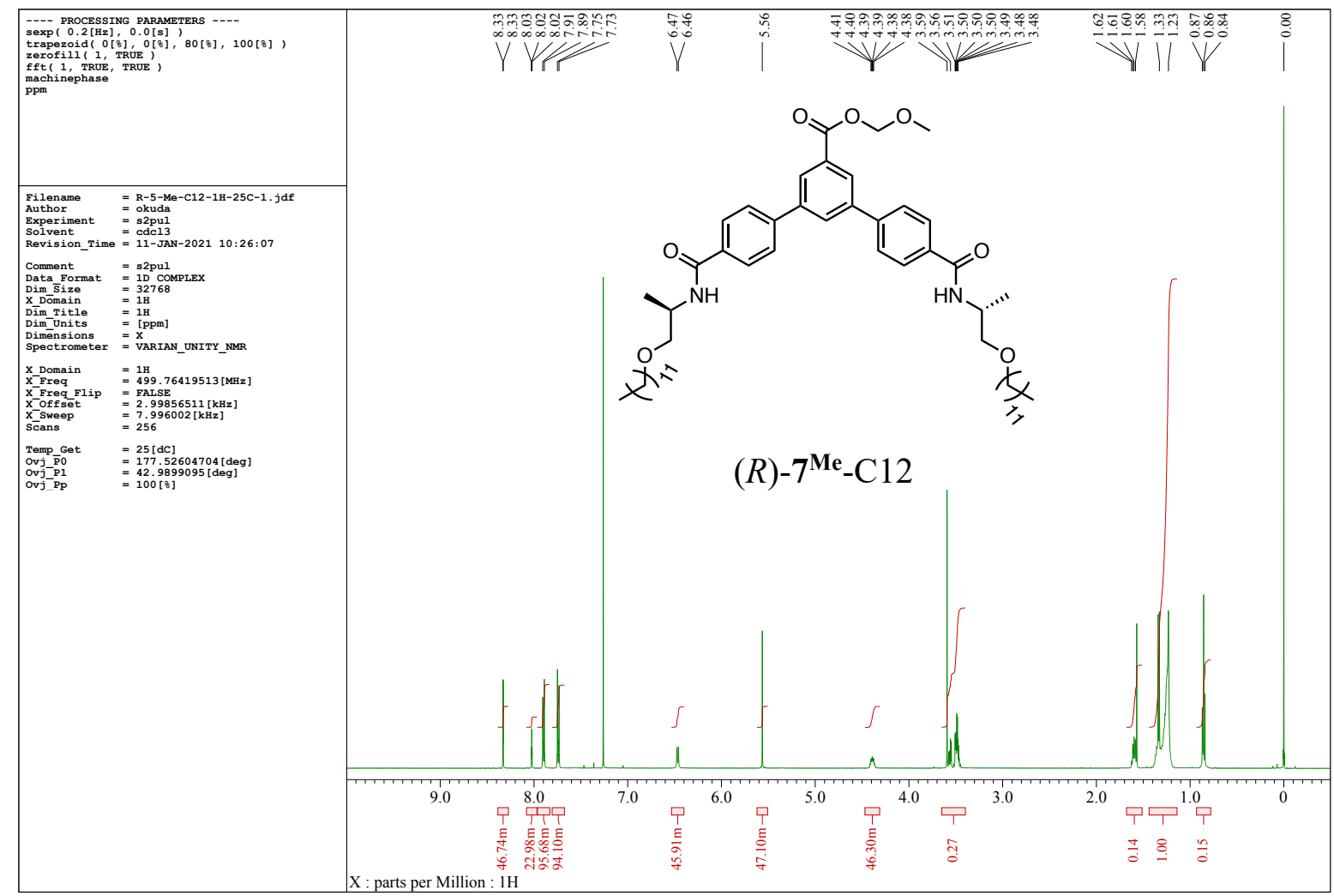

Figure S94. ${ }^{1} \mathrm{H}$ NMR spectrum of $(R)-7^{\mathrm{Me}}-\mathrm{C} 12$ in $\mathrm{CDCl}_{3}$ at $25^{\circ} \mathrm{C}$.

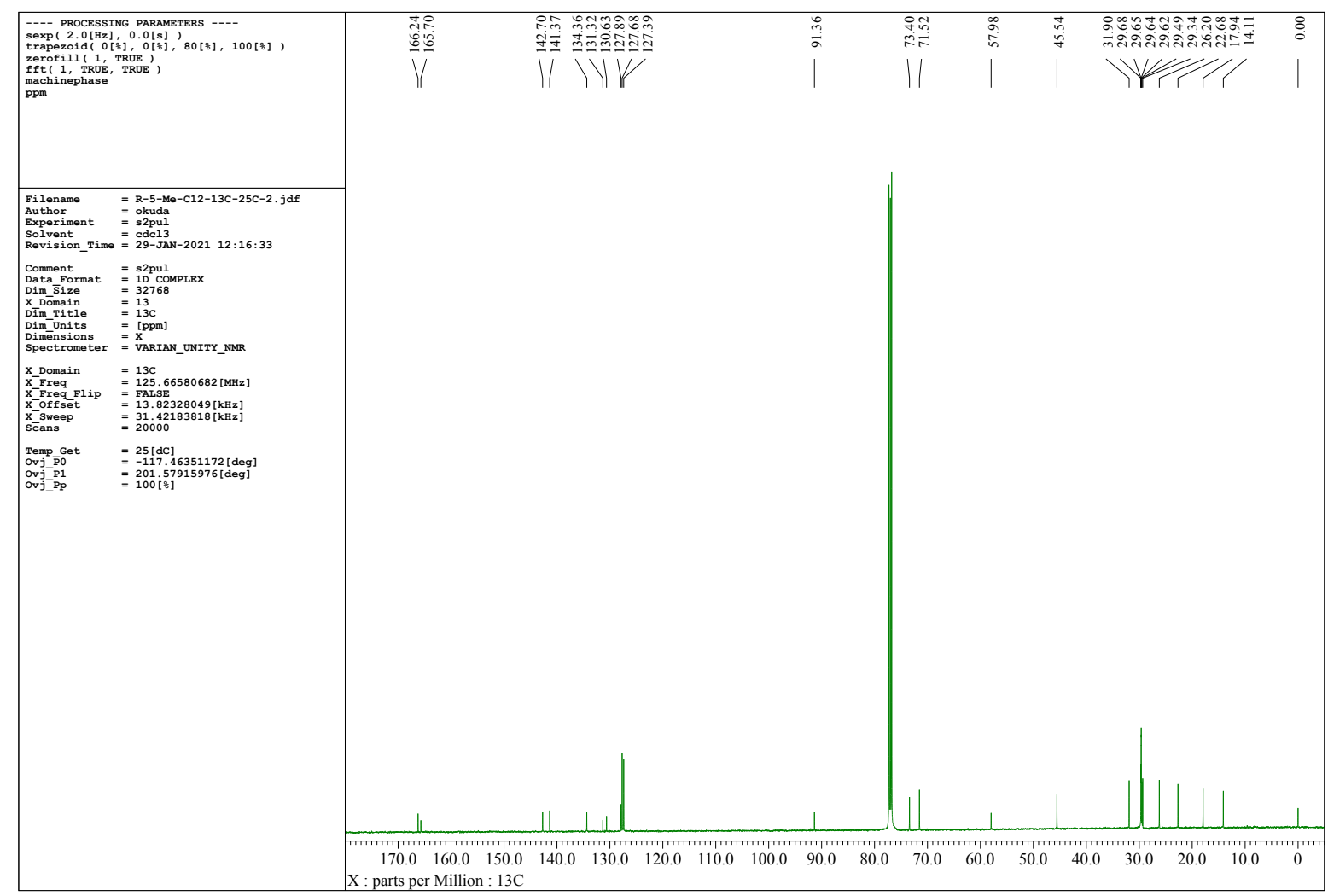

Figure S95. ${ }^{13} \mathrm{C}$ NMR spectrum of $(R)-7^{\mathrm{Me}}-\mathrm{C} 12$ in $\mathrm{CDCl}_{3}$ at $25^{\circ} \mathrm{C}$. 


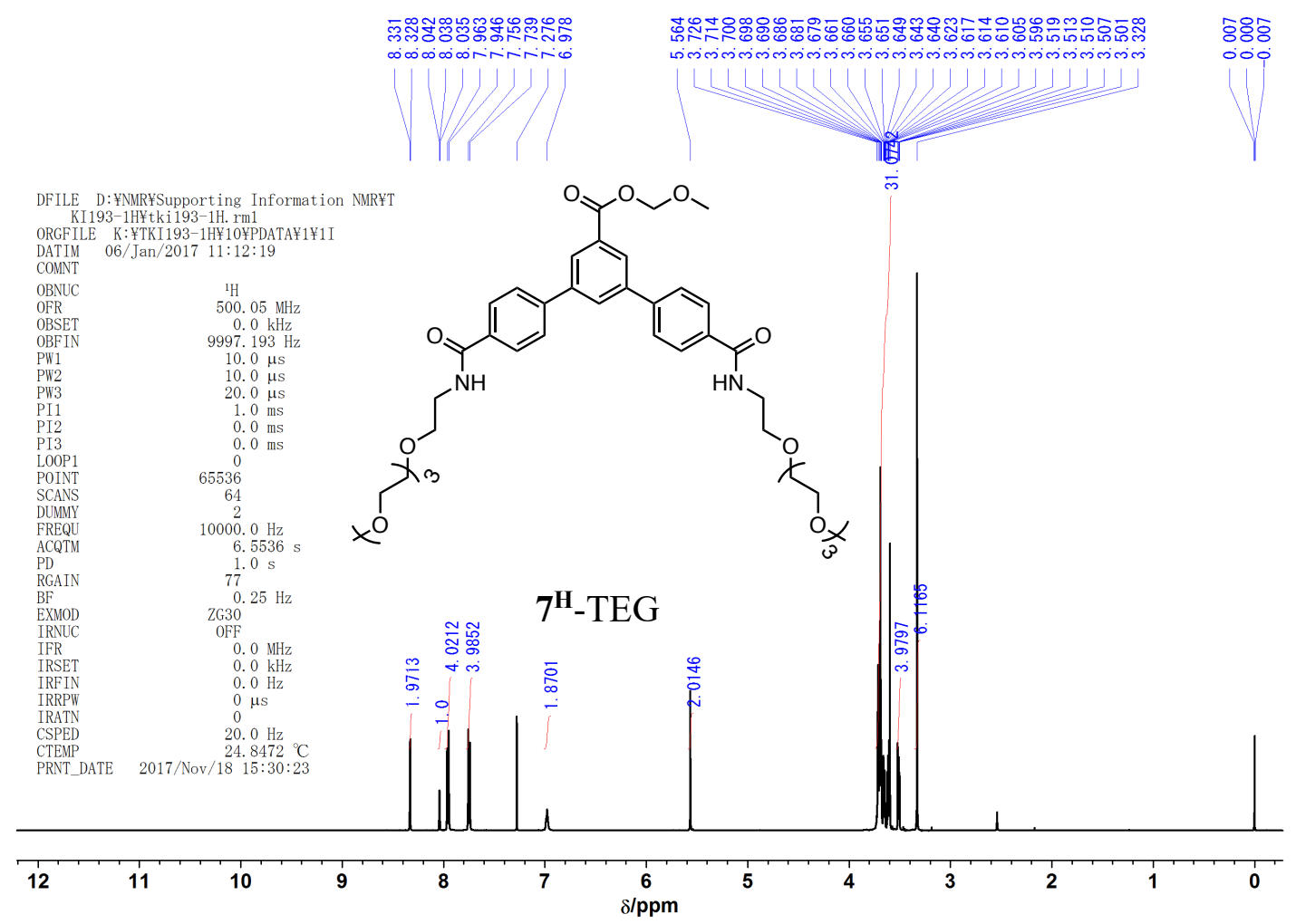

Figure S96. ${ }^{1} \mathrm{H}$ NMR spectrum of $7^{\mathrm{H}}-\mathrm{TEG}$ in $\mathrm{CDCl}_{3}$ at $25^{\circ} \mathrm{C}$.

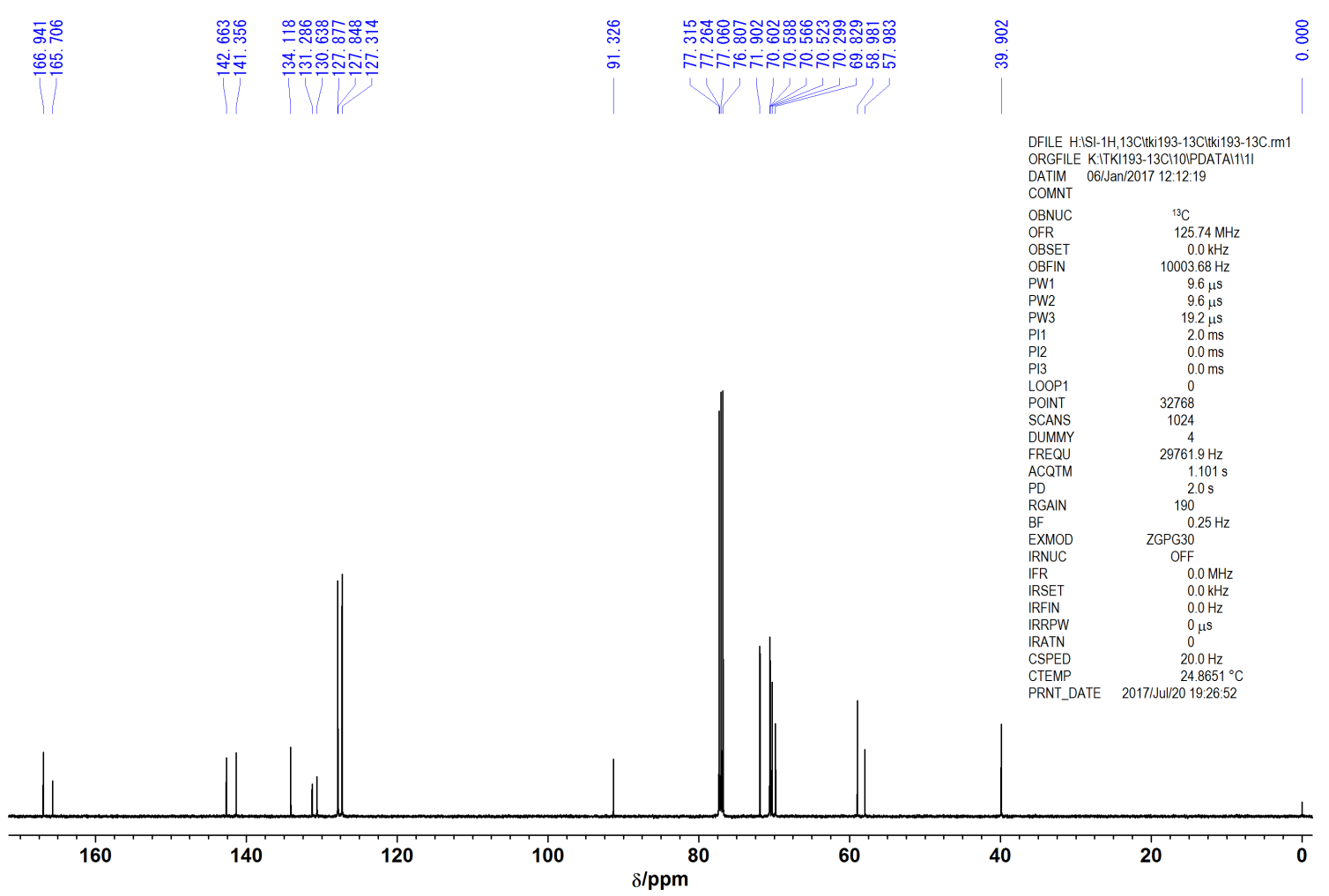

Figure S97. ${ }^{13} \mathrm{C}$ NMR spectrum of $7^{\mathrm{H}}-\mathrm{TEG}$ in $\mathrm{CDCl}_{3}$ at $25^{\circ} \mathrm{C}$. 


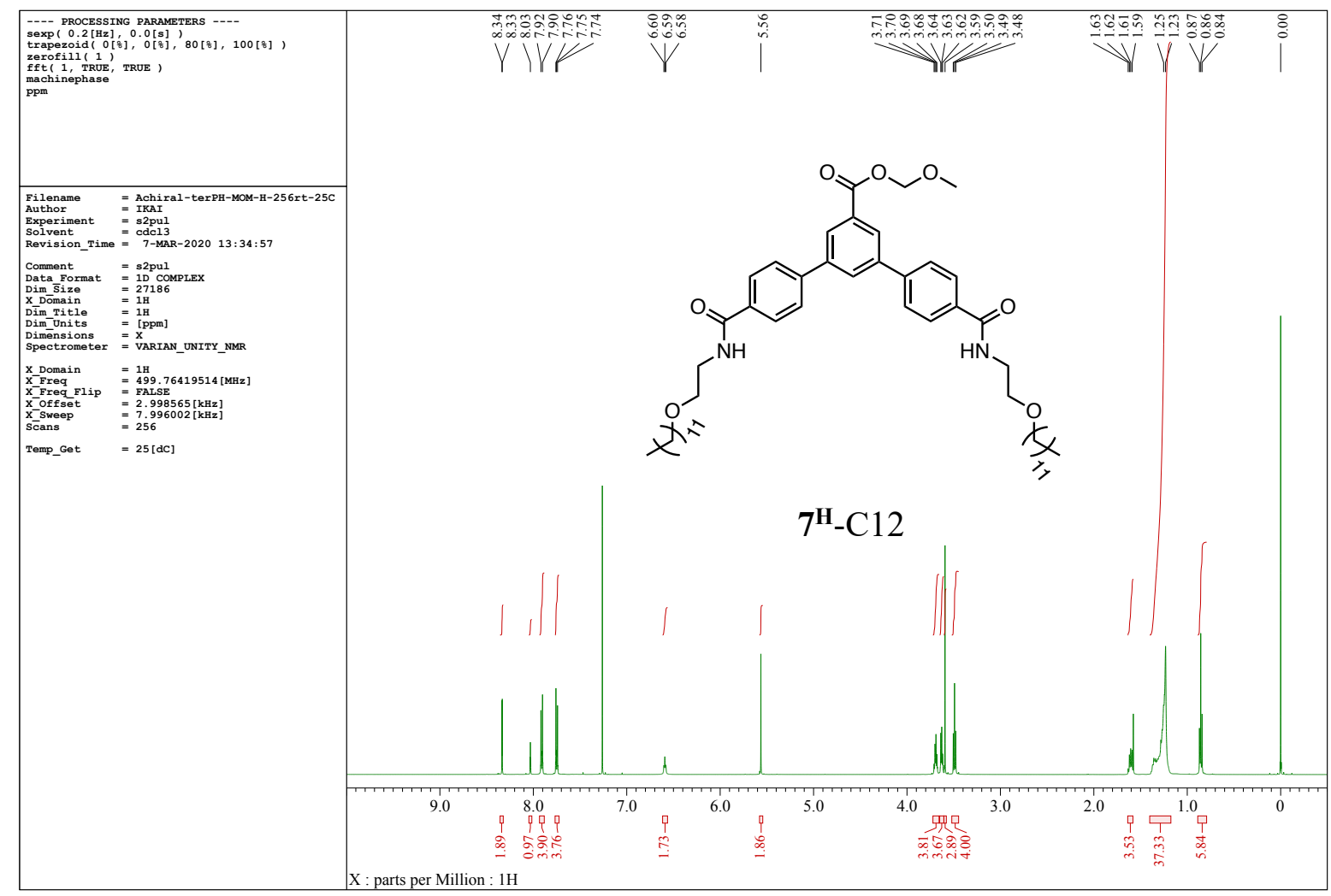

Figure S98. ${ }^{1} \mathrm{H}$ NMR spectrum of $7^{\mathrm{H}_{-}} \mathrm{C} 12$ in $\mathrm{CDCl}_{3}$ at $25{ }^{\circ} \mathrm{C}$.

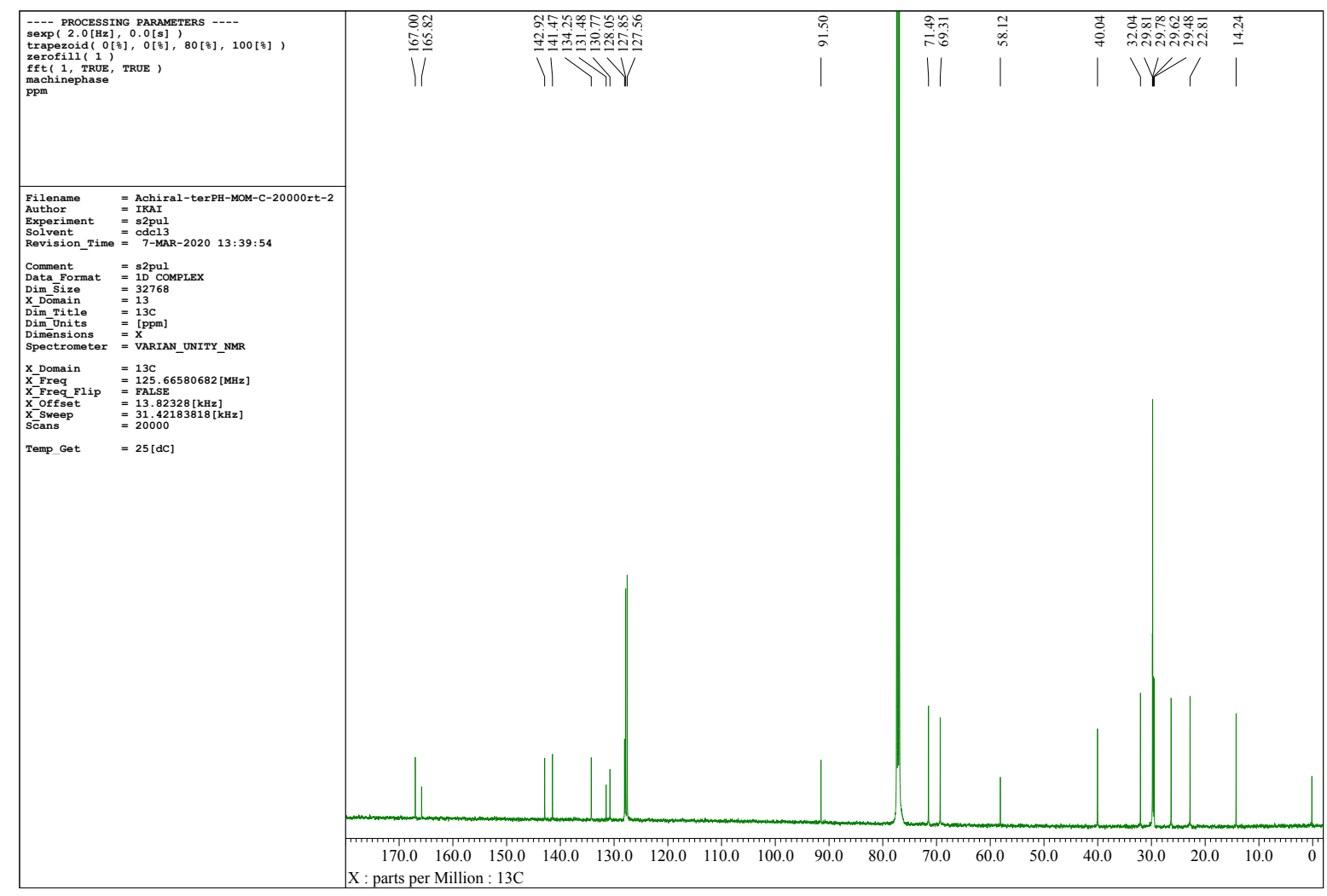

Figure S99. ${ }^{13} \mathrm{C}$ NMR spectrum of $7^{\mathrm{H}_{-}} \mathrm{C} 12$ in $\mathrm{CDCl}_{3}$ at $25{ }^{\circ} \mathrm{C}$. 


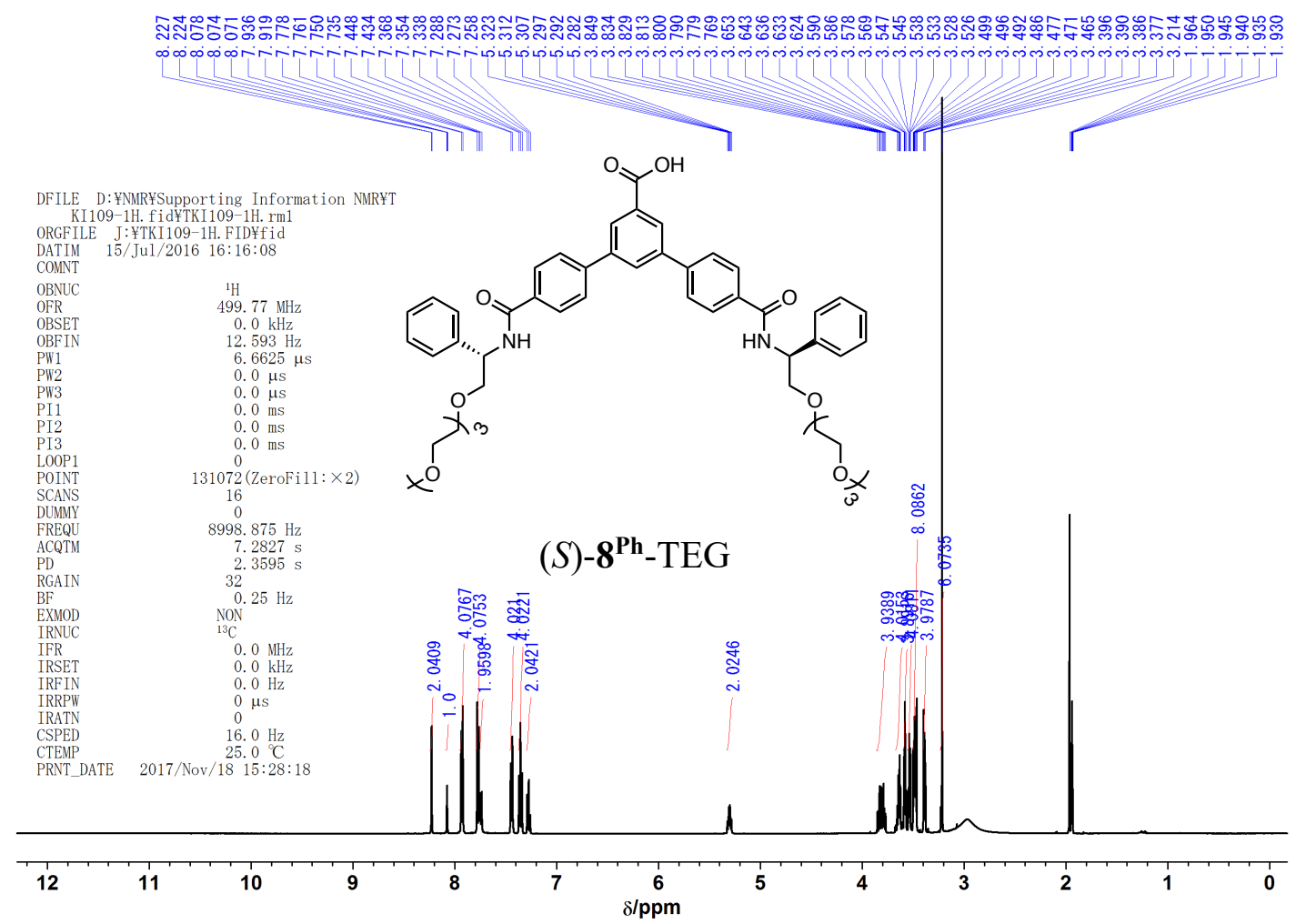

Figure S100. ${ }^{1} \mathrm{H}$ NMR spectrum of $(S)-8^{\mathrm{Ph}}-\mathrm{TEG}$ in $\mathrm{CD}_{3} \mathrm{CN}$ at $25^{\circ} \mathrm{C}$.

DFILE H:ISI-1H,13CITK1109-13C.fid1109-1 3C.rm1
ORGFILE H:ISI-1H,13CITKI109-13C.FIDI11 PDATAl1111
DATIM 15/Jul/2016 00:00:00

C13 std parameter $\begin{array}{ll}\text { OBNUC } & { }^{13} \mathrm{C} \\ \text { OFR } & 125.68 \mathrm{MHz}\end{array}$ $\begin{array}{lc}\text { OFR } & 125.68 \mathrm{MHz} \\ \text { OBSET } & 0.0 \mathrm{kHz} \\ \text { OBFIN } & 10001.2 \mathrm{~Hz} \\ \text { PW1 } & 6.7 \mu \mathrm{s} \\ \text { PW2 } & 134 \mu \mathrm{s}\end{array}$

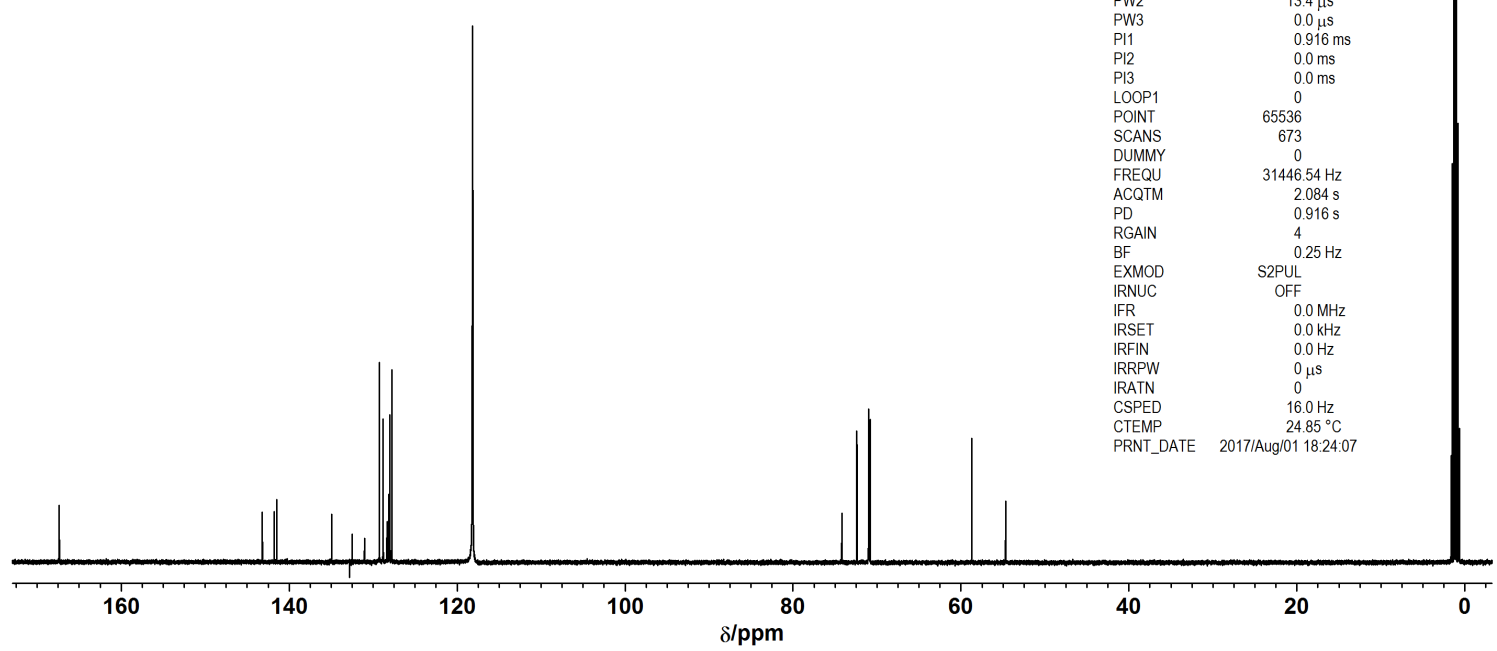

Figure S101. ${ }^{13} \mathrm{C}$ NMR spectrum of $(S)-8^{\mathrm{Ph}}-\mathrm{TEG}$ in $\mathrm{CD}_{3} \mathrm{CN}$ at $25{ }^{\circ} \mathrm{C}$. 

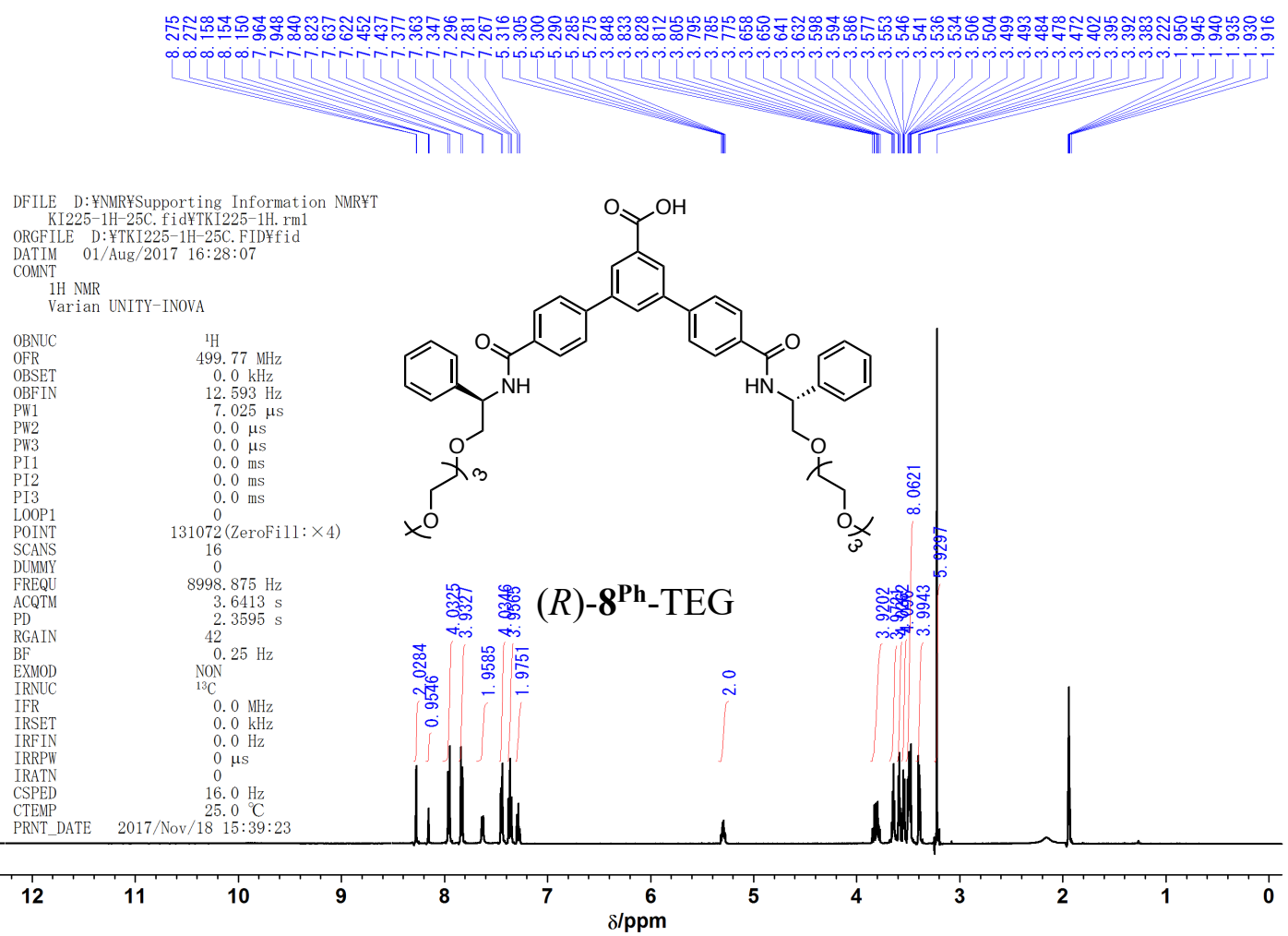

Figure S102. ${ }^{1} \mathrm{H}$ NMR spectrum of $(R)-8^{\mathrm{Ph}}-\mathrm{TEG}$ in $\mathrm{CD}_{3} \mathrm{CN}$ at $25^{\circ} \mathrm{C}$.

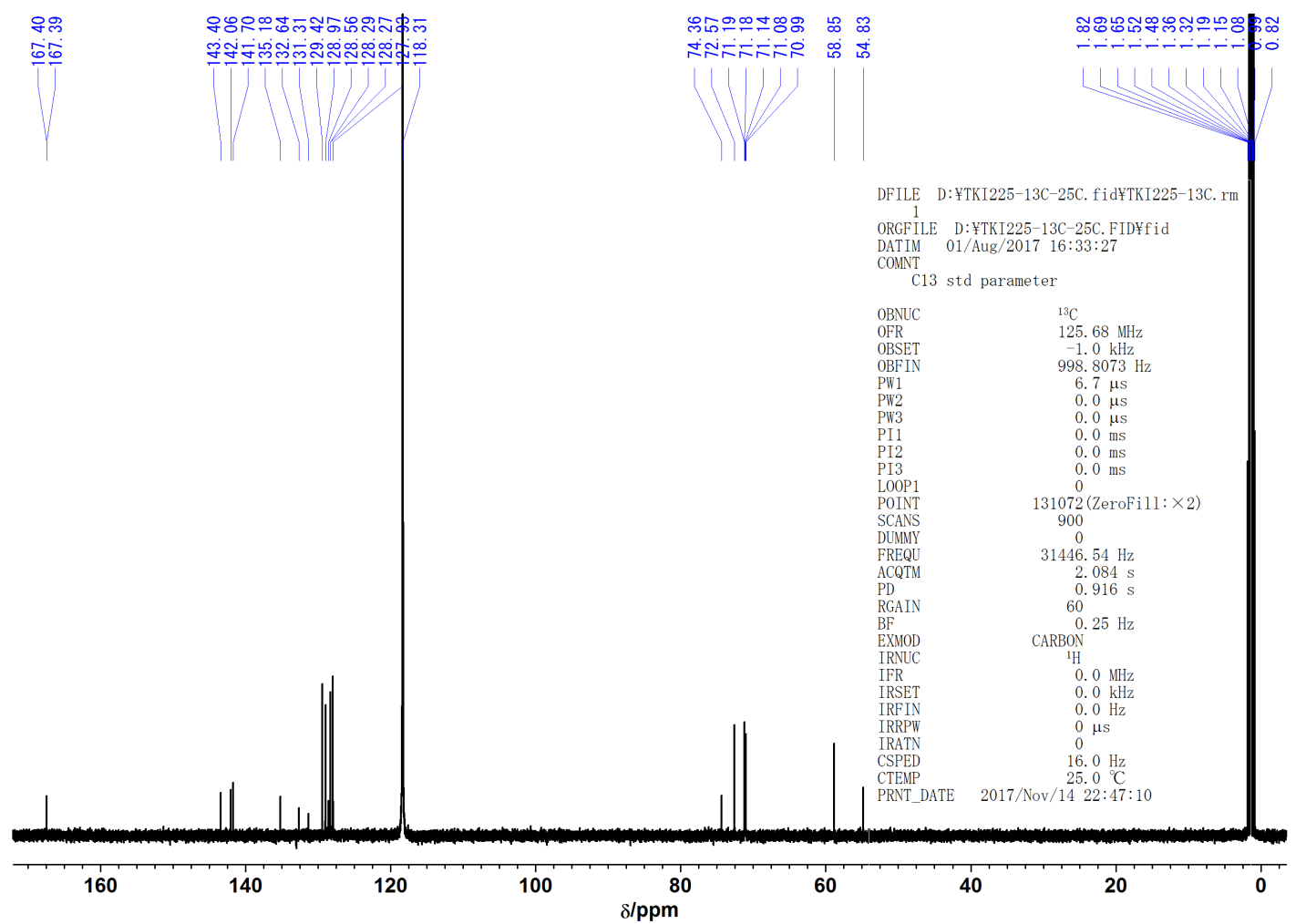

Figure S103. ${ }^{13} \mathrm{C}$ NMR spectrum of $(R)-8^{\mathrm{Ph}}-\mathrm{TEG}$ in $\mathrm{CD}_{3} \mathrm{CN}$ at $25{ }^{\circ} \mathrm{C}$. 


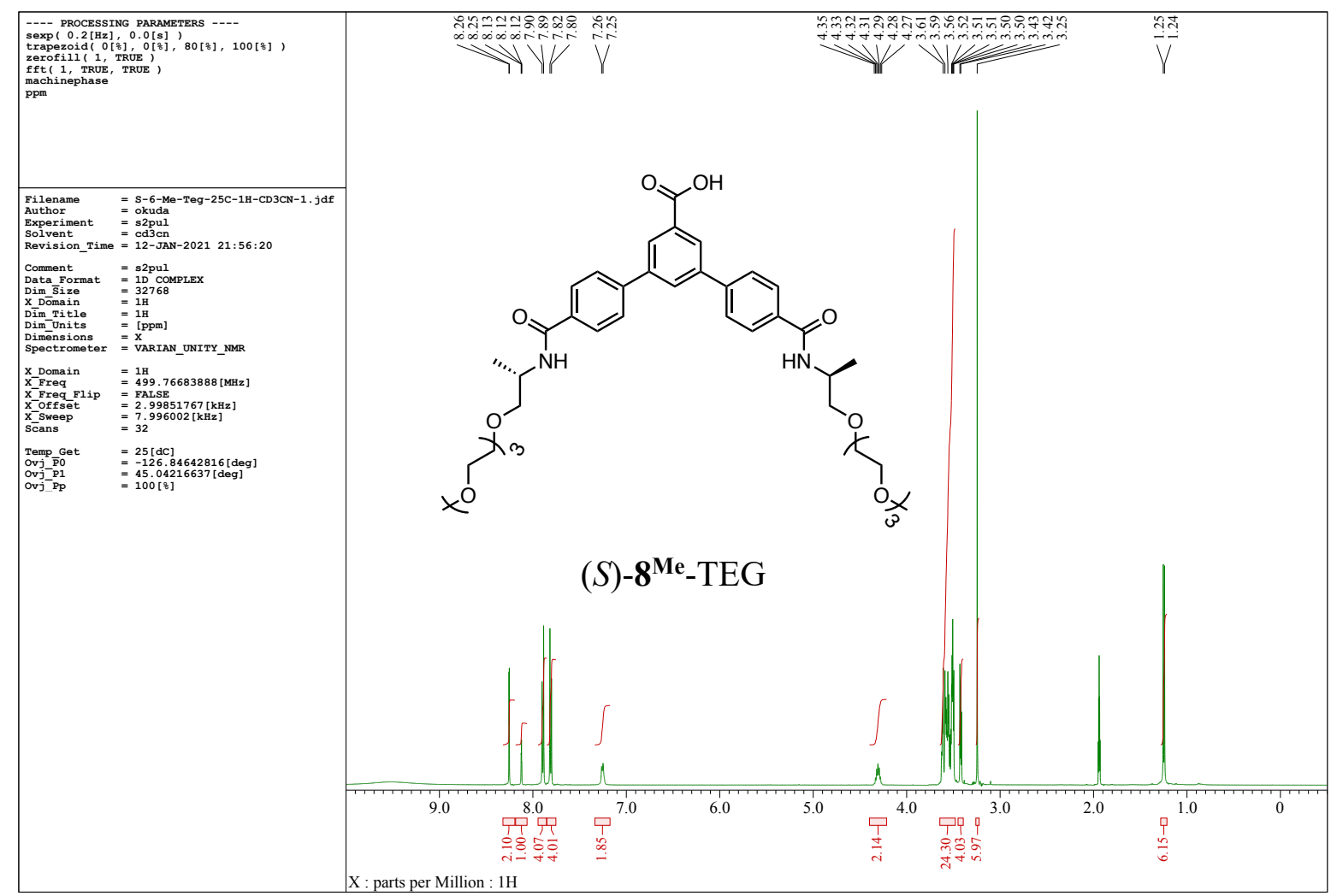

Figure S104. ${ }^{1} \mathrm{H}$ NMR spectrum of $(S)-8^{\mathrm{Me}}-\mathrm{TEG}$ in $\mathrm{CD}_{3} \mathrm{CN}$ at $25^{\circ} \mathrm{C}$.

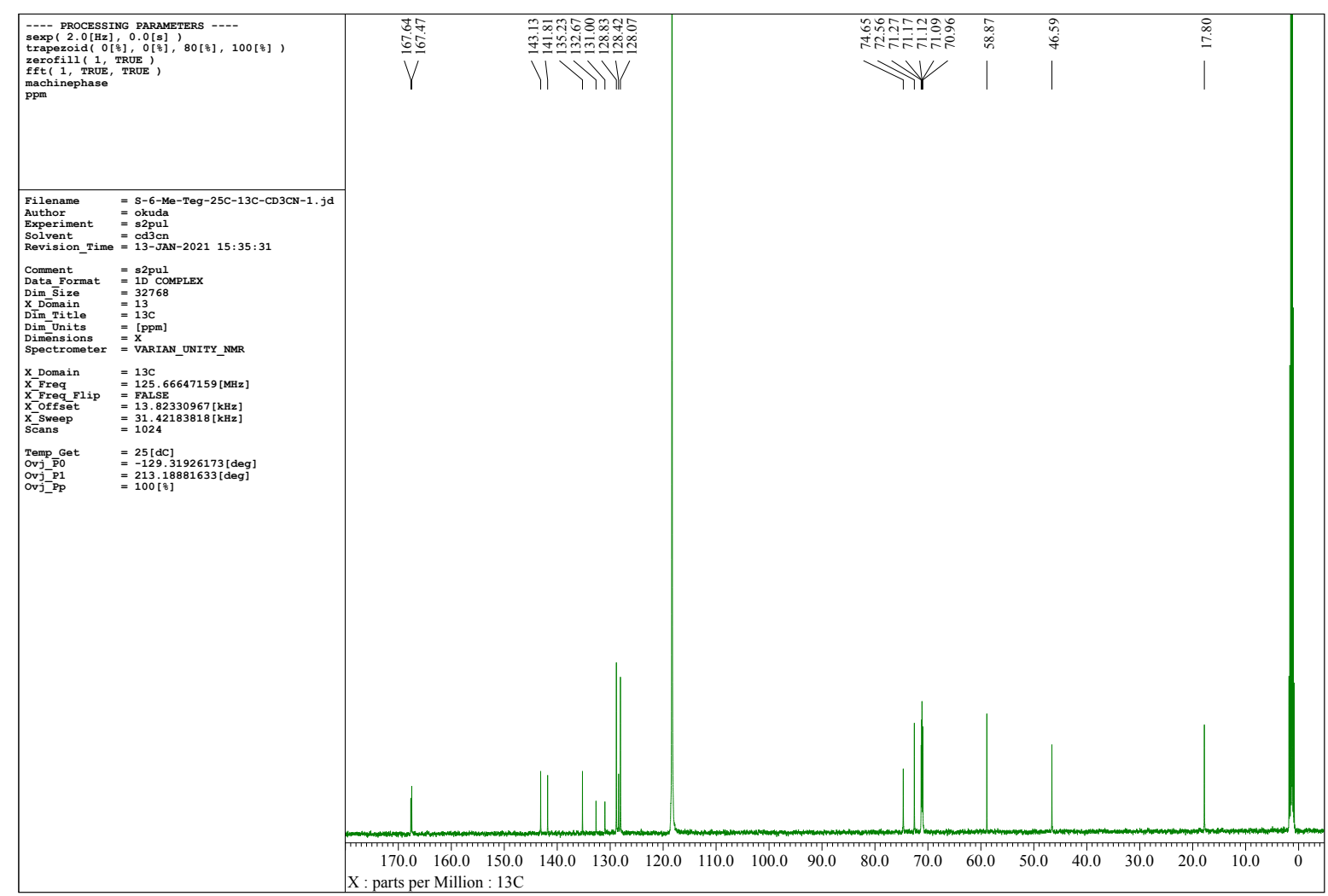

Figure S105. ${ }^{13} \mathrm{C}$ NMR spectrum of $(S)-8^{\mathrm{Me}}-\mathrm{TEG}$ in $\mathrm{CD}_{3} \mathrm{CN}$ at $25{ }^{\circ} \mathrm{C}$. 


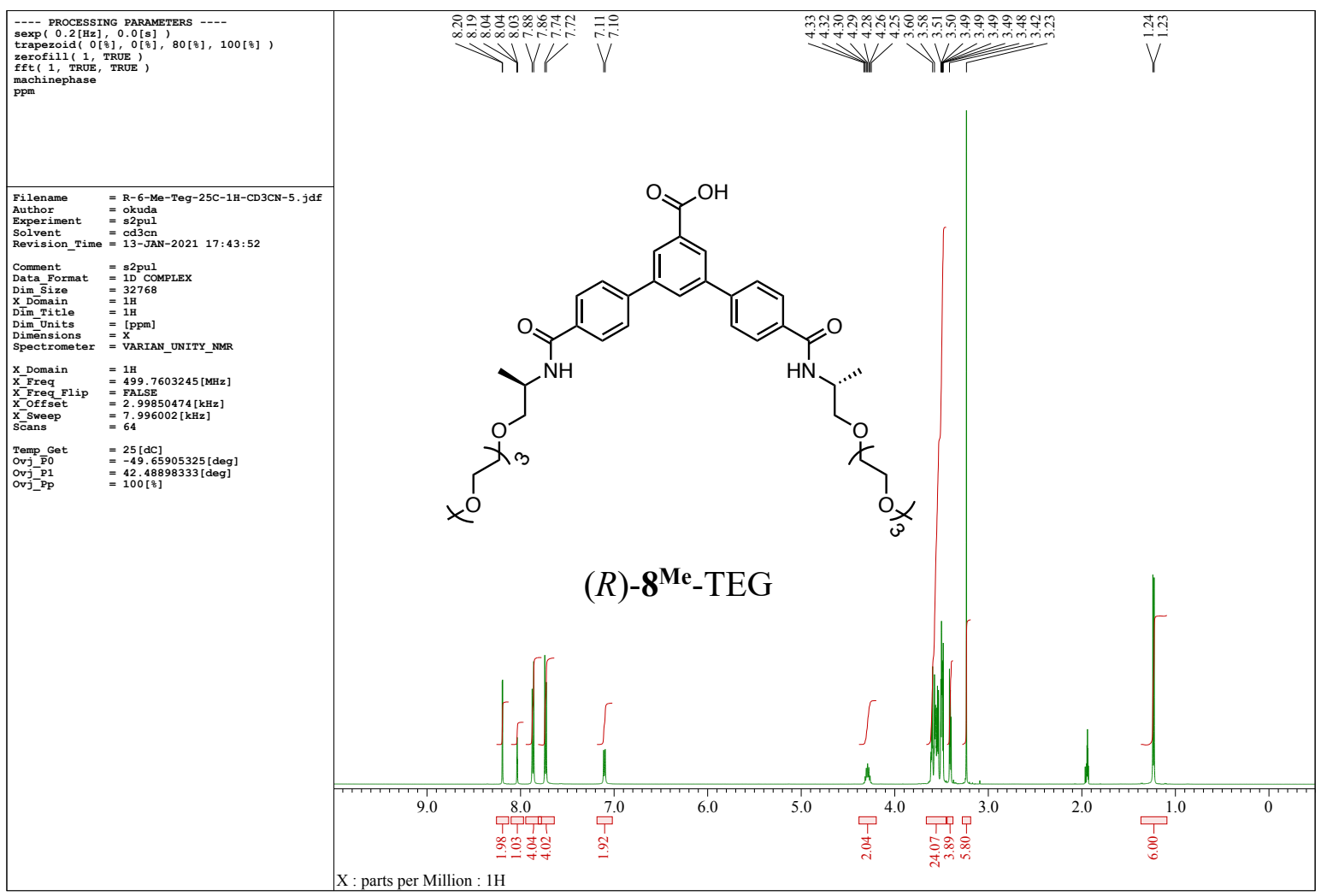

Figure S106. ${ }^{1} \mathrm{H}$ NMR spectrum of $(R)-8^{\mathrm{Me}}-\mathrm{TEG}$ in $\mathrm{CD}_{3} \mathrm{CN}$ at $25^{\circ} \mathrm{C}$.

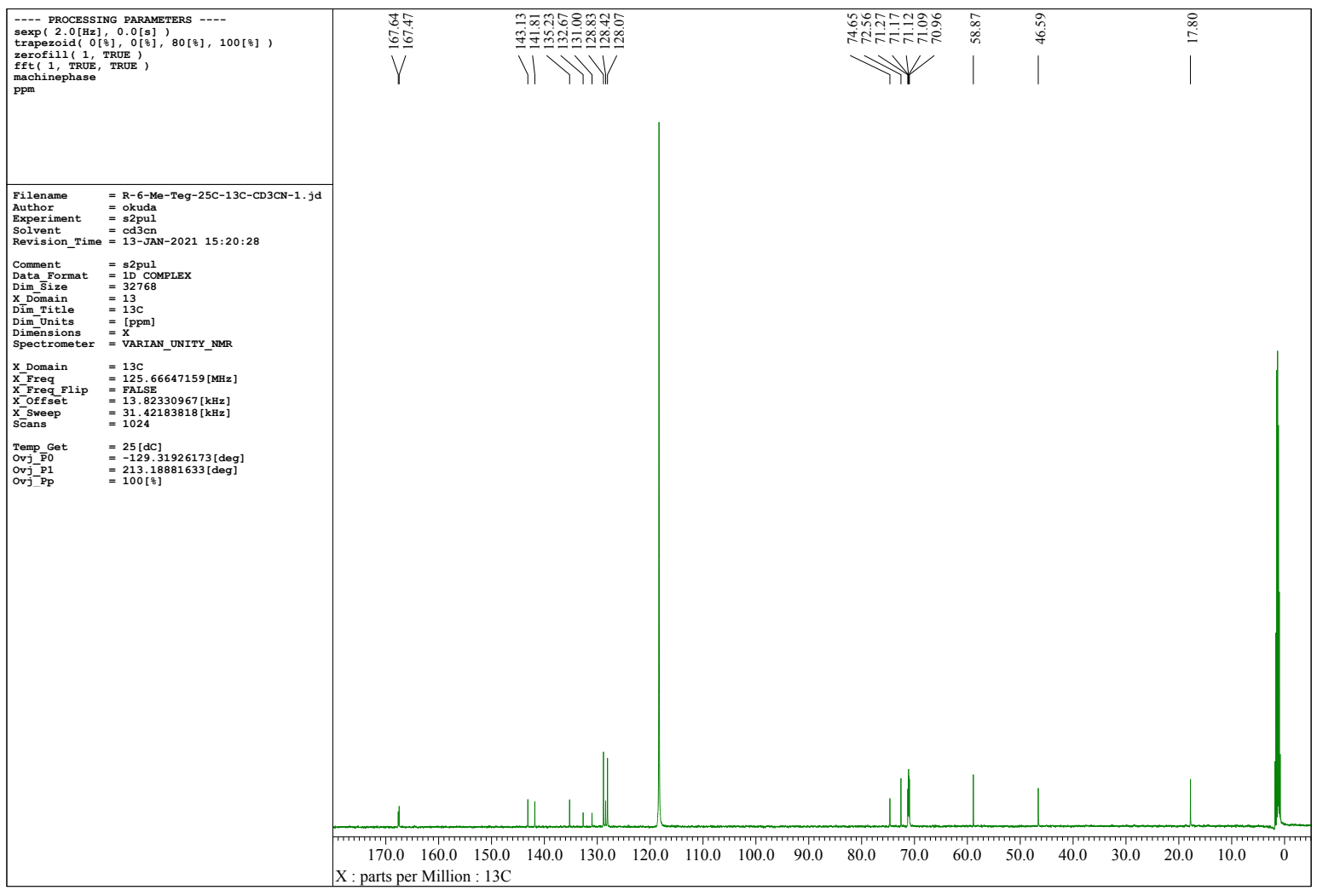

Figure S107. ${ }^{13} \mathrm{C}$ NMR spectrum of $(R)-8^{\mathrm{Me}}-\mathrm{TEG}$ in $\mathrm{CD}_{3} \mathrm{CN}$ at $25^{\circ} \mathrm{C}$. 


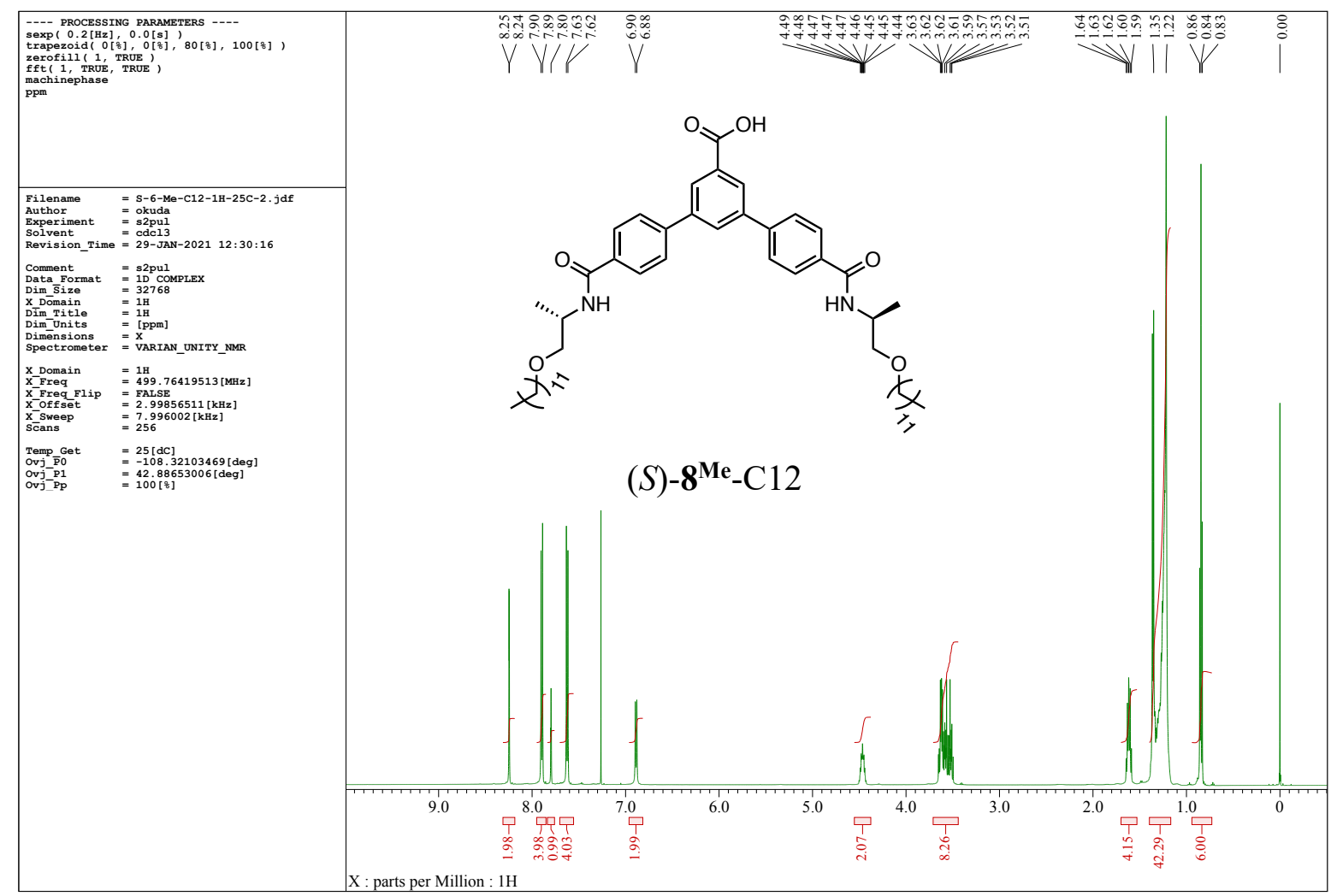

Figure S108. ${ }^{1} \mathrm{H}$ NMR spectrum of $(S)-8^{\mathrm{Me}}-\mathrm{C} 12$ in $\mathrm{CDCl}_{3}$ at $25^{\circ} \mathrm{C}$.

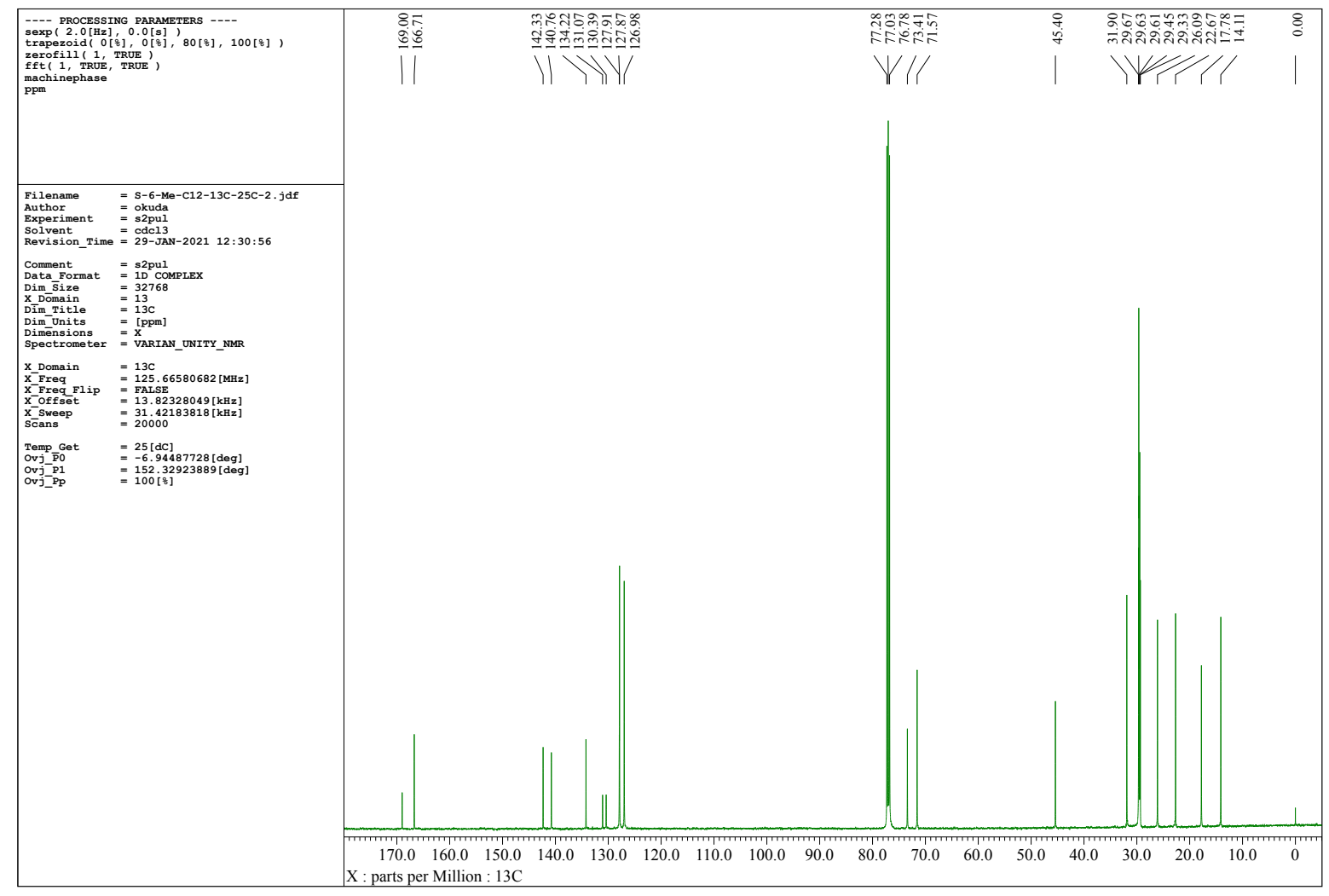

Figure S109. ${ }^{13} \mathrm{C}$ NMR spectrum of $(S)-8^{\mathrm{Me}}-\mathrm{C} 12$ in $\mathrm{CDCl}_{3}$ at $25^{\circ} \mathrm{C}$. 


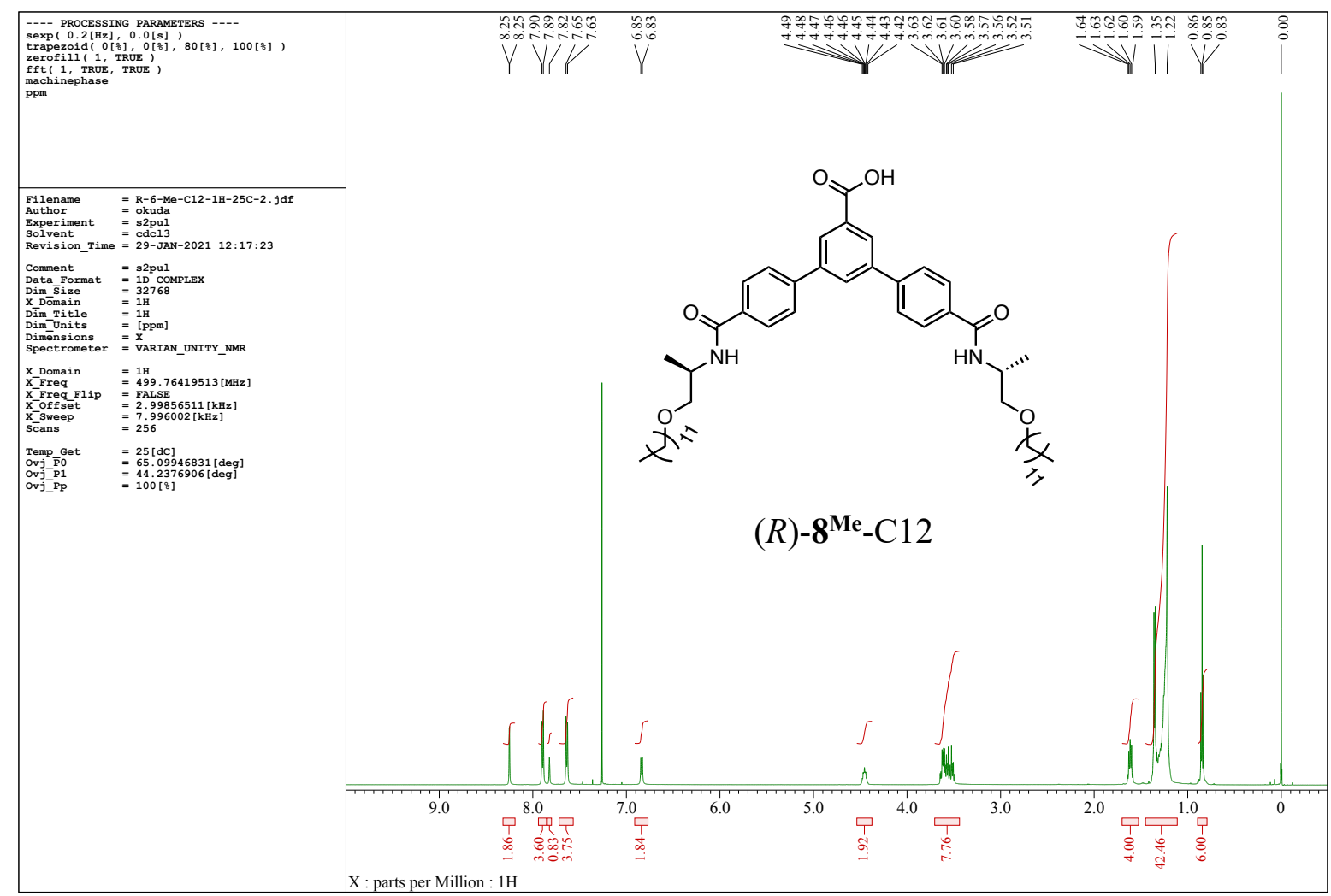

Figure S110. ${ }^{1} \mathrm{H}$ NMR spectrum of $(R)-8^{\mathrm{Me}}-\mathrm{C} 12$ in $\mathrm{CDCl}_{3}$ at $25^{\circ} \mathrm{C}$.

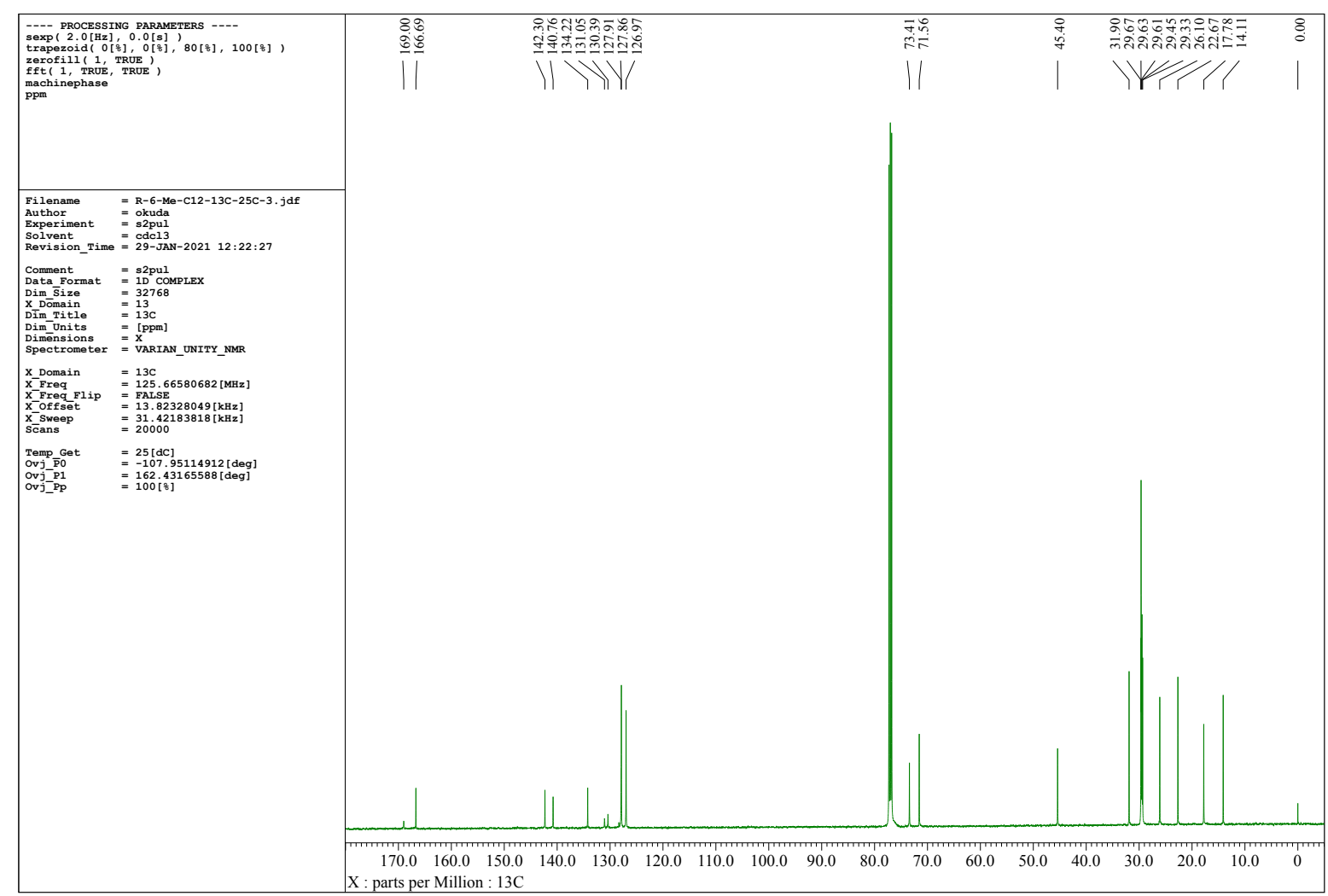

Figure S111. ${ }^{13} \mathrm{C}$ NMR spectrum of $(R)-8^{\mathrm{Me}}-\mathrm{C} 12$ in $\mathrm{CDCl}_{3}$ at $25^{\circ} \mathrm{C}$. 

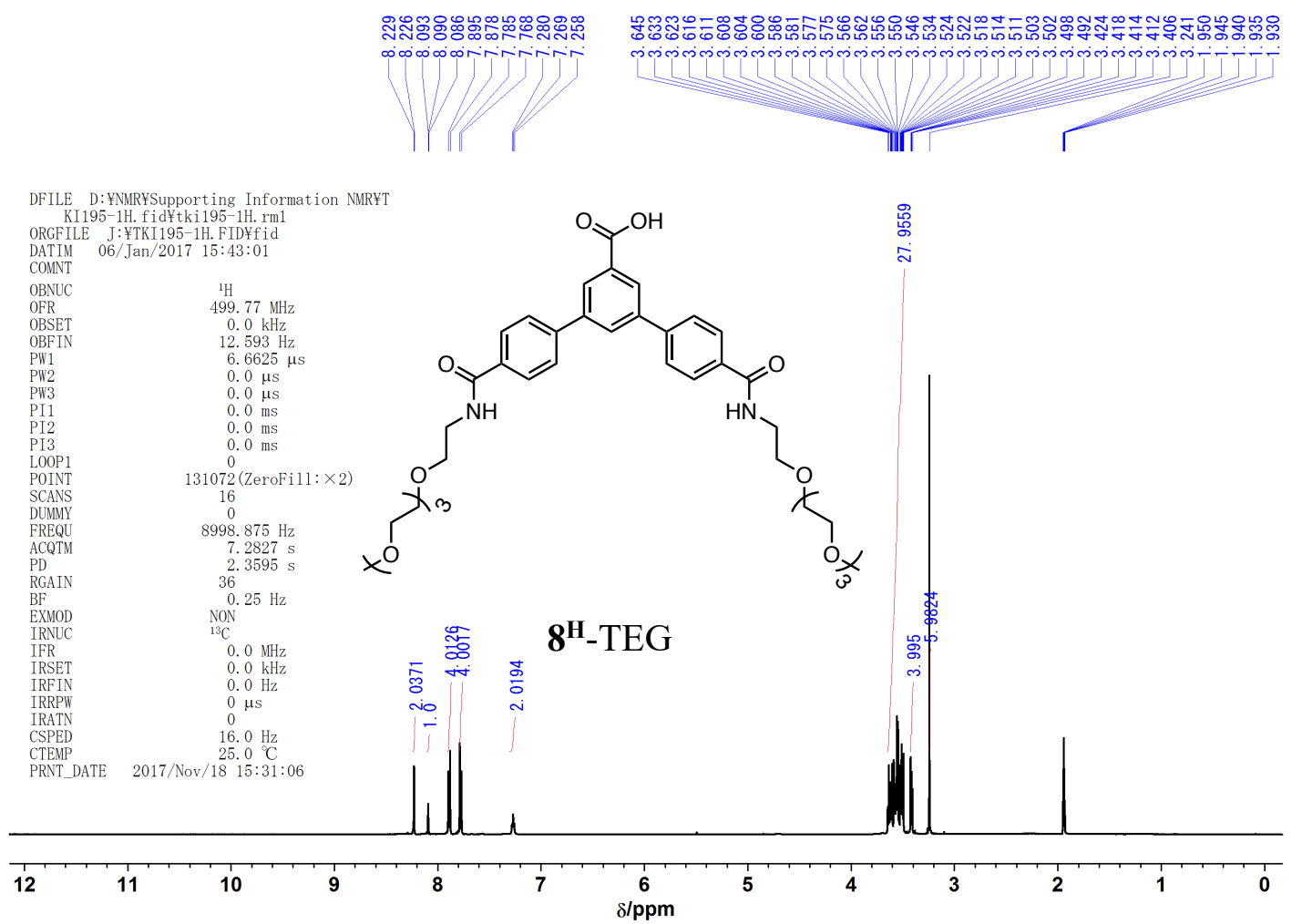

Figure S112. ${ }^{1} \mathrm{H}$ NMR spectrum of $\mathbf{8}^{\mathrm{H}}-\mathrm{TEG}$ in $\mathrm{CD}_{3} \mathrm{CN}$ at $25^{\circ} \mathrm{C}$.

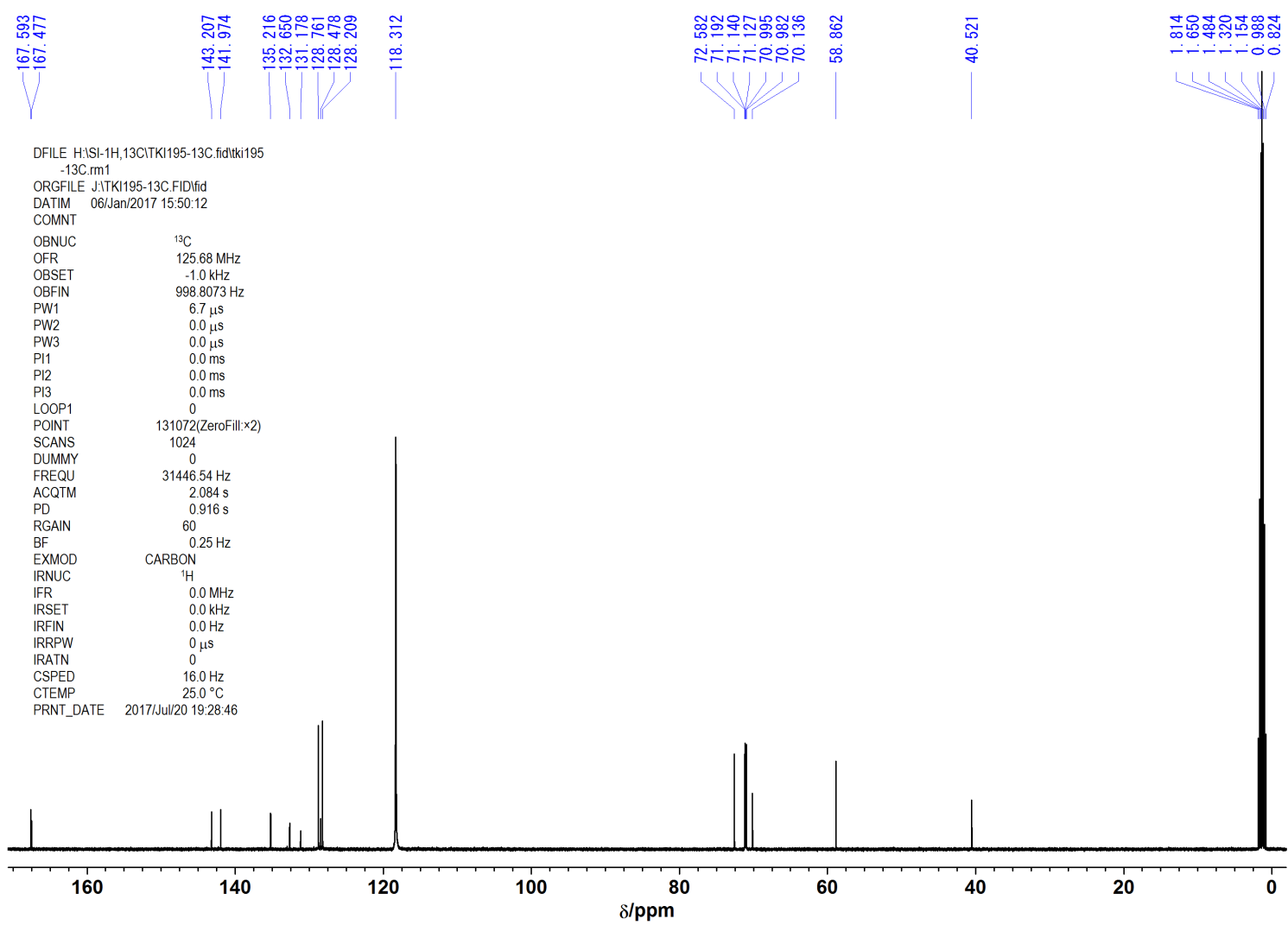

Figure S113. ${ }^{13} \mathrm{C}$ NMR spectrum of $8^{\mathrm{H}}-\mathrm{TEG}$ in $\mathrm{CD}_{3} \mathrm{CN}$ at $25{ }^{\circ} \mathrm{C}$. 


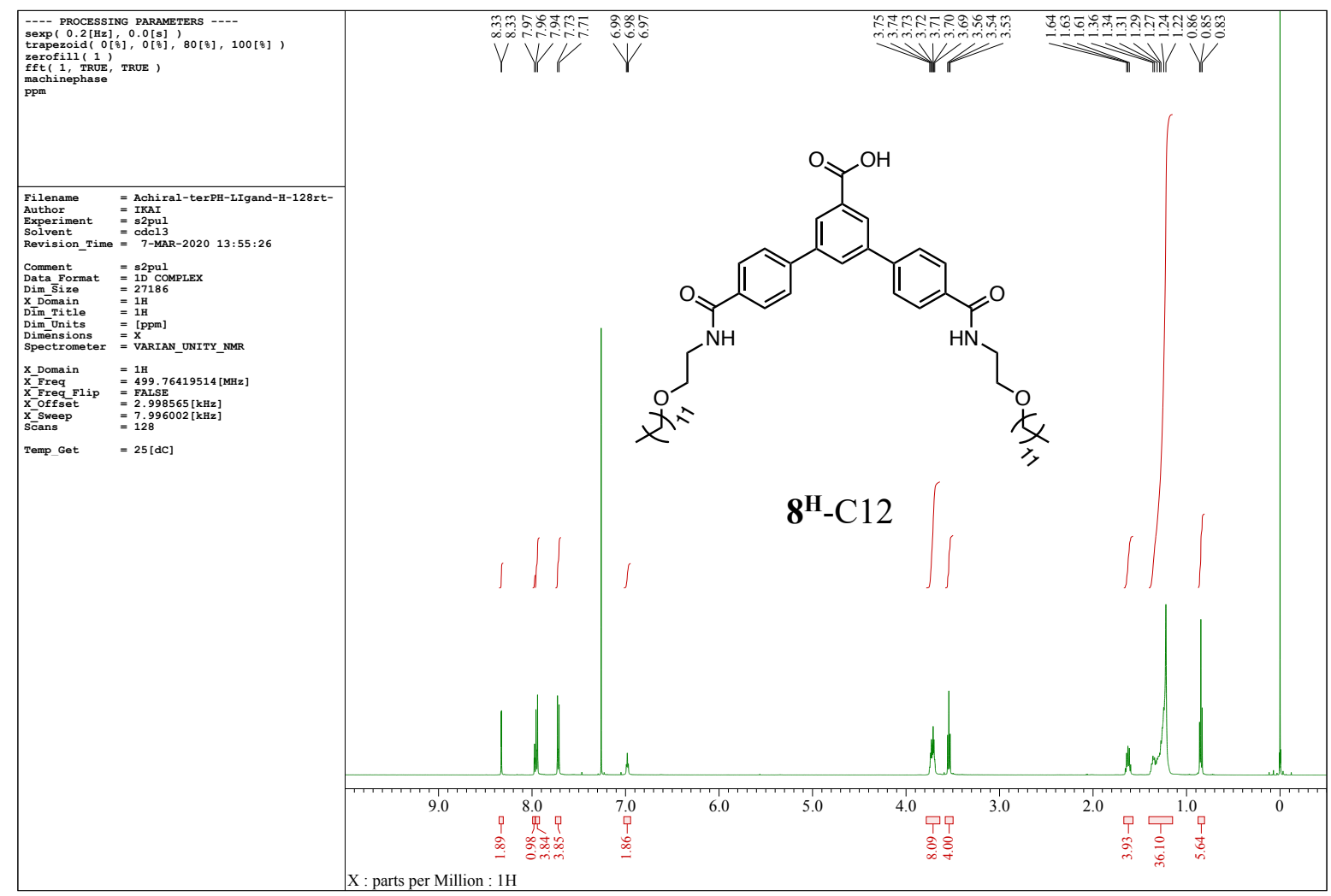

Figure S114. ${ }^{1} \mathrm{H}$ NMR spectrum of $\mathbf{8}^{\mathbf{H}}-\mathrm{C} 12$ in $\mathrm{CDCl}_{3}$ at $25^{\circ} \mathrm{C}$.

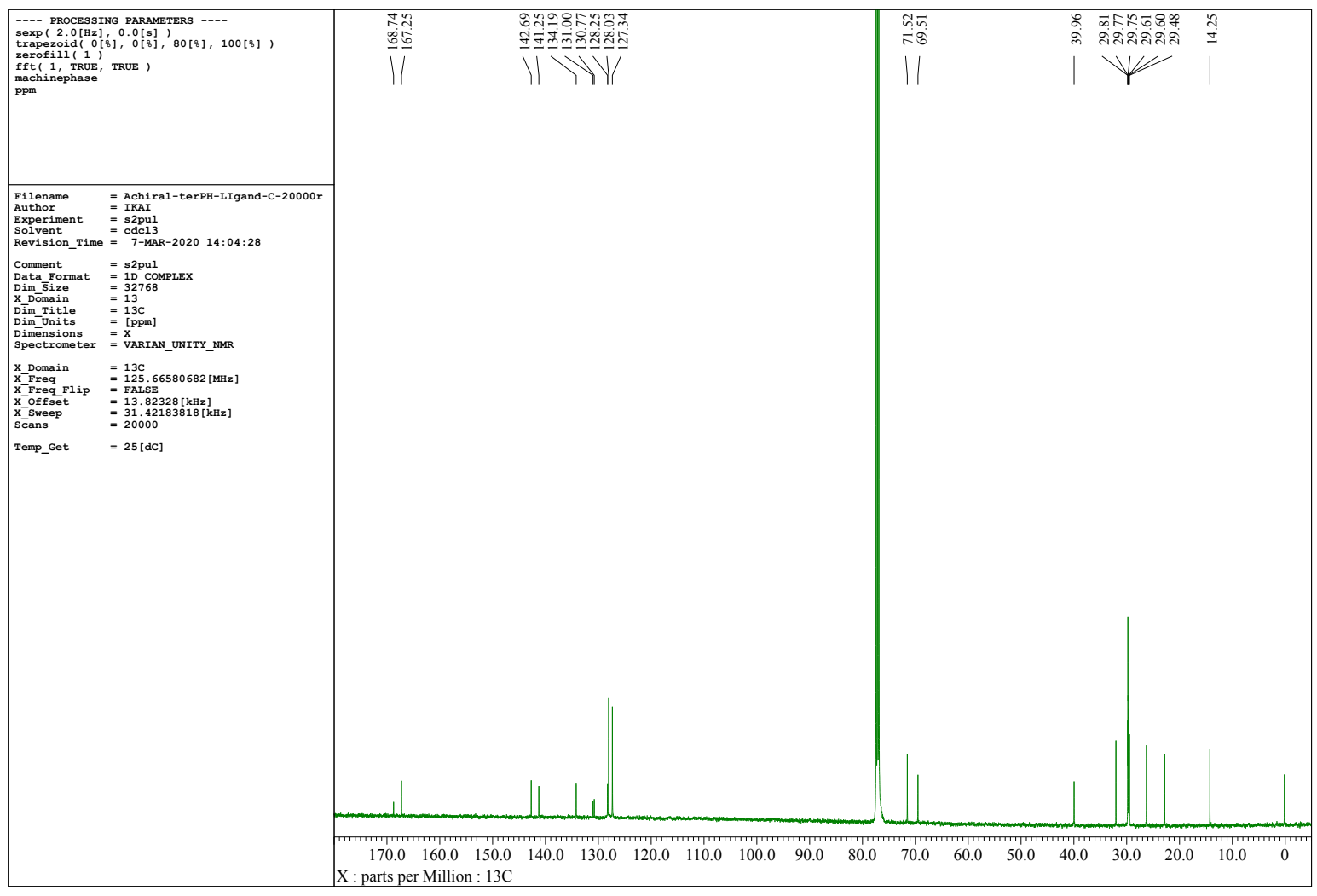

Figure S115. ${ }^{13} \mathrm{C}$ NMR spectrum of $\mathbf{8}^{\mathbf{H}}-\mathrm{C} 12$ in $\mathrm{CDCl}_{3}$ at $25{ }^{\circ} \mathrm{C}$. 


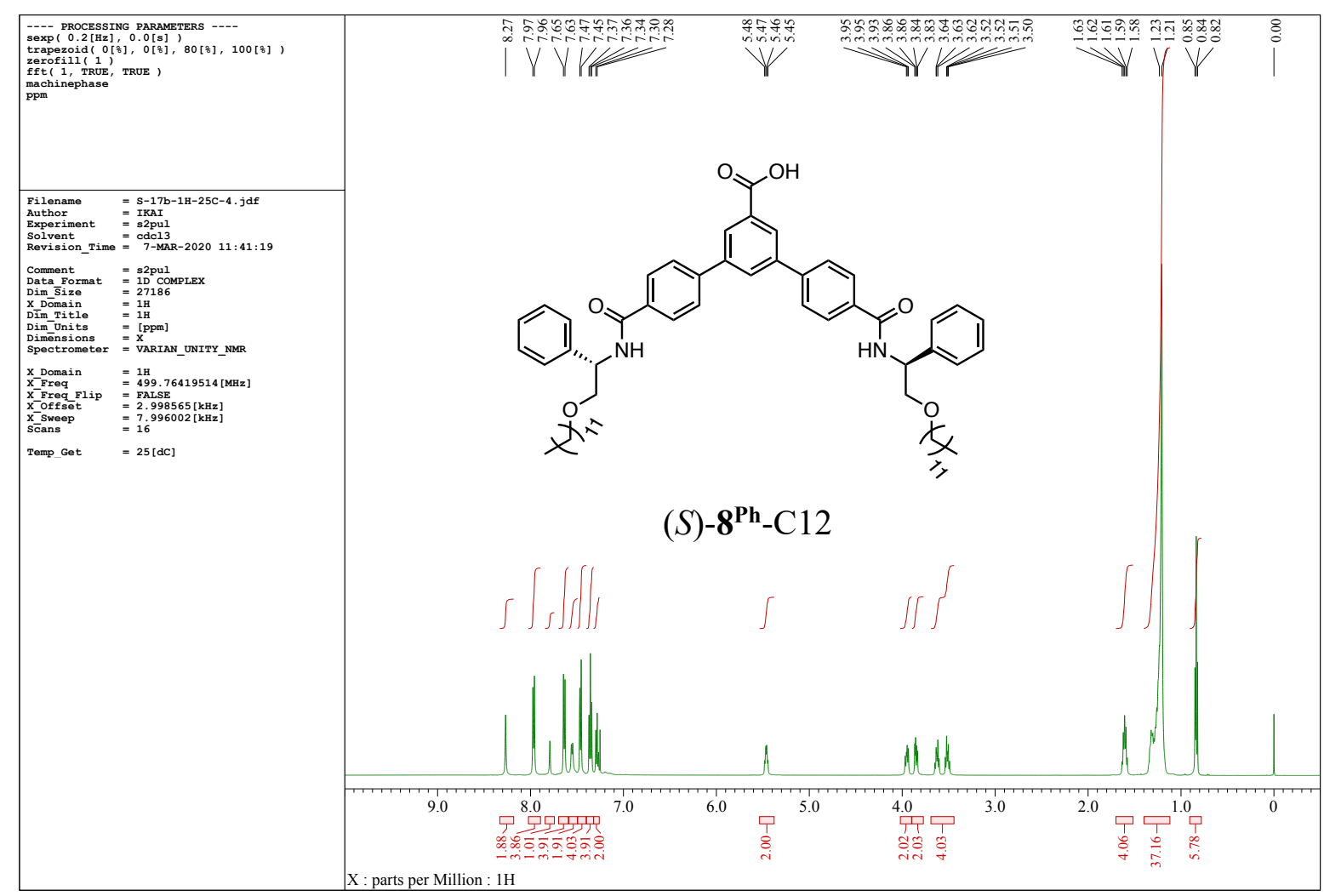

Figure S116. ${ }^{1} \mathrm{H}$ NMR spectrum of $(S)-8^{\mathrm{Ph}}-\mathrm{C} 12$ in $\mathrm{CDCl}_{3}$ at $25^{\circ} \mathrm{C}$.

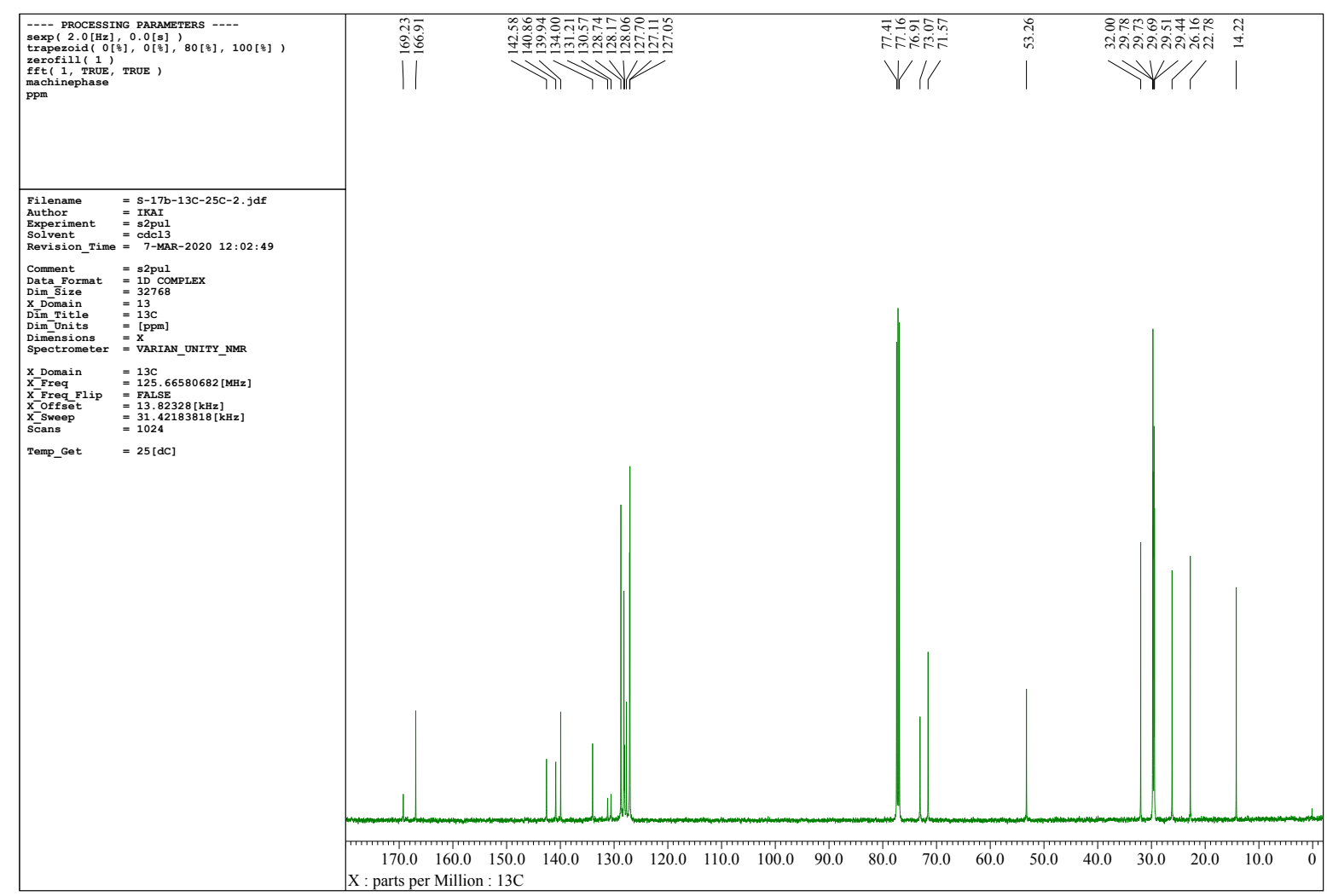

Figure S117. ${ }^{13} \mathrm{C}$ NMR spectrum of $(S)-8^{\mathrm{Ph}}-\mathrm{C} 12$ in $\mathrm{CDCl}_{3}$ at $25{ }^{\circ} \mathrm{C}$. 


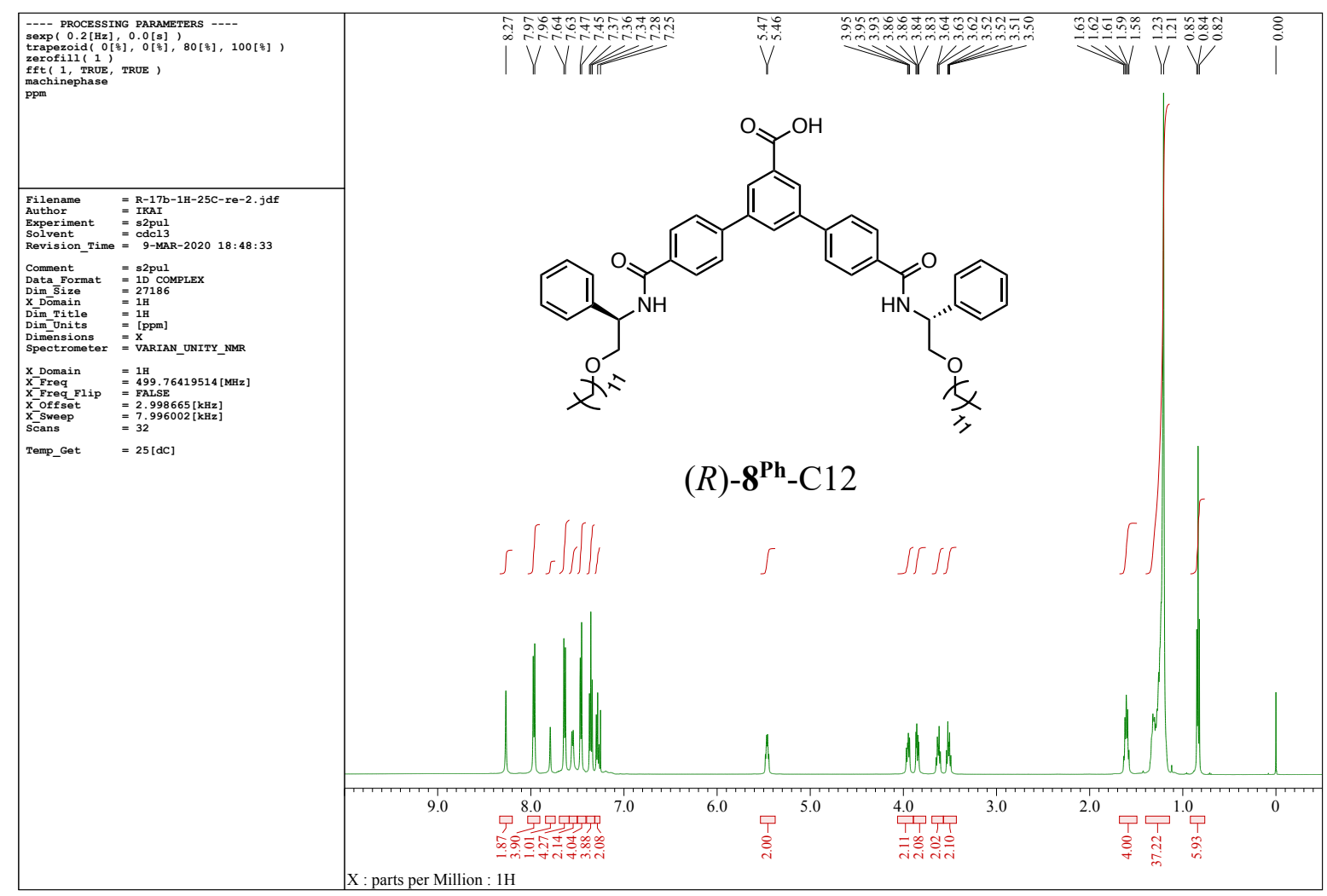

Figure S118. ${ }^{1} \mathrm{H}$ NMR spectrum of $(R)-8^{\mathrm{Ph}}-\mathrm{C} 12$ in $\mathrm{CDCl}_{3}$ at $25^{\circ} \mathrm{C}$.

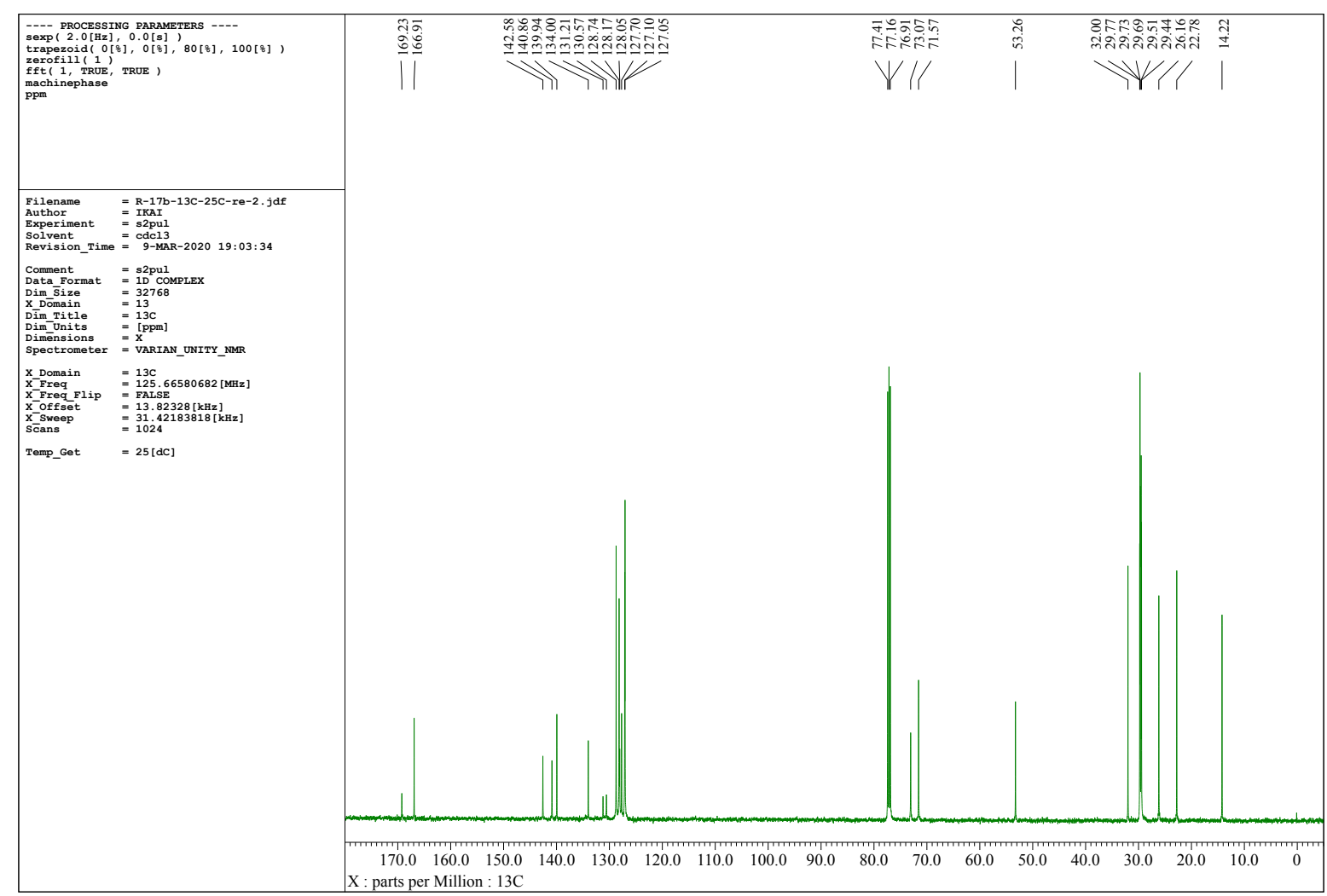

Figure S119. ${ }^{13} \mathrm{C}$ NMR spectrum of $(R)-8^{\mathrm{Ph}}-\mathrm{C} 12$ in $\mathrm{CDCl}_{3}$ at $25^{\circ} \mathrm{C}$. 


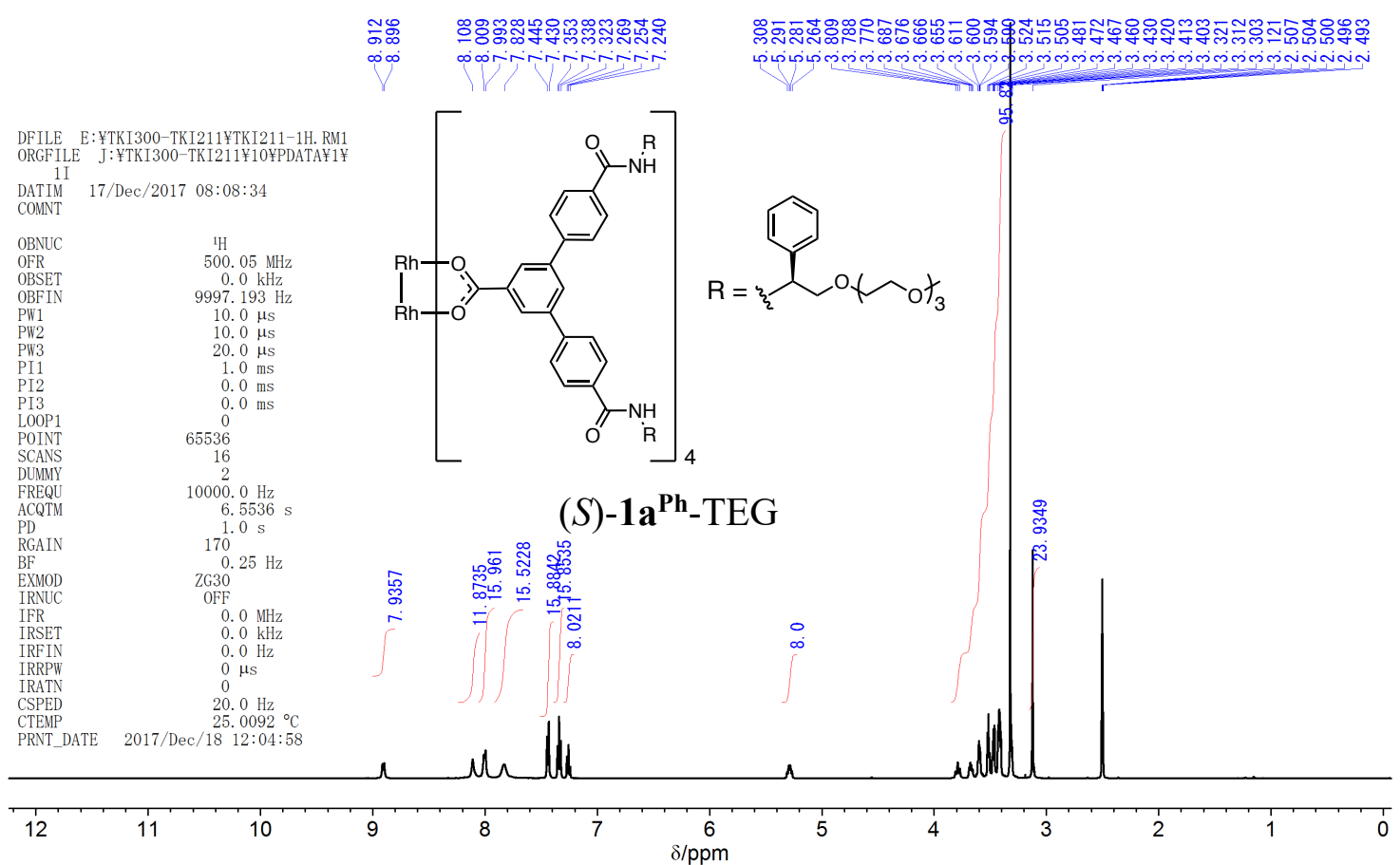

Figure S120. ${ }^{1} \mathrm{H}$ NMR spectrum of $(S)-1 \mathbf{a}^{\mathrm{Ph}}-\mathrm{TEG}$ in DMSO- $d_{6}$ at $25^{\circ} \mathrm{C}$.

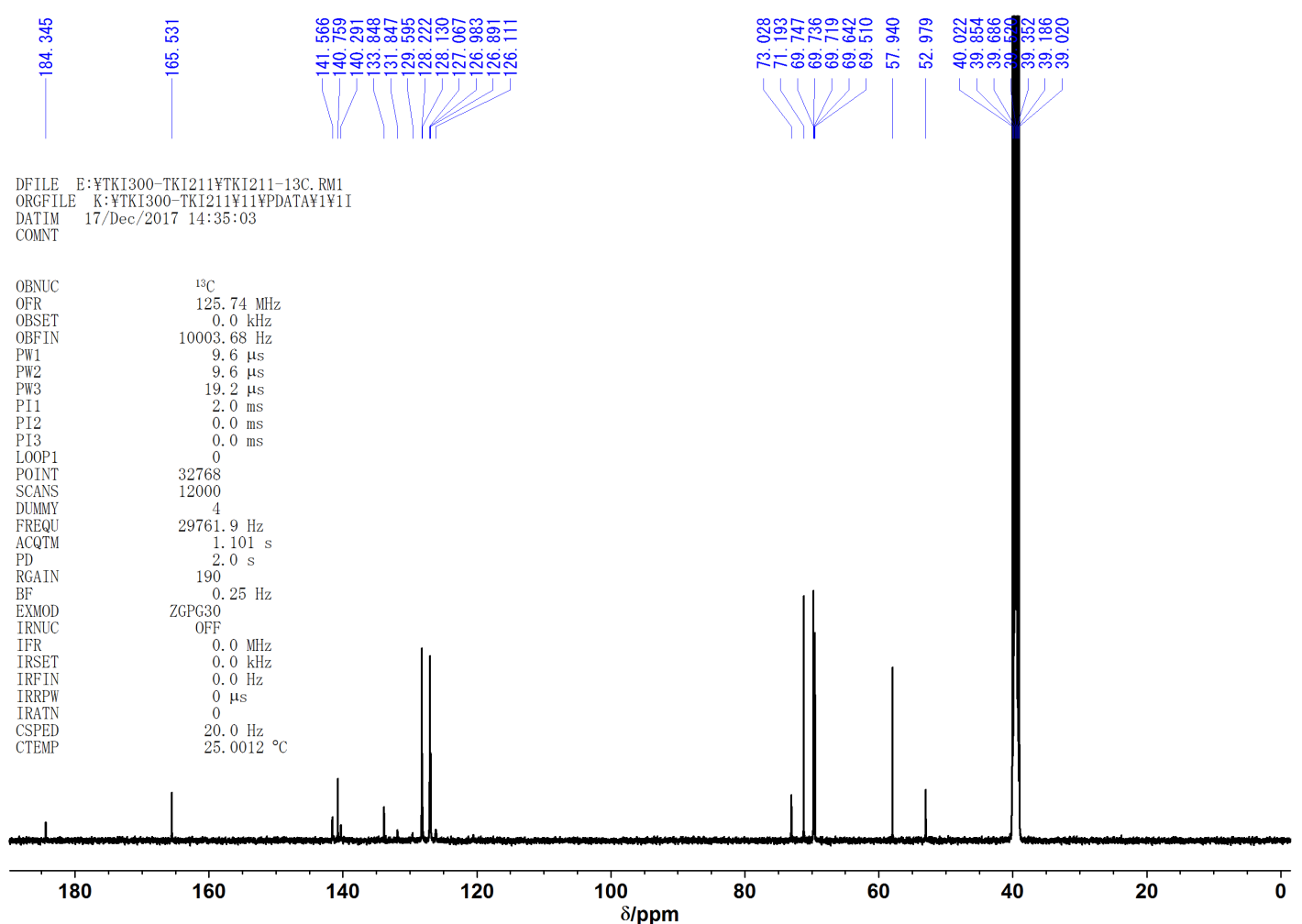

Figure S121. ${ }^{13} \mathrm{C}$ NMR spectrum of $(S)-1 \mathbf{a}^{\mathrm{Ph}}-\mathrm{TEG}$ in DMSO- $d_{6}$ at $25{ }^{\circ} \mathrm{C}$. 


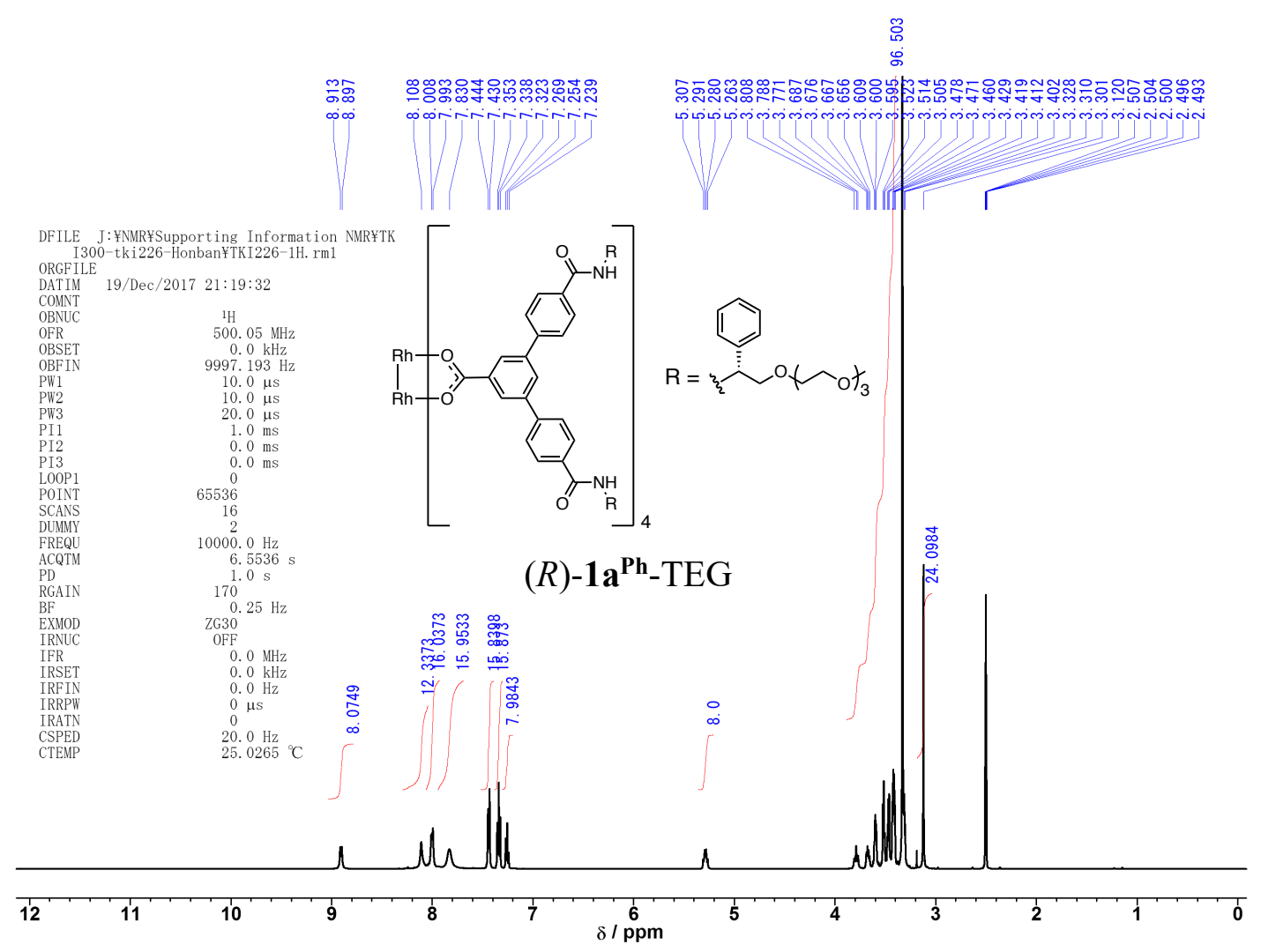

Figure S122. ${ }^{1} \mathrm{H}$ NMR spectrum of $(R)-\mathbf{1 \mathbf { a } ^ { \mathrm { Ph } }}$-TEG in DMSO- $d_{6}$ at $25^{\circ} \mathrm{C}$.

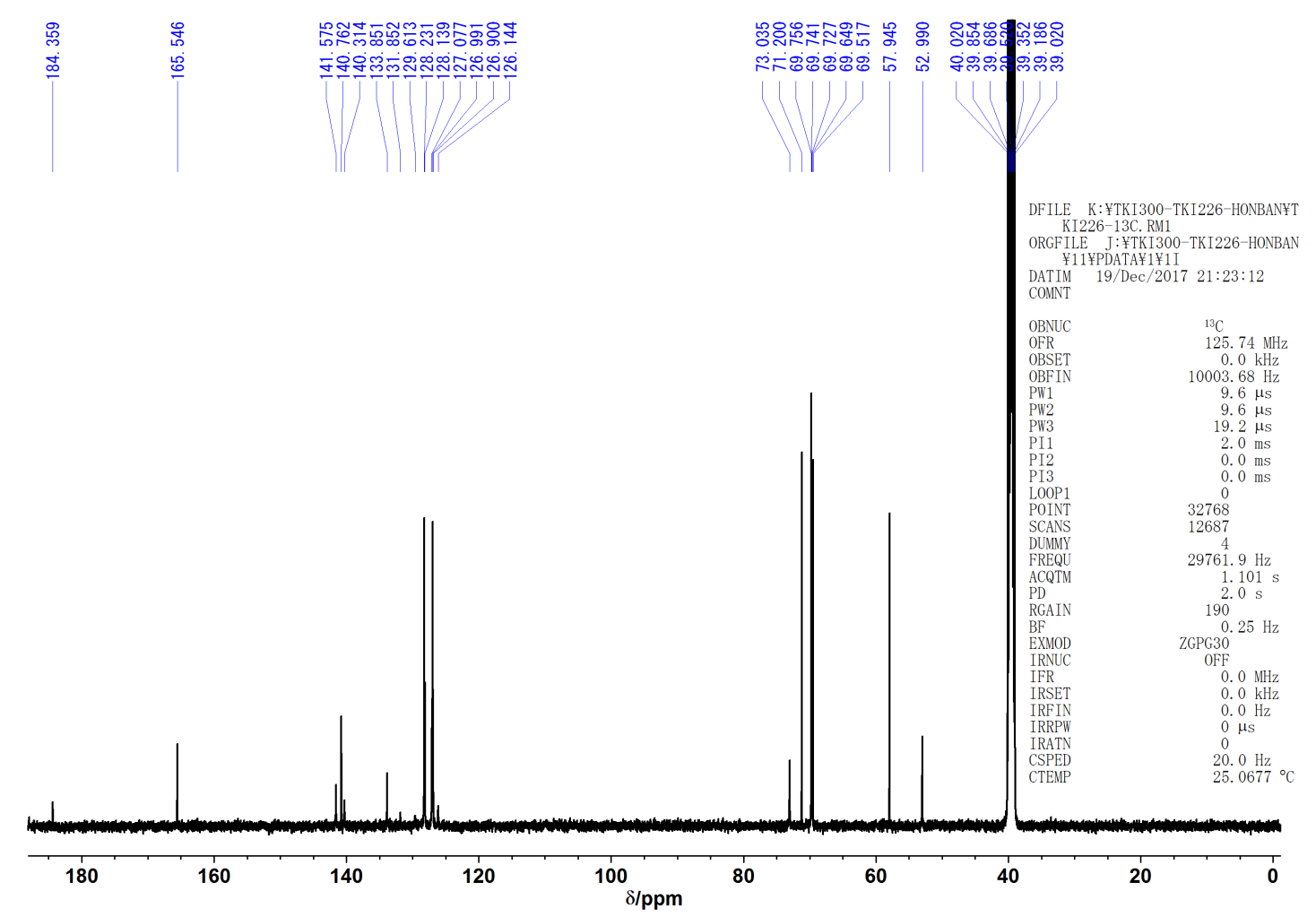

Figure S123. ${ }^{13} \mathrm{C}$ NMR spectrum of $(R)-\mathbf{1 a}{ }^{\mathrm{Ph}}-\mathrm{TEG}$ in DMSO- $d_{6}$ at $25{ }^{\circ} \mathrm{C}$. 


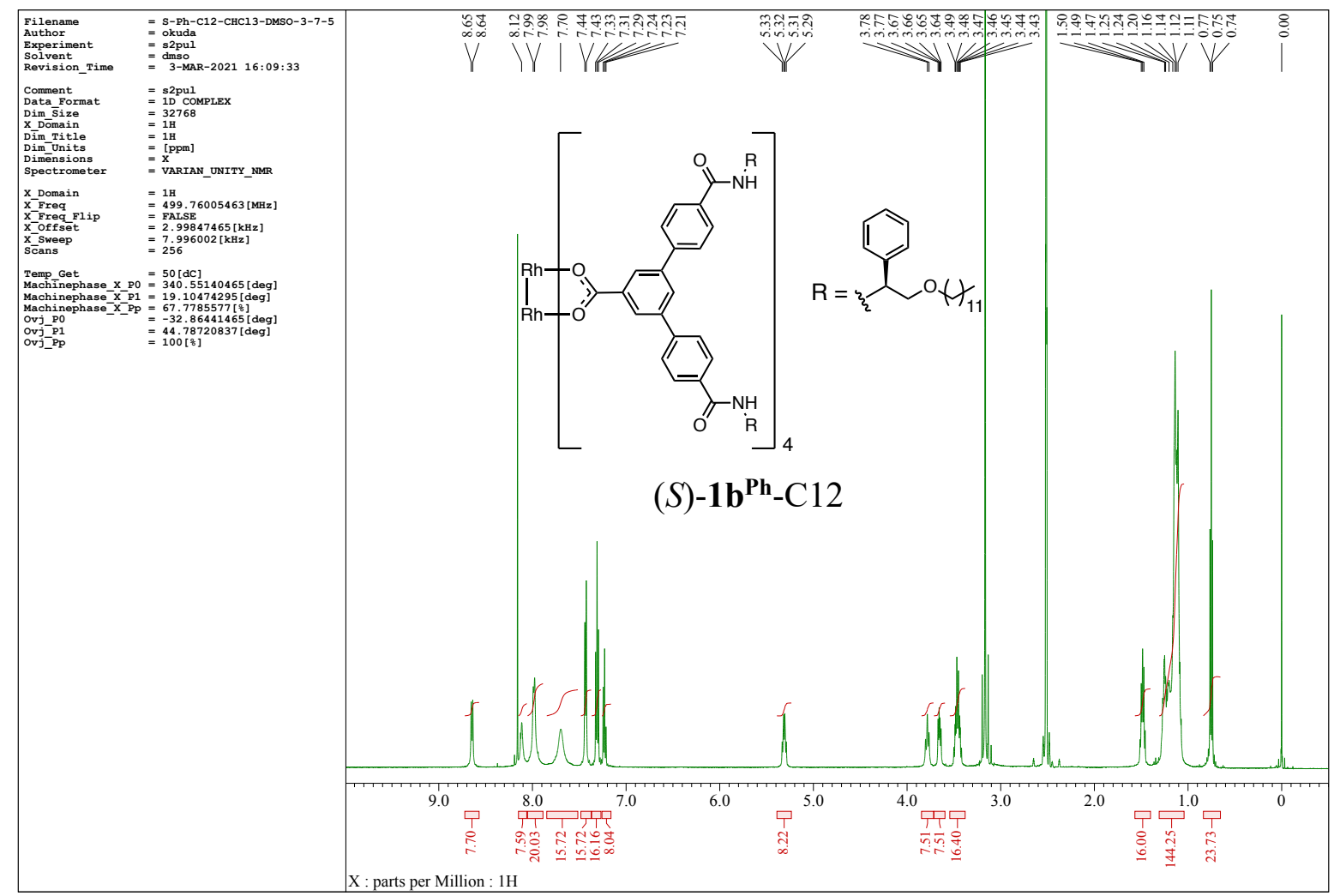

Figure S124. ${ }^{1} \mathrm{H}$ NMR spectrum of $(S)-\mathbf{1} \mathbf{b}^{\mathrm{Ph}}-\mathrm{C} 12$ in $\mathrm{CDCl}_{3} / \mathrm{DMSO}-d_{6}=3 / 7(\mathrm{v} / \mathrm{v})$ at $50{ }^{\circ} \mathrm{C}$.

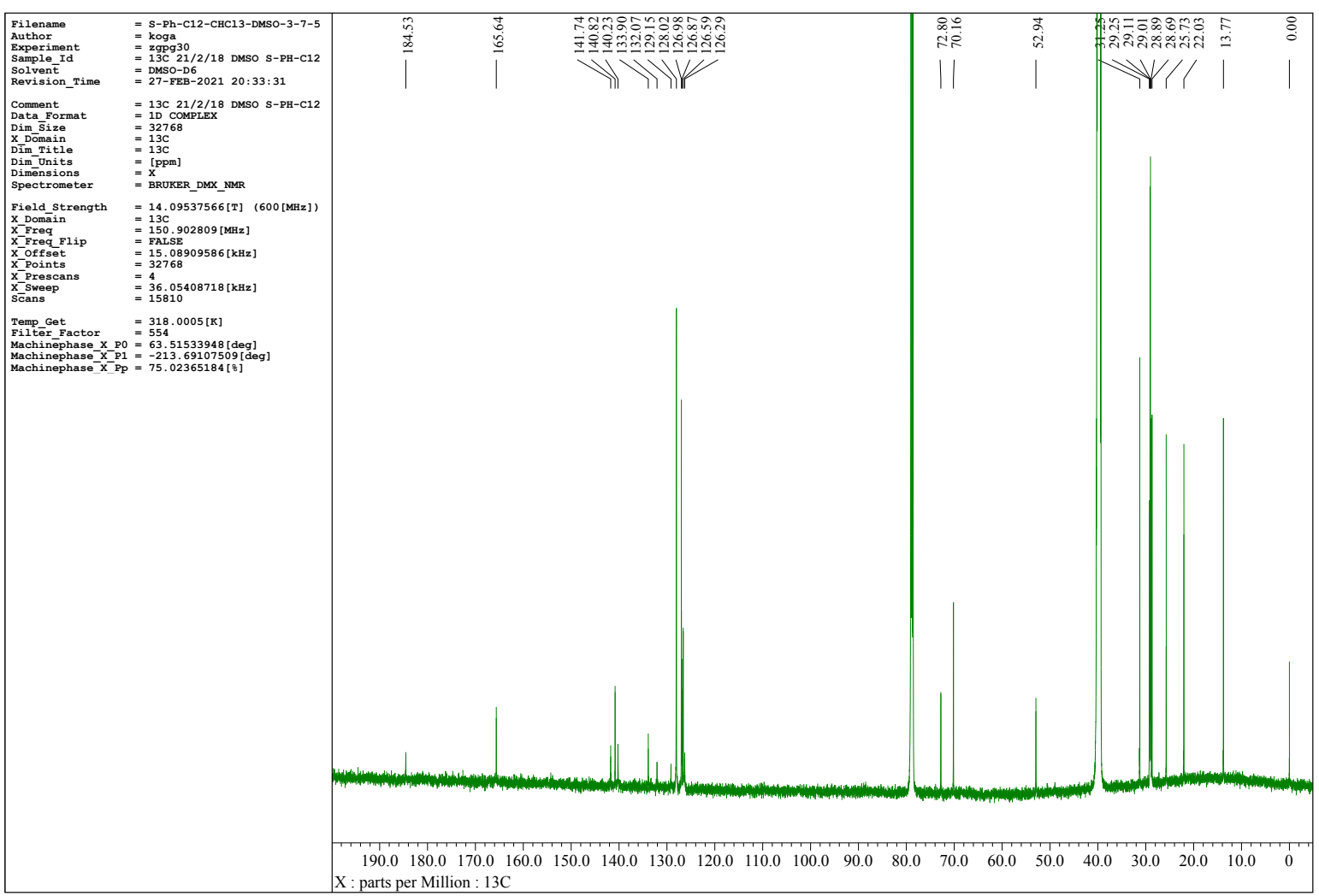

Figure S125. ${ }^{13} \mathrm{C}$ NMR spectrum of $(S)-\mathbf{1 b}^{\mathrm{Ph}}-\mathrm{C} 12$ in $\mathrm{CDCl}_{3} / \mathrm{DMSO}-d_{6}=3 / 7(\mathrm{v} / \mathrm{v})$ at $50{ }^{\circ} \mathrm{C}$. 


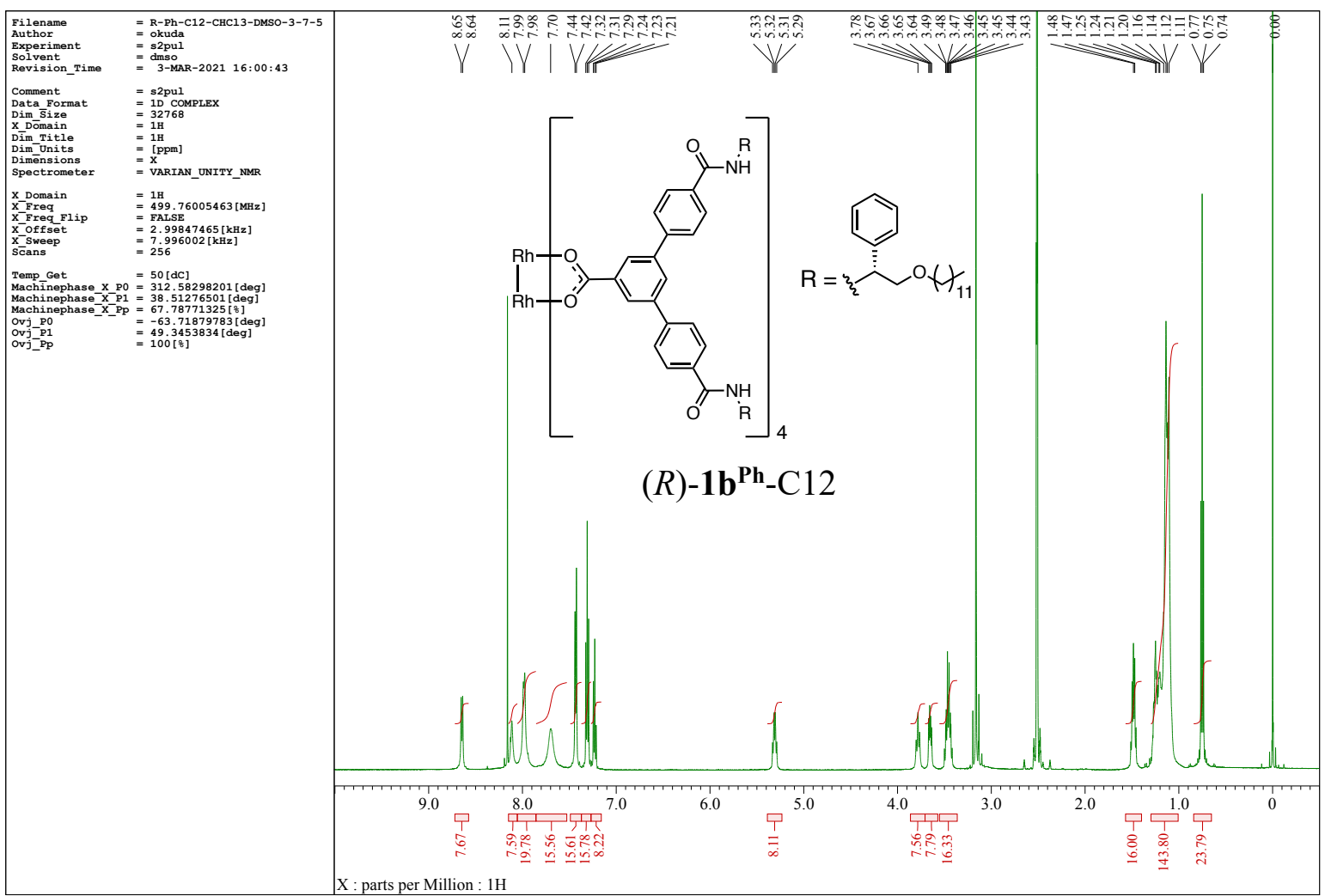

Figure S126. ${ }^{1} \mathrm{H}$ NMR spectrum of $(R)-\mathbf{1 b}^{\mathrm{Ph}}-\mathrm{C} 12$ in $\mathrm{CDCl}_{3} / \mathrm{DMSO}-d_{6}=3 / 7(\mathrm{v} / \mathrm{v})$ at $50{ }^{\circ} \mathrm{C}$.

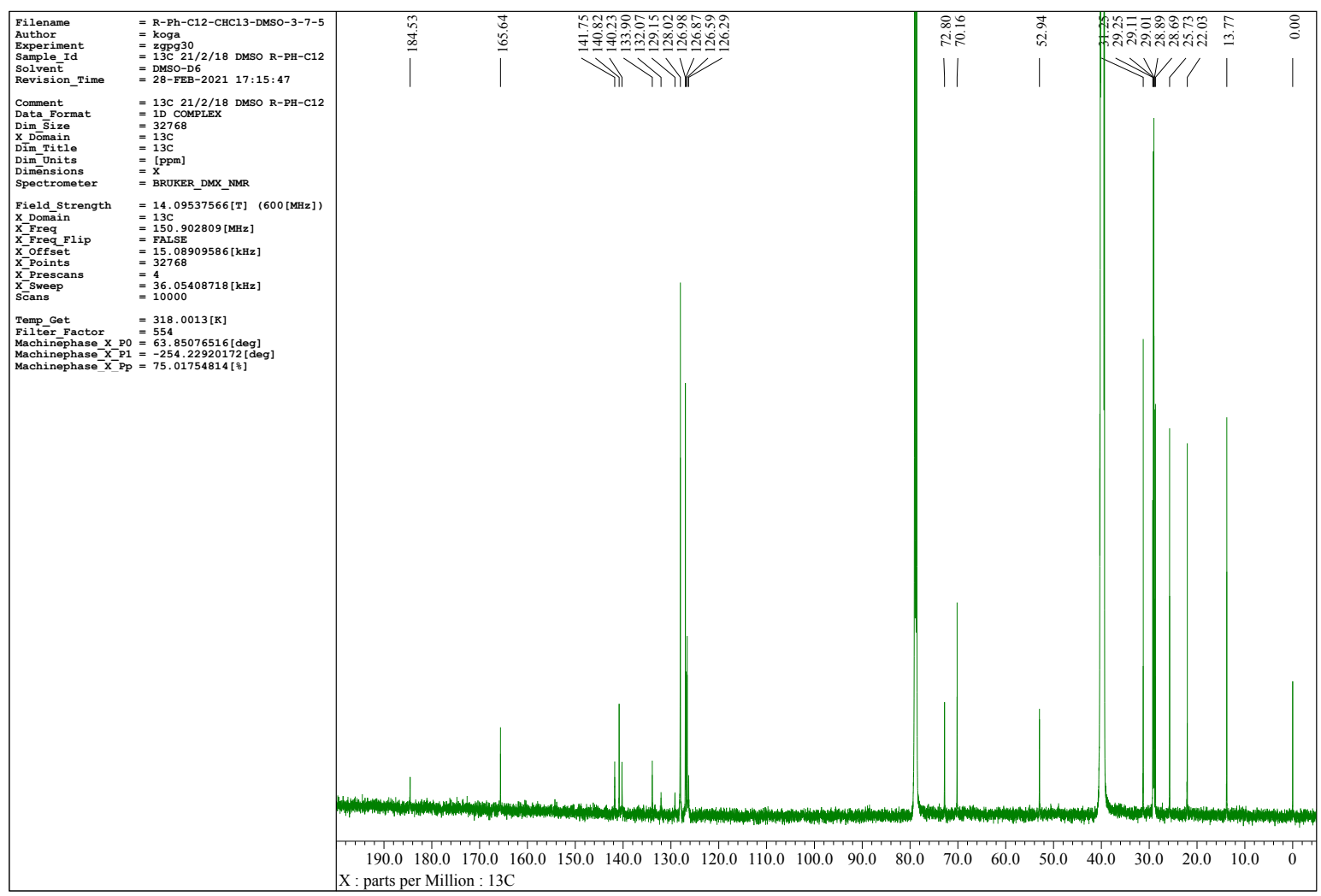

Figure S127. ${ }^{13} \mathrm{C}$ NMR spectrum of $(R)-\mathbf{1 b}{ }^{\mathrm{Ph}}-\mathrm{C} 12$ in $\mathrm{CDCl}_{3} / \mathrm{DMSO}-d_{6}=3 / 7(\mathrm{v} / \mathrm{v})$ at $50{ }^{\circ} \mathrm{C}$. 


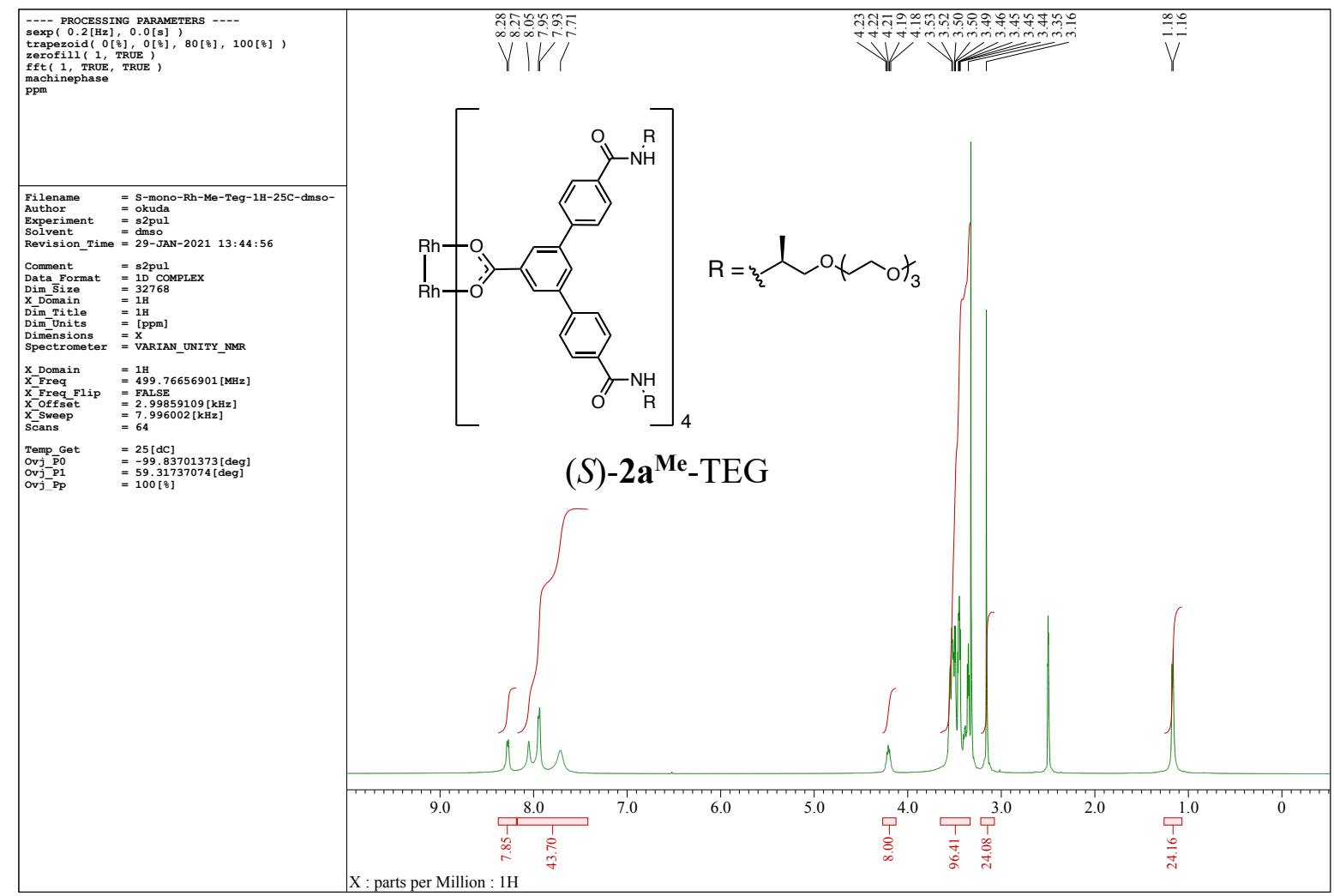

Figure S128. ${ }^{1} \mathrm{H}$ NMR spectrum of $(S)-\mathbf{2} \mathbf{a}^{\mathrm{Me}}-\mathrm{TEG}$ in DMSO- $d_{6}$ at $25^{\circ} \mathrm{C}$.

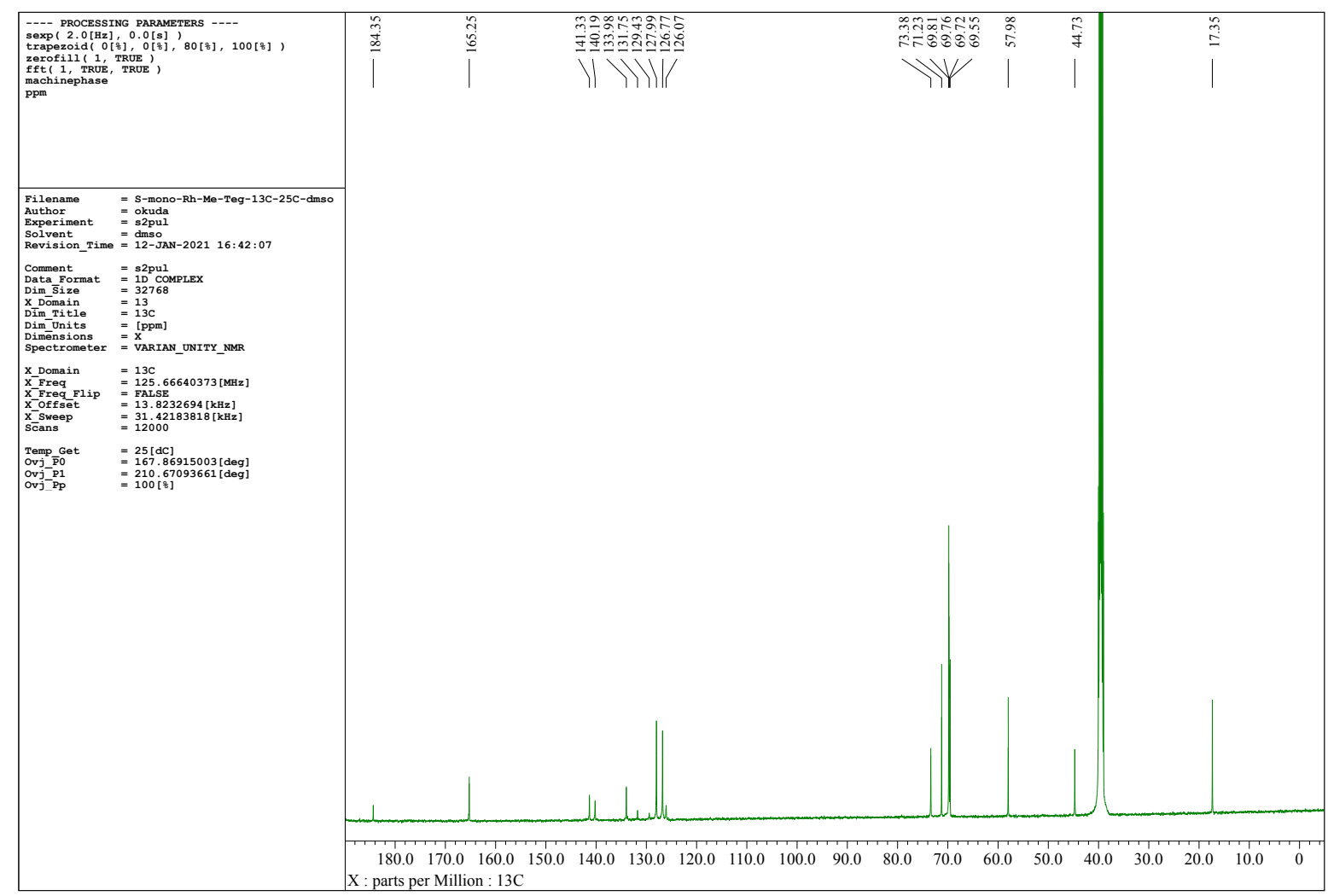

Figure S129. ${ }^{13} \mathrm{C}$ NMR spectrum of $(S)-\mathbf{2 a} \mathbf{a}^{\mathrm{Me}}-\mathrm{TEG}$ in DMSO- $d_{6}$ at $25{ }^{\circ} \mathrm{C}$. 


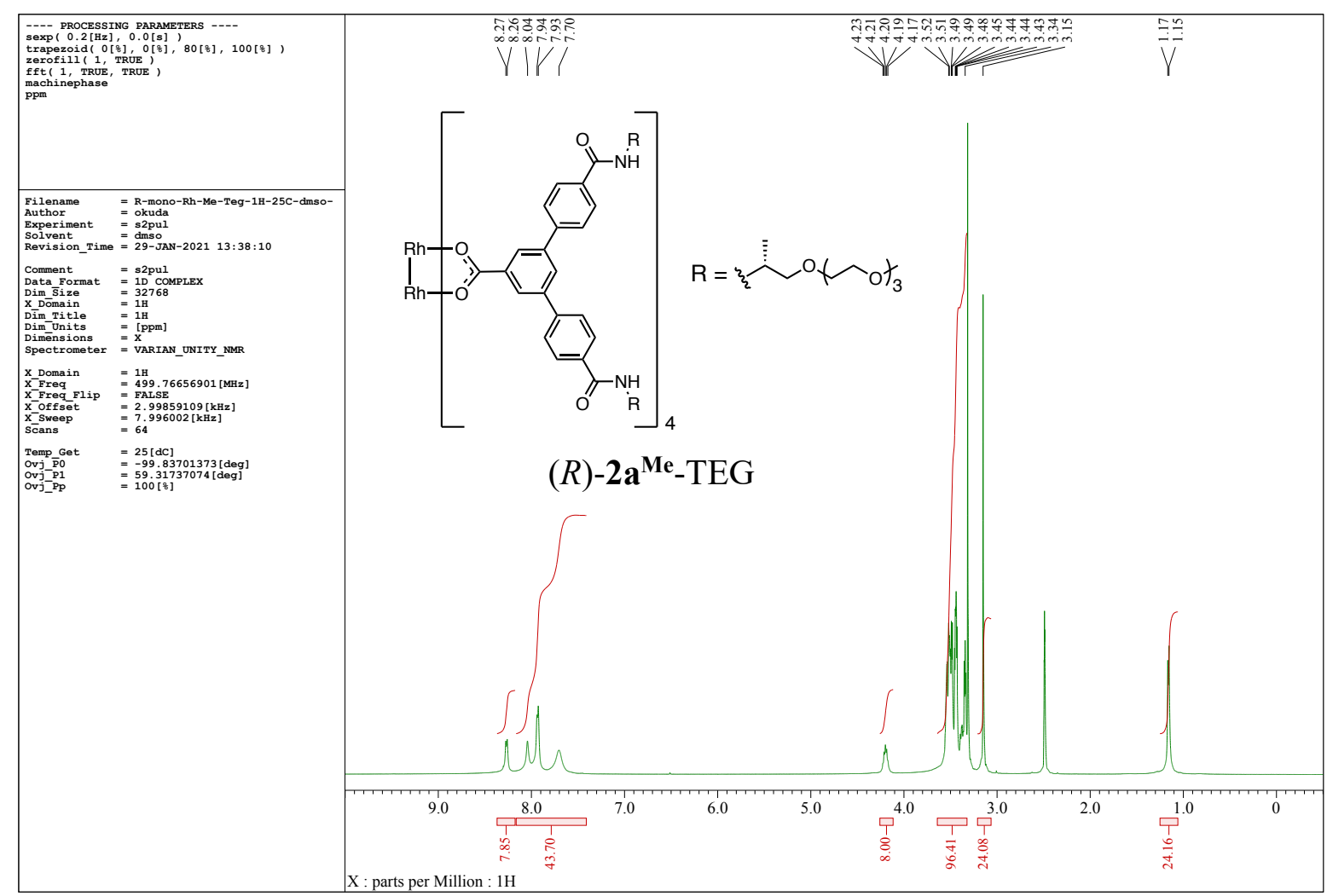

Figure S130. ${ }^{1} \mathrm{H}$ NMR spectrum of $(R)-2 \mathbf{a}^{\mathrm{Me}}-\mathrm{TEG}$ in DMSO- $d_{6}$ at $25^{\circ} \mathrm{C}$.

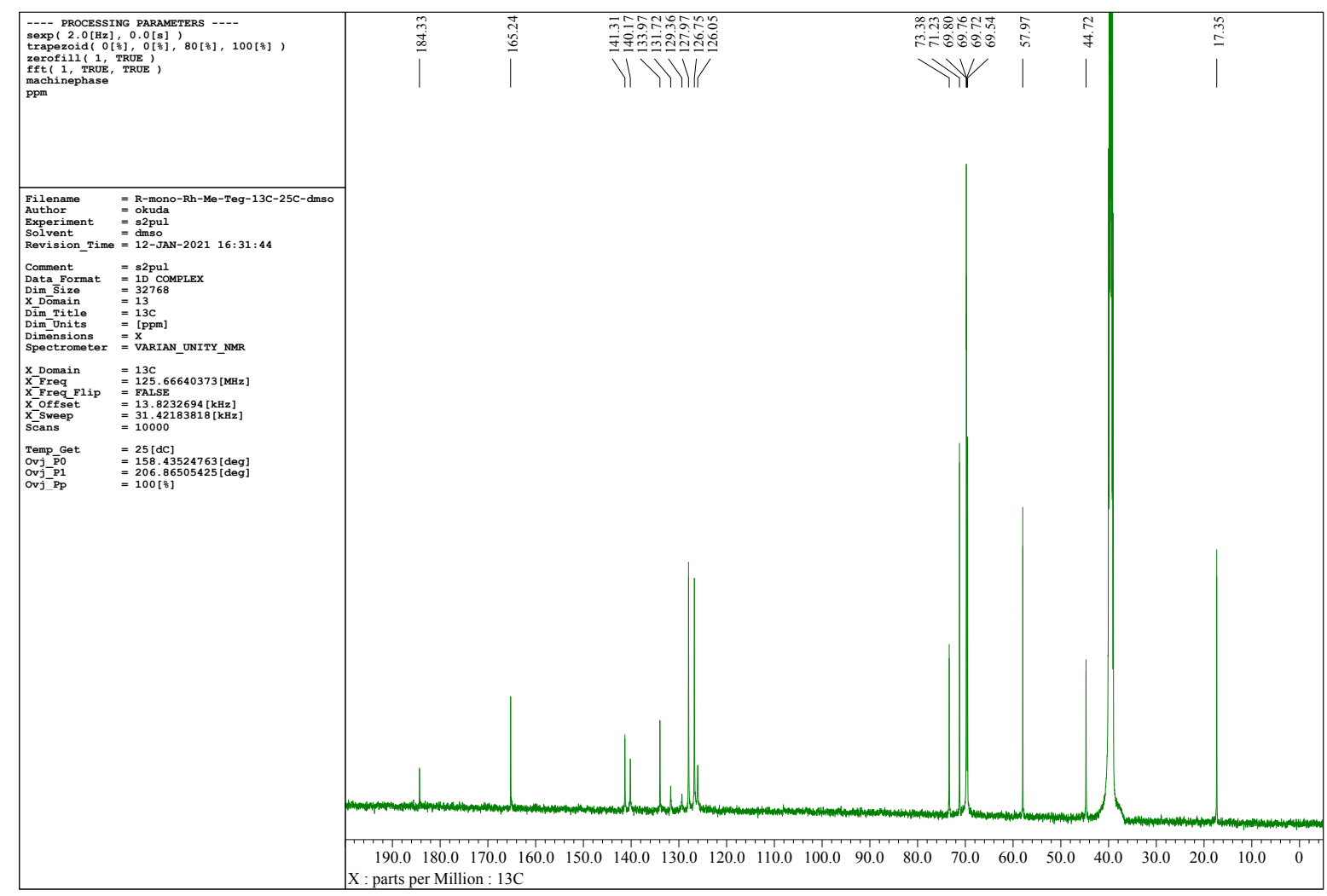

Figure S131. ${ }^{13} \mathrm{C}$ NMR spectrum of $(R)-\mathbf{2} \mathbf{a}^{\mathrm{Me}}-\mathrm{TEG}$ in DMSO- $d_{6}$ at $25{ }^{\circ} \mathrm{C}$. 


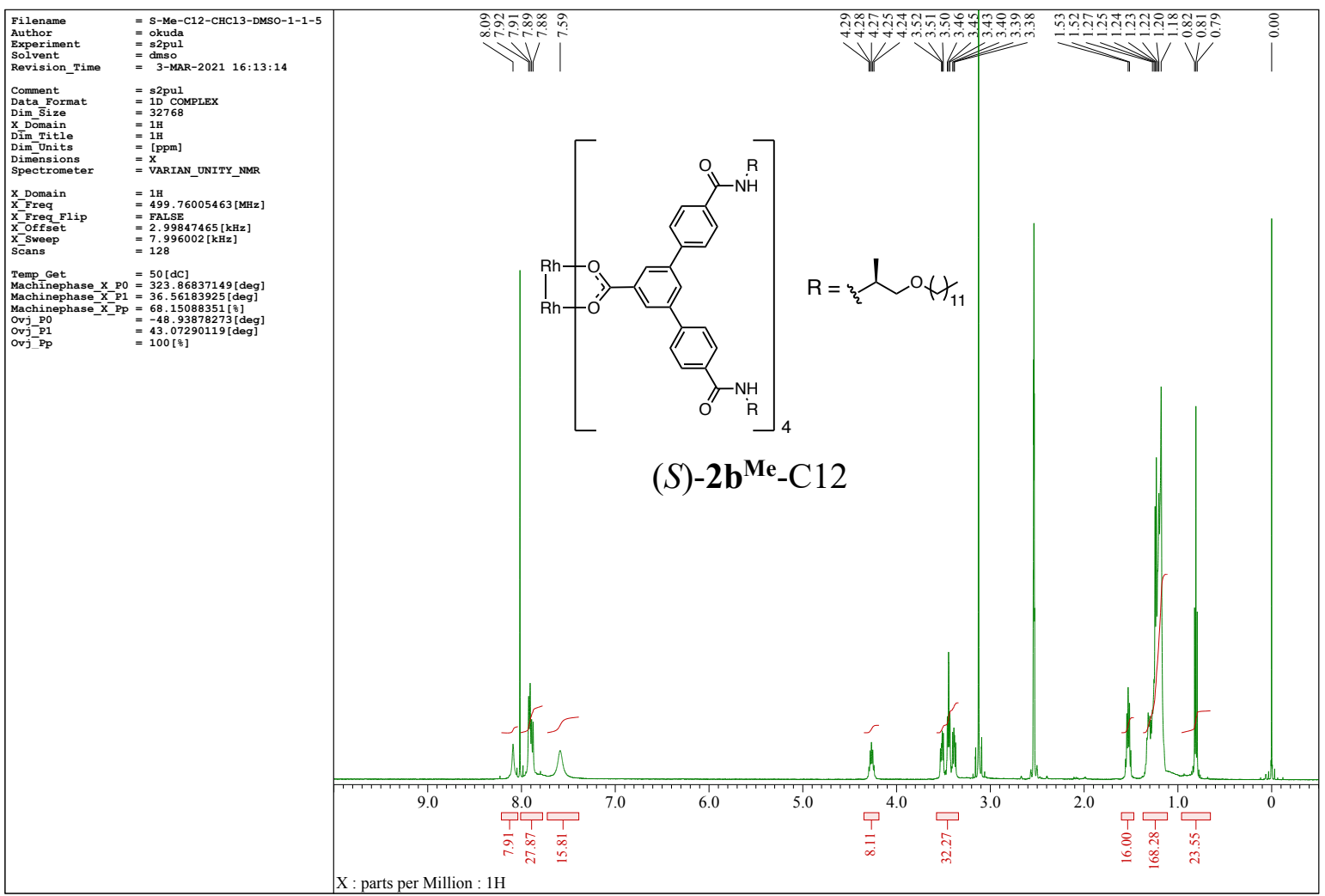

Figure S132. ${ }^{1} \mathrm{H}$ NMR spectrum of $(S)-2 \mathbf{b}^{\mathrm{Me}}-\mathrm{C} 12$ in $\mathrm{CDCl}_{3} / \mathrm{DMSO}-d_{6}=1 / 1(\mathrm{v} / \mathrm{v})$ at $50{ }^{\circ} \mathrm{C}$.

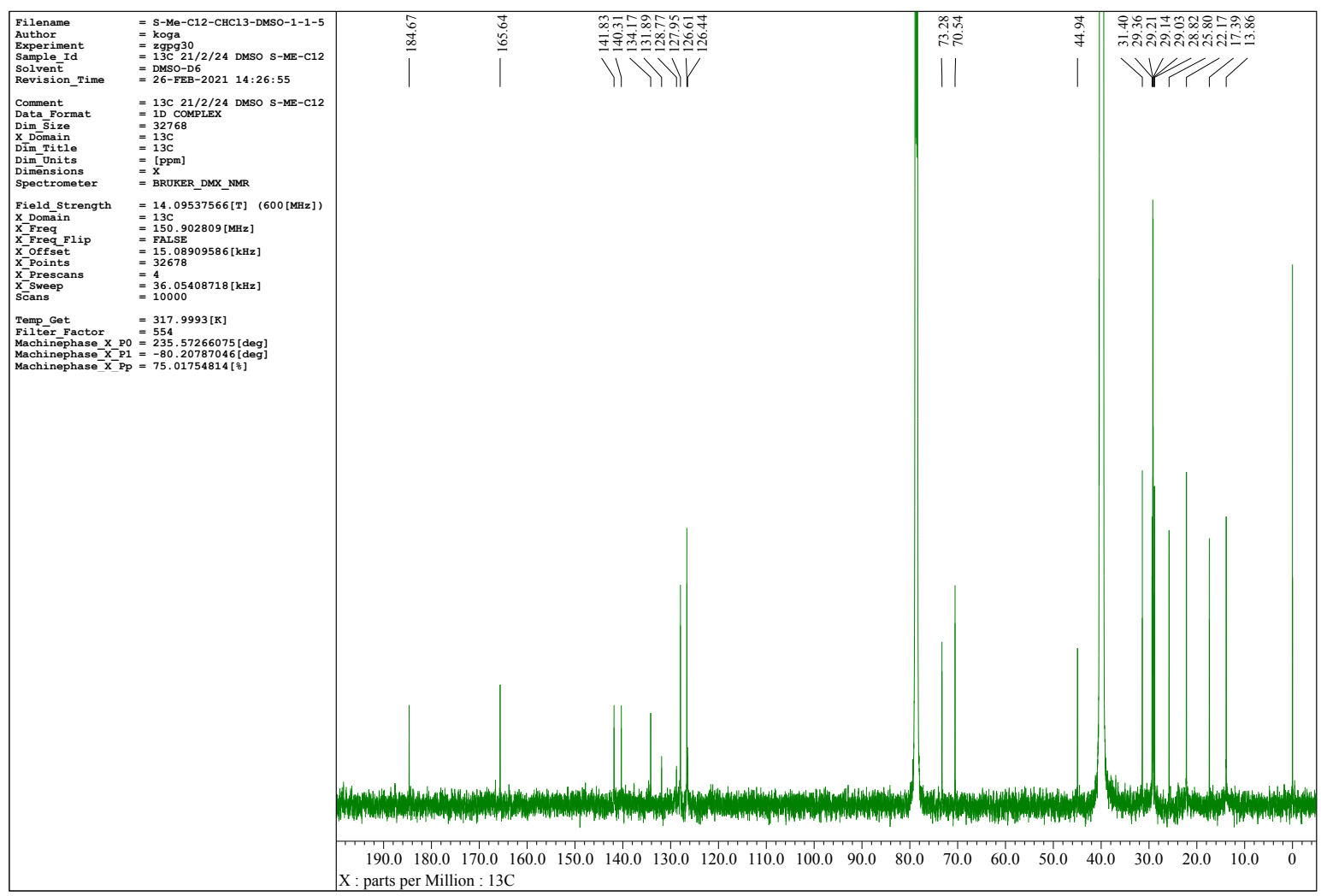

Figure S133. ${ }^{13} \mathrm{C}$ NMR spectrum of $(S)-2 \mathbf{b}^{\mathrm{Me}}-\mathrm{C} 12$ in $\mathrm{CDCl}_{3} / \mathrm{DMSO}-d_{6}=1 / 1(\mathrm{v} / \mathrm{v})$ at $50{ }^{\circ} \mathrm{C}$. 


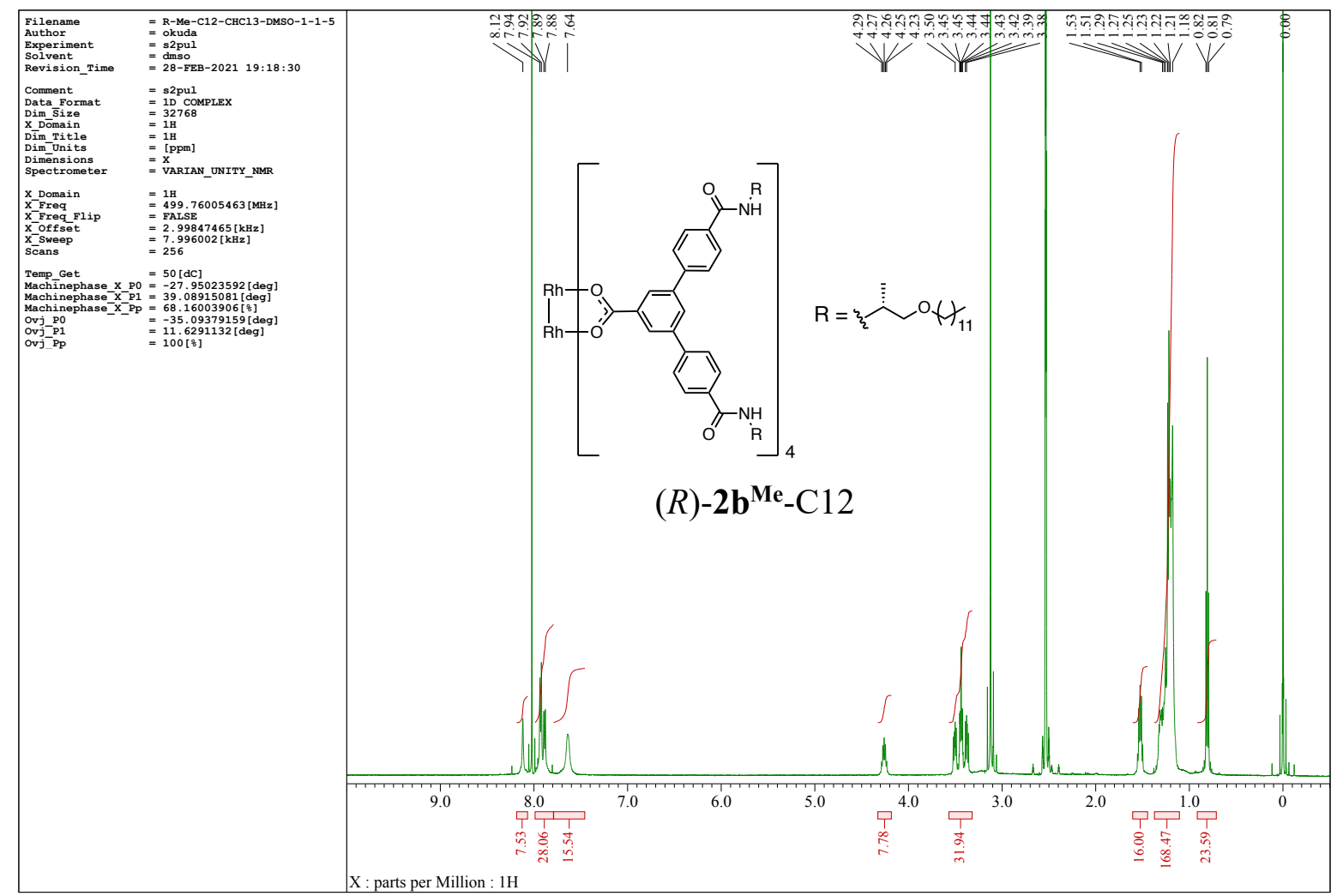

Figure S134. ${ }^{1} \mathrm{H}$ NMR spectrum of $(R)-2 \mathbf{b}^{\mathrm{Me}}-\mathrm{C} 12$ in $\mathrm{CDCl}_{3} / \mathrm{DMSO}-d_{6}=1 / 1(\mathrm{v} / \mathrm{v})$ at $50{ }^{\circ} \mathrm{C}$.

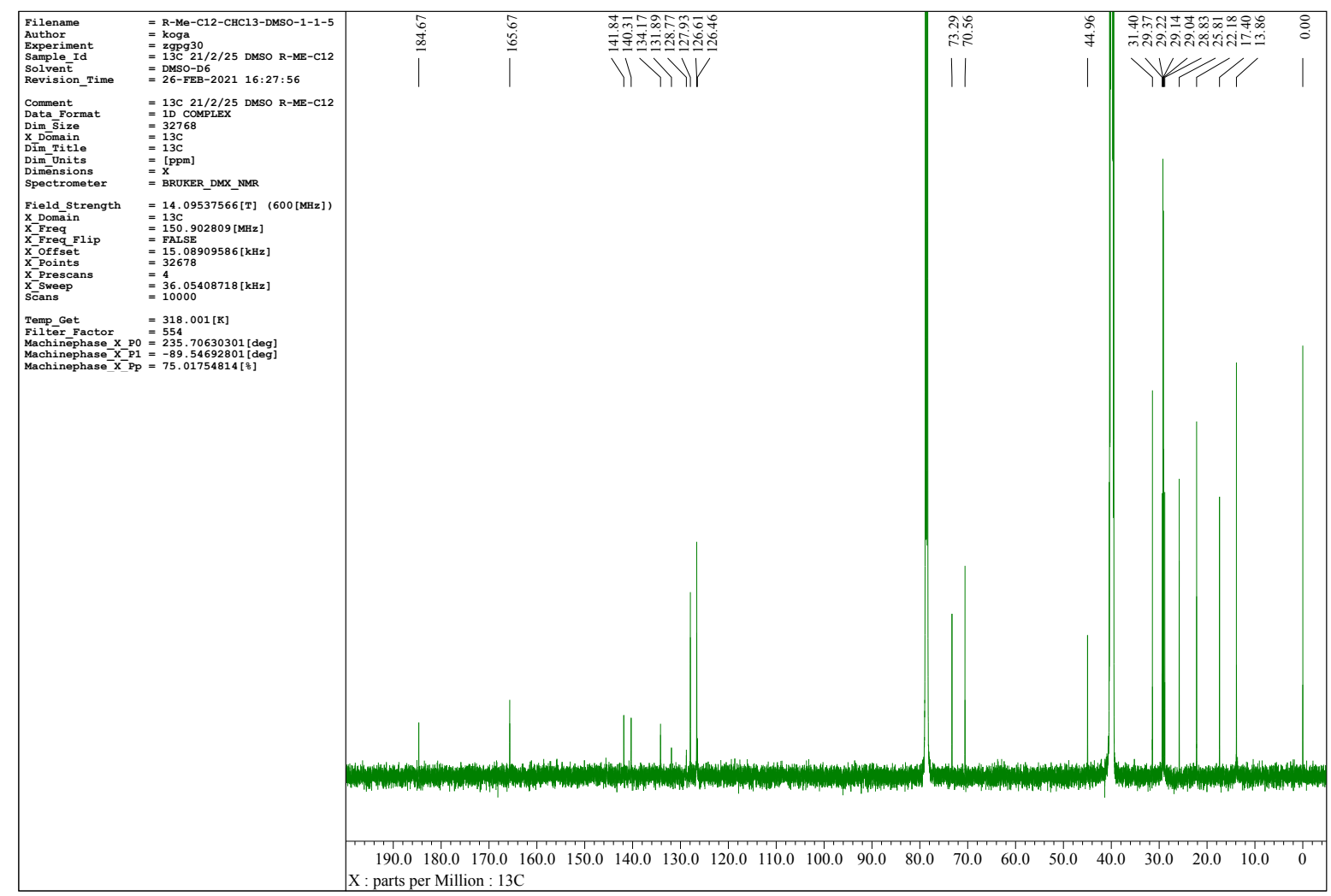

Figure S135. ${ }^{13} \mathrm{C}$ NMR spectrum of $(R)-2 \mathbf{b}^{\mathrm{Me}}-\mathrm{C} 12$ in $\mathrm{CDCl}_{3} / \mathrm{DMSO}-d_{6}=1 / 1(\mathrm{v} / \mathrm{v})$ at $50{ }^{\circ} \mathrm{C}$. 


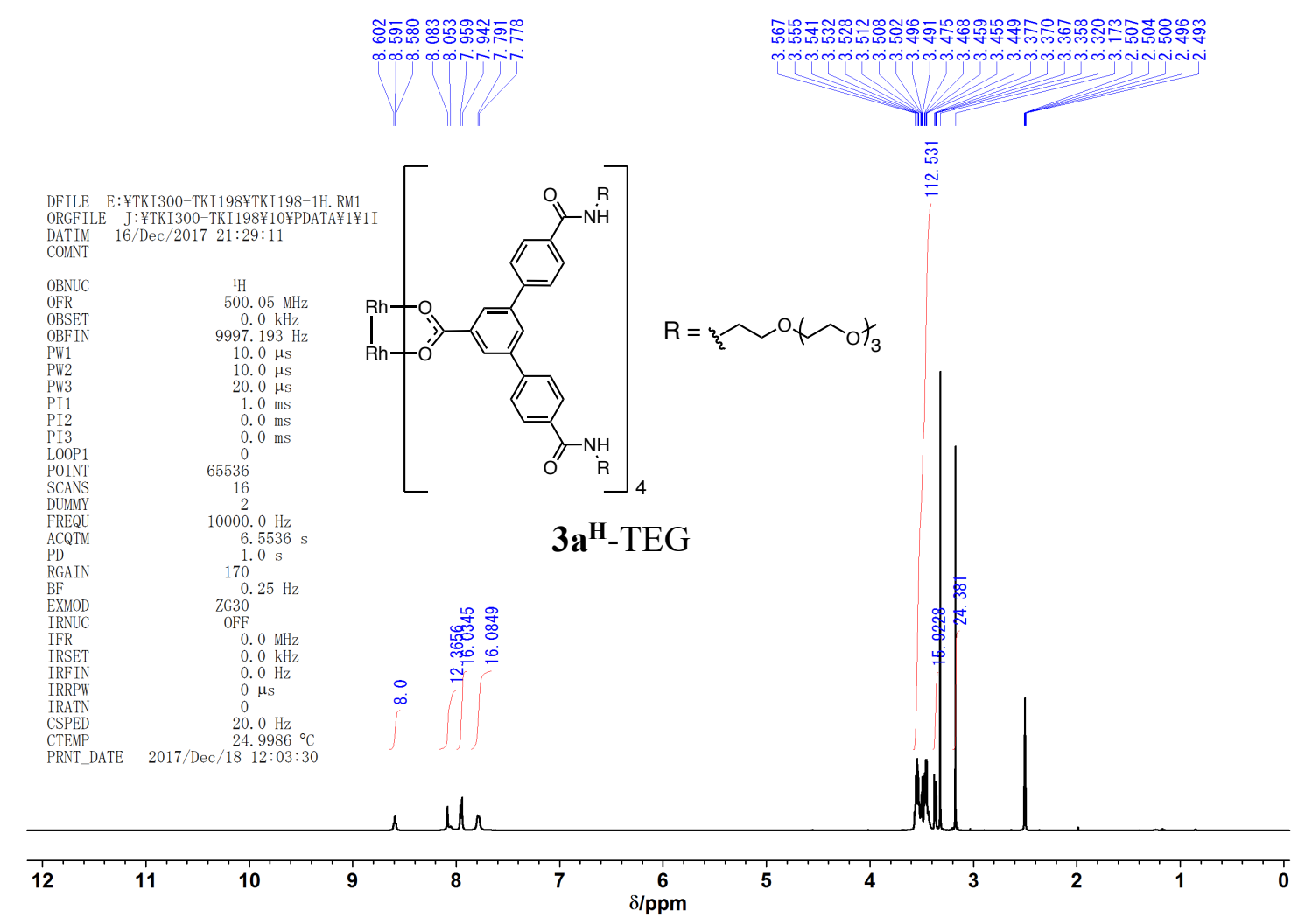

Figure S136. ${ }^{1} \mathrm{H}$ NMR spectrum of $\mathbf{3 a}^{\mathbf{H}}-\mathrm{TEG}$ in DMSO- $d_{6}$ at $25^{\circ} \mathrm{C}$.

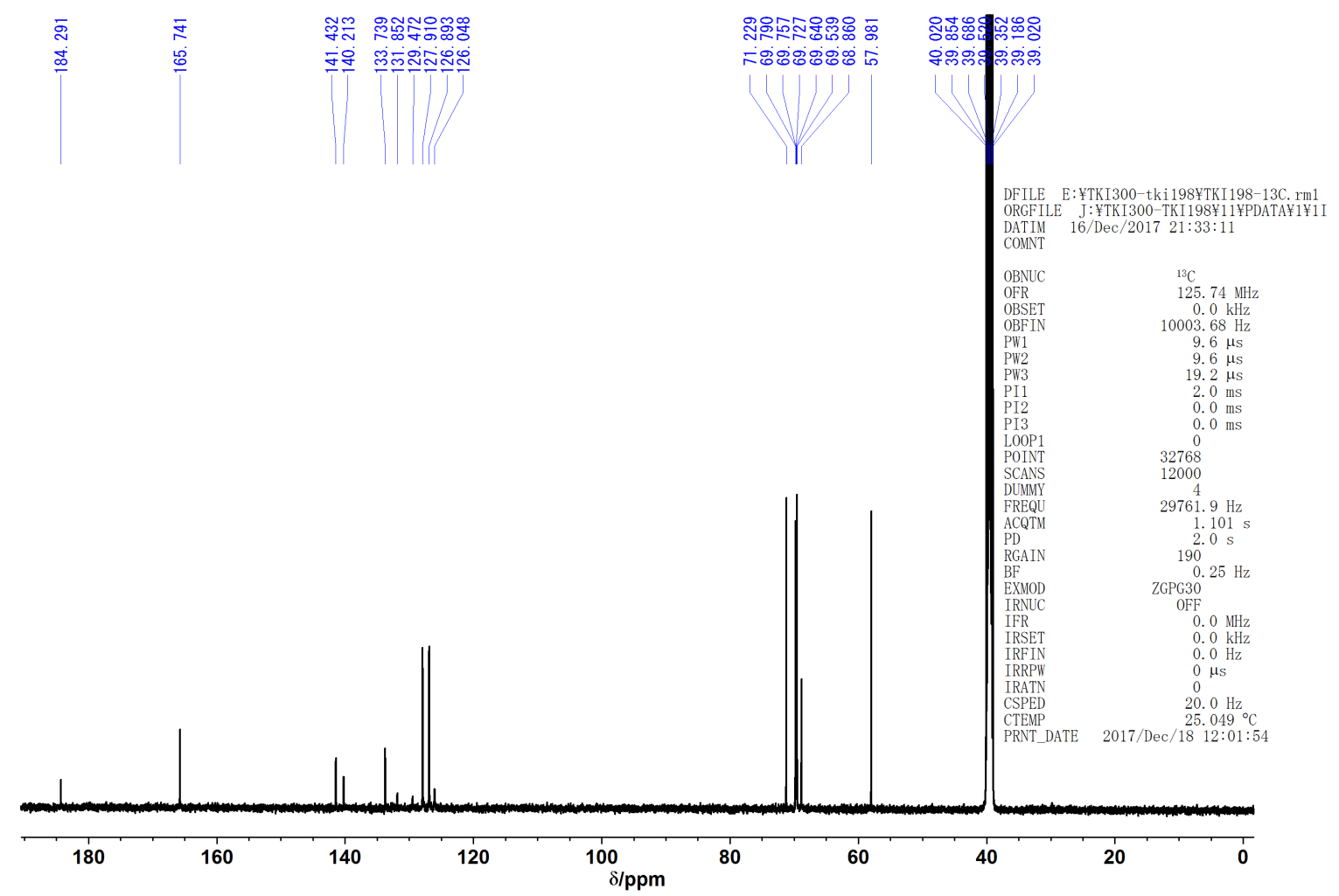

Figure S137. ${ }^{13} \mathrm{C}$ NMR spectrum of $\mathbf{3} \mathbf{a}^{\mathrm{H}}-\mathrm{TEG}$ in DMSO- $d_{6}$ at $25^{\circ} \mathrm{C}$. 


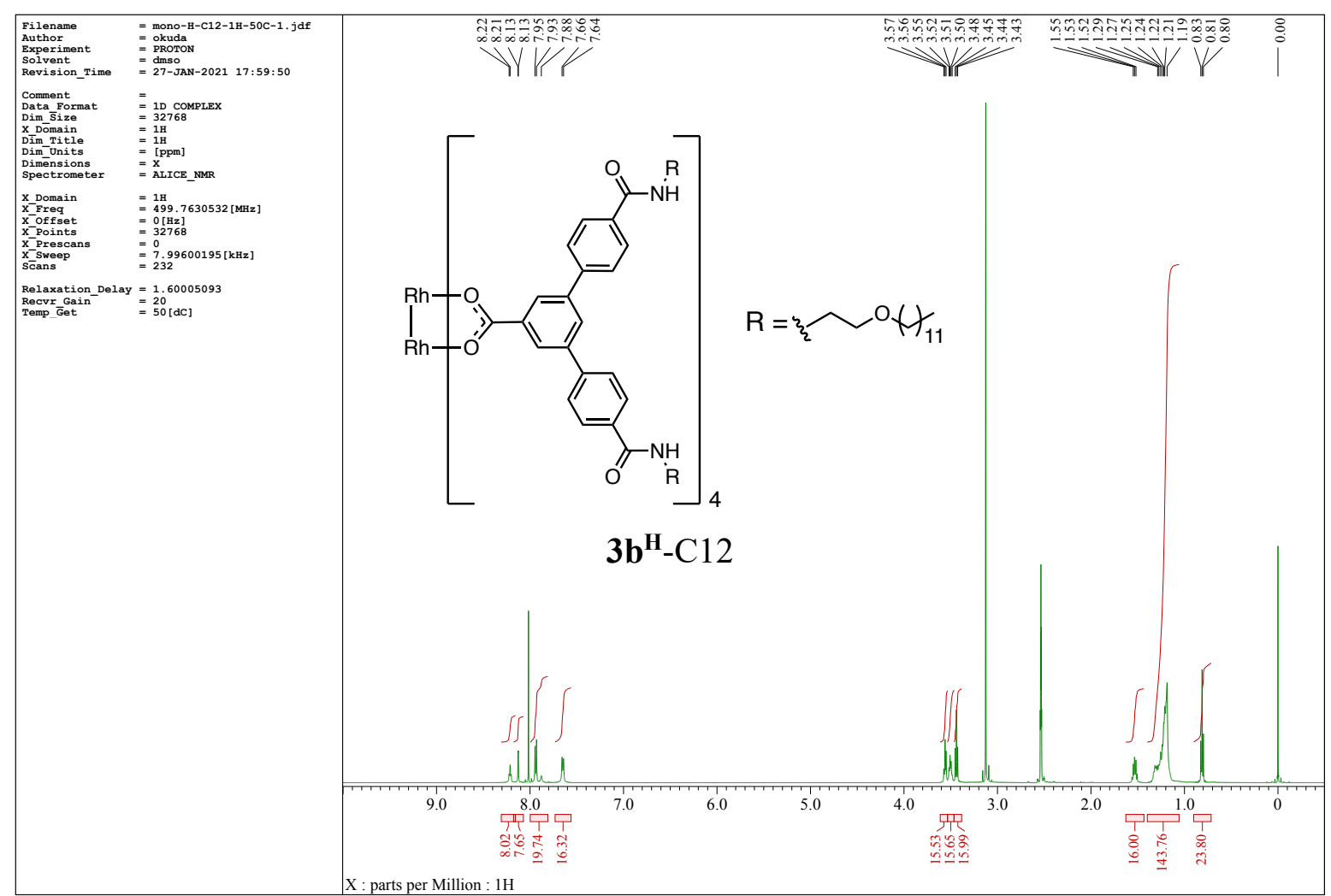

Figure S138. ${ }^{1} \mathrm{H}$ NMR spectrum of $\mathbf{3} \mathbf{b}^{\mathbf{H}}-\mathrm{C} 12$ in $\mathrm{CDCl}_{3} / \mathrm{DMSO}-d_{6}=1 / 1(\mathrm{v} / \mathrm{v})$ at $50{ }^{\circ} \mathrm{C}$.

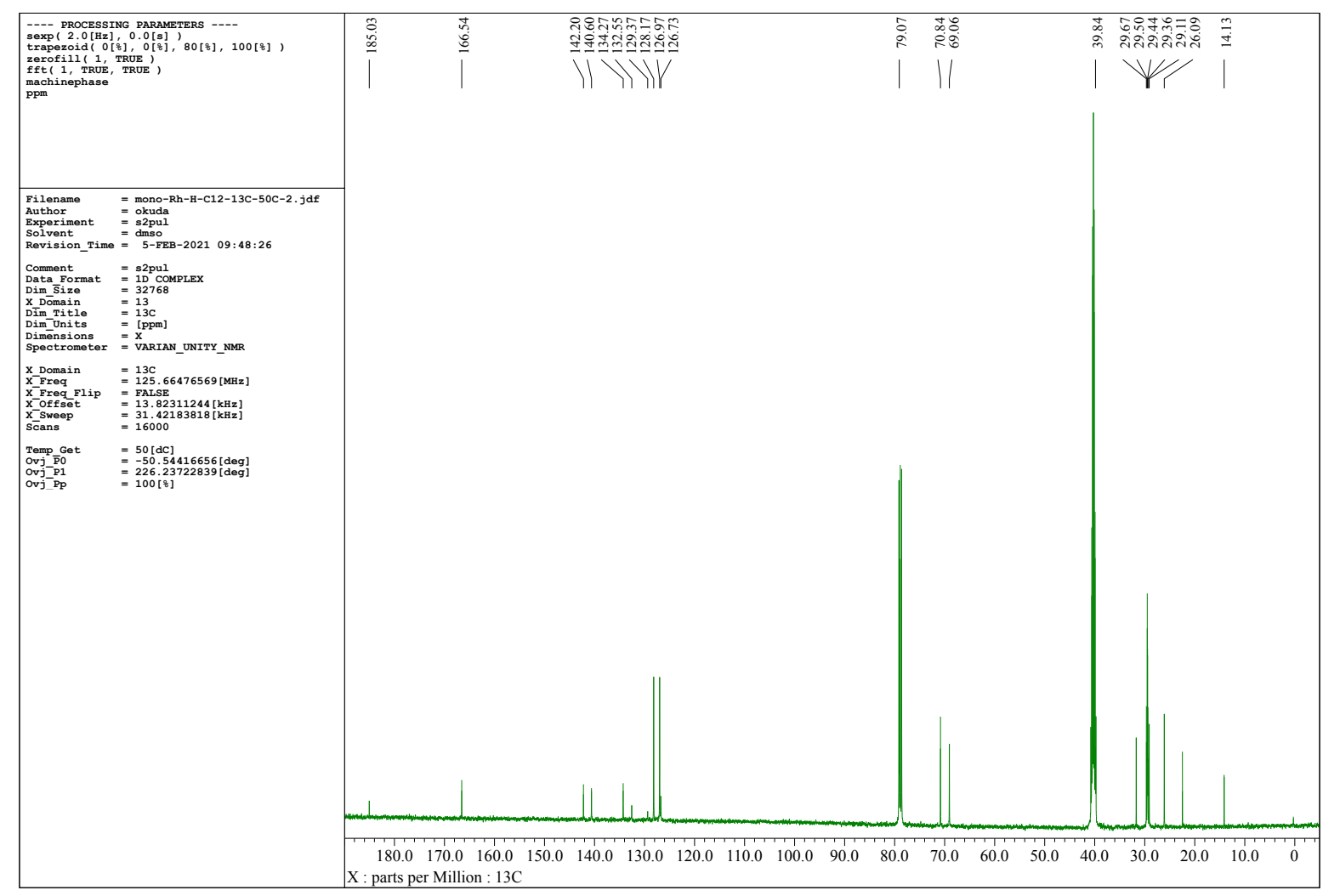

Figure S139. ${ }^{13} \mathrm{C}$ NMR spectrum of $3 \mathbf{b}^{\mathrm{H}}-\mathrm{C} 12$ in $\mathrm{CDCl}_{3} / \mathrm{DMSO}-d_{6}=1 / 1(\mathrm{v} / \mathrm{v})$ at $50{ }^{\circ} \mathrm{C}$. 


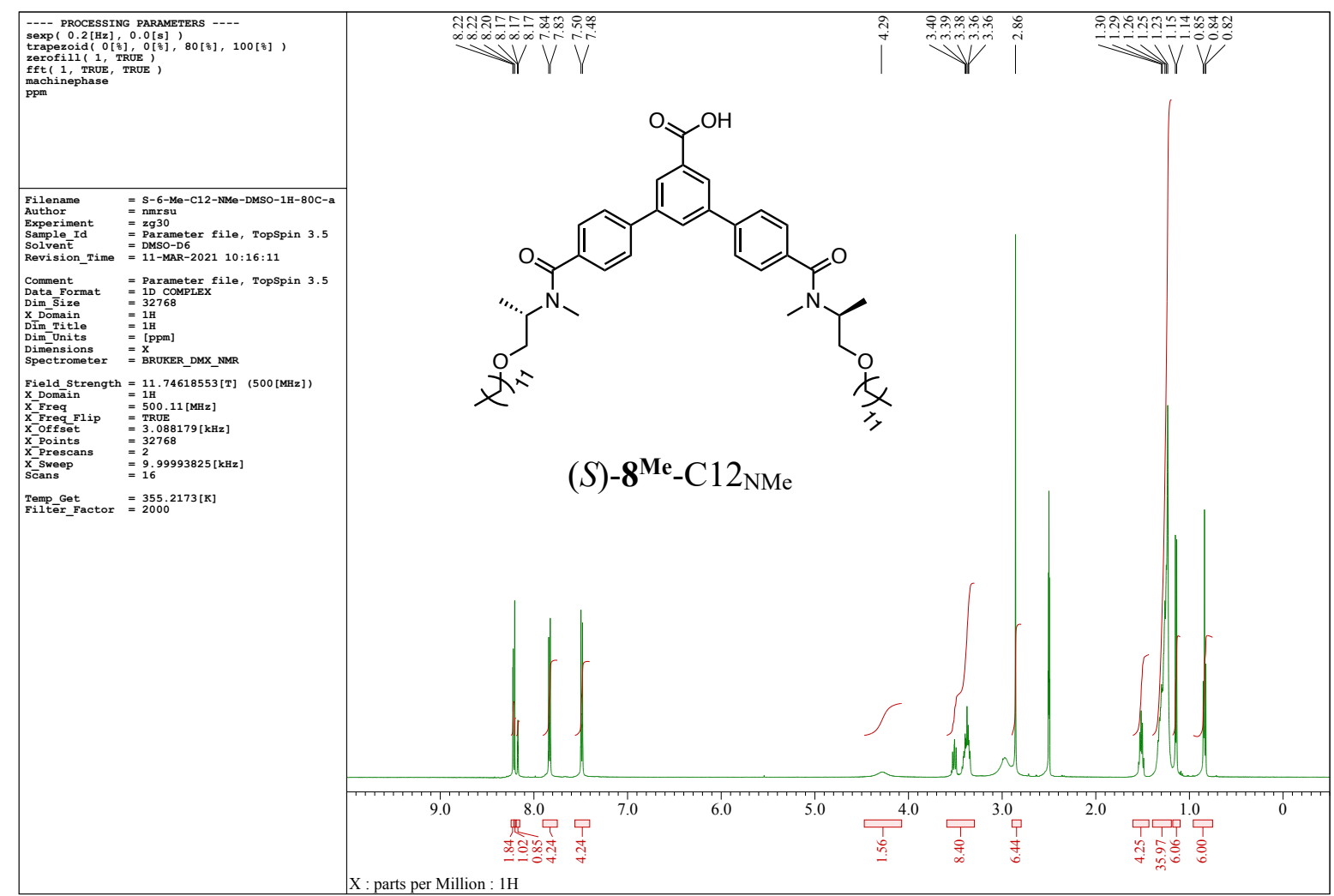

Figure S140. ${ }^{1} \mathrm{H}$ NMR spectrum of $(S)-8^{\mathrm{Me}}-\mathrm{C} 12_{\mathrm{NMe}}$ in DMSO- $d_{6}$ at $80{ }^{\circ} \mathrm{C}$.

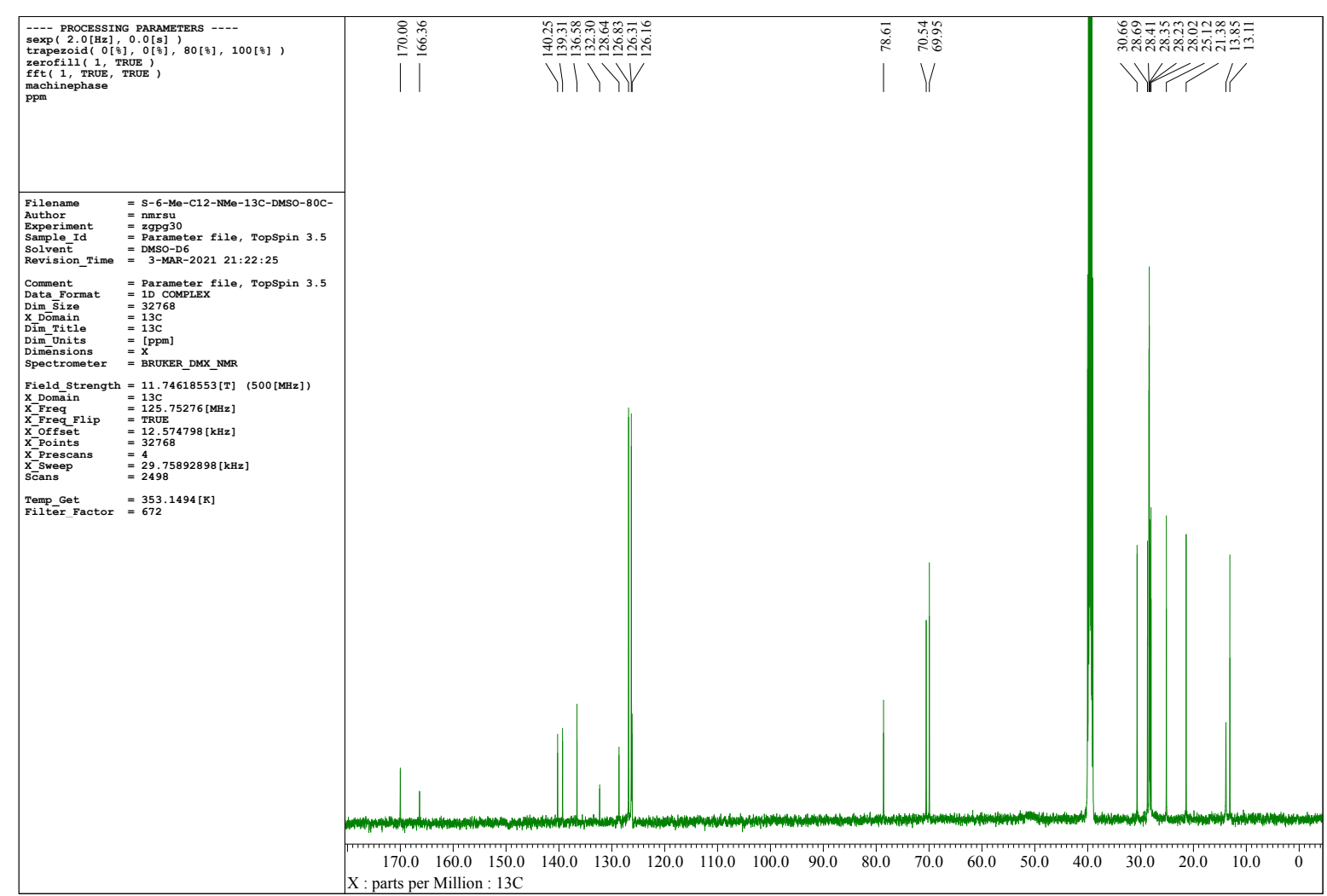

Figure S141. ${ }^{13} \mathrm{C}$ NMR spectrum of $(S)-8^{\mathrm{Me}}-\mathrm{C} 12_{\mathrm{NMe}}$ in DMSO- $d_{6}$ at $80{ }^{\circ} \mathrm{C}$. 


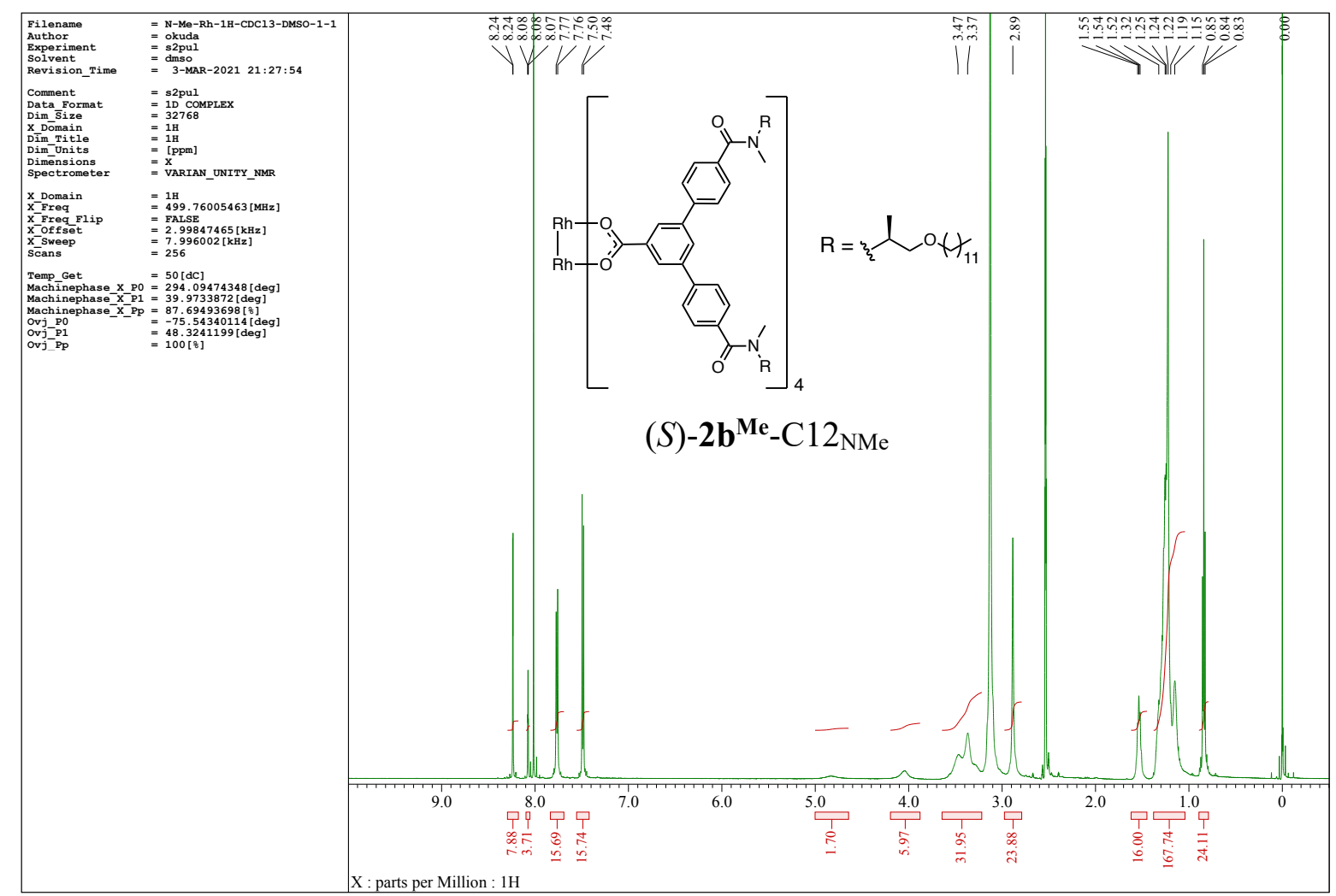

Figure S142. ${ }^{1} \mathrm{H}$ NMR spectrum of $(S)-2 \mathbf{b}^{\mathrm{Me}}-\mathrm{C} 12_{\mathrm{NMe}}$ in $\mathrm{CDCl}_{3} / \mathrm{DMSO}-d_{6}=1 / 1(\mathrm{v} / \mathrm{v})$ at $50{ }^{\circ} \mathrm{C}$

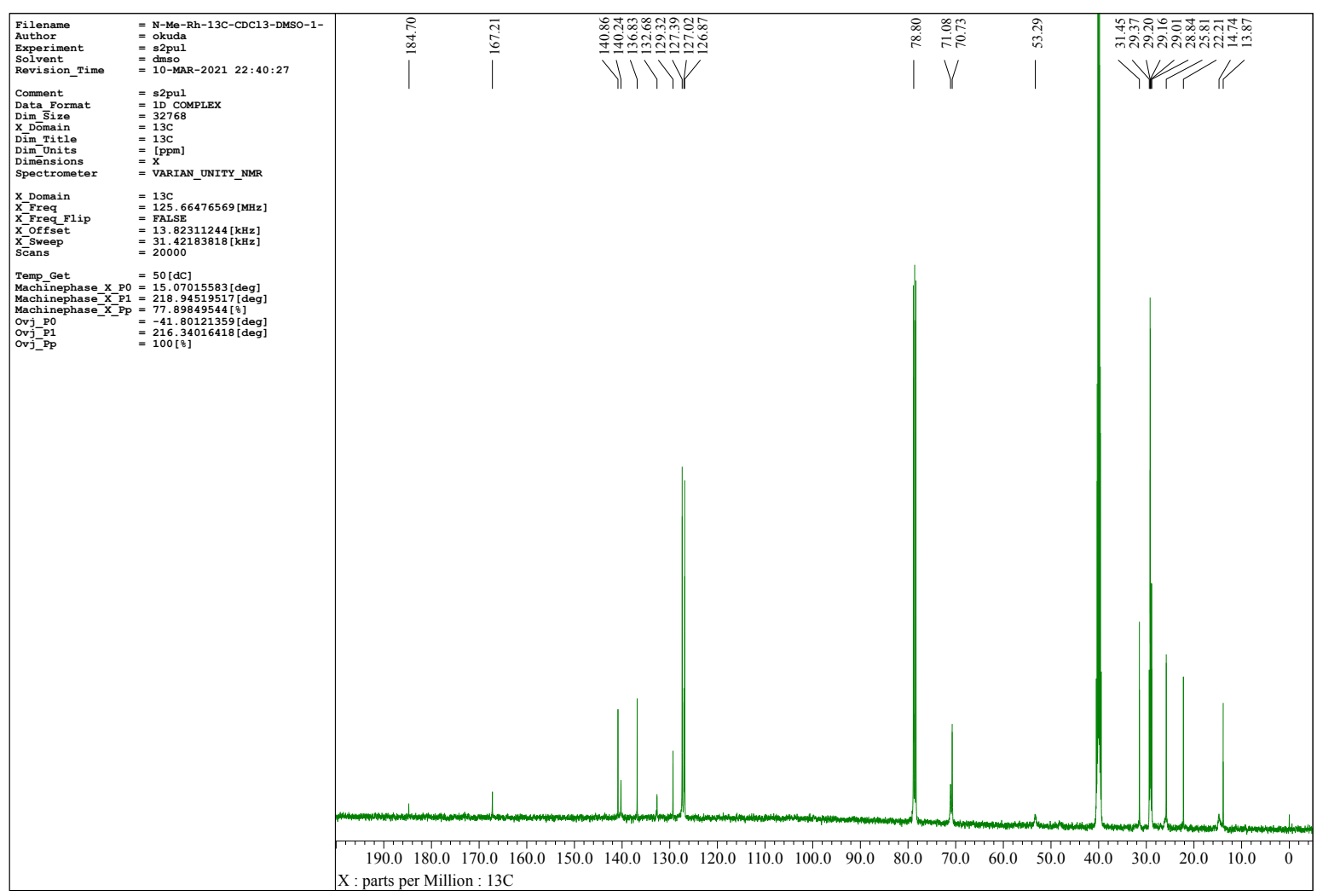

Figure S143. ${ }^{13} \mathrm{C}$ NMR spectrum of $(S)-2 \mathbf{b}^{\mathrm{Me}}-\mathrm{C} 12_{\mathrm{NMe}}$ in $\mathrm{CDCl}_{3} / \mathrm{DMSO}-d_{6}=1 / 1(\mathrm{v} / \mathrm{v})$ at $50{ }^{\circ} \mathrm{C}$. 